\title{
Labeling preferences of diazirines with protein biomolecules
}

\author{
Alexander V. West, Giovanni Muncipinto, Hung-Yi Wu, Andrew C. Huang, Matthew T. \\ Labenski, Lyn H. Jones, Christina M. Woo
}

Journal of the American Chemical Society

Supporting Information

Index

Supplementary Figures $\quad$ Page

Figure S1. ${ }^{1} \mathrm{H}$ NMR yields and representative products from NMR S3 assignment for the alkyl diazirine 3 .

Figure S2. ${ }^{19} \mathrm{~F}$ NMR spectra for neat reactions between the aryl $\quad$ S4

diazirine 2 and protected amino acids.

Figure S3. Representative ${ }^{1} \mathrm{H}$ NMR of aqueous reactions between $\quad$ S5

diazirine 3 and protected amino acids.

Figure S4. Reaction with aryl diazirine 2 and $\beta$-mercaptoethanol in S6 aqueous conditions.

Figure S5. ${ }^{1} \mathrm{H}$ NMR yields of aqueous photolysis of the alkyl diazirine 3 S7 and its tetra-deuterated analog S5.

Figure S6. Full gels of single protein labeling of BSA with diazirine S8 probes $\mathbf{3}$ and $\mathbf{5}$ in Tris buffer.

Figure S7. Single protein labeling of BSA by the alkyl diazirine 3 in $\quad$ S9

acidic phosphate buffer at varying concentrations.

Figure S8. PAL probe structures and their corresponding dosing S10

groups to SK-N-SH cells.

Figure S9. Photoaffinity labeling of 12 PAL probes in HEK293T cells S11

visualized by Azide-Fluor 488 and in-gel fluorescence.

Figure S10. Photoaffinity labeling of 12 PAL probes in HEK293T S12

lysates visualized by Azide-Fluor 488 and in-gel fluorescence.

Figure S11. Representative binding site spectra of PAL probes with S13

Vimentin and Cathepsin B.

Figure S12. Meta-analysis of proteomics data.

S14

Figure S13. Single protein and amino acid labeling by JN942 and $\quad$ S15

JN935.

\section{Supplementary Methods}

General Synthetic Procedures $\quad$ S16

Chemical Materials $\quad$ S16

Biological Materials $\quad$ S16

Instrumentation $\quad$ S16

$\begin{array}{ll}\text { Software } & \text { S17 }\end{array}$

Aqueous Photoreactions with Diazirine Probes $\quad$ S18

Experimental Procedures with Individual Amino Acids $\quad \mathrm{S} 18$

Experimental Procedures with Purified Proteins $\quad$ S20

Experimental Procedures with Whole Cells $\quad$ S21

Mass Spectrometry Procedures $\quad$ S24

Synthetic Procedures $\quad$ S26

West, A. et al. Labeling preferences of diazirines with protein biomolecules, J. Am. Chem. S1 Soc. 2021. 
Catalog of nuclear magnetic resonance and infrared spectra for

synthetic compounds

Catalog of nuclear magnetic resonance and liquid chromatograms for

analytical reactions

S107

Catalog of binding site spectra

S147

References

S205

Quantitative and binding site proteomics data are available in Supplementary Tables 1-5:

Table S1: Quantitative proteomics for JN26 v JN1

Table S2: Quantitative proteomics for JN33 v JN1

Table S3: Quantitative proteomics for JN938 v JN1

Table S4: Quantitative proteomics for JN939 v JN1

Table S5: Binding stie data for all 32 PAL probes

West, A. et al. Labeling preferences of diazirines with protein biomolecules, J. Am. Chem. 


\section{SI Figures:}

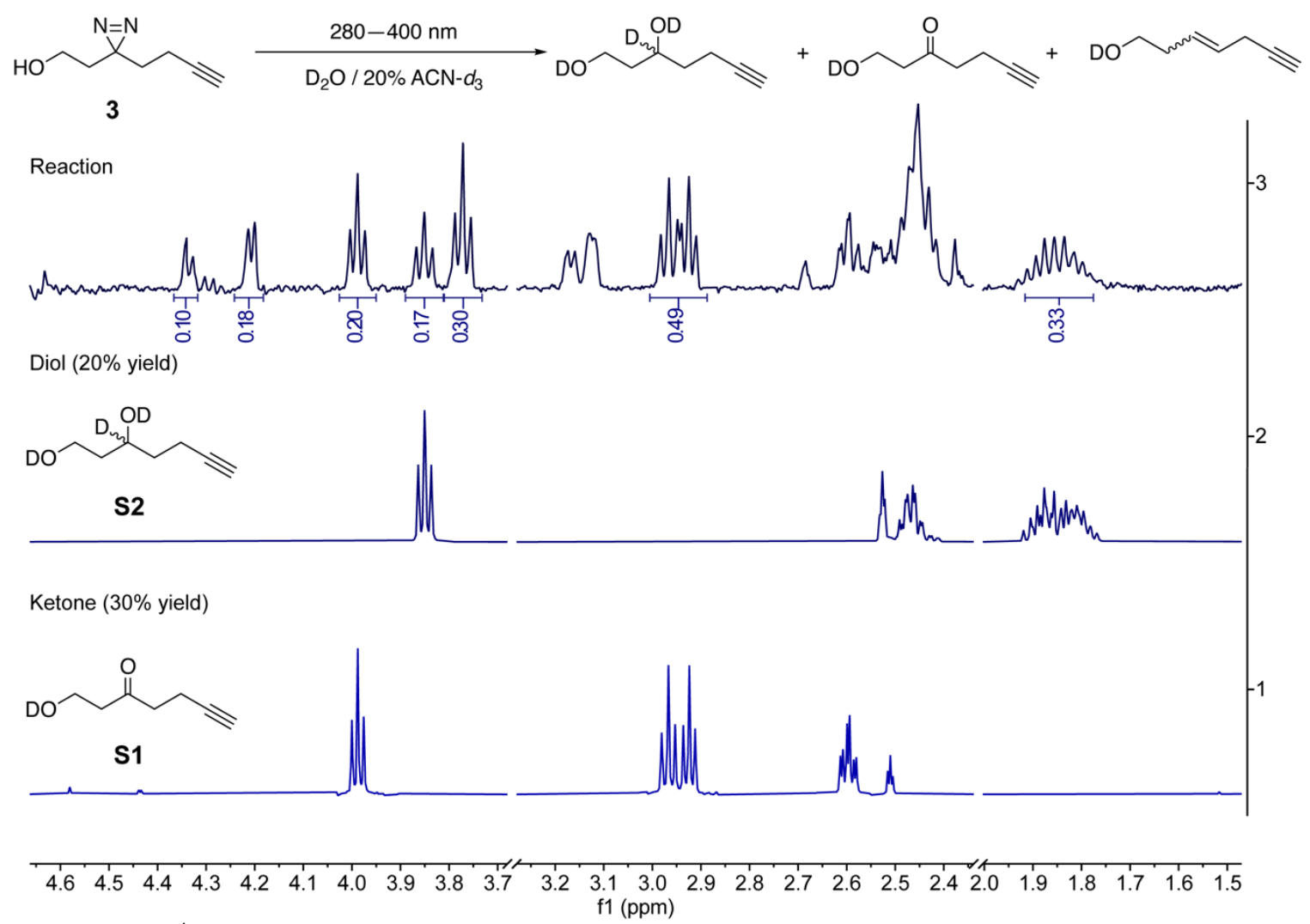

Figure S1. ${ }^{1} \mathrm{H}$ NMR yields and representative products from NMR assignment for the alkyl diazirine 3 . 

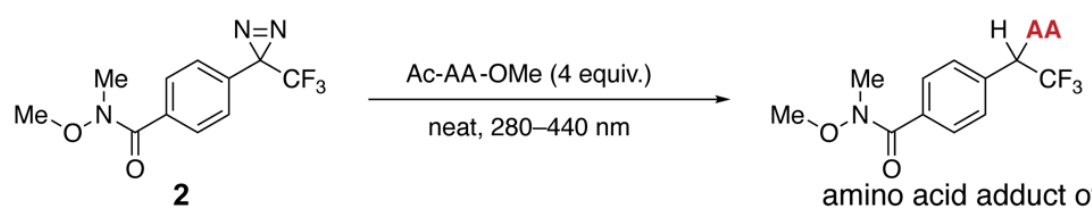

amino acid adduct of 2

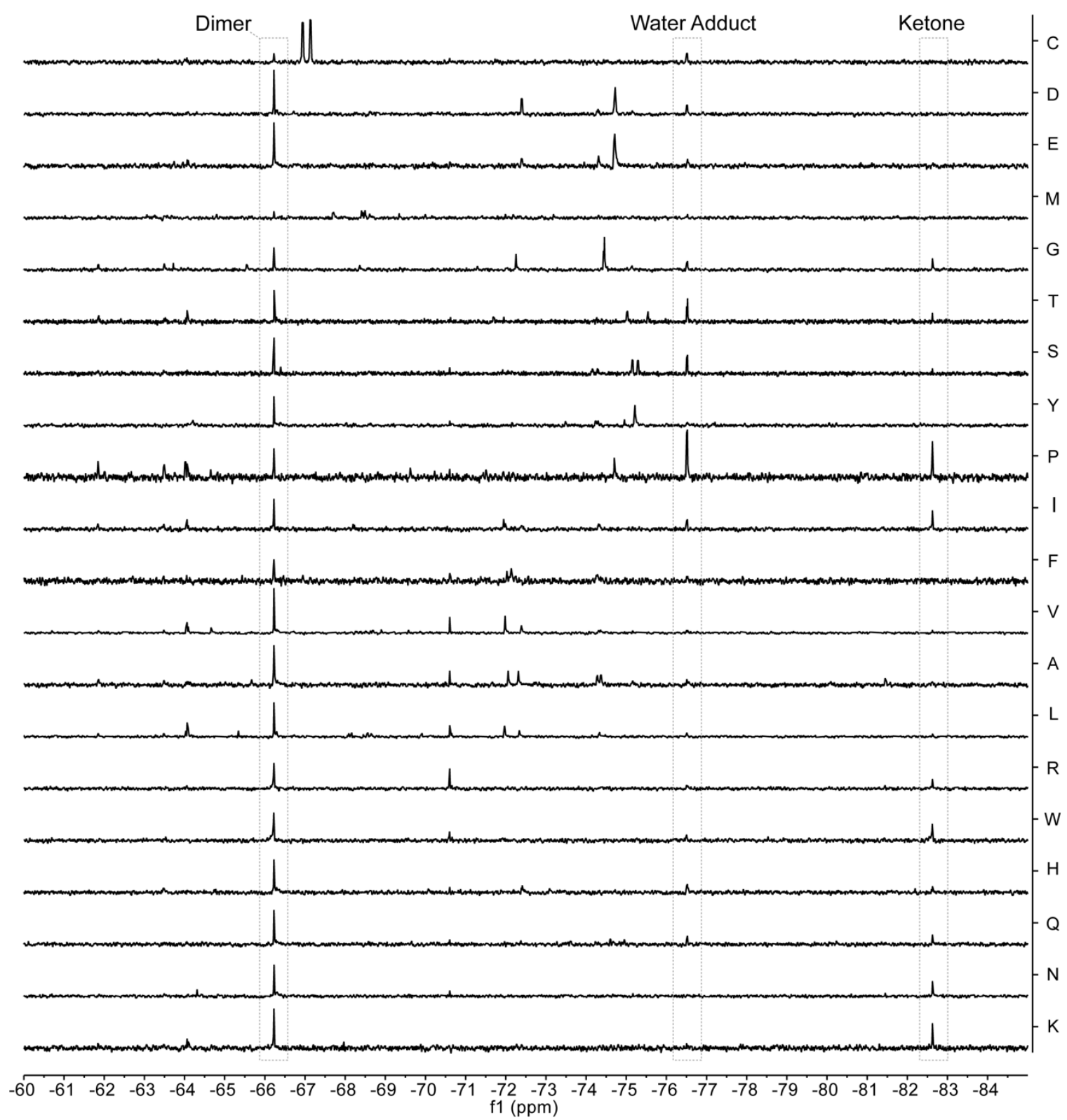

Figure S2. ${ }^{19} \mathrm{~F}$ NMR spectra for neat reactions between the aryl-trifluorodiazirine 2 and the 20 protected amino acids. Common products are highlighted in the grey boxes. The integrals of the unique peaks were used to estimate the yield of amino acid adducts. 

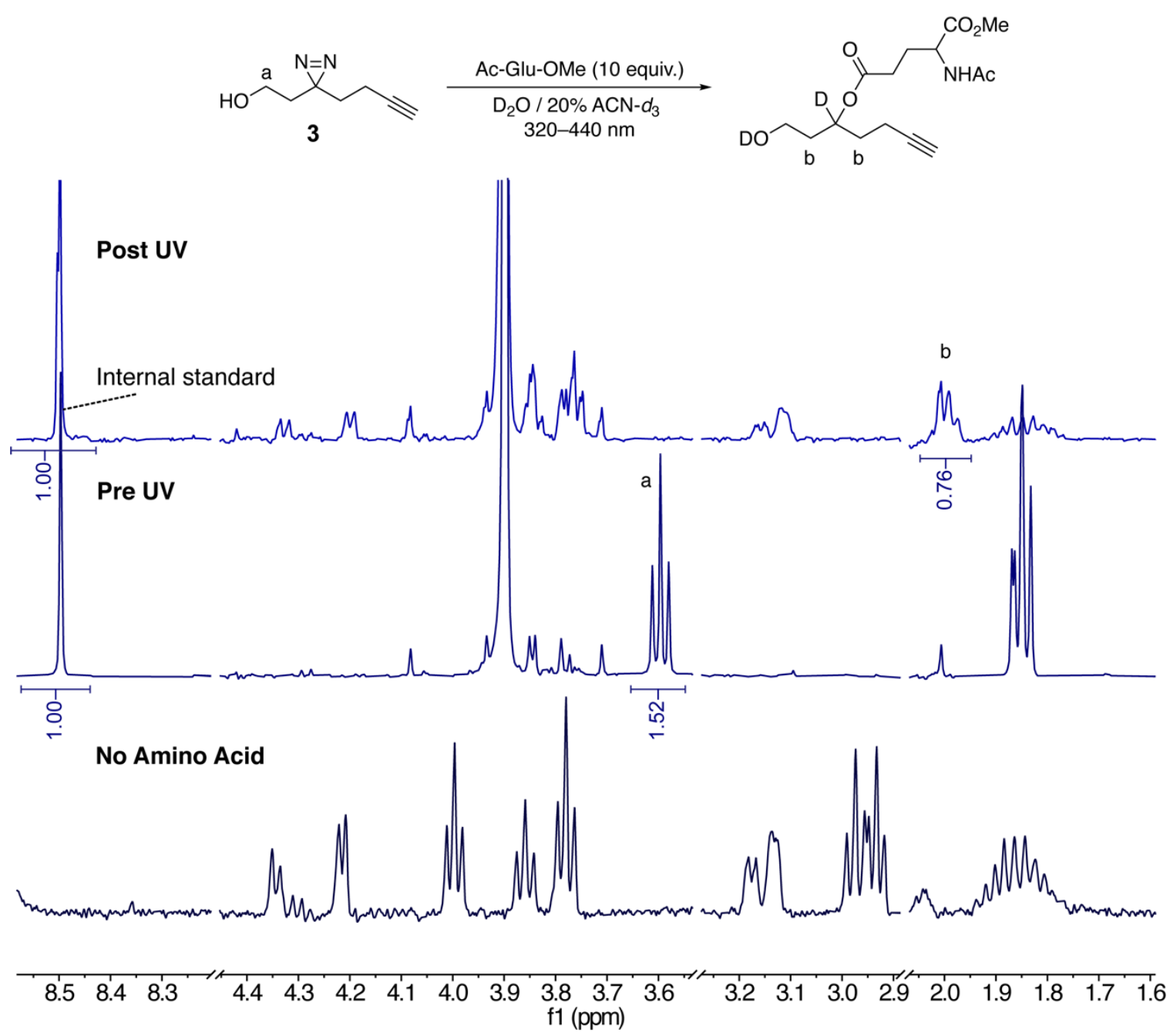

Figure S3. Representative ${ }^{1} \mathrm{H}$ NMR of aqueous reactions between the alkyl diazirine $\mathbf{3}$ and protected amino acids. Putative products are shown along with the integrated proton signals used to estimate their yields. Product formation was usually observed along with the loss of ketone formation. 

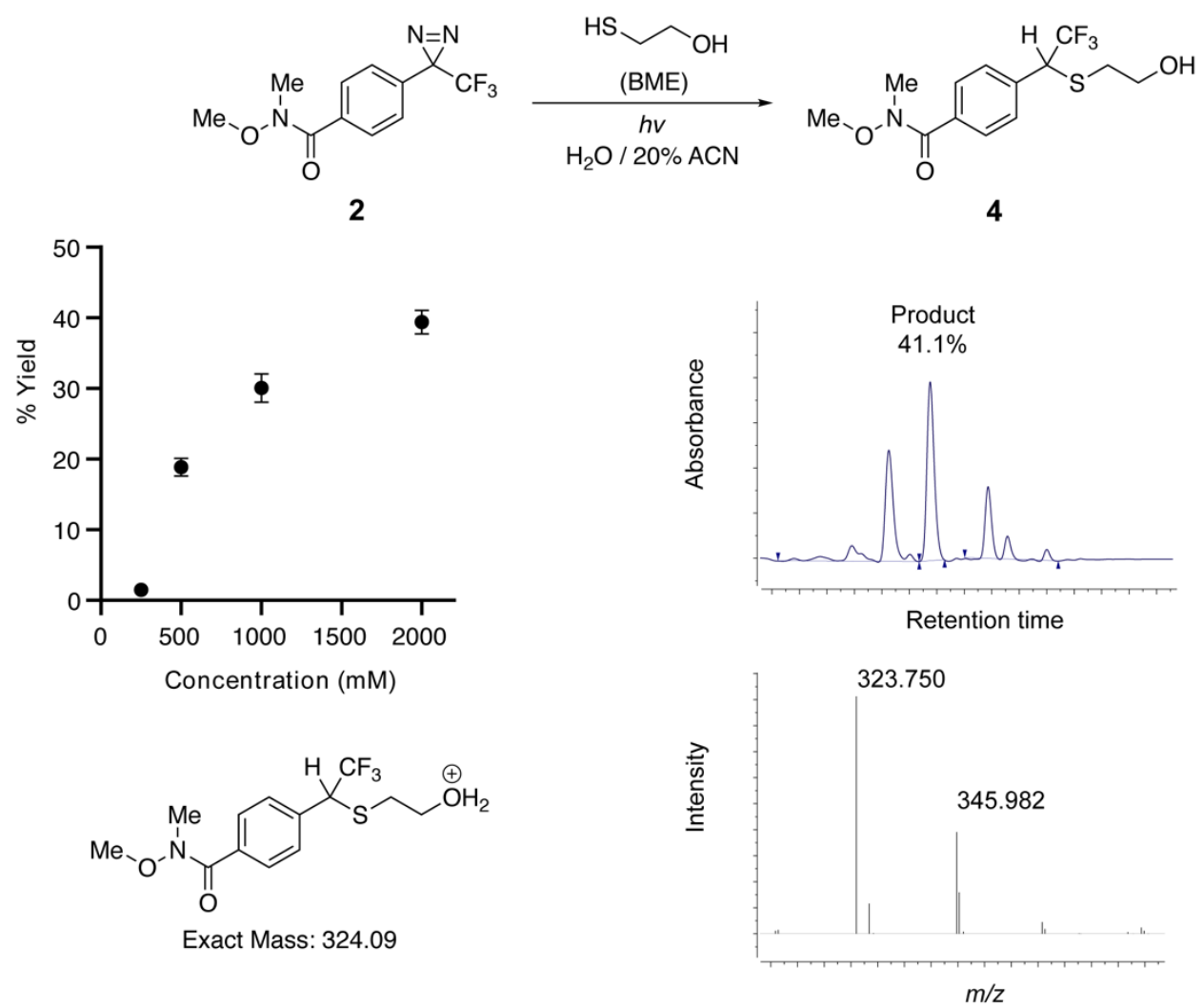

Figure S4. Reaction with aryl diazirine 2 and $\beta$-mercaptoethanol (BME) in aqueous conditions. LC-MS spectra from reaction with $2 \mathrm{M} \mathrm{BME}$ are shown. Detectible product formation begins at $250 \mathrm{mM}$ concentration of BME. Yields were calculated using UV-Vis peak integration of the BME adduct peak relative to other products.

West, A. et al. Labeling preferences of diazirines with protein biomolecules, J. Am. Chem. S6 Soc. 2021. 


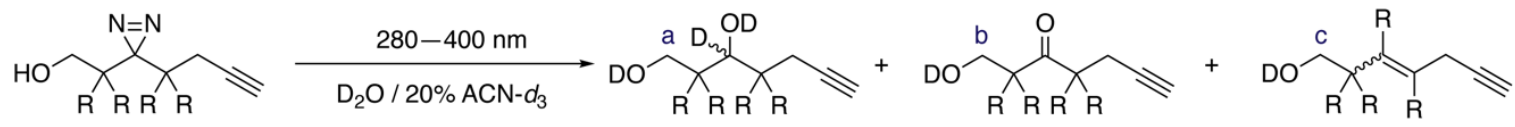

3: $\mathrm{R}=\mathrm{H}$

S3: $R=D$

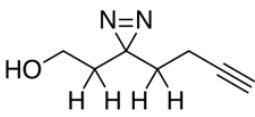

3

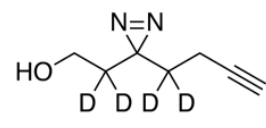

S3
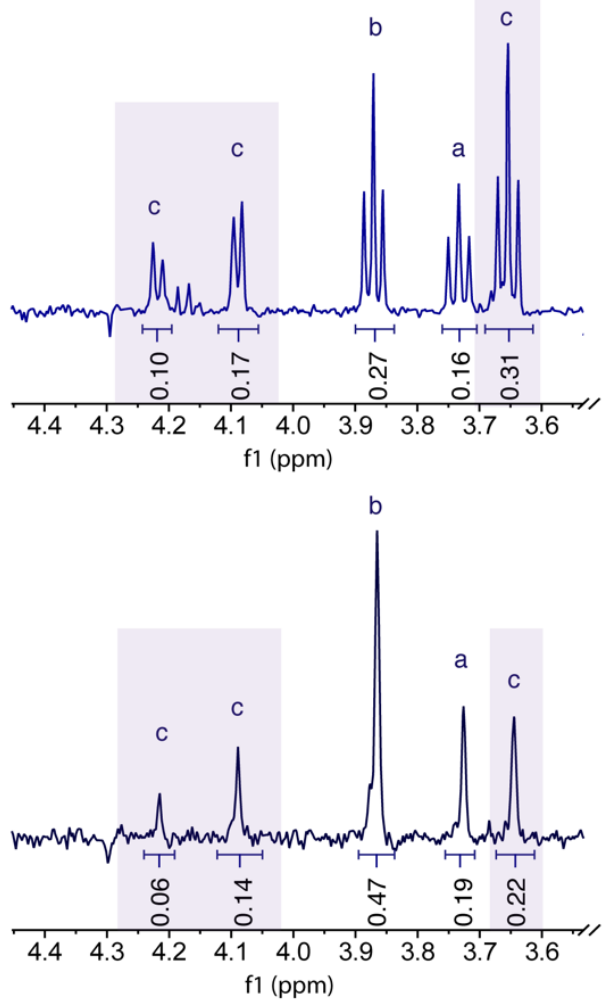

S1
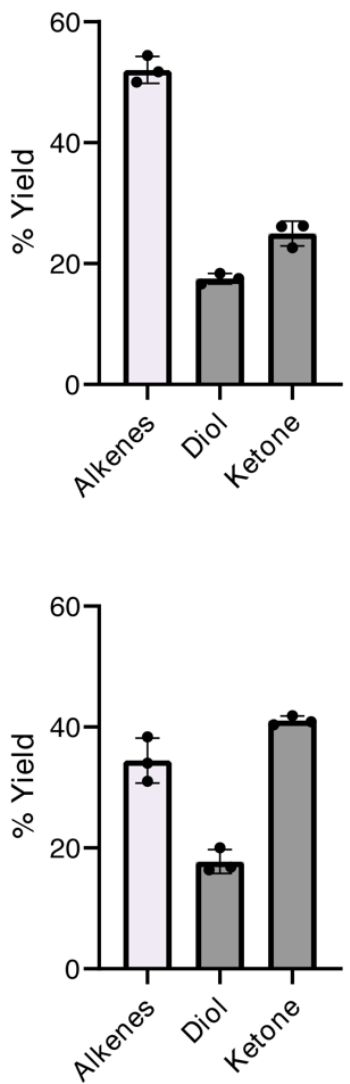

Figure S5. ${ }^{1} \mathrm{H}$ NMR yields of aqueous photolysis of the diazirine 3 and its tetra-deuterated analog S3. Peaks corresponding to alkene products are highlighted. Yields were calculated using ${ }^{1} \mathrm{H}$ NMR peak integration relative to an internal standard. 

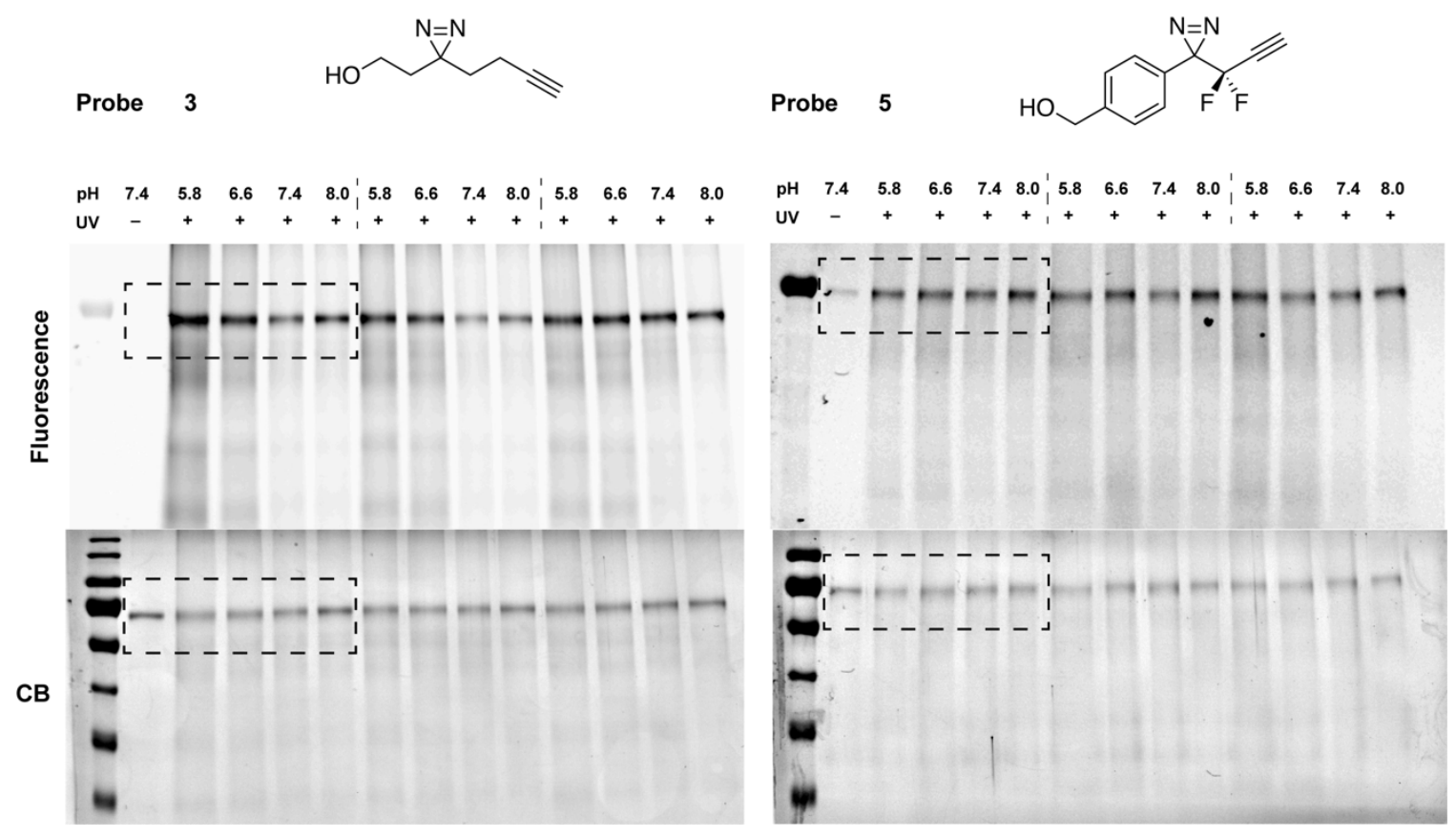

Figure S6. Full gels of single protein labeling of BSA with diazirine probes $\mathbf{3}$ and $\mathbf{5}$ in Tris buffer. Boxed regions are shown in Fig 3. Labeling was quantified by dividing AzideFluor 488 fluorescence by coomassie blue signal (CB). 
a.
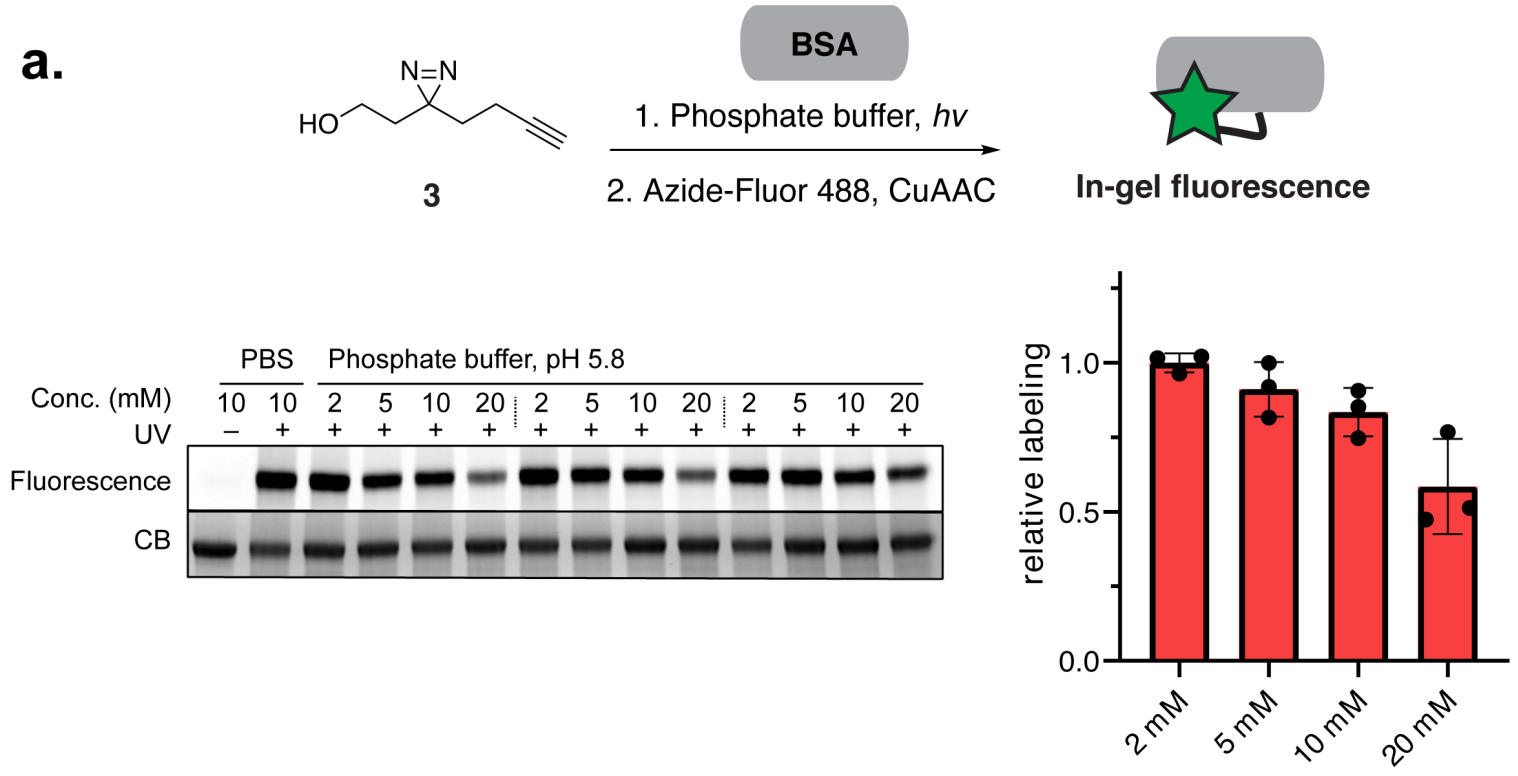

b.

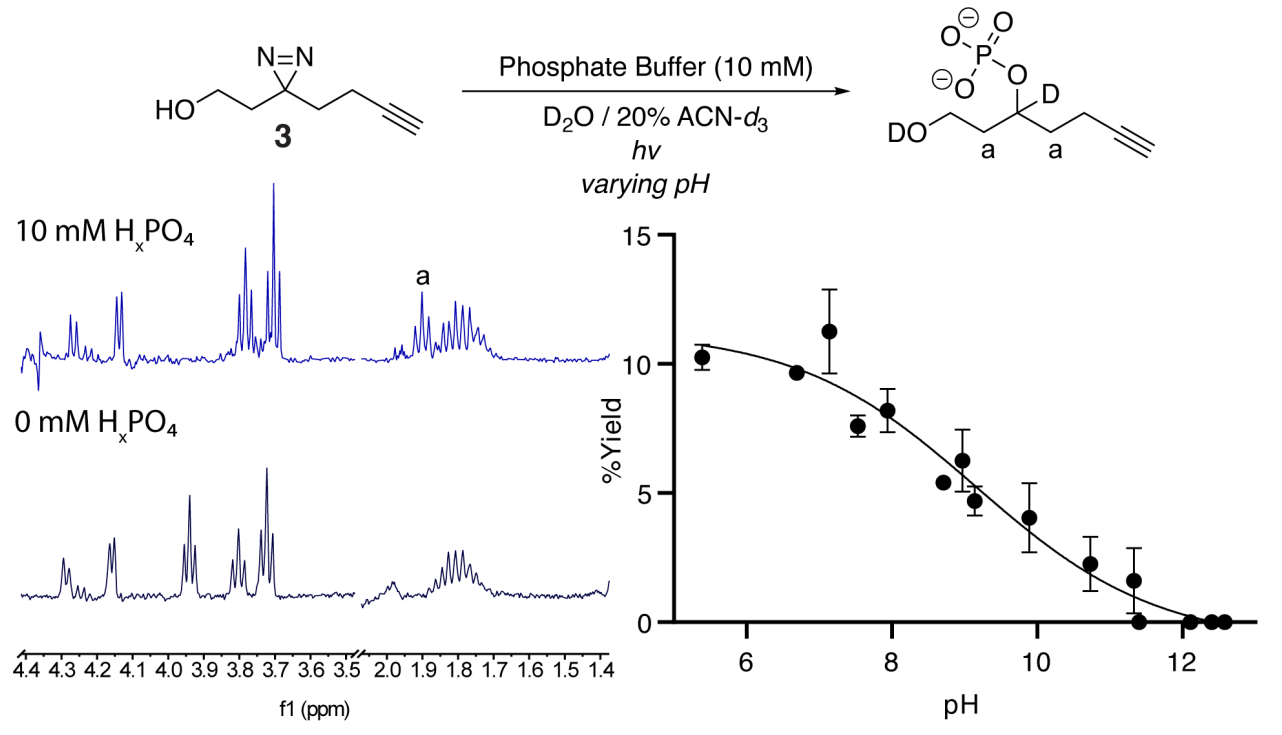

Figure S7. (a) Single protein labeling of BSA by the diazirine 3 in acidic phosphate buffer at varying concentration. Relative labeling was quantified with Azide-Fluor 488 fluorescence relative to coomassie (CB) signal. (b) Photolysis of the diazirine $\mathbf{3}$ in phosphate buffer produces $\mathrm{pH}$-dependent yields of a phosphate adduct. Yields were calculated using ${ }^{1} \mathrm{H}$ NMR peak integration relative to an internal standard. 


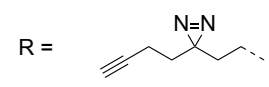

Group 1<smiles>[R]C(=O)NC</smiles>

JN0001
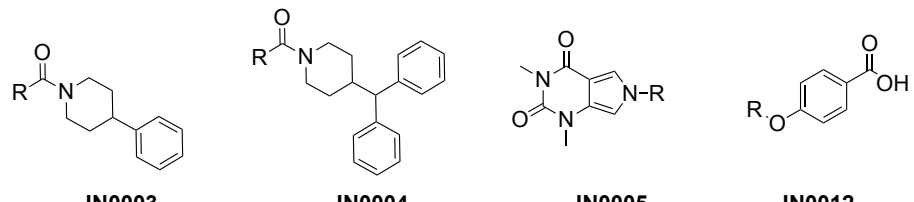

Group 2
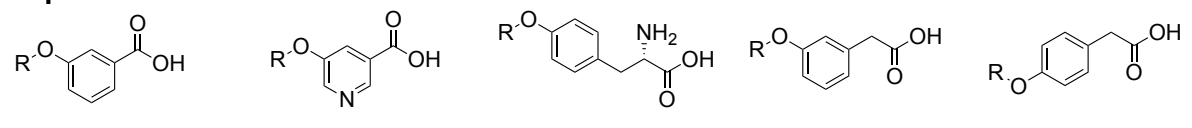

JN0020

JN0013

JN0021

JN0022

JN0017

\section{Group 3}
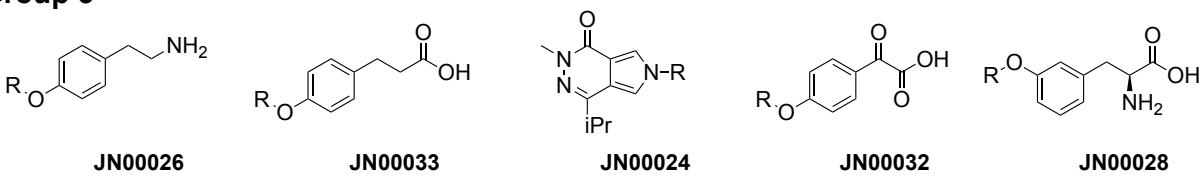

\section{Group 4}<smiles>O=C(O)c1ccc2[nH]ccc2c1</smiles>

JN00247

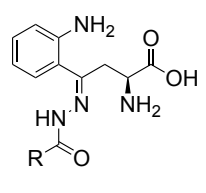

JN00248

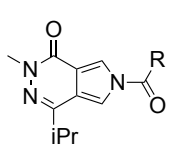

JN835

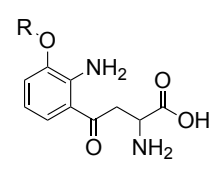

JN00245

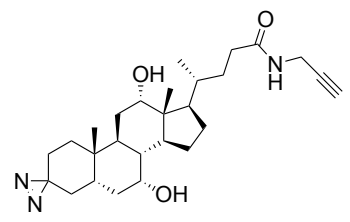

JN00038

\section{Group 5}

(N)

JN935

JN846

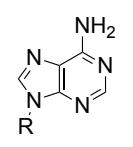

JN847

$$
\sum_{n}^{N} \overbrace{}^{\mathrm{N}}
$$

JN845

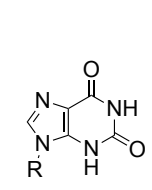

JN936<smiles>[R]C(=O)Nc1ccc(OCCN(C)CC(=O)O)cc1</smiles>

\section{Group 6}

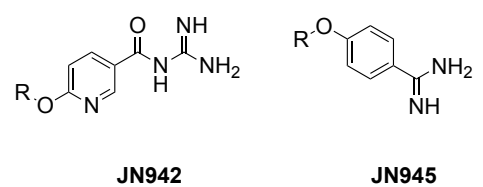

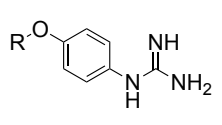

JN940

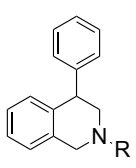

JN849

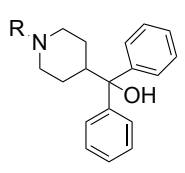

JN938

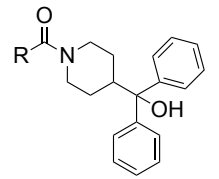

JN939

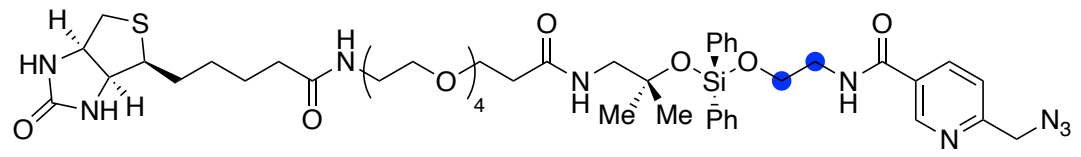

S4 - $=3: 1{ }^{13} \mathrm{C}_{2}:{ }^{12} \mathrm{C}_{2}$

Figure S8. PAL probe structures and their corresponding dosing groups to SK-N-SH cells, and the isotopically-labeled cleavable picolyl biotin azide probe $\mathbf{S 4}{ }^{1}$ 


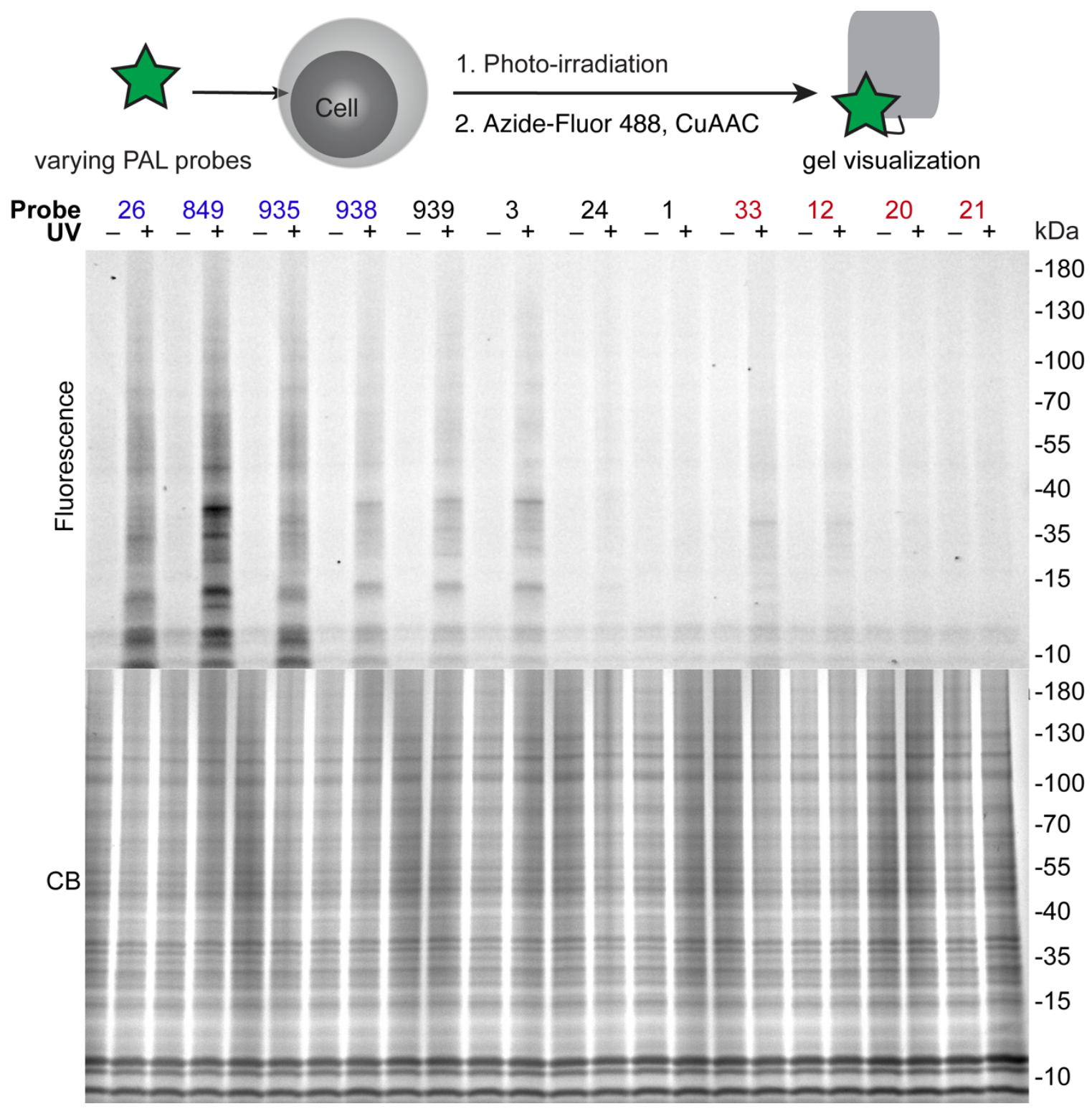

Figure S9. Photoaffinity labeling yields of 12 PAL probes in HEK293T cells visualized by Azide-Fluor 488 and in-gel fluorescence. Top image is Azide-Fluor 488 fluorescence, bottom is coomassie blue stain. 


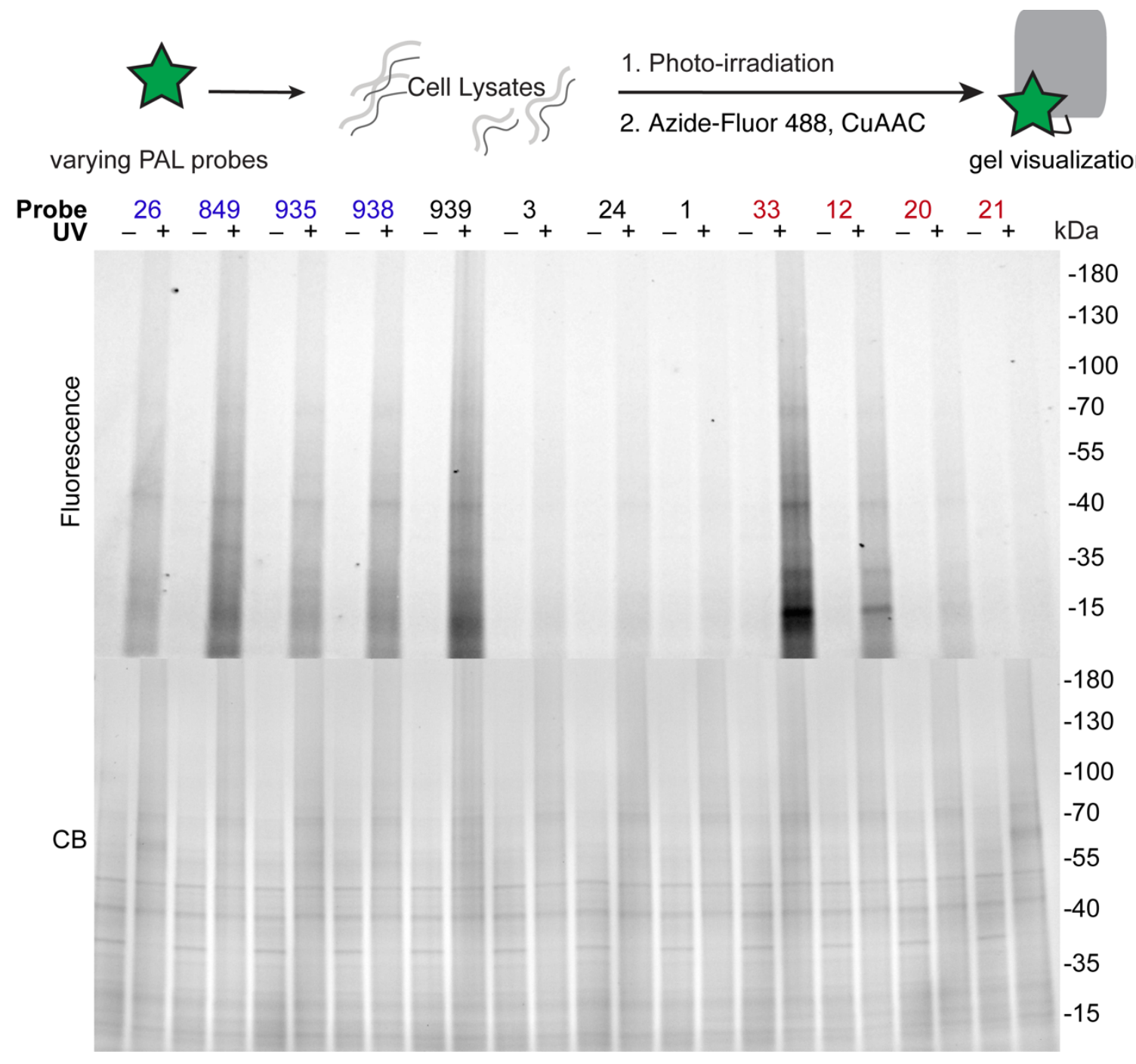

Figure S10. Photoaffinity labeling yields of $12 \mathrm{PAL}$ probes in nondenatured HEK293T lysates visualized by Azide-Fluor 488 and in-gel fluorescence. Top image is Azide-Fluor 488 Fluorescence, bottom is coomassie blue stain. 


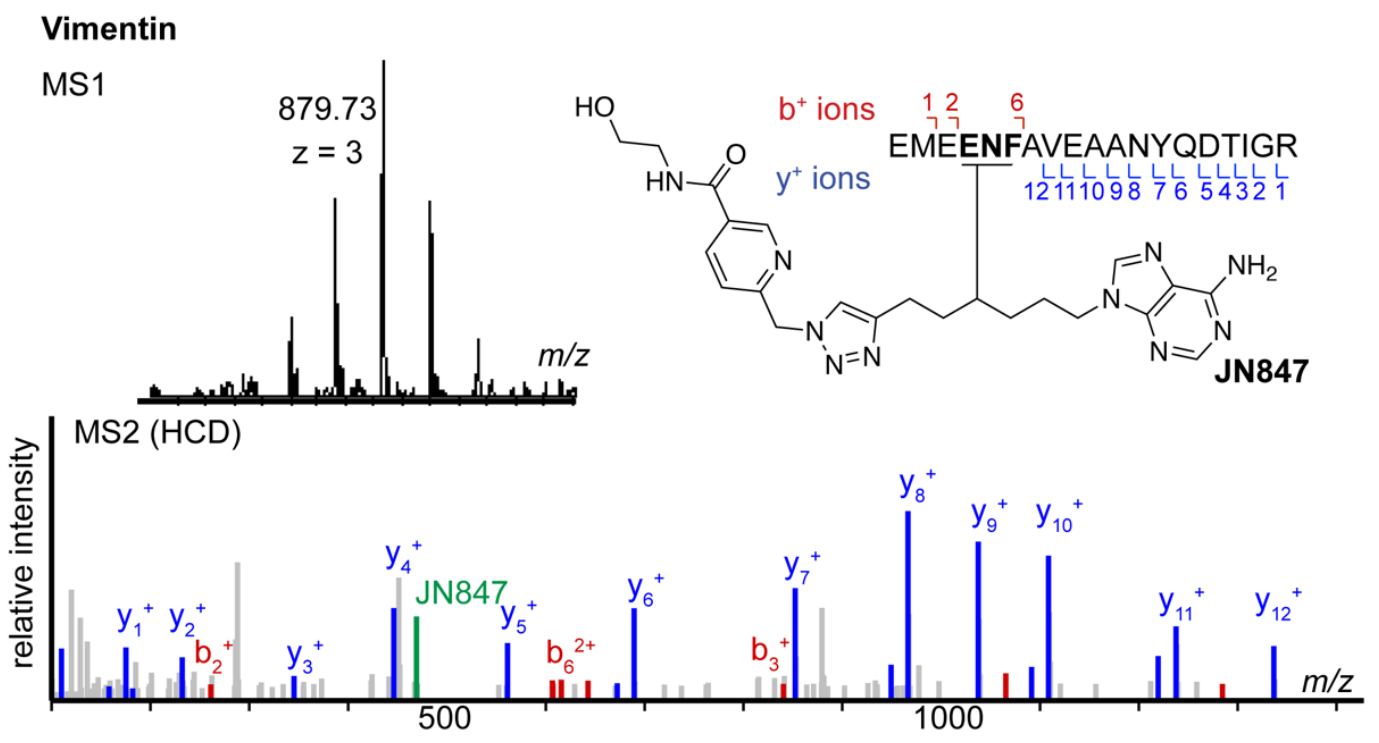

\section{Cathepsin B}

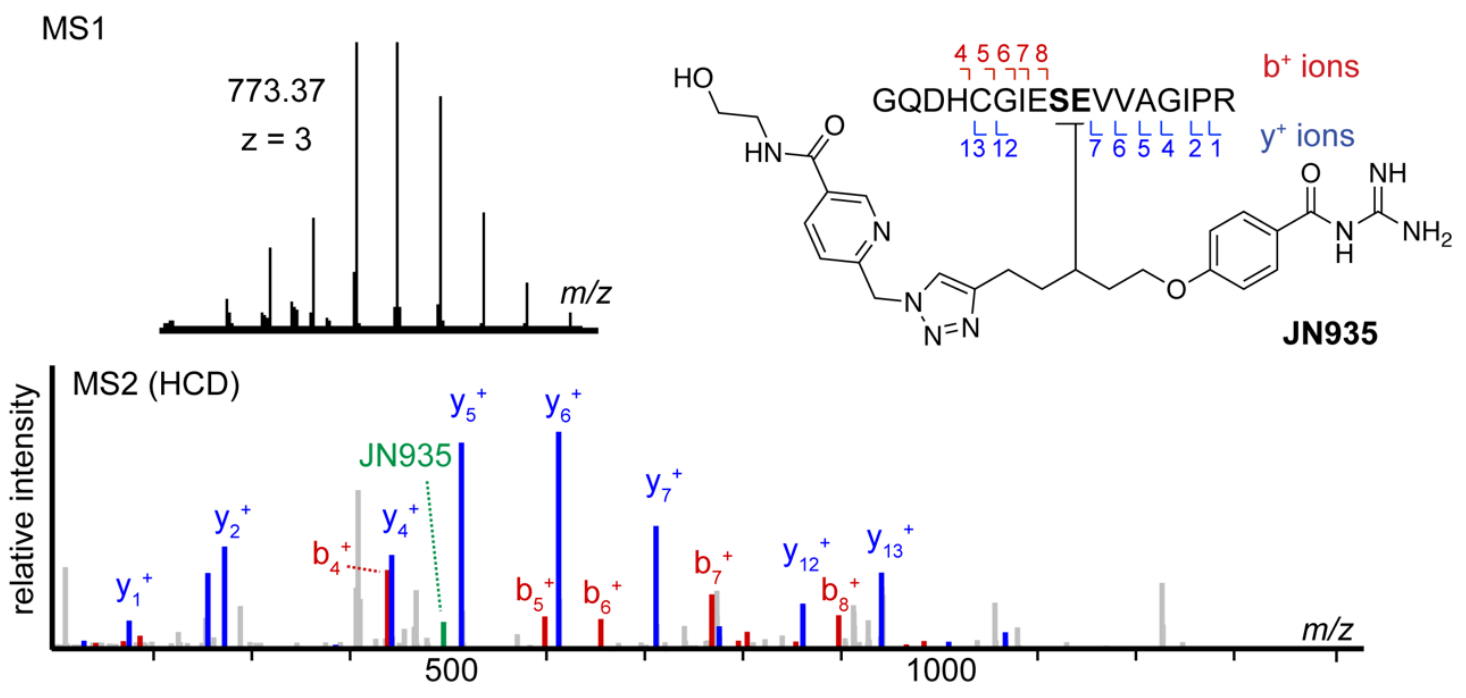

Figure S11. Annotated binding sites for Vimentin and Cathepsin B. 
a.<smiles>C#CCCC1(CCC(=O)C(C)(C)C)N=N1</smiles><smiles>[R]NCc1ccc2sccc2c1</smiles><smiles>CPNc1ccc2c(c1)CCC(=O)N2[N+](=O)[O-]</smiles><smiles>[R]N1CCC(c2ccccc2)CC1</smiles><smiles>[R]Nc1ccc(C2CCNCC2)cc1</smiles><smiles>PN1CCN(C(c2ccccc2)c2ccccc2)CC1</smiles>

F6

F8

F9

F11<smiles>CCNc1ccc(CN2CCN(C)CC2)cc1</smiles><smiles>PN1CCCCC1Cc1ccccc1</smiles><smiles>PNC12CC3CC(C3)C1C2</smiles>

F12

F13

F14<smiles>[R]NCCc1ccc2c(c1)OCO2</smiles>

F15

b.

Percentage of proteins with SILAC ratio $=20$

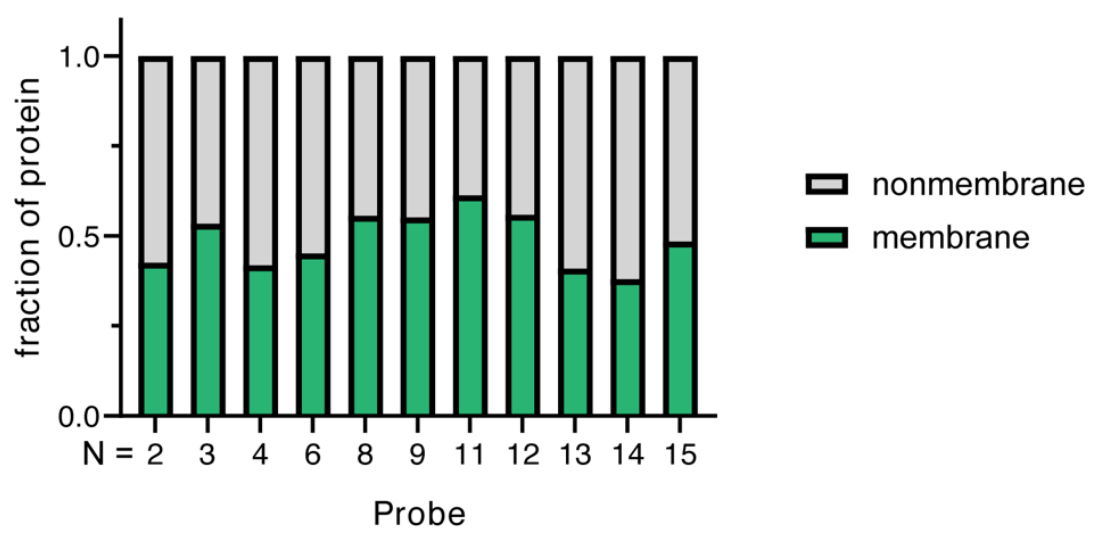

Figure S12. Meta-analysis of proteomics data in Parker et al. ${ }^{2}$ (a) Structures of fully functionalized fragments (FFFs). (b) Analysis of data from FFFs showing the fraction of membrane proteins with SILAC ratios greater than or equal to 20 . These data were collected from HEK293T cells at $200 \mu \mathrm{M}$ dosing of FFF's. ${ }^{2}$ 
a.

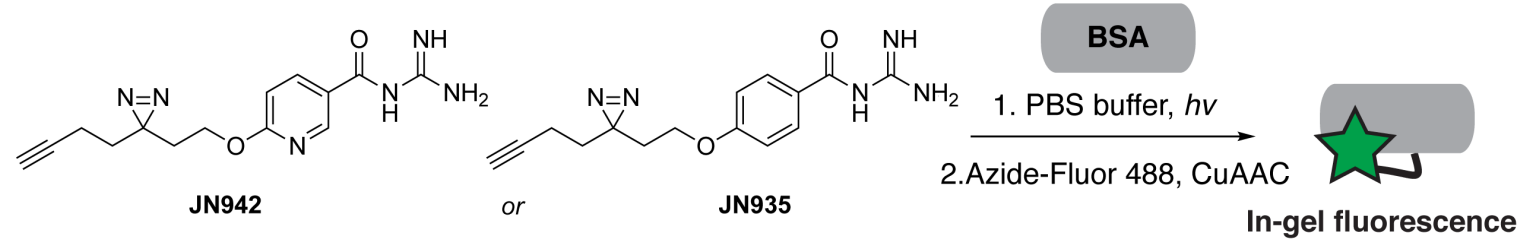

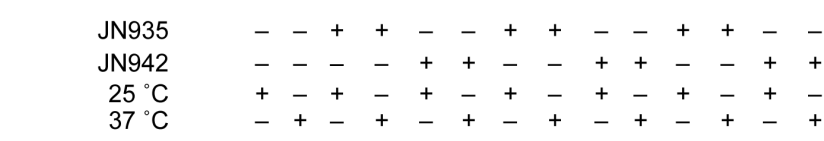
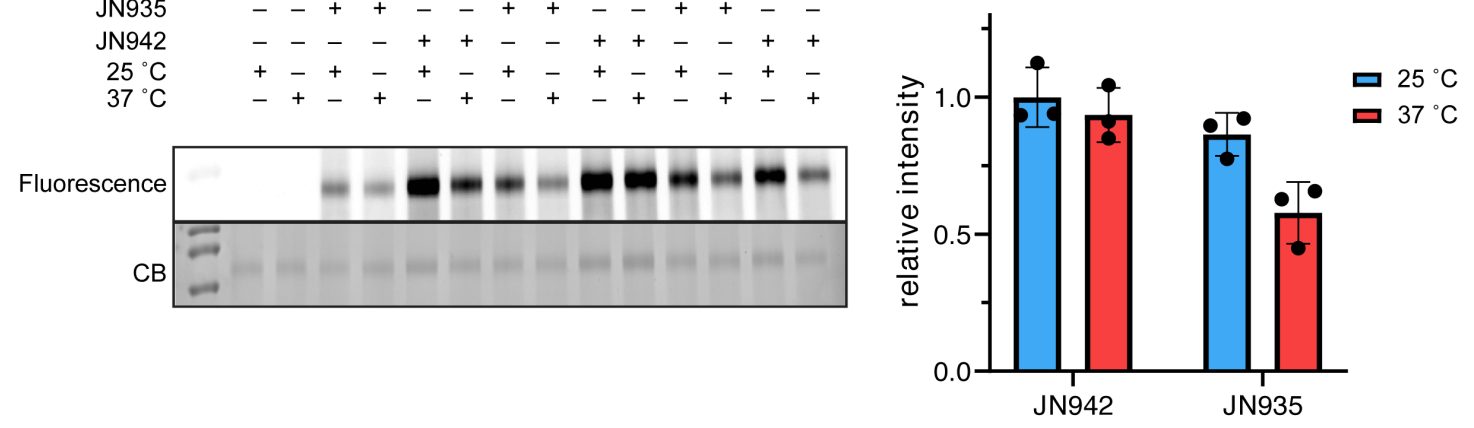

b.

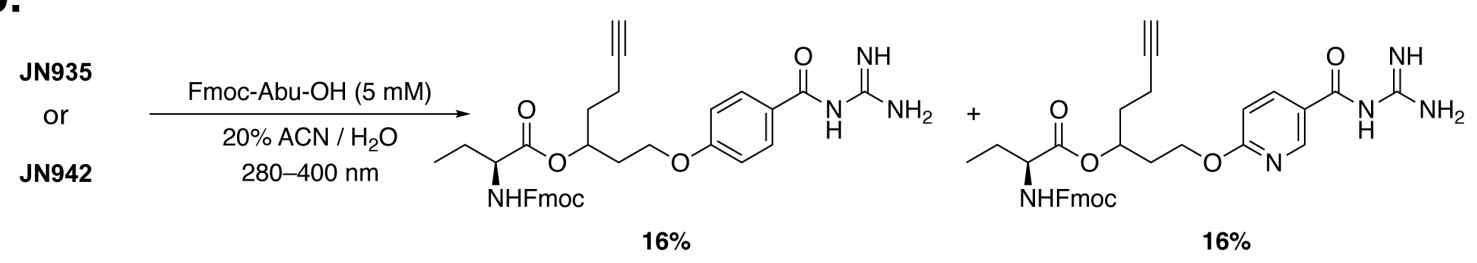

Figure S13. Single protein and amino acid labeling by JN942 and JN935. (a) Labeling efficiency of BSA in PBS and $25^{\circ} \mathrm{C}$ or $37^{\circ} \mathrm{C}$ was calculated with in gel fluorescence AzideFluor 488 signal relative to coomassie blue signal. Plotted values show the mean with error bars representing standard deviation with $n=3$. (b) Reactivity of the PAL probe with the amino acid Fmoc-Abu-OH. Amino acid reaction yields were calculated by UPLC-MS UV-Vis peak integration. 


\section{Supplementary Methods}

General Synthetic Procedures. All reactions were performed in single-neck, oven-dried, round-bottomed flasks fitted with rubber septa under a positive pressure of nitrogen, unless otherwise noted. Air- and moisture-sensitive liquids were transferred via syringe or stainless-steel cannula. Organic solutions were concentrated by rotary evaporation at $30-33{ }^{\circ} \mathrm{C}$. Normal phase flash-column chromatography was performed as described by

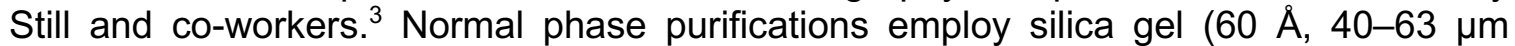
particle size) purchased from Silicycle (Quebec, Canada). Analytical thin-layer chromatography (TLC) was performed using glass plates pre-coated with silica gel $(0.25$ $\mathrm{mm}, 60 \AA$ pore size) impregnated with a fluorescent indicator $(254 \mathrm{~nm})$. TLC plates were visualized by exposure to ultraviolet light (UV), iodine $\left(\mathrm{I}_{2}\right)$, and/or submersion in $p$ anisaldehyde or ninhydrin followed by brief heating with a heat gun (10-15 s).

Chemical Materials. Commercial solvents and reagents were used as received with the following exceptions. Dichloromethane (DCM) was purified according to the method of Grubbs and co-workers. ${ }^{4}$ Acetonitrile was dried over calcium hydride for at least $24 \mathrm{~h}$ prior to use. Minimalist tag 3 was prepared according to the method of Yao and co-workers. ${ }^{5}$ The cleavable biotin azide probe was synthesized according to the method of Woo and co-workers. ${ }^{1}$ Tris(3-hydroxypropyltriazolylmethyl)amine (THPTA) was obtained from Sigma-Aldrich (\#762342). Azide-Fluor 488 was obtained from Sigma-Aldrich (\#760765).

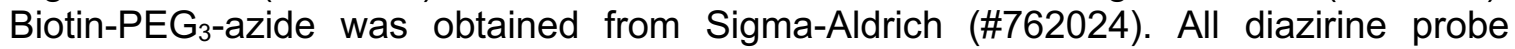
concentrations are based on calculated concentration after dilution from a dimethylsulfoxide stock, acetonitrile or $\mathrm{D}_{2} \mathrm{O}$ stock solutions as specified in their corresponding experimental sections.

Biological Materials. SK-N-SH and HEK293T cells were a gift from the Bertozzi lab (Stanford University) and were acquired from the ATCC. GelCode Blue Stain Reagent (Thermo Scientific, \#24590) was used to image total protein in polyacrylamide gels. For Western blotting, IRDye 800CW Streptavidin (LICOR \#926-32230), Streptavidin-HRP (Thermo Fisher, 21130) and BSA (Sigma-Aldrich \#A7906) were used. Protease inhibitor tablets (Roche EDTA-free cOmplete tablets, Sigma-Aldrich, \#11836170001) were resuspended as a $25 \mathrm{x}$ stock in $2 \mathrm{~mL}$ PBS and stored at $-20{ }^{\circ} \mathrm{C}$. For LC-MS/MS analysis, proteins were digested with Sequencing grade trypsin (Promega, \#V5111) using S-Trap mini (Protifi, \#C02-mini) or micro devices (Protifi, \#C02-micro). C18 Ziptips (Millipore, \#ZTC18S096) were used for sample desalting, or Mini Bio-Spin columns (Bio-Rad, \#7326207). Triton X-114 (Thomas Scientific, \#C987Q42) was used as a nondenaturing buffer for cell lysate experiments.

Instrumentation. Proton nuclear magnetic resonance spectra $\left({ }^{1} \mathrm{H}\right.$ NMR) were recorded at 500 or $400 \mathrm{MHz}$ at $24{ }^{\circ} \mathrm{C}$. Chemical shifts are expressed in parts per million (ppm, $\delta$ scale) downfield from tetramethylsilane and are referenced to residual protium in the NMR solvent $\left[\mathrm{CHCl}_{3}, \delta 7.26\right]$ or $\left[\mathrm{D}_{2} \mathrm{O}, \delta 4.79\right]$. Data are represented as follows: chemical shift, multiplicity $(\mathrm{s}=$ singlet, $\mathrm{d}=$ doublet, $\mathrm{t}=$ triplet, $\mathrm{q}=$ quartet, quin $=$ quintet, $\mathrm{m}=$ multiplet and/or multiple resonances, $\mathrm{br}=$ broad, $\mathrm{app}=$ apparent), integration, coupling constant in Hertz, and assignment. Proton-decoupled carbon nuclear magnetic resonance spectra $\left({ }^{13} \mathrm{C}\right.$ NMR) were recorded at $125 \mathrm{MHz}$ at $24{ }^{\circ} \mathrm{C}$, unless otherwise noted. Chemical shifts are expressed in parts per million (ppm, $\delta$ scale) downfield from tetramethylsilane and are referenced to the carbon resonances of the solvent $\left(\mathrm{CDCl}_{3}, \delta 77.0\right) .{ }^{13} \mathrm{C} \mathrm{NMR}$ and data are represented as follows: chemical shift, carbon type. Chemical shifts are expressed in parts per million (ppm, $\delta$ scale) downfield from tetramethylsilane. Infrared (IR) spectra 
were obtained using a Shimadzu 8400 S FT-IR spectrometer referenced to a polystyrene standard. Data are represented as follows: frequency of absorption $\left(\mathrm{cm}^{-1}\right)$, intensity of absorption ( $\mathrm{s}=$ strong, $\mathrm{m}=$ medium, $\mathrm{w}=$ weak, $\mathrm{br}=$ broad). Small molecule highresolution mass spectrometry (HRMS) measurements were obtained at the Harvard University Chemistry and Chemical Biology Department Mass Spectrometry Facility using an Agilent 1260 UPLC-MS Bruker connected in line with a microTOF-Q II hybrid quadrupole-time of flight MS. Low-resolution mass spectrometry (LRMS) measurements were obtained on a Waters ACQUITY UPLC equipped with SQ Detector 2 mass spectrometer. Ultrapure water was obtained using a GenPure UV/UF xCAD plus system (Thermo Scientific). All UV irradiation was performed using a Dymax ECE 5000 UV LightCuring Flood Lamp system (\#41060). The lamp was warmed up for at least 15 minutes prior to use. Irradiation was performed in a $4{ }^{\circ} \mathrm{C}$ cold room for specified time. For irradiation, samples were placed either in $100 \mu \mathrm{L}$ or $500 \mu \mathrm{L}$ PPE PCR tubes (20170-010) or in quartz NMR tubes (528-PP-7QTZ). Transfer of proteins from SDS-PAGE gels to membranes was performed using a iBlot 2 dry blotting system (Invitrogen). In-gel fluorescence, GelCode Blue staining, IR blots, and Ponceau were imaged by an Azure Imager C600 (Azure Biosystems, Inc., Dublin, CA). Peptides were dried using a Vacufuge plus (Eppendorf). Proteomics mass spectrometry instrumentation is described in the "Proteomics Mass Spectrometry and Data Processing Procedures" section.

Software. Data was analyzed and visualized using Microsoft Excel (v16.22) and GraphPad Prism (v8.1.1), in addition to software listed by experiment. Pymol v2.1.0 (Schrodinger) was used to visualize protein structures. DNA and protein sequences were analyzed using Geneious (v10.0.7). Images were made using ImageJ (NIH, v1.52q), Adobe Photoshop (v19.1.5), and Adobe Illustrator (v23.1.1). 


\section{Experimental Procedures with Individual Amino Acids}

Photolysis of the diazirine in aqueous conditions. A quartz NMR tube was charged with a solution of the diazirine $3(1 \mathrm{mM})$ and calcium formate $(1 \mathrm{mM})$ in $20 \%$ acetonitrile$d_{3}$-deuterium oxide $(500 \mu \mathrm{L})$. The resulting solution was analyzed via ${ }^{1} \mathrm{H}$ NMR and then irradiated $(280-320 \mathrm{~nm})$ for $2 \times 90 \mathrm{sec}$ at $4{ }^{\circ} \mathrm{C}$. The irradiated sample was reanalyzed via ${ }^{1} \mathrm{H}$ NMR and the product distribution was integrated against calcium formate as the internal standard. For the diazirine $\mathbf{3}$, the ketone $\mathbf{S 1}$ and the diol $\mathbf{S 2}$ products were analogous to synthetic standards.

General procedure for neat photolysis of the diazirine with $\mathrm{N}$-acetyl O-methyl amino acids. Stock solutions of the $20 \mathrm{~N}$-acetyl O-methyl amino acids and the diazirine probes 1 or $2(100 \mathrm{mM})$ were prepared in acetonitrile and dried over activated $3 \AA$ molecular sieves for $24 \mathrm{~h}$ immediately before use. The diazirine probe 1 or 2 (5.00 $\mu \mathrm{L}, 1$ equiv) and the amino acid ( $20.0 \mu \mathrm{L}, 4.00$ equiv) were added in sequence to a PCR tube (Part number) in quadruplicate. The resulting solution was concentrated to dryness by vacufuge for $1 \mathrm{~h}$ at $30{ }^{\circ} \mathrm{C}$ to afford an oil emulsion, with the exception of mixtures prepared with Asn, Gln, and Trp, which afforded a white crystalline solid. The neat mixture was degassed, and the PCR tube was sealed under nitrogen in a glovebox. Three of the four sealed tubes were irradiated with a broadband UV lamp $(280-400 \mathrm{~nm})$ for $6 \times 90 \mathrm{~s}$ at $4{ }^{\circ} \mathrm{C}$. The remaining sealed tube was not irradiated and kept in the dark.

Note: Drying the stock solution and sealing the PCR tube under nitrogen is required to reduce UV products arising from residual water or oxygen. For Arg and Asn, methanol was used to prepare the stock solutions.

Analysis of the alkyl diazirine 1 photolysis by UPLC-MS. The irradiated samples were unsealed and dissolved in dimethylsulfoxide (DMSO) $(100 \mu \mathrm{L})$. Half of the dissolved sample $(50.0 \mu \mathrm{L})$ was diluted with a $4 \mathrm{mM}$ solution of L-2-(Fmoc-amino)butyric acid (50 $\mu \mathrm{L}, 2 \mathrm{mM}$ final) used as an internal standard. The resulting solution was analyzed via UPLC-MS (5-90\% acetonitrile-water, $10 \mathrm{~min}$ ), and monitored by UV-Vis absorbance over 250-270 nm. The product identity for each peak was determined by the $\mathrm{m} / \mathrm{z}$ in the ES+ mass spectra. Product yields were determined using peak integrals relative to the internal standard.

Analysis of the aryl diazirine 2 photolysis by NMR. The irradiated samples were dissolved in $500 \mu \mathrm{L}$ of DMSO- $d_{6}$ containing $1 \mathrm{mM}$ fumaric acid and $1 \mathrm{mM}$ hexafluorobenzene and transferred to a NMR tube (final concentration $\sim 1 \mathrm{mM}$ diazirine). The samples were analyzed via ${ }^{1} \mathrm{H}$ NMR and ${ }^{19} \mathrm{~F}$ NMR. Product identities were inferred from unique peaks in the ${ }^{19} \mathrm{~F}$ NMR spectra relative to the non-irradiated control. Product yields were calculated from ${ }^{19} \mathrm{~F}$ NMR peak integration relative to hexafluorobenzene internal standard.

Aqueous photo-reactions with individual amino acids. Stock solutions of the $20 \mathrm{~N}$ acetyl O-methyl amino acids (50 mM) and the diazirine probe $3(10 \mathrm{mM})$ were prepared in $50 \%$ acetonitrile- $d_{3}-\mathrm{D}_{2} \mathrm{O}$ prior to use. The amino acid was added at a equimolar ratio (10.0 $\mu \mathrm{L}, 1.00 \mathrm{mM}, 1.00$ equiv.) or 10 -fold excess $(100 \mu \mathrm{L}, 10.0 \mathrm{mM}, 10.0$ equiv.) to a solution of the diazirine probe $3(50.0 \mu \mathrm{L}, 1.00 \mathrm{mM}, 1$ equiv.) and calcium formate $(5.00 \mu \mathrm{L}, 1.00$ $\mathrm{mM}, 1.00$ equiv.) in $20 \%$ acetonitrile- $d_{3}-\mathrm{D}_{2} \mathrm{O}$ in a quartz NMR tube in quadruplicate. Three of the four samples were irradiated with either a broadband UV lamp (280-400 nm) for 2 
x 90 seconds, or a filtered UV lamp $(320-400 \mathrm{~nm})$ for $4 \times 90$ seconds at $4{ }^{\circ} \mathrm{C}$. The remaining sample was not irradiated and kept in the dark as a control. The photolyzed samples were analyzed by ${ }^{1} \mathrm{H}$ NMR and UPLC-MS.

For ${ }^{1} \mathrm{H}$ NMR, reactions were examined by comparing the spectra of irradiated samples to the spectra of the no-UV control and spectra from aqueous photolysis of diazirine probe 3 alone. Unique peaks not belonging to starting materials or diazirine $\mathbf{3}$ photolysis products were observed only with reactions with Glu, Asp, Tyr, and Cys (Figure S3). To calculate product yields, clearly-resolved peaks were integrated relative to the calcium formate internal standard. All samples were additionally analyzed without further dilution via UPLCMS, (5-90\% acetonitrile in water over $10 \mathrm{~min})$. Product identities were determined from the $\mathrm{m} / \mathrm{z}$ in the ES+ mass spectra. $\mathrm{m} / \mathrm{z}$ matching expected $\mathrm{XH}$ insertion products were found for Glu, Asp, Tyr, and Cys but no other amino acids.

pH titration reactions with individual amino acids. Stock solutions of the $\mathrm{N}$-acetyl Omethyl Tyr, Lys, His and Glu, and the Fmoc diazirine probe 1 were prepared $(100 \mathrm{mM})$ in acetonitrile prior to use. The diazirine $(5.00 \mu \mathrm{L}, 1.00 \mathrm{mM}, 1$ equiv) and the amino acid $(50.0 \mu \mathrm{L}, 10.0 \mathrm{mM}, 10.0$ equiv) were added in sequence to $20 \%$ acetonitrile-water (445 $\mu \mathrm{L})$. The $\mathrm{pH}$ of the solution was adjusted using $\mathrm{KOH}(100 \mathrm{mM}$ in water) or $\mathrm{HCl}(100 \mathrm{mM}$ in water) and measured. The resulting solution was aliquoted into $4 \times 100 \mu \mathrm{L}$ samples in PCR tubes. Three of the samples were irradiated $(320-400 \mathrm{~nm})$ for $4 \times 90 \mathrm{~s}$ at $4{ }^{\circ} \mathrm{C}$. The remaining sample was kept in the dark. After UV treatment, Fmoc-Ser(tBu)-OH (1 mM final) was added to each sample as an internal standard. The resulting solutions were analyzed via UPLC-MS, (5-90\% acetonitrile-water, $10 \mathrm{~min}$ ), and monitored by UV absorbance (250-270 nm). Product yields were determined using peak integrals relative to the internal standard.

pH titration reactions with phosphate. Stock solutions of monobasic phosphate (100 $\mathrm{mM})$ in $\mathrm{D}_{2} \mathrm{O}$ and alkyl diazirine probe $3(10 \mathrm{mM})$ were prepared in $50 \%$ acetonitrile- $d_{3}-$ $\mathrm{D}_{2} \mathrm{O}$ prior to use. The phosphate solution was added at 10 -fold excess $(100 \mu \mathrm{L}, 10.0 \mathrm{mM}$, 10.0 equiv.) to a solution of the diazirine probe $3(50.0 \mu \mathrm{L}, 1.00 \mathrm{mM}, 1$ equiv.) and calcium formate $\left(5.00 \mu \mathrm{L}, 1.00 \mathrm{mM}, 1.00\right.$ equiv.) in $20 \%$ acetonitrile- $d_{3}-\mathrm{D}_{2} \mathrm{O}$. The solution was $\mathrm{pH}-$ adjusted with $\mathrm{HCl}\left(100 \mathrm{mM}\right.$ in $\left.\mathrm{D}_{2} \mathrm{O}\right)$ then partitioned into four quartz NMR tubes. Three of the four samples were irradiated $(280-400 \mathrm{~nm})$ for $2 \times 90$ seconds at $4{ }^{\circ} \mathrm{C}$. The remaining sample was not irradiated and kept in the dark as a control. The photolyzed samples were analyzed by ${ }^{1} \mathrm{H}$ NMR. Reactions were examined by comparing the spectra of irradiated samples to the spectra of the no-UV control and spectra from aqueous photolysis of diazirine probe 3 alone. Unique peaks not belonging to starting materials or diazirine 3 photolysis products were observed and attributed to putative phosphate adduct.

Aqueous photo-reactions with $\beta$-mercapto ethanol. Solutions of $\beta$-mercapto ethanol (BME) in $50 \%$ acetonitrile-water were prepared via serial dilution $(2 \mathrm{M}, 1 \mathrm{M}, 500 \mathrm{mM}, 250$ $\mathrm{mM}, 125 \mathrm{mM})$. A stock solution of aryl diazirine $2(10 \mathrm{mM})$ in $50 \%$ acetonitrile was prepared, and $5 \mu \mathrm{L}$ added to $45 \mu \mathrm{L}$ aliquots of respective BME solutions in quadruplicate in PCR tubes. Three of the four samples per concentration were irradiated $(280-400 \mathrm{~nm})$ $2 \times 90 \mathrm{sec}$ at $4{ }^{\circ} \mathrm{C}$. The samples were then analyzed via UPLC-MS (5-90\% acetonitrile in water, $6 \mathrm{~min}$ ). Product identities were determined from the $\mathrm{m} / \mathrm{z}$ in the ES+ mass spectra. Yields were calculated using relative peak integration in the UV-Vis chromatogram.

West, A. et al. Labeling preferences of diazirines with protein biomolecules, J. Am. Chem. 


\section{Experimental Procedures with Purified Proteins}

pH-dependent labeling of BSA with diazirine probes 3 and 5. Tris ( $\mathrm{pH} 7.4$ and 8.0) and bis-tris (pH 5.8 and 6.6) buffers were prepared at $100 \mathrm{mM}$ with $1 \%$ SDS and $\mathrm{pH}$ adjusted. All experiments done with tris and bis-tris buffers were performed at $20 \mathrm{mM}$ concentration. Phosphate buffers were prepared at $100 \mathrm{mM}$ concentration with $1 \%$ SDS and $\mathrm{pH}$ adjusted to $\mathrm{pH} 5.8,6.6$ and 7.4 , then diluted to $20 \mathrm{mM}, 10 \mathrm{mM}, 5 \mathrm{mM}$ and $2 \mathrm{mM}$. 20x stock solutions of purified BSA $(1 \mu \mathrm{L})$ were added to $18 \mu \mathrm{L}(0.1 \mu \mathrm{g} / \mathrm{mL})$ of variable buffer in a PCR tube. 20x solutions of diazirine probes 3 or 5 in DMSO $(1 \mu \mathrm{L})$ were added to each sample $(100 \mu \mathrm{M})$ and the samples incubated at $24{ }^{\circ} \mathrm{C}$ for $30 \mathrm{~min}$. The samples were irradiated for $30 \mathrm{~s}$ at $4{ }^{\circ} \mathrm{C}$ on a bed of ice. Following irradiation, samples were diluted with acetone $(100 \mu \mathrm{L})$ and transferred to $1.7 \mathrm{~mL}$ Eppendorf tubes. An additional $100 \mu \mathrm{L}$ of acetone was used to rinse the samples into the new tubes. The protein was precipitated at $-80{ }^{\circ} \mathrm{C}$ for $30 \mathrm{~min}$, then pelleted via centrifugation $\left(10 \mathrm{~min}, 21,000 \mathrm{~g}, 4{ }^{\circ} \mathrm{C}\right)$. The supernatant was discarded and the pellets were dried under air for $5 \mathrm{~min}$. Samples were resuspended in $50 \mu \mathrm{L}$ of $5 \times$ PBS-1\%SDS and click reacted with a mixture of Azide-Fluor $488(25 \mu \mathrm{M})$, copper (II) sulfate $(250 \mu \mathrm{M})$, THPTA $(250 \mu \mathrm{M})$, sodium ascorbate $(2.0 \mathrm{mM})$ for $60 \mathrm{~min}$ at $24{ }^{\circ} \mathrm{C}$ with rotation. The reaction was quenched with the addition of acetone $(300 \mu \mathrm{L})$ followed by precipitation at $-20{ }^{\circ} \mathrm{C}$ for $12 \mathrm{~h}$. Proteins were pelleted by centrifugation $\left(21,000 \mathrm{~g}, 10 \mathrm{~min}, 4{ }^{\circ} \mathrm{C}\right)$. The supernatant was discarded and the pellets dried under air for $5 \mathrm{~min}$. The pellets were suspended in 1x SDS-PAGE gel loading buffer $(30 \mu \mathrm{L})$ and stored at $-20{ }^{\circ} \mathrm{C}$. Thawed samples were heated for $5 \mathrm{~min}$ at $95{ }^{\circ} \mathrm{C}$ prior to separation via SDS-PAGE $12 \%$ gel. Probe labeling was visualized by analyzing fluorescence signal and protein loading visualized with coomassie blue staining. 


\section{Experimental Procedures with Whole Cells}

Photo-labeling in HEK293T cells with photo probes for in-gel fluorescence imaging. Cells were grown to $80-90 \%$ confluency in $3.5 \mathrm{~cm}$, six-well plates and incubated with 10 $\mu \mathrm{M}$ of each corresponding probe in FBS-free media for $1 \mathrm{~h}$ at $37^{\circ} \mathrm{C}$. Following incubation, half of the plates were irradiated for $60 \mathrm{~s}$ at $4{ }^{\circ} \mathrm{C}$. The cells were harvested via scraping, collected in PBS $(2 \times 1 \mathrm{~mL})$, pelleted $(500 \mathrm{~g}, 5 \mathrm{~min})$, and washed with PBS $(1 \mathrm{~mL})$. The pellets were resuspended in PBS $(200 \mu \mathrm{L})$ with $1 \%$ SDS and proteasome inhibitor then sonicated with a probe tip sonicator to lyse the cells. The lysates were cleared via centrifugation and the concentration of protein adjusted to $1.8 \mathrm{mg} / \mathrm{mL}$ using the BCA assay. $50 \mu \mathrm{g}$ of protein $(60 \mu \mathrm{L})$ from each sample was taken and reacted with a mixture of Azide-Fluor $488(25 \mu \mathrm{M})$, copper (II) sulfate $(250 \mu \mathrm{M})$, THPTA $(250 \mu \mathrm{M})$, sodium ascorbate $(2.0 \mathrm{mM})$ for $90 \mathrm{~min}$ at $24{ }^{\circ} \mathrm{C}$ with rotation. The reaction was quenched with the addition of acetone $(300 \mu \mathrm{L})$ followed by precipitation at $-20{ }^{\circ} \mathrm{C}$ for $12 \mathrm{~h}$. Proteins were pelleted by centrifugation $\left(21,000 \mathrm{~g}, 10 \mathrm{~min}, 4{ }^{\circ} \mathrm{C}\right)$. The supernatant was discarded and the pellets dried under air for $5 \mathrm{~min}$. The pellets were suspended in 1x SDS-PAGE gel loading buffer $(50 \mu \mathrm{L})$ and stored at $-20^{\circ} \mathrm{C}$. Thawed samples were heated for $5 \mathrm{~min}$ at 95 ${ }^{\circ} \mathrm{C}$ prior to separation on a 4\%-15\% Criterion TGX Precast gel (\#5671085). Probe labeling was visualized by analyzing fluorescence signal and protein loading visualized with coomassie blue staining.

Photo-labeling in HEK293T lysates with photo probes for in-gel fluorescence imaging. Cells were grown to $80-90 \%$ confluency in $15 \mathrm{~cm}$ plates. The cells were washed with PBS $(10 \mathrm{~mL})$ then treated with trypsin $(3 \mathrm{~mL})$ and incubated at $37{ }^{\circ} \mathrm{C}$ to detach. The cells were collected in PBS $(7 \mathrm{~mL})$, pelleted $(500 \mathrm{~g}, 5 \mathrm{~min})$, and washed with PBS $(10 \mathrm{~mL})$. The pellets were resuspended in PBS $(1000 \mu \mathrm{L})$ with $0.3 \%$ Triton and proteasome inhibitor then sonicated with a probe tip sonicator to lyse the cells. The lysates were cleared via centrifugation and the concentration of protein adjusted to $1.0 \mathrm{mg} / \mathrm{mL}$ using the BCA assay. $50 \mu \mathrm{g}$ aliquots were prepared in PCR tubes, dosed with each corresponding probe and incubated at $24{ }^{\circ} \mathrm{C}$ for $30 \mathrm{~min}$. Half of the samples were irradiated for $30 \mathrm{~s}$ at $4{ }^{\circ} \mathrm{C}$. Following irradiation, $10 \%$ SDS-PBS $(5 \mu \mathrm{L})$ was added to denature proteins and the samples were reacted with a mixture of Azide-Fluor $488(25 \mu \mathrm{M})$, copper (II) sulfate (250 $\mu \mathrm{M})$, THPTA $(250 \mu \mathrm{M})$, sodium ascorbate $(2.0 \mathrm{mM})$ for $90 \mathrm{~min}$ at $24{ }^{\circ} \mathrm{C}$ with rotation. The reactions were transferred with acetone $(100 \mu \mathrm{L})$ to $1.7 \mathrm{~mL}$ Eppendorf tubes and diluted with an additional $200 \mu \mathrm{L}$, followed by precipitation at $-20{ }^{\circ} \mathrm{C}$ for $12 \mathrm{~h}$. Proteins were pelleted by centrifugation $\left(21,000 \mathrm{~g}, 10 \mathrm{~min}, 4{ }^{\circ} \mathrm{C}\right)$. The supernatant was discarded and the pellets dried under air for $5 \mathrm{~min}$. The pellets were suspended in 1x SDS-PAGE gel loading buffer $(50 \mu \mathrm{L})$ and stored at $-20{ }^{\circ} \mathrm{C}$. Thawed samples were heated for $5 \mathrm{~min}$ at 95 ${ }^{\circ} \mathrm{C}$ prior to separation on a 4\%-15\% Criterion TGX Precast gel (\#5671085). Probe labeling was visualized by analyzing fluorescence signal and protein loading visualized with coomassie blue staining.

Photo-labeling of SK-N-SH cells with photo-probes for quantitative LC-MS/MS. Cells were grown to $90-100 \%$ confluency in $10 \mathrm{~cm}$ plates then incubated with $10 \mu \mathrm{M}$ of each probe in the treatment group (or $0.5 \% \mathrm{DMSO}$ ) in FBS-free media for $2 \mathrm{~h}$ at $37^{\circ} \mathrm{C}$. Following incubation, the plates were irradiated for $60 \mathrm{~s}$ at $4{ }^{\circ} \mathrm{C}$. The cells were harvested via scraping, collected in PBS (10 mL), pelleted $(2000 \mathrm{~g}, 5 \mathrm{~min})$, and washed with PBS (10 $\mathrm{mL})$. The pellets were resuspended in PBS $(500 \mu \mathrm{L})$ with $1 \%$ SDS and proteasome inhibitor then sonicated with a probe tip sonicator to lyse the cells. The lysates were cleared via centrifugation and the concentration of protein adjusted using the BCA assay (to concentrations of $\sim 2 \mathrm{mg} / \mathrm{mL}$ ). The lysates were reacted with a mixture of picolyl biotin

West, A. et al. Labeling preferences of diazirines with protein biomolecules, J. Am. Chem. 
azide probe $\mathbf{S 4}(100 \mu \mathrm{M})$, copper (II) sulfate $(250 \mu \mathrm{M})$, THPTA $(250 \mu \mathrm{M})$, sodium ascorbate $(2.5 \mathrm{mM})$ for $90 \mathrm{~min}$ at $24{ }^{\circ} \mathrm{C}$ with rotation. The reaction was quenched with the addition of $\mathrm{MeOH}(1200 \mu \mathrm{L})$ followed by precipitation at $-80{ }^{\circ} \mathrm{C}$ for $1 \mathrm{~h}$. Proteins were pelleted by centrifugation $\left(21,000 \mathrm{~g}, 10 \mathrm{~min}, 4^{\circ} \mathrm{C}\right)$. The supernatant was discarded and the pellets were dried under air for $5 \mathrm{~min}$.

Enrichment of photo-crosslinked proteins for quantitative LC-MS/MS. Protein pellets were resuspended in $1 \%$ SDS in PBS $(500 \mu \mathrm{L})$ with brief sonication. $20 \mu \mathrm{L}$ of sample was taken as the "load fraction." Streptavidin-agarose resin $(200 \mu \mathrm{L}$, washed $3 \times 1 \mathrm{~mL}$ with PBS) was added to each sample, which was then incubated for $18 \mathrm{~h}$ at $24{ }^{\circ} \mathrm{C}$ with rotation. The slurries were transferred to mini biospin column (\#7326207) and centrifuged (1 min, $1000 \mathrm{~g}$ ). The supernatant collected as the "supernatant fraction." The columns were transferred to a vacuum manifold and washed with $1 \%$ SDS in PBS (1 mL), $8 \mathrm{M}$ fresh urea in PBS $(5 \times 1 \mathrm{~mL})$ and PBS $(5 \times 1 \mathrm{~mL})$. The beads were resuspended in PBS $(200 \mu \mathrm{L})$, reduced with $5 \mathrm{mM}$ dithiothreitol (DTT) for $30 \mathrm{~min}$ at $24{ }^{\circ} \mathrm{C}$, then alkylated with $10 \mathrm{mM}$ iodoacetamide for $30 \mathrm{~min}$ at $24{ }^{\circ} \mathrm{C}$ in the dark with rotation. The columns were transferred to the vacuum manifold and washed with PBS $(1 \mathrm{~mL})$ and $50 \mathrm{mM}$ HEPES $(1 \mathrm{~mL}, \mathrm{pH} 8.5)$. The beads were centrifuged then washed with PBS $(1 \mathrm{~mL})$ and resuspended in $250 \mu \mathrm{L}$ $0.5 \mathrm{M}$ guanidine- $\mathrm{HCl} / 50 \mathrm{mM}$ HEPES (pH 8.5). $2 \mu \mathrm{L}$ of $0.5 \mathrm{mg} / \mathrm{mL}$ Trypsin was added to each sample. Samples were incubated at $37{ }^{\circ} \mathrm{C}$ for $12 \mathrm{~h}$. The beads were pelleted via centrifugation and washed water $(200 \mu \mathrm{L})$, and $50 \%$ acetonitrile / water $(200 \mu \mathrm{L})$. The supernatant and washes were collected as part of the "trypsin fraction." The trypsin fraction was concentrated to dryness using a SpeedVac heated to $45^{\circ} \mathrm{C}$. Trypsin fractions were suspended in $25 \mu \mathrm{L}$ water, the corresponding TMT reagent $(2 \mu \mathrm{L}, 20 \mu \mathrm{g} / \mu \mathrm{L}$ in ACN) was added, and the solutions rotated for $1 \mathrm{~h}$ at $24{ }^{\circ} \mathrm{C}$. Hydroxyammonia $(6 \mu \mathrm{L}, 5 \%)$ was added to each sample, which was rotated an additional 15 min to quench the reactions. All trypsin fractions were combined and dried via SpeedVac at $45^{\circ} \mathrm{C}$. The trypsin fraction was fractionated using Thermo Pierce High pH Reversed-Phase Peptide Fractionation Kit (\#84868). The fractions were dried and dissolved in $20 \mu \mathrm{L}$ of $0.1 \%$ formic acid.

Multiplexed photo-labeling of SK-N-SH cells with photo-probes for binding site LCMS/MS. Probes were sorted into six separate treatment groups according to Figure S8. Cells were grown to $90-100 \%$ confluency in $10 \mathrm{~cm}$ plates, then incubated with $10 \mu \mathrm{M}$ of each probe in the treatment group, or $0.5 \%$ DMSO (two plates per treatment group) in FBS-free media for $2 \mathrm{~h}$ at $37^{\circ} \mathrm{C}$. Following incubation, the plates were irradiated for $60 \mathrm{~s}$ at $4{ }^{\circ} \mathrm{C}$. The cells were harvested via scraping, collected in PBS $(10 \mathrm{~mL})$, pelleted $(2000$ g, $5 \mathrm{~min})$, and washed with PBS $(10 \mathrm{~mL})$. The pellets were resuspended in PBS $(500 \mu \mathrm{L})$ with $1 \%$ SDS and proteasome inhibitor then sonicated with a probe tip sonicator to lyse the cells. The lysates were cleared via centrifugation and the concentration of protein adjusted using the BCA assay (to concentrations of $\sim 1-1 \mathrm{mg} / \mathrm{mL}$ ). The lysates were reacted with a mixture of cleavable picolyl biotin azide probe S4 (100 $\mu \mathrm{M})$, copper (II) sulfate $(250 \mu \mathrm{M})$, THPTA $(250 \mu \mathrm{M})$, sodium ascorbate $(2.5 \mathrm{mM})$ for $3 \mathrm{~h}$ at $24{ }^{\circ} \mathrm{C}$ with rotation. The reaction was quenched with the addition of $\mathrm{MeOH}(1 \mathrm{~mL})$ followed by precipitation at $-80{ }^{\circ} \mathrm{C}$ for $1 \mathrm{~h}$. Proteins were pelleted by centrifugation $(21,000 \mathrm{~g}, 10 \mathrm{~min}$, $4{ }^{\circ} \mathrm{C}$ ). The supernatant was discarded and the pellets were dried under air for $5 \mathrm{~min}$.

Enrichment of photo-crosslinked proteins for binding site LC-MS/MS. Protein pellets were resuspended in $1 \%$ SDS in PBS $(500 \mu \mathrm{L})$ with brief sonication. $20 \mu \mathrm{L}$ of sample was taken as the "load fraction." Streptavidin-agarose resin $(200 \mu \mathrm{L}$, washed $3 \times 1 \mathrm{~mL}$ with PBS) was added to each sample, which was then incubated for $18 \mathrm{~h}$ at $24{ }^{\circ} \mathrm{C}$ with rotation. The slurries were transferred to mini biospin column (\#7326207) and centrifuged (1 min,

West, A. et al. Labeling preferences of diazirines with protein biomolecules, J. Am. Chem. 
$1000 \mathrm{~g}$ ). The supernatant collected as the "supernatant fraction." The columns were transferred to a vacuum manifold and washed with $1 \%$ SDS in PBS (1 mL), $8 \mathrm{M}$ fresh urea in PBS $(5 \times 1 \mathrm{~mL})$ and PBS $(5 \times 1 \mathrm{~mL})$. The beads were resuspended in PBS $(200 \mu \mathrm{L})$, reduced with $5 \mathrm{mM}$ dithiothreitol (DTT) for $30 \mathrm{~min}$ at $24{ }^{\circ} \mathrm{C}$, then alkylated with $10 \mathrm{mM}$ iodoacetamide for $30 \mathrm{~min}$ at $24{ }^{\circ} \mathrm{C}$ in the dark with rotation. The columns were transferred to the vacuum manifold and washed with PBS $(1 \mathrm{~mL})$ and $50 \mathrm{mM}$ HEPES $(1 \mathrm{~mL}, \mathrm{pH} 8.5)$. The beads were centrifuged then washed with PBS $(1 \mathrm{~mL})$ and resuspended in $250 \mu \mathrm{L}$ $0.5 \mathrm{M}$ guanidine- $\mathrm{HCl} / 50 \mathrm{mM}$ HEPES ( $\mathrm{pH} 8.5$ ). $2 \mu \mathrm{L}$ of $0.5 \mathrm{mg} / \mathrm{mL}$ Trypsin was added to each sample. Samples were incubated at $37{ }^{\circ} \mathrm{C}$ for $12 \mathrm{~h}$. The beads were pelleted via centrifugation and washed water $(200 \mu \mathrm{L})$, and $50 \%$ acetonitrile / water $(200 \mu \mathrm{L})$. The supernatant and washes were collected as part of the "trypsin fraction." The beads were

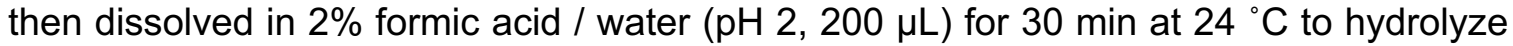
the cleavable biotin probe $\mathbf{S 4}$. These samples were centrifuged and the supernatant collected as the "cleavage fraction." Both the cleavage fractions were concentrated to dryness using a SpeedVac heated to $45^{\circ} \mathrm{C}$. The cleavage fraction samples were desalted using a C18 Ziptip according to the previously described procedure with elution in $0.1 \%$ formic acid $/ 75 \%$ acetonitrile/water. ${ }^{6}$ The elutions were concentrated to dryness using a SpeedVac and resuspended in $20 \mu \mathrm{L} 0.1 \%$ formic acid/water. 


\section{Mass Spectrometry Procedures.}

Mass spectrometry procedures. The desalted samples were resuspended in $0.1 \%$ formic acid/water $(20 \mu \mathrm{L})$. The sample $(10.0 \mu \mathrm{L})$ was loaded onto a C18 trap column (3 $\mathrm{cm}, 3 \mu \mathrm{m}$ particle size C10 Dr. Maisch $150 \mu \mathrm{m}$ I.D) and then separated on an analytical column (Thermo Scientific Acclaim PepMap 100, 2 um particle size, $250 \mathrm{~mm}$ length, 75 $\mu \mathrm{m}$ internal diameter) at $150 \mathrm{~nL} / \mathrm{min}$ with a Thermo Scientific Ultimate 3000 system connected in line to a Thermo Scientific Orbitrap Fusion Tribrid. The column temperature was maintained at $50{ }^{\circ} \mathrm{C}$. The tryptic peptides were separated via a step-wise gradient from $5 \%$ to $98 \%$ of $0.1 \%$ formic acid/acetonitrile over $120 \mathrm{~min}(0-1 \mathrm{~min}, 0-5 \%$; $1-91 \mathrm{~min}$, 5-27\%; 91-115 min, 27-98\%; 115-120 min, 98\%-0\%). The cleavage peptides were separated via the same gradient described above. Survey scans of peptide precursors were performed at $120 \mathrm{~K}$ FWHM resolution $(\mathrm{m} / \mathrm{z}=200)$. Tandem MS was performed on the most abundant precursors exhibiting a charge state from 2 to 6 at a resolving power settings of $50 \mathrm{~K}$. HCD fragmentation was applied with $35 \%$ collision energy and resulting fragments accumulated for up to $100 \mathrm{~ms}$.

\section{Data Analysis Procedures.}

Quantitative analysis of photolabeled SK-N-SH proteome. Analysis was performed in Thermo Scientific Proteome Discoverer version 2.3. HCD spectra with a signal-to-noise ratio greater than 1.5 were searched against a database containing the Uniprot 2016 annotated human proteome (Swissprot) and contaminant proteins using Sequest HT with a mass tolerance of $10 \mathrm{ppm}$ for the precursor and $0.02 \mathrm{Da}$ for fragment ions with specific trypsin digestion, 2 missed cleavages, variable oxidation on methionine residues $(+15.995$ $\mathrm{Da})$, static carboxyamidomethylation of cysteine residues (+57.021 Da), and static TMT labeling at lysine residues and N-termini. Assignments were validated using Percolator. The resulting peptide spectral matches (PSMs) were filtered to include medium and high confidence matches, and TMT reporter ions were quantified using the Reporter lons Quantifier. PSMs were filtered based on if a PSM is in only one protein group with an Isolation Interference under $70 \%$. Empty abundances were filled in with minimum noise level computed by taking the minimum for each channel in Control and minimum for each channel in Treatment. 2000 centroids were generated at random from the absolute max in Control and Treatment and the absolute min in Control and Treatment, and a minimum noise level was generated using a K-means clustering method. If one abundance was missing, then the instance was filled with the geometric mean of the PSM for Control or Treatment. If all abundances were missing for Control and Treatment or the variance between existing abundances was above 30\%, the PSM was removed. Any empty abundance missing completely at random, missing not at random, or missing at random any valid instances were filled with the appropriate method described above. P-values for enrichment ratios were calculated using the t-test (background) method.

Binding site analysis. Data analysis was performed with Proteome Discoverer version 2.3 using SEQUEST HT, allowing for variable modifications (methionine oxidation: +15.995 Da; cysteine carbamidomethylation: +57.021 Da; asparagine/glutamine deamidation: +0.984 ; and $\mathrm{JN}$ compound masses from Table S6), up to two missed cleavages and a mass tolerance of $10 \mathrm{ppm}$ for the precursor ion and $0.02 \mathrm{Da}$ for fragment ions from HCD. Searching was performed against the Swiss-Prot human database and a contaminant protein database. For binding sites of JN probes, MS/MS data from the cleavage fraction were searched by SEQUEST HT against the Uniprot 2016 annotated human proteome (Swissprot) and contaminant proteins. The high and medium confidence 
peptide assignments (false discovery rate $<5 \%$ ) were analyzed using IsoStamp for the precursor isotope pattern and filtered based on manual validation.

Table S6. List of probes and modification masses

\begin{tabular}{|c|c|c|c|}
\hline Probe & $\begin{array}{c}\text { Treatment } \\
\text { Group }\end{array}$ & JNXX_CBPA_si0 & JNXX_CBPA_si2 \\
\hline JN12 & 1 & 451.1856 & 453.1923 \\
\hline JN5 & 1 & 492.2234 & 494.2301 \\
\hline JN4 & 1 & 592.3162 & 594.3229 \\
\hline JN3 & 1 & 502.2696 & 504.2759 \\
\hline JN1 & 1 & 372.1910 & 374.1977 \\
\hline JN22 & 2 & 494.2278 & 496.2345 \\
\hline JN21 & 2 & 452.1808 & 454.1875 \\
\hline JN20 & 2 & 451.1856 & 453.1923 \\
\hline JN17 & 2 & 465.2012 & 467.2079 \\
\hline JN13 & 2 & 465.2012 & 467.2079 \\
\hline JN24 & 3 & 518.2754 & 520.2821 \\
\hline JN33 & 3 & 479.2169 & 481.2236 \\
\hline JN32 & 3 & 479.1805 & 481.1872 \\
\hline JN28 & 3 & 494.2278 & 496.2345 \\
\hline JN26 & 3 & 450.2379 & 452.2446 \\
\hline JN835 & 4 & 546.2703 & 548.2770 \\
\hline JN248 & 4 & 563.2605 & 565.2672 \\
\hline JN247 & 4 & 474.2016 & 476.2083 \\
\hline JN245 & 4 & 537.2336 & 539.2403 \\
\hline JN38 & 4 & 648.3999 & 650.4066 \\
\hline JN936 & 5 & 465.1873 & 467.1940 \\
\hline JN935 & 5 & 492.2234 & 494.2301 \\
\hline JN847 & 5 & 448.2084 & 450.2151 \\
\hline JN846 & 5 & 464.2033 & 466.2100 \\
\hline JN845 & 5 & 449.1924 & 451.1991 \\
\hline JN836 & 5 & 565.2649 & 567.2716 \\
\hline JN945 & 6 & 449.2175 & 451.2242 \\
\hline JN942 & 6 & 493.2186 & 495.2253 \\
\hline JN940 & 6 & 464.2284 & 466.2351 \\
\hline JN939 & 6 & 608.3111 & 610.3178 \\
\hline JN938 & 6 & 580.3162 & 582.3229 \\
\hline JN849 & 6 & 522.2743 & 524.2810 \\
\hline
\end{tabular}




\section{Synthetic Procedures:}

Ketone $\mathbf{S} 1$ and amine $\mathbf{S} 6$ were prepared according to Li et al. ${ }^{5}$ Trifluoromethyl diazirine S8 was synthesized following procedures adapted from Nassal, M. ${ }^{7}$ Difluoro ester $\mathbf{S 1 0}$ was prepared according to Fustero et al. ${ }^{8}$

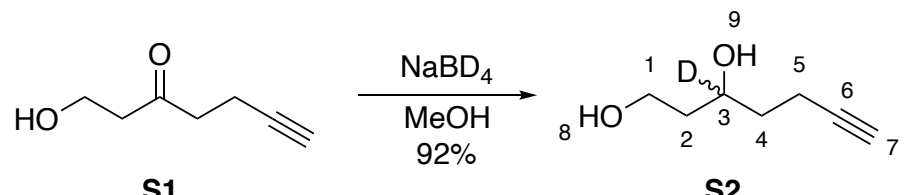

S1

S2

\section{Synthesis of deuterated diol (S2):}

Sodium borodeuteride $(5.0 \mathrm{mg}, 119 \mu \mathrm{mol}, 1.50$ equiv) was added to a solution of the ketone $\mathbf{S} 1(9.9 \mathrm{mg}, 79.0 \mu \mathrm{mol}, 1$ equiv) in $\mathrm{MeOH}(1.2 \mathrm{~mL})$. The resulting solution was stirred at $24{ }^{\circ} \mathrm{C}$ for $30 \mathrm{~min}$. The reaction was concentrated via $\mathrm{N}_{2}$ flow, then diluted with water $(1 \mathrm{~mL})$ and extracted in situ with dichloromethane / $i-\mathrm{PrOH}(5 \times 1 \mathrm{~mL}, 3: 1$ mixture). The organic layers were combined, washed with brine $(1 \mathrm{~mL})$ and dried over $\mathrm{Na}_{2} \mathrm{SO}_{4}$. The dried organic layer was filtered over a cotton plug and concentrated in vacuo. The crude material was purified via flash chromatography $(0-5 \% \mathrm{MeOH}$ in DCM) to yield $\mathbf{S 2}(9.4 \mathrm{mg}$, $728 \mu \mathrm{mol}, 93 \%)$ as a clear oil.

$\mathbf{R}_{\mathbf{f}}=0.3$ (50\% EtOAc in hexane, $p$-anisaldehyde stain) ${ }^{1} \mathbf{H} \mathbf{N M R}\left(500 \mathrm{MHz}, \mathrm{CDCl}_{3}\right) \delta 4.02$ - $3.76\left(\mathrm{~m}, 2 \mathrm{H}, \mathrm{H}_{1}\right), 2.82\left(\mathrm{~s}, 1 \mathrm{H}, \mathrm{O}_{8}\right), 2.41\left(\mathrm{~s}, 1 \mathrm{H}, \mathrm{O}_{9}\right), 2.34\left(\mathrm{tt}, J=6.8,2.7 \mathrm{~Hz}, 2 \mathrm{H}, \mathrm{H}_{5}\right), 1.98$ $\left(\mathrm{t}, J=2.7 \mathrm{~Hz}, 1 \mathrm{H}, \mathrm{H}_{7}\right), 1.81-1.64\left(\mathrm{~m}, 4 \mathrm{H}, \mathrm{H}_{2}\right.$ and $\left.\mathrm{H}_{4}\right) \cdot{ }^{13} \mathrm{C} \mathrm{NMR}\left(126 \mathrm{MHz}, \mathrm{CDCl}_{3}\right) \delta 83.38$ (s, $\left.\mathrm{C}_{6}\right), 70.48\left(\mathrm{t}, J=22.1 \mathrm{~Hz}, \mathrm{C}_{3}\right), 67.71\left(\mathrm{~s}, \mathrm{C}_{7}\right), 61.61\left(\mathrm{~s}, \mathrm{C}_{1}\right), 38.64\left(\mathrm{~s}, \mathrm{C}_{2}\right), 34.97\left(\mathrm{~s}, \mathrm{C}_{4}\right)$, 14.80 (s, C 5 ). IR (ATR-FTIR) $\mathrm{cm}^{-1}:$ 3373.93, 3283.47, 2922.55, 1436.59, 1111.62, 1059.01. HRMS-ESI $(\mathrm{m} / \mathrm{z}):[\mathrm{M}+\mathrm{H}]^{+}$calculated for $\mathrm{C}_{7} \mathrm{H}_{11} \mathrm{DO}_{2} \mathrm{H}^{+}=130.0973$; found 130.0973

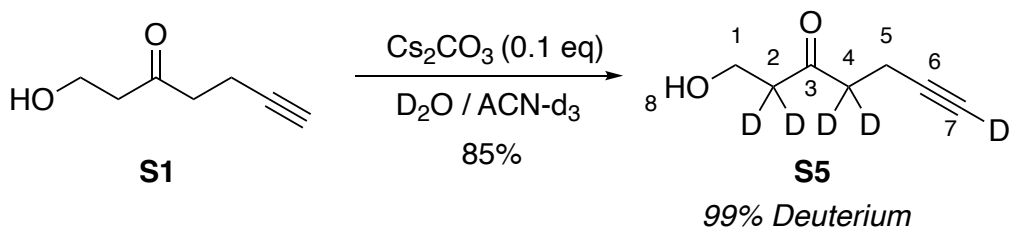

\section{Synthesis of deuterated ketone (S5):}

Cesium carbonate ( $24.0 \mathrm{mg}, 74 \mu \mathrm{mol}, 0.10$ equiv) was added to a solution of the ketone S1 (93.0 mg, $737 \mu \mathrm{mol}, 1$ equiv) in $20 \%$ acetonitrile- $d_{3}$ in $\mathrm{D}_{2} \mathrm{O}(2.9 \mathrm{~mL})$. The solution was stirred at $24{ }^{\circ} \mathrm{C}$ under $\mathrm{N}_{2}$ for $18 \mathrm{~h}$. When the \% deuterium was above $99 \%$ as determined by ${ }^{1} \mathrm{H} N M R$, the reaction was extracted with dichloromethane $(5 \times 5 \mathrm{~mL})$. The organic layers were combined, washed with brine $(5 \mathrm{~mL})$ and dried over $\mathrm{Na}_{2} \mathrm{SO}_{4}$. The dried organic layer was filtered over cotton and concentrated in vacuo to afford S5 $(82 \mathrm{mg}, 628 \mu \mathrm{mol}$, $85 \%)$ as a clear oil. 
$\mathbf{R}_{\mathbf{f}}=0.35$ (50\% EtOAc in hexane, $p$-anisaldehyde stain) ${ }^{1} \mathbf{H}$ NMR $\left(500 \mathrm{MHz}, \mathrm{CDCl}_{3}\right) \delta 3.86$ $\left(\mathrm{d}, J=7.9 \mathrm{~Hz}, 2 \mathrm{H}, \mathrm{H}_{1}\right), 2.46\left(\mathrm{~s}, 2 \mathrm{H}, \mathrm{H}_{5}\right), 2.30$ (t, $\left.J=7.9 \mathrm{~Hz}, 1 \mathrm{H}, \mathrm{H}_{8}\right) .{ }^{13} \mathrm{C}$ NMR $(126 \mathrm{MHz}$, $\mathrm{CDCl}_{3}$ ) $\delta 209.29\left(\mathrm{~s}, \mathrm{C}_{3}\right), 82.28\left(\mathrm{t}, J=8.2 \mathrm{~Hz}, \mathrm{C}_{6}\right), 68.73\left(\mathrm{t}, J=38.0 \mathrm{~Hz}, \mathrm{C}_{7}\right), 57.65\left(\mathrm{~s}, \mathrm{C}_{1}\right)$, $43.91\left(\mathrm{t}, J=19.2 \mathrm{~Hz}, \mathrm{C}_{2}\right), 41.14\left(\mathrm{t}, J=20.0,18.4 \mathrm{~Hz}, \mathrm{C}_{4}\right), 12.62\left(\mathrm{C}_{5}\right)$. IR (ATR-FTIR) $\mathrm{cm}^{-1}$ : 3550.19, 2948.89, 2582.74, 1972.29, 1699.22, 1433.69, 1286.12, 1226.34, 1060.86, 1007.56. HRMS-ESI $(\mathrm{m} / \mathrm{z})$ : $[\mathrm{M}+\mathrm{H}]^{+}$calculated for $\mathrm{C}_{7} \mathrm{H}_{5} \mathrm{D}_{5} \mathrm{O}_{2}+\mathrm{H}^{+}:=132.1067$; found 132.1067

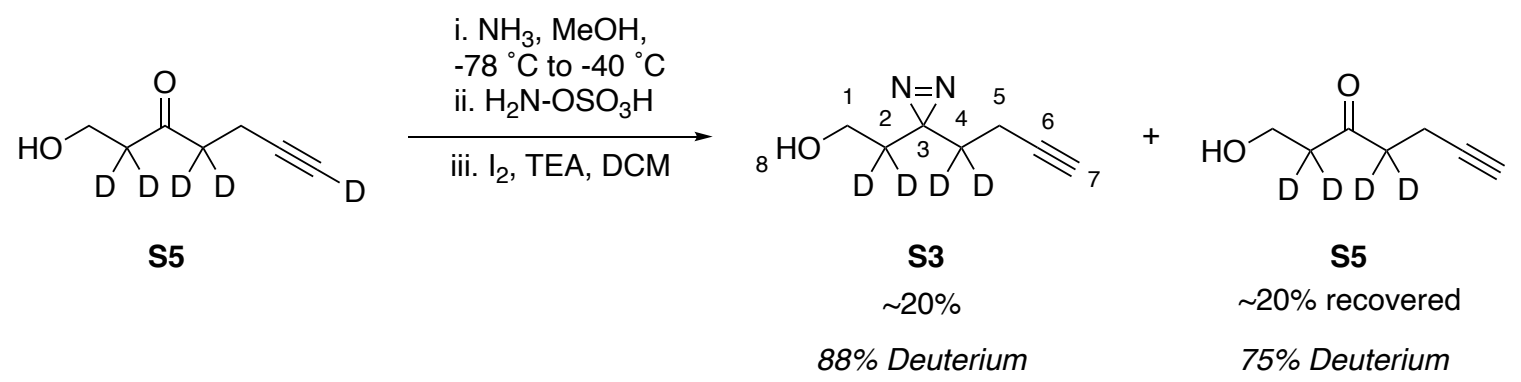

Synthesis of deuterated diazirine (S3):

A solution of deuterated ketone $\mathbf{S 5}(55.0 \mathrm{mg}, 420 \mu \mathrm{mol}, 1$ equiv) in $\mathrm{MeOH}(1.1 \mathrm{~mL})$ was cooled to $-78{ }^{\circ} \mathrm{C}$ under $\mathrm{N}_{2}$ flow. Ammonia was condensed into the flask $(\sim 5 \mathrm{~mL})$ and the reaction stirred for $30 \mathrm{~min}$ at $-78{ }^{\circ} \mathrm{C}$. The solution was warmed to $-40{ }^{\circ} \mathrm{C}$ and stirred an additional $8 \mathrm{~h}$. A solution of hydroxylamine-O-sulfonic acid ( $56.0 \mathrm{mg}, 500 \mu \mathrm{mol}, 1.20$ equiv) in $\mathrm{MeOH}(500 \mu \mathrm{L})$ was added and the reaction warmed to $24{ }^{\circ} \mathrm{C}$ overnight with a bubbler to vent pressure. The reaction was concentrated in vacuo to afford a white film which was dissolved in dichloromethane / MeOH $\left(0.1 \mathrm{M}, 1: 1\right.$ mixture) and cooled to $0{ }^{\circ} \mathrm{C}$. Triethylamine $(115 \mu \mathrm{L}, 830 \mu \mathrm{mol}, 1.50$ equiv) was added followed by titration with a solution of $\mathrm{I}_{2}$ in dichloromethane / $\mathrm{MeOH}(1 \mathrm{M}, 2: 1$ mixture) until a brown color persists in solution. The reaction was stirred an additional $30 \mathrm{~min}$, then quenched with sat sodium thiosulfate $(1 \mathrm{~mL})$. The solution was diluted with sat $\mathrm{NH}_{4} \mathrm{Cl}(10 \mathrm{~mL})$ and extracted with dichloromethane $(3 \times 10 \mathrm{~mL})$. The organic layers were washed with brine $(10 \mathrm{~mL})$, dried over $\mathrm{Na}_{2} \mathrm{SO}_{4}$, filtered and concentrated in vacuo. The crude mixture was purified via flash chromatography to afford S3 (12 mg, $80 \mu \mathrm{mol}, 20 \%)$ as a clear oil and starting material S5 without deuterium on the alkyne (11 mg, $80 \mu \mathrm{mol}, 20 \%)$. Notably, the percentage of deuterium in product $\mathbf{S 3}$ was $88 \%$ while the percentage in recovered starting material $\mathbf{S 5}$ dropped to $75 \%$.

$\mathbf{R}_{\mathbf{f}}=0.6$ (50\% EtOAc in hexane, $p$-anisaldehyde stain). ${ }^{1} \mathbf{H}$ NMR $\left(500 \mathrm{MHz}, \mathrm{CDCl}_{3}\right) \delta 3.49$ (d, J = 4.7 Hz, 2H, $\mathrm{H}_{1}$ ), $2.03\left(\mathrm{~s}, 2 \mathrm{H}, \mathrm{H}_{5}\right), 2.00\left(\mathrm{t}, J=2.7 \mathrm{~Hz}, 1 \mathrm{H}, \mathrm{H}_{7}\right), 1.44(\mathrm{t}, J=5.3 \mathrm{~Hz}$, $\left.1 \mathrm{H}, \mathrm{H}_{8}\right) .{ }^{13} \mathrm{C}$ NMR $\left(126 \mathrm{MHz}, \mathrm{CDCl}_{3}\right) \delta 82.84\left(\mathrm{~s}, \mathrm{C}_{6}\right), 69.20\left(\mathrm{~s}, \mathrm{C}_{7}\right), 57.31\left(\mathrm{~s}, \mathrm{C}_{1}\right), 34.77(\mathrm{t}$, $\left.J=20.7 \mathrm{~Hz}, \mathrm{C}_{2}\right), 32.27\left(\mathrm{t}, J=20.5 \mathrm{~Hz}, \mathrm{C}_{3}\right), 26.38\left(\mathrm{~s}, \mathrm{C}_{3}\right), 13.05\left(\mathrm{~s}, \mathrm{C}_{5}\right)$. IR (ATR-FTIR) $\mathrm{cm}^{-}$ 1: 3294.79, 2921.87, 2888.40, 2587.17, 2214.59, 2118.45, 1694.02, 1584.84, 1470.99, 1433.20, 1378.32, 1314.45, 1046.86. HRMS-ESI $(\mathrm{m} / \mathrm{z}):[\mathrm{M}+\mathrm{H}]^{+}$calculated for $\mathrm{C}_{7} \mathrm{H}_{6} \mathrm{D}_{4} \mathrm{~N}_{2} \mathrm{O}+\mathrm{H}^{+}=143.1117$; found 143.1116

West, A. et al. Labeling preferences of diazirines with protein biomolecules, J. Am. Chem. 


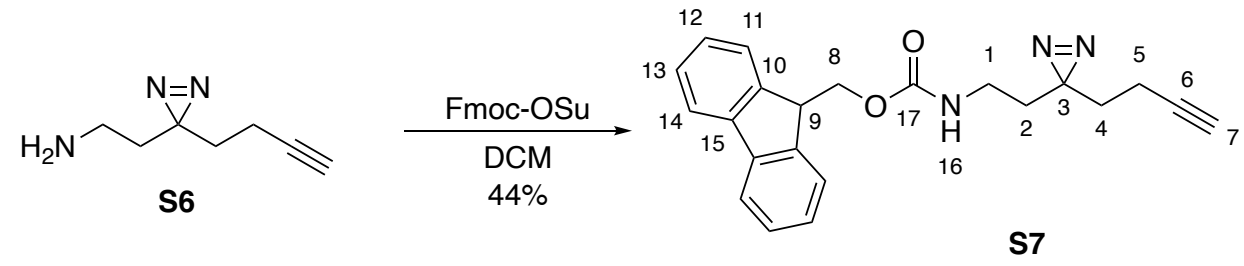

\section{Synthesis of Fmoc diazirine (S7):}

Fmoc-OSu (53.0 mg, $0.16 \mathrm{mmol}, 1.20$ equiv) was added to a solution of amine $\mathbf{S 6}$ (18.0 $\mathrm{mg}, 0.13 \mathrm{mmol}, 1$ equiv) in DCM $(1.6 \mathrm{~mL})$. The reaction was stirred at $24{ }^{\circ} \mathrm{C}$ under $\mathrm{N}_{2}$ for 2 hours. The reaction was diluted with sat. $\mathrm{NH}_{4} \mathrm{Cl}(5 \mathrm{~mL})$ and washed with dichloromethane $(3 \times 5 \mathrm{~mL})$. The organic layers were combined, washed with brine $(5 \mathrm{~mL})$ and dried over $\mathrm{Na}_{2} \mathrm{SO}_{4}$ The dried organic layer was filtered through a cotton plug and concentrated in vacuo. The crude material was purified via column chromatography (0$30 \%$ EtOAc in hexane over $40 \mathrm{CV}$ ) to yield $\mathbf{S 7}(25 \mathrm{mg}, 70 \mu \mathrm{mol}, 44 \%)$ as a clear oil.

$\mathbf{R}_{\mathbf{f}}=0.3\left(20 \%\right.$ EtOAc in hexane, UV) ${ }^{1} \mathrm{H}$ NMR $\left(500 \mathrm{MHz}, \mathrm{CDCl}_{3}\right) \delta 7.77(\mathrm{~d}, J=7.5 \mathrm{~Hz}, 2 \mathrm{H}$, $\mathrm{H}_{11}$ ), 7.60 (d, J = 7.6 Hz, 2H, $\left.\mathrm{H}_{14}\right), 7.41\left(\mathrm{t}, J=7.5 \mathrm{~Hz}, 2 \mathrm{H}, \mathrm{H}_{12}\right), 7.32$ (t, J = $7.4 \mathrm{~Hz}, 2 \mathrm{H}$, $\left.\mathrm{H}_{13}\right), 4.85\left(\mathrm{~s}, 1 \mathrm{H}, \mathrm{H}_{16}\right), 4.41\left(\mathrm{~d}, J=7.0 \mathrm{~Hz}, 2 \mathrm{H}, \mathrm{H}_{8}\right), 4.22\left(\mathrm{t}, J=6.9 \mathrm{~Hz}, 1 \mathrm{H}, \mathrm{H}_{9}\right), 3.06(\mathrm{q}, J$ $\left.=6.5 \mathrm{~Hz}, 2 \mathrm{H}, \mathrm{H}_{1}\right), 2.02\left(\mathrm{td}, J=7.0,2.1 \mathrm{~Hz}, 2 \mathrm{H}, \mathrm{H}_{5}\right), 1.99\left(\mathrm{t}, J=2.4 \mathrm{~Hz}, 1 \mathrm{H}, \mathrm{H}_{7}\right), 1.70(\mathrm{t}, J$ $\left.=6.7 \mathrm{~Hz}, 2 \mathrm{H}, \mathrm{H}_{2}\right), 1.66\left(\mathrm{t}, J=7.3 \mathrm{~Hz}, 2 \mathrm{H}, \mathrm{H}_{4}\right) \cdot{ }^{13} \mathrm{C} \mathrm{NMR}\left(101 \mathrm{MHz}, \mathrm{CDCl}_{3}\right) \delta 156.17(\mathrm{~s}$, $\mathrm{C}_{17}$ ), 143.88 (s, $\mathrm{C}_{10}$ ), 141.33 (s, $\mathrm{C}_{15}$ ), 127.69 (s, $\left.\mathrm{C}_{13}\right), 127.04$ (s, $\left.\mathrm{C}_{14}\right), 125.01$ (s, $\mathrm{C}_{12}$ ), $119.98\left(\mathrm{~s}, \mathrm{C}_{11}\right), 82.60\left(\mathrm{~s}, \mathrm{C}_{6}\right), 69.42\left(\mathrm{~s}, \mathrm{C}_{7}\right), 66.67\left(\mathrm{~s}, \mathrm{C}_{8}\right), 47.24\left(\mathrm{~s}, \mathrm{C}_{1}\right), 35.76\left(\mathrm{~s}, \mathrm{C}_{2}\right), 32.93$ (s, $\left.\mathrm{C}_{4}\right), 32.21\left(\mathrm{~s}, \mathrm{C}_{9}\right), 26.63\left(\mathrm{~s}, \mathrm{C}_{3}\right), 13.20\left(\mathrm{~s}, \mathrm{C}_{5}\right)$. IR (ATR-FTIR) cm $\mathrm{cm}^{-1}:$ 3295.13, 3064.69, 2943.49, 2117.31, 1698.29, 1520.51, 1472.58, 1443.92, 1370.08, 1321.89, 1241.86, 1137.97, 1021.76. HRMS-ESI $(\mathrm{m} / \mathrm{z})$ : $[\mathrm{M}+\mathrm{H}]^{+}$calculated for $\mathrm{C}_{22} \mathrm{H}_{21} \mathrm{~N}_{3} \mathrm{O}_{2}+\mathrm{H}^{+}=360.1707$; found 360.1708<smiles>O=C(O)c1ccc(C2(C(F)(F)F)N=N2)cc1</smiles>

S8

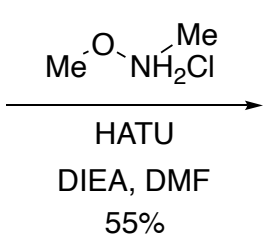

$55 \%$

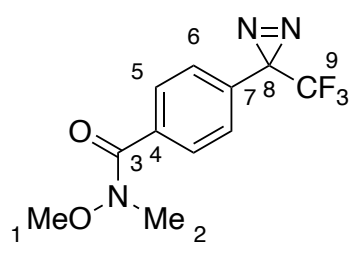

S9

\section{Synthesis of amide trifluoromethyl diazirine (S9):}

(1-[Bis(dimethylamino)methylene]-1H-1,2,3-triazolo[4,5-b]pyridinium

3-oxide hexafluorophosphate (HATU) $(148 \mathrm{mg}, 0.39 \mathrm{mmol}, 1.50$ equiv) diisopropylethylamine (68.0 $\mu \mathrm{L}, 0.39 \mathrm{mmol}, 1.50$ equiv) and Weinreb salt $(38.0 \mathrm{mg}, 0.39 \mathrm{mmol}, 1.50$ equiv) were added in sequence to a solution of the acid $\mathbf{S} 8(60.0 \mathrm{mg}, 0.26 \mathrm{mmol}, 1$ equiv) in DMF (1.3 $\mathrm{mL})$. The reaction was stirred at $24{ }^{\circ} \mathrm{C}$ under Ar for 3 hours then concentrated in vacuo. The crude mixture was dissolved in dichloromethane $(3 \mathrm{~mL})$, washed with $\mathrm{HCl}(1 \mathrm{~N}, 3 \mathrm{~mL})$, 
sat. $\mathrm{NaHCO}_{3}(1 \mathrm{~mL})$ and brine $(1 \mathrm{~mL})$. The organic layer was dried over $\mathrm{Na}_{2} \mathrm{SO}_{4}$ and concentrated to dryness. The crude material was purified via column chromatography to afford diazirine $\mathbf{S 9}$ (39 $\mathrm{mg}, 0.14 \mathrm{mmol}, 55 \%$ ).

$\mathbf{R}_{\mathbf{f}}=0.4$ (20\% EtOAc in hexane, UV). ${ }^{1} \mathrm{H}$ NMR (500 MHz, $\left.\mathrm{CDCl}_{3}\right) \delta 7.72\left(\mathrm{~d}, 2 \mathrm{H}, \mathrm{H}_{6}\right), 7.22$ (d, $2 \mathrm{H}, \mathrm{H}_{5}$ ), 3.52 (s, 3H, $\mathrm{H}_{1}$ ), 3.36 (s, 3H, $\left.\mathrm{H}_{2}\right) .{ }^{19} \mathrm{~F} \mathrm{NMR}\left(471 \mathrm{MHz}, \mathrm{CDCl}_{3}\right) \delta-65.00$ (s, $\left.\mathrm{C}_{9}\right)$.

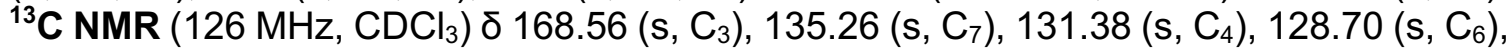
126.04 (s, C 5 ), 121.96 (q, J = $274.9 \mathrm{~Hz}, \mathrm{C}_{9}$ ), 61.20 (s, C 1 ), 33.38 (s, C 2 ). IR (ATR-FTIR) $\mathrm{cm}^{-1}: 1643.41,1418.99,1378.66,1285.49,1150.80$. HRMS-ESI $(\mathrm{m} / \mathrm{z}):[\mathrm{M}+\mathrm{H}]^{+}$calculated for $\mathrm{C}_{11} \mathrm{H}_{10} \mathrm{~F}_{3} \mathrm{~N}_{3} \mathrm{O}_{2}+\mathrm{H}^{+}=274.0798$; found 274.0800

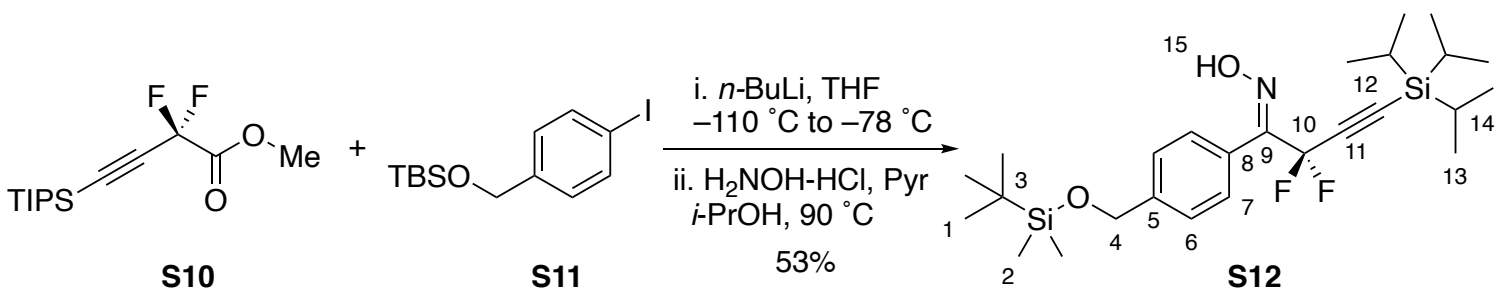

\section{Synthesis of difluoro oxime (S12):}

$n$-butyl lithium (2.03 M, $142 \mu \mathrm{L}, 0.29 \mathrm{mmol}, 1$ equiv) in hexane was added to a solution of $\mathbf{S} 10$ (84.0 mg, $0.29 \mathrm{mmol}, 1$ equiv) and $\mathbf{S} 11$ (101 $\mathrm{mg}, 0.29 \mathrm{mmol}, 1.00$ equiv) in THF (5.8 $\mathrm{mL}$ ) over 5 minutes, stirring at $-110{ }^{\circ} \mathrm{C}$ under $\mathrm{N}_{2}$. The reaction was warmed to $-78{ }^{\circ} \mathrm{C}$ and stirred for $30 \mathrm{~min}$. The reaction was quenched with sat. $\mathrm{NH}_{4} \mathrm{Cl}(10 \mathrm{~mL})$ and washed with ethyl acetate (EtOAc) $(3 \times 10 \mathrm{~mL})$. The organic layers were combined, washed with brine $(10 \mathrm{~mL})$, dried over $\mathrm{Na}_{2} \mathrm{SO}_{4}$ and concentrated in vacuo. The crude oil was dissolved in $i-$ $\mathrm{PrOH}$ (1.45 mL), $\mathrm{H}_{2} \mathrm{NOH}-\mathrm{HCl}$ (101 mg, $1.45 \mathrm{mmol}, 5.00$ equiv) and pyridine (233 $\mu \mathrm{L}, 299.4$ $\mathrm{mg}, 2.9 \mathrm{mmol}, 10.0$ equiv) were added and the solution warmed to $90{ }^{\circ} \mathrm{C}$ stirring under $\mathrm{N}_{2}$ for $2 \mathrm{~h}$. The reaction was cooled to $0{ }^{\circ} \mathrm{C}$, diluted with $1 \mathrm{~N} \mathrm{HCl}$ and washed with dichloromethane. The organic layers were combined, washed with brine and dried over $\mathrm{Na}_{2} \mathrm{SO}_{4}$. The crude oil was purified via column chromatography $(0-5 \%$ EtOAc in hexane over $30 \mathrm{CV}$ ) to yield $76 \mathrm{mg}$ of $\mathbf{S 1 2}(76 \mathrm{mg}, 0.15 \mathrm{mmol}, 53 \%)$ as an orange solid.

*Note: purification of the ketone from the initial Li-l exchange proved difficult due to very similar $R_{f}$ between the ketone and both starting materials. The oxime formation could be performed on the crude product of the first reaction, and was much more amenable to purification.

$\mathbf{R}_{\mathbf{f}}=0.4$ (3\% EtOAc in hexane, UV). ${ }^{1} \mathbf{H}$ NMR $\left(500 \mathrm{MHz}, \mathrm{CDCl}_{3}\right) \delta 8.26\left(\mathrm{~s}, 1 \mathrm{H}, \mathrm{H}_{15}\right), 7.45$ $\left(\mathrm{d}, J=8.3 \mathrm{~Hz}, 2 \mathrm{H}, \mathrm{H}_{7}\right), 7.42-7.36\left(\mathrm{~m}, 2 \mathrm{H}, \mathrm{H}_{6}\right), 4.77\left(\mathrm{~s}, 3 \mathrm{H}, \mathrm{H}_{4}\right), 1.09-0.99\left(\mathrm{~m}, 20 \mathrm{H}, \mathrm{H}_{13}\right)$, $0.98-0.89\left(\mathrm{~m}, 9 \mathrm{H}, \mathrm{H}_{1}\right), 0.15-0.05\left(\mathrm{~m}, 6 \mathrm{H}, \mathrm{H}_{2}\right) .{ }^{13} \mathrm{C}$ NMR $\left(126 \mathrm{MHz}, \mathrm{CDCl}_{3}\right) \delta 152.77(\mathrm{t}$, $\left.J=29.9 \mathrm{~Hz}, \mathrm{C}_{9}\right), 143.50$ (s, $\left.\mathrm{C}_{5}\right), 128.80\left(\mathrm{~s}, \mathrm{C}_{6}\right), 125.84\left(\mathrm{~s}, \mathrm{C}_{7}\right), 125.61$ (s, $\left.\mathrm{C}_{8}\right), 110.29$ (t, J $\left.=233.3 \mathrm{~Hz}, \mathrm{C}_{10}\right), 96.74\left(\mathrm{t}, J=37.5 \mathrm{~Hz}, \mathrm{C}_{11}\right), 94.09$ (t, $\left.\mathrm{C}_{12}\right), 64.49$ (s, C $\left.)_{4}\right), 25.93\left(\mathrm{~s}, \mathrm{C}_{1}\right)$, 18.66 (s, $\mathrm{C}_{2}$ ), 18.38 (s, $\left.\mathrm{C}_{13}\right), 10.87\left(\mathrm{~s}, \mathrm{C}_{14}\right),-5.31\left(\mathrm{~s}, \mathrm{C}_{2}\right) .{ }^{19} \mathrm{~F} \mathrm{NMR}\left(471 \mathrm{MHz}, \mathrm{CDCl}_{3}\right) \delta-$ 80.90 (s, F $\mathrm{F}_{10}$ ). IR (ATR-FTIR) $\mathrm{cm}^{-1}:$ 3276.67, 2945.83, 2928.29, 2892.67, 2865.44, 1702.95, 1686.50, 1625.42, 1613.35, 1462.74. HRMS-ESI $(\mathrm{m} / \mathrm{z}):[\mathrm{M}+\mathrm{H}]^{+}$calculated for $\mathrm{C}_{26} \mathrm{H}_{43} \mathrm{~F}_{2} \mathrm{NO}_{2} \mathrm{Si}_{2}+\mathrm{H}^{+}=492.2873$; found 496.2872 


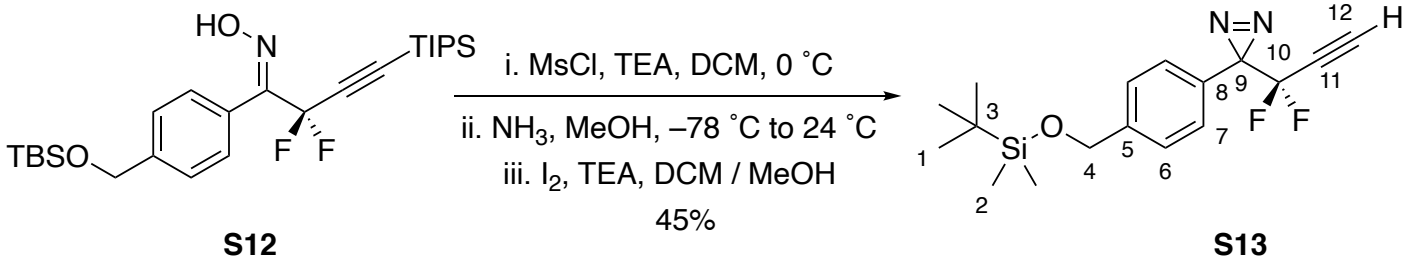

\section{Synthesis of protected aryl difluoro diazirine (S13):}

Mesyl chloride ( $9.84 \mu \mathrm{L}, 14.6 \mathrm{mg}, 0.127 \mathrm{mmol}, 1.50$ equiv) was added to a solution of $\mathbf{S 1 2}$ $(42.0 \mathrm{mg}, 85.0 \mu \mathrm{mol}, 1$ equiv) in dichloromethane $(850 \mu \mathrm{L})$ and triethylamine $(22.5 \mu \mathrm{L}$, $17.2 \mathrm{mg}, 0.169 \mathrm{mmol}, 2.00$ equiv) at $0{ }^{\circ} \mathrm{C}$ under $\mathrm{N}_{2}$. The reaction was warmed to $24{ }^{\circ} \mathrm{C}$ over 2 hours, then diluted with sat $\mathrm{NH}_{4} \mathrm{Cl}$ and washed with ethyl acetate. The combined organic layers were washed with brine, dried over $\mathrm{Na}_{2} \mathrm{SO}_{4}$ and concentrated to dryness in vacuo. The crude oil was redissolved in $\mathrm{MeOH}(1 \mathrm{~mL})$ and cooled to $-78{ }^{\circ} \mathrm{C}$ under $\mathrm{N}_{2}$. Ammonia $(\sim 5 \mathrm{~mL})$ was condensed into the reaction, which was allowed to warm to $24{ }^{\circ} \mathrm{C}$ gradually over $18 \mathrm{~h}$ with a light flow of $\mathrm{N}_{2}$ and a bubbler to vent pressure. The reaction was concentrated in vacuo, then redissolved in dichloromethane and $\mathrm{MeOH}$ (2:1 mixture, $1.5 \mathrm{~mL})$. Triethylamine $(47.2 \mu \mathrm{L}, 34.3 \mathrm{mg}, 0.339 \mathrm{mmol}, 4$ equiv) was add to the reaction, stirring under $\mathrm{N}_{2}$ at $0{ }^{\circ} \mathrm{C}$. A solution of iodine in $\mathrm{MeOH}(1 \mathrm{M})$ was added dropwise to the reaction until a brown color persisted in solution. The reaction was stirred $30 \mathrm{~min}$, then sat. sodium thiosulfate was added until the brown color of the reaction turned clear. The solution was diluted with sat $\mathrm{NH}_{4} \mathrm{Cl}(3 \mathrm{~mL})$ and washed with ethyl acetate $(3 \times 3 \mathrm{~mL})$. The organic layers were combined, washed with brine $(3 \mathrm{~mL})$ and dried over $\mathrm{Na}_{2} \mathrm{SO}_{4}$. The crude oil was purified via column chromatography $(0-10 \%$ EtOAc in hexane over $30 \mathrm{CV})$ to yield $14 \mathrm{mg}$ of $\mathbf{S} 13$ (14 $\mathrm{mg}, 0.040 \mathrm{mmol}, 45 \%$ ) as a yellow oil.

*Note: deprotection of the TIPS group was unintentional, and a small amount of the product with the TIPS still intact is formed and has a similar $R_{f}$ to the protonated alkyne.

$\mathbf{R}_{\mathbf{f}}=0.5$ (5\% EtOAc in hexane, UV). ${ }^{1} \mathrm{H}$ NMR $\left(500 \mathrm{MHz}, \mathrm{CDCl}_{3}\right) \delta 7.32(\mathrm{~d}, \mathrm{~J}=8.0 \mathrm{~Hz}, 2 \mathrm{H}$, $\left.\mathrm{H}_{7}\right), 7.24\left(\mathrm{~d}, 2 \mathrm{H}, \mathrm{H}_{6}\right), 4.72\left(\mathrm{~s}, 2 \mathrm{H}, \mathrm{H}_{4}\right), 2.94\left(\mathrm{t}, J=5.1 \mathrm{~Hz}, 1 \mathrm{H}, \mathrm{H}_{12}\right), 0.92\left(\mathrm{~s}, 9 \mathrm{H}, \mathrm{H}_{1}\right), 0.08$ (s, 6H, H2). ${ }^{13} \mathrm{C}$ NMR (126 MHz, CDCl $) \delta 142.91$ (s, $\mathrm{C}_{5}$ ), 129.03 (s, $\mathrm{C}_{8}$ ), 127.01 (s, $\mathrm{C}_{6}$ ), 126.06 (s, $\mathrm{C}_{7}$ ), 110.47 (t, J=241.0 Hz, C 10 ), 79.98 (t, $J=6.4$ Hz, $\mathrm{C}_{12}$ ), 73.97 (t, J=40.6 $\left.\mathrm{Hz}, \mathrm{C}_{11}\right), 64.34\left(\mathrm{~s}, \mathrm{C}_{4}\right), 31.31$ (t, J=35.0 Hz, C 9 ), 25.91 (s, C 1 ), $17.98\left(\mathrm{~s}, \mathrm{C}_{3}\right),-5.31\left(\mathrm{~s}, \mathrm{C}_{2}\right)$. ${ }^{19} \mathrm{~F}$ NMR $\left(471 \mathrm{MHz}, \mathrm{CDCl}_{3}\right) \delta-82.47\left(\mathrm{~d}, J=4.8 \mathrm{~Hz}, \mathrm{~F}_{10}\right)$. IR (ATR-FTIR) cm' ${ }^{-1}$ 2953.98, 2929.23, 2895.80, 2858.01, 2137.55, 1462.44. HRMS-ESI $(\mathrm{m} / \mathrm{z}):\left[\mathrm{M}+\mathrm{NH}_{4}\right]^{+}$calculated for $\mathrm{C}_{17} \mathrm{H}_{26} \mathrm{~F}_{2} \mathrm{~N}_{3} \mathrm{OSi}^{+}=354.1808$; found 354.1800
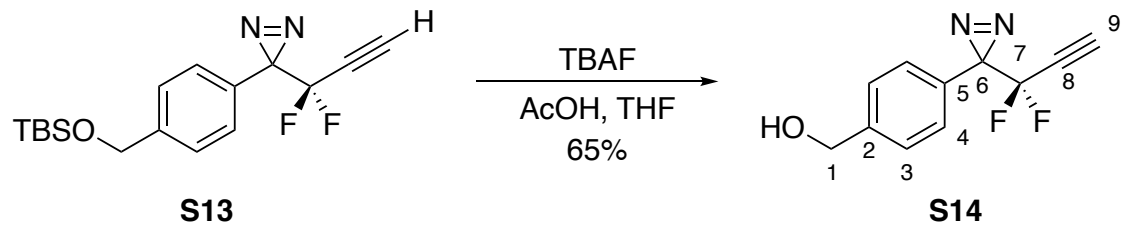

\section{Synthesis of aryl difluoro diazirine (S14):}

Tetrabutylammonium fluoride ( $1 \mathrm{M}, 92.0 \mu \mathrm{L}, 92.0 \mu \mathrm{mol}, 2.20$ equiv) was added to a solution of $\mathbf{S} 13$ (14.0 mg, $0.42 \mathrm{mmol}, 1$ equiv) in tetrahydrofuran $(830 \mu \mathrm{L})$ and acetic acid 
(6 $\mu \mathrm{L}, 0.104 \mathrm{mmol}, 2.50$ equiv) at $0{ }^{\circ} \mathrm{C}$ under Ar. The reaction was warmed to $24{ }^{\circ} \mathrm{C}$ over $18 \mathrm{~h}$, then diluted with water $(1 \mathrm{~mL})$ and extracted with ethyl acetate $(3 \times 1 \mathrm{~mL})$. The combined organic layers were washed with brine $(1 \mathrm{~mL})$, dried over $\mathrm{Na}_{2} \mathrm{SO}_{4}$ and concentrated in vacuo. The crude oil was purified via column chromatography $(20-50 \%$ EtOAc in hexane over $30 \mathrm{CV}$ ) to yield $6 \mathrm{mg}$ of $\mathbf{S 1 4}(6 \mathrm{mg}, 0.027 \mathrm{mmol}, 65 \%)$ as a clear oil.

$\mathbf{R}_{\mathbf{f}}=0.3\left(20 \%\right.$ EtOAc in hexane, UV). ${ }^{1} \mathbf{H}$ NMR $\left(500 \mathrm{MHz}, \mathrm{CDCl}_{3}\right) \delta 7.41-7.31(\mathrm{~m}, 2 \mathrm{H}$, $\left.\mathrm{H}_{4}\right), 7.26\left(\mathrm{~d}, J=8.6 \mathrm{~Hz}, 2 \mathrm{H}, \mathrm{H}_{3}\right), 4.70\left(\mathrm{~s}, 2 \mathrm{H}, \mathrm{H}_{1}\right), 2.95\left(\mathrm{t}, J=5.1 \mathrm{~Hz}, 1 \mathrm{H}, \mathrm{H}_{9}\right) .{ }^{13} \mathrm{C} \mathrm{NMR}$ (126 MHz, $\left.\mathrm{CDCl}_{3}\right) \delta 142.23$ (s, $\left.\mathrm{C}_{5}\right), 129.91\left(\mathrm{~s}, \mathrm{C}_{2}\right), 127.42\left(\mathrm{~s}, \mathrm{C}_{4}\right), 127.05\left(\mathrm{~s}, \mathrm{C}_{3}\right), 110.52$ $\left(\mathrm{t}, J=236.1 \mathrm{~Hz}, \mathrm{C}_{7}\right), 80.19\left(\mathrm{t}, J=6.4 \mathrm{~Hz}, \mathrm{C}_{9}\right), 73.96\left(\mathrm{t}, J=40.6 \mathrm{~Hz}, \mathrm{C}_{8}\right), 64.72\left(\mathrm{~s}, \mathrm{C}_{1}\right)$, $31.35\left(\mathrm{t}, J=34.8 \mathrm{~Hz}, \mathrm{C}_{6}\right) .{ }^{19} \mathrm{~F}$ NMR $\left(471 \mathrm{MHz} \mathrm{CDCl}_{3}\right) \delta-82.42\left(\mathrm{~d}, J=4.6 \mathrm{~Hz}, \mathrm{~F}_{7}\right)$. IR (ATRFTIR) $\mathrm{cm}^{-1}: 3297.45,2928.69,2873.72,2136.25,1610.02$ HRMS-ESI $(\mathrm{m} / \mathrm{z}):[\mathrm{M}+\mathrm{H}]^{+}$ calculated for $\mathrm{C}_{11} \mathrm{H}_{8} \mathrm{~F}_{2} \mathrm{~N}_{2} \mathrm{O}_{1}+\mathrm{H}^{+}=221.0530$; found 221.0532 
Synthetic Procedures for PAL Probes:<smiles>C#CCCC1(CCC(=O)NC)NN1</smiles>

Preparation of $\mathbf{J N}-0001, \mathbf{J N}-0003$ and $\mathbf{J N}-0004$ was performed as reported in Parker et al. $^{2}$

Synthesis of $\mathrm{JN}-0013$<smiles>COC(=O)Cc1ccc(O)cc1</smiles><smiles>C#CCCC1(CCI)N=N1</smiles><smiles>C#CCCC1(CCOc2ccc(CC(=O)OC)cc2)N=N1</smiles><smiles>O[Mg]O</smiles><smiles>C#CCCC1(CCOc2ccc(CC(=O)O)cc2)N=N1</smiles>

$\mathrm{JN}-0013$

\section{Step 1}

3-but-3-ynyl-3-(2-iodoethyl)diazirine (26.9 mg, $108.3 \mu \mathrm{mol}, 1.2$ equiv) was added to a solution of Methyl 2-(4-hydroxyphenyl)acetate (15 mg, $90.3 \mu \mathrm{mol}, \quad 1.0$ equiv) in dimethylformamide (DMF) (902.7 uL), and potassium carbonate $(25.0 \mathrm{mg}, 180.5$ $\mu \mathrm{mol}, 2$ equiv). The reaction mixture was stirred at $24{ }^{\circ} \mathrm{C} 12 \mathrm{~h}$. The reaction mixture was filtered, diluted with DMSO and purified by prep HPLC using $0.1 \%$ formic acid in water / $0.1 \%$ formic acid in acetonitrile as eluent to give pure intermediate 1 as a white solid (26 $\mathrm{mg}, 23 \%)$.

\section{Step 2}

$2 \mathrm{M}$ aq. $\mathrm{LiOH}(2.51 \mathrm{mg}, 104.8 \mathrm{umol}, 52 \mu \mathrm{L}, 2.00$ equiv) was added to a solution of intermediate 1 (6 mg, $21.0 \mu \mathrm{mol}, 1$ equiv) in a mixture of THF (293.4 $\mu \mathrm{L})$ and water $(125.7$ $\mu \mathrm{L})$. The reaction mixture was stirred at $24{ }^{\circ} \mathrm{C}$ overnight. $1 \mathrm{M}$ aq. $\mathrm{HCl}$ was slowly added and the resulting mixture was washed with dichloromethane $(x 3)$. The combined organic layers were dried over $\mathrm{Na}_{2} \mathrm{SO}_{4}$, filtered, and concentrated under vacuum. The crude was dissolved in DMSO and purified by prep HPLC using $0.1 \%$ formic acid in water $/ 0.1 \%$ formic acid in acetonitrile as eluent to give pure $\mathrm{JN}-0013$ as a white solid ( $3 \mathrm{mg}, 50 \%$ ). LC/MS (m/z): $[\mathrm{M}+\mathrm{H}]^{+}$273. ${ }^{1} \mathrm{H}$ NMR (400 MHz, DMSO- $\left.\mathrm{d}_{6}\right) \delta 7.16(\mathrm{~d}, J=8.0 \mathrm{~Hz}, 2 \mathrm{H}), 6.85$ $(\mathrm{d}, J=8.0 \mathrm{~Hz}, 2 \mathrm{H}), 3.79(\mathrm{t}, J=8.0 \mathrm{~Hz}, 2 \mathrm{H}), 3.48(\mathrm{~s}, 2 \mathrm{H}), 2.84(\mathrm{dd}, J=4.0,4.0 \mathrm{~Hz}, 1 \mathrm{H})$, $2.04(\mathrm{td}, J=8.0,4.0 \mathrm{~Hz}, 2 \mathrm{H}), 1.86(\mathrm{t}, J=8.0 \mathrm{~Hz}, 2 \mathrm{H}), 1.65(\mathrm{t}, J=8.0 \mathrm{~Hz}, 2 \mathrm{H})$. 
$\mathrm{JN}-0017, \mathrm{JN}-0020, \mathrm{JN}-0021$, and $\mathrm{JN}-0032$ were prepared using the same procedure as JN-0013.

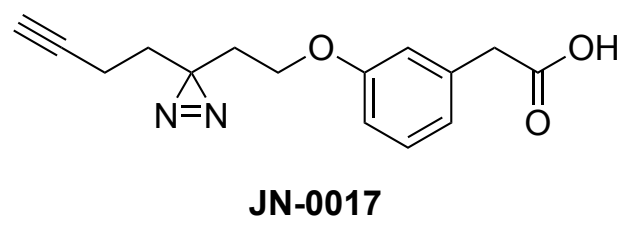

LC/MS (m/z): [M+H] ${ }^{+} 273 .{ }^{1} \mathrm{H} \mathrm{NMR}\left(400 \mathrm{MHz}, \mathrm{CDCl}_{3}\right) \delta 7.23(\mathrm{~s}, 1 \mathrm{H}), 6.89(\mathrm{~d}, J=7.8 \mathrm{~Hz}$, $1 \mathrm{H}), 6.86-6.76(\mathrm{~m}, 2 \mathrm{H}), 3.83(\mathrm{t}, J=6.2 \mathrm{~Hz}, 2 \mathrm{H}), 3.64(\mathrm{~s}, 2 \mathrm{H}), 2.11-2.02(\mathrm{~m}, 2 \mathrm{H}), 1.99$ (t, $J=2.7 \mathrm{~Hz}, 1 \mathrm{H}), 1.88(\mathrm{t}, J=6.2 \mathrm{~Hz}, 2 \mathrm{H}), 1.74(\mathrm{t}, J=7.5 \mathrm{~Hz}, 2 \mathrm{H})$.<smiles></smiles>

LC/MS (m/z): $[\mathrm{M}+\mathrm{H}]^{+}$259. ${ }^{1} \mathrm{H}$ NMR $\left(400 \mathrm{MHz}, \mathrm{DMSO}-\mathrm{d}_{6}\right) \delta 7.53(\mathrm{dd}, J=7.7,1.3 \mathrm{~Hz}, 1 \mathrm{H})$, $7.44-7.37(\mathrm{~m}, 2 \mathrm{H}), 7.17(\mathrm{dd}, J=8.6,2.6 \mathrm{~Hz}, 1 \mathrm{H}), 3.88(\mathrm{t}, J=6.0 \mathrm{~Hz}, 2 \mathrm{H}), 2.84(\mathrm{t}, J=2.6$ $\mathrm{Hz}, 1 \mathrm{H}), 2.04(\mathrm{td}, J=7.4,2.7 \mathrm{~Hz}, 2 \mathrm{H}), 1.89(\mathrm{t}, J=6.0 \mathrm{~Hz}, 2 \mathrm{H}), 1.67(\mathrm{t}, J=7.4 \mathrm{~Hz}, 2 \mathrm{H})$.<smiles>C#CCCC1(CCOc2cncc(C(=O)O)c2)N=NON(OC)O1</smiles>

LC/MS (m/z): $[\mathrm{M}+\mathrm{H}]^{+}$260. ${ }^{1} \mathrm{H}$ NMR (400 MHz, DMSO- $\left.\mathrm{d}_{6}\right) \delta 8.67(\mathrm{~d}, J=1.6 \mathrm{~Hz}, 1 \mathrm{H}), 8.46$ $(\mathrm{d}, J=2.9 \mathrm{~Hz}, 1 \mathrm{H}), 7.73(\mathrm{dd}, J=3.0,1.7 \mathrm{~Hz}, 1 \mathrm{H}), 3.99(\mathrm{t}, J=6.1 \mathrm{~Hz}, 2 \mathrm{H}), 2.84(\mathrm{t}, J=2.7$ $\mathrm{Hz}, 1 \mathrm{H}), 2.04(\mathrm{td}, J=7.3,2.6 \mathrm{~Hz}, 2 \mathrm{H}), 1.91(\mathrm{t}, J=6.1 \mathrm{~Hz}, 2 \mathrm{H}), 1.67(\mathrm{t}, J=7.4 \mathrm{~Hz}, 2 \mathrm{H})$.

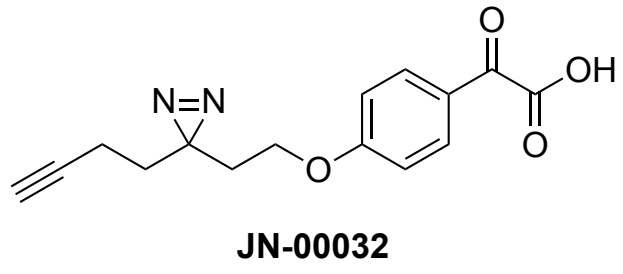

LC/MS (m/z): [M-H] $285 .{ }^{1} \mathrm{H}$ NMR (400 MHz, DMSO- $\left.d_{6}\right) \delta 7.87$ (d, J = 8.0 Hz, 2H), 7.10 $(\mathrm{d}, J=8.0 \mathrm{~Hz}, 2 \mathrm{H}), 3.96(\mathrm{t}, J=6.1 \mathrm{~Hz}, 2 \mathrm{H}), 2.84(\mathrm{t}, J=2.7 \mathrm{~Hz}, 1 \mathrm{H}), 2.04(\mathrm{td}, J=7.3,2.7$ $\mathrm{Hz}, 2 \mathrm{H}), 1.91(\mathrm{t}, J=6.0 \mathrm{~Hz}, 2 \mathrm{H}), 1.67(\mathrm{t}, J=7.4 \mathrm{~Hz}, 2 \mathrm{H})$.

\section{Synthesis of JN-0022}


<smiles>CC(C)(C)OC(=O)[C@H](Cc1ccc(O)cc1)NC(=O)OCc1ccccc1</smiles><smiles>C#CCCC1(CCI)N=N1</smiles>

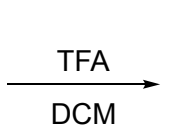<smiles>C#CCCC1(CCOc2ccc(C[C@H](NC(=O)OCc3ccccc3)C(=O)OC(C)(C)C)cc2)N=N1</smiles><smiles>C#CCCC1(CCOc2ccc(C[C@H](N)C(=O)O)cc2)N=N1</smiles>

\section{Step 1}

3-but-3-ynyl-3-(2-iodoethyl)diazirine (12.1 mg, $48.9 \mu \mathrm{mol}, 1.50$ equiv) was added to a solution of tert-butyl (2S)-2-(tert-butoxycarbonylamino)-3-(4-hydroxyphenyl)propanoate (11 mg, $32.6 \mu \mathrm{mol}, 1$ equiv) and potassium carbonate (6.76 mg $48.9 \mu \mathrm{mol}, 1.50$ equiv) in DMF $(326 \mu \mathrm{L})$. The reaction mixture was stirred at $24^{\circ} \mathrm{C}$ overnight. Water was added and the resulting mixture was washed with dichloromethane $(x 3)$. The combined organic layers were dried over $\mathrm{Na}_{2} \mathrm{SO}_{4}$, filtered, and concentrated under vacuum. The crude intermediate 1 was used for next step without any further purification.

\section{Step 2}

Crude intermediate 1 (15 mg, $32.78 \mu \mathrm{mol}, 1$ equiv) was dissolved in DCM (327.83 $\mu \mathrm{L})$, then trifluoroacetic acid (TFA) $(74.76 \mathrm{mg}, 655.65 \mu \mathrm{mol}, 50.51 \mu \mathrm{L}, 20.4$ equiv) was added and the reaction was stirred at $24{ }^{\circ} \mathrm{C}$ overnight. The reaction was concentrated under vacuum, then the crude was dissolved in DMSO and purified by prep HPLC using $0.1 \%$ formic acid in water $/ 0.1 \%$ formic acid in acetonitrile as eluent to give pure $\mathrm{JN}-0022$ as a white solid (2 mg, 15\% yield over 2 steps) LC/MS (m/z): $[\mathrm{M}+\mathrm{H}]^{+} 302 .{ }^{1} \mathrm{H}$ NMR (400 $\left.\mathrm{MHz}, \mathrm{DMSO}-d_{6}\right) \delta 7.17(\mathrm{~d}, J=8.4 \mathrm{~Hz}, 2 \mathrm{H}), 6.84(\mathrm{~d}, J=8.4 \mathrm{~Hz}, 2 \mathrm{H}), 3.94$ (brs, $\left.1 \mathrm{H}\right), 3.77$ $(\mathrm{t}, J=6.0 \mathrm{~Hz}, 2 \mathrm{H}), 3.07(\mathrm{dd}, J=12.04 .0 \mathrm{~Hz}, 1 \mathrm{H}), 2.85(\mathrm{t}, J=2.5 \mathrm{~Hz}, 1 \mathrm{H}), 2.83-2.73(\mathrm{~m}$, $1 \mathrm{H}), 2.11-1.98(\mathrm{~m}, 2 \mathrm{H}), 1.87(\mathrm{t}, J=6.0 \mathrm{~Hz}, 2 \mathrm{H}), 1.67(\mathrm{t}, J=7.3 \mathrm{~Hz}, 2 \mathrm{H})$.

JN-0012, JN-00026, JN-00028, and JN-00945 were prepared using the same procedure as $\mathrm{JN}-0022$

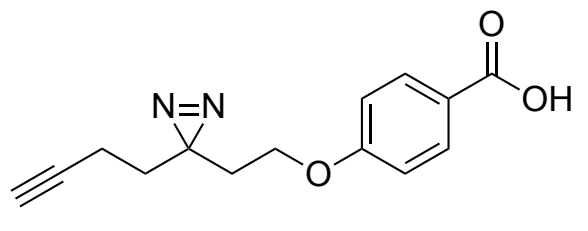

\section{$\mathrm{JN}-0012$}

LC/MS (m/z): $[\mathrm{M}+\mathrm{H}]^{+}$259. ${ }^{1} \mathrm{H}$ NMR (400 MHz, DMSO- $\left.d_{6}\right) \delta 7.89(\mathrm{~d}, J=8.0 \mathrm{~Hz}, 2 \mathrm{H}), 7.01$ $(\mathrm{d}, J=8.0 \mathrm{~Hz}, 2 \mathrm{H}), 3.90(\mathrm{t}, J=6.1 \mathrm{~Hz}, 2 \mathrm{H}), 2.84(\mathrm{t}, J=2.6 \mathrm{~Hz}, 1 \mathrm{H}), 2.04(\mathrm{td}, J=7.3,2.7$ $\mathrm{Hz}, 2 \mathrm{H}), 1.90(\mathrm{t}, J=6.1 \mathrm{~Hz}, 2 \mathrm{H}), 1.67(\mathrm{t}, J=7.4 \mathrm{~Hz}, 2 \mathrm{H})$. 


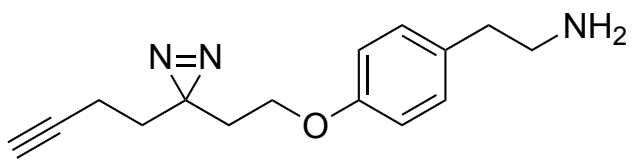

JN-00026

LC/MS (m/z): $[\mathrm{M}+\mathrm{H}]^{+}$258. ${ }^{1} \mathrm{H}$ NMR (400 MHz, DMSO- $\left.\mathrm{d}_{6}\right) \delta 8.40$ (brs, $\left.1 \mathrm{H}\right), 7.15$ (d, J = 8.2 $\mathrm{Hz}, 2 \mathrm{H}), 6.87(\mathrm{~d}, J=8.5 \mathrm{~Hz}, 2 \mathrm{H}), 3.78(\mathrm{t}, J=6.0 \mathrm{~Hz}, 3 \mathrm{H}), 2.94(\mathrm{t}, J=7.7 \mathrm{~Hz}, 2 \mathrm{H}), 2.84(\mathrm{t}$, $J=2.7 \mathrm{~Hz}, 1 \mathrm{H}), 2.75(\mathrm{t}, J=7.9 \mathrm{~Hz}, 2 \mathrm{H}), 2.03(\mathrm{td}, J=7.4,2.6 \mathrm{~Hz}, 2 \mathrm{H}), 1.87(\mathrm{t}, J=6.0 \mathrm{~Hz}$, $2 \mathrm{H}), 1.65(\mathrm{t}, J=7.4 \mathrm{~Hz}, 2 \mathrm{H})$.<smiles>C#CCCC1(CCOc2cccc(C[C@H](N)C(=O)O)c2)N=N1</smiles>

\section{$\mathrm{JN}-00028$}

LC/MS (m/z): $[\mathrm{M}+\mathrm{H}]^{+}$302. ${ }^{1} \mathrm{H}$ NMR $\left(400 \mathrm{MHz}\right.$, DMSO- $\left.d_{6}\right) \delta 7.19(\mathrm{t}, J=7.9 \mathrm{~Hz}, 1 \mathrm{H}), 6.83$ $(\mathrm{d}, J=7.0 \mathrm{~Hz}, 2 \mathrm{H}), 6.79-6.73(\mathrm{~m}, 1 \mathrm{H}), 3.79(\mathrm{t}, J=6.1 \mathrm{~Hz}, 2 \mathrm{H}), 3.16-3.09(\mathrm{~m}, 2 \mathrm{H}), 2.86$ $(\mathrm{t}, J=2.7 \mathrm{~Hz}, 1 \mathrm{H}), 2.78(\mathrm{dd}, J=14.3,8.6 \mathrm{~Hz}, 1 \mathrm{H}), 2.04(\mathrm{td}, J=7.4,2.7 \mathrm{~Hz}, 2 \mathrm{H}), 1.87(\mathrm{t}, J$ $=6.1 \mathrm{~Hz}, 2 \mathrm{H}), 1.66(\mathrm{t}, J=7.4 \mathrm{~Hz}, 2 \mathrm{H})$.<smiles>C#CCCC1(CCOc2ccc(C(=N)N)cc2)N=N1</smiles>

\section{$\mathrm{JN}-00945$}

LC/MS (m/z): $[\mathrm{M}+\mathrm{H}]^{+}$257. ${ }^{1} \mathrm{H}$ NMR (400 MHz, DMSO- $\left.d_{6}\right) \delta 8.46(\mathrm{~s}, 1 \mathrm{H}), 7.80(\mathrm{~d}, J=8.8$ $\mathrm{Hz}, 2 \mathrm{H}), 7.14(\mathrm{~d}, J=8.8 \mathrm{~Hz}, 2 \mathrm{H}), 3.94(\mathrm{t}, J=6.1 \mathrm{~Hz}, 2 \mathrm{H}), 2.85(\mathrm{t}, J=2.7 \mathrm{~Hz}, 1 \mathrm{H}), 2.04$ (td, $J=7.4,2.7 \mathrm{~Hz}, 2 \mathrm{H}), 1.92(\mathrm{t}, J=6.0 \mathrm{~Hz}, 2 \mathrm{H}), 1.67(\mathrm{t}, J=7.4 \mathrm{~Hz}, 2 \mathrm{H})$.

\section{Synthesis of $\mathrm{JN}-0005$}<smiles>Cn1c(=O)c2c[nH]cc2n(C)c1=O</smiles><smiles>C#CCCC1(CCI)N=N1</smiles><smiles>C#CCCC1(CCn2cc3c(=O)n(C)c(=O)n(C)c3c2)N=N1</smiles>

JN-0005

Sodium hydride (60\% dispersion in mineral oil, $4.63 \mathrm{mg}, 120.94 \mu \mathrm{mol}, 1.50$ equiv) was added to a solution of 1,3-dimethyl-6H-pyrrolo[3,4-d]pyrimidine-2,4-dione $(21.67 \mathrm{mg}$, $120.94 \mu \mathrm{mol}, 1.50$ equiv) in dry dimethylformamide $(806.25 \mu \mathrm{L})$ at $0{ }^{\circ} \mathrm{C}$. The reaction mixture was stirred at $0{ }^{\circ} \mathrm{C}$ for 15 minutes, then 3-but-3-ynyl-3-(2-iodoethyl)diazirine (20 $\mathrm{mg}, 80.62 \mu \mathrm{mol}, 1$ equiv) was added and the reaction was slowly warmed to $24{ }^{\circ} \mathrm{C}$ and stirred until completion. $1 \mathrm{M}$ aq. $\mathrm{HCl}$ was added and the mixture was washed with dichloromethane (x3). The combined organic layers were dried over $\mathrm{Na}_{2} \mathrm{SO}_{4}$, filtered, and 
concentrated under vacuum. The crude was then purified by prep HPLC using $0.1 \%$ formic acid in water $/ 0.1 \%$ formic acid in acetonitrile as eluent to give pure $\mathrm{JN}-0005$ as a white solid (6mg, 25\%) LC/MS (m/z): [M+H] ${ }^{+} 300 .{ }^{1} \mathrm{H}$ NMR (400 MHz, DMSO-d $) \delta 7.58(\mathrm{~s}, 1 \mathrm{H})$, $6.86(\mathrm{~s}, 1 \mathrm{H}), 3.94(\mathrm{t}, J=7.4 \mathrm{~Hz}, 2 \mathrm{H}), 3.26(\mathrm{~s}, 3 \mathrm{H}), 3.19(\mathrm{~s}, 3 \mathrm{H}), 2.85(\mathrm{~s}, 1 \mathrm{H}), 1.98(\mathrm{td}, J=$ 7.0, 3.0 Hz, 2H), $1.95-1.85(\mathrm{~m}, 2 \mathrm{H}), 1.59-1.49(\mathrm{~m}, 2 \mathrm{H})$.

JN-00024 was prepared using the same procedure as $\mathrm{JN}-0005$

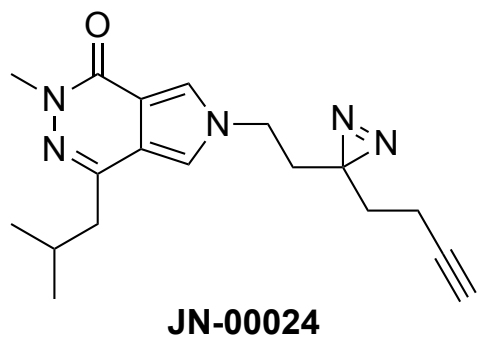

LC/MS (m/z): $[\mathrm{M}+\mathrm{H}]^{+}$326. ${ }^{1} \mathrm{H}$ NMR (400 MHz, DMSO-d $) \delta 7.70(\mathrm{~d}, J=2.0 \mathrm{~Hz}, 1 \mathrm{H}), 7.59$ $(\mathrm{d}, J=2.0 \mathrm{~Hz}, 1 \mathrm{H}), 4.11(\mathrm{t}, J=7.2 \mathrm{~Hz}, 2 \mathrm{H}), 3.54(\mathrm{~s}, 3 \mathrm{H}), 2.83(\mathrm{t}, J=2.6 \mathrm{~Hz}, 1 \mathrm{H}), 2.10$ (dq, $J=13.5,6.7 \mathrm{~Hz}, 1 \mathrm{H}), 1.96$ (ddt, $J=7.5,5.3,2.4 \mathrm{~Hz}, 4 \mathrm{H}), 1.51$ (t, $J=7.4 \mathrm{~Hz}, 2 \mathrm{H}), 0.91$ (d, $J=6.6 \mathrm{~Hz}, 6 \mathrm{H})$.

\section{Synthesis of JN-00935}<smiles>CC(C)(C)OC(=O)c1ccc(O)cc1</smiles><smiles>C#CCCC1(CCI)N=N1</smiles><smiles></smiles>

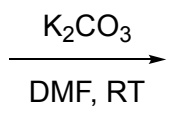<smiles>C#CCCC1(CCOc2ccc(C(=O)OC(C)(C)C)cc2)N=N1</smiles><smiles>N=C(N)N</smiles>
RT<smiles>C#CCCC1(CCOc2ccc(C(=O)NC(=N)N)cc2)N=N1</smiles>

\section{Step 1}

3-but-3-ynyl-3-(2-iodoethyl)diazirine (38.32 mg, $154.46 \mu \mathrm{mol}, 1.5$ equiv) was added to a solution of tert-butyl 4-hydroxybenzoate $(20 \mathrm{mg}, 102.97 \mu \mathrm{mol}, 1$ equiv) in dimethylformamide $(343.24 \mu \mathrm{L})$, and potassium carbonate $(42.70 \mathrm{mg}, 308.92 \mu \mathrm{mol}, 18.64$ $\mu \mathrm{L}, 3.00$ equiv). The reaction mixture was stirred at $24{ }^{\circ} \mathrm{C}$ overnight. Brine was added and the resulting mixture was then washed with ethyl acetate $(x 3)$. The combined organic layers were dried on $\mathrm{Na}_{2} \mathrm{SO}_{4}$, filtered, and concentrated under vacuum. The crude was purified by flash column chromatography using heptane / ethyl acetate as eluent to give pure intermediate 1 as a white solid (24 mg, 74\%)

\section{Step 2}


Intermediate 1 (13 mg, $41.35 \mu \mathrm{mol}, 1$ equiv) was dissolved in dichloromethane (413.51 $\mu \mathrm{L})$, then TFA (94.30 mg, $827.03 \mu \mathrm{mol}, 63.71 \mu \mathrm{L}, 20$ equiv) was added and the reaction was stirred at $24^{\circ} \mathrm{C}$ overnight. The reaction was then concentrated under vacuum and the crude product was azeotroped with toluene $(x 3)$ to remove the excess of TFA, and used for next step without any further purification.

\section{Step 3}

Intermediate 2 (3 mg, $11.62 \mu \mathrm{mol}, 1$ equiv) was dissolved in dimethylformamide (116.16 $\mu \mathrm{L})$, then HATU $(5.30 \mathrm{mg}, 13.94 \mu \mathrm{mol}, 1.20$ equiv) was added followed by diisopropylethylamine $(6.00 \mathrm{mg}, 46.46 \mu \mathrm{mol}, 8.09 \mu \mathrm{L}, 4.00$ equiv). The reaction was stirred at $24{ }^{\circ} \mathrm{C}$ for $10 \mathrm{~min}$, then guanidine- $\mathrm{HCl}(2.22 \mathrm{mg}, 23.23 \mu \mathrm{mol}, 2.00$ equiv) was added and the reaction mixture was stirred overnight. The reaction mixture was then purified by prep HPLC using $0.1 \%$ formic acid in water / $0.1 \%$ formic acid in acetonitrile as eluent to give pure $\mathrm{JN}-00935$ as a white solid (3mg, 70\% yield over two steps) LC/MS $(\mathrm{m} / \mathrm{z}):[\mathrm{M}+\mathrm{H}]^{+}$300. ${ }^{1} \mathrm{H}$ NMR $(400 \mathrm{MHz}$, DMSO-d $) \delta 8.33(\mathrm{~s}, 2 \mathrm{H}), 8.01(\mathrm{~d}, J=8.7 \mathrm{~Hz}, 2 \mathrm{H})$, $6.90(\mathrm{~d}, J=8.8 \mathrm{~Hz}, 2 \mathrm{H}), 3.86(\mathrm{t}, J=6.1 \mathrm{~Hz}, 3 \mathrm{H}), 2.85(\mathrm{t}, J=2.6 \mathrm{~Hz}, 1 \mathrm{H}), 2.05(\mathrm{td}, J=7.4$, $2.8 \mathrm{~Hz}, 2 \mathrm{H}), 1.89(\mathrm{t}, J=6.1 \mathrm{~Hz}, 2 \mathrm{H}), 1.67(\mathrm{t}, J=7.4 \mathrm{~Hz}, 2 \mathrm{H})$.

JN-00942 was prepared using same procedure as JN-00935.

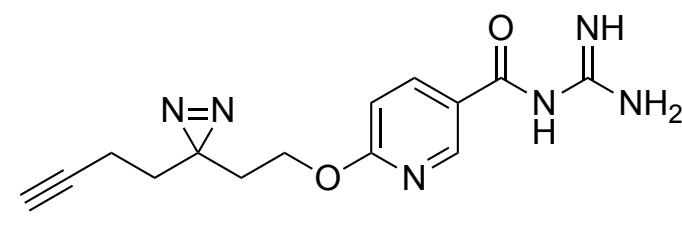

JN-00942

LC/MS (m/z): $[\mathrm{M}+\mathrm{H}]^{+}$301. ${ }^{1} \mathrm{H}$ NMR (400 MHz, DMSO-d $) \delta 8.41(\mathrm{~d}, J=2.5 \mathrm{~Hz}, 1 \mathrm{H}), 8.14$ $(\mathrm{s}, 1 \mathrm{H}), 7.96(\mathrm{dd}, J=9.5,2.5 \mathrm{~Hz}, 1 \mathrm{H}), 6.55(\mathrm{~s}, 1 \mathrm{H}), 6.37(\mathrm{~d}, J=9.5 \mathrm{~Hz}, 1 \mathrm{H}), 3.90(\mathrm{t}, J=$ $7.5 \mathrm{~Hz}, 2 \mathrm{H}$ ), $2.84(\mathrm{t}, J=2.7 \mathrm{~Hz}, 1 \mathrm{H}), 2.02(\mathrm{td}, J=7.4,2.7 \mathrm{~Hz}, 2 \mathrm{H}), 1.73(\mathrm{t}, J=7.5 \mathrm{~Hz}, 2 \mathrm{H})$, $1.62(\mathrm{t}, J=7.4 \mathrm{~Hz}, 2 \mathrm{H})$.

Synthesis of JN-00033<smiles>O=C(O)CCc1ccc(O)cc1</smiles><smiles>C#CCCC1(CCI)N=N1</smiles><smiles>C#CCCC1(CCOc2ccc(CCC(=O)O)cc2)N=N1</smiles>

$\mathrm{NaOH}$ 6M (18.05 mg, $451.34 \mu \mathrm{mol}, 75 \mathrm{uL}, 3.00$ equiv) was added to a solution of 3-(4hydroxyphenyl)propanoic acid (25 mg, $150.45 \mu \mathrm{mol}, 1$ equiv) in methanol (300.89 $\mu \mathrm{L}$ ) followed by 3-but-3-ynyl-3-(2-iodoethyl)diazirine $(55.98 \mathrm{mg}, 225.67 \mu \mathrm{mol}, 1.50$ equiv). The reaction was then stirred at $50{ }^{\circ} \mathrm{C}$ overnight. $1 \mathrm{M}$ aq. $\mathrm{HCl}$ was added and the resulting acidic mixture was washed with ethyl acetate $(x 2)$. The combined organic layers were dried over $\mathrm{Na}_{2} \mathrm{SO}_{4}$, filtered, and concentrated under vacuum. The crude product was dissolved in DMSO and purified by prep HPLC using $0.1 \%$ formic acid in water $/ 0.1 \%$ formic acid in acetonitrile as eluent to give pure $\mathrm{JN}-00033$ as a white solid $(10 \mathrm{mg}, 23 \%$ 
yield). LC/MS (m/z): $[\mathrm{M}+\mathrm{H}]^{+}$287. ${ }^{1} \mathrm{H}$ NMR (400 MHz, DMSO- $\left.d_{6}\right) \delta 7.12(\mathrm{~d}, J=8.3 \mathrm{~Hz}, 2 \mathrm{H})$, $6.82(\mathrm{~d}, J=8.4 \mathrm{~Hz}, 2 \mathrm{H}), 3.77(\mathrm{t}, J=6.1 \mathrm{~Hz}, 2 \mathrm{H}), 2.84(\mathrm{t}, J=2.6 \mathrm{~Hz}, 1 \mathrm{H}), 2.73(\mathrm{t}, J=7.6$ $\mathrm{Hz}, 2 \mathrm{H}), 2.45(\mathrm{t}, J=7.6 \mathrm{~Hz}, 2 \mathrm{H}), 2.03(\mathrm{td}, J=7.4,2.6 \mathrm{~Hz}, 2 \mathrm{H}), 1.85(\mathrm{t}, J=6.1 \mathrm{~Hz}, 2 \mathrm{H})$, $1.65(\mathrm{t}, \mathrm{J}=7.4 \mathrm{~Hz}, 2 \mathrm{H})$.

\section{Synthesis of JN-00038}<smiles>COC(=O)CC[C@H](C)[C@H]1CC[C@H]2[C@H]3[C@H](C[C@H](O)[C@]21C)[C@@]1(C)CCC(=O)C[C@H]1C[C@H]3O</smiles>

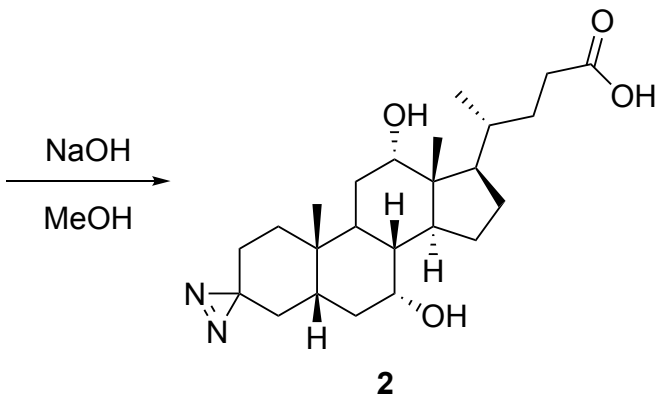

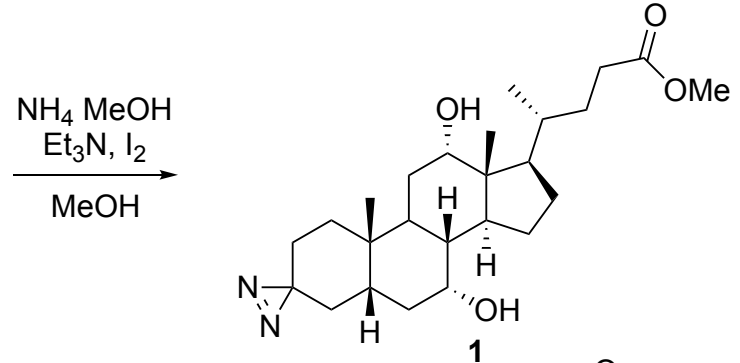
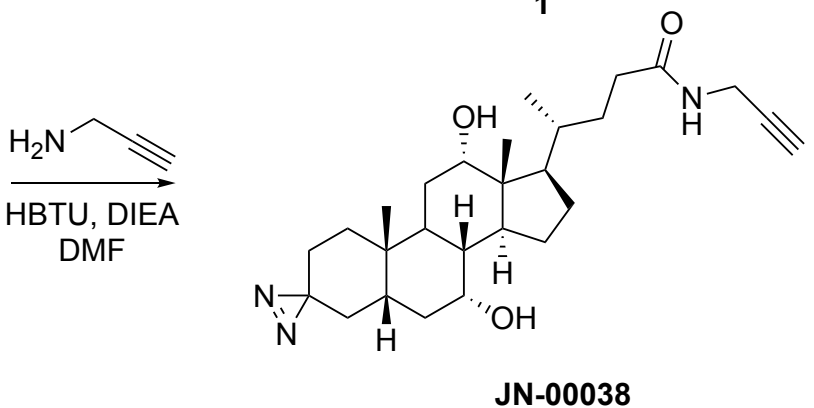

\section{Step 1}

Methyl (4R)-4-[(5R,7R,8R,10S,12S,13R,14S,17R)-7,12-dihydroxy-10,13-dimethyl-3-oxo$1,2,4,5,6,7,8,9,11,12,14,15,16,17$-tetradecahydrocyclopenta[a]phenanthren-17-

yl]pentanoate (100 mg, $237.77 \mu \mathrm{mol}, 1$ equiv) was dissolved in ammonia $7.0 \mathrm{M}$ in methanol $\left(237.77 \mu \mathrm{mol}, 1.25 \mathrm{~mL}, 1.00\right.$ equiv) at $-20{ }^{\circ} \mathrm{C}$ and the resulting reaction mixture was stirred for 1 hour. The temperature was then raised to $24{ }^{\circ} \mathrm{C}$ and the reaction was stirred for additional 2 hours. The temperature was then lowered to $-15{ }^{\circ} \mathrm{C}$ and amino hydrogen sulfate $(32.5 \mathrm{mg}, 287.37 \mu \mathrm{mol}, 1.200$ equiv $-6.5 \mathrm{mg} \times 5$ times were added every 30 minutes). When the addition was completed the reaction was warmed to $24{ }^{\circ} \mathrm{C}$ and stirred for additional 14 hours. The reaction was concentrated under reduced pressure, and the residue was dissolved in methanol $(1.25 \mathrm{~mL})$. Triethylamine $(40.90 \mathrm{mg}, 404.20$ $\mu \mathrm{mol}, 56.34 \mu \mathrm{L}, 1.50$ equiv) was then added at $0{ }^{\circ} \mathrm{C}$ followed by lodine (108.62 $\mathrm{mg}, 427.98$ $\mu \mathrm{mol}, 1.80$ equiv) portion wise. The resulting mixture was then warmed to $24^{\circ} \mathrm{C}$ and stirred for additional 30 minutes. Saturated aq. $\mathrm{Na}_{2} \mathrm{~S}_{2} \mathrm{O}_{3}$ solution was added to quench the excess of lodine, then the mixture was washed with ethyl acetate $(x 3)$. The combined organic layers were dried over $\mathrm{Na}_{2} \mathrm{SO}_{4}$, filtered, and concentrated under vacuum. The crude product was purified by flash column chromatography using heptane / ethyl acetate as eluent to give pure intermediate $1(68 \mathrm{mg}, 66 \%)$.

\section{Step 2}

Intermediate 1 (68 mg, $157.19 \mu \mathrm{mol}, 1$ equiv) was suspended in methanol (1.57 $\mathrm{mL}$ ) and $\mathrm{NaOH}\left(62.88 \mathrm{mg}, 1.57 \mathrm{mmol}, 10\right.$ equiv) and the reaction was stirred at $24{ }^{\circ} \mathrm{C}$ overnight. The reaction mixture was then acidified with $1 \mathrm{M}$ aq. $\mathrm{HCl}$ and washed with EtOAc (x3). The combined organic layers were dried over $\mathrm{Na}_{2} \mathrm{SO}_{4}$, filtered, and concentrated 
under vacuum. The crude product was used for next step without any further purification (49 $\mathrm{mg}, 75 \%)$.

\section{Step 3}

Intermediate 2 (49 $\mathrm{mg}, 117.07 \mu \mathrm{mol}, 1$ equiv) was dissolved in dimethylformamide (1.17 $\mathrm{mL}$ ), then HBTU (66.59 mg, $175.60 \mu \mathrm{mol}, 1.50$ equiv), prop-2-yn-1-amine (19.34 mg, $351.20 \mu \mathrm{mol}, 22.49 \mu \mathrm{L}, 3.00$ equiv.) and diisopropylethylamine $(45.39 \mathrm{mg}, 351.20 \mu \mathrm{mol}$, $61.17 \mu \mathrm{L}, 3.00$ equiv) were added and the reaction was stirred at $24{ }^{\circ} \mathrm{C}$ for $18 \mathrm{~h}$. Water was then added and the resulting mixture was washed with EtOAc (x3). The combined organic layers were washed with brine, dried over $\mathrm{Na}_{2} \mathrm{SO}_{4}$, filtered, and concentrated under vacuum. The crude was then purified by flash column chromatography using DCM/DCM:MeOH 9:1 to give pure JN-00038 as a white solid (32mg, 61\%) LC/MS (m/z): $[\mathrm{M}+\mathrm{H}]^{+}$456. ${ }^{1} \mathrm{H}$ NMR $(400 \mathrm{MHz}$, Chloroform- $d$ ) $\delta 5.68(\mathrm{~s}, 1 \mathrm{H}), 4.05$ (ddd, $J=5.3,2.6,0.8$ $\mathrm{Hz}, 2 \mathrm{H}), 4.02(\mathrm{dd}, J=2.8 \mathrm{~Hz}, 1 \mathrm{H}), 3.88-3.85(\mathrm{~m}, 1 \mathrm{H}), 2.92(\mathrm{dd}, J=15.0,13.4 \mathrm{~Hz}, 1 \mathrm{H})$, $2.32-2.08(\mathrm{~m}, 4 \mathrm{H}), 1.98-1.86(\mathrm{~m}, 4 \mathrm{H}), 1.85-1.53(\mathrm{~m}, 10 \mathrm{H}), 1.52-1.10(\mathrm{~m}, 7 \mathrm{H}), 1.00$ $-0.98(\mathrm{~m}, 5 \mathrm{H}), 0.72(\mathrm{~s}, 3 \mathrm{H}), 0.32-0.16(\mathrm{~m}, 2 \mathrm{H})$.

\section{Synthesis of JN-00247}<smiles>COC(=O)c1ccc2[nH]ccc2c1</smiles><smiles>C#CCCC1(CCI)N=N1</smiles>

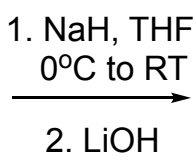<smiles>C#CCCC1(CCn2ccc3cc(C(=O)O)ccc32)N=N1</smiles>

JN-00247

Sodium hydride (3.94 mg, $102.75 \mu \mathrm{mol}, 60 \%$ dispersion in mineral oil, 1.20 equiv) was added to a solution of methyl $1 \mathrm{H}$-indole-5-carboxylate $(15 \mathrm{mg}, 85.62 \mu \mathrm{mol}, 1$ equiv) in dry THF $(856.24 \mu \mathrm{L})$ at $0{ }^{\circ} \mathrm{C}$, and the reaction was stirred for 30 minutes. 3-but-3-ynyl-3-(2iodoethyl)diazirine ( $25.49 \mathrm{mg}, 102.75 \mu \mathrm{mol}, 1.20$ equiv) was then added and the reaction was slowly warmed to RT and stirred for 48 hours. Some ester hydrolysis was detected along with the desired product. $2 \mathrm{M}$ aq. $\mathrm{LiOH}(428.12 \mu \mathrm{mol}, 0.214 \mathrm{~mL})$ was added and the reaction was stirred at $50{ }^{\circ} \mathrm{C}$ for $18 \mathrm{~h}$. $1 \mathrm{M}$ aq. $\mathrm{HCl}$ was added until the $\mathrm{pH}$ was 2-3, then the mixture was washed with EtOAc $(x 3)$. The combined organic layers were dried over $\mathrm{Na}_{2} \mathrm{SO}_{4}$, filtered, and concentrated under vacuum. The crude product was dissolved in DMSO and purified by prep HPLC using $0.1 \%$ formic acid in water $/ 0.1 \%$ formic acid in acetonitrile as eluent to give pure $\mathrm{JN}-00247$ as a white solid $(6.7 \mathrm{mg}, 28 \%)$. LC/MS $(\mathrm{m} / \mathrm{z})$ : $[\mathrm{M}+\mathrm{H}]^{+}$282. ${ }^{1} \mathrm{H}$ NMR $\left(400 \mathrm{MHz}, \mathrm{DMSO}_{-} d_{6}\right) \delta 8.22(\mathrm{~d}, J=1.5 \mathrm{~Hz}, 1 \mathrm{H}), 7.75(\mathrm{dd}, J=8.7$, $1.7 \mathrm{~Hz}, 1 \mathrm{H}), 7.54(\mathrm{dd}, J=6.0,2.8 \mathrm{~Hz}, 2 \mathrm{H}), 6.61(\mathrm{~d}, J=3.1 \mathrm{~Hz}, 1 \mathrm{H}), 4.15(\mathrm{t}, J=7.2 \mathrm{~Hz}$, $2 \mathrm{H}), 2.83(\mathrm{t}, J=2.6 \mathrm{~Hz}, 1 \mathrm{H}), 1.90(\mathrm{td}, J=7.3,4.8 \mathrm{~Hz}, 4 \mathrm{H}), 1.49(\mathrm{t}, J=7.3 \mathrm{~Hz}, 2 \mathrm{H})$.

\section{Synthesis of $\mathrm{JN}-00248$}
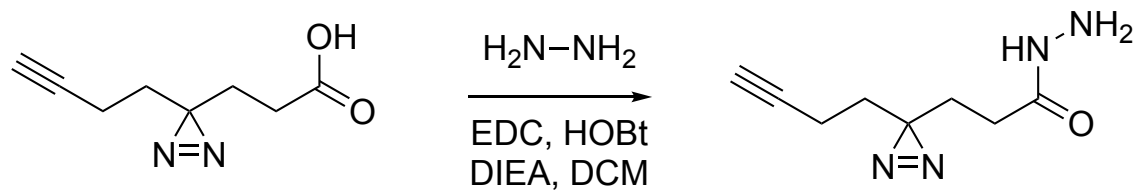

A

West, A. et al. Labeling preferences of diazirines with protein biomolecules, J. Am. Chem. 
<smiles>Nc1ccccc1C(=O)CC(N)C(=O)O</smiles><smiles>CO[N+](=O)[O-]</smiles><smiles>CC(C)(C)OC(=O)NC(CC(=O)c1ccccc1N)C(=O)O</smiles>

1<smiles>C#CCCC1(CCC(=O)NN)N=N1</smiles>

A<smiles>C#CCCC1(CCC(=O)N/N=C(/C[C@H](NC(=O)OCc2ccccc2)C(=O)O)c2ccccc2N)N=N1</smiles>

\section{Synthesis of A}

Hydrazine (19.28 mg, $601.77 \mu \mathrm{mol}, 18.91 \mu \mathrm{L}, 2.00$ equiv), diisopropylethylamine (38.89 $\mathrm{mg}, 300.88 \mu \mathrm{mol}, 52.41 \mu \mathrm{L}, 1.00$ equiv), 1-Ethyl-3-(3-dimethylaminopropyl)carbodiimide (86.52 mg, $451.33 \mu \mathrm{mol}, 1.50$ equiv), and HOBt (60.98 mg, $451.33 \mu \mathrm{mol}, 1.50$ equiv) were added in sequence to a solution of 3-[3-azidopropyl(methyl)amino]-6,6-dimethyl-5,7dihydro-2H-indol-4-one (50 mg, $300.88 \mu \mathrm{mol}, 1$ equiv) in dichloromethane $(5.01 \mathrm{~mL})$, and the reaction was stirred at $24{ }^{\circ} \mathrm{C}$ for $12 \mathrm{~h}$. The reaction was diluted with dichloromethane then washed with saturated aq. $\mathrm{NH}_{4} \mathrm{Cl}$ and brine. The organic layer was then dried over $\mathrm{Na}_{2} \mathrm{SO}_{4}$, filtered, and concentrated under vacuum. The crude intermediate $\mathrm{A}$ was used for next step without any further purification (54 mg, 74\%). LC/MS (m/z): $[\mathrm{M}+\mathrm{H}]^{+} 181$.

\section{Step 1}

(2S)-2-amino-4-(2-aminophenyl)-4-oxo-butanoic acid $(200 \mathrm{mg}, 960.55 \mu \mathrm{mol}, 1$ equiv) and Di-tert-butyl dicarbonate (440.24 mg, $2.02 \mathrm{mmol}, 462.92 \mu \mathrm{L}, 2.20$ equiv) were dissolved in acetonitrile $(2 \mathrm{~mL})$ and water $(4 \mathrm{~mL})$. Sodium bicarbonate $(338.91 \mathrm{mg}, 4.03$ mmol 4.00 equiv) was added and the reaction mixture was stirred at $24{ }^{\circ} \mathrm{C}$ for 60 hours. $5-10 \%$ of bis-protected product was observed along with the desired product. The reaction was acidified with $1 \mathrm{~N} \mathrm{HCl}$ and washed with EtOAc $(3 \times 10 \mathrm{~mL})$. The combined organic layers were dried over $\mathrm{Na}_{2} \mathrm{SO}_{4}$, filtered, and concentrated under vacuum. The crude intermediate 1 was used for next step without any further purification ( $270 \mathrm{mg}, 91 \%$ ).

\section{Step 2}

Crude intermediate 1 (15 mg, $48.65 \mu \mathrm{mol}, 1$ equiv) and intermediate A (10.52 mg, 58.38 $\mu \mathrm{mol}, 1.2$ equiv) were dissolved in ethanol $(243.25 \mu \mathrm{L})$, and the reaction mixture was stirred at $50{ }^{\circ} \mathrm{C} 12 \mathrm{~h}$. The solvent was then evaporated and the crude was dissolved in DMSO and purified by prep HPLC using $0.1 \%$ formic acid in water $/ 0.1 \%$ formic acid in Acetonitrile as eluent to give pure intermediate 2 as a white solid ( $7 \mathrm{mg}, 31 \%)$.

\section{Step 3}

West, A. et al. Labeling preferences of diazirines with protein biomolecules, J. Am. Chem. 
Intermediate $2(7 \mathrm{mg}, 14.88 \mu \mathrm{mol})$ was dissolved in DCM $(148.77 \mu \mathrm{L})$ then TFA (33.93 $\mathrm{mg}, 297.54 \mu \mathrm{mol}, 22.92 \mu \mathrm{L}$ ) was added and the reaction was stirred at $24{ }^{\circ} \mathrm{C}$. The reaction was concentrated in vacuo then azeotroped with Toluene $(x 3)$ to remove the excess TFA. The crude material was dissolved in DMSO and purified by prep HPLC using $0.1 \%$ formic acid in water $/ 0.1 \%$ formic acid in acetonitrile as eluent to give pure $\mathrm{JN}-00248$ as a light yellow solid (2 mg, 26\%). LC/MS (m/z): $[\mathrm{M}+\mathrm{H}]^{+} 371 .{ }^{1} \mathrm{H}$ NMR $\left(400 \mathrm{MHz}\right.$, DMSO- $\left.d_{6}\right) \delta 7.48$ $(\mathrm{d}, J=8.1 \mathrm{~Hz}, 1 \mathrm{H}), 7.27(\mathrm{~s}, 2 \mathrm{H}), 7.03(\mathrm{t}, J=7.7 \mathrm{~Hz}, 1 \mathrm{H}), 6.70(\mathrm{~d}, J=8.0 \mathrm{~Hz}, 1 \mathrm{H}), 6.52(\mathrm{t}$, $J=7.4 \mathrm{~Hz}, 1 \mathrm{H}), 2.89(\mathrm{~d}, J=14.5 \mathrm{~Hz}, 1 \mathrm{H}), 2.84(\mathrm{t}, J=2.6 \mathrm{~Hz}, 1 \mathrm{H}), 2.11(\mathrm{t}, J=7.8 \mathrm{~Hz}, 2 \mathrm{H})$, $2.02(\mathrm{td}, J=7.3,2.8 \mathrm{~Hz}, 3 \mathrm{H}), 1.76(\mathrm{t}, J=7.7 \mathrm{~Hz}, 2 \mathrm{H}), 1.62(\mathrm{t}, J=7.3 \mathrm{~Hz}, 3 \mathrm{H})$.

\section{Synthesis of JN-00835}<smiles>CC(C)Cc1nn(C)c(=O)c2c[nH]cc12</smiles><smiles>C#CCCC1(CCC(=O)O)N=N1</smiles>

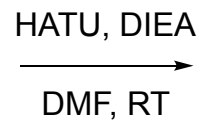<smiles>C#CCCC1(CCC(=O)n2cc3c(CC(C)C)nn(C)c(=O)c3c2)N=N1</smiles>

JN-00835

3-(3-but-3-ynyldiazirin-3-yl)propanoic acid (18.22 $\mathrm{mg}, 109.62 \mu \mathrm{mol}, 1.50$ equiv) and HATU (41.68 mg, $109.62 \mu \mathrm{mol}, 1.50$ equiv) were dissolved in dimethylformamide $(365.40 \mu \mathrm{L})$ then diisopropylethylamine $(23.61 \mathrm{mg}, 182.70 \mu \mathrm{mol}$, $31.82 \mu \mathrm{L}$ ) was added and the reaction was stirred at $24{ }^{\circ} \mathrm{C}$ for $5-10$ minutes. 1-isobutyl3-methyl-6H-pyrrolo[3,4-d]pyridazin-4-one (15 mg, $73.08 \mu \mathrm{mol}, 1$ equiv) was then added and the reaction was stirred at $24{ }^{\circ} \mathrm{C}$ for 10 minutes. The reaction mixture was then diluted with DMSO and purified by prep HPLC using $0.1 \%$ formic acid in water $/ 0.1 \%$ formic acid in acetonitrile as eluent to give pure $\mathrm{JN}-00835$ as a white solid $(11.5 \mathrm{mg}, 45 \%)$. LC/MS $(\mathrm{m} / \mathrm{z}):[\mathrm{M}+\mathrm{H}]^{+}$354. ${ }^{1} \mathrm{H}$ NMR $\left(400 \mathrm{MHz}, \mathrm{DMSO}_{-} \mathrm{d}_{6}\right) \delta 8.24(\mathrm{~d}, J=4.8 \mathrm{~Hz}, 1 \mathrm{H}), 8.15(\mathrm{~d}, J=$ $4.5 \mathrm{~Hz}, 1 \mathrm{H}), 3.55(\mathrm{~s}, 3 \mathrm{H}), 3.17$ (brs, $2 \mathrm{H}), 2.89-2.80(\mathrm{~m}, 1 \mathrm{H}), 2.59-2.57$ (brm, 2H), 2.16 - $1.95(\mathrm{~m}, 4 \mathrm{H}), 1.83-1.77$ (brm, 2H), $1.71-1.65$ (brm, 2H), $0.97-0.90$ (brm, 6H).

JN-00939 was prepared using same procedure as JN-00835

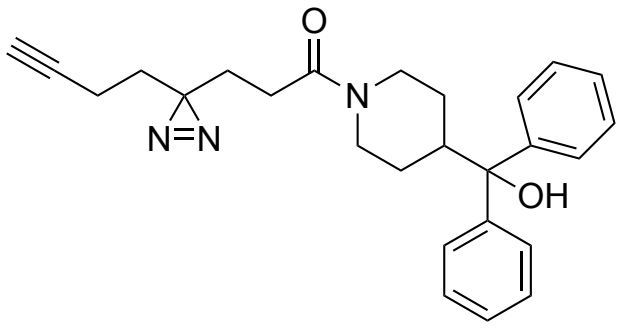

JN-00939

LC/MS (m/z): $[\mathrm{M}+\mathrm{H}]^{+}$416. ${ }^{1} \mathrm{H}$ NMR (400 MHz, DMSO- $\left.d_{6}\right) \delta 7.53$ (dd, J = 7.7, $\left.3.7 \mathrm{~Hz}, 4 \mathrm{H}\right)$, $7.27(\mathrm{t}, J=7.6 \mathrm{~Hz}, 4 \mathrm{H}), 7.14(\mathrm{t}, J=7.3 \mathrm{~Hz}, 2 \mathrm{H}), 5.32(\mathrm{~s}, 1 \mathrm{H}), 4.39(\mathrm{~d}, J=12.8 \mathrm{~Hz}, 1 \mathrm{H})$, $3.81(\mathrm{~d}, J=13.1 \mathrm{~Hz}, 1 \mathrm{H}), 3.02-2.89(\mathrm{~m}, 1 \mathrm{H}), 2.85-2.73(\mathrm{~m}, 2 \mathrm{H}), 2.10(\mathrm{t}, J=7.6 \mathrm{~Hz}$, $2 \mathrm{H}), 1.98(\mathrm{td}, J=7.4,2.7 \mathrm{~Hz}, 2 \mathrm{H}), 1.59(\mathrm{q}, J=7.7 \mathrm{~Hz}, 4 \mathrm{H}), 1.39-1.18(\mathrm{~m}, 4 \mathrm{H})$.

\section{Synthesis of JN-00245}

West, A. et al. Labeling preferences of diazirines with protein biomolecules, J. Am. Chem. 
<smiles>Nc1c(O)cccc1C(=O)CC(N)C(=O)O</smiles>

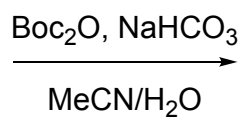<smiles>C#CCCC1(CCOC(=O)C(CC(=O)c2cccc(OCCC3(CCC#C)N=N3)c2N)NC(=O)OC(C)(C)C)N=N1</smiles><smiles>CC(C)(C)OC(=O)NC(CC(=O)c1cccc(O)c1N)C(=O)O</smiles>

1

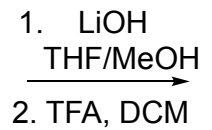

RT<smiles>C#CCCC1(CCI)N=N1</smiles><smiles>C#CCCC1(CCOc2cccc(C(=O)CC(N)C(=O)O)c2N)N=N1</smiles>

\section{Step 1}

2-amino-4-(2-amino-3-hydroxy-phenyl)-4-oxo-butanoic acid (25 mg, $111.50 \mu \mathrm{mol}, 1$ equiv) and Di-tert-butyl dicarbonate $(51.10 \mathrm{mg}, 234.15 \mu \mathrm{mol}, 53.74 \mu \mathrm{L}, 2.00$ equiv) were dissolved in a mixture of acetonitrile $(0.5 \mathrm{~mL})$ and water $(0.5 \mathrm{~mL})$, then sodium bicarbonate (39.34 mg, $468.31 \mu \mathrm{mol}, 4.00$ equiv) was added and the reaction mixture was stirred at RT for 3 hours. Some bis-Boc protected product was observed along with the desired product. $1 \mathrm{M}$ aq. $\mathrm{HCl}$ was added and the mixture was washed with EtOAc $(x 3)$. The combined organic layers were dried over $\mathrm{Na}_{2} \mathrm{SO}_{4}$, filtered, and concentrated under vacuum. The crude intermediate 1 was used for next step without any further purification (10 mg, 28\%).

\section{Step 2}

Intermediate 1 (10 mg, $30.83 \mu \mathrm{mol}, 1$ equiv) was dissolved in DMF (308.33 $\mu \mathrm{L})$, then potassium carbonate $(21.31 \mathrm{mg}, 154.16 \mu \mathrm{mol}, 5.00$ equiv) was added followed by 3-but-3-ynyl-3-(2-iodoethyl)diazirine (19.12 mg, $77.08 \mu \mathrm{mol}, 2.20$ equiv). The reaction mixture was stirred at $24{ }^{\circ} \mathrm{C}$ overnight and the main product was the bis-propargylated derivative. Saturated aq. $\mathrm{NaHCO} 3$ solution was then added and the mixture was extracted with EtOAc (x3). The combined organic layers were dried over $\mathrm{Na}_{2} \mathrm{SO}_{4}$, filtered, and concentrated under vacuum. The crude intermediate 2 was used for next step without any further purification.

\section{Step 3}

Intermediate 2 (17.5 mg, $30.99 \mu \mathrm{mol}, 1$ equiv) was dissolved in THF (247.95 $\mu \mathrm{L})$ and methanol $(61.99 \mu \mathrm{L})$, then $2 \mathrm{M}$ aq. $\mathrm{LiOH}(3.71 \mathrm{mg}, 154.97 \mu \mathrm{mol}, 78 \mathrm{uL}, 5.00$ equiv) was added and the reaction mixture was stirred at $24{ }^{\circ} \mathrm{C}$ for $30 \mathrm{~min} .1 \mathrm{M}$ aq. $\mathrm{HCl}$ was added and the mixture was washed with EtOAc (x3). The combined organic layers were dried 
over $\mathrm{Na}_{2} \mathrm{SO}_{4}$, filtered, and concentrated under vacuum. The crude intermediate was used for next step without any further purification.

\section{Step 4}

The above crude intermediate (13.8 mg, $31.05 \mu \mathrm{mol}, 1$ equiv) was dissolved in dichloromethane, then TFA (70.80 mg, $620.95 \mu \mathrm{mol}, 47.84 \mu \mathrm{L}, 20$ equiv) was added and the reaction was stirred at $24{ }^{\circ} \mathrm{C}$ for $1 \mathrm{~h}$. The reaction was concentrated in vacuo and the crude product was dissolved in DMSO and purified by prep HPLC using $0.1 \%$ formic acid in water $/ 0.1 \%$ formic acid in acetonitrile as eluent to give pure $\mathrm{JN}-00245$ as a white solid (2 mg, 15\% yield over 4 steps). LC/MS (m/z): $[\mathrm{M}+\mathrm{H}]^{+} 345 .{ }^{1} \mathrm{H}$ NMR (400 MHz, DMSOd6) $\delta 7.29(\mathrm{~d}, J=8.3 \mathrm{~Hz}, 1 \mathrm{H}), 6.91(\mathrm{~d}, J=7.7 \mathrm{~Hz}, 1 \mathrm{H}), 6.78(\mathrm{~s}, 2 \mathrm{H}), 6.47(\mathrm{t}, J=7.9 \mathrm{~Hz}$, $1 \mathrm{H}), 3.79(\mathrm{t}, J=6.0 \mathrm{~Hz}, 2 \mathrm{H}), 2.78(\mathrm{t}, J=2.7 \mathrm{~Hz}, 1 \mathrm{H}), 1.97(\mathrm{td}, J=7.3,2.6 \mathrm{~Hz}, 2 \mathrm{H}), 1.88$ $(\mathrm{t}, J=6.1 \mathrm{~Hz}, 2 \mathrm{H}), 1.61(\mathrm{t}, J=7.3 \mathrm{~Hz}, 2 \mathrm{H})$.

\section{Synthesis of JN-00836}<smiles>CNCCO</smiles><smiles>COC(OC)C(C)(C)OC(=O)CBr</smiles><smiles>CN(CCO)CC(=O)OC(C)(C)C</smiles>

A<smiles>O=C(Nc1ccc(O)cc1)c1ccccc1</smiles><smiles>CN(CCO)CC(=O)OC(C)(C)C</smiles>

A<smiles>CC(C)N(CC(=O)Oc1ccc(NC(=O)c2ccccc2)cc1)CC(=O)OC(C)(C)C</smiles><smiles>CN(CCOc1ccc(N)cc1)CC(=O)OC(C)(C)C</smiles><smiles>C=CCCC(N)(CCC)CCC(=O)O</smiles>
$\underset{\mathrm{DMF}, \mathrm{RT}}{\stackrel{\text { HATU, DIEA }}{\longrightarrow}}$<smiles>C#CCCC(N)(O)CCCC(C)(CCC(=O)Nc1ccc(OCCN(C)CC(=O)O)cc1)N=O</smiles>

\section{Synthesis of Intermediate A}

2-(methylamino)ethanol (88.57 mg, $1.18 \mathrm{mmol}, 94.32 \mu \mathrm{L} 1.10$ equiv), tert-butyl 2bromoacetate $(200 \mathrm{mg}, 1.03 \mathrm{mmol}, 150.38 \mu \mathrm{L}, 1.00$ equiv), were added to a solution of triethylamine (103.76 mg, $1.03 \mathrm{mmol}, 142.91 \mu \mathrm{L}, 1.00$ equiv) in methanol (2.05 mL), and stirred at $24{ }^{\circ} \mathrm{C}$ for $12 \mathrm{~h}$. The concentrated in vacuo then the crude product was dissolved in water and mixture washed with EtOAc (x3). The combined organic layers were dried over $\mathrm{Na}_{2} \mathrm{SO}_{4}$, filtered, and concentrated in vacuo. The crude product was purified by flash column chromatography using $\mathrm{DCM} / \mathrm{MeOH}$ as eluent to give intermediate $\mathrm{A}$ as a white solid (134 mg, 69\%). LC/MS (m/z): [M+H] ${ }^{+}$190. ${ }^{1} \mathrm{H}$ NMR (400 MHz, DMSO-d $) \delta 4.40$ (brs, $1 \mathrm{H}), 3.47-3.44$ (brm, 2H), 3.20 (brs, 2H), $2.61-2.54$ (m, 2H), 2.31 (s, 3H), $1.42(\mathrm{~s}, 9 \mathrm{H})$.

\section{Step 1}

West, A. et al. Labeling preferences of diazirines with protein biomolecules, J. Am. Chem. 
tert-butyl N-(4-hydroxyphenyl)carbamate (25 mg, $119.48 \mu \mathrm{mol}, 1$ equiv) and Intermediate A $\left(29.40 \mathrm{mg}, 155.32 \mu \mathrm{mol}, 1.40\right.$ equiv) were dissolved in dry THF (1.19 mL), then $\mathrm{Ph}_{3} \mathrm{P}$ (47.01 mg, $179.22 \mu \mathrm{mol}, 1.5$ equiv) was added and the resulting solution was brought to $0{ }^{\circ} \mathrm{C}$ before diisopropyl azodicarboxylate $(36.24 \mathrm{mg}, 179.22 \mu \mathrm{mol}, 35.18 \mu \mathrm{L})$ was added dropwise. The reaction was slowly warmed to $24^{\circ} \mathrm{C}$ for $12 \mathrm{~h}$. The solvent was removed in vacuo and the crude product purified by flash column chromatography using heptane I EtOAc as eluent to give the pure intermediate 1 as a white solid (40 mg, 88\%).

\section{Step 2}

TFA (119.87 mg, $1.05 \mathrm{mmol}, 80.99 \mu \mathrm{L}, 10$ equiv) was added to a solution of intermediate 1 (40 mg, $105.13 \mu \mathrm{mol}, 1$ equiv) in dichloromethane $(1.05 \mathrm{~mL})$ and the reaction stirred at $24{ }^{\circ} \mathrm{C}$ for 6 hours. The solvent was removed in vacuo and the crude product was azeotroped with toluene $(x 3)$ to remove the excess of TFA. Intermediate 2 was used for next step without any further purification.

\section{Step 3}

3-(3-but-3-ynyldiazirin-3-yl)propanoic acid (9.48 mg, $57.05 \mu \mathrm{mol}, 1.50$ equiv) was added to a solution of HATU (20.25 mg, $53.25 \mu \mathrm{mol}, 1.40$ equiv) and diisopropylethylamine (24.58 mg, $190.17 \mu \mathrm{mol}, 33.12 \mu \mathrm{L}, 5.00$ equiv) in DMF $(190.17 \mu \mathrm{L})$, and the reaction was stirred at $24{ }^{\circ} \mathrm{C}$ for $10 \mathrm{~min}$. The above crude intermediate $2(15 \mathrm{mg}, 38.03 \mu \mathrm{mol}$, TFA, 1 equiv) was then added and the reaction was complete in $5 \mathrm{~min}$. The reaction mixture was purified by prep HPLC using $0.1 \%$ formic acid in water $/ 0.1 \%$ formic acid in acetonitrile as eluent to give pure intermediate 3 as a white solid (6 mg, 40\%).

\section{Step 4}

TFA (31.93 mg, $280.03 \mu \mathrm{mol}, 21.57 \mu \mathrm{L}, 20$ equiv) was added to a solution of intermediate 3 (6 mg, $14.00 \mu \mathrm{mol}, 1$ equiv) in DCM (140.02 $\mu \mathrm{L})$, and the reaction was stirred at $24{ }^{\circ} \mathrm{C}$ for 24 hours. The solvent was then removed in vacuo, and the crude azeotroped with toluene $(x 3)$ to remove the excess of TFA and purified by prep HPLC using $0.1 \%$ formic acid in water $/ 0.1 \%$ formic acid in acetonitrile as eluent to give $\mathrm{JN}-00836$ as a white solid (1.1 mg, 20\%). LC/MS (m/z): $[\mathrm{M}+\mathrm{H}]^{+}$373. ${ }^{1} \mathrm{H}$ NMR (400 MHz, DMSO-d $\left.d_{6}\right) \delta 9.80(\mathrm{~s}, 1 \mathrm{H})$, $7.46(\mathrm{~d}, J=8.1 \mathrm{~Hz}, 2 \mathrm{H}), 6.87(\mathrm{~d}, J=8.5 \mathrm{~Hz}, 2 \mathrm{H}), 4.06-4.02(\mathrm{brm}, 2 \mathrm{H}), 3.24-3.21$ (brm, $2 \mathrm{H}$ ), 3.00 - 2.92 (brm, 2H), $2.86-2.84$ (brs, 1H), 2.43 (s, 3H), $2.11-2.07$ (brm, 2H), 2.02 - 1.99 (brm, 2H), $1.77-1.72$ (brm, 2H), $1.63-1.58$ (brm, 2H).

\section{Synthesis of JN-00846 and JN-00936}


<smiles>Nc1nc(Cl)c2nc[nH]c2n1</smiles><smiles>C#CCCC1(CCI)N=N1</smiles><smiles>C#CCCC1(CCn2cnc3c(Cl)nc(N)nc32)N=N1</smiles>

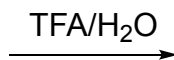<smiles>C#CCCC1(CCn2cnc3c(=O)[nH]c(N)nc32)N=N1</smiles>

$\mathrm{JN}-00846$<smiles>[C][C+](C)(O)O[N+](=O)[O-]</smiles>

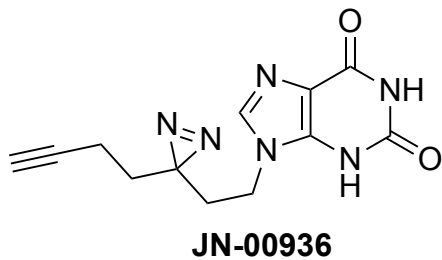

\section{Step 1}

Cesium carbonate ( $57.64 \mathrm{mg}, 176.92 \mu \mathrm{mol}, 1.50$ equiv) was added to a solution of 6chloro-9H-purin-2-amine (20 mg, $117.94 \mu \mathrm{mol}, 1$ equiv) in DMF $(589.72 \mu \mathrm{L})$ followed by $3-$ but-3-ynyl-3-(2-iodoethyl)diazirine (35.11 mg, $141.53 \mu \mathrm{mol}, 1.2$ equiv). The reaction mixture was stirred at $24{ }^{\circ} \mathrm{C}$ for $12 \mathrm{~h}$. The reaction was diluted with brine and the mixture was washed with DCM (x3). The combined organic layers were dried over $\mathrm{Na}_{2} \mathrm{SO}_{4}$, filtered, and concentrated in vacuo. The crude intermediate 1 was used for next step without any further purification.

\section{Step 2}

Intermediate 1 (34 mg, $117.35 \mu \mathrm{mol}, 1$ equiv) was dissolved in a mixture of TFA (880.15 $\mu \mathrm{L})$ and water $(293.38 \mu \mathrm{L})$, and the reaction was stirred at $24^{\circ} \mathrm{C}$ for 48 hours. The solvents were evaporated in vacuo, then the crude azeotroped with toluene $(x 3)$ to remove excess of TFA and water. The obtained solid was then washed with diethyl ether to give pure JN00846 as a white precipitate that was filtered and dried under vacuum (12 $\mathrm{mg}, 38 \%$ ). LC/MS (m/z): $[\mathrm{M}+\mathrm{H}]^{+}$272. ${ }^{1} \mathrm{H}$ NMR (400 MHz, DMSO-d $\left.\mathrm{d}_{6}\right) \delta 10.94(\mathrm{~s}, 1 \mathrm{H}), 8.22(\mathrm{~s}, 1 \mathrm{H})$, $6.71(\mathrm{~s}, 2 \mathrm{H}), 3.93(\mathrm{t}, J=7.3 \mathrm{~Hz}, 2 \mathrm{H}), 2.85(\mathrm{dt}, J=4.6,2.6 \mathrm{~Hz}, 1 \mathrm{H}), 1.99(\mathrm{td}, J=7.3,2.6$ $\mathrm{Hz}, 2 \mathrm{H}), 1.93(\mathrm{t}, J=7.3 \mathrm{~Hz}, 2 \mathrm{H}), 1.62(\mathrm{t}, J=7.1 \mathrm{~Hz}, 3 \mathrm{H})$.

\section{Synthesis of JN-00936}

$\mathrm{JN}-00846$ (20 mg, $73.73 \mu \mathrm{mol}, 1$ equiv) was resuspended in water $(1.84 \mathrm{~mL})$, then acetic acid $(66.41 \mathrm{mg}, 1.11 \mathrm{mmol}, 63.25 \mu \mathrm{L}, 15 \mathrm{eq})$ was added followed by Sodium Nitrite $(45.78$ $\mathrm{mg}, 663.53 \mu \mathrm{mol})$. The reaction mixture was stirred at $40{ }^{\circ} \mathrm{C} 12 \mathrm{~h}$. Brine was added and the resulting mixture was washed with EtOAc $(x 3)$. The combined organic layers were dried over $\mathrm{Na}_{2} \mathrm{SO}_{4}$, filtered, and concentrated in vacuo. The crude was dissolved in DMSO and purified by prep HPLC using $0.1 \%$ formic acid in water $/ 0.1 \%$ formic acid in acetonitrile as eluent to to give pure $\mathrm{JN}-00936$ as a white solid $(5 \mathrm{mg}, 25 \%)$. LC/MS (m/z): $[\mathrm{M}+\mathrm{H}]^{+}$ 273. ${ }^{1} \mathrm{H}$ NMR (400 MHz, DMSO- $\left.d_{6}\right) \delta 10.66(\mathrm{~s}, 1 \mathrm{H}), 7.70(\mathrm{~s}, 1 \mathrm{H}), 3.95(\mathrm{t}, J=7.5 \mathrm{~Hz}, 2 \mathrm{H})$, $2.85(\mathrm{t}, J=2.7 \mathrm{~Hz}, 1 \mathrm{H}), 1.98(\mathrm{td}, J=7.4,2.6 \mathrm{~Hz}, 2 \mathrm{H}), 1.82(\mathrm{dd}, J=8.2,6.8 \mathrm{~Hz}, 2 \mathrm{H}), 1.62$ $(\mathrm{t}, J=7.4 \mathrm{~Hz}, 2 \mathrm{H})$.

\section{Synthesis of JN-00847 and JN-00845}


<smiles>Nc1ncnc2[nH]cnc12</smiles><smiles>C#CCCC1(CCI)N=N1</smiles><smiles>C#CCCC1(CCn2cnc3c(N)ncnc32)N=N1</smiles><smiles>O=[W]O[N+](=O)[O-]</smiles><smiles>C#CCCC1(CCn2cnc3c(=O)[nH]cnc32)N=N1</smiles>

Cesium carbonate ( $54.25 \mathrm{mg}, 166.51 \mu \mathrm{mol} .1 .50$ equiv) was added to a solution of $9 \mathrm{H}-$ purin-6-amine (15 mg, $111.01 \mu \mathrm{mol}, 1$ equiv) in DMF $(222.01 \mu \mathrm{L})$, followed by 3-but-3ynyl-3-(2-iodoethyl)diazirine (33.04 mg, $133.21 \mu \mathrm{mol}, 1.20$ equiv), then the reaction mixture was stirred at $24{ }^{\circ} \mathrm{C} 12 \mathrm{~h}$. Water was added and the resulting mixture was washed with EtOAc (x3). The combined organic layers were dried over $\mathrm{Na}_{2} \mathrm{SO}_{4}$, filtered, and concentrated in vacuo. The crude material was treated with DCM to give pure JN-00847 as a white solid $(10 \mathrm{mg}, 35 \%)$. LC/MS $(\mathrm{m} / \mathrm{z}):[\mathrm{M}+\mathrm{H}]^{+}$256. ${ }^{1} \mathrm{H}$ NMR $\left(400 \mathrm{MHz}, \mathrm{DMSO}-d_{6}\right)$ $\delta 8.16(\mathrm{~s}, 1 \mathrm{H}), 8.13(\mathrm{~s}, 1 \mathrm{H}), 7.22(\mathrm{~s}, 1 \mathrm{H}), 4.10(\mathrm{t}, J=7.1 \mathrm{~Hz}, 2 \mathrm{H}), 2.83(\mathrm{t}, J=2.7 \mathrm{~Hz}, 1 \mathrm{H})$, $1.97(\mathrm{td}, J=7.3,2.9 \mathrm{~Hz}, 4 \mathrm{H}), 1.59(\mathrm{~d}, J=14.8 \mathrm{~Hz}, 2 \mathrm{H})$.

Crude JN-00847 (22 mg, $86.18 \mu \mathrm{mol})$ was resuspended in water $(2.15 \mathrm{~mL})$, then sodium nitrite $(53.51 \mathrm{mg}, 775.63 \mu \mathrm{mol}, 24.66 \mu \mathrm{L})$ was added, followed by acetic acid $(77.63 \mathrm{mg}$, $1.29 \mathrm{mmol}, 73.93 \mu \mathrm{L}$ ). The reaction mixture was stirred at $40{ }^{\circ} \mathrm{C} 12$. The reaction mixture was concentrated in vacuo, then the crude was azeotroped with toluene $(x 3)$ to remove excess of water and acetic acid. The crude was then dissolved in DMSO and purified by prep HPLC using $0.1 \%$ formic acid in water / $0.1 \%$ formic acid in acetonitrile as eluent to give pure $\mathrm{JN}-00845$ as a white solid $(8 \mathrm{mg}, 36 \%)$. LC/MS (m/z): $[\mathrm{M}+\mathrm{H}]^{+} 257 .{ }^{1} \mathrm{H}$ NMR (400 $\left.\mathrm{MHz}, \mathrm{DMSO}-d_{6}\right) \delta 8.12(\mathrm{~s}, 1 \mathrm{H}), 8.04(\mathrm{~s}, 1 \mathrm{H}), 4.11(\mathrm{t}, J=7.2 \mathrm{~Hz}, 2 \mathrm{H}), 2.84(\mathrm{t}, J=2.7 \mathrm{~Hz}$, $1 \mathrm{H}), 2.01-1.91(\mathrm{~m}, 4 \mathrm{H}), 1.59(\mathrm{t}, J=7.4 \mathrm{~Hz}, 2 \mathrm{H})$.

\section{Synthesis of JN-00938}<smiles>OC(c1ccccc1)(c1ccccc1)C1CCNCC1</smiles><smiles>C#CCCC1(CCI)N=N1</smiles><smiles>C#CCCC1(CCN2CCC(C(O)(c3ccccc3)c3ccccc3)CC2)N=N1</smiles>

JN-00938

Potassium carbonate (11.63 mg, $84.15 \mu \mathrm{mol}, 5.08 \mu \mathrm{L}, 1.50$ equiv) was added to a solution of diphenyl(4-piperidyl)methanol (15 mg, $56.10 \mu \mathrm{mol}, 1$ equiv) in acetone $(561.03 \mu \mathrm{L})$, followed by 3-but-3-ynyl-3-(2-iodoethyl)diazirine (16.70 mg, $67.32 \mu \mathrm{mol}, 1.20$ equiv). The reaction was then stirred at $24{ }^{\circ} \mathrm{C} 12 \mathrm{~h}$. The reaction was concentrated in vacuo and the 
crude was dissolved in DMSO, filtered and purified by prep HPLC using $0.1 \%$ formic acid in water $/ 0.1 \%$ formic acid in acetonitrile as eluent to give pure $\mathrm{JN}-00938$ as a white solid (9 mg, 37\%). LC/MS (m/z): $[\mathrm{M}+\mathrm{H}]^{+} 388 .{ }^{1} \mathrm{H}$ NMR (400 MHz, DMSO-d 6$) \delta 7.50$ (d, J = 7.5 $\mathrm{Hz}, 4 \mathrm{H}), 7.26(\mathrm{t}, J=7.6 \mathrm{~Hz}, 4 \mathrm{H}), 7.12(\mathrm{t}, J=7.3 \mathrm{~Hz}, 2 \mathrm{H}), 2.83-2.77(\mathrm{~m}, 3 \mathrm{H}), 2.06(\mathrm{t}, J=$ $7.5 \mathrm{~Hz}, 2 \mathrm{H}), 1.99(\mathrm{td}, J=7.4,2.7 \mathrm{~Hz}, 2 \mathrm{H}), 1.84(\mathrm{t}, J=11.5 \mathrm{~Hz}, 2 \mathrm{H}), 1.60-1.39(\mathrm{~m}, 6 \mathrm{H})$, $1.26-1.15(\mathrm{~m}, 2 \mathrm{H})$.

JN-00849 was prepared using the same procedure as $\mathrm{JN}-00938$

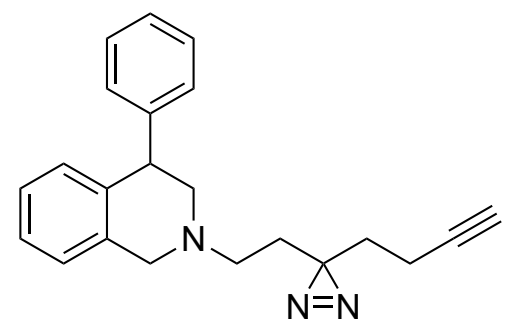

$\mathrm{JN}-00849$

LC/MS (m/z): $[\mathrm{M}+\mathrm{H}]^{+} 330 .{ }^{1} \mathrm{H}$ NMR $\left(400 \mathrm{MHz}, \mathrm{CDCl}_{3}\right) \delta 7.31-7.28(\mathrm{~m}, 1 \mathrm{H}), 7.25-7.17$ $(\mathrm{m}, 3 \mathrm{H}), 7.16-7.03(\mathrm{~m}, 3 \mathrm{H}), 6.87(\mathrm{~d}, J=7.5 \mathrm{~Hz}, 1 \mathrm{H}), 4.29-4.17(\mathrm{~m}, 1 \mathrm{H}), 3.79-3.61$ $(\mathrm{m}, 2 \mathrm{H}), 3.00(\mathrm{ddd}, J=11.4,5.4,1.2 \mathrm{~Hz}, 1 \mathrm{H}), 2.62(\mathrm{dd}, J=11.5,8.0 \mathrm{~Hz}, 1 \mathrm{H}), 2.36(\mathrm{t}, J=$ $7.4 \mathrm{~Hz}, 2 \mathrm{H}), 2.00-1.91(\mathrm{~m}, 3 \mathrm{H}), 1.68-1.55(\mathrm{~m}, 4 \mathrm{H})$.

\section{Synthesis of JN-00940}<smiles>O=C(Nc1ccc(O)cc1)Oc1ccccc1</smiles><smiles>C#CCCC1(CCI)N=N1</smiles><smiles>[R]OC(=O)C1(C(=O)O)OC1=O</smiles>

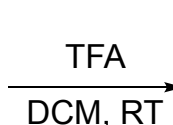

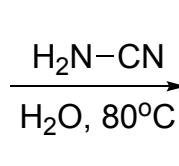

2<smiles>C#CCCC1(CCOc2ccc(NC(=O)OC(C)(C)C)cc2)N=N1</smiles>

1<smiles>C#CCCC1(CCOc2ccc(NC(=N)N)cc2)N=N1</smiles>

JN-00940

\section{Step 1}

Cesium carbonate ( $58.39 \mathrm{mg}, 179.22 \mu \mathrm{mol}, 1.50$ equiv) was added to a solution of tertbutyl N-(4-hydroxyphenyl)carbamate (25 mg, $119.48 \mu \mathrm{mol}, 1$ equiv) d in DMF (1.19 mL), followed by 3-but-3-ynyl-3-(2-iodoethyl)diazirine (35.57 mg, $143.37 \mu \mathrm{mol}, 1.20$ equiv). The resulting mixture was stirred at $24{ }^{\circ} \mathrm{C} 12 \mathrm{~h}$. Brine was added and the mixture was washed with diethyl ether (x3). The combined organic layers were dried over $\mathrm{Na}_{2} \mathrm{SO}_{4}$, filtered, and concentrated in vacuo. The crude was purified by flash column chromatography using heptane / EtOAc as eluent to give pure intermediate 1 as a white solid (15 mg, 38\%).

\section{Step 2}


Intermediate 1 (15 mg, $45.54 \mu \mathrm{mol}, 1$ equiv) was dissolved in DCM $(455.38 \mu \mathrm{L})$, then TFA (103.85 mg, $910.77 \mu \mathrm{mol}, 70.17 \mu \mathrm{L}, 20.0$ equiv) was added and the reaction was stirred at $24{ }^{\circ} \mathrm{C}$ for $12 \mathrm{~h}$. The reaction was concentrated in vacuo, then the crude was azeotroped with toluene $(x 3)$ to remove the excess of TFA. The crude intermediate 2 was used for next step without any further purification.

\section{Step 3}

Intermediate $2(11 \mathrm{mg}, 47.98 \mu \mathrm{mol})$ was dissolved in water $(479.77 \mu \mathrm{L})$, then cyanamide $(10.08 \mathrm{mg}, 239.88 \mu \mathrm{mol}, 7.88 \mu \mathrm{L})$ was added and the reaction mixture was stirred at 80 ${ }^{\circ} \mathrm{C}$ overnight. The reaction was concentrated in vacuo, then the crude product was dissolved in DMSO and purified by prep HPLC using $0.1 \%$ formic acid in water $/ 0.1 \%$ formic acid in acetonitrile as eluent to give pure $\mathrm{JN}-00940$ as a white solid (3 $\mathrm{mg}, 20 \%$ ). LC/MS (m/z): $[\mathrm{M}+\mathrm{H}]^{+}$272. ${ }^{1} \mathrm{H}$ NMR (400 MHz, DMSO-d $)$ $\delta 8.42(\mathrm{~s}, 1 \mathrm{H}), 7.55(\mathrm{~s}, 4 \mathrm{H}), 7.12$ $(\mathrm{d}, J=9.2 \mathrm{~Hz}, 2 \mathrm{H}), 6.96(\mathrm{~d}, J=8.9 \mathrm{~Hz}, 2 \mathrm{H}), 3.80(\mathrm{t}, J=6.0 \mathrm{~Hz}, 2 \mathrm{H}), 2.85(\mathrm{t}, J=2.7 \mathrm{~Hz}$, $1 \mathrm{H}), 2.04$ (td, $J=7.4,2.6 \mathrm{~Hz}, 2 \mathrm{H}), 1.89(\mathrm{t}, J=6.0 \mathrm{~Hz}, 2 \mathrm{H}), 1.66(\mathrm{t}, J=7.4 \mathrm{~Hz}, 2 \mathrm{H})$. 
Catalog of nuclear magnetic resonance and infrared spectra for synthesized compounds:

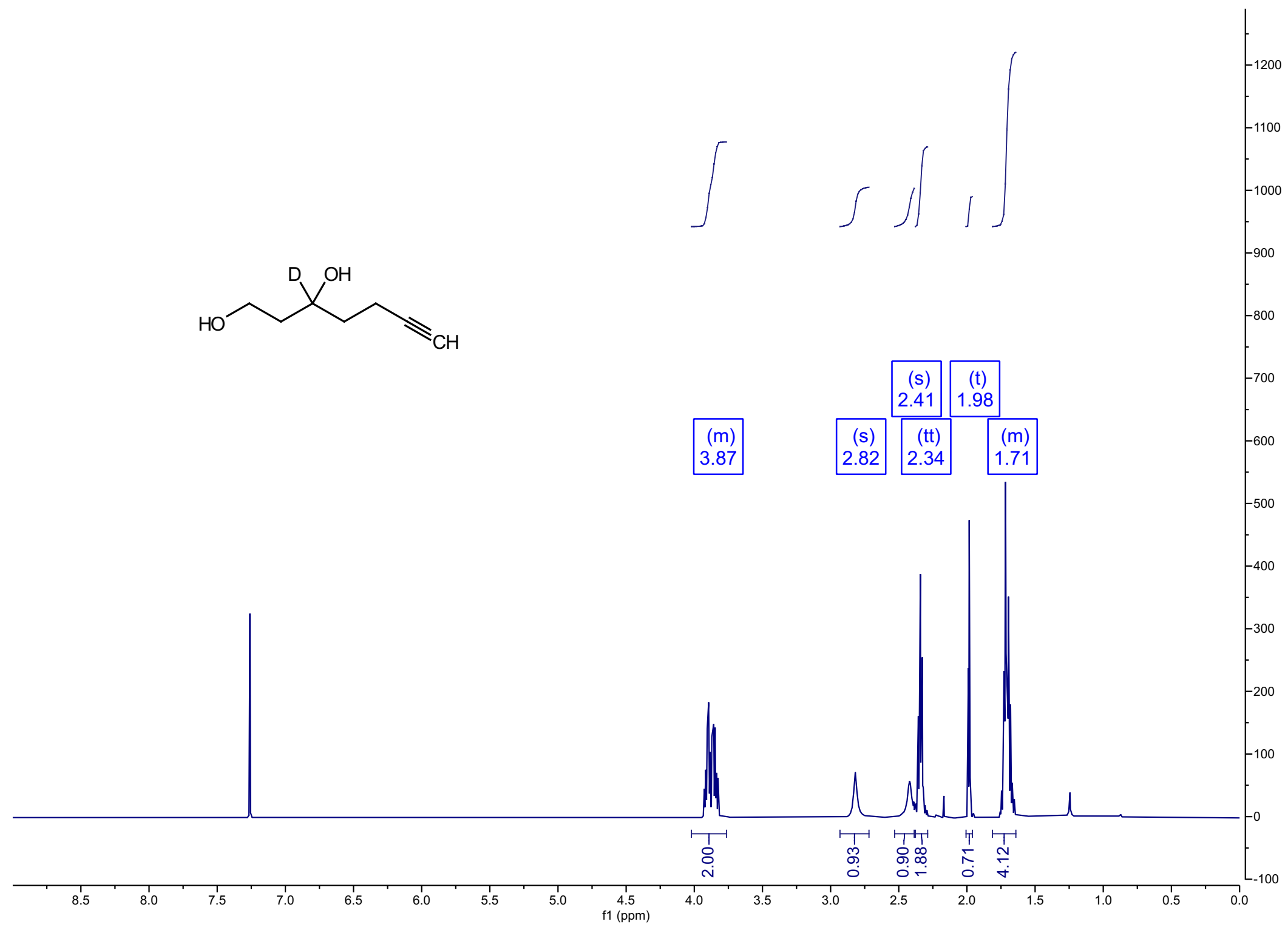




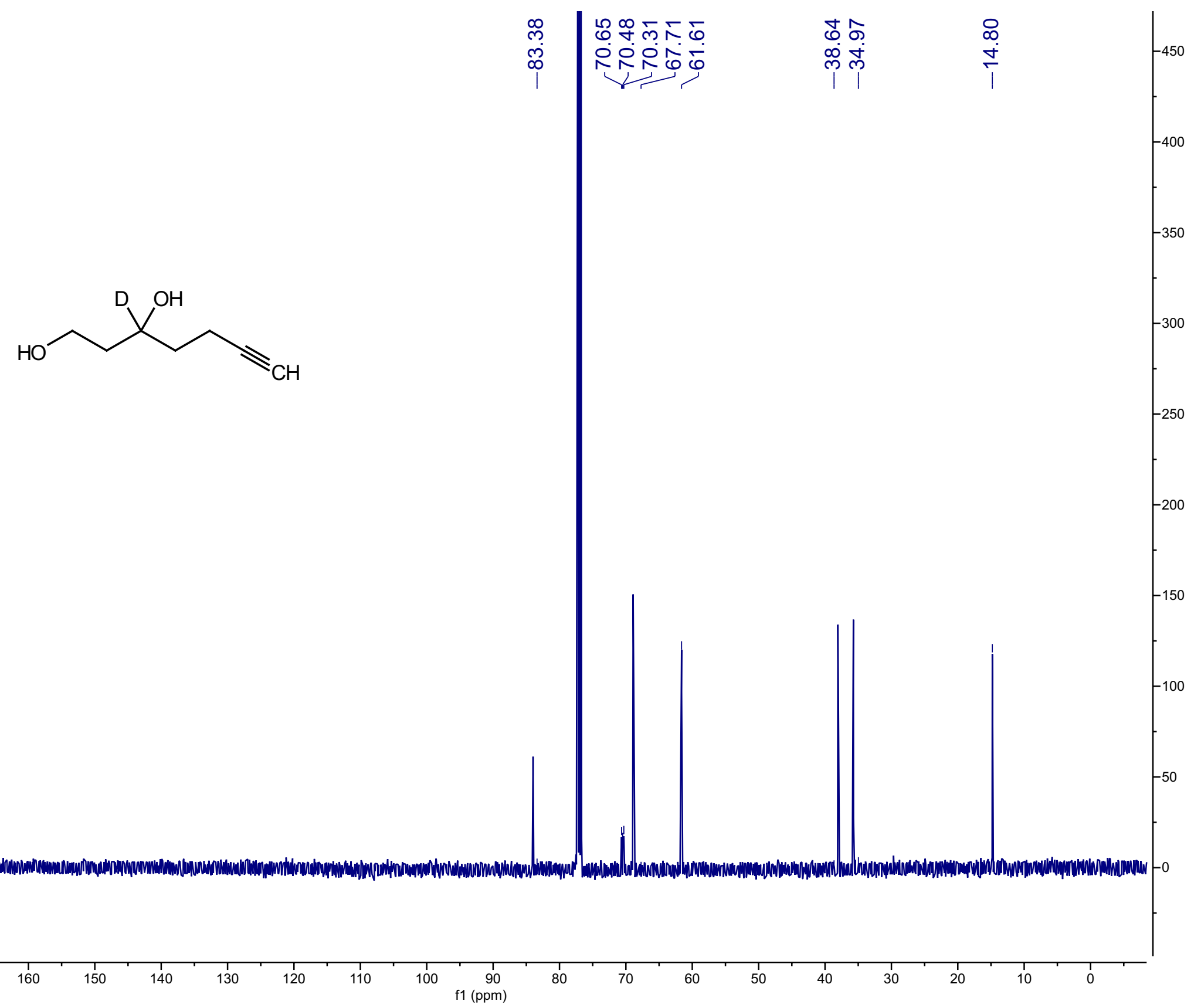




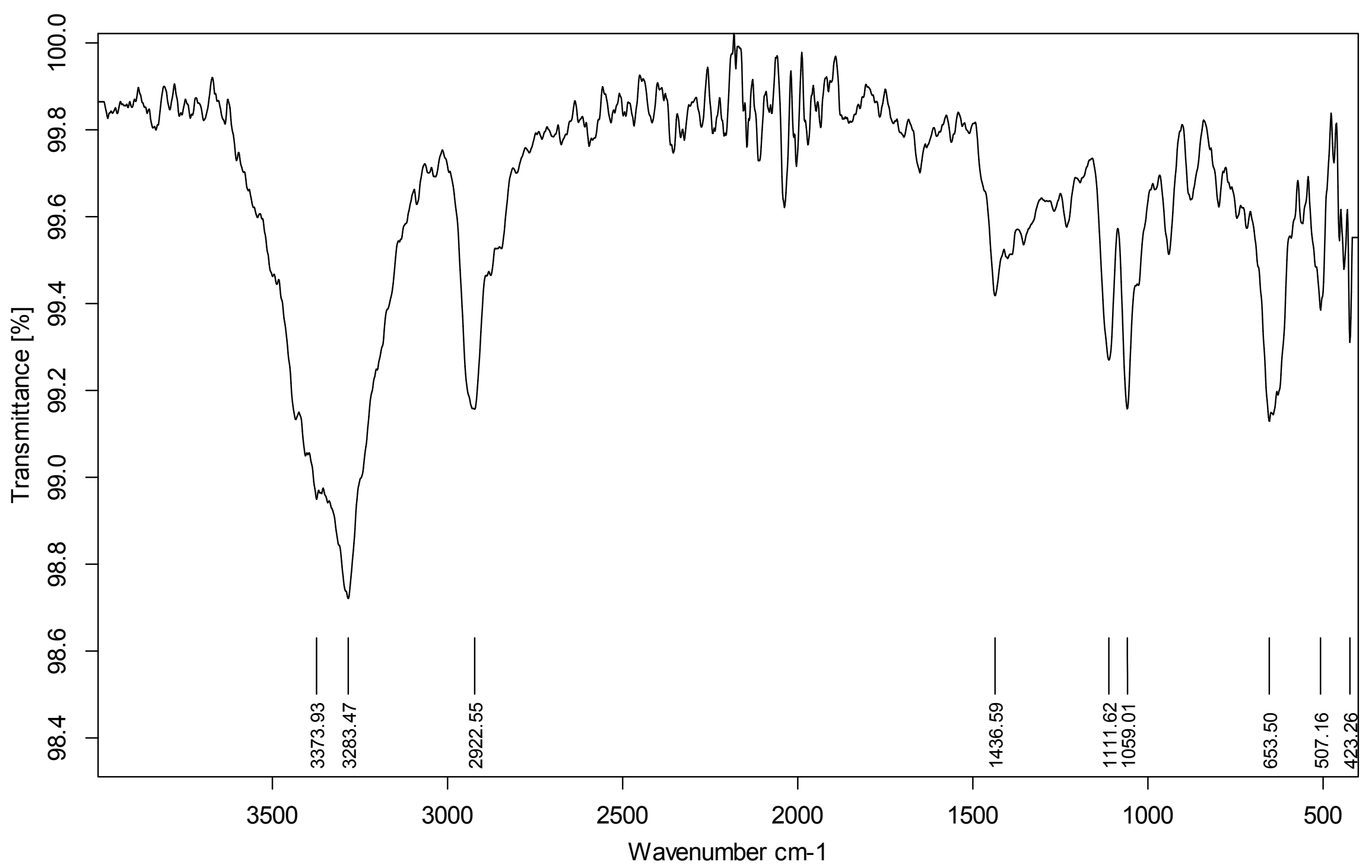

Z:IAlpha FTIR DatalWoolAlex\AW_I_51.0

AW_I51

Diamond ATR

Page 1/2 


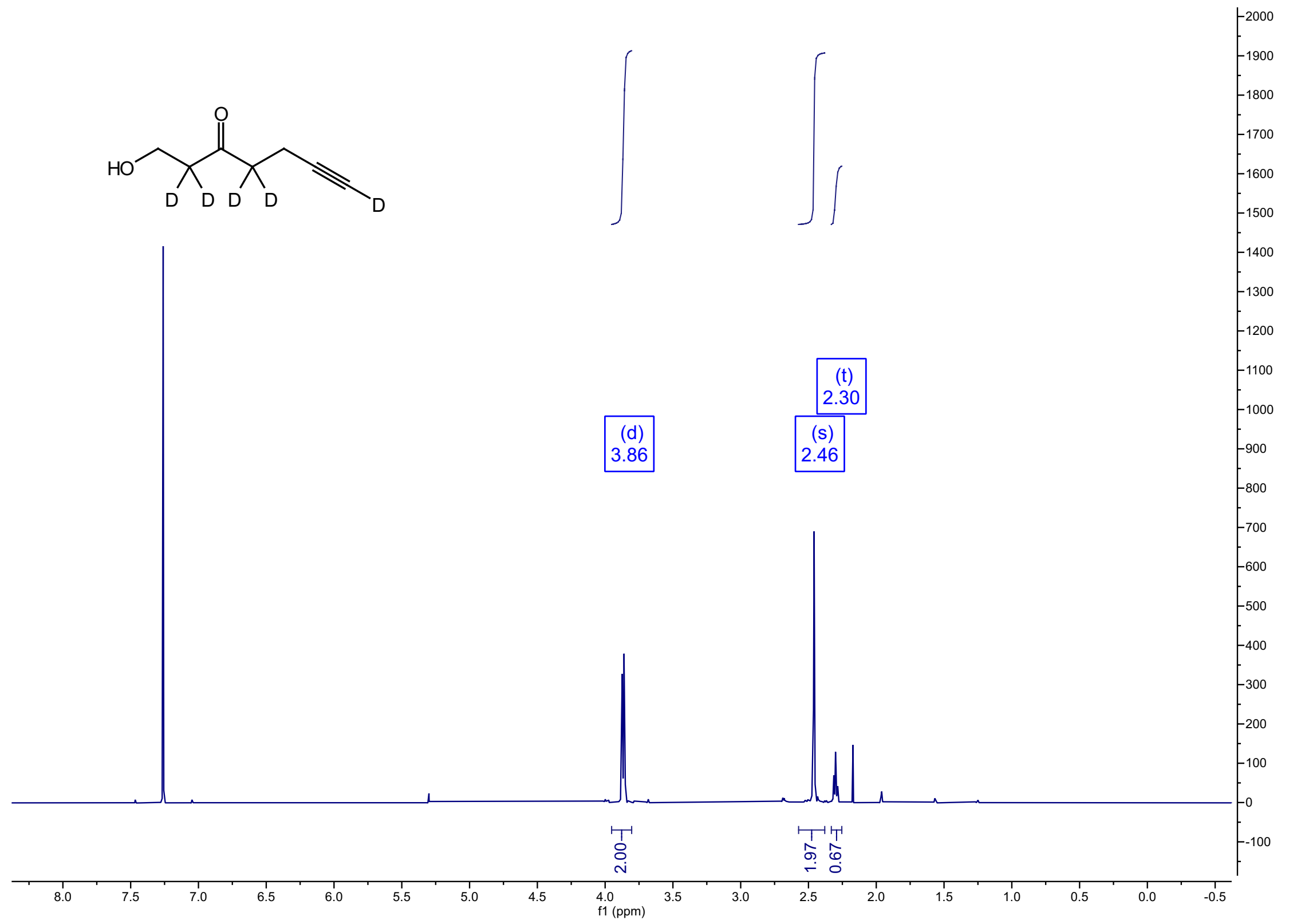

West, A. et al. Labeling preferences of diazirines with protein biomolecules, J. Am. Chem. Soc. 2021. 

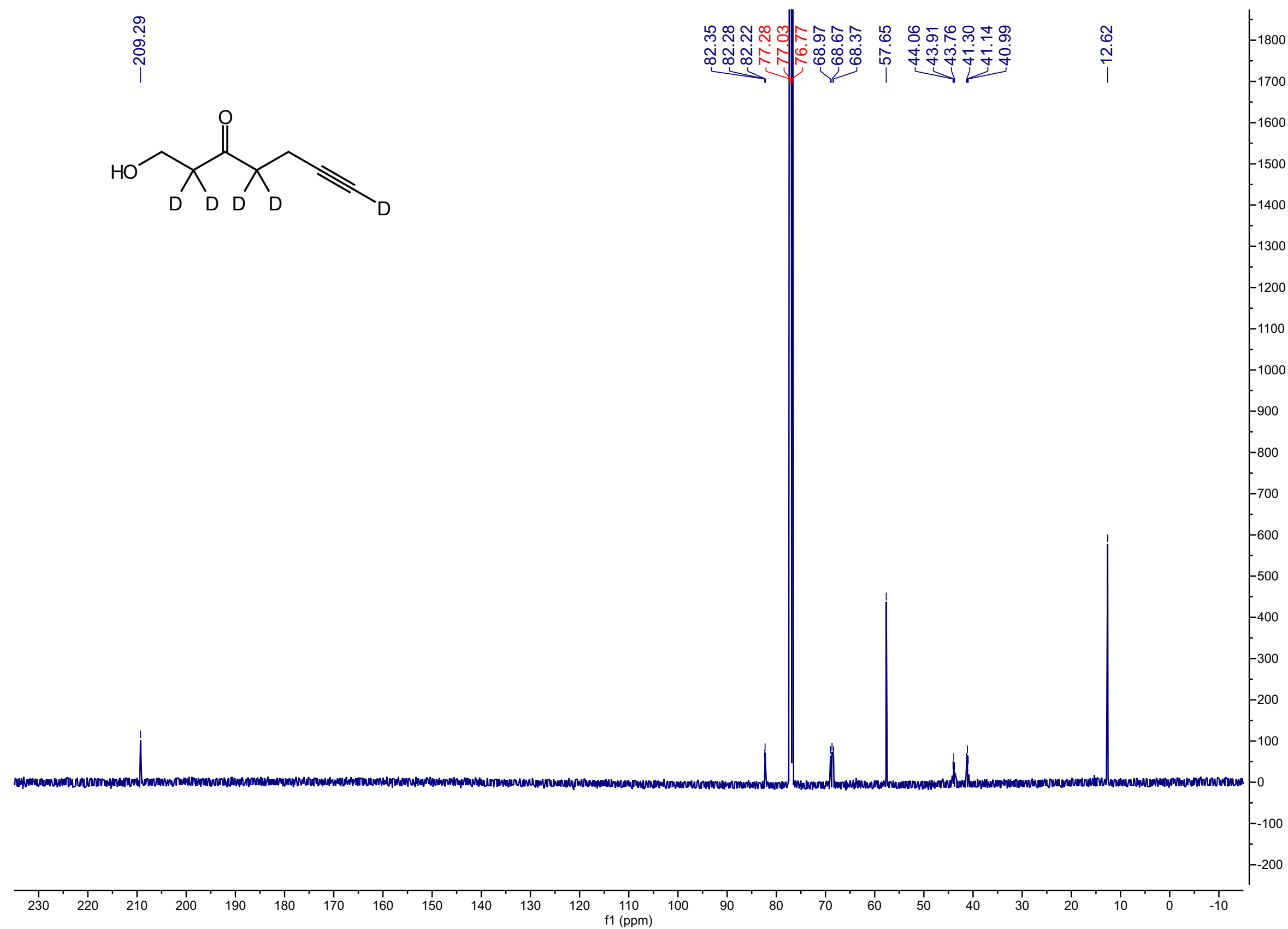


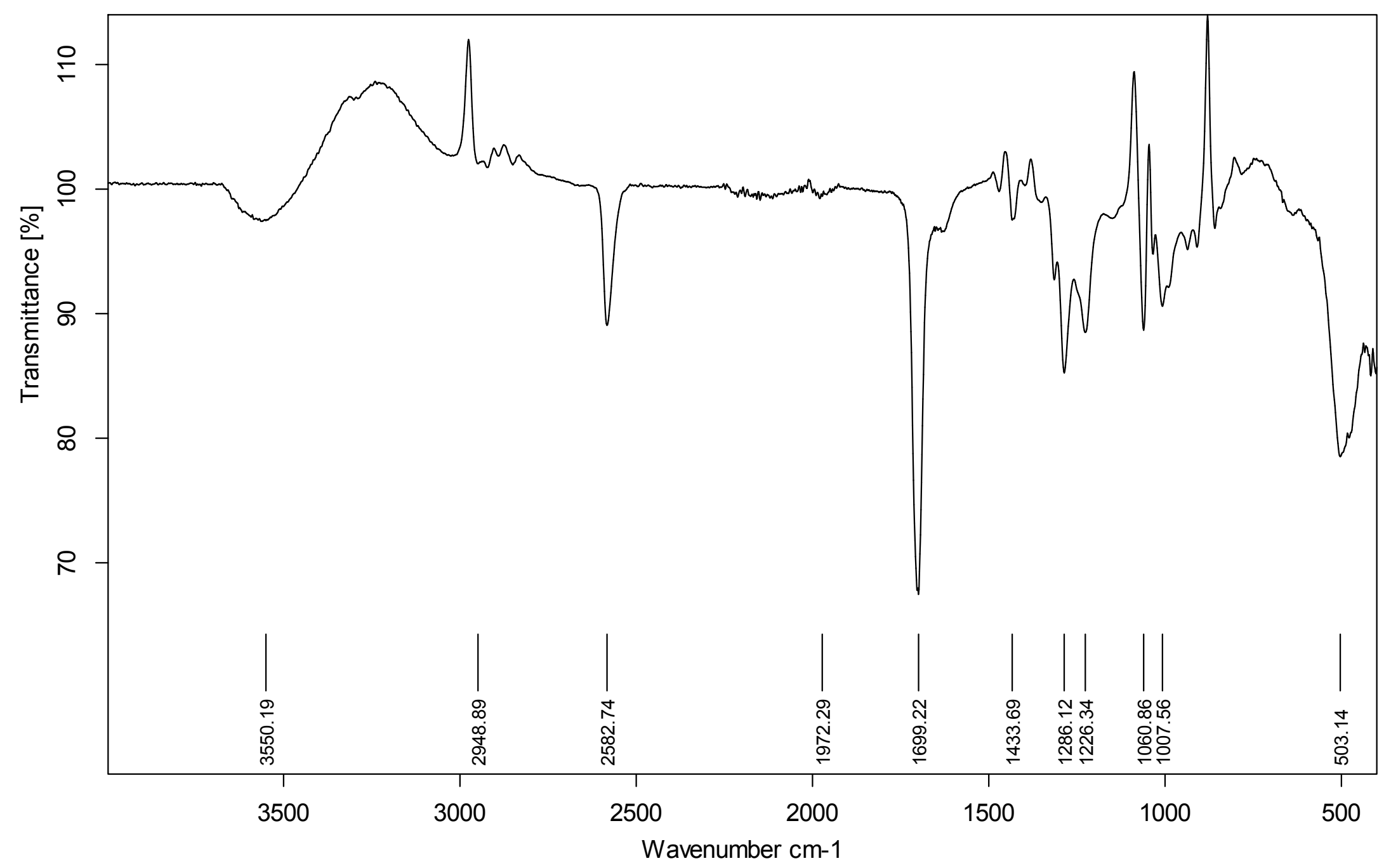

Z:IAlpha FTIR DatalWoolAlexIAW_I63.0 AW_I_63 Diamond ATR

Page 1/2 

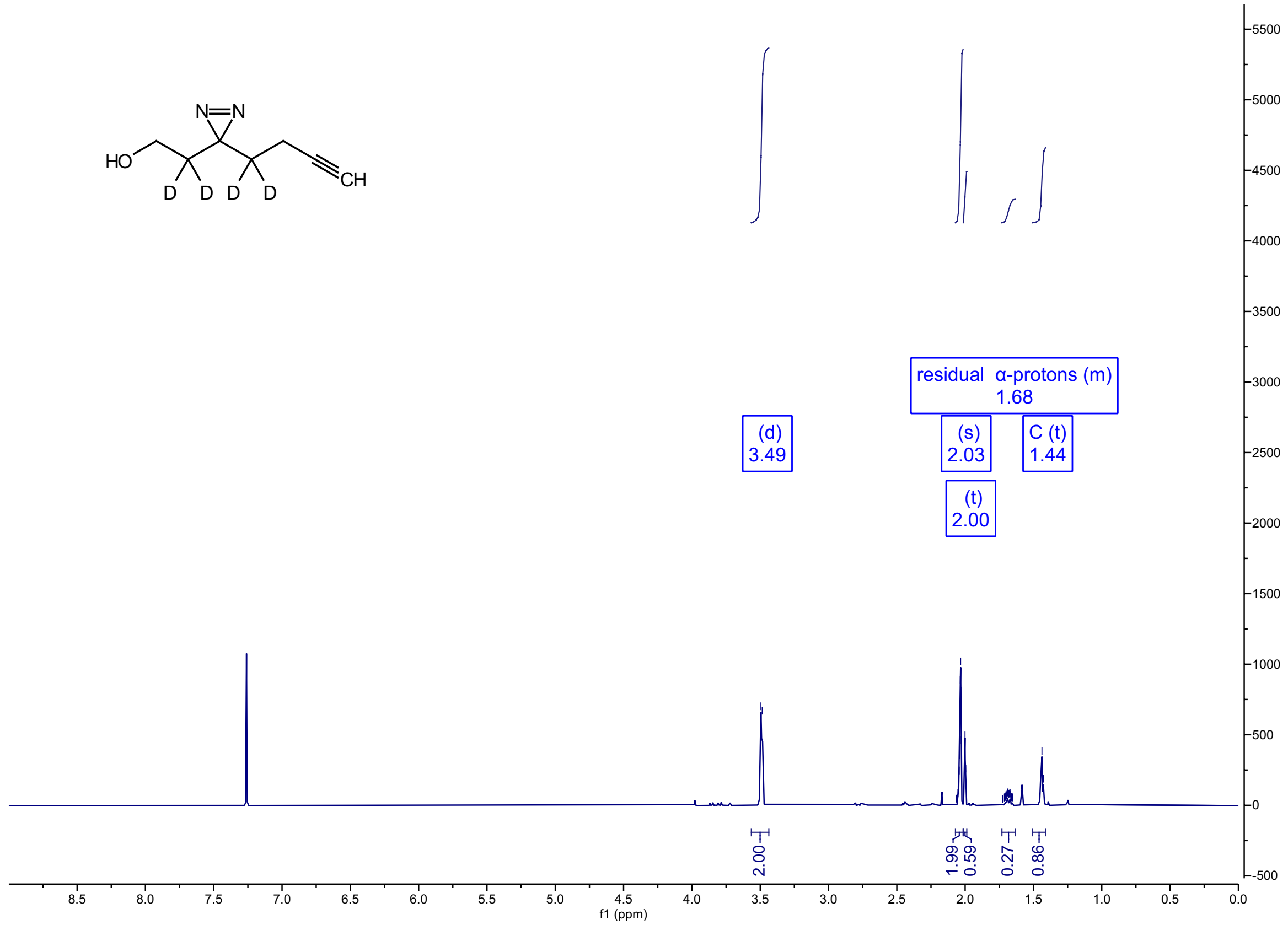

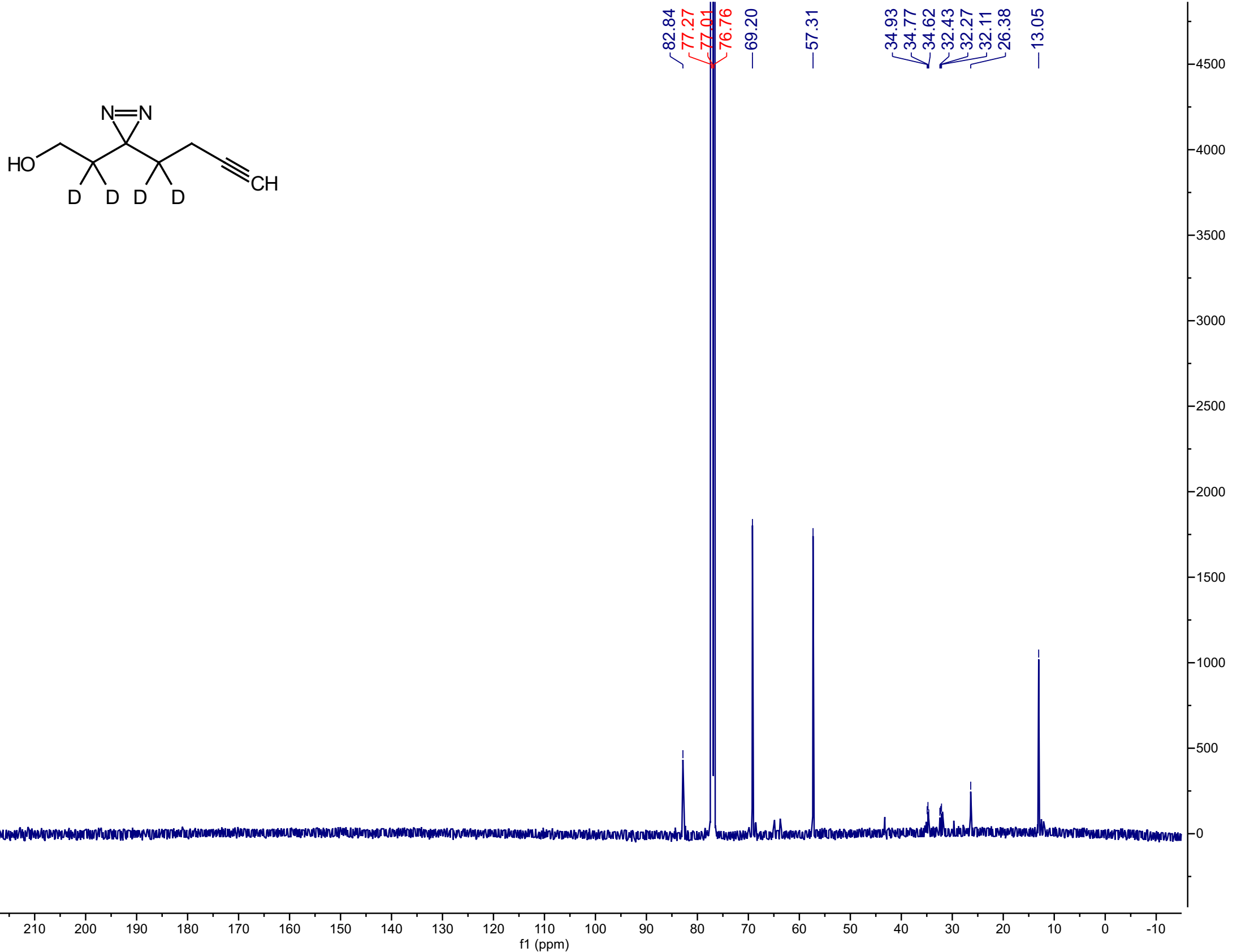


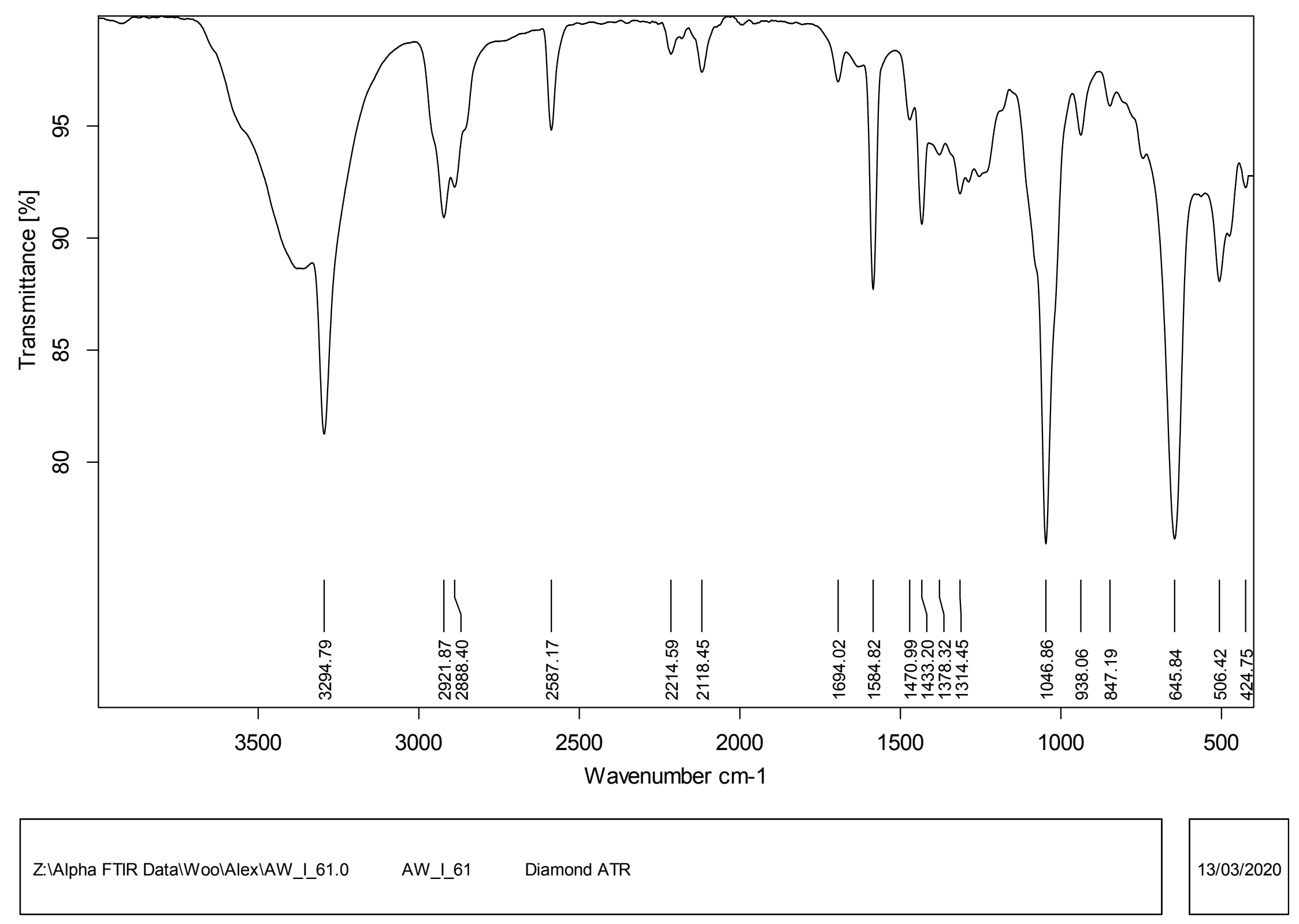

Page 1/2 


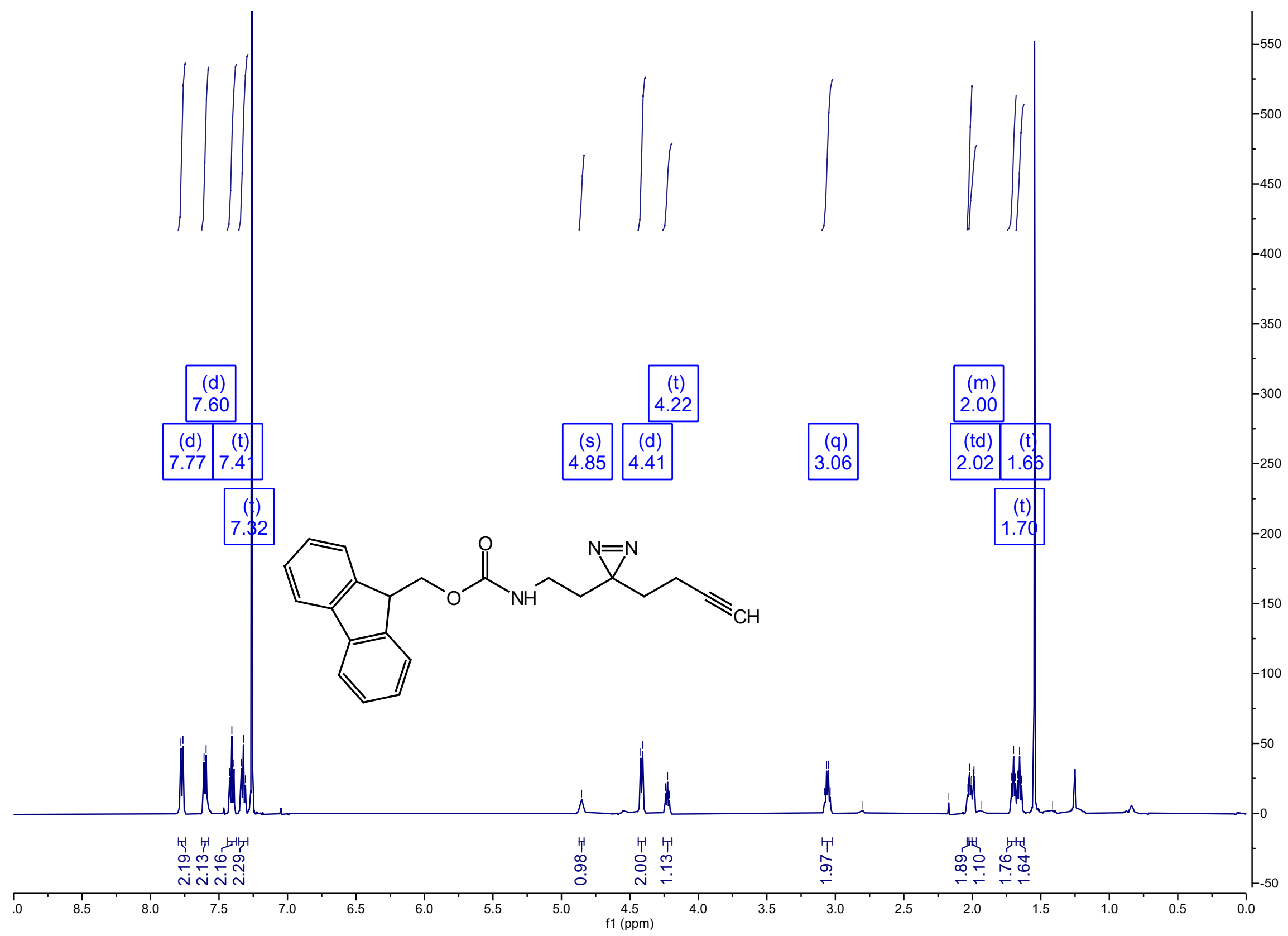

West, A. et al. Labeling preferences of diazirines with protein biomolecules, J. Am. Chem. Soc. 2021. 


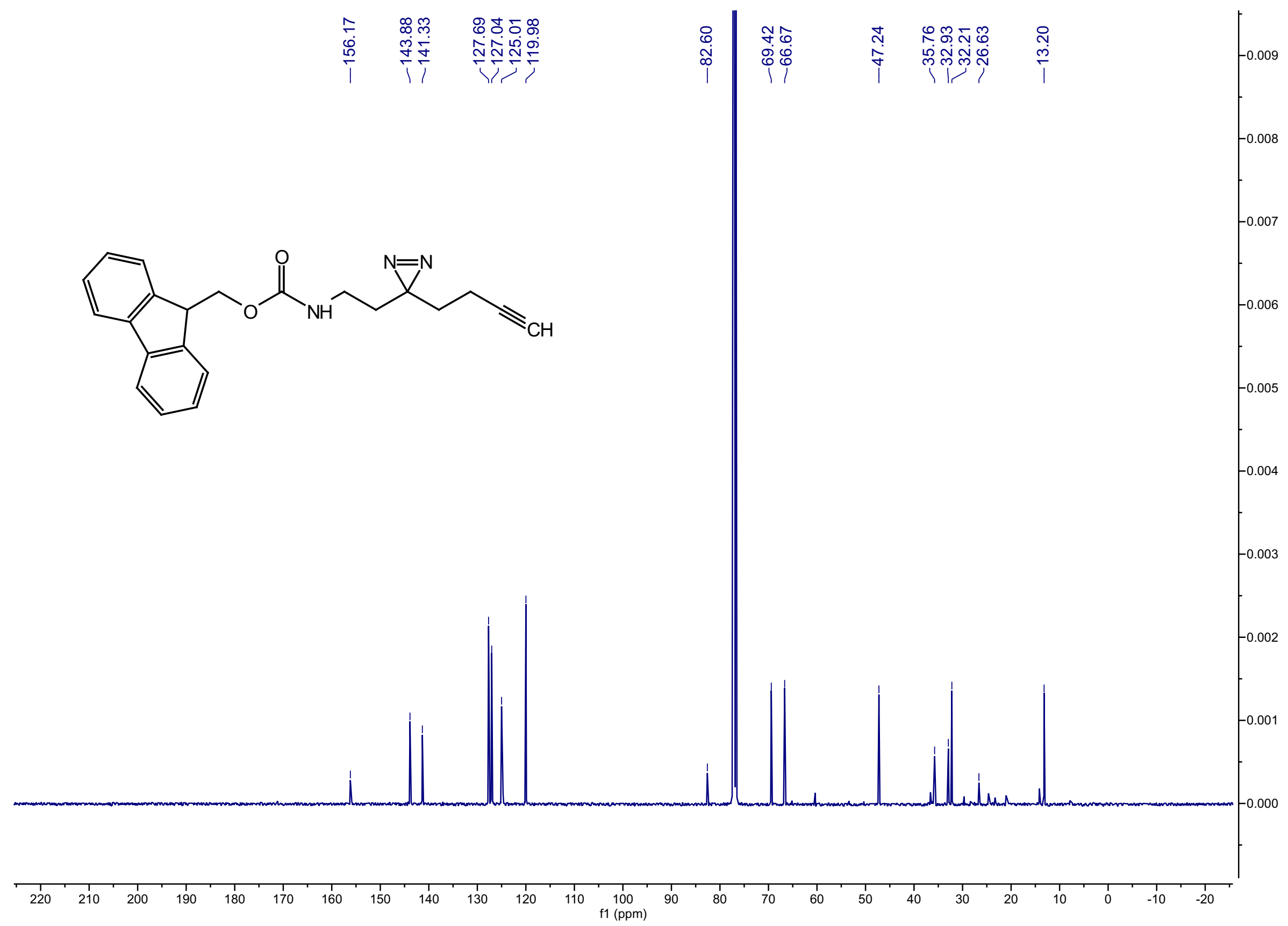




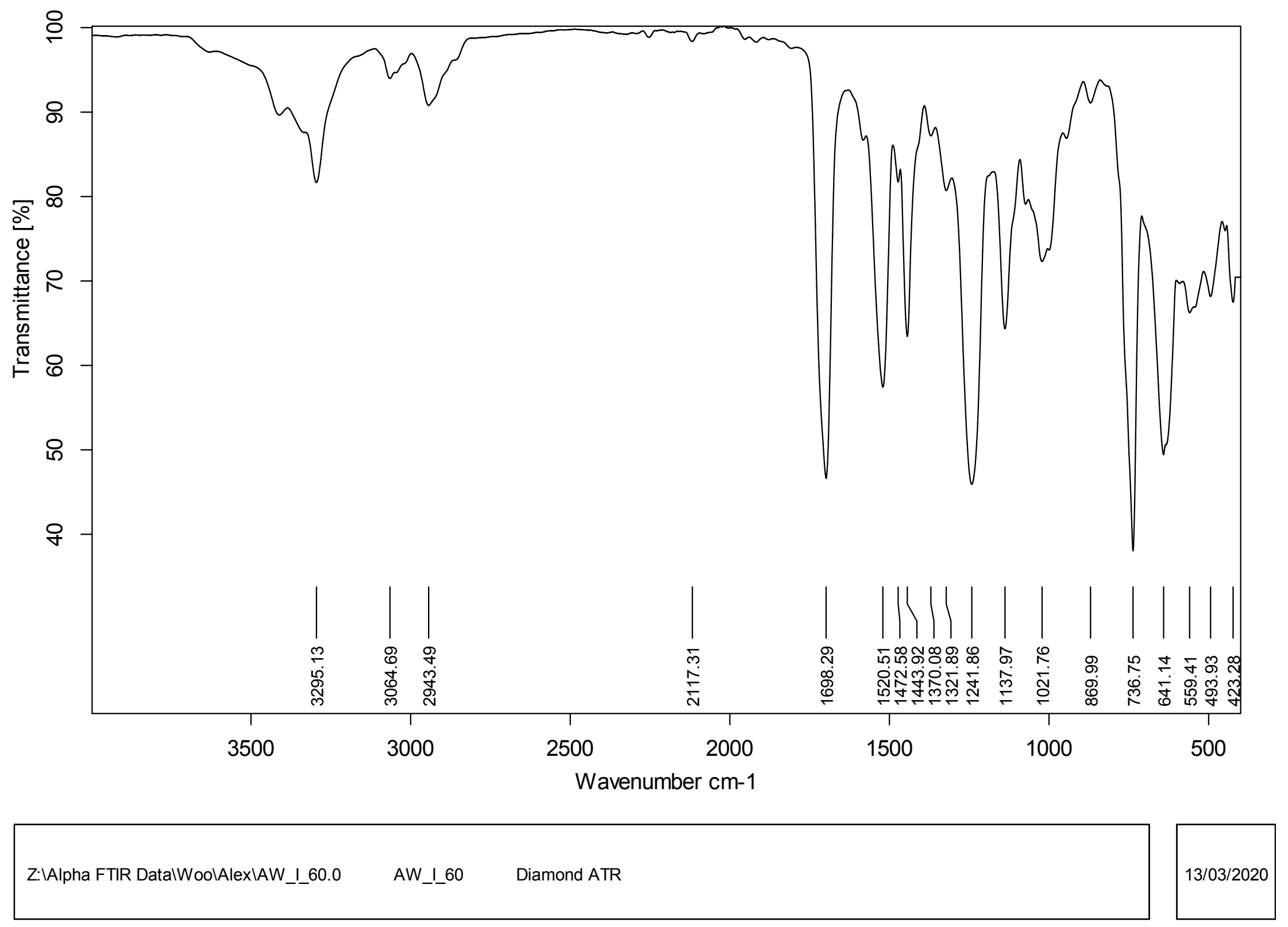

Page 1/2 


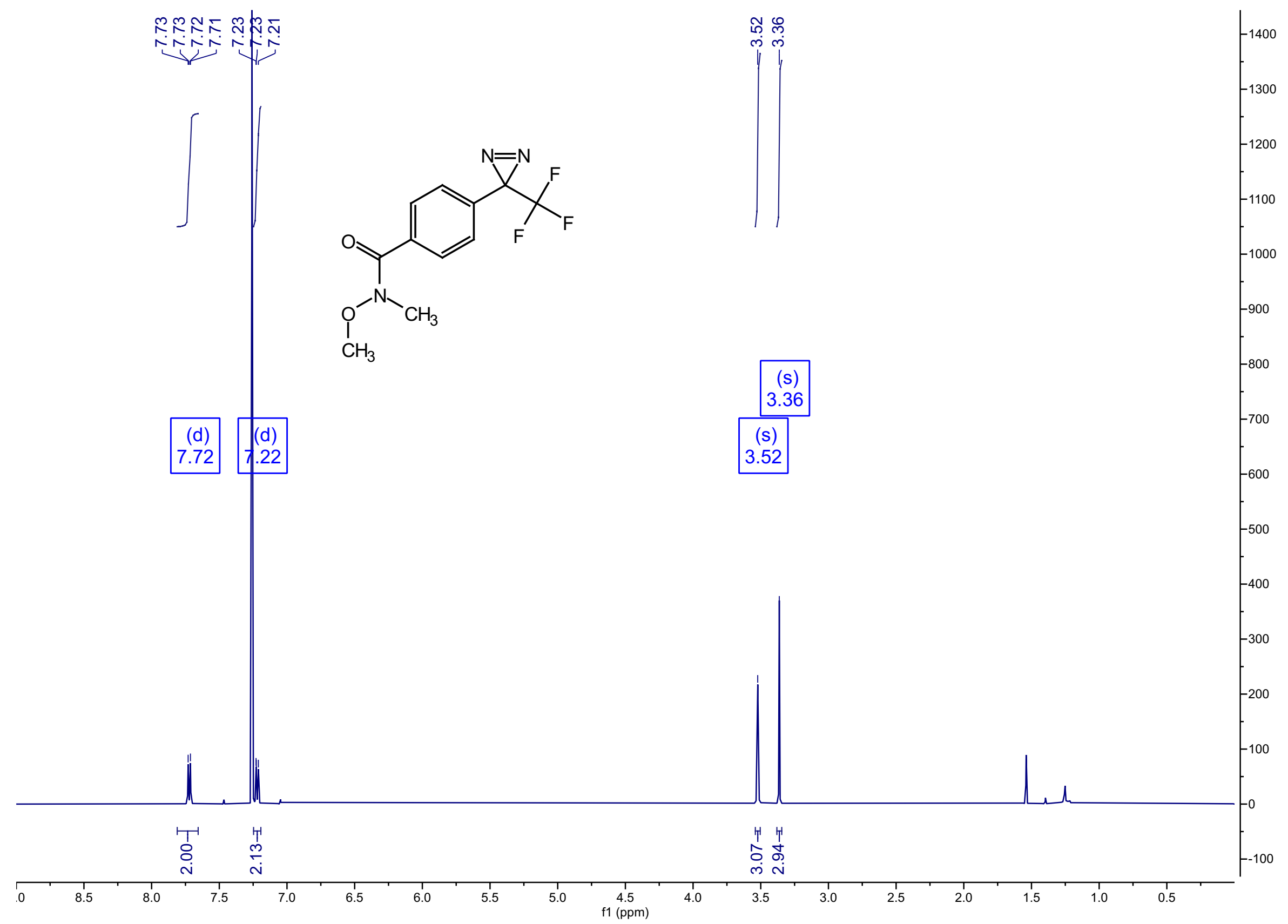

West, A. et al. Labeling preferences of diazirines with protein biomolecules, J. Am. Chem. Soc. 2021. 


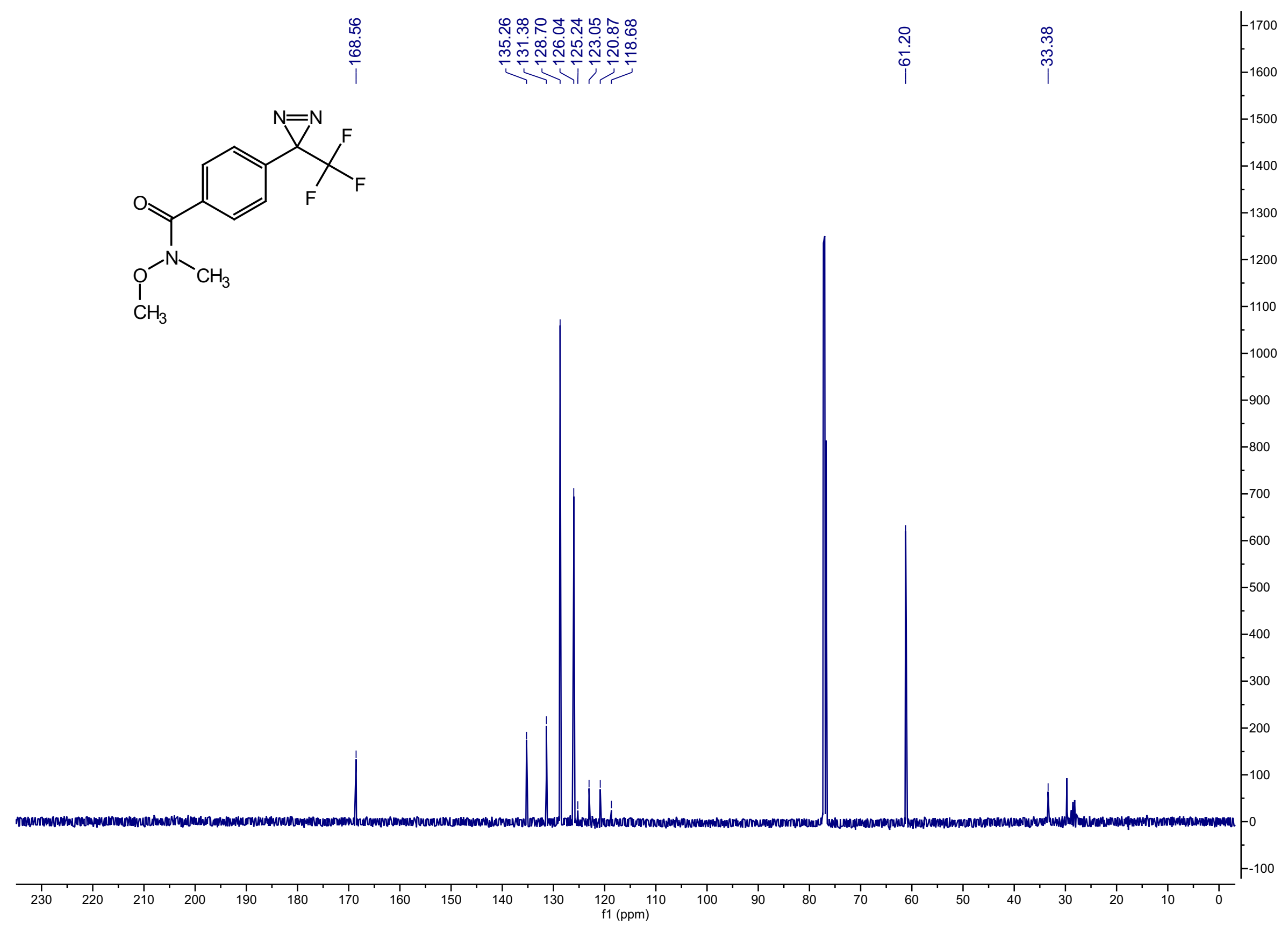




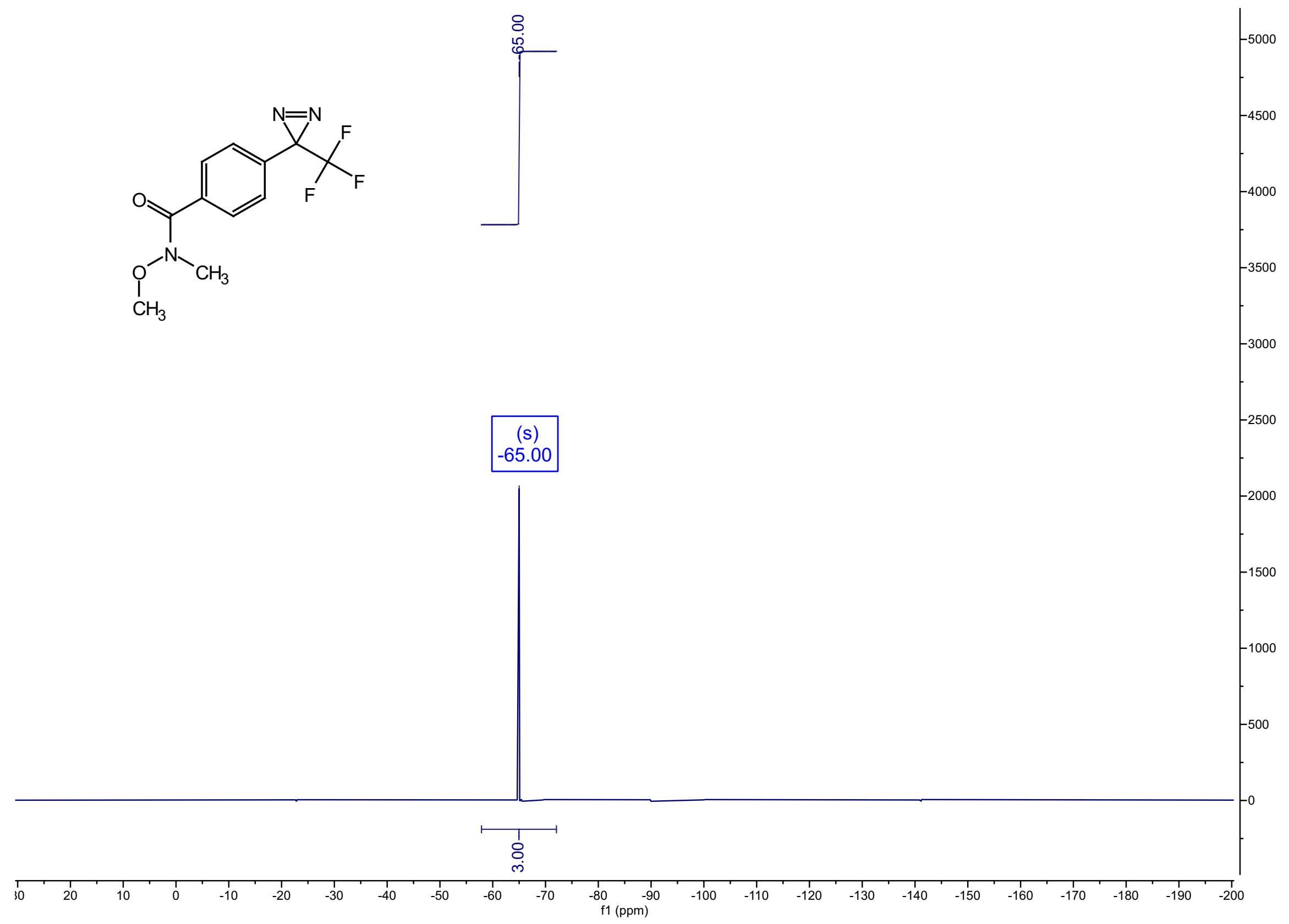




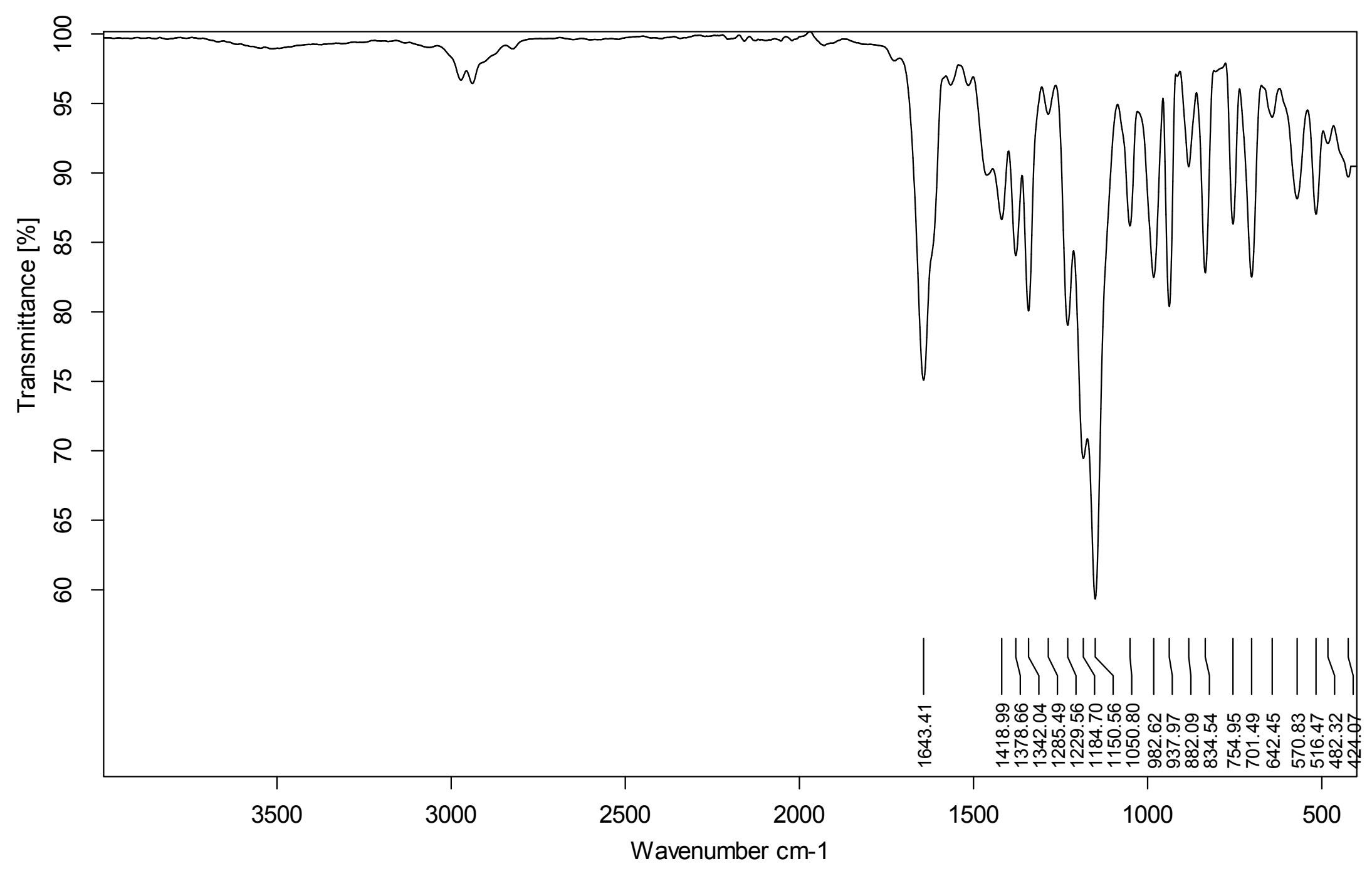

Z:IAlpha FTIR DatalWoolAlex\AW_II_22.0

AW_II22

Diamond ATR

Page 1/2 


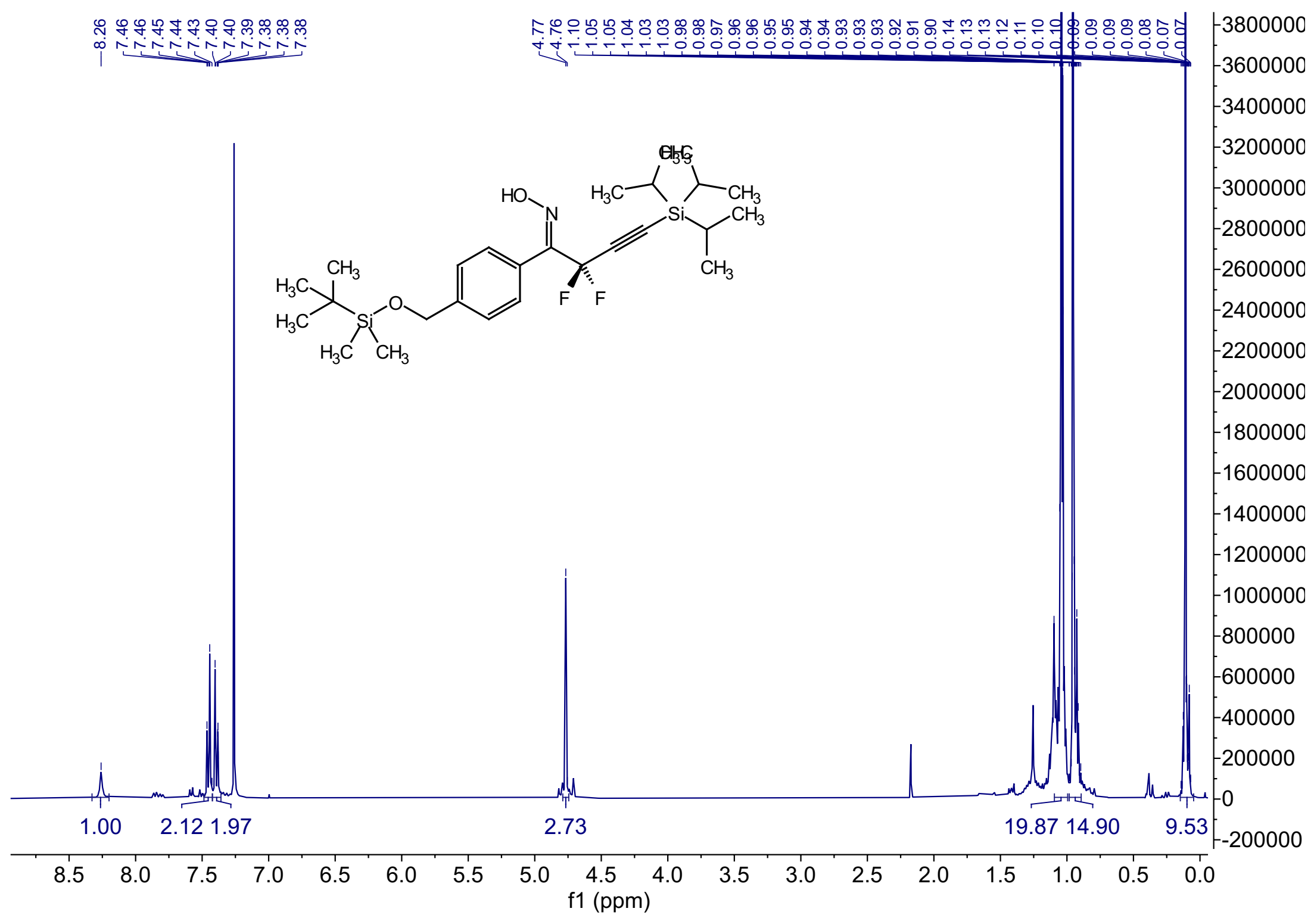




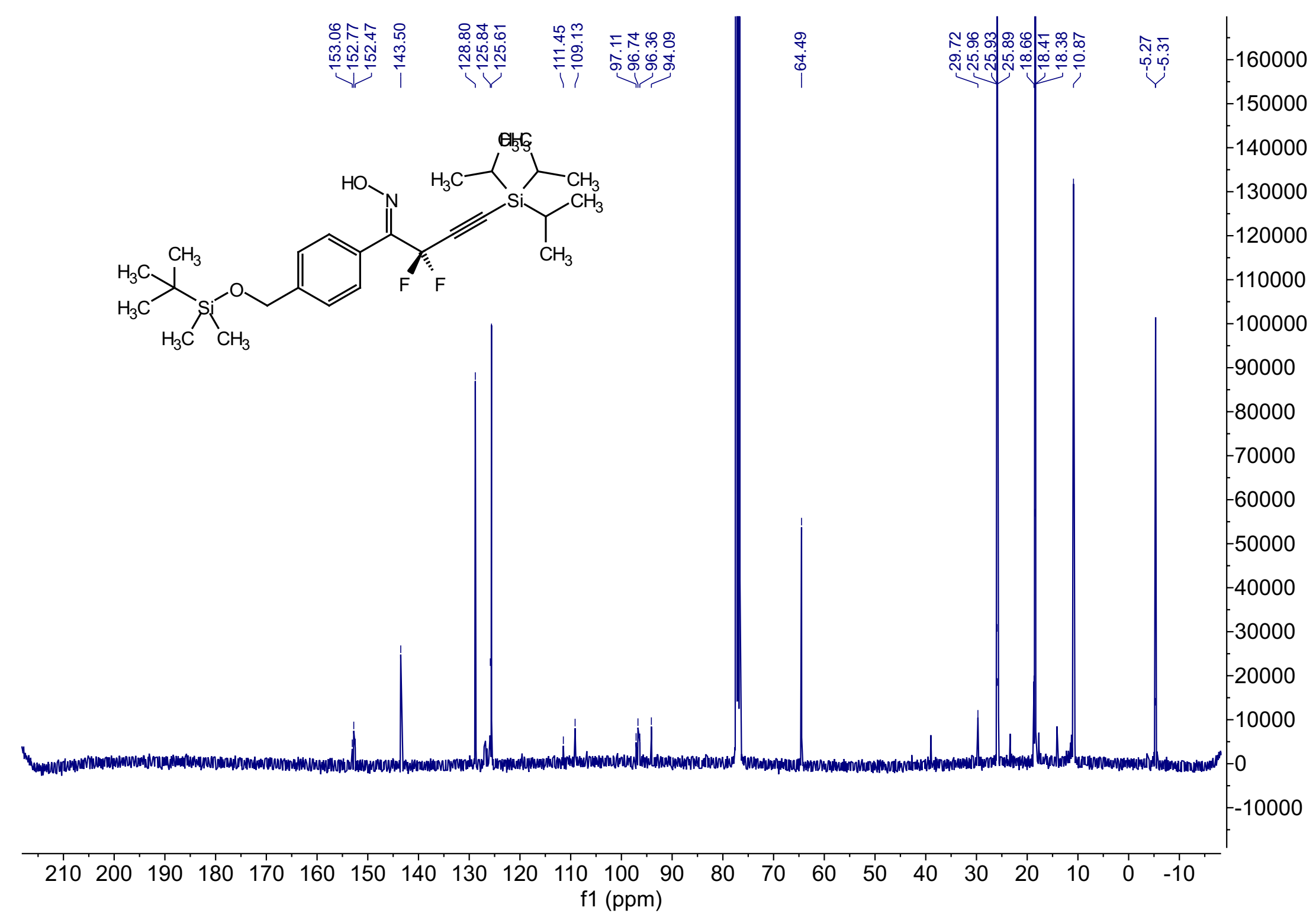

West, A. et al. Labeling preferences of diazirines with protein biomolecules, J. Am. Chem. Soc. 2021. 


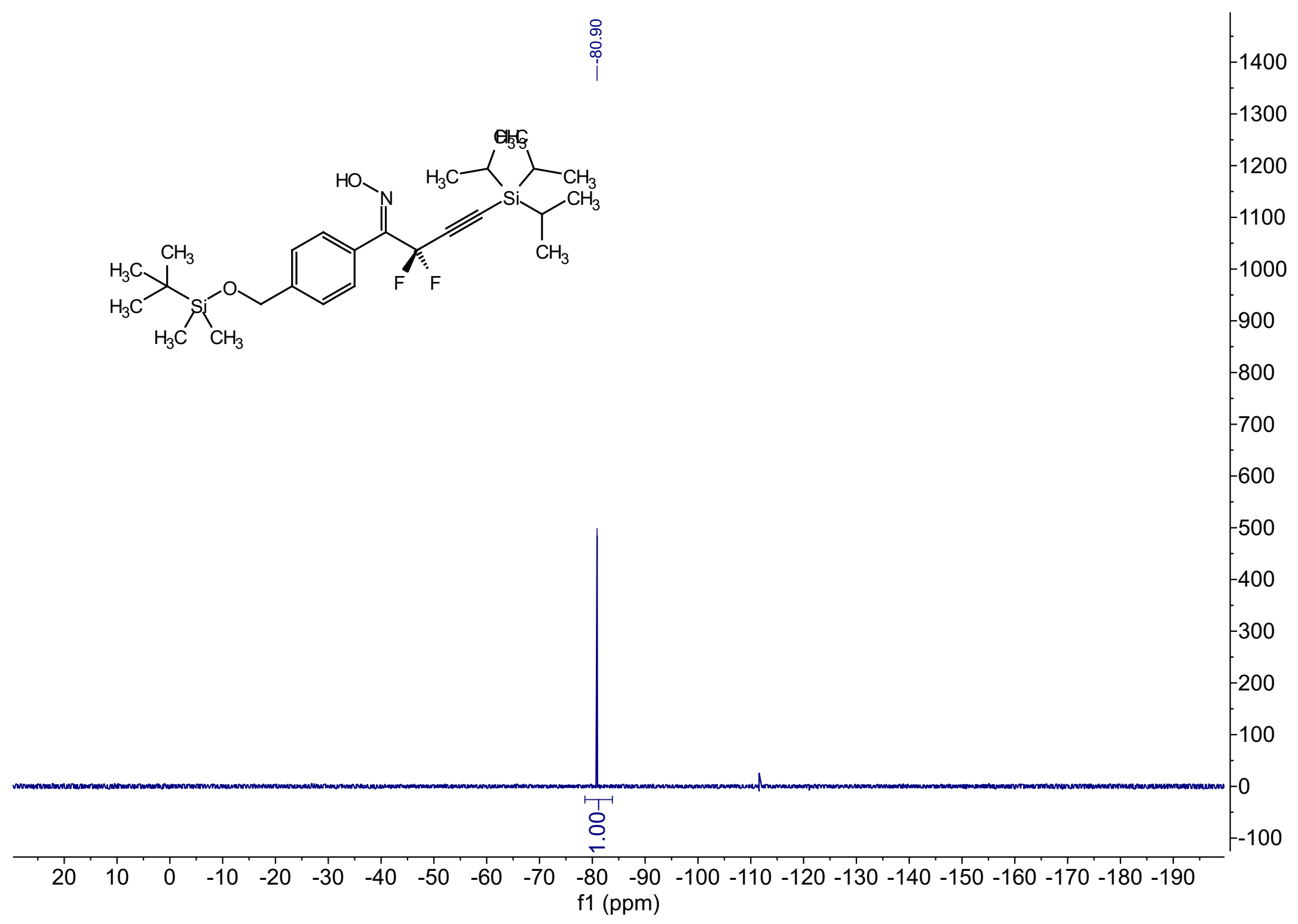



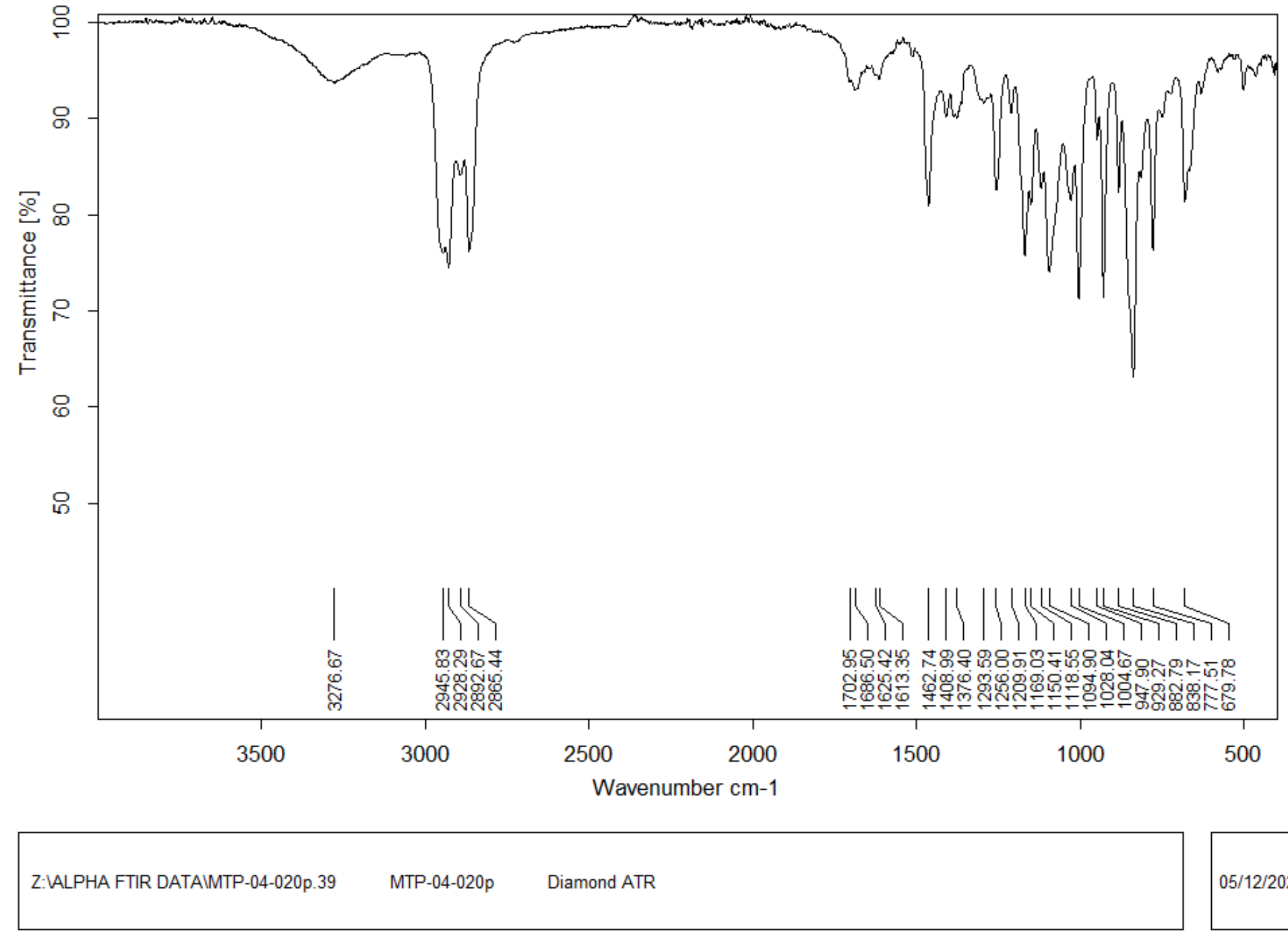

$05 / 12 / 2020$

Page 1/2 


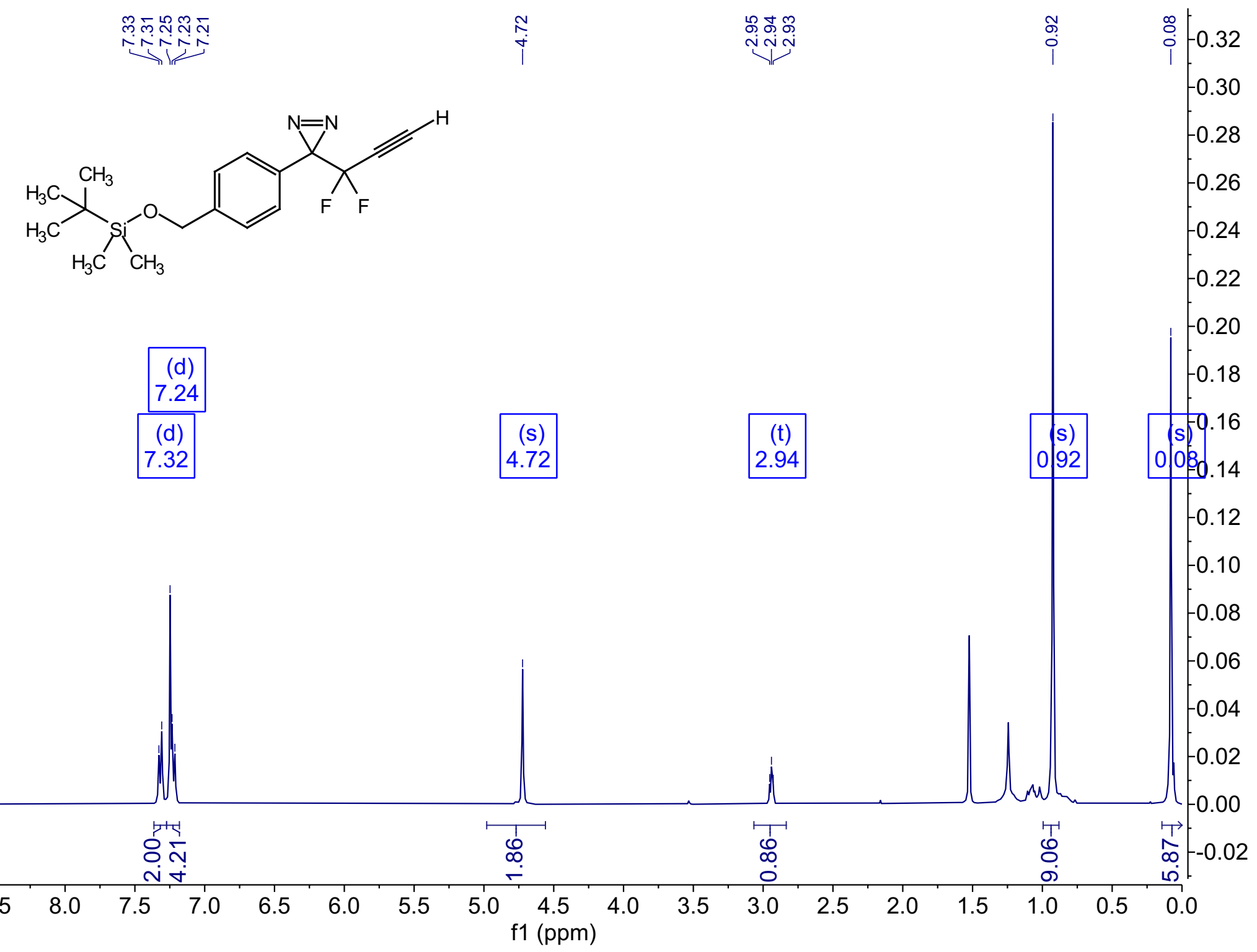

West, A. et al. Labeling preferences of diazirines with protein biomolecules, J. Am. Chem. Soc. 2021. 


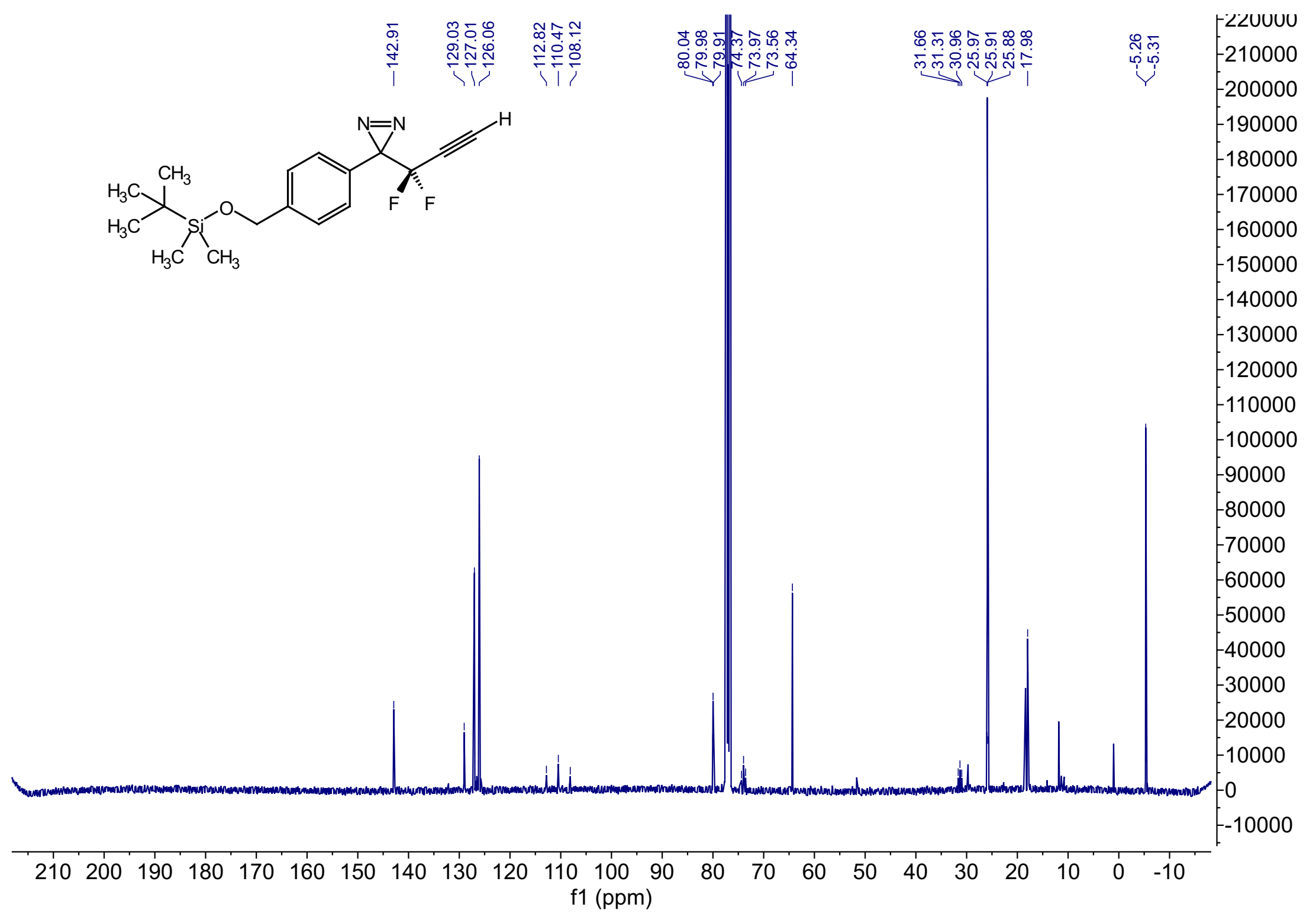

West, A. et al. Labeling preferences of diazirines with protein biomolecules, J. Am. Chem. Soc. 2021. 


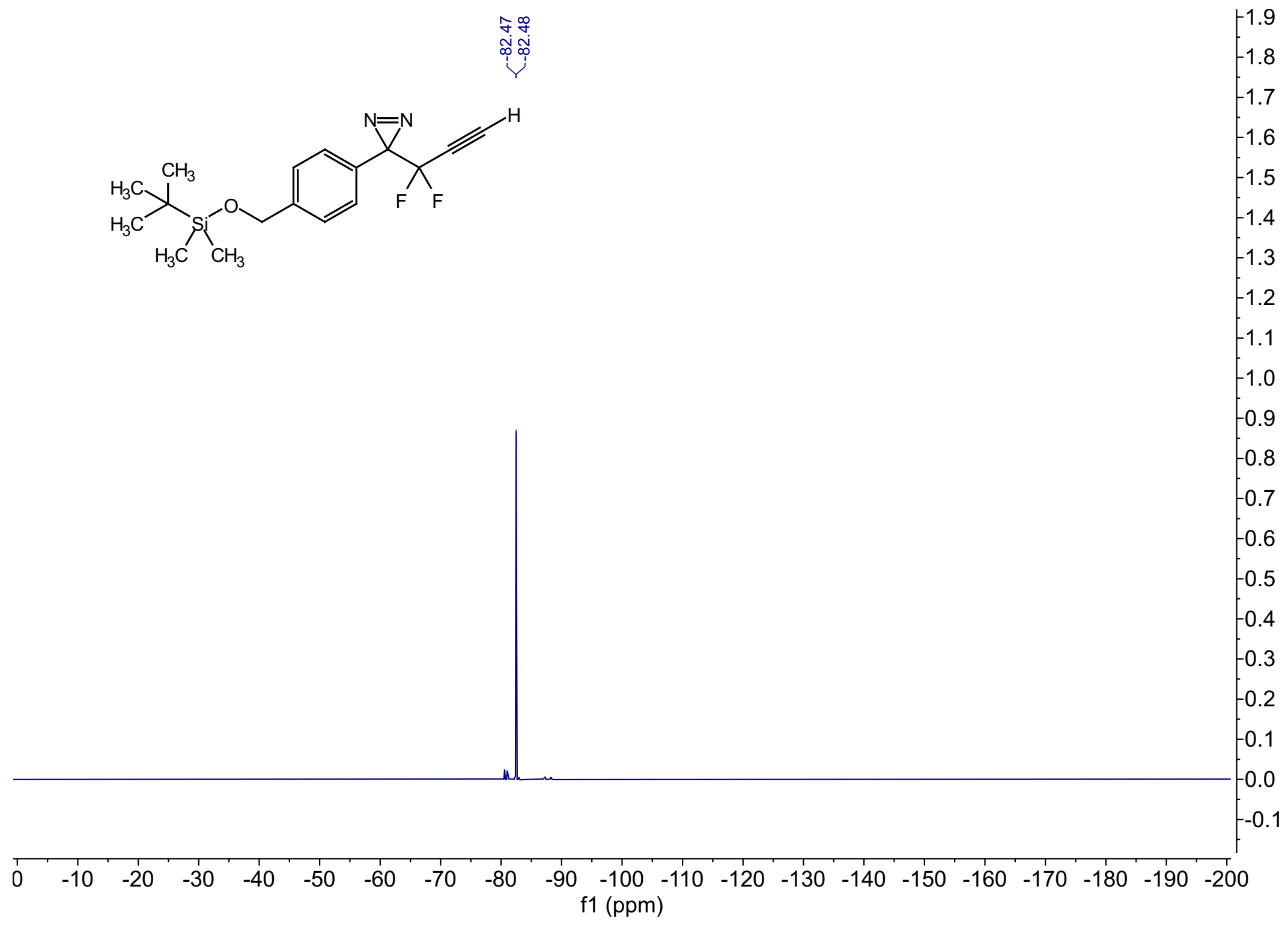




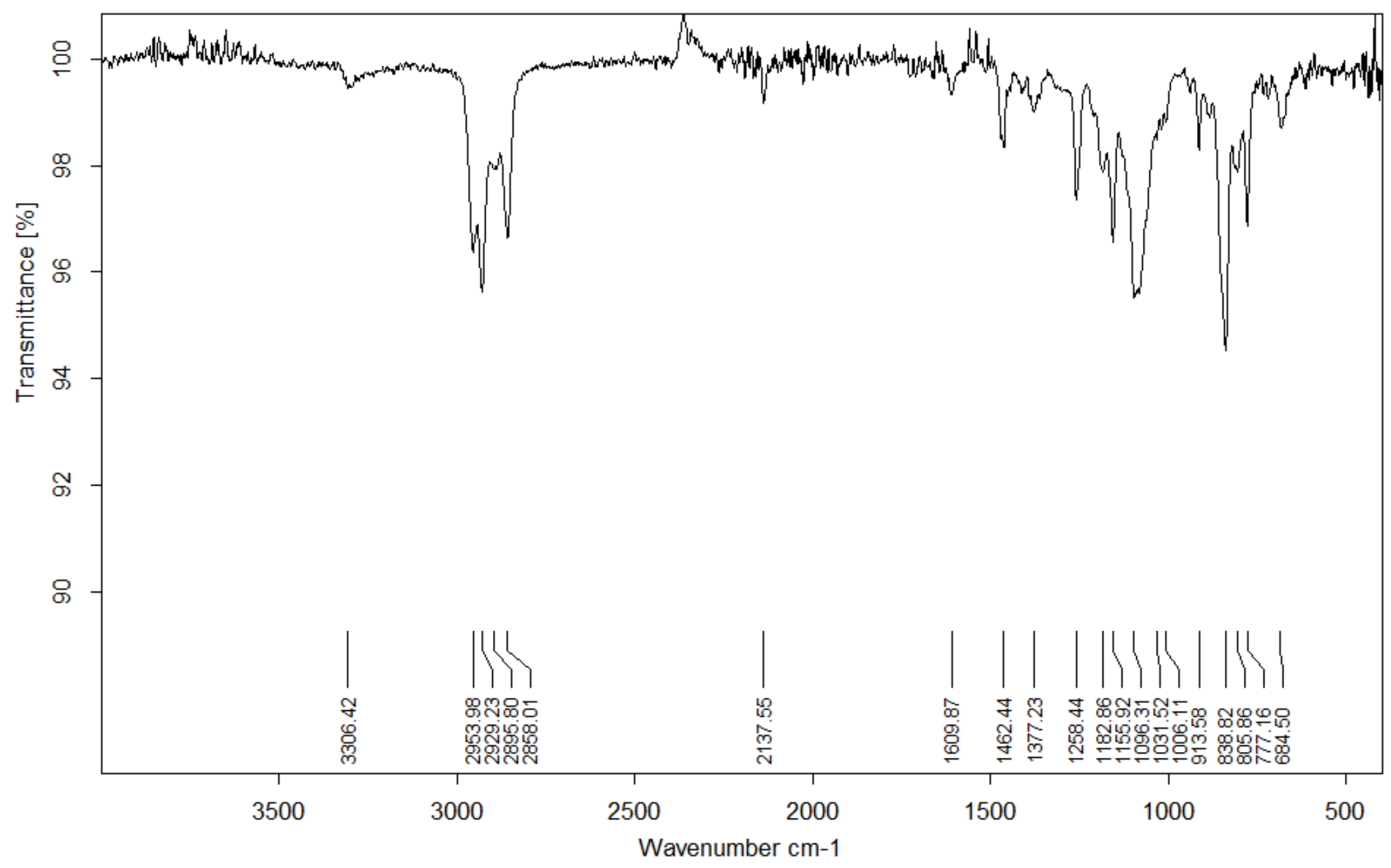

Z:VLLPHA FTIR DATAIMTP-04-020p.42

MTP-04-020p

Diamond ATR

Page 1/2 


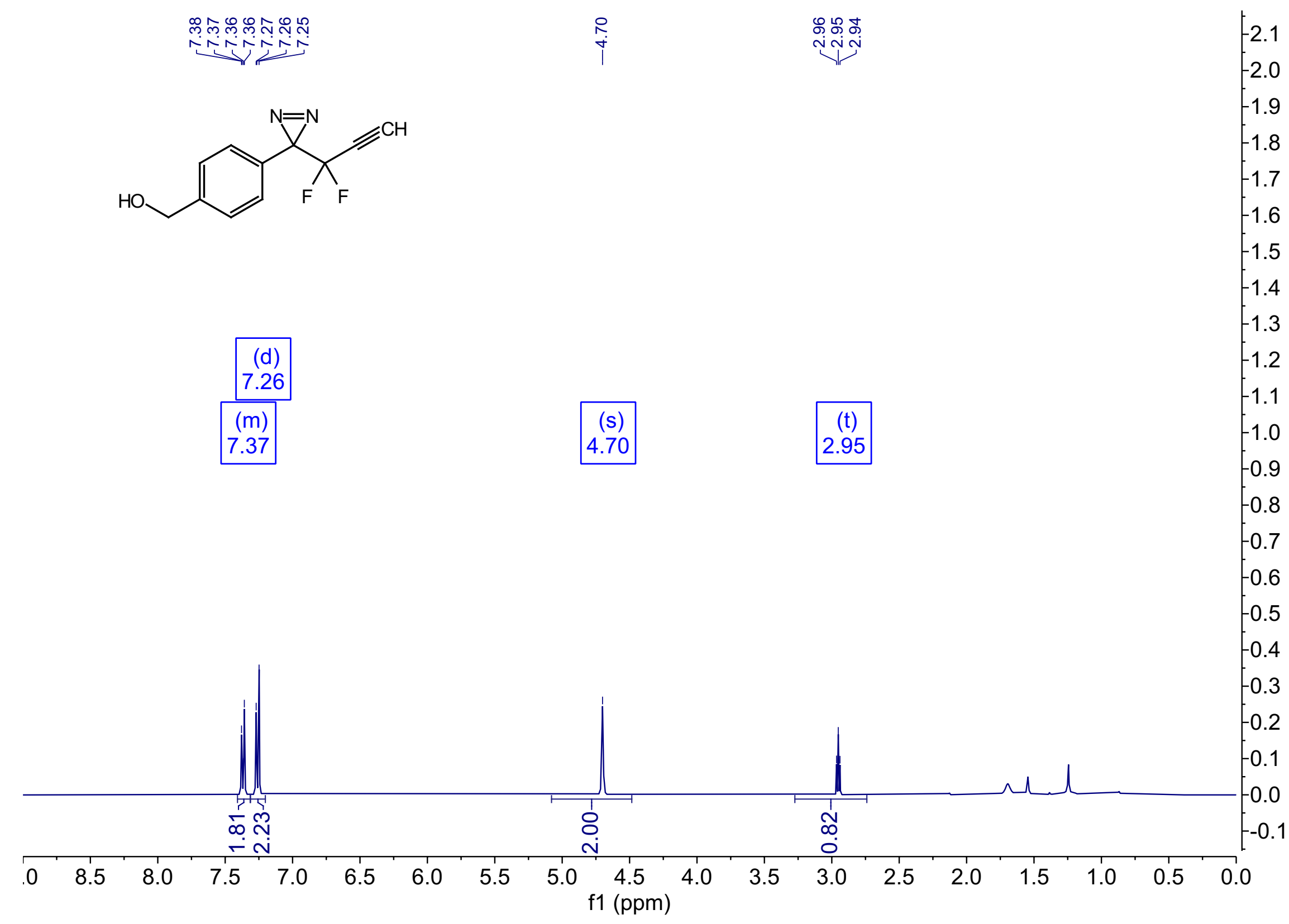

West, A. et al. Labeling preferences of diazirines with protein biomolecules, J. Am. Chem. Soc. 2021. 


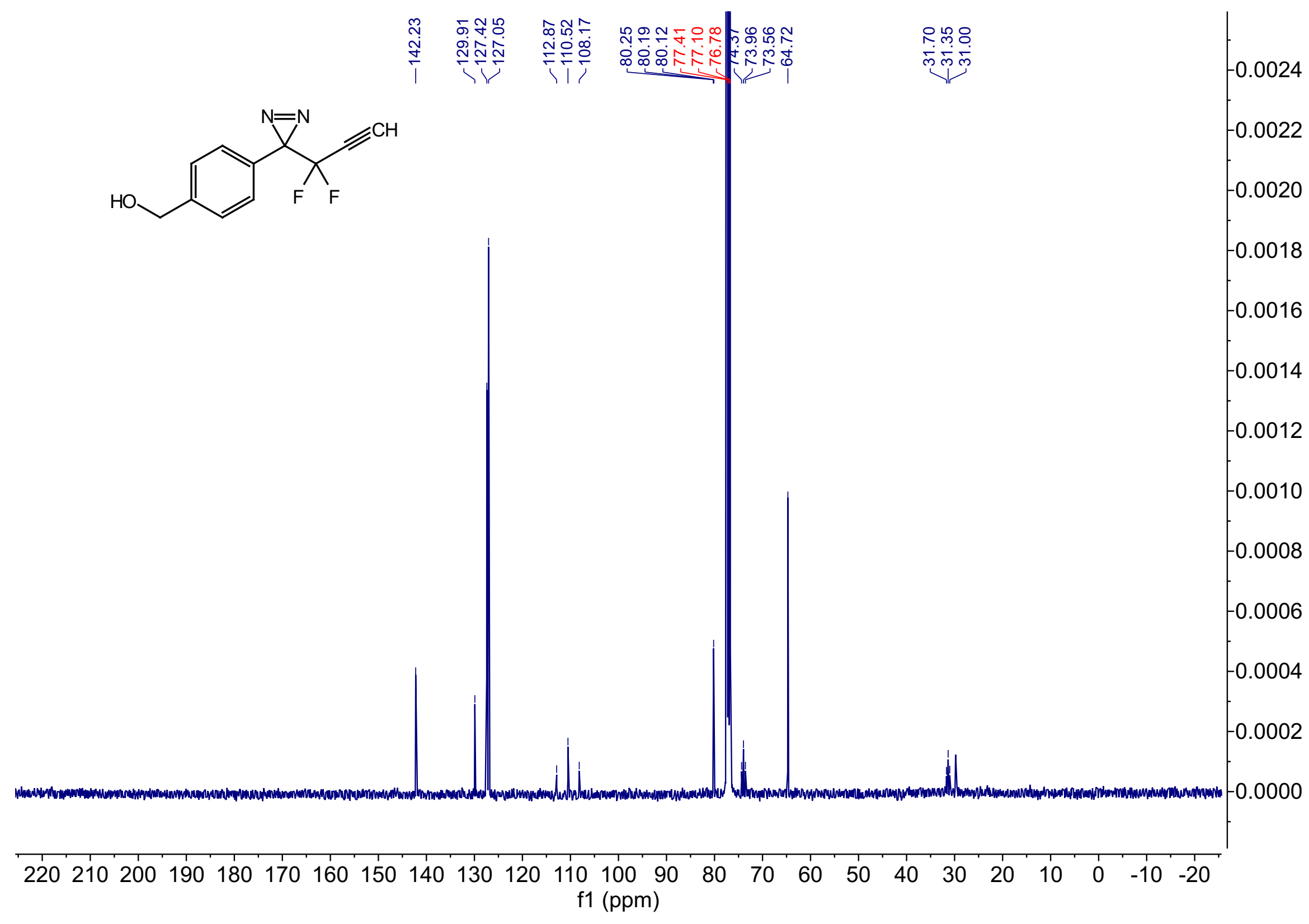

West, A. et al. Labeling preferences of diazirines with protein biomolecules, J. Am. Chem. Soc. 2021. 


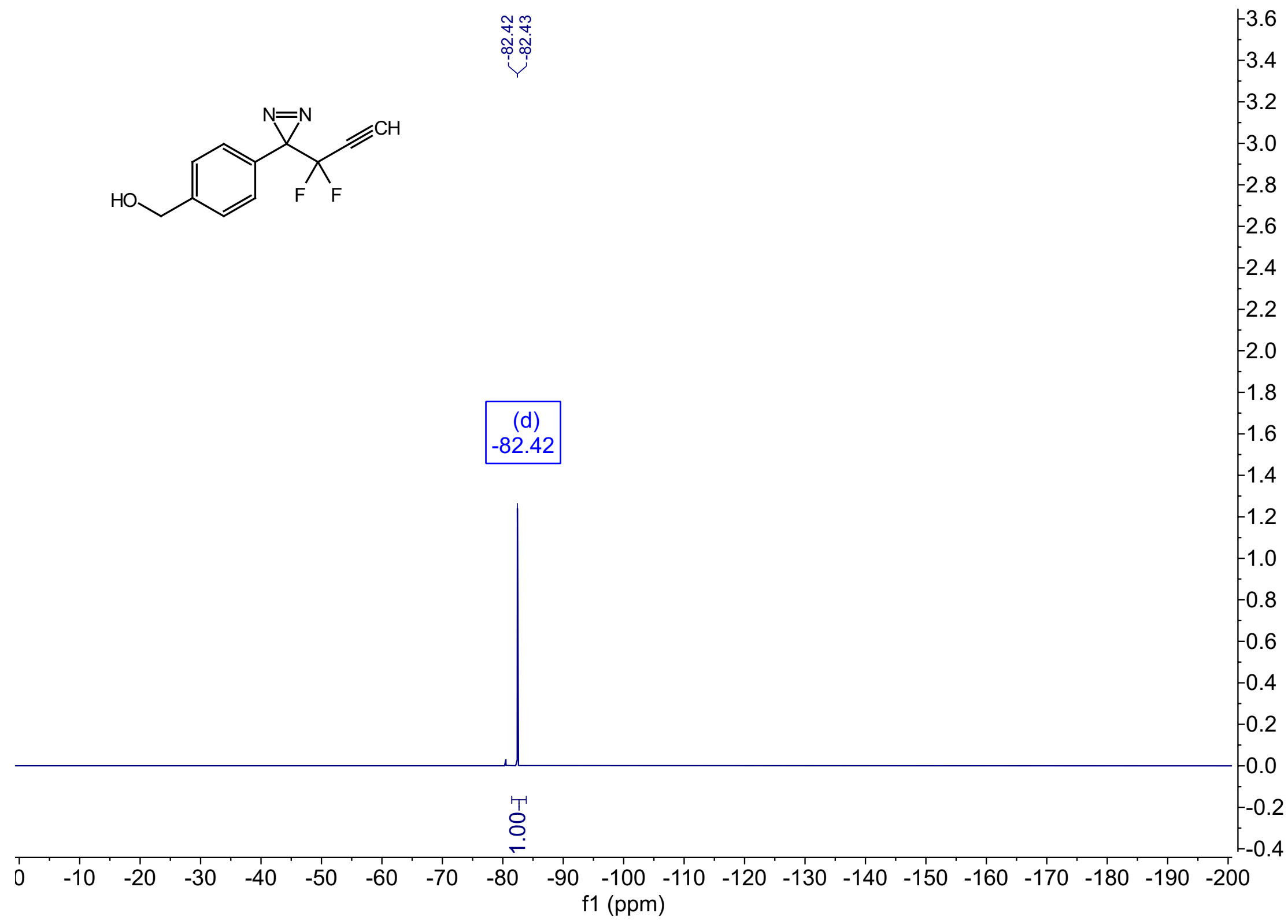




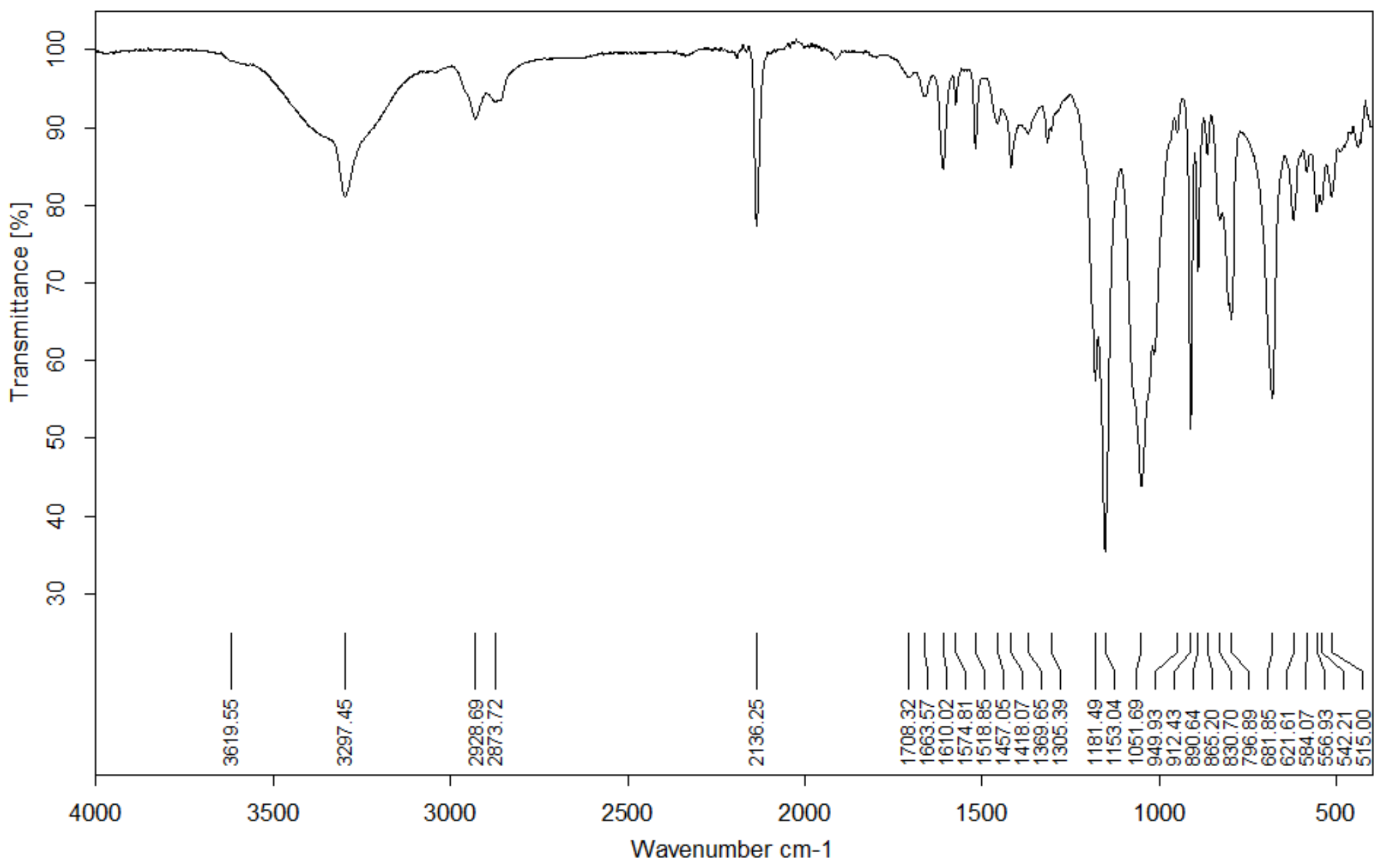

Z:VLPHA FTIR DATAIMTP-04-020p.44 MTP-04-020p Diamond ATR

Page 1/2 

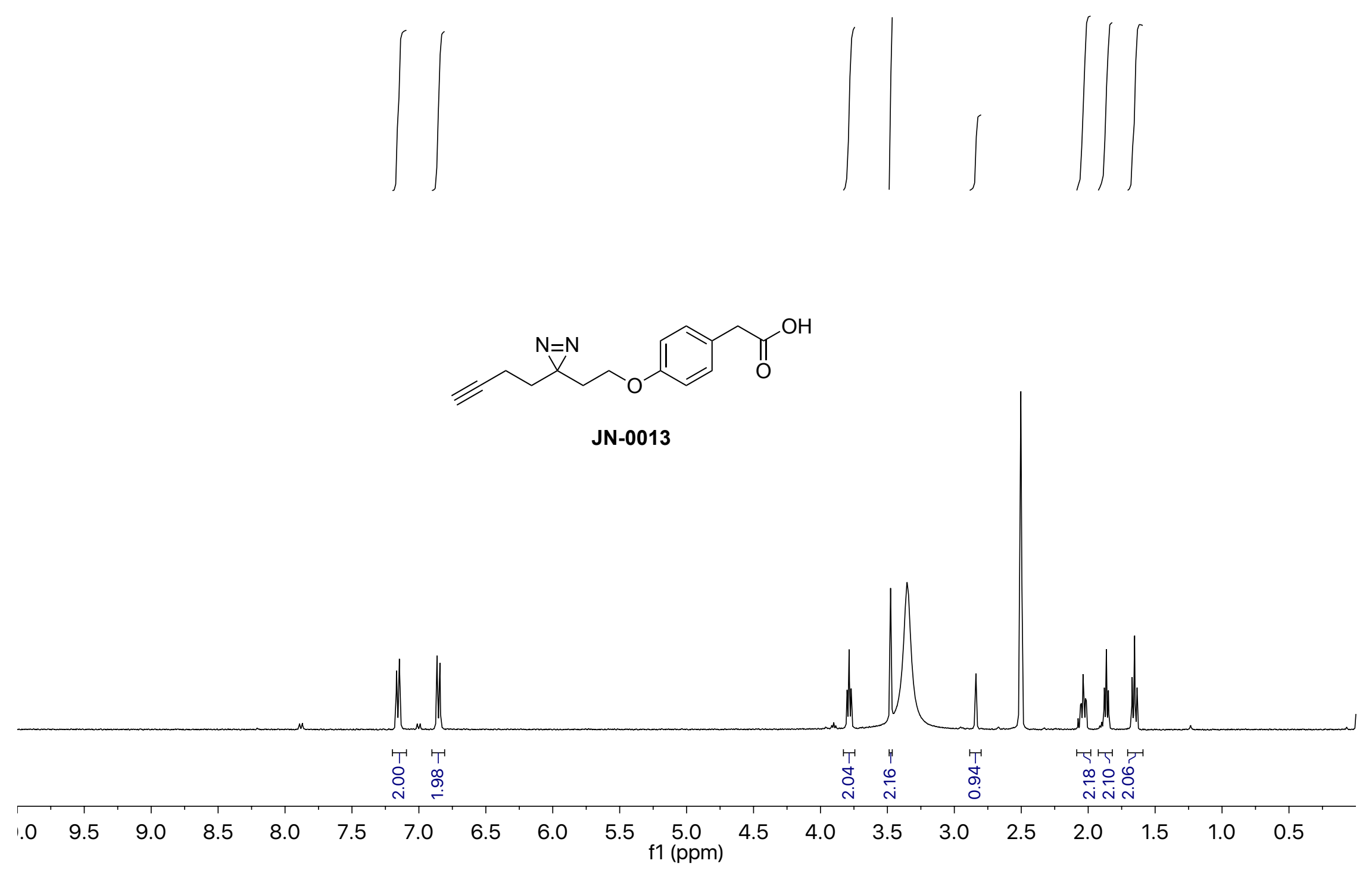


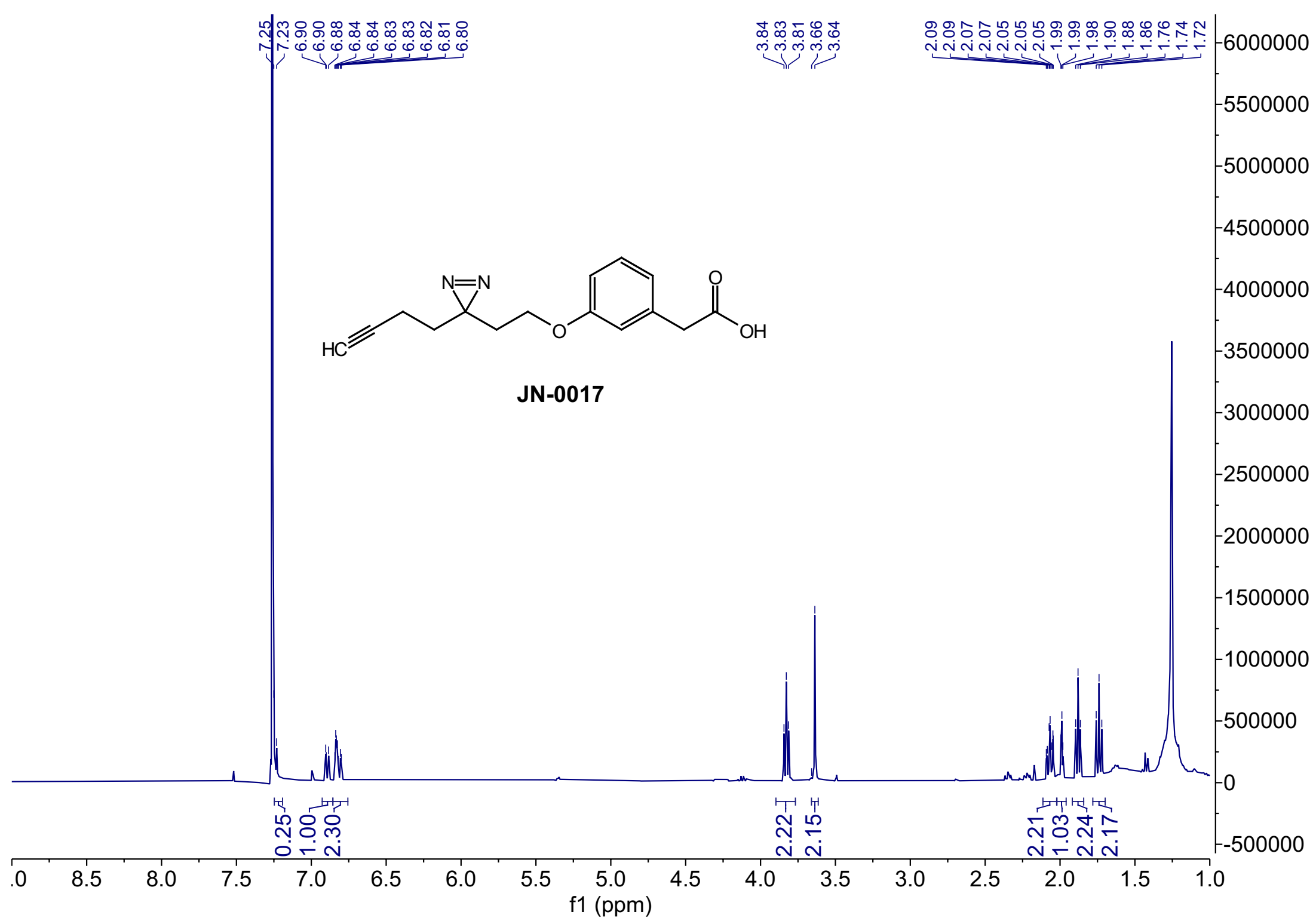




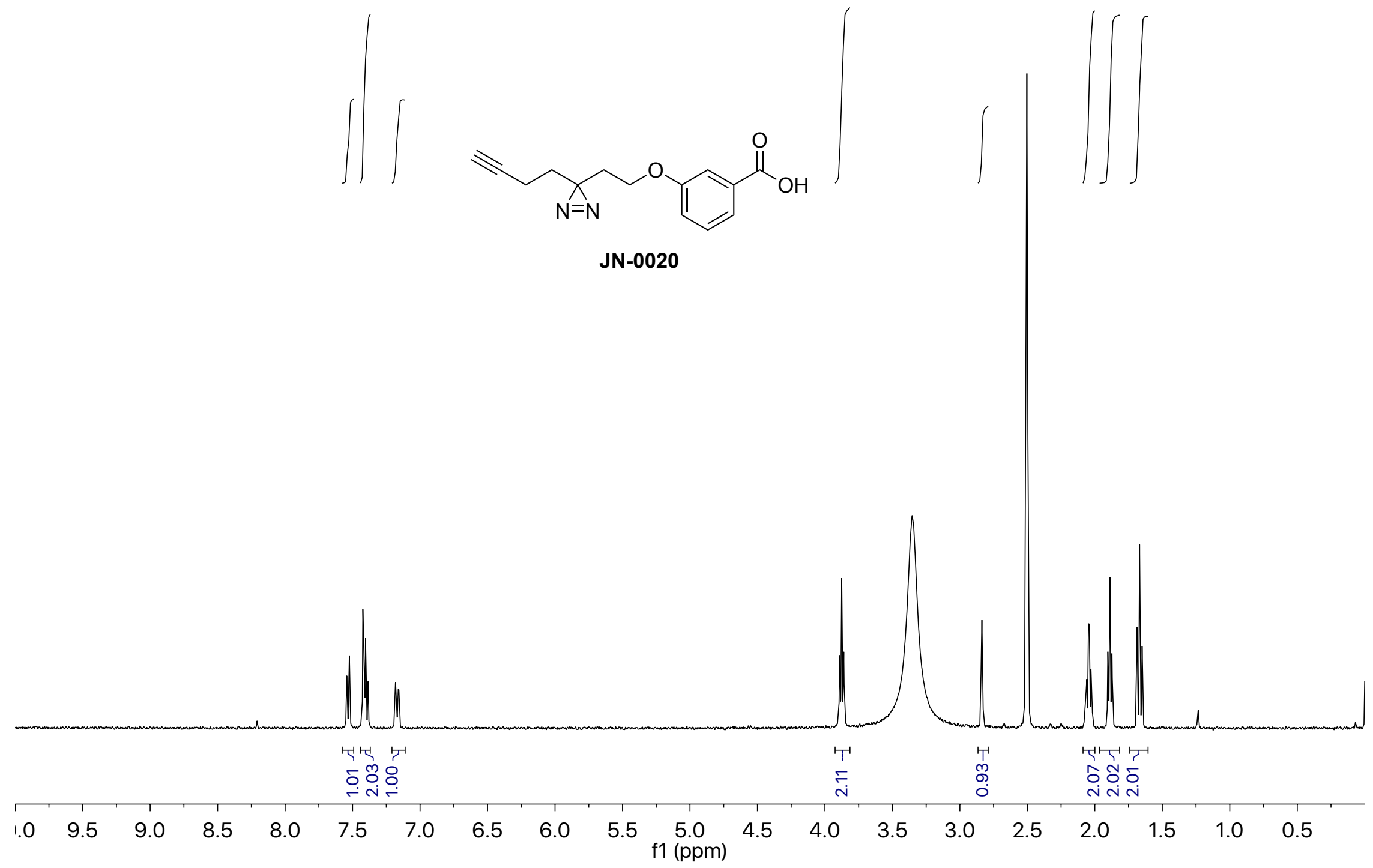

West, A. et al. Labeling preferences of diazirines with protein biomolecules, J. Am. Chem. Soc. 2021. 


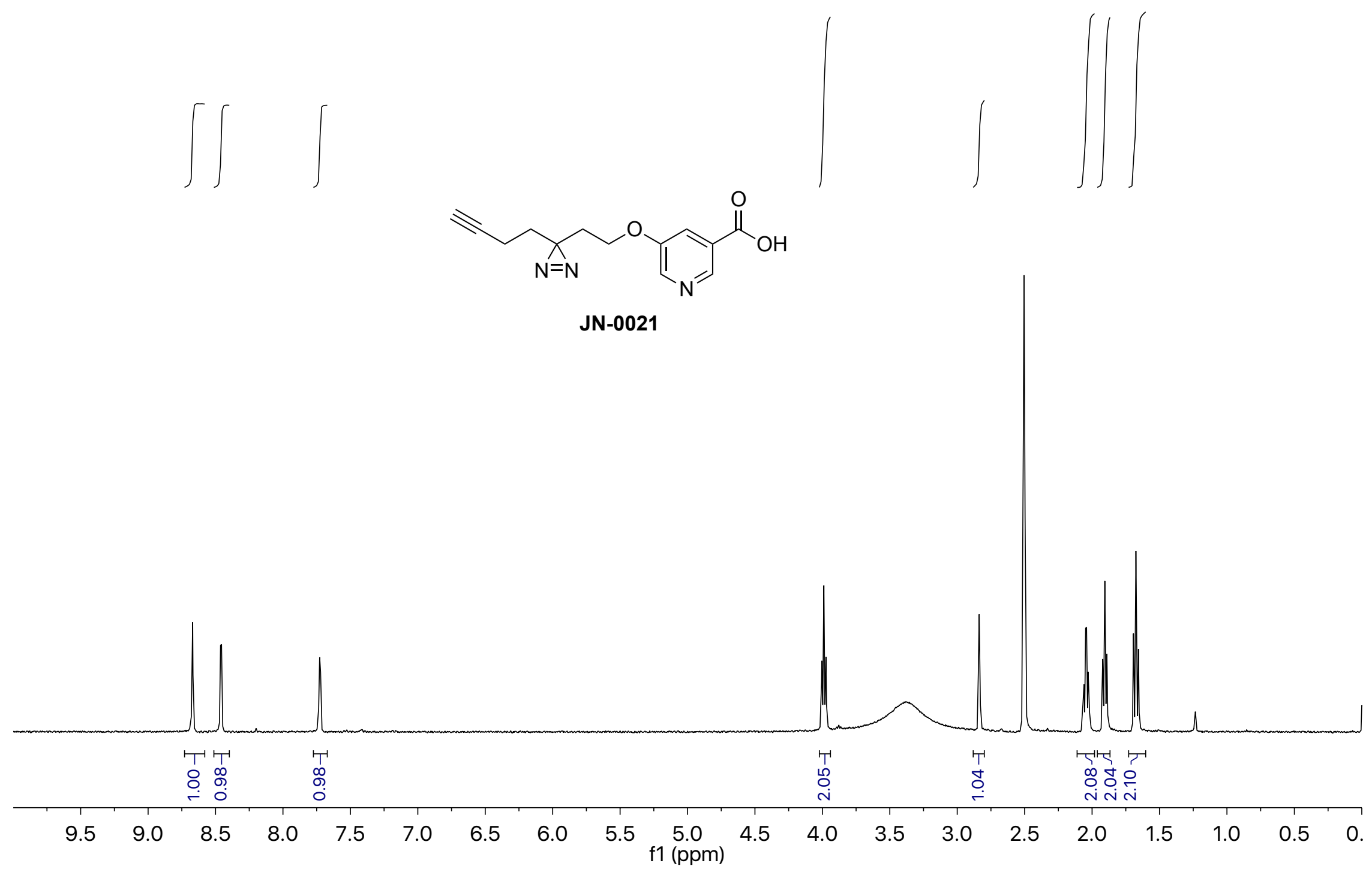

West, A. et al. Labeling preferences of diazirines with protein biomolecules, J. Am. Chem. Soc. 2021. 


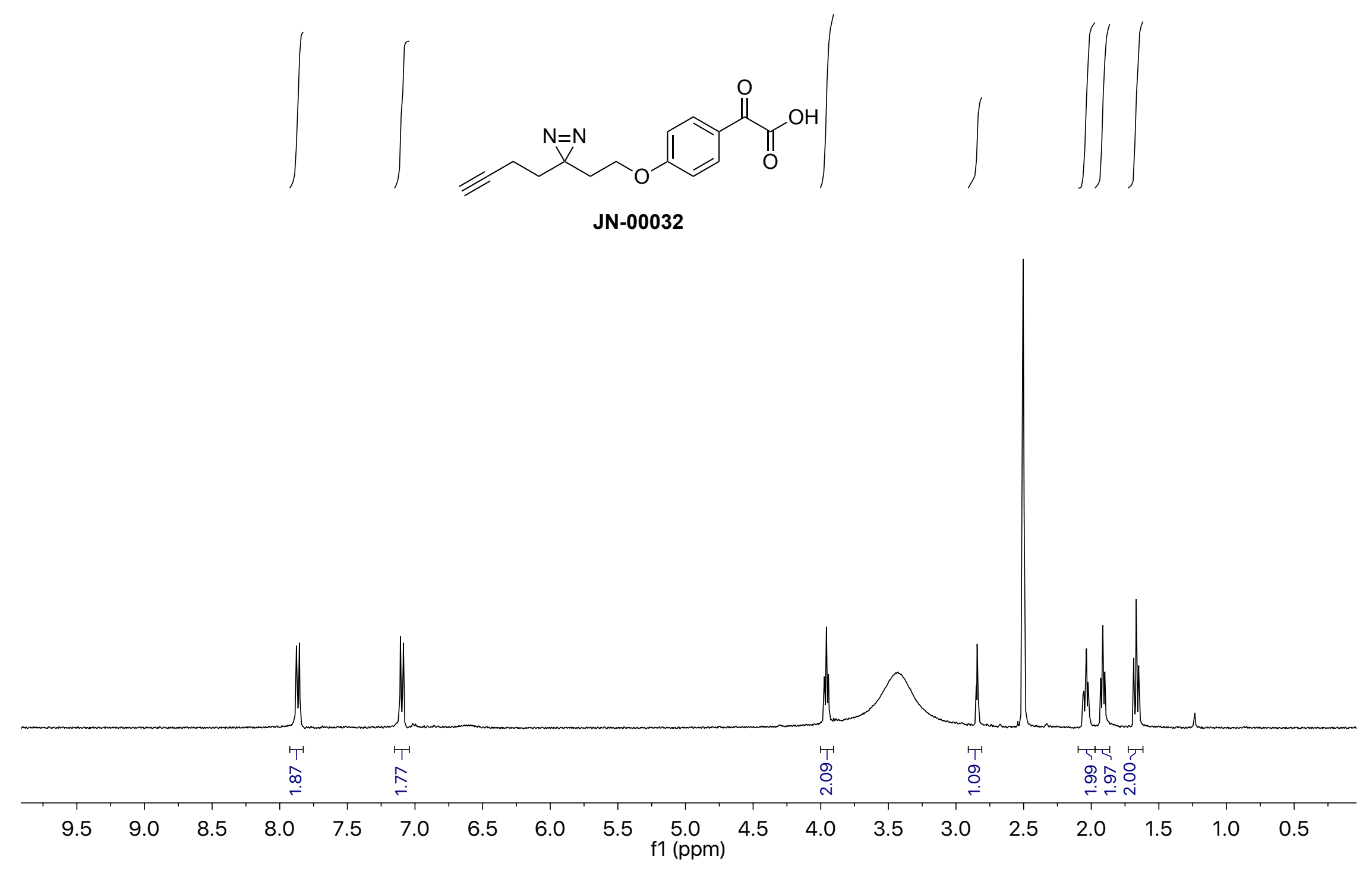

West, A. et al. Labeling preferences of diazirines with protein biomolecules, J. Am. Chem. Soc. 2021. 


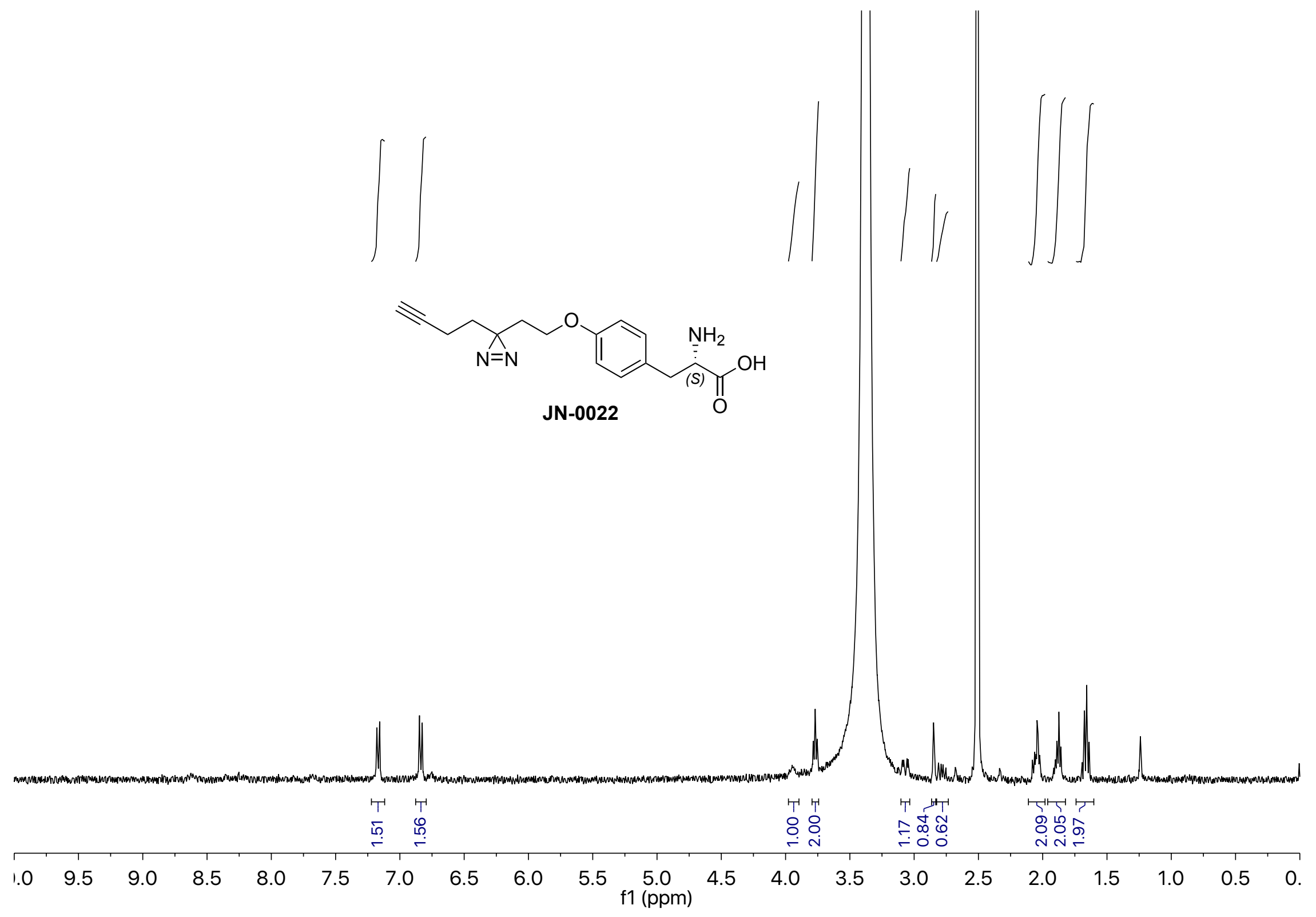

West, A. et al. Labeling preferences of diazirines with protein biomolecules, J. Am. Chem. Soc. 2021. 


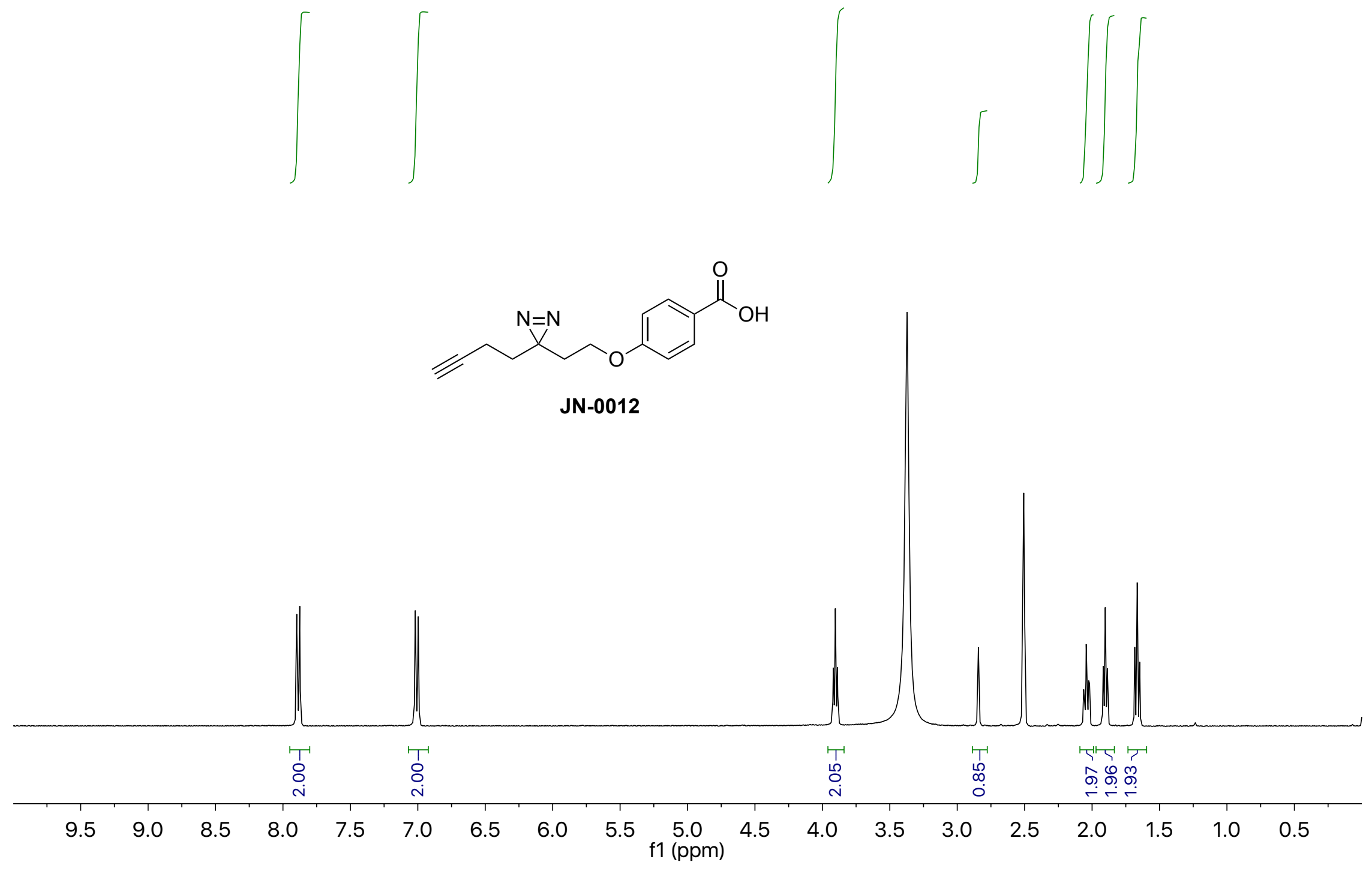

West, A. et al. Labeling preferences of diazirines with protein biomolecules, J. Am. Chem. Soc. 2021. 


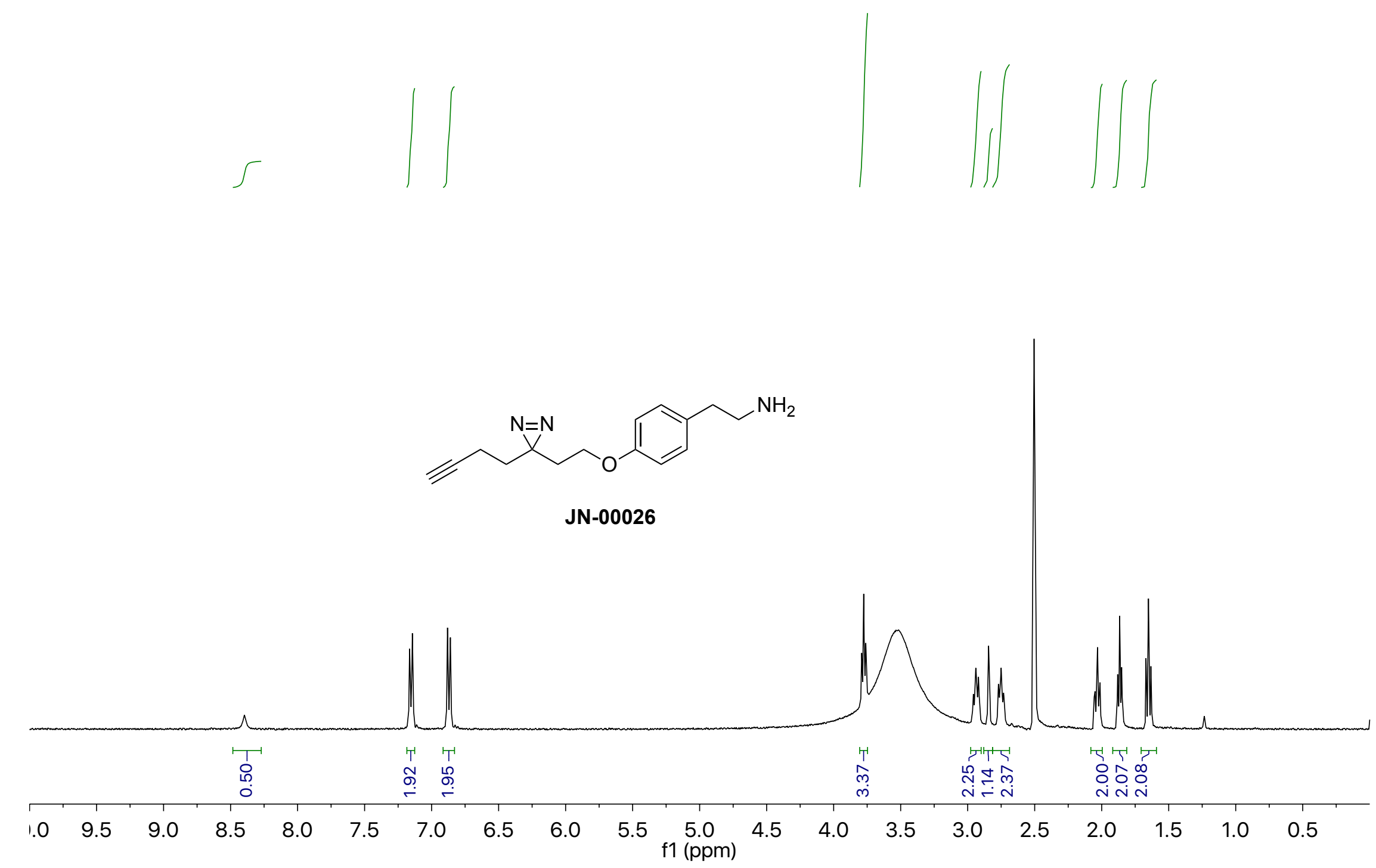




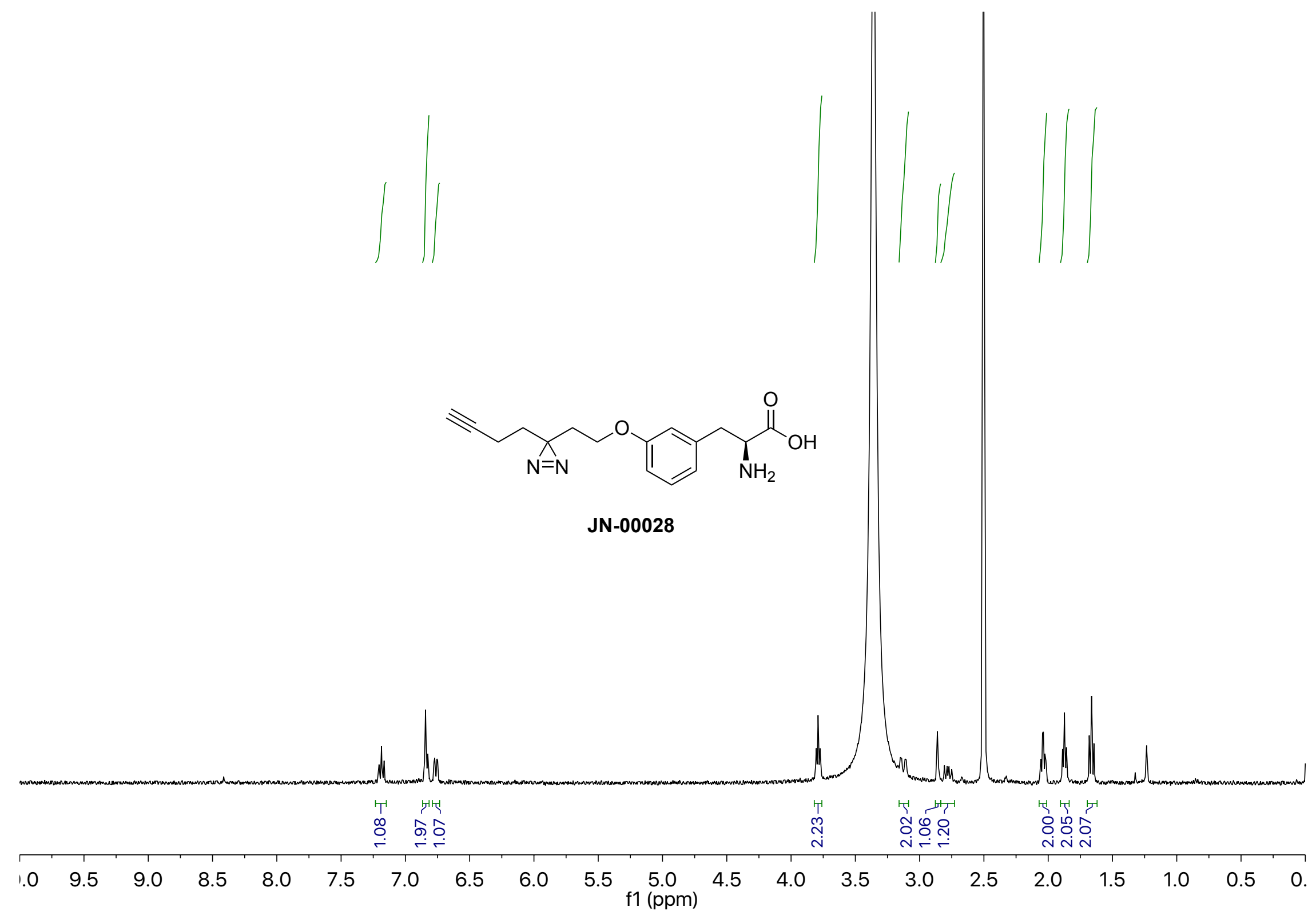

West, A. et al. Labeling preferences of diazirines with protein biomolecules, J. Am. Chem. Soc. 2021. 


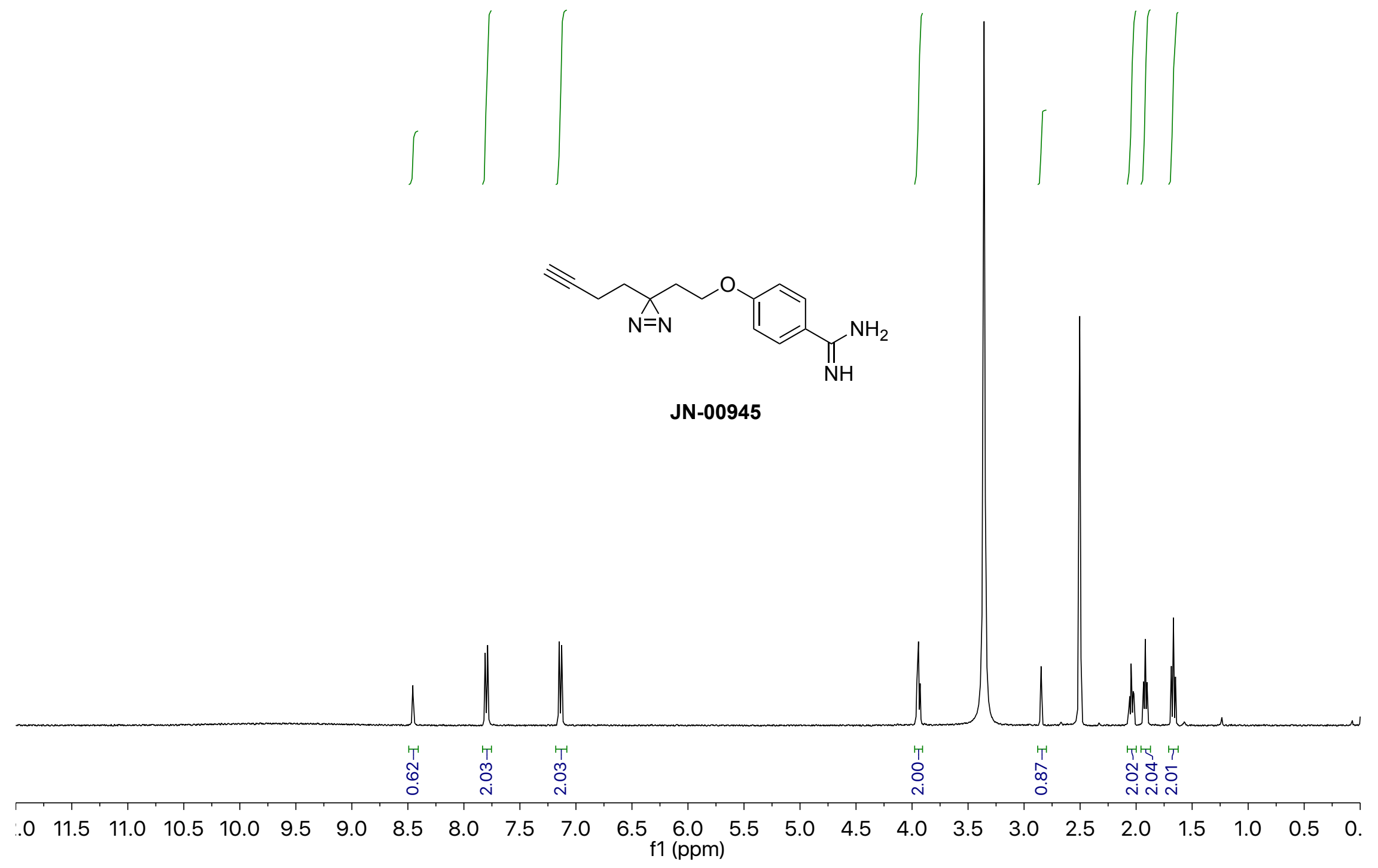

West, A. et al. Labeling preferences of diazirines with protein biomolecules, J. Am. Chem. Soc. 2021. 


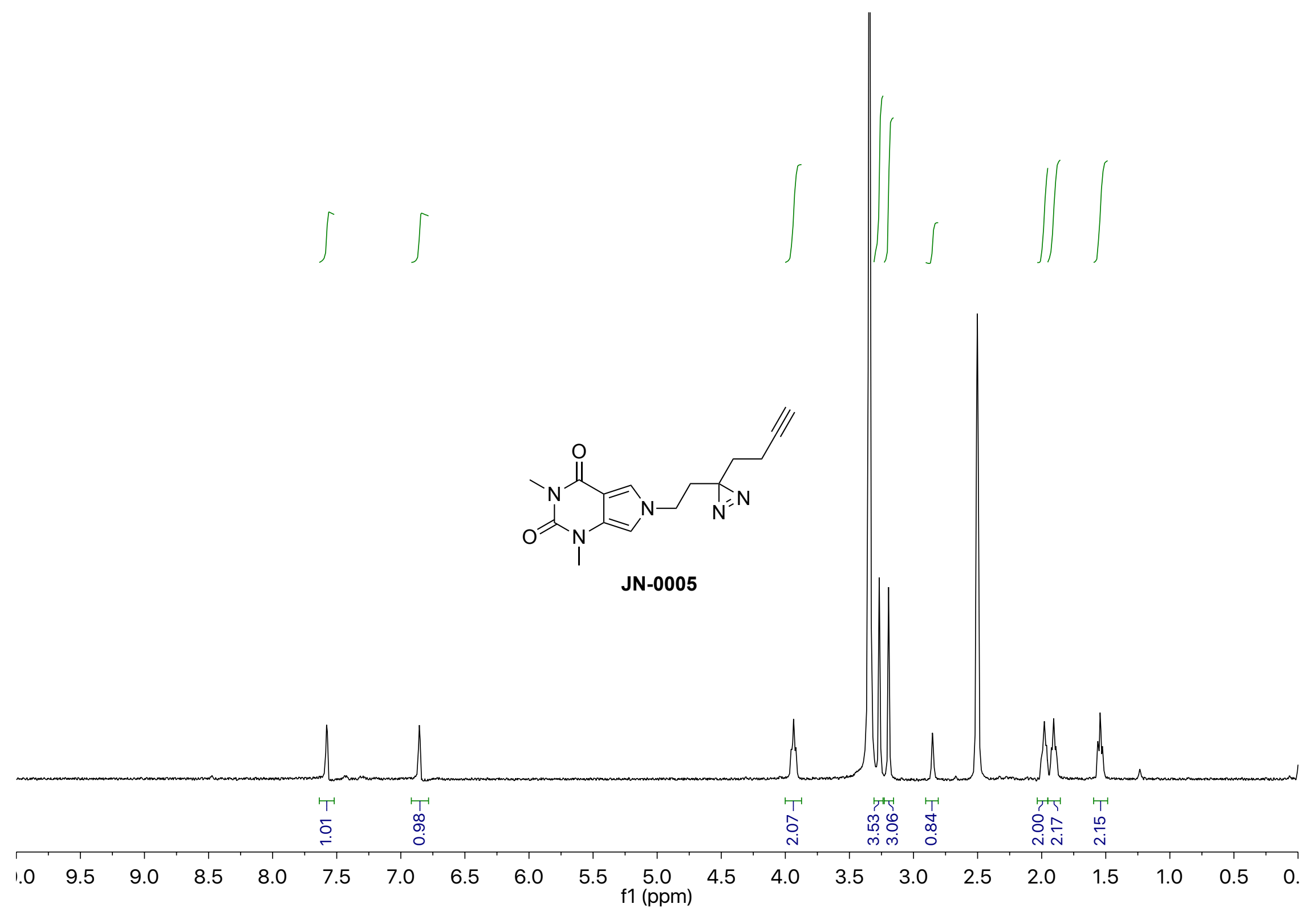




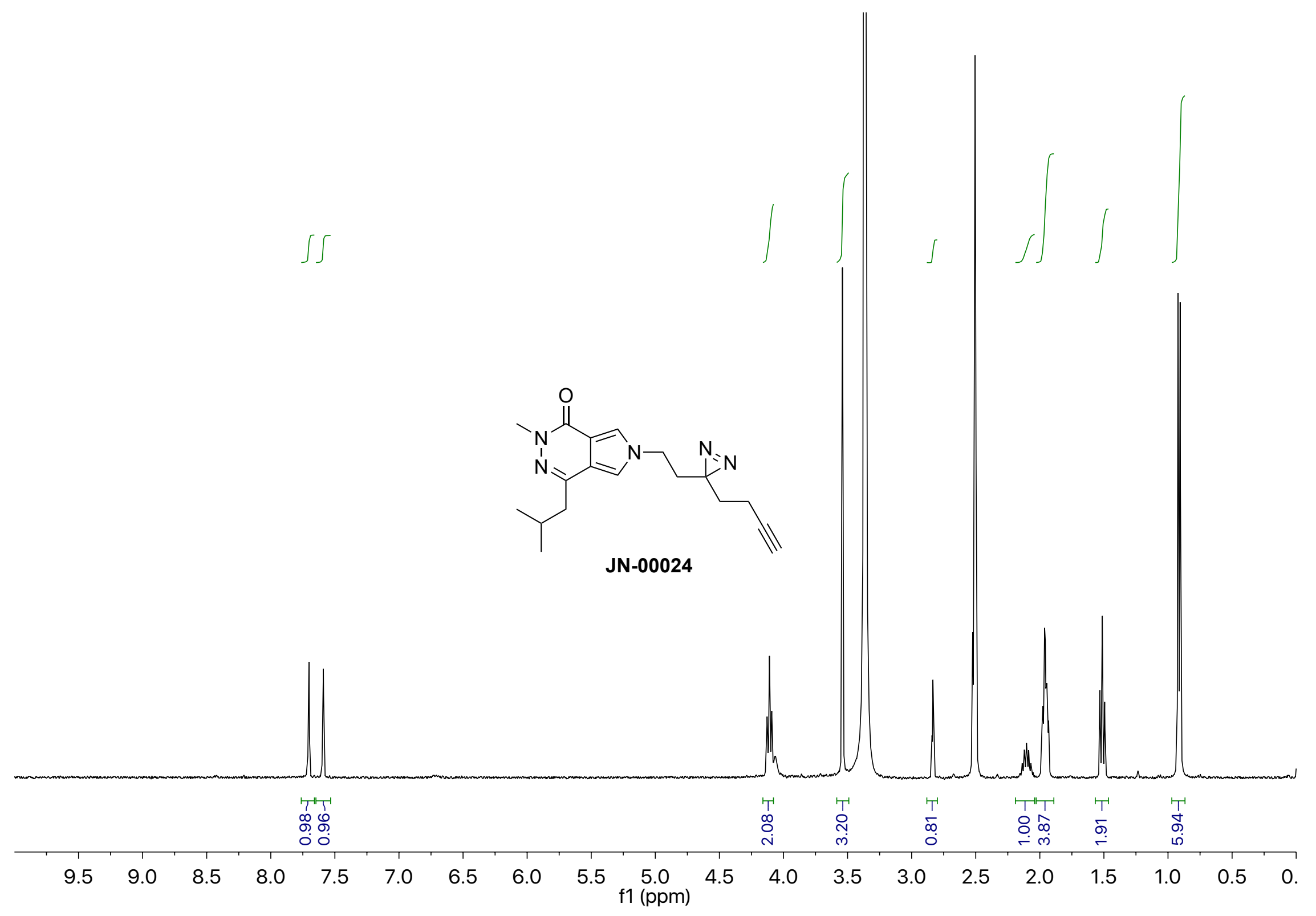

West, A. et al. Labeling preferences of diazirines with protein biomolecules, J. Am. Chem. Soc. 2021. 


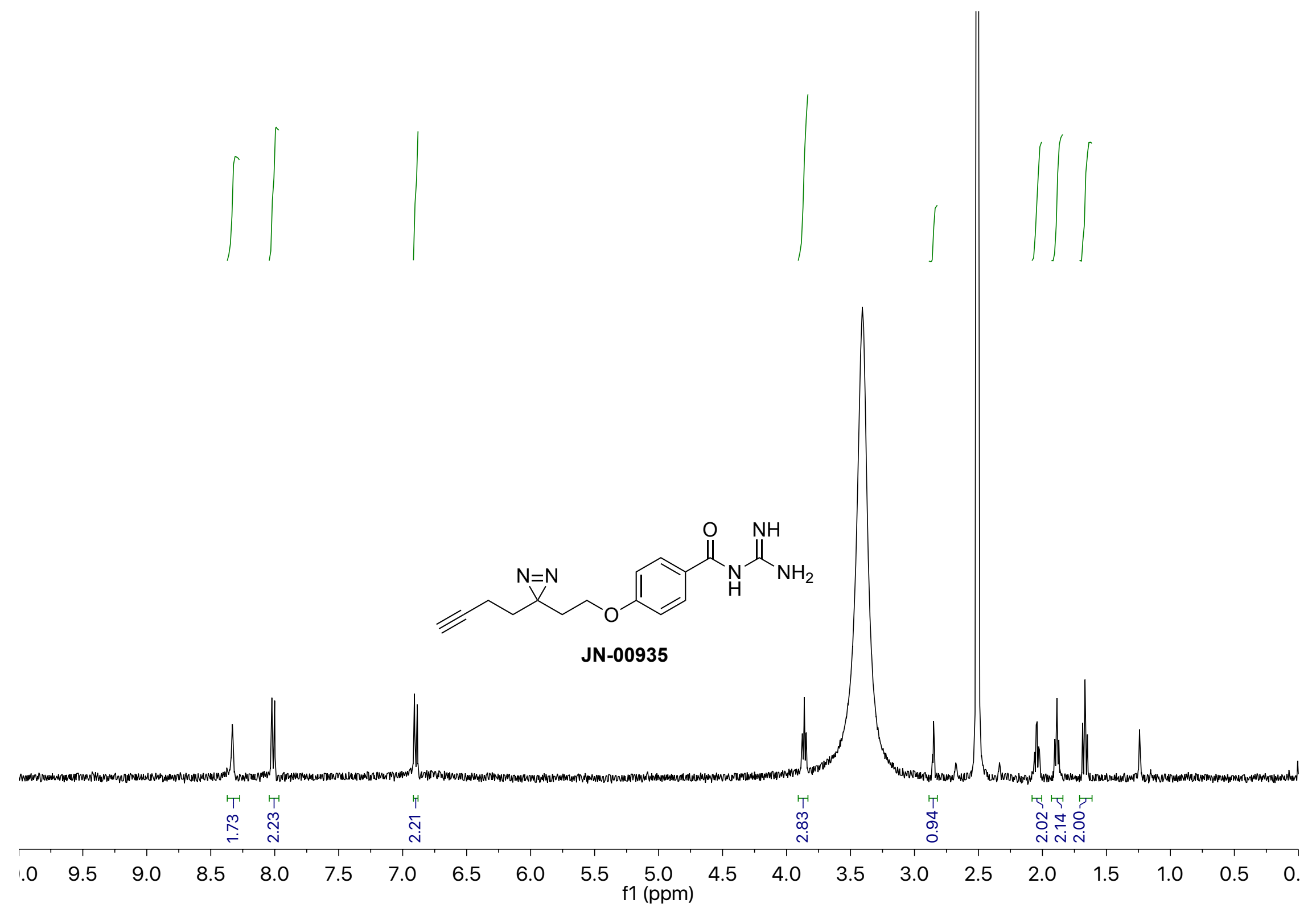




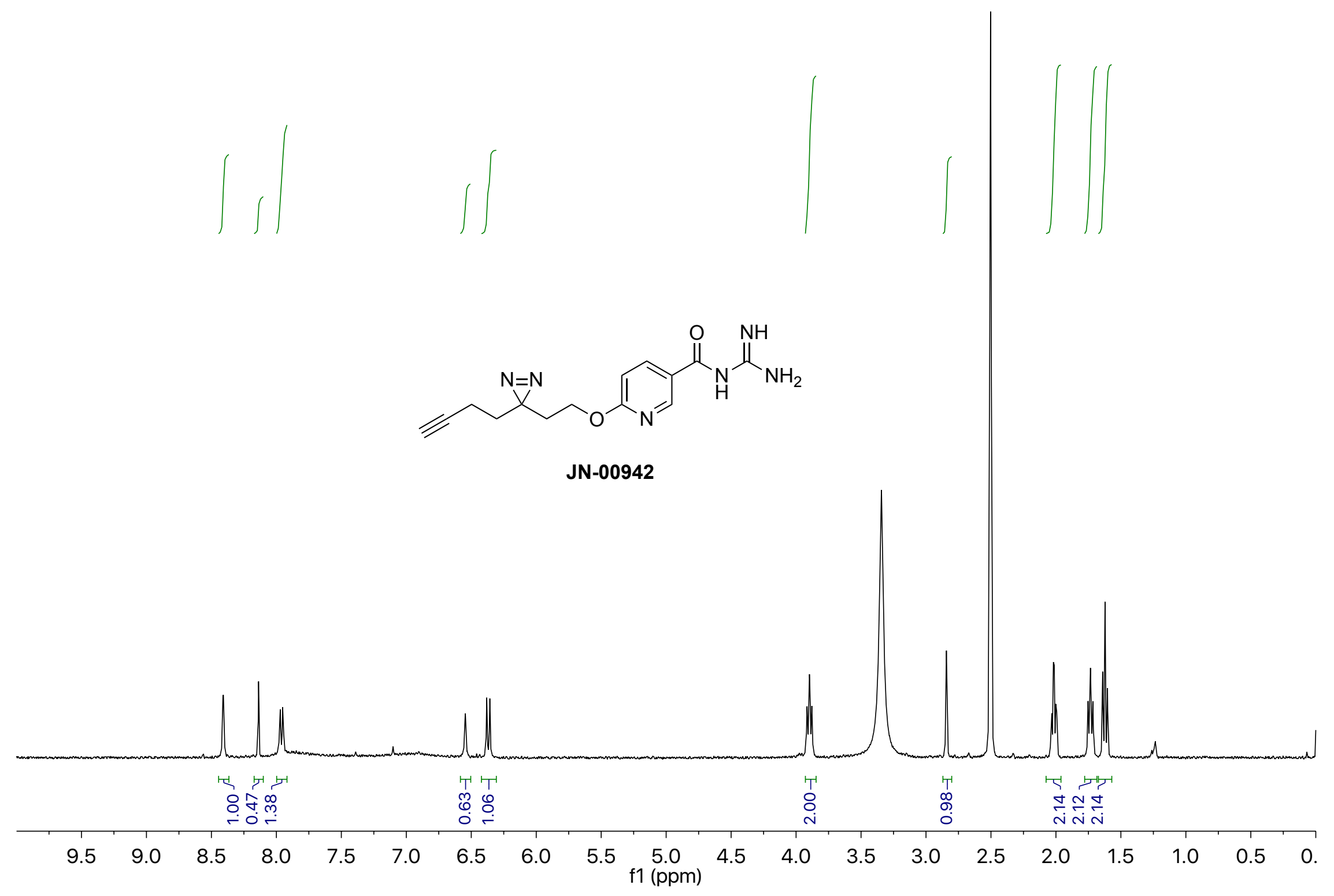

West, A. et al. Labeling preferences of diazirines with protein biomolecules, J. Am. Chem. Soc. 2021. 

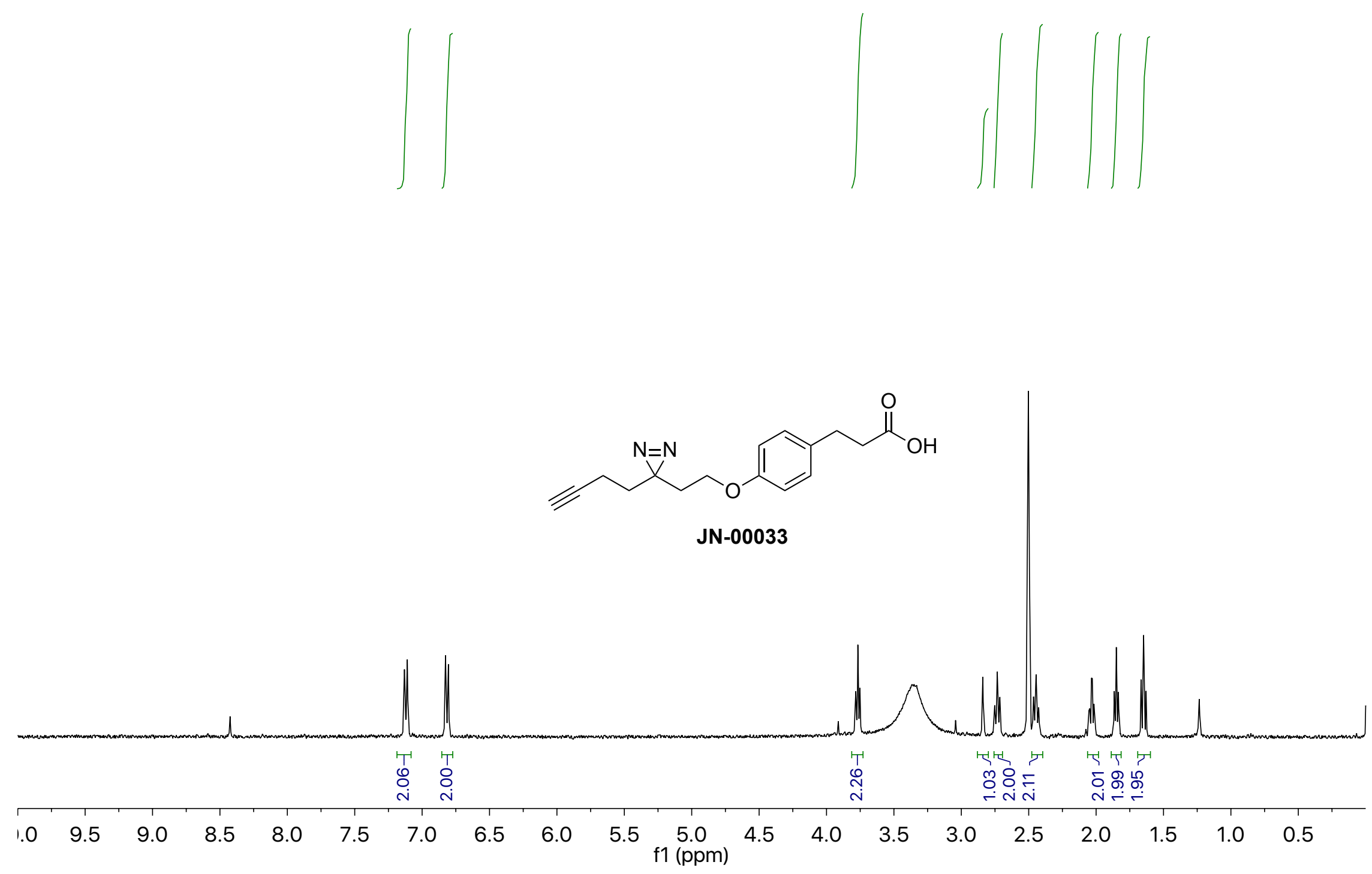


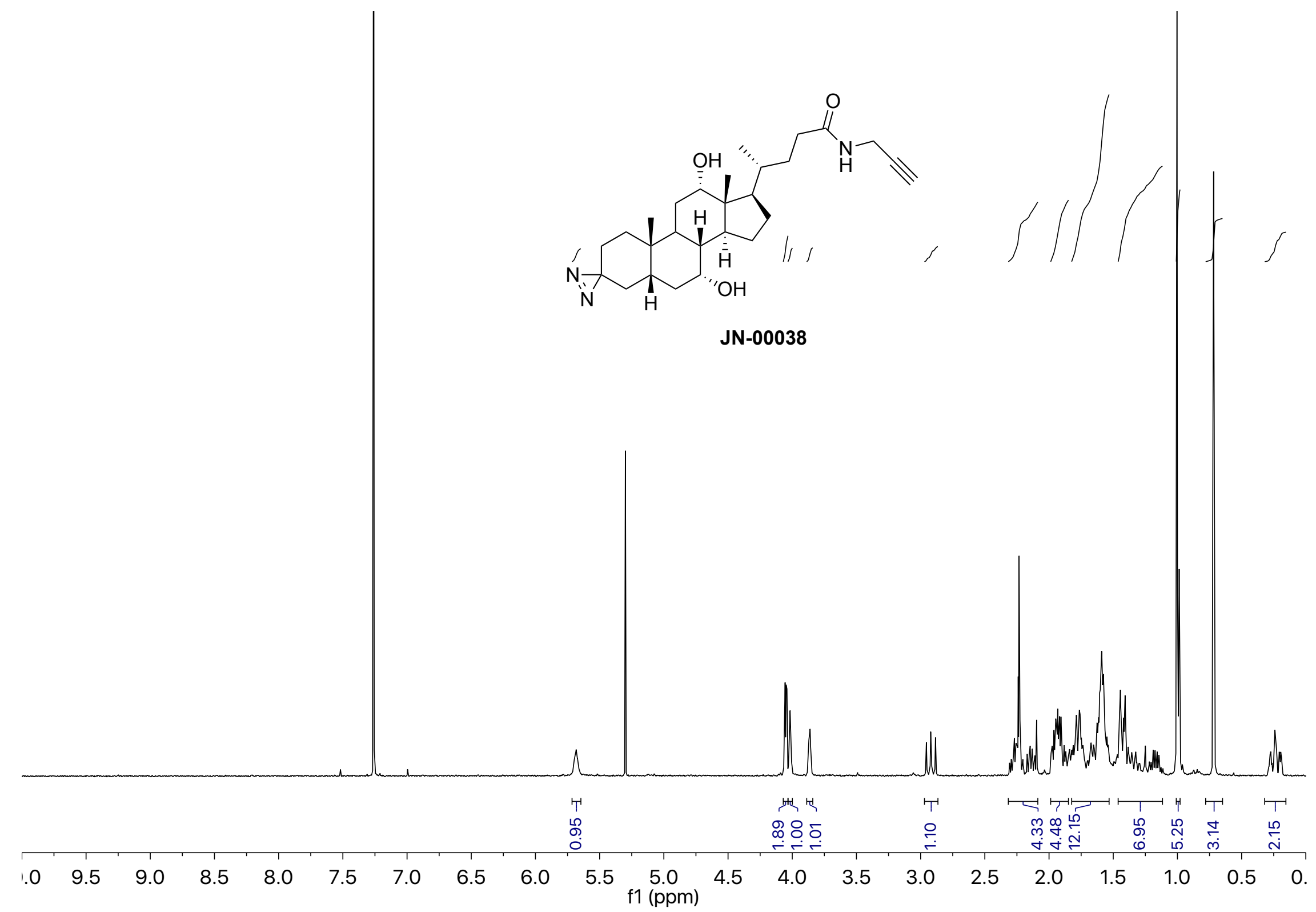




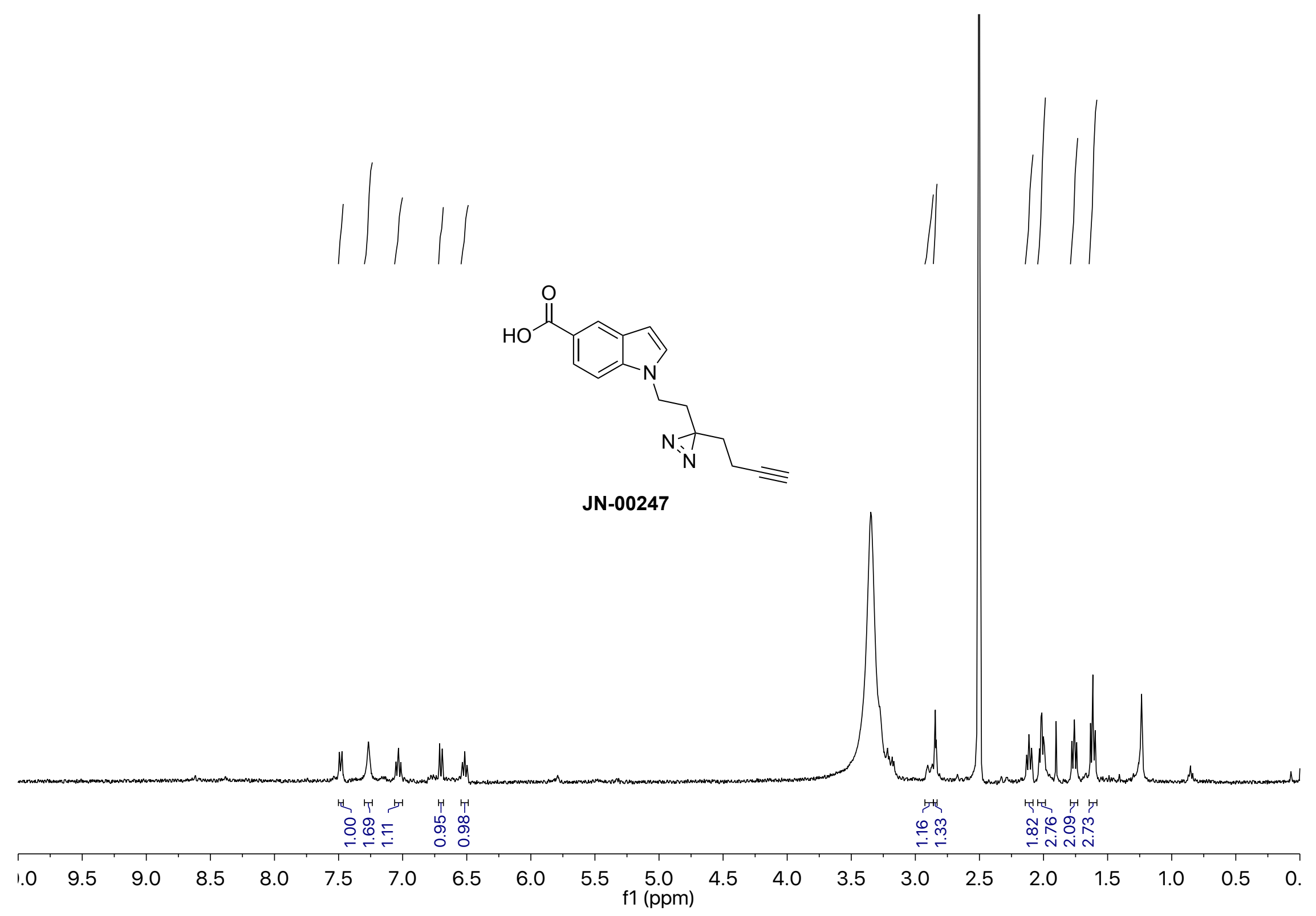

West, A. et al. Labeling preferences of diazirines with protein biomolecules, J. Am. Chem. Soc. 2021. 


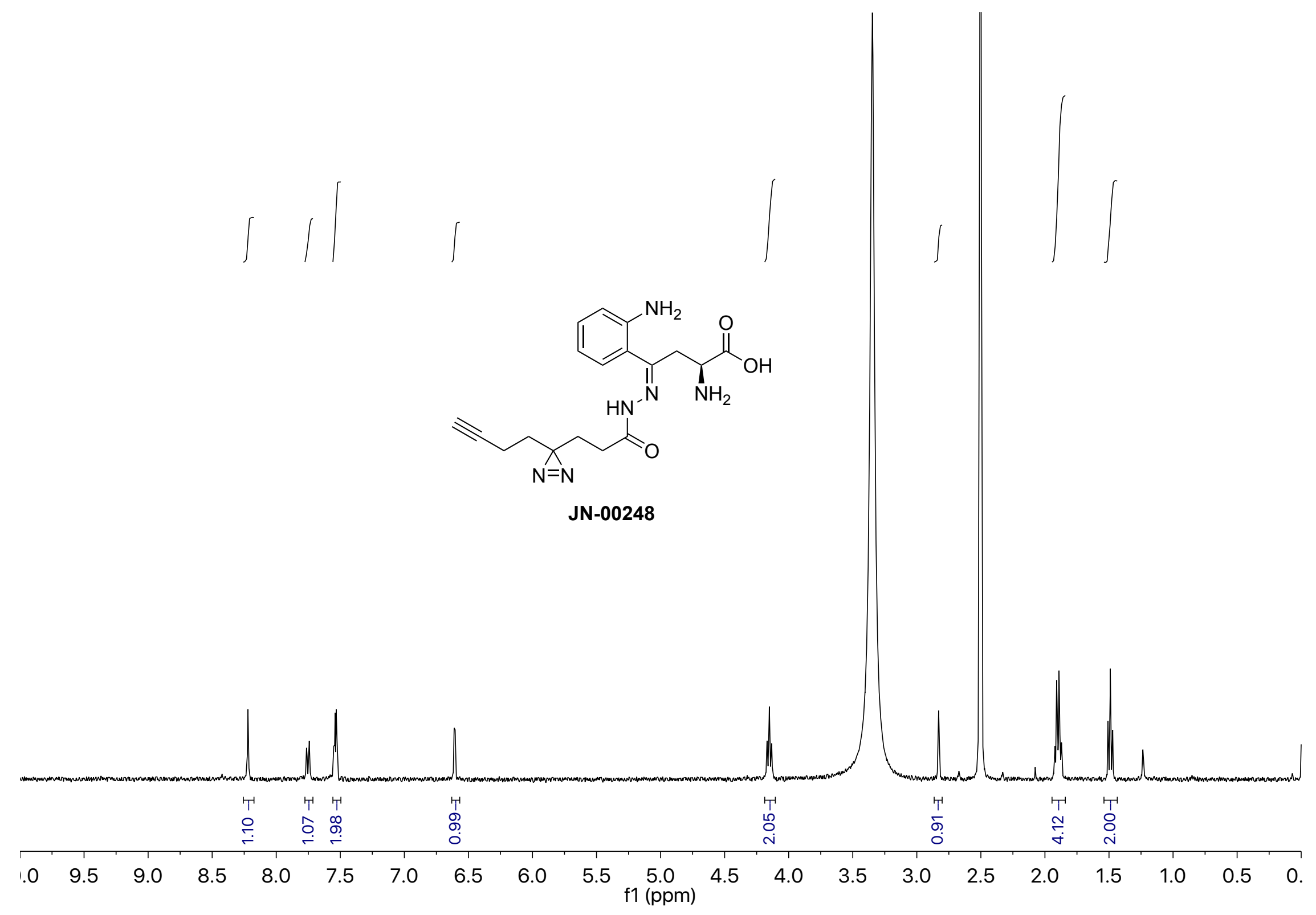




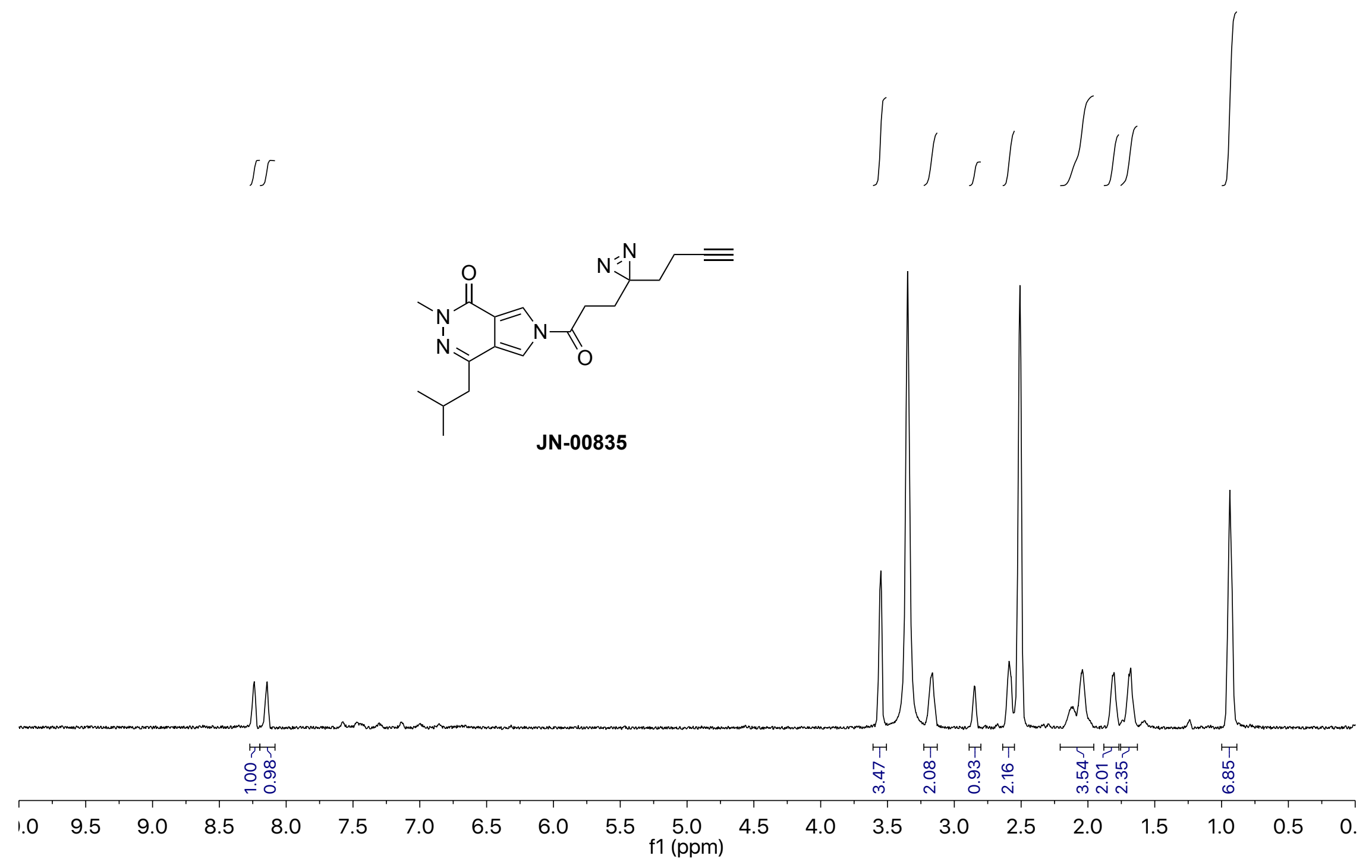

West, A. et al. Labeling preferences of diazirines with protein biomolecules, J. Am. Chem. Soc. 2021. 


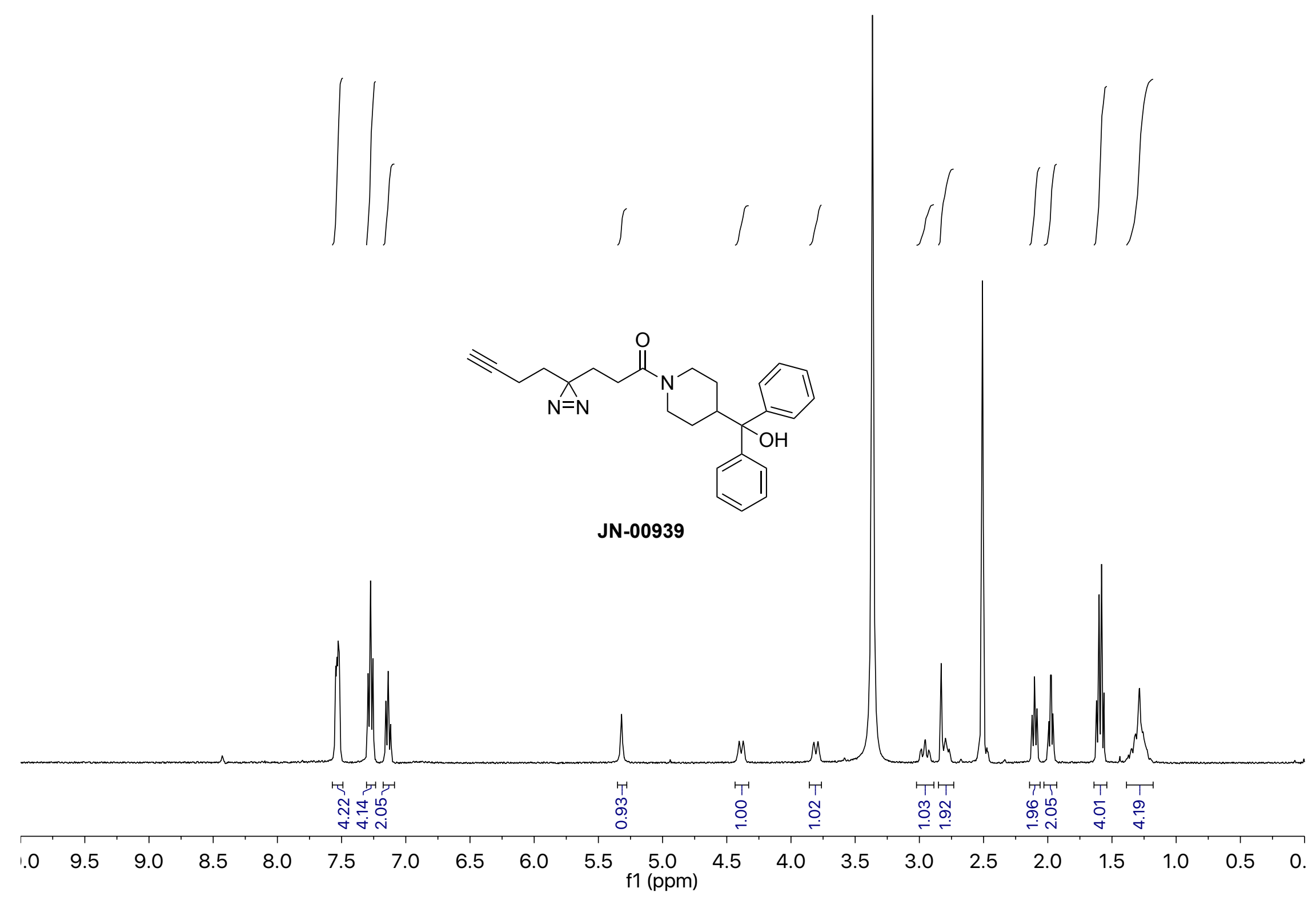




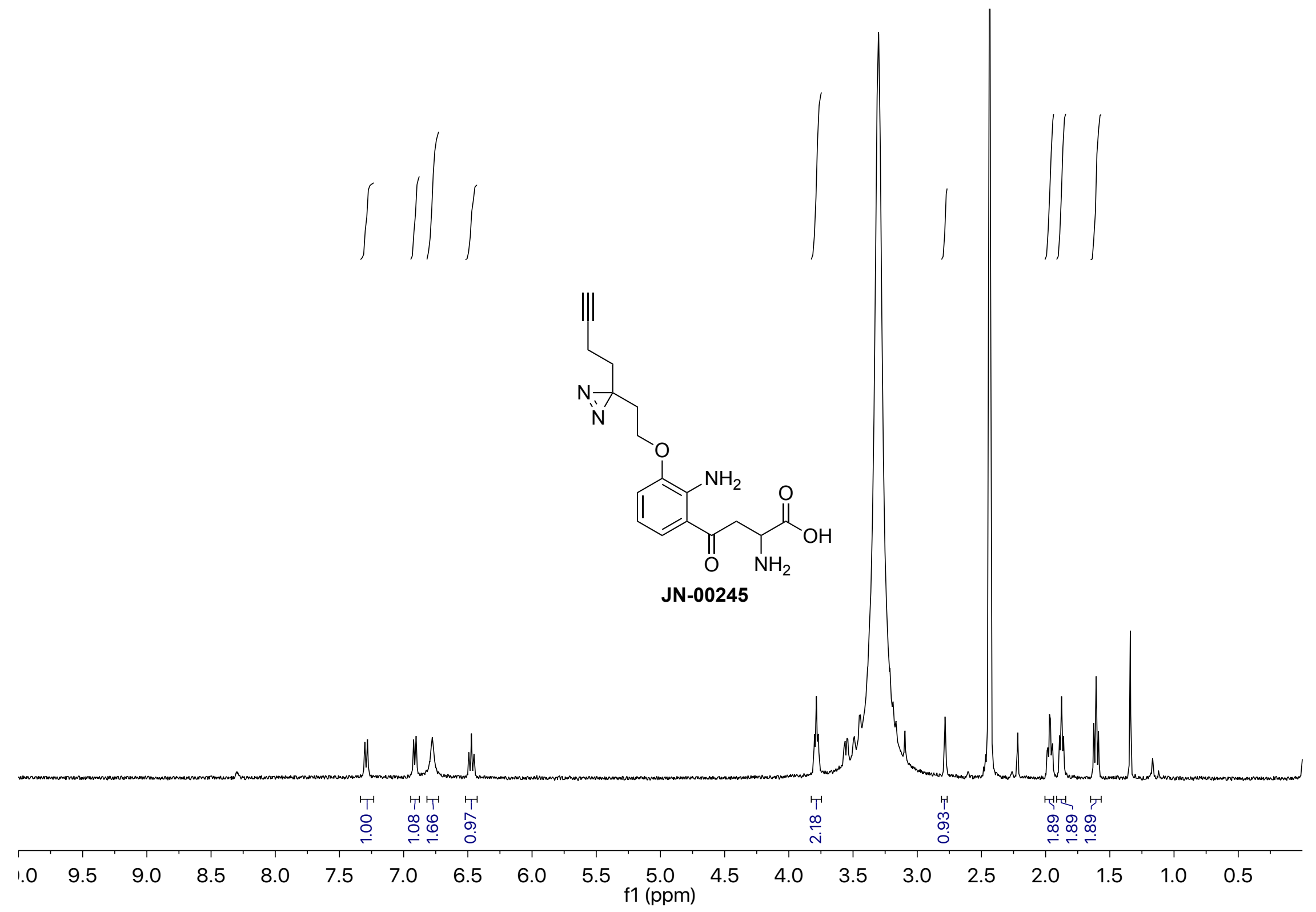




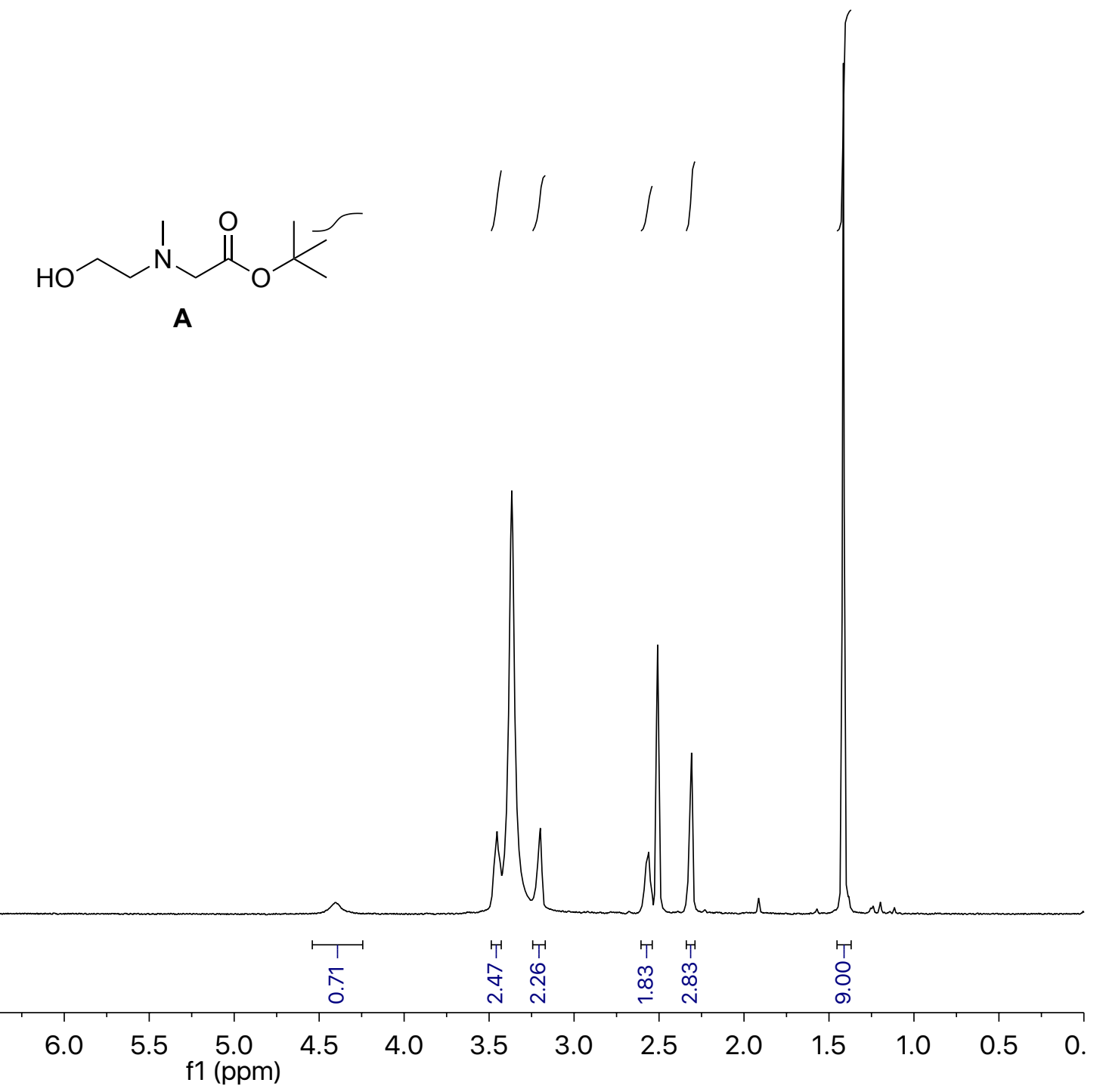

West, A. et al. Labeling preferences of diazirines with protein biomolecules, J. Am. Chem. Soc. 2021. 
I
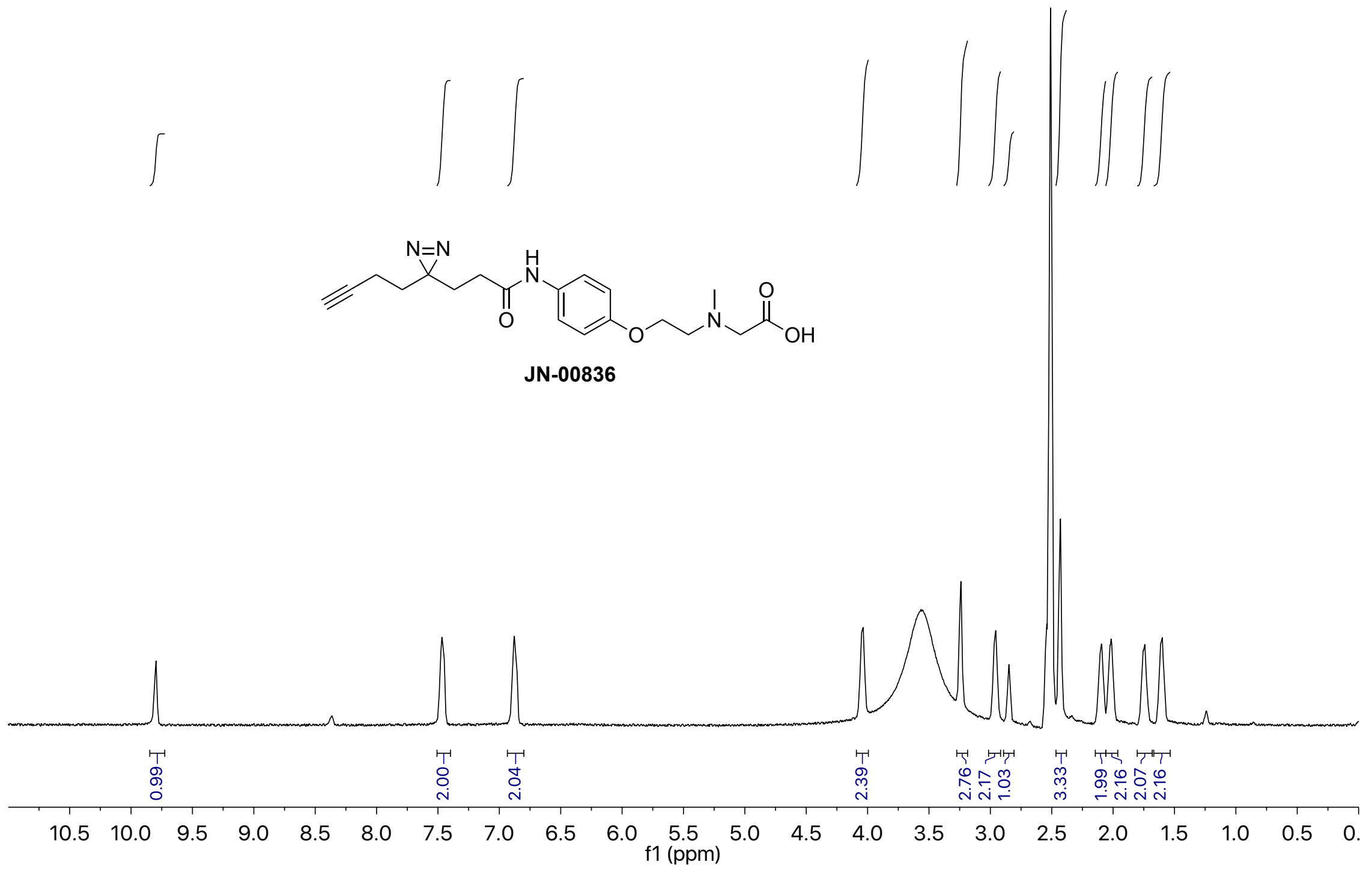

West, A. et al. Labeling preferences of diazirines with protein biomolecules, J. Am. Chem. Soc. 2021.

S99 


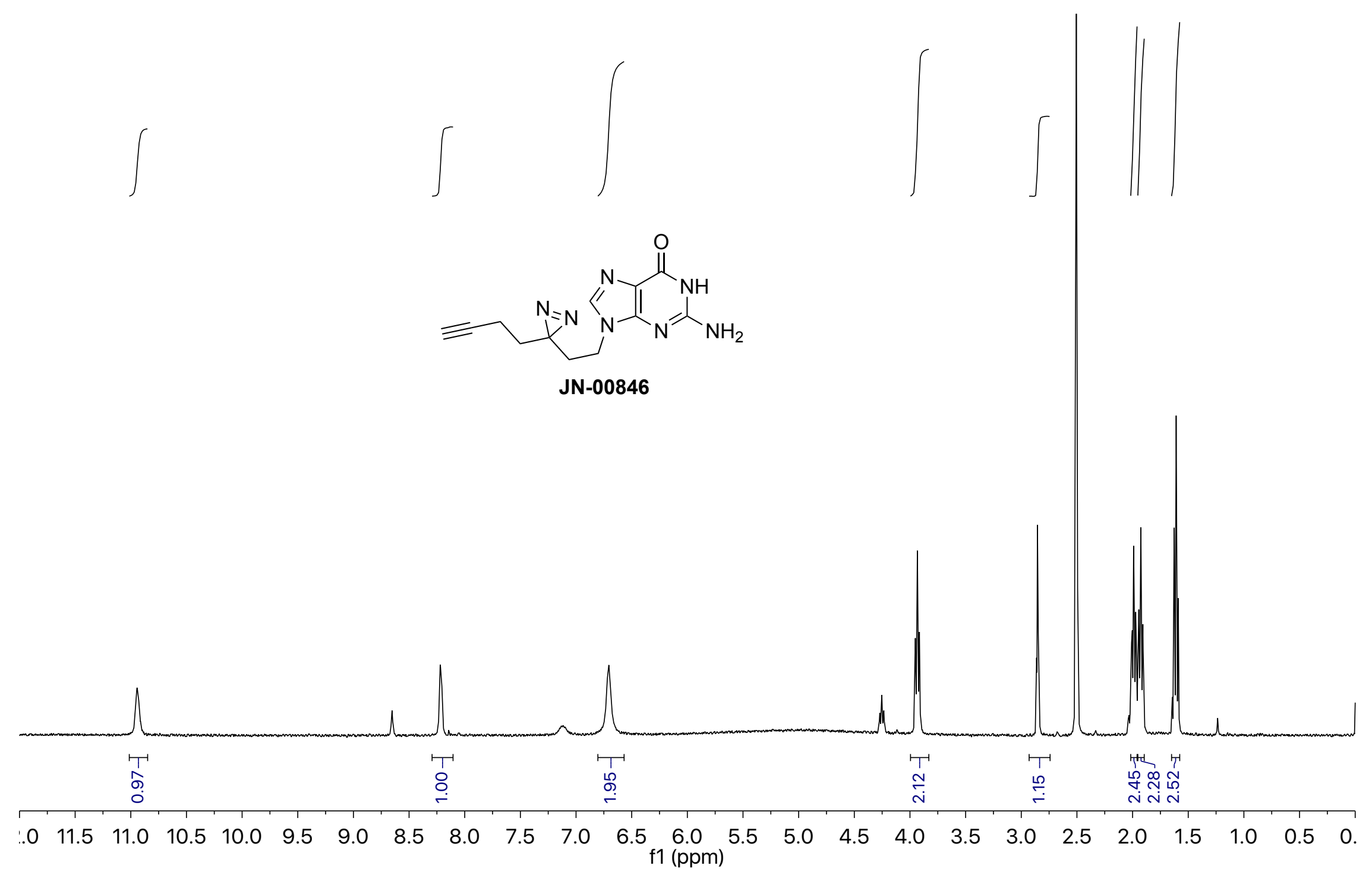

West, A. et al. Labeling preferences of diazirines with protein biomolecules, J. Am. Chem. Soc. 2021. 


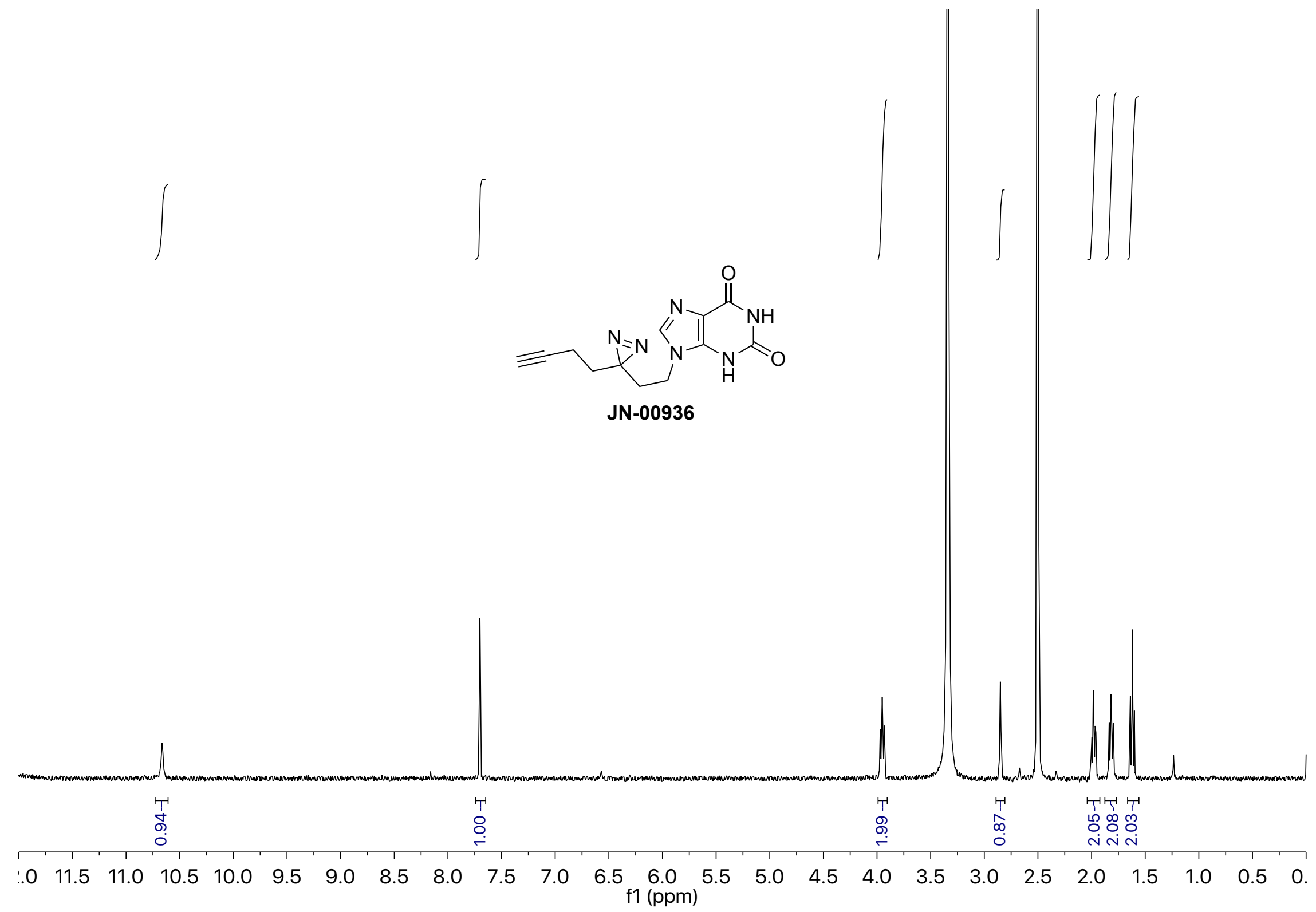

West, A. et al. Labeling preferences of diazirines with protein biomolecules, J. Am. Chem. Soc. 2021. 


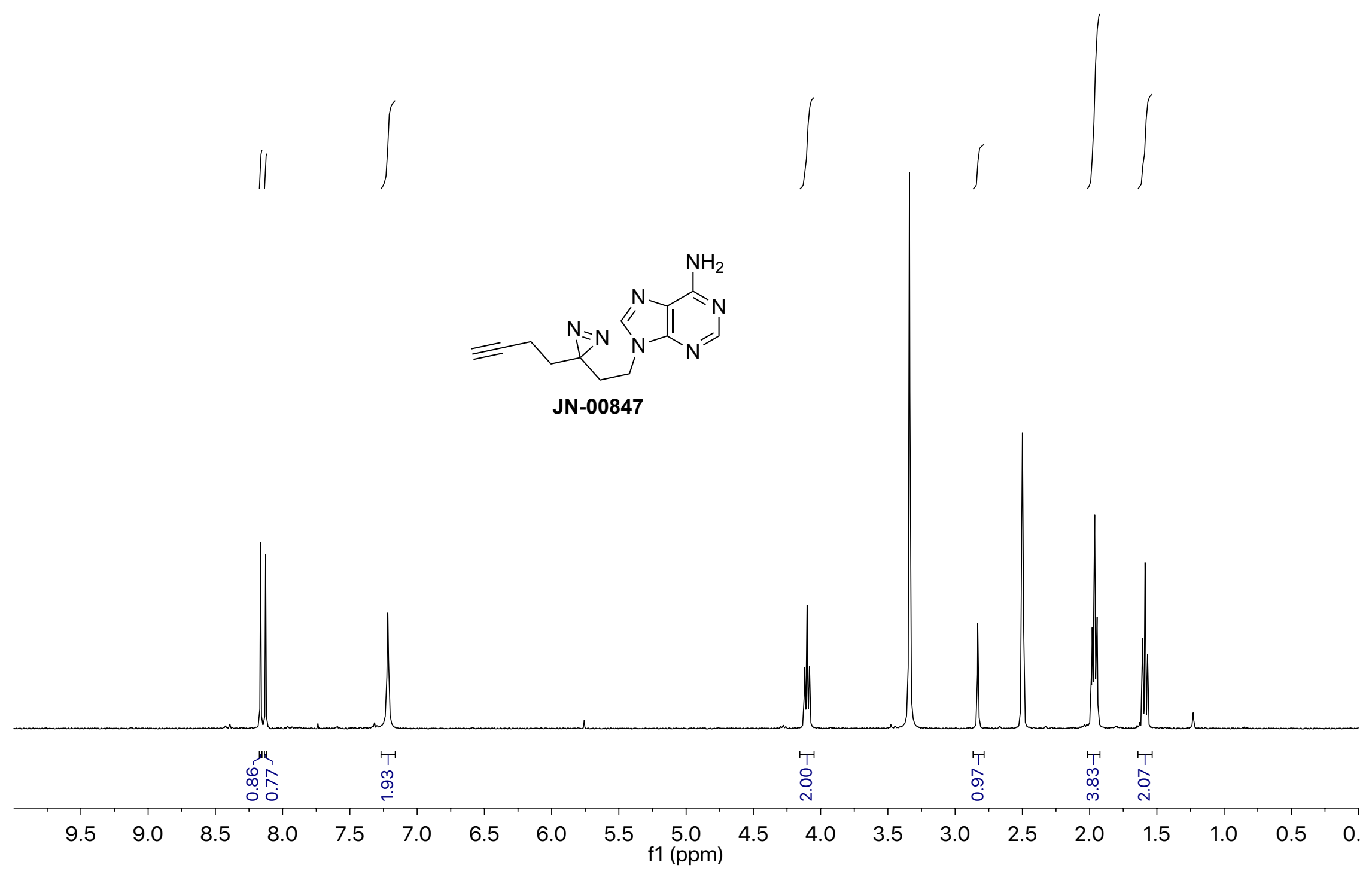

West, A. et al. Labeling preferences of diazirines with protein biomolecules, J. Am. Chem. Soc. 2021. 


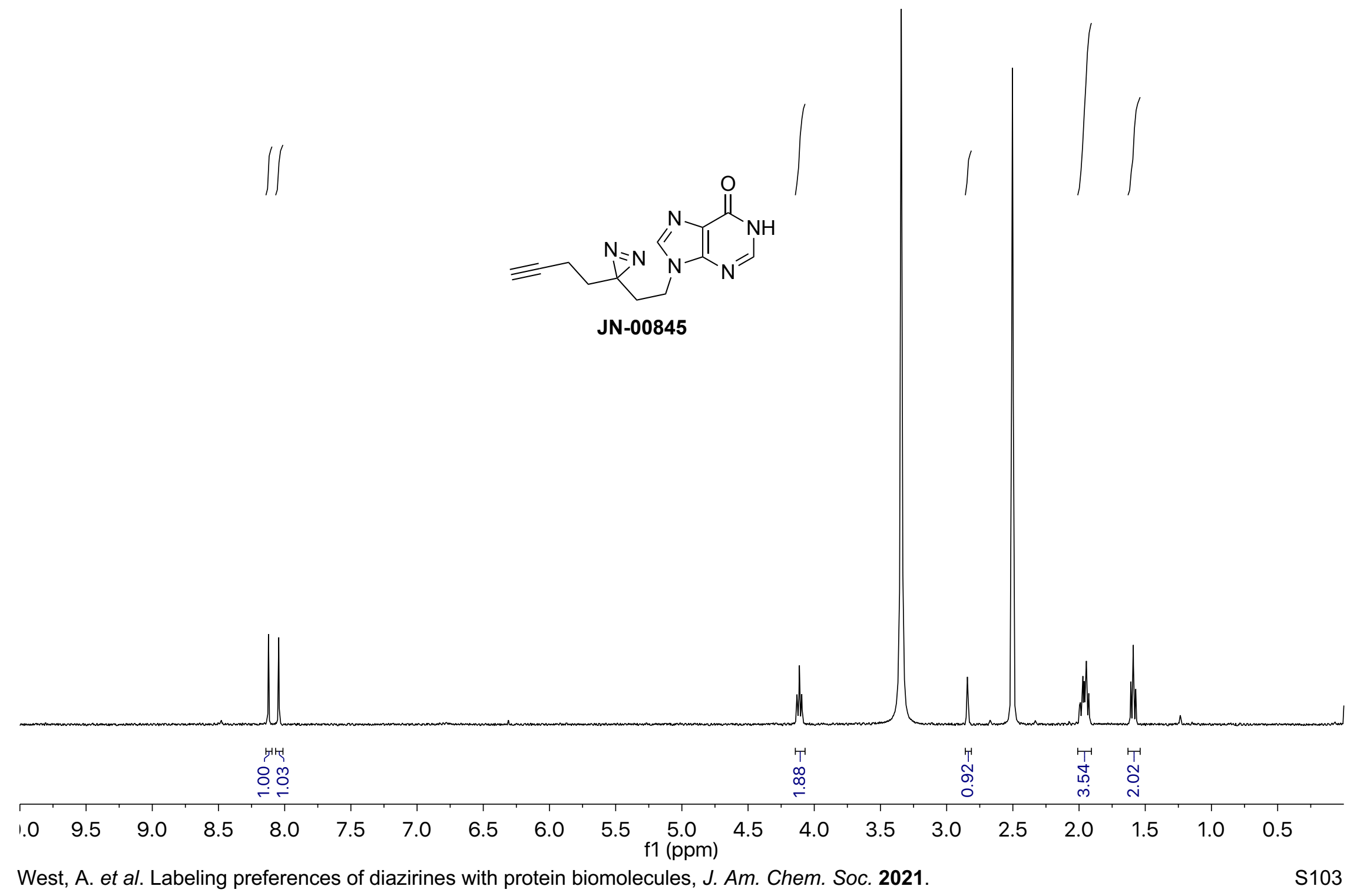




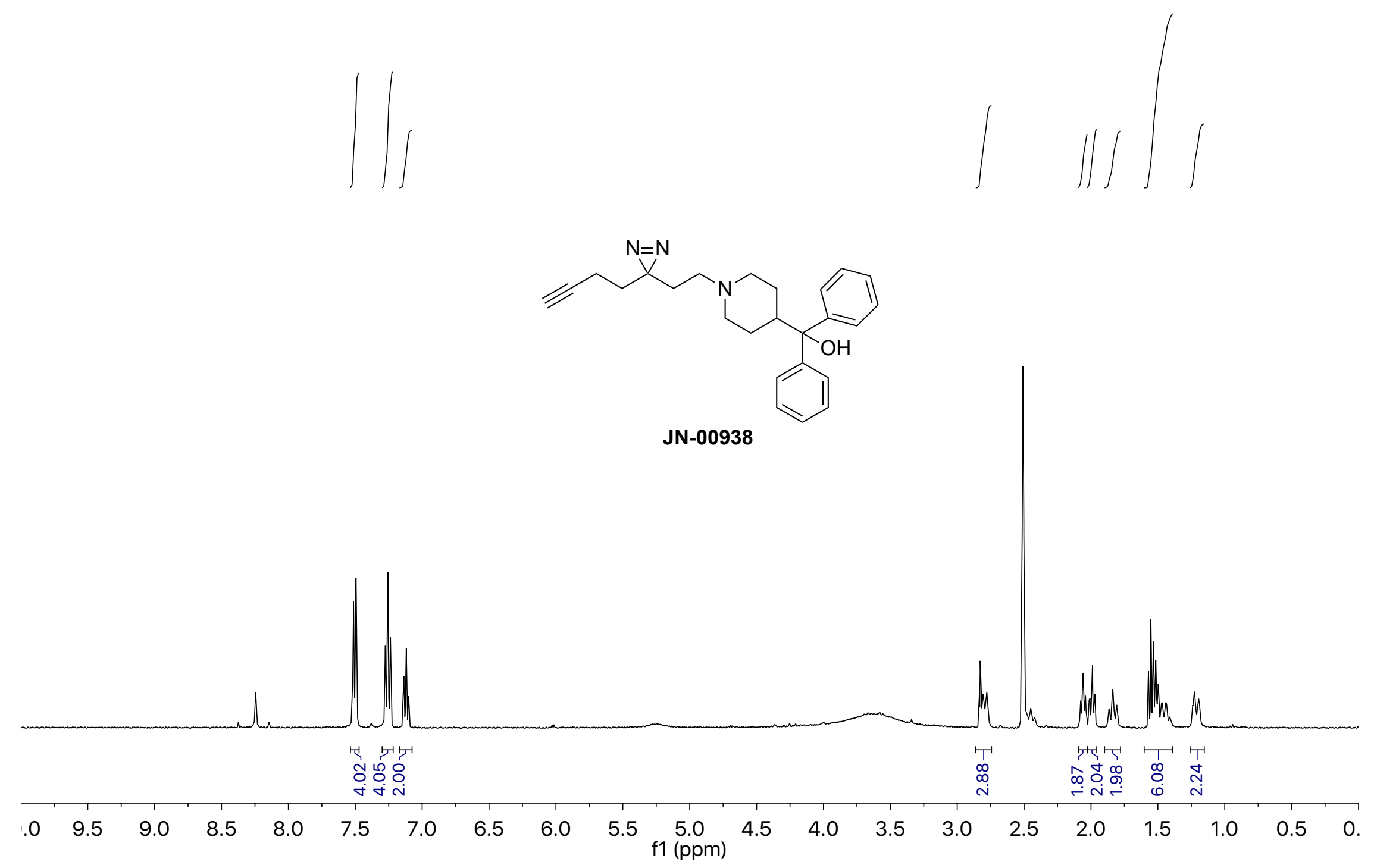

West, A. et al. Labeling preferences of diazirines with protein biomolecules, J. Am. Chem. Soc. 2021. 


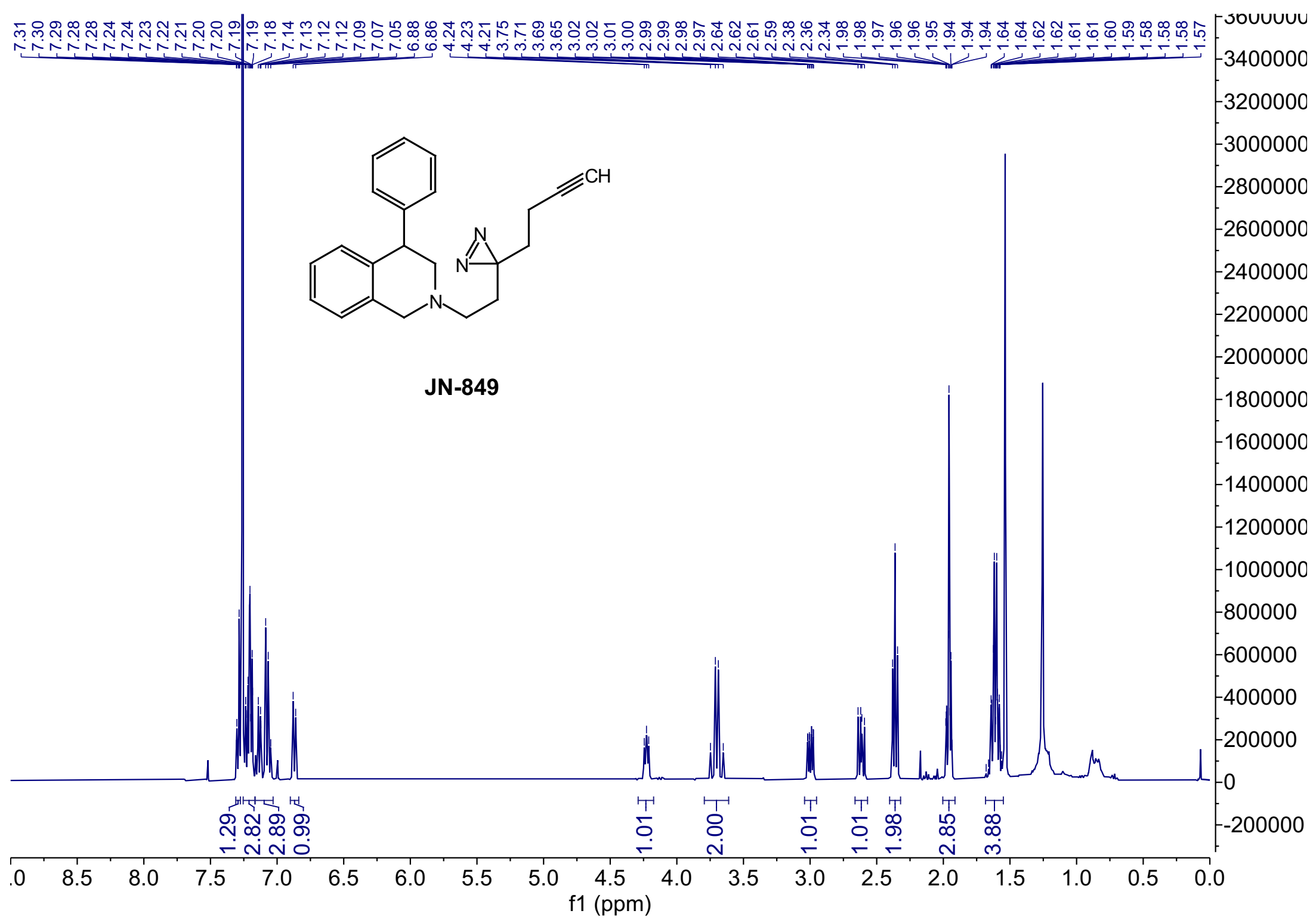




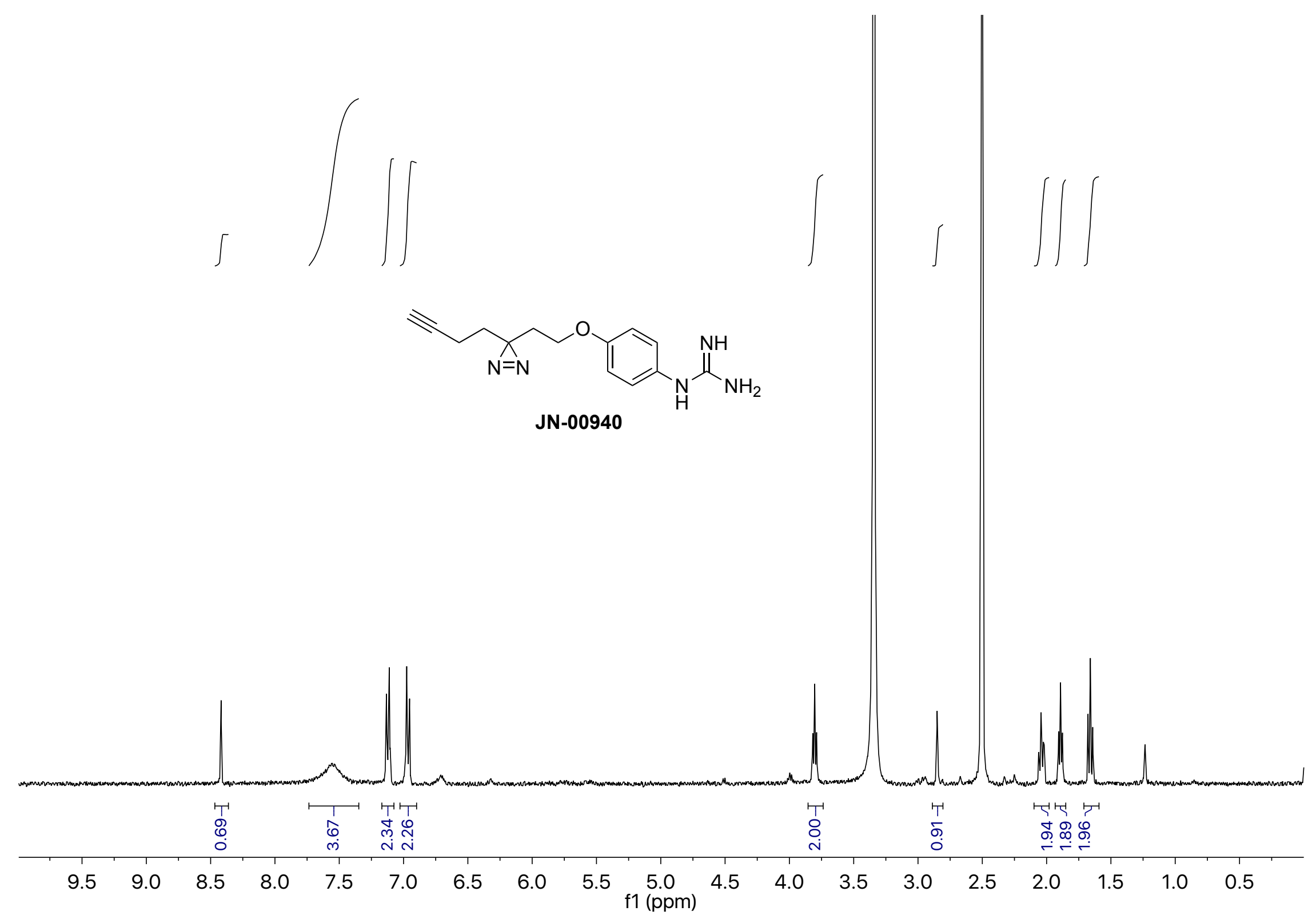


Catalog of nuclear magnetic resonance and liquid chromatograms for analytical reactions:

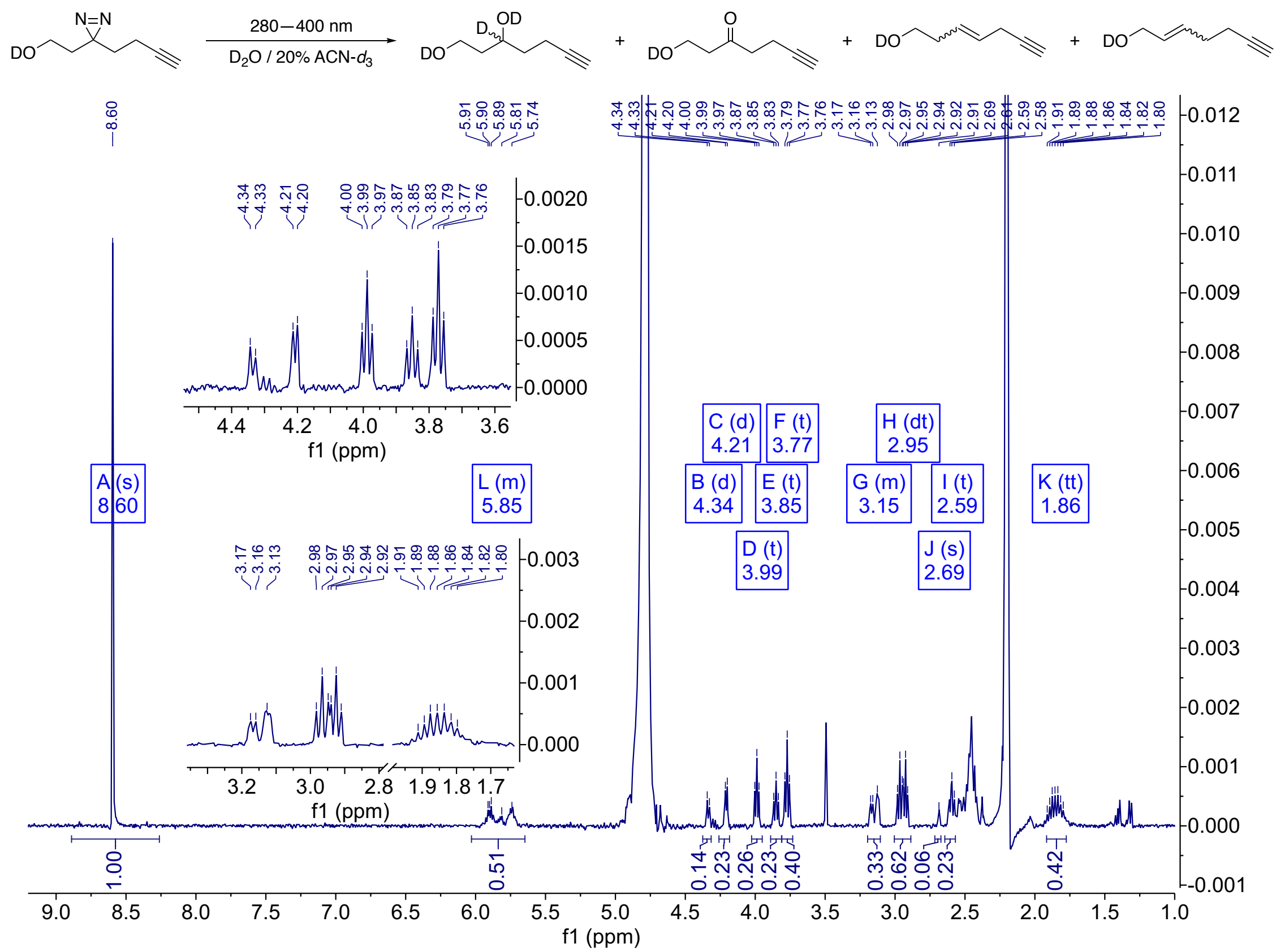




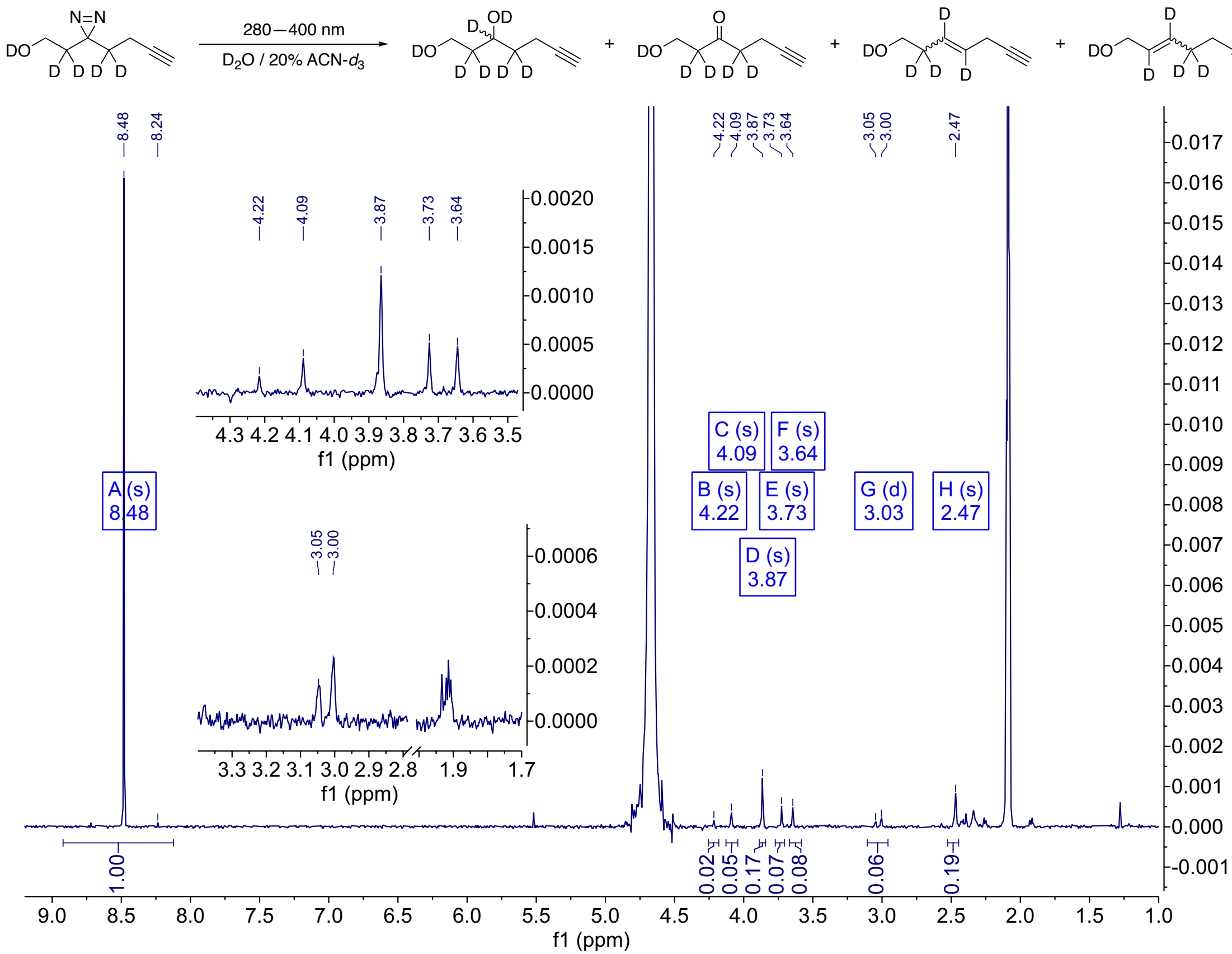

West, A. et al. Labeling preferences of diazirines with protein biomolecules, J. Am. Chem. Soc. 2021. 


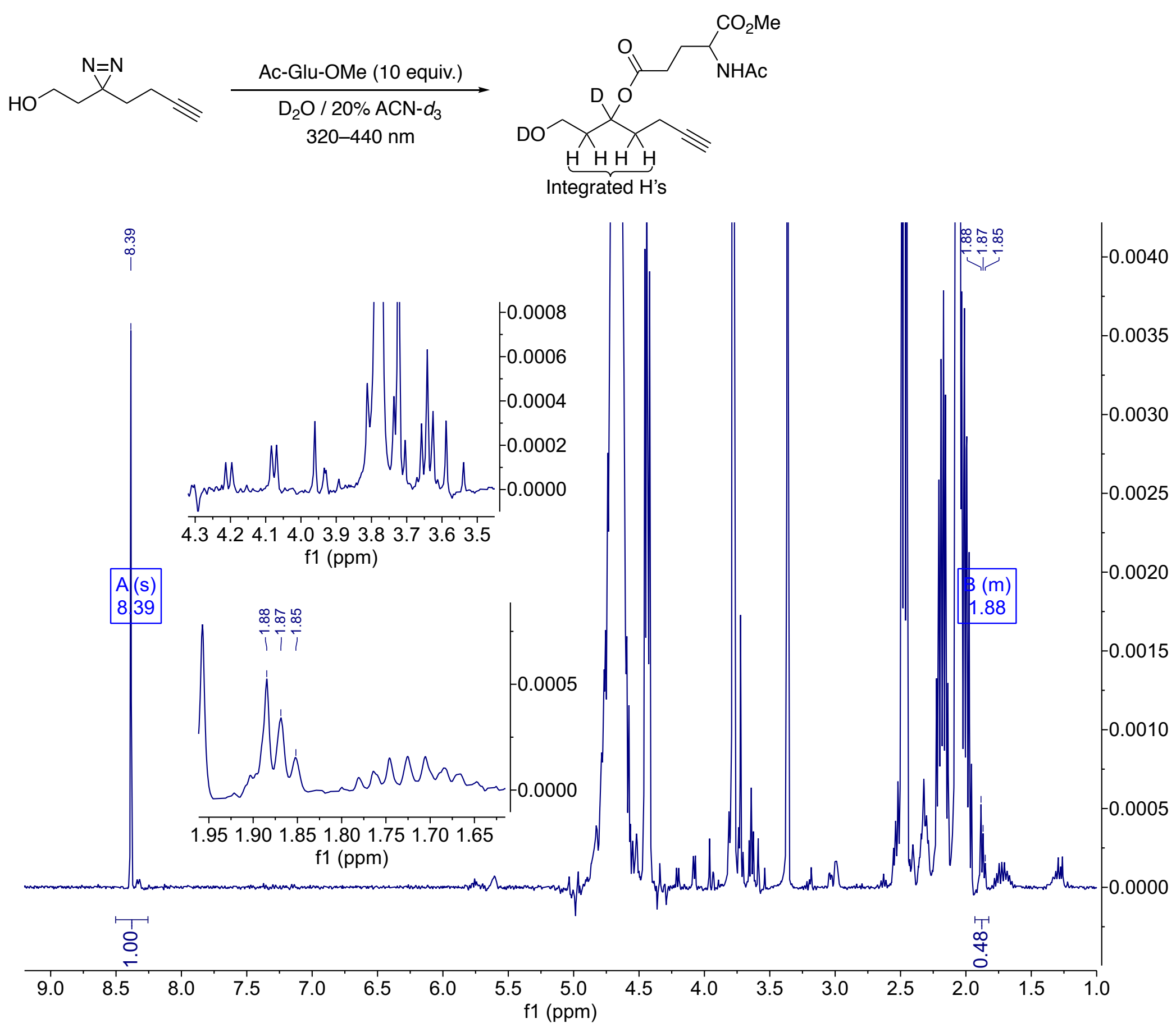

West, A. et al. Labeling preferences of diazirines with protein biomolecules, J. Am. Chem. Soc. 2021. 


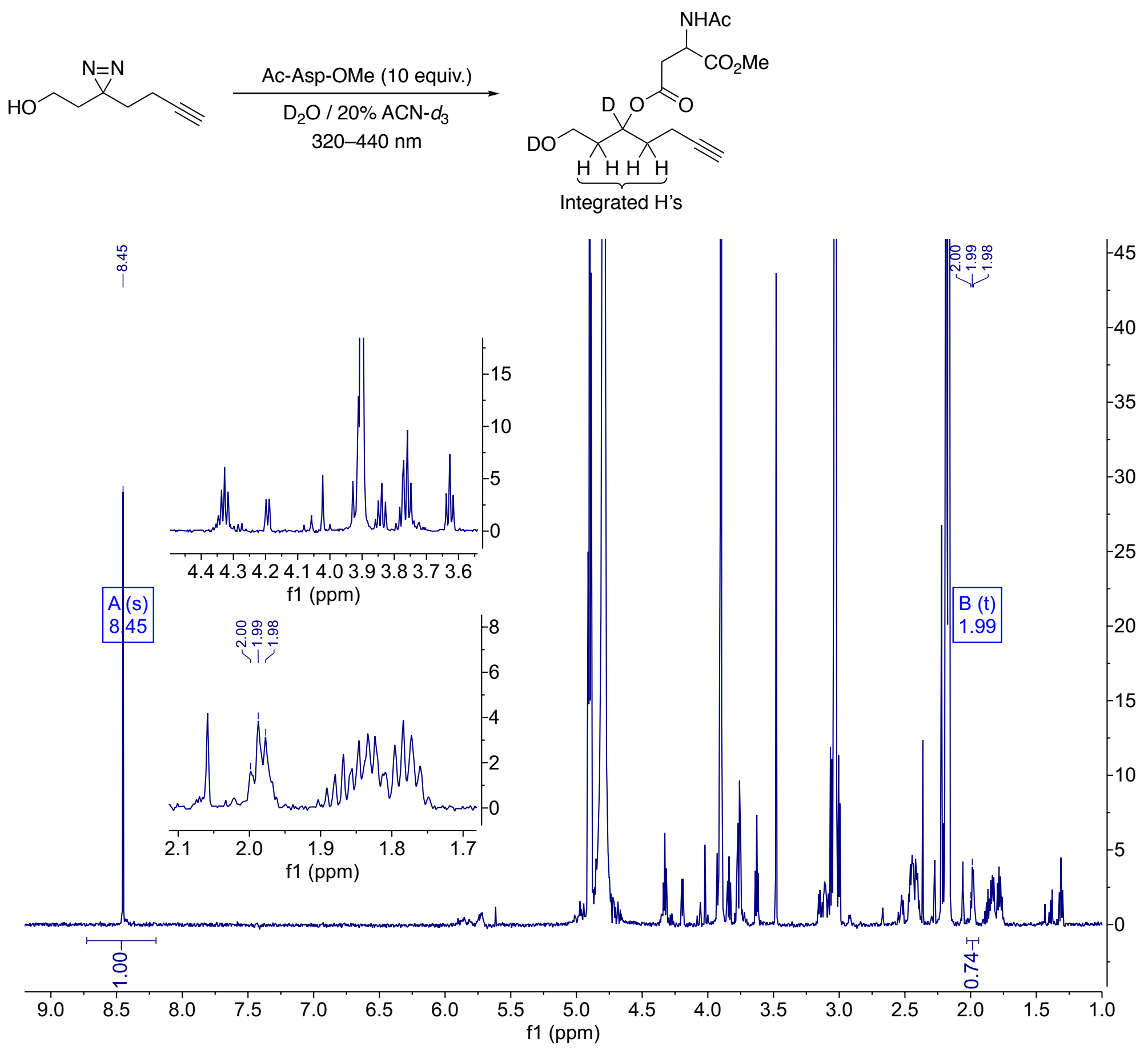

West, A. et al. Labeling preferences of diazirines with protein biomolecules, J. Am. Chem. Soc. 2021. 

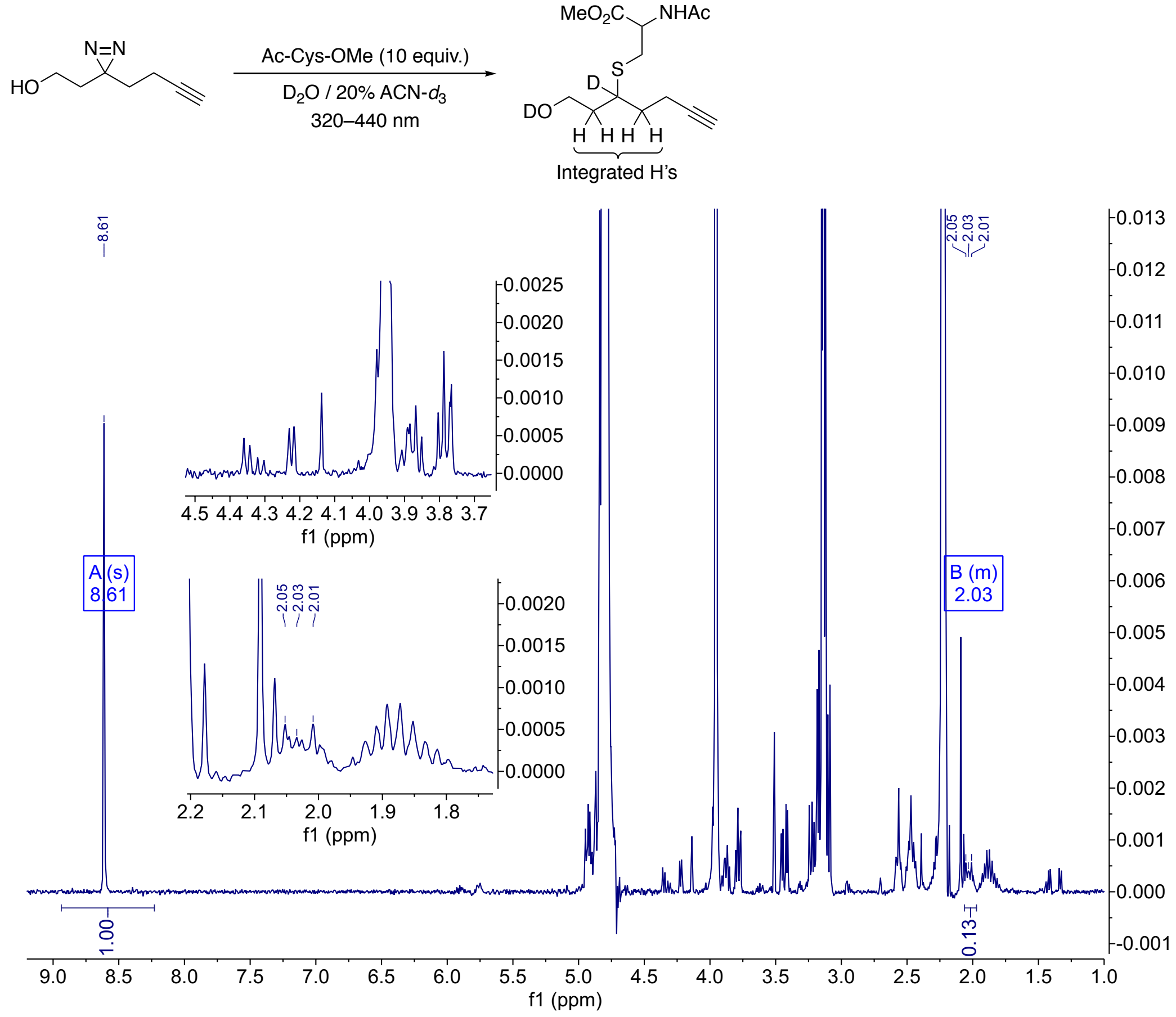

West, A. et al. Labeling preferences of diazirines with protein biomolecules, J. Am. Chem. Soc. 2021. 


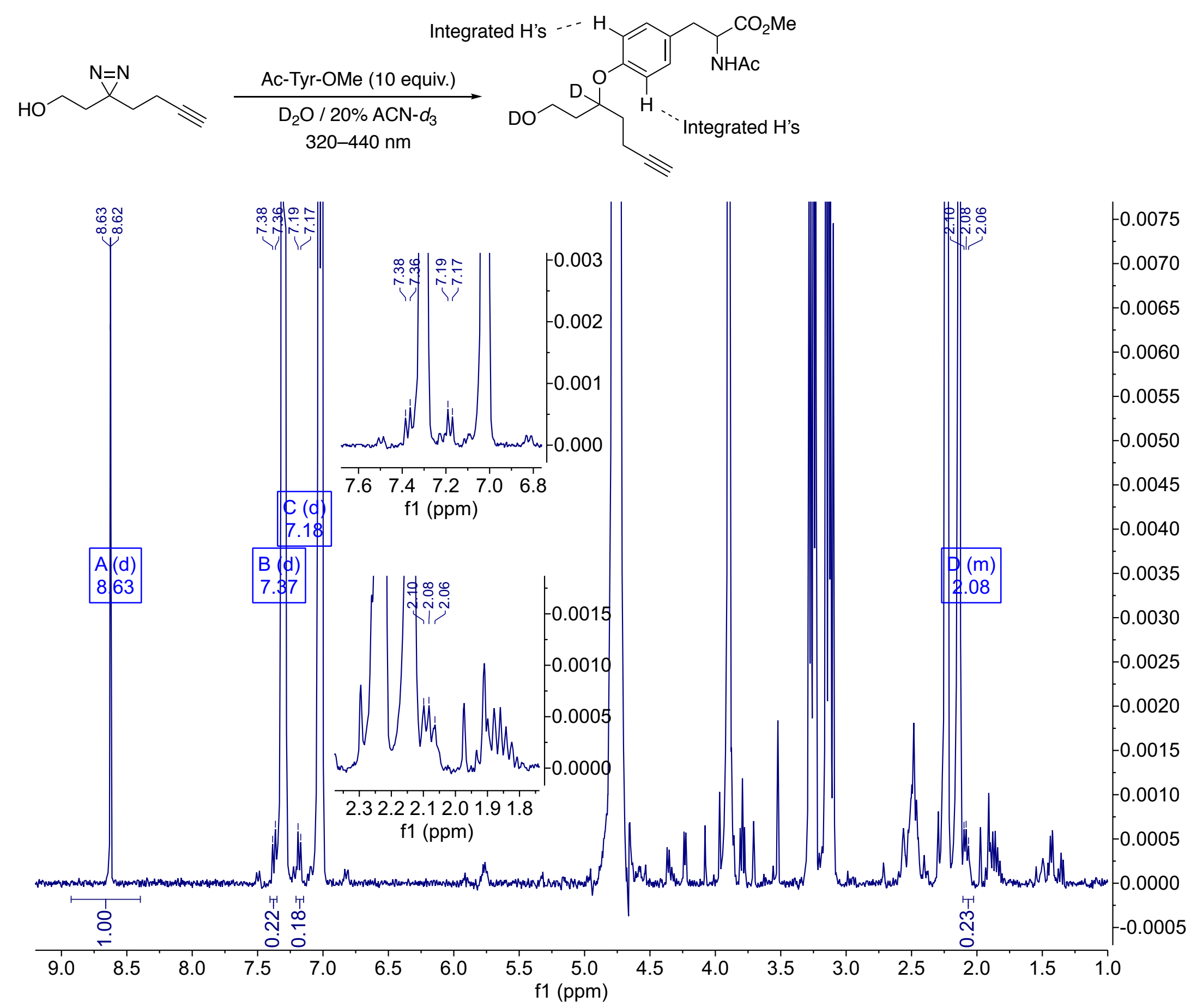

West, A. et al. Labeling preferences of diazirines with protein biomolecules, J. Am. Chem. Soc. 2021. 


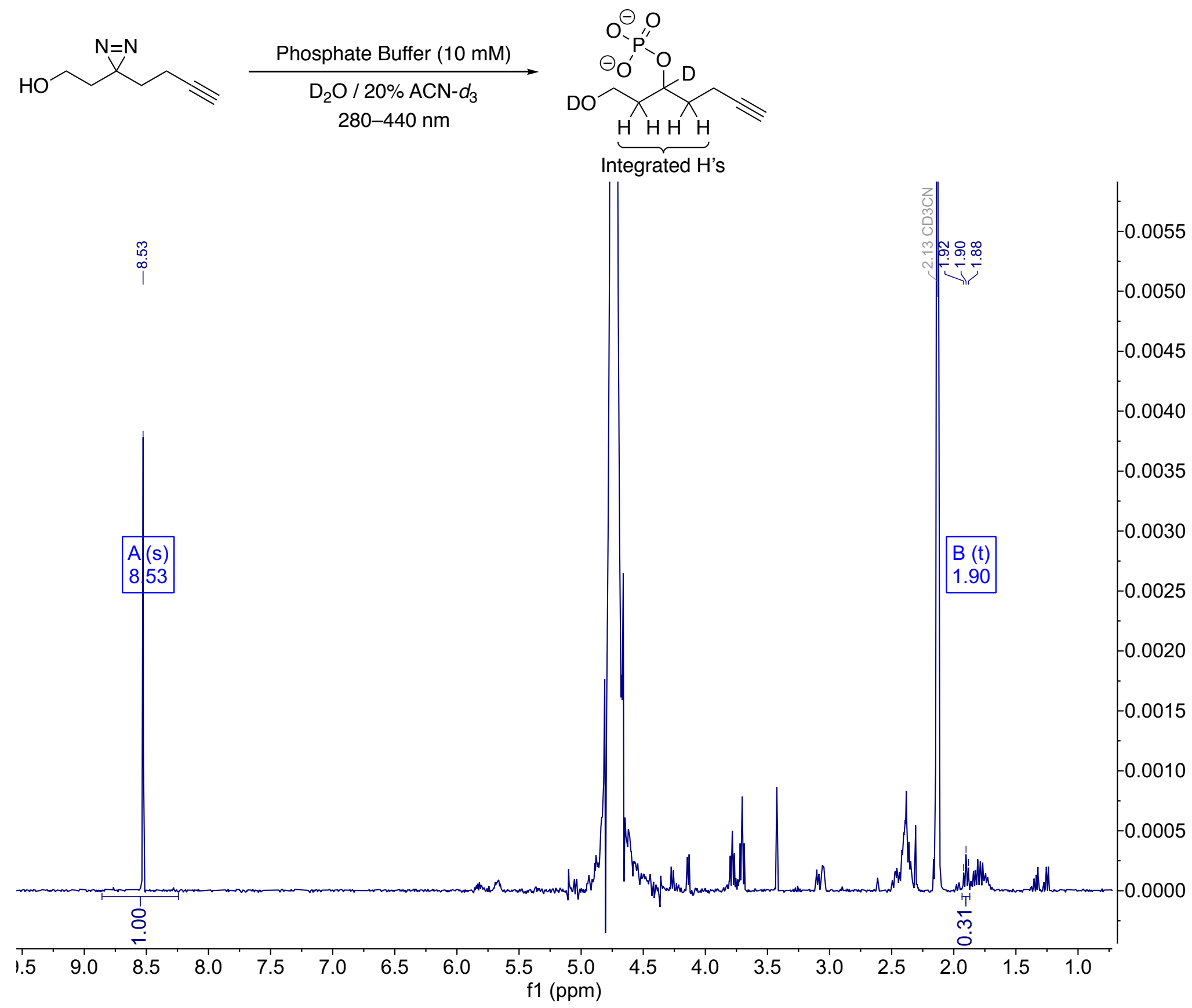

West, A. et al. Labeling preferences of diazirines with protein biomolecules, J. Am. Chem. Soc. 2021. 
<smiles>COC(=O)c1ccc(C2(C(F)(F)F)N=N2)cc1</smiles>
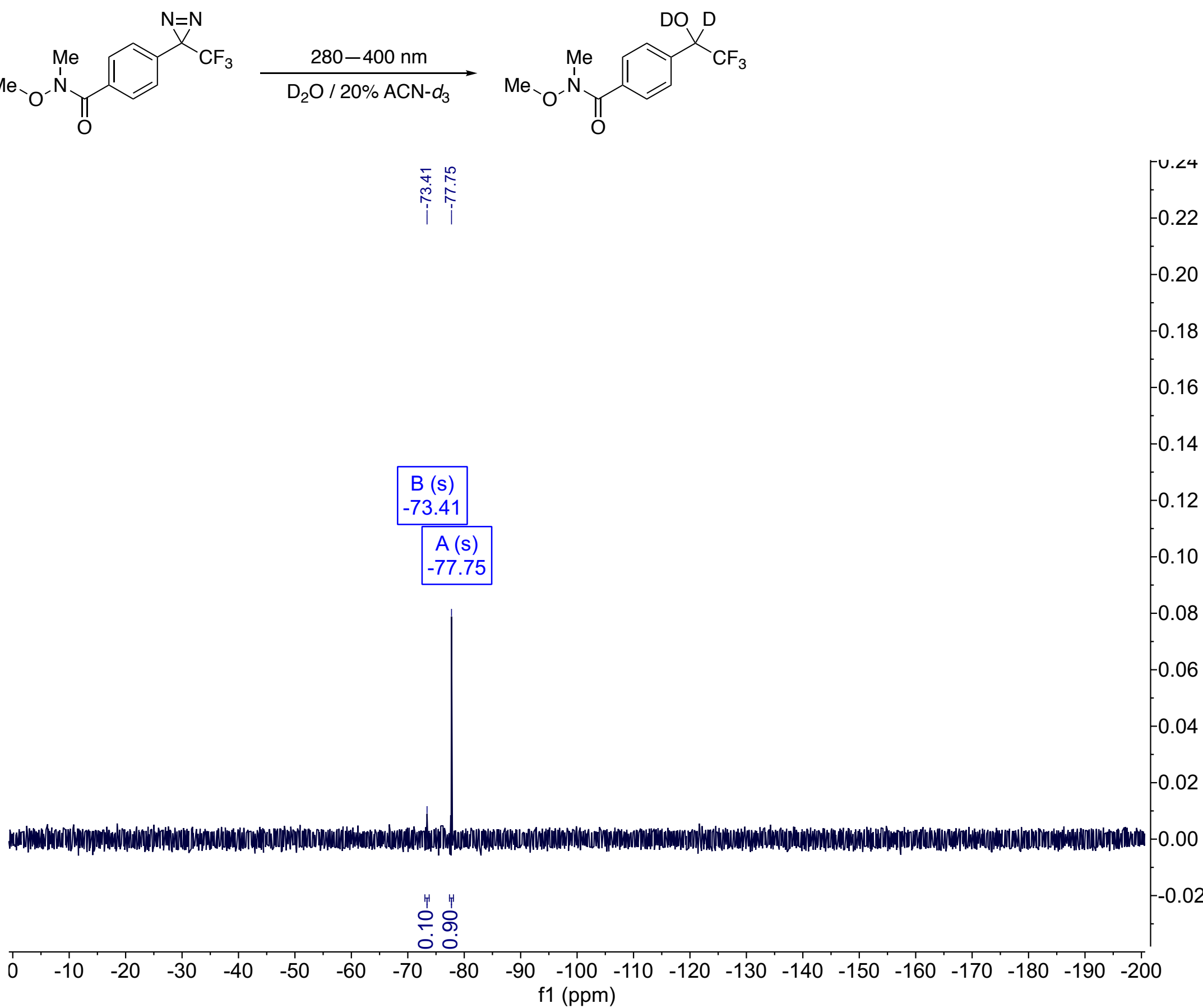
<smiles>COC(=O)c1ccc(C2(C(F)(F)F)N=N2)cc1</smiles>

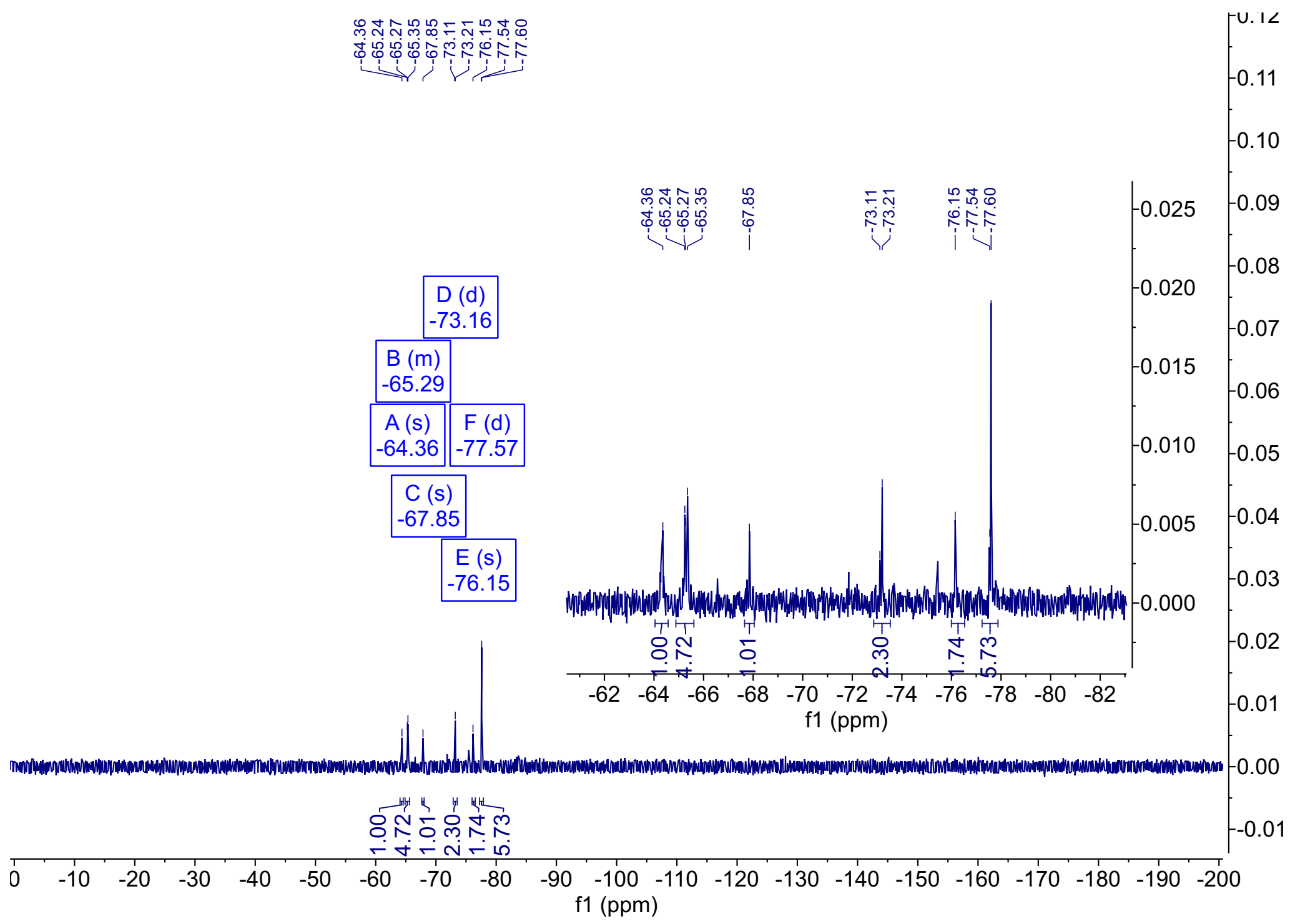

West, A. et al. Labeling preferences of diazirines with protein biomolecules, J. Am. Chem. Soc. 2021. 
$\mathrm{Me}_{\mathrm{O}-\mathrm{N}_{\mathrm{O}}^{\mathrm{N}}}^{\mathrm{N}=\mathrm{N}}$

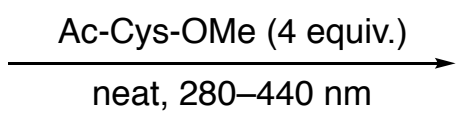

ஜำ ำ

نं

$B(d d)$

$-67.04$

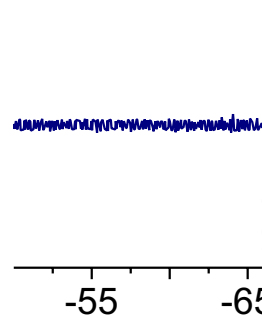

กิ
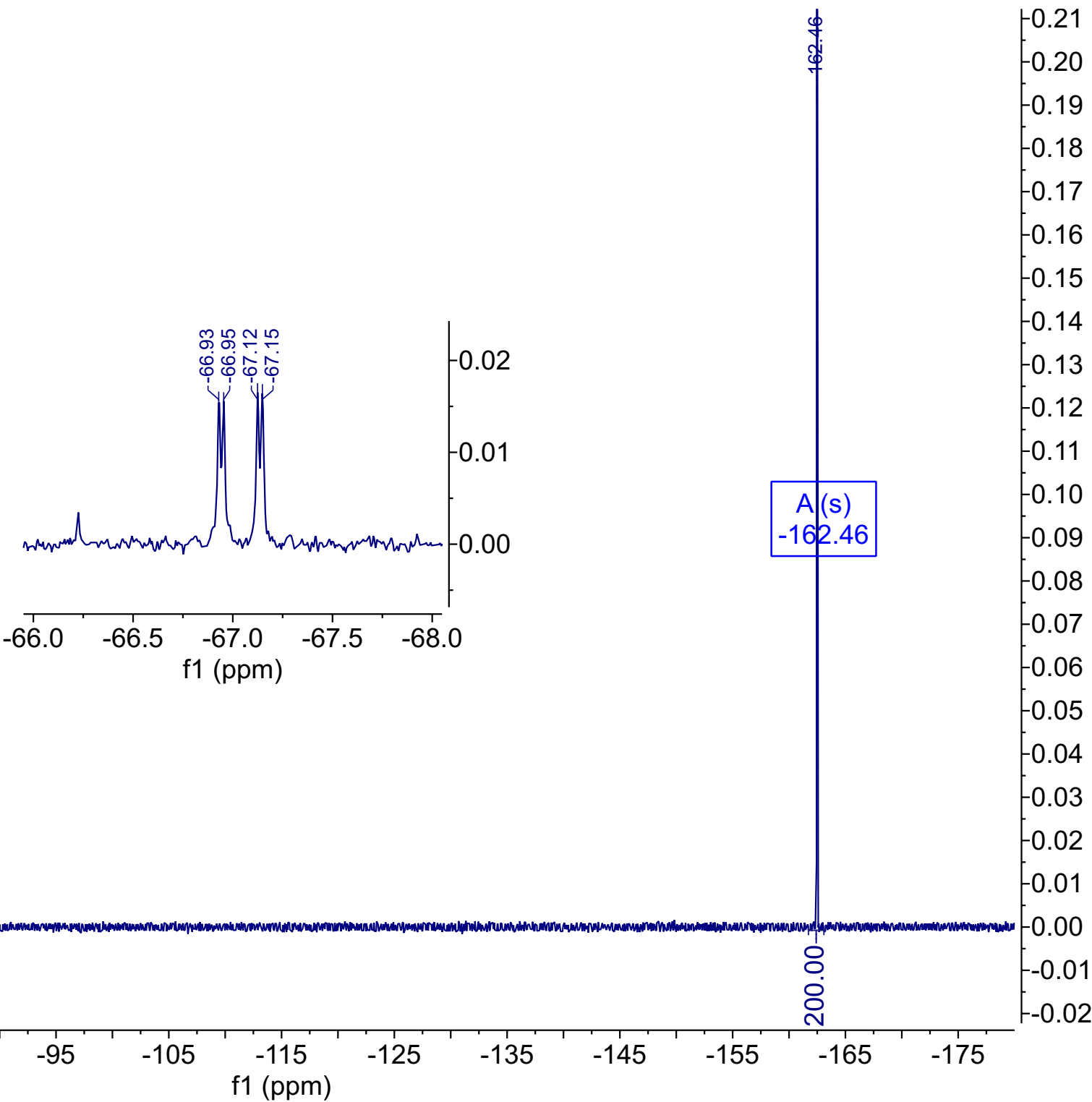

$\mathrm{Me}_{\mathrm{O}_{-}^{-1}}^{1}$

West, A. et al. Labeling preferences of diazirines with protein biomolecules, J. Am. Chem. Soc. 2021. 
Me

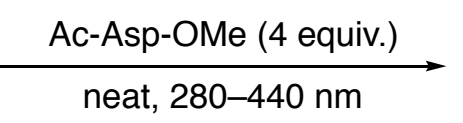

กุ

i
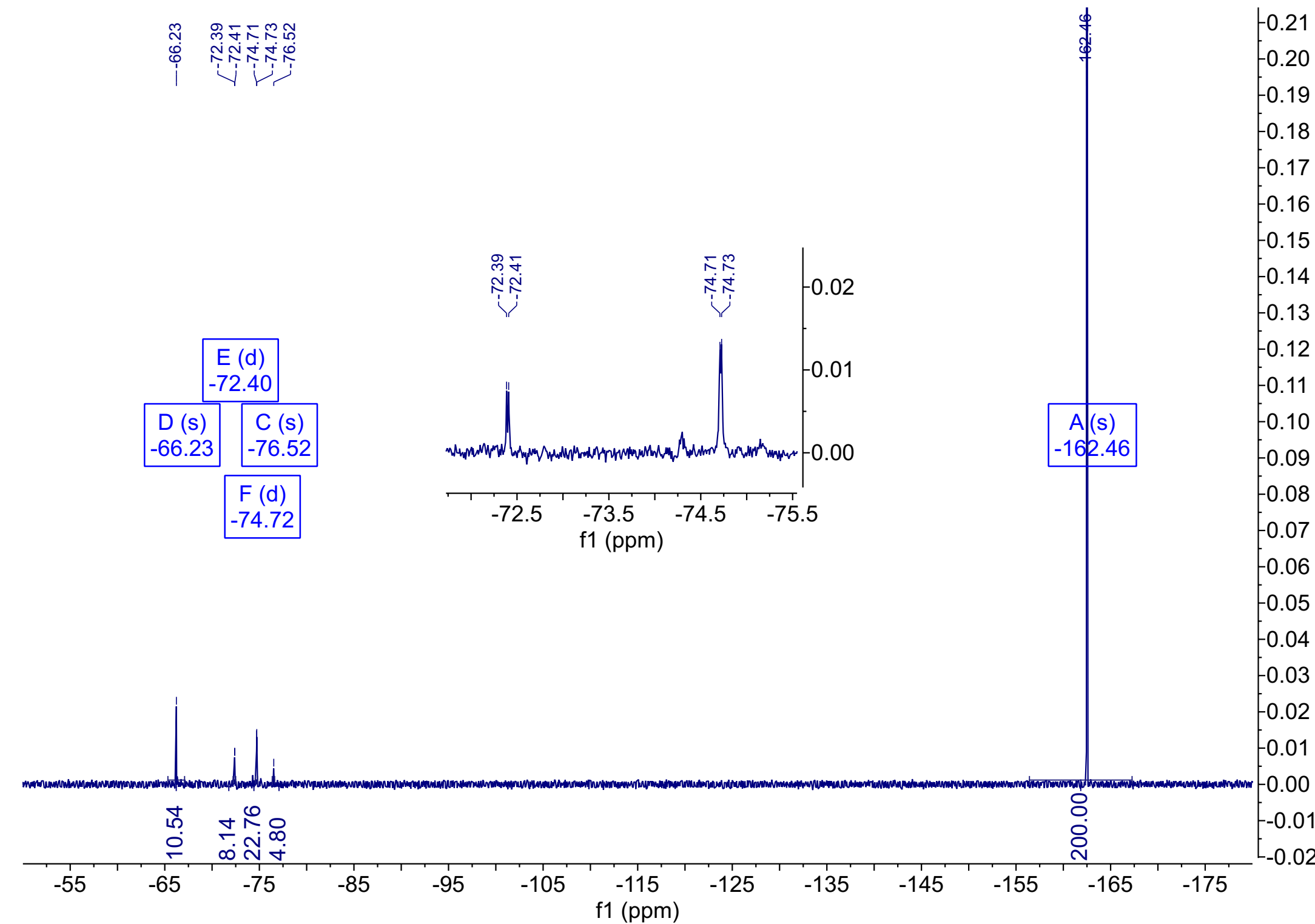

(1)

West, A. et al. Labeling preferences of diazirines with protein biomolecules, J. Am. Chem. Soc. 2021. 
${ }_{\mathrm{O}}^{\mathrm{C}}$<smiles>CC(C)(C)CCCCOC(C)(C)C</smiles><smiles>CON(C)C(=O)c1ccc(C(Cl)(Cl)C(F)(F)F)cc1</smiles>

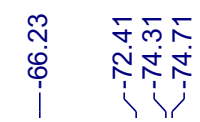
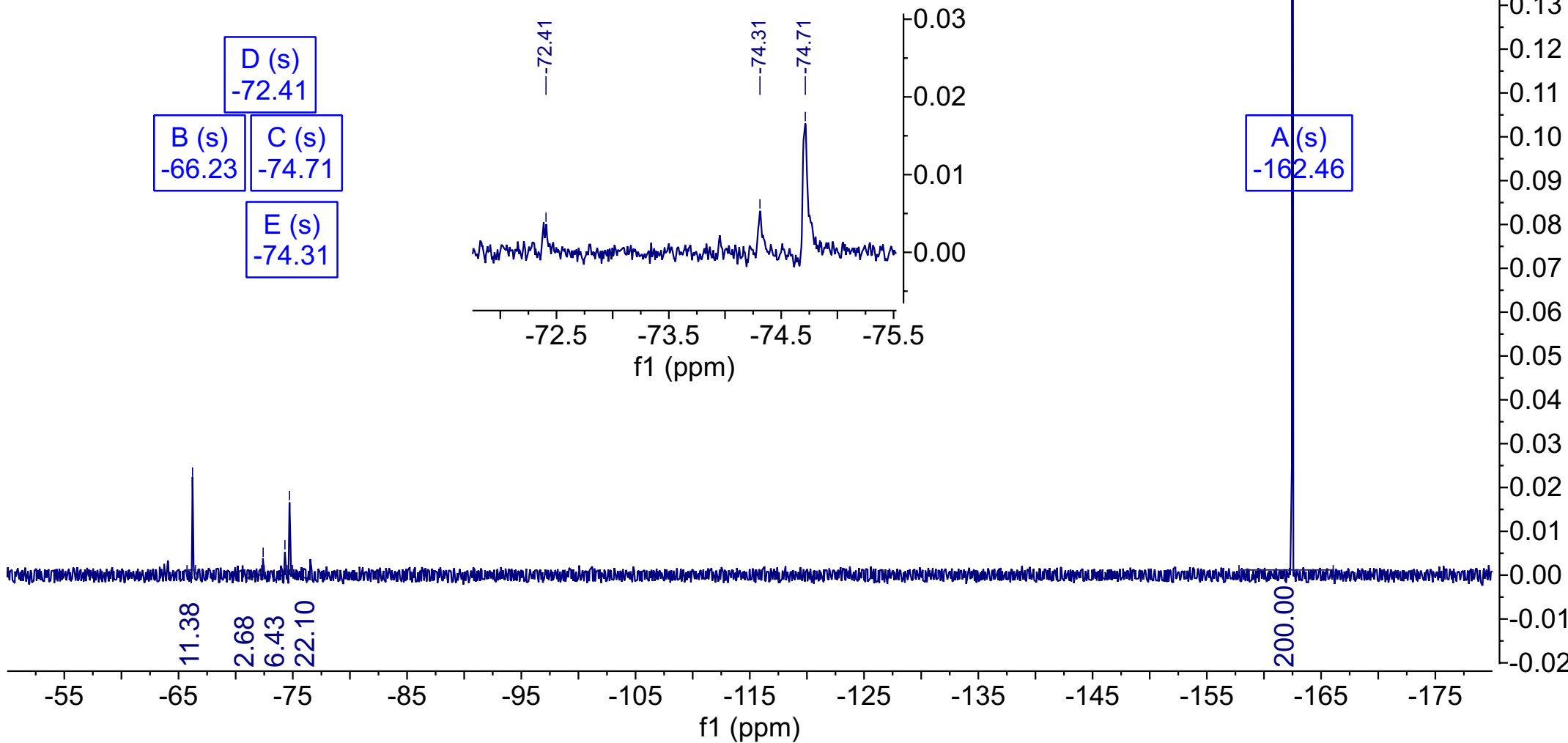

West, A. et al. Labeling preferences of diazirines with protein biomolecules, J. Am. Chem. Soc. 2021. 
$\mathrm{Me}_{\mathrm{O}_{-}^{-N}}^{1}$

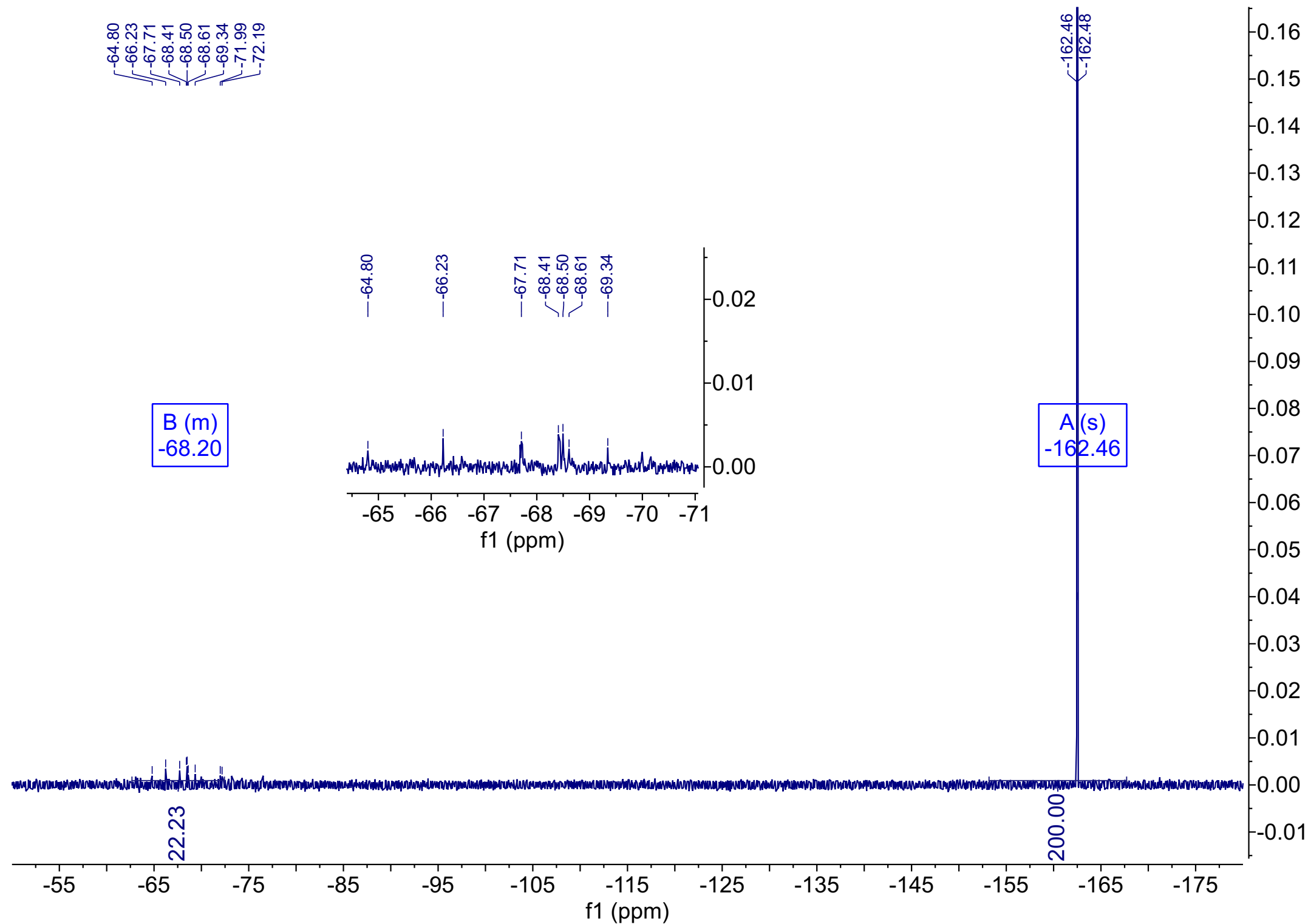


<smiles>CON(C)C(=O)c1ccc(C2(C(F)(F)F)N=N2)cc1</smiles><smiles>CC(C)(C)OCCOC(=O)OCc1ccccc1</smiles>

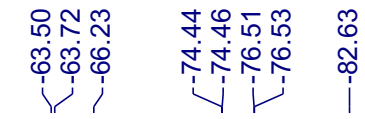

\begin{tabular}{|c|c|}
\hline $\begin{array}{r}D(s) \\
-66.23 \\
\end{array}$ & $\begin{array}{r}E(d) \\
-76.52 \\
\end{array}$ \\
\hline 3 (d) & $\mid \begin{array}{c}C(d) \\
-74.45\end{array}$ \\
\hline
\end{tabular}<smiles>CON(C)C(=O)c1ccc(C(Cl)(Cl)C(F)(F)F)cc1</smiles>

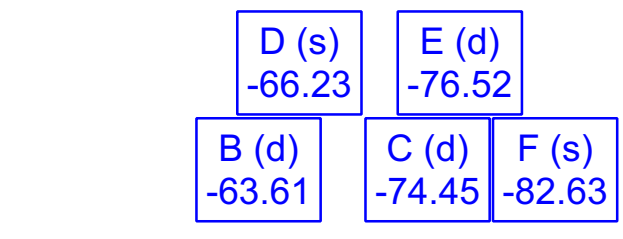

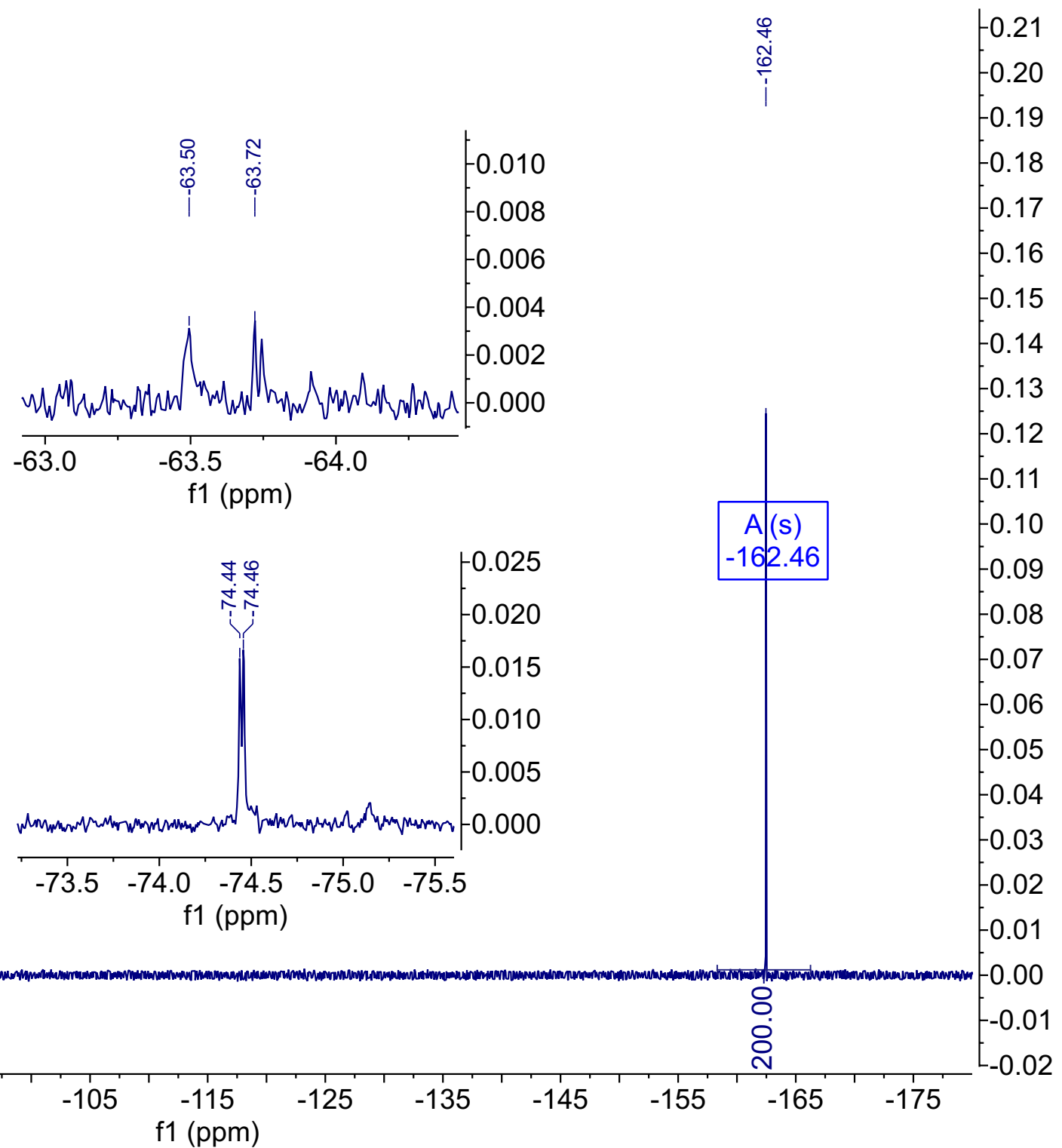

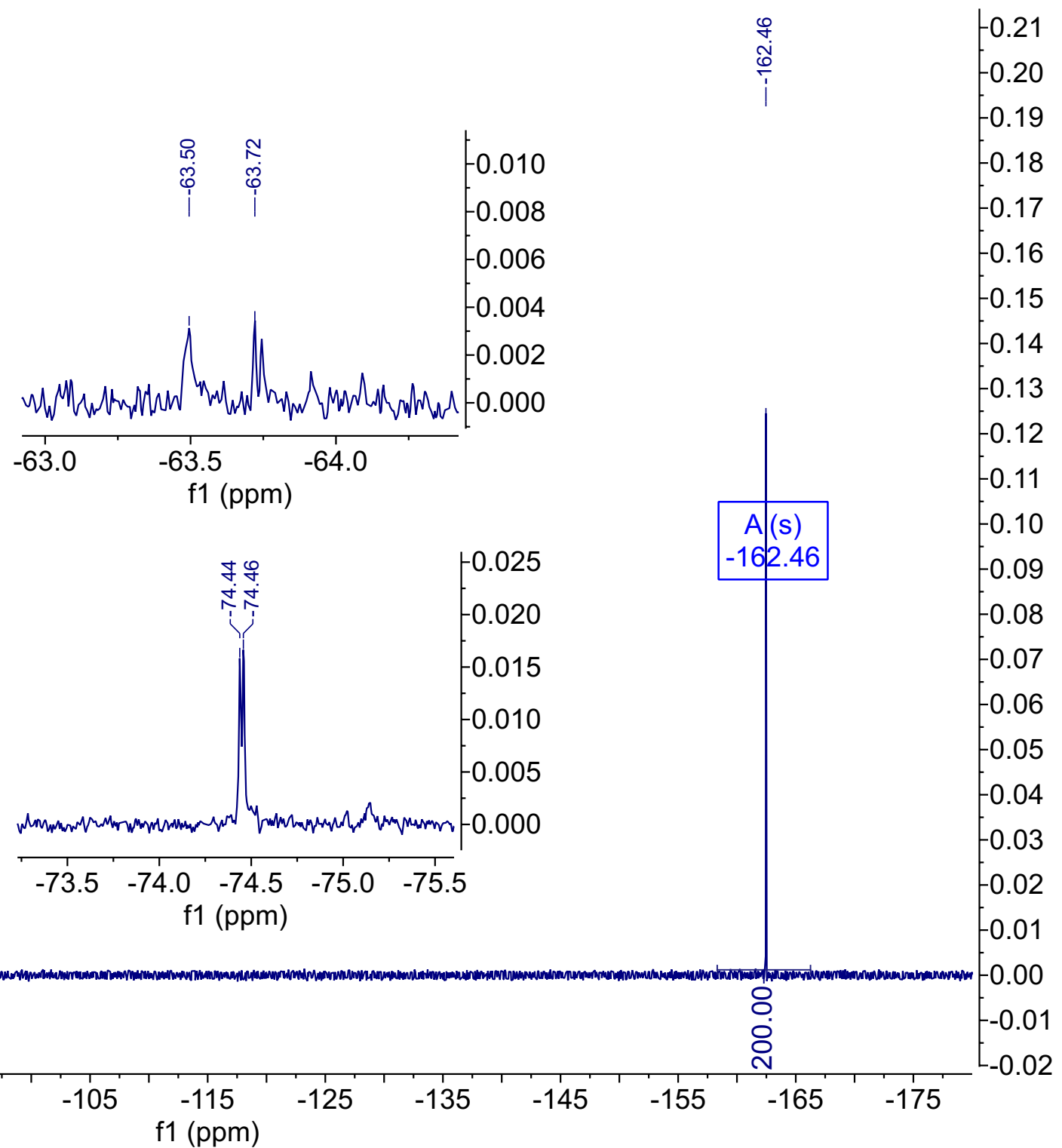

West, A. et al. Labeling preferences of diazirines with protein biomolecules, J. Am. Chem. Soc. 2021. 
Meror

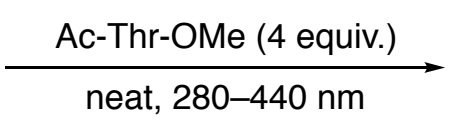

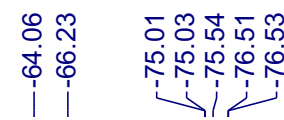

\begin{tabular}{|c|c|}
\hline $\begin{array}{c}C(s) \\
-66.23\end{array}$ & $\begin{array}{c}E(d) \\
-76.52\end{array}$ \\
\hline $\begin{array}{l}\text { (s) } \\
4.06\end{array}$ & \begin{tabular}{|c|}
$\mathrm{D}(\mathrm{m})$ \\
-75.20
\end{tabular} \\
\hline
\end{tabular}

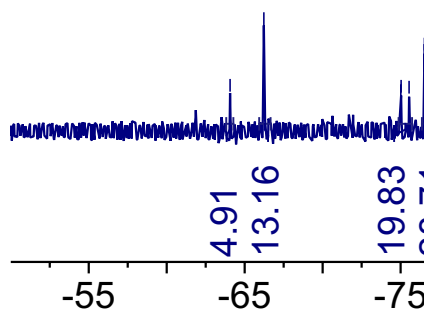

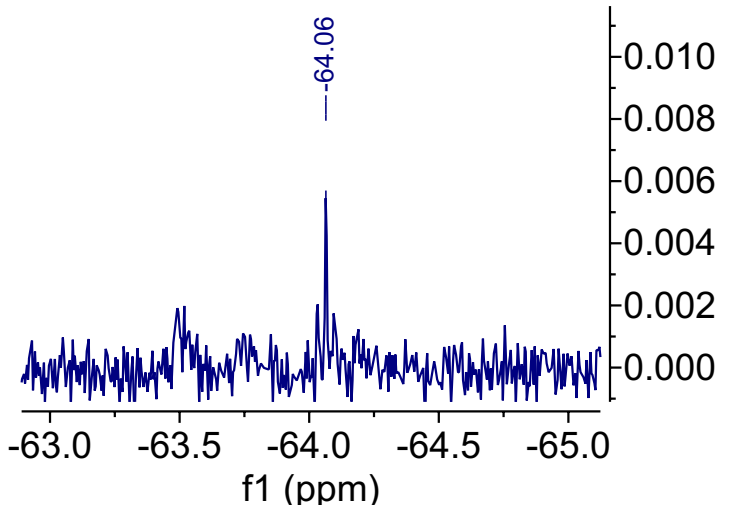

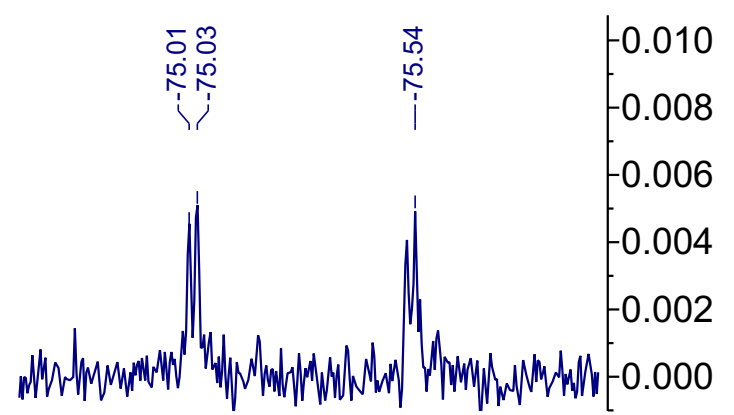

-74.8-75.0-75.2-75.4-75.6-75.8

f1 (ppm)
0.18

$-0.17$

$-0.16$

$-0.15$

$-0.14$

$-0.13$

$-0.12$

0.11

$-0.10$

0.09

$-0.08$

$-0.07$

$-0.06$

$-0.05$

0.04

$-0.03$

$-0.02$

$-0.01$

0.00

$-0.01$ 

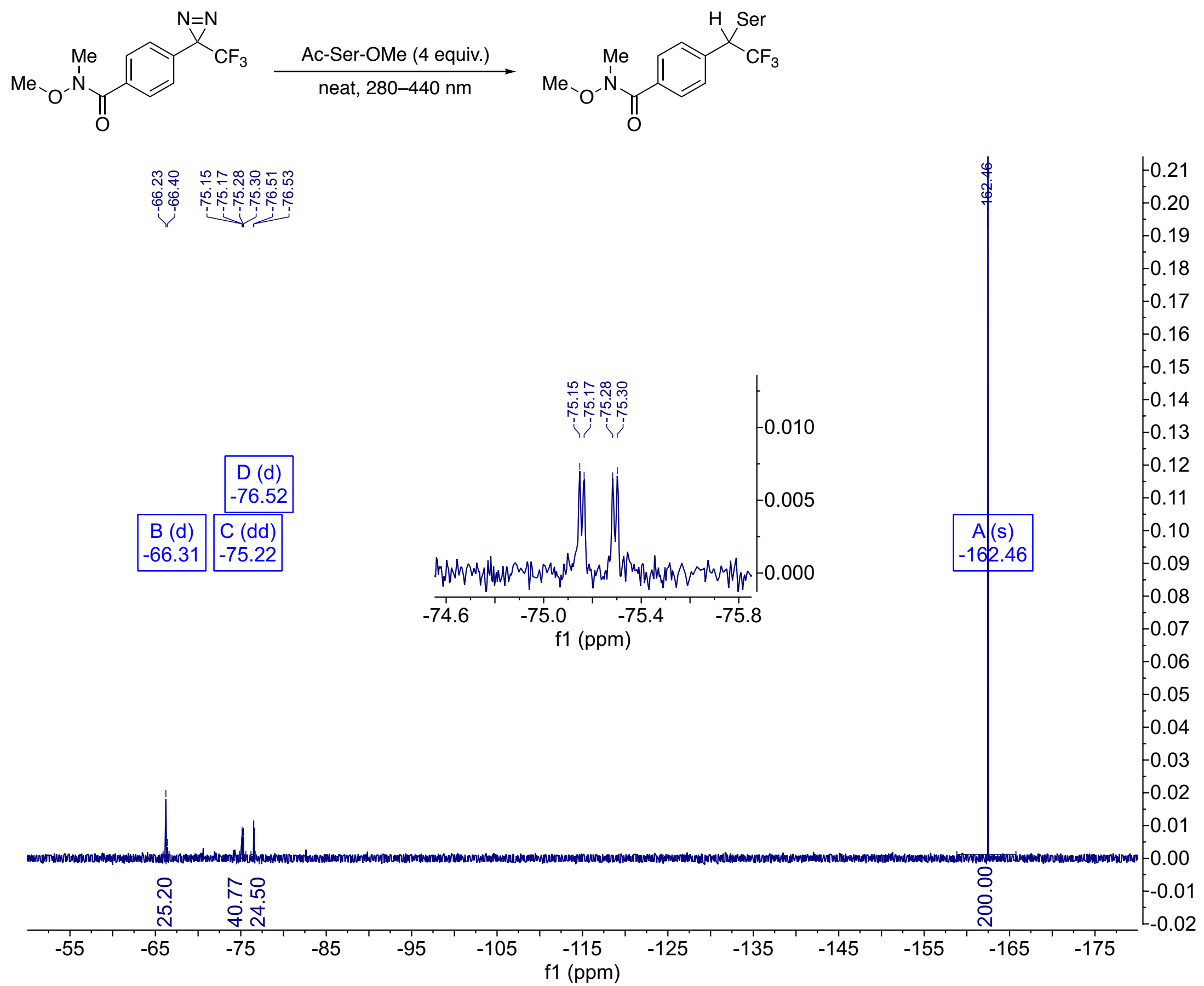

West, A. et al. Labeling preferences of diazirines with protein biomolecules, J. Am. Chem. Soc. 2021. 
neat, 280-440 nm

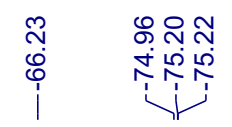

\begin{tabular}{c|c|}
\hline C (s) & B (m) \\
-66.23 & -75.12 \\
\hline
\end{tabular}
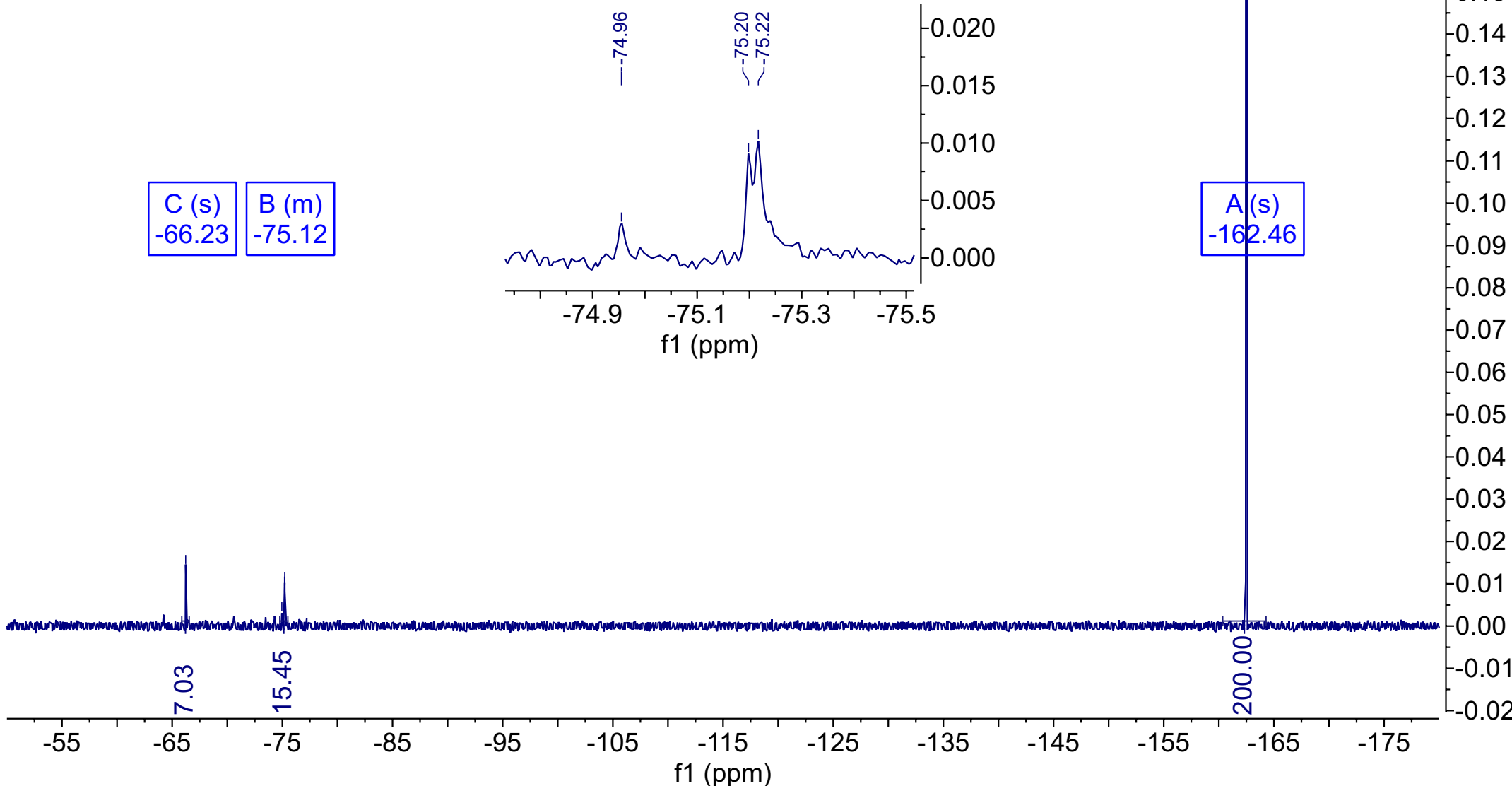

West, A. et al. Labeling preferences of diazirines with protein biomolecules, J. Am. Chem. Soc. 2021. 
<smiles>COC(=O)N(OC)C(=O)c1ccc(C2(C(F)(F)F)N=N2)cc1</smiles>

Ac-Pro-OMe (4 equiv.)

neat, $280-440 \mathrm{~nm}$<smiles>CON(C)C(=O)c1ccc(C(c2ccccc2)C(F)(F)F)cc1</smiles>

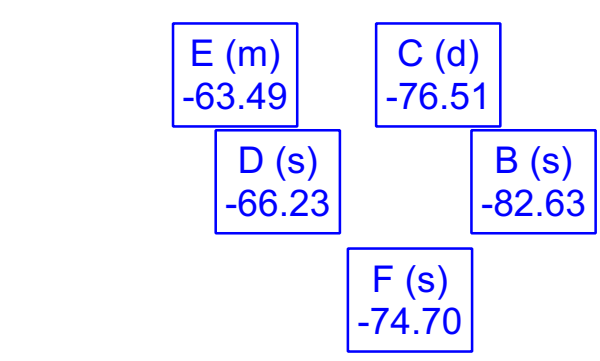

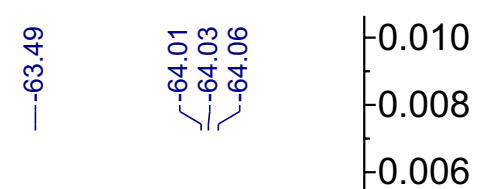$$
\begin{aligned}
-0.004 \\
-0.002
\end{aligned}
$$

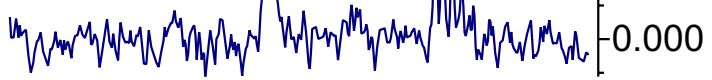$$
\begin{array}{lllll}
-62.8 & -63.2 & -63.6 & -64.0 & -64.4 \\
& & f 1(p p m) &
\end{array}
$$

$$
\text { i }
$$

0.010

0.008

$-0.006$ $-0.004$

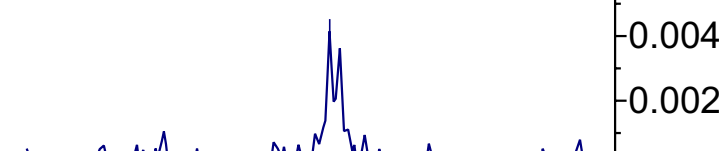

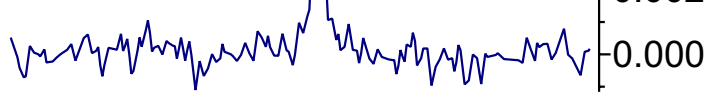$$
\begin{array}{llllllll} 
& -74.2 & -74.4 & -74.6 & -74.8 & -75.0 & -75.2
\end{array}
$$$$
\text { f1 (ppm) }
$$ 
<smiles>CON(C)C(=O)c1ccc(C2(C(F)(F)F)N=N2)cc1</smiles><smiles>CCCCCCCCOC(=O)OCc1ccccc1</smiles>

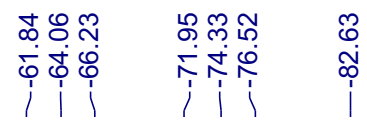

\begin{tabular}{|c|c|c|}
\hline \multicolumn{2}{|c|}{\begin{tabular}{c|c}
$D(s)$ & $F$ \\
-66.23 & -7 \\
\end{tabular}} & \\
\hline & $\begin{array}{l}\text { B (s) } \\
-71.95\end{array}$ & $\begin{array}{c}E(s) \\
-82.63\end{array}$ \\
\hline $\begin{array}{l}H(s) \\
-64.06\end{array}$ & & \\
\hline
\end{tabular}

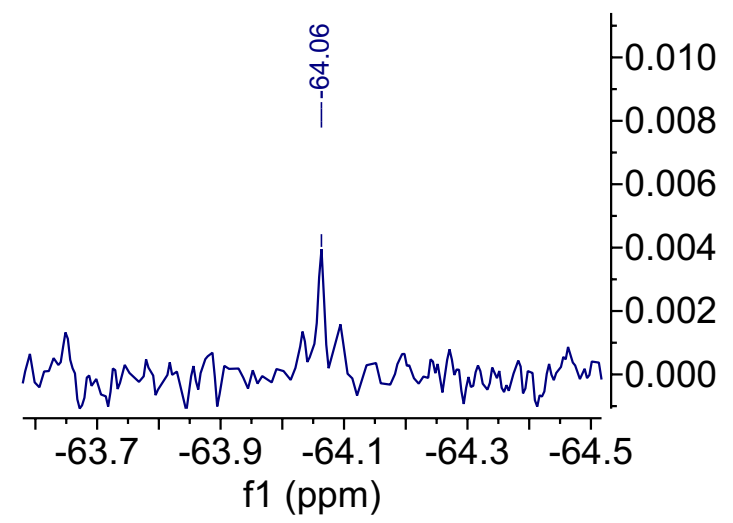<smiles>CN(O[Al])C(=O)c1ccc(C([AlH2])C(F)(F)F)cc1</smiles>

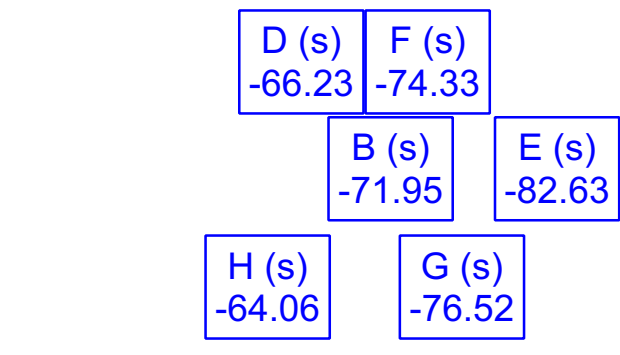

$$
\begin{array}{ccccc}
-63.7 & -63.9 & -64.1 & -64.3 & -64.5 \\
\text { f1 (ppm) } & &
\end{array}
$$

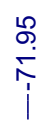

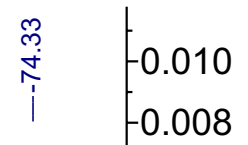

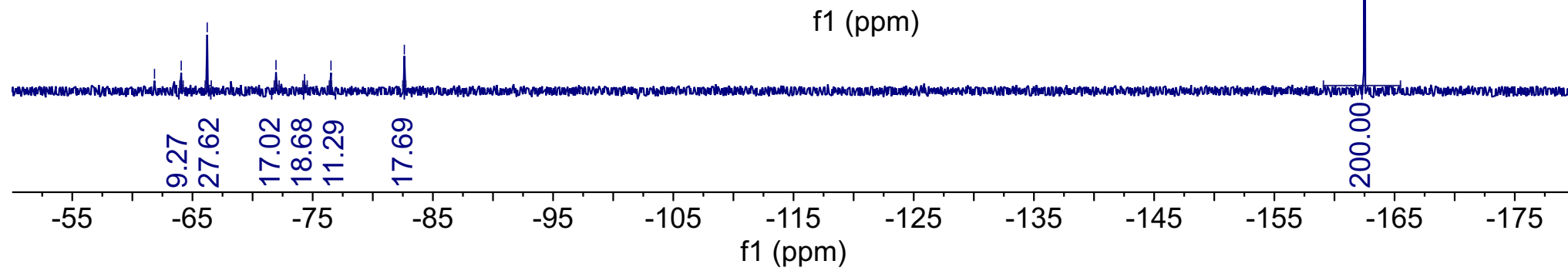


neat, 280-440 nm

î̉
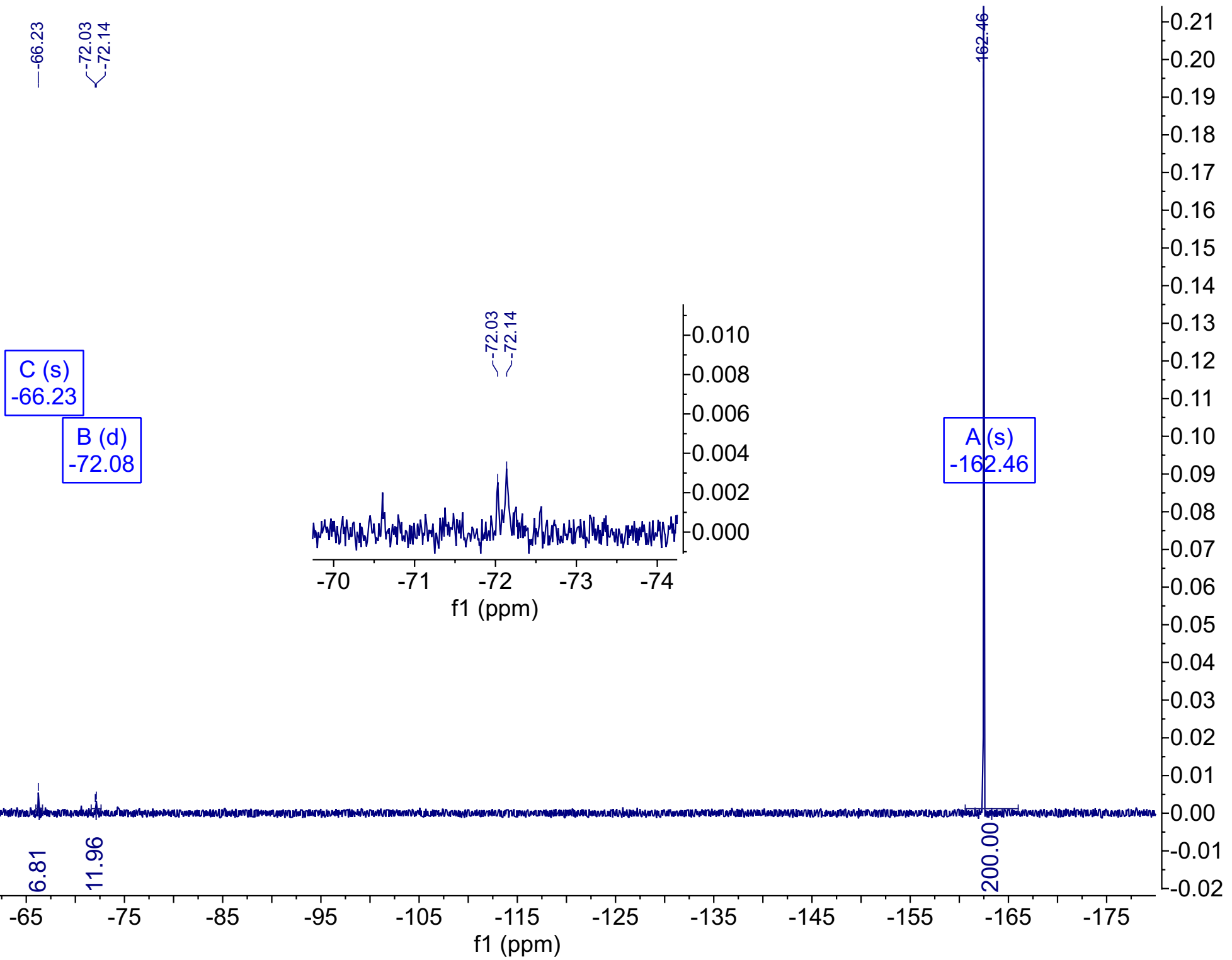

West, A. et al. Labeling preferences of diazirines with protein biomolecules, J. Am. Chem. Soc. 2021. 


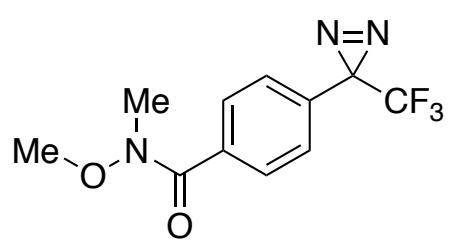

Ac-Val-OMe (4 equiv.)

m

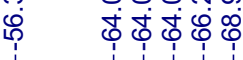
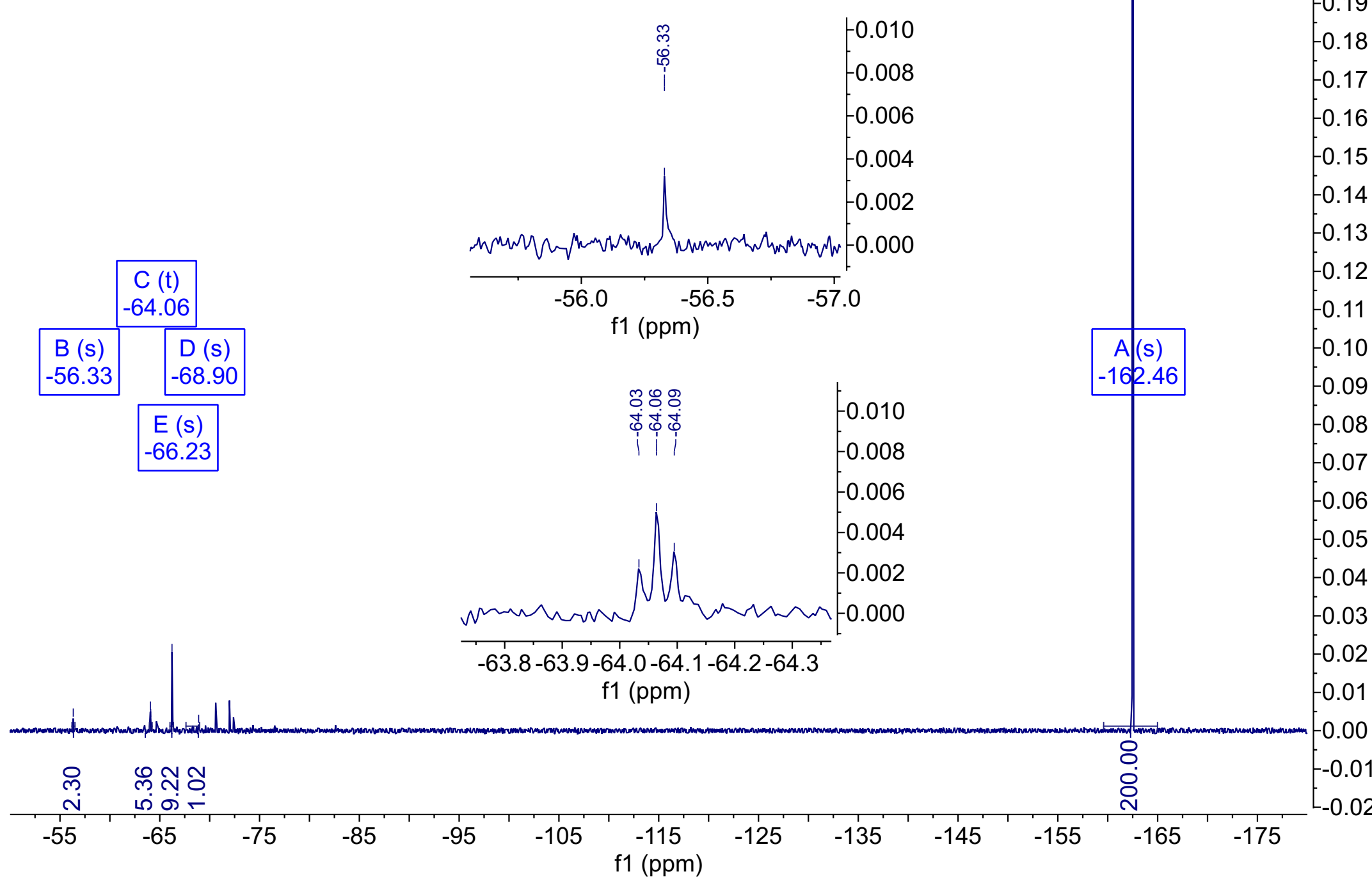

West, A. et al. Labeling preferences of diazirines with protein biomolecules, J. Am. Chem. Soc. 2021. \begin{tabular}{l}
-0.21 \\
-0.20 \\
-0.19 \\
-0.18 \\
-0.17 \\
-0.16 \\
-0.15 \\
-0.14 \\
-0.13 \\
-0.12 \\
-0.11 \\
-0.10 \\
-0.09 \\
-0.08 \\
-0.07 \\
-0.06 \\
-0.05 \\
\hline 0.04 \\
-0.03 \\
-0.02 \\
-0.01 \\
-0.00 \\
-0.01 \\
-0.02 \\
\hline
\end{tabular} 02 
neat, 280-440 nm

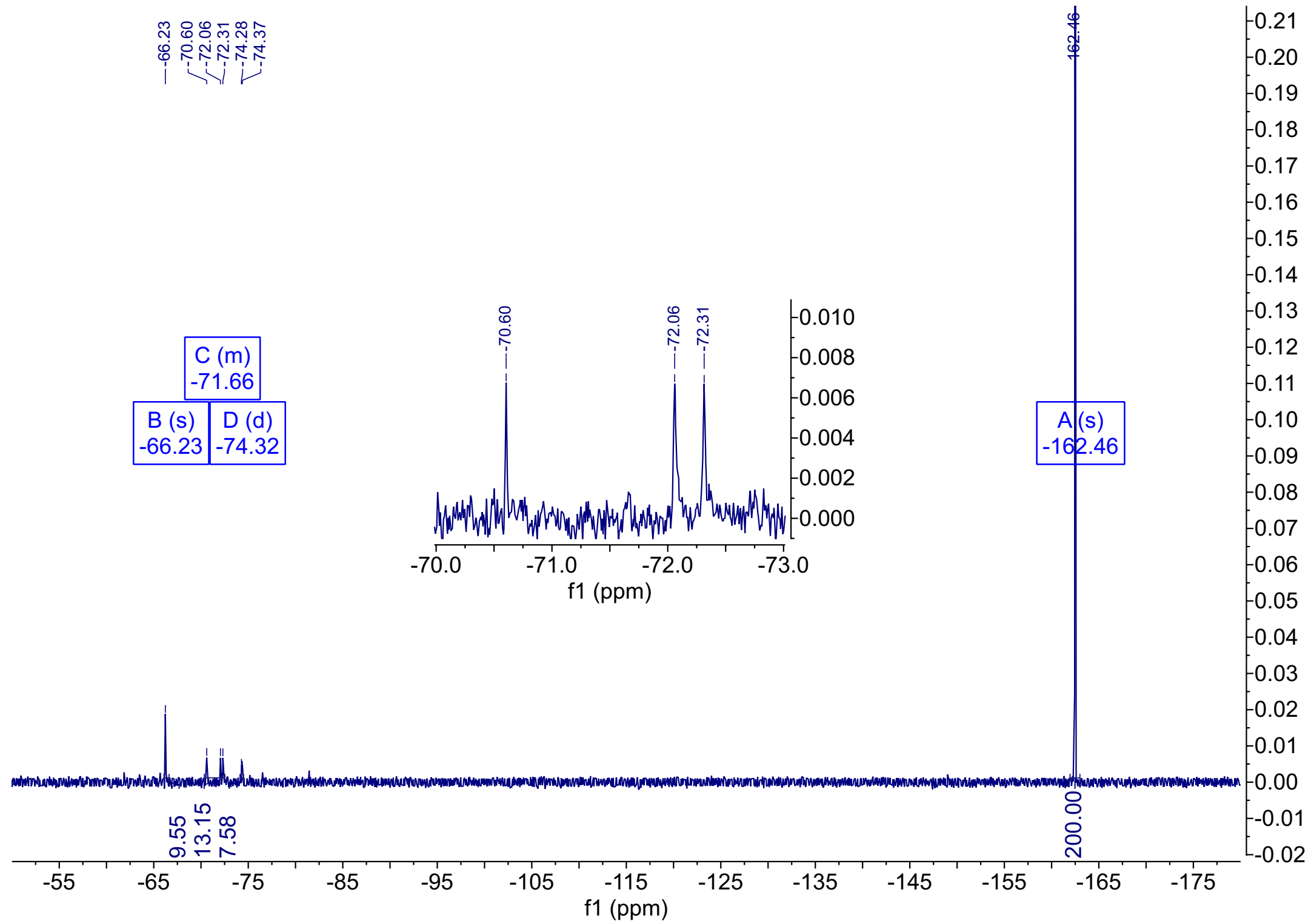

West, A. et al. Labeling preferences of diazirines with protein biomolecules, J. Am. Chem. Soc. 2021. 


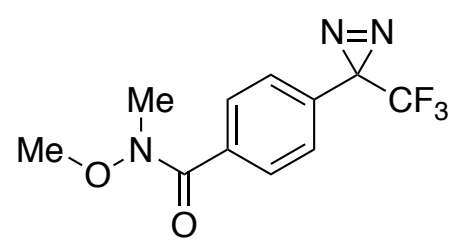

$\underset{\text { neat, } 280-440 \mathrm{~nm}}{\stackrel{\text { Ac-Leu-OMe (4 equiv.) }}{\longrightarrow}}$

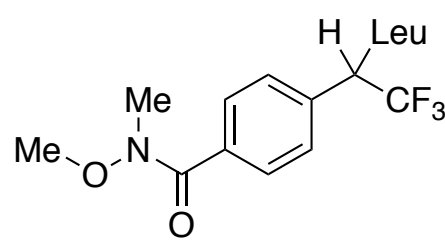

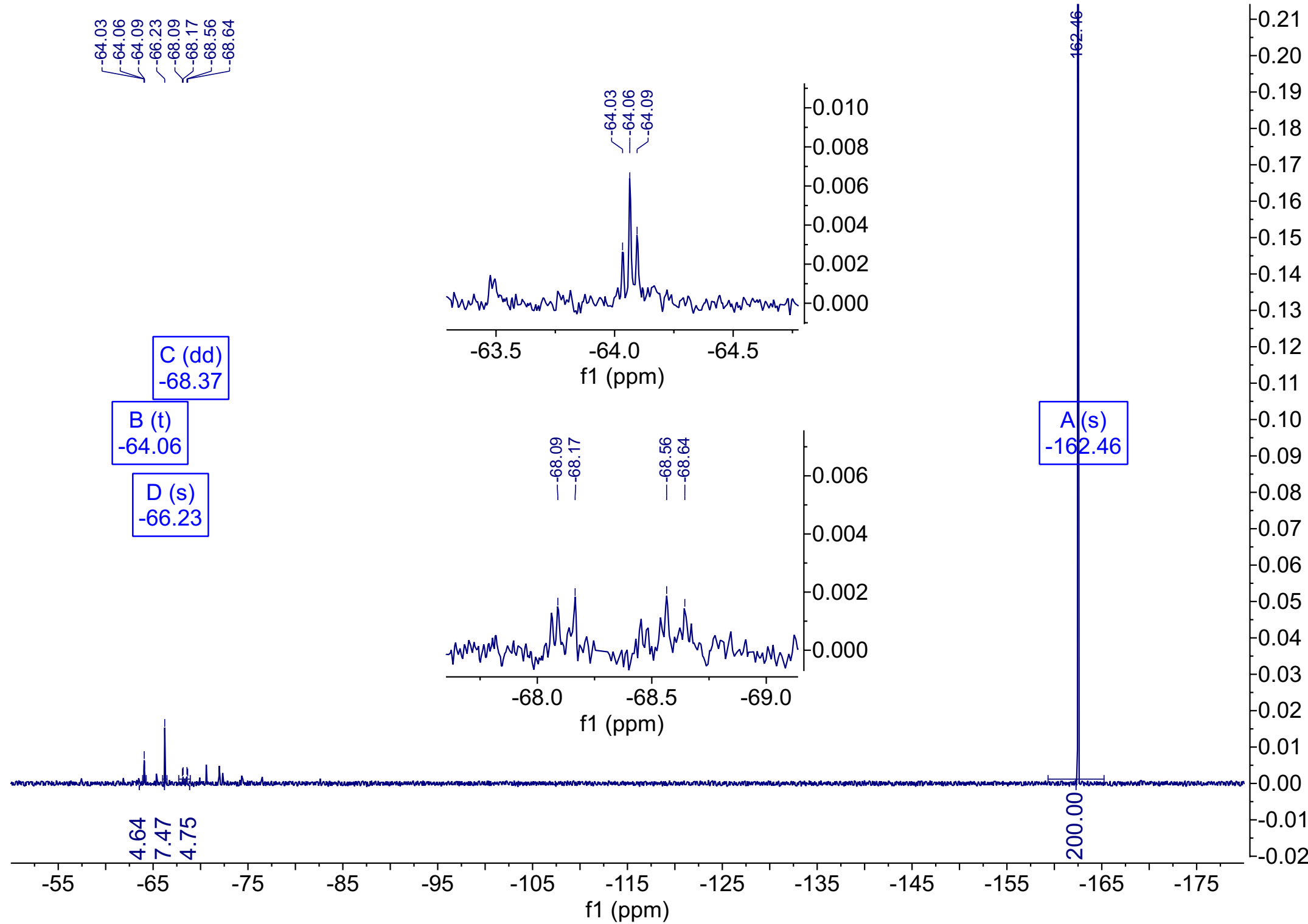

West, A. et al. Labeling preferences of diazirines with protein biomolecules, J. Am. Chem. Soc. 2021. 
<smiles>CON(C)C(=O)c1ccc(C2(C(F)(F)F)N=N2)cc1</smiles>

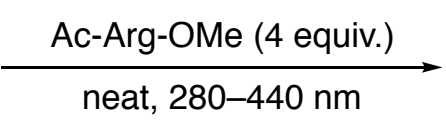<smiles>CON(C)C(=O)c1ccc(C([AlH2])([AlH2])C(F)(F)F)cc1</smiles>
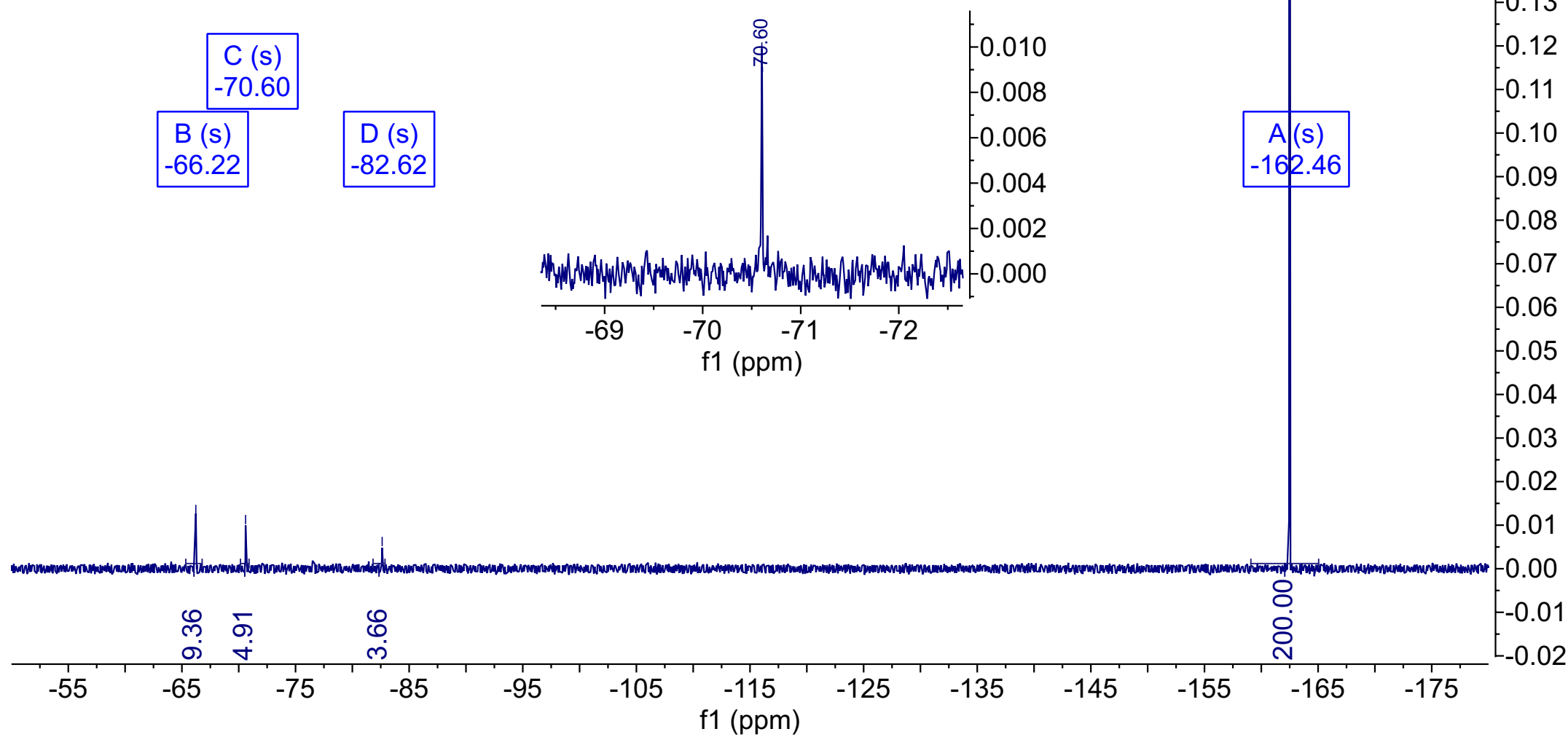

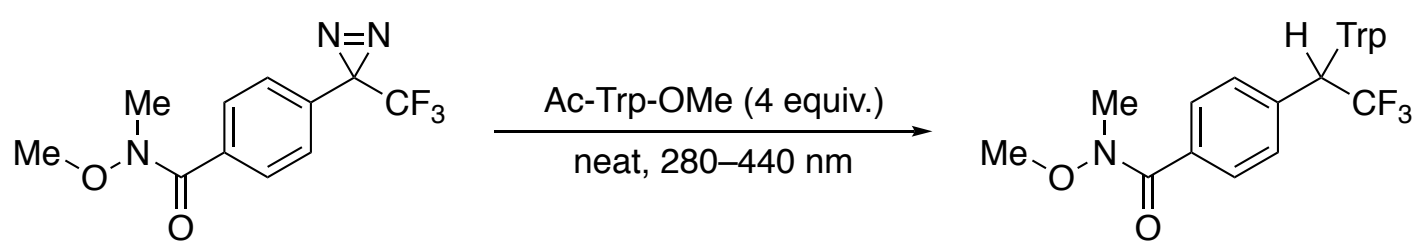

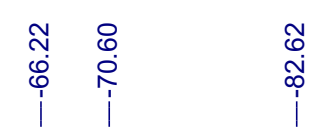
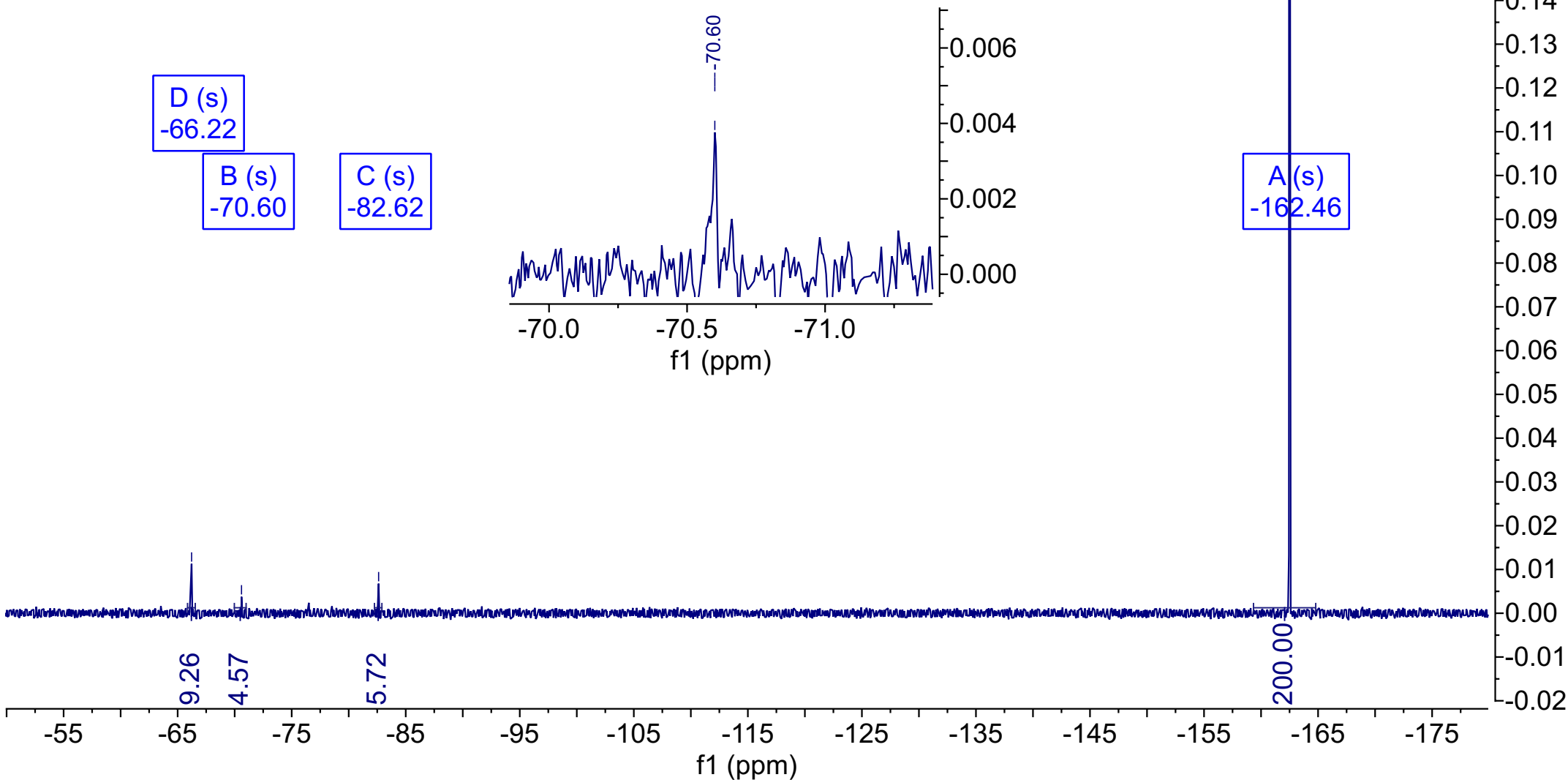

West, A. et al. Labeling preferences of diazirines with protein biomolecules, J. Am. Chem. Soc. 2021. 
neat, 280-440 nm

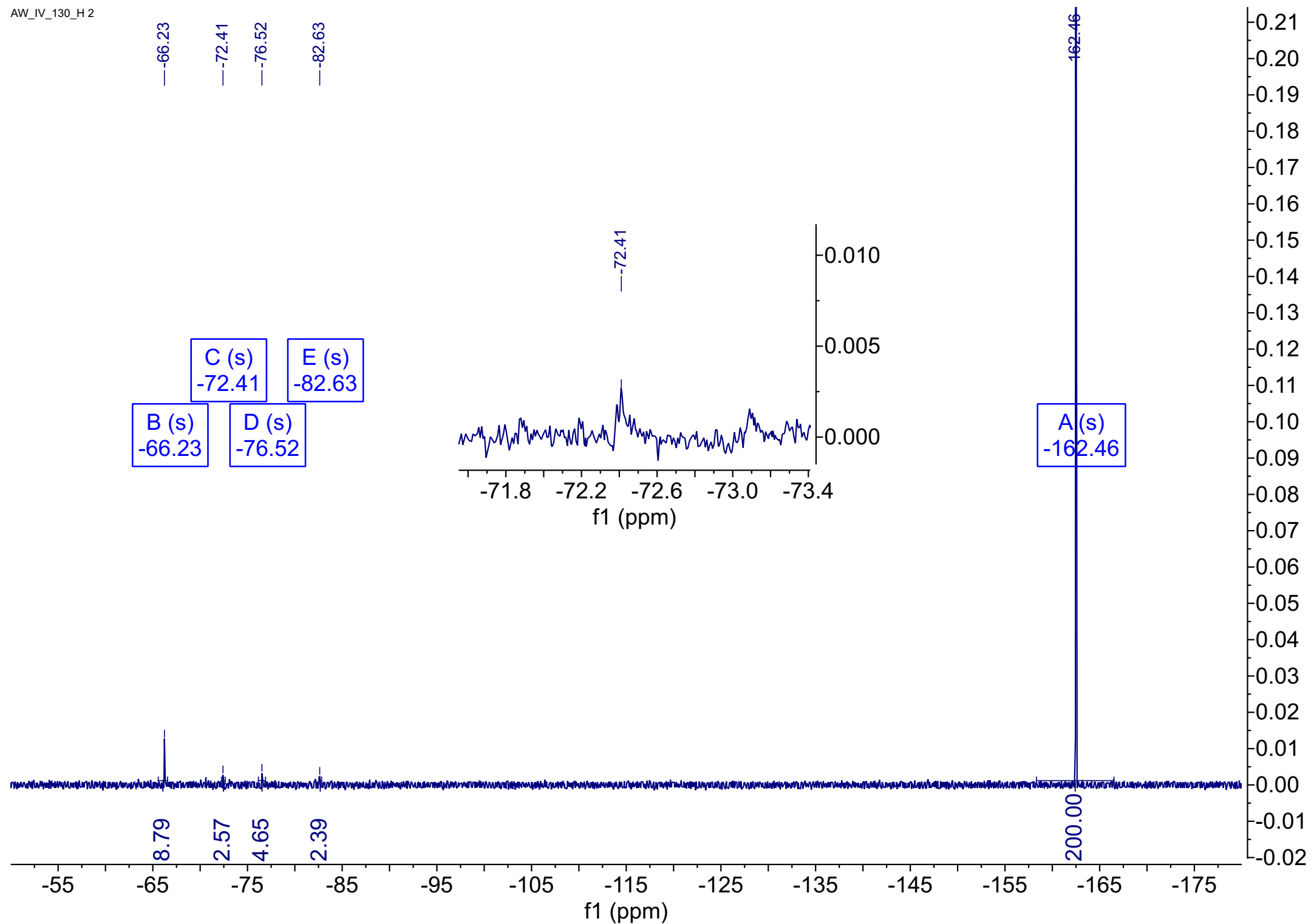


neat, 280-440 nm
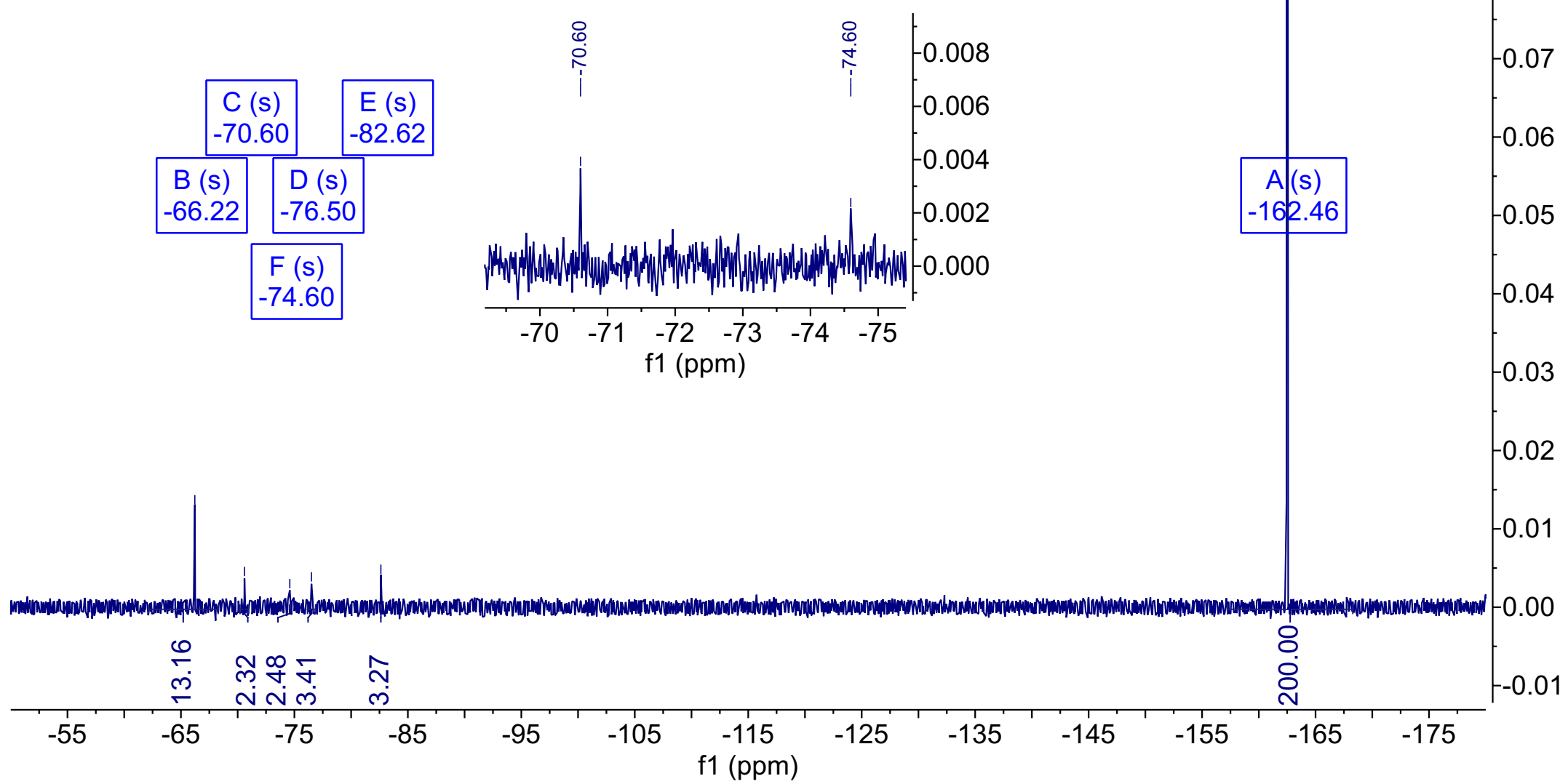
<smiles>CON(C)C(=O)c1ccc(C2(C(F)(F)F)N=N2)cc1</smiles>

Ac-Asn-OMe (4 equiv.)

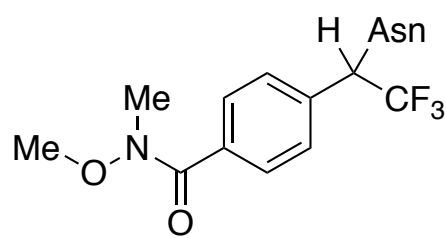

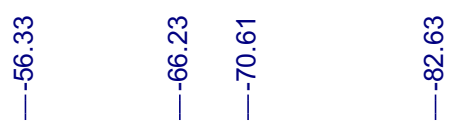
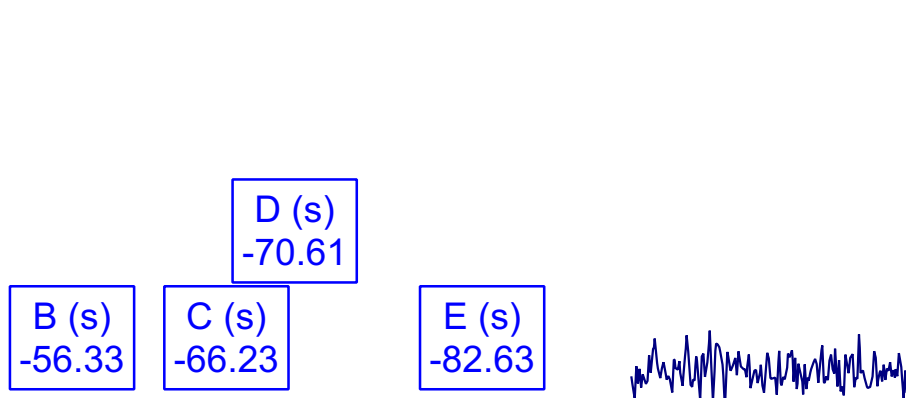

i্

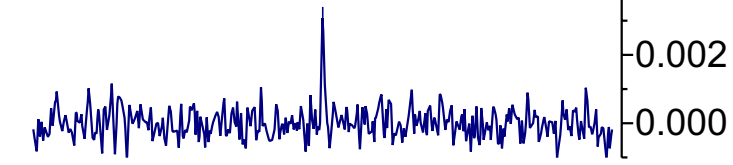

-0.010
-0.008

$-0.006$

$-0.004$

$-0.002$

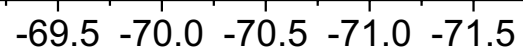

f1 (ppm)

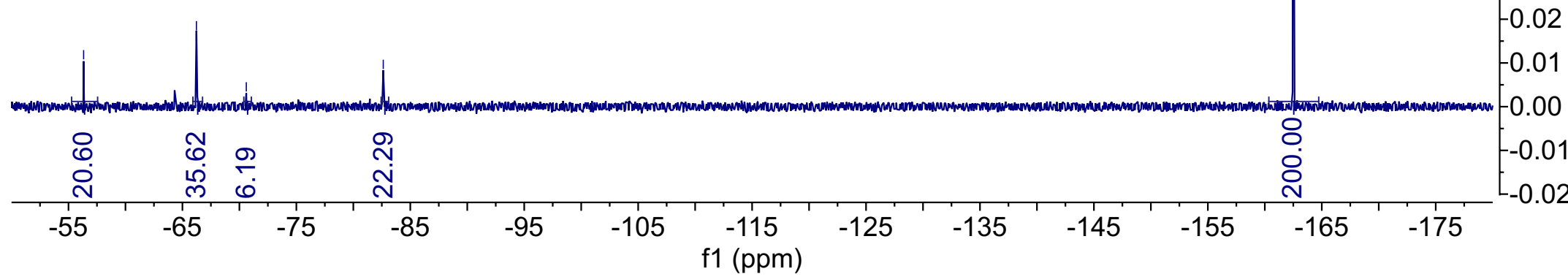

West, A. et al. Labeling preferences of diazirines with protein biomolecules, J. Am. Chem. Soc. 2021. 
<smiles>CON(C)C(=O)c1ccc(C(F)(F)F)cc1</smiles>

$\underset{\text { neat, } 280-440 \mathrm{~nm}}{\text { Ac-Lys-OMe (4 equiv.) }}$

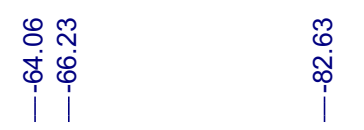

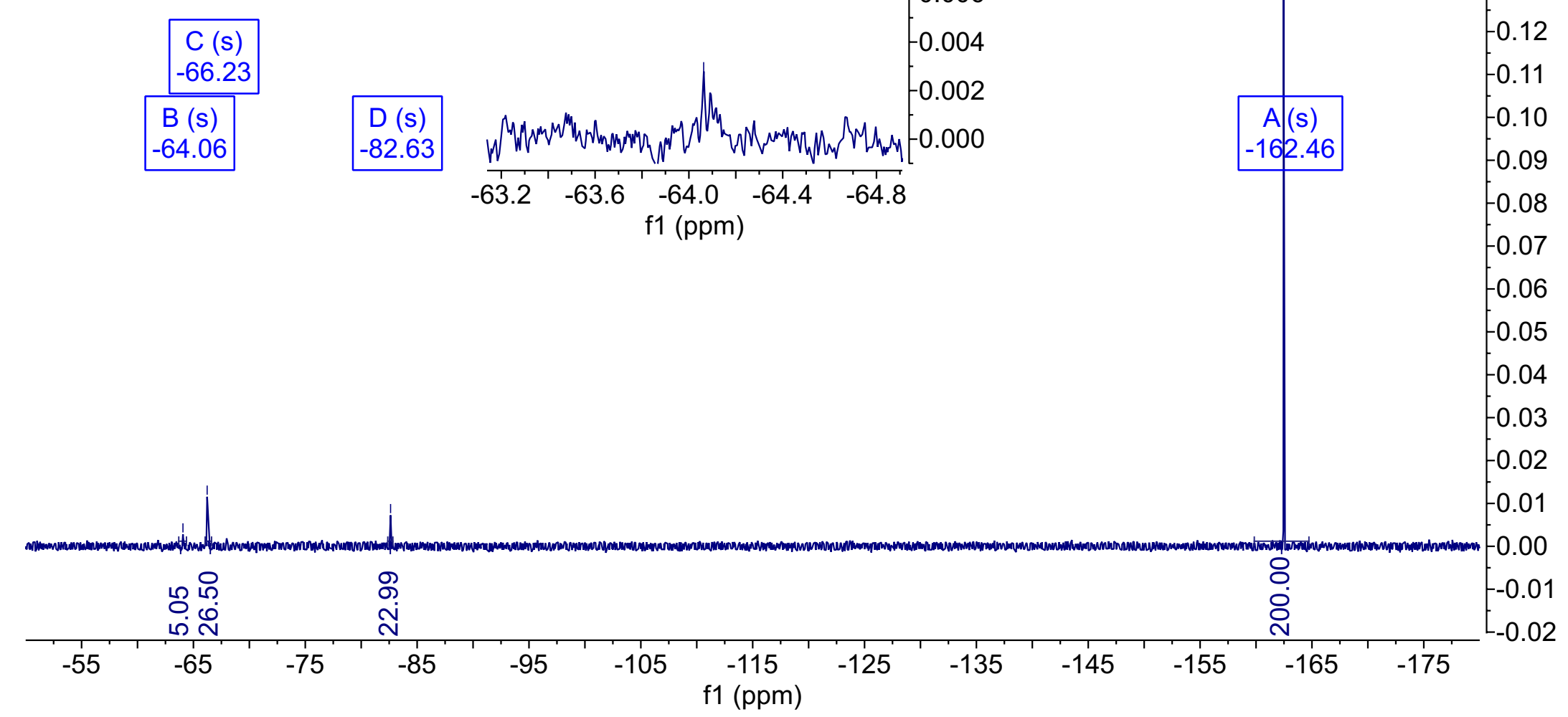

West, A. et al. Labeling preferences of diazirines with protein biomolecules, J. Am. Chem. Soc. 2021. 

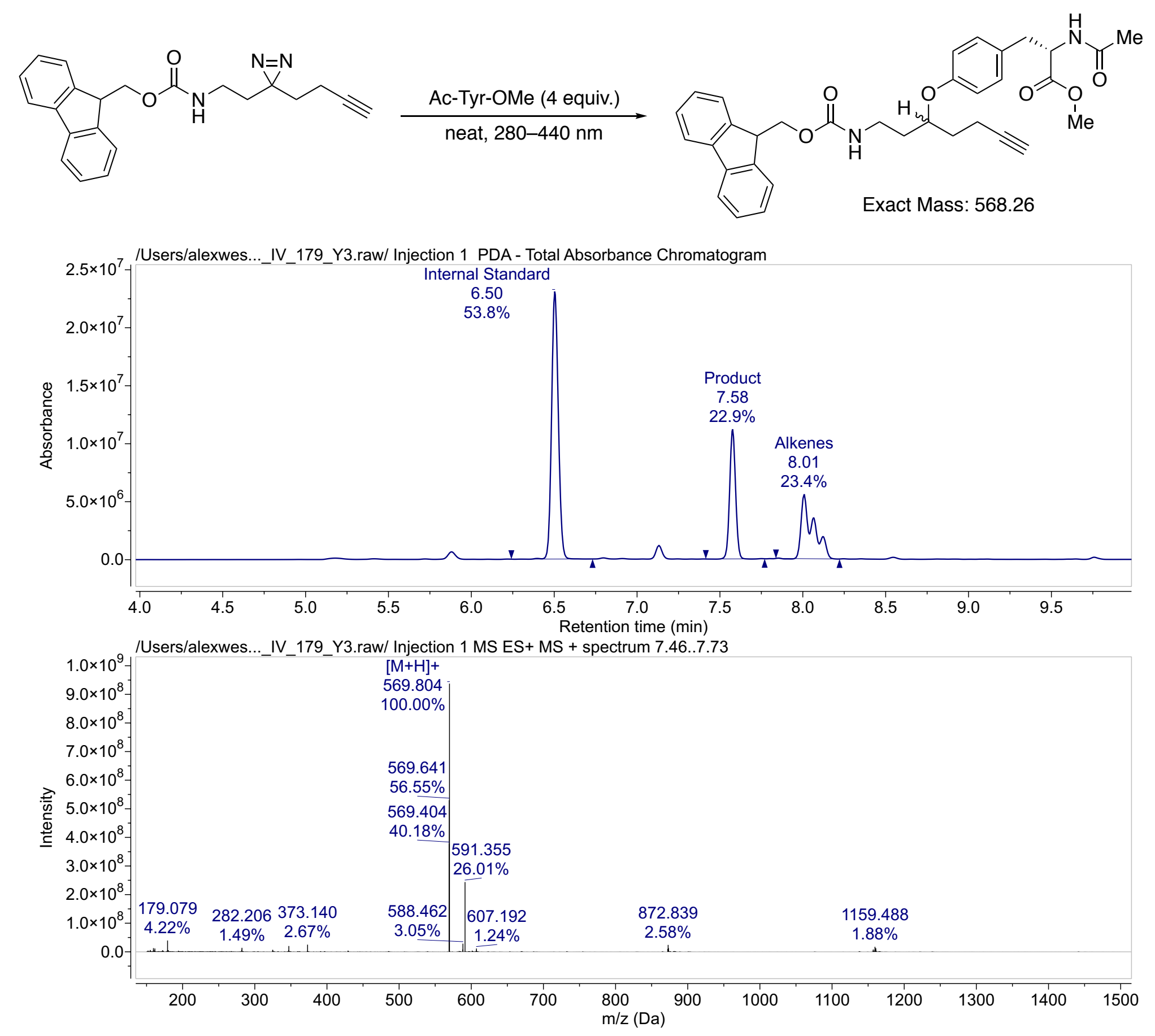

West, A. et al. Labeling preferences of diazirines with protein biomolecules, J. Am. Chem. Soc. 2021. 

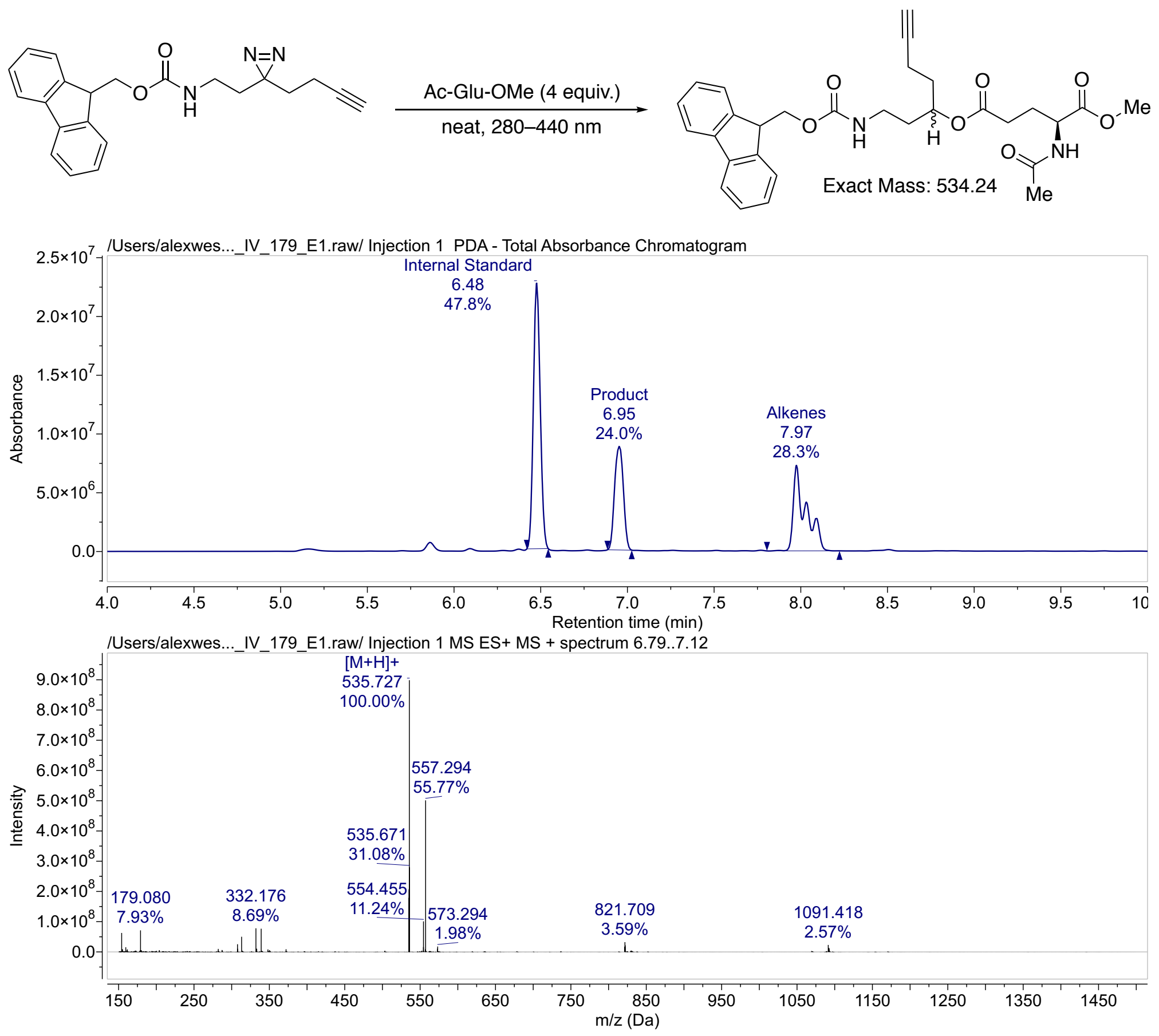

West, A. et al. Labeling preferences of diazirines with protein biomolecules, J. Am. Chem. Soc. 2021. 

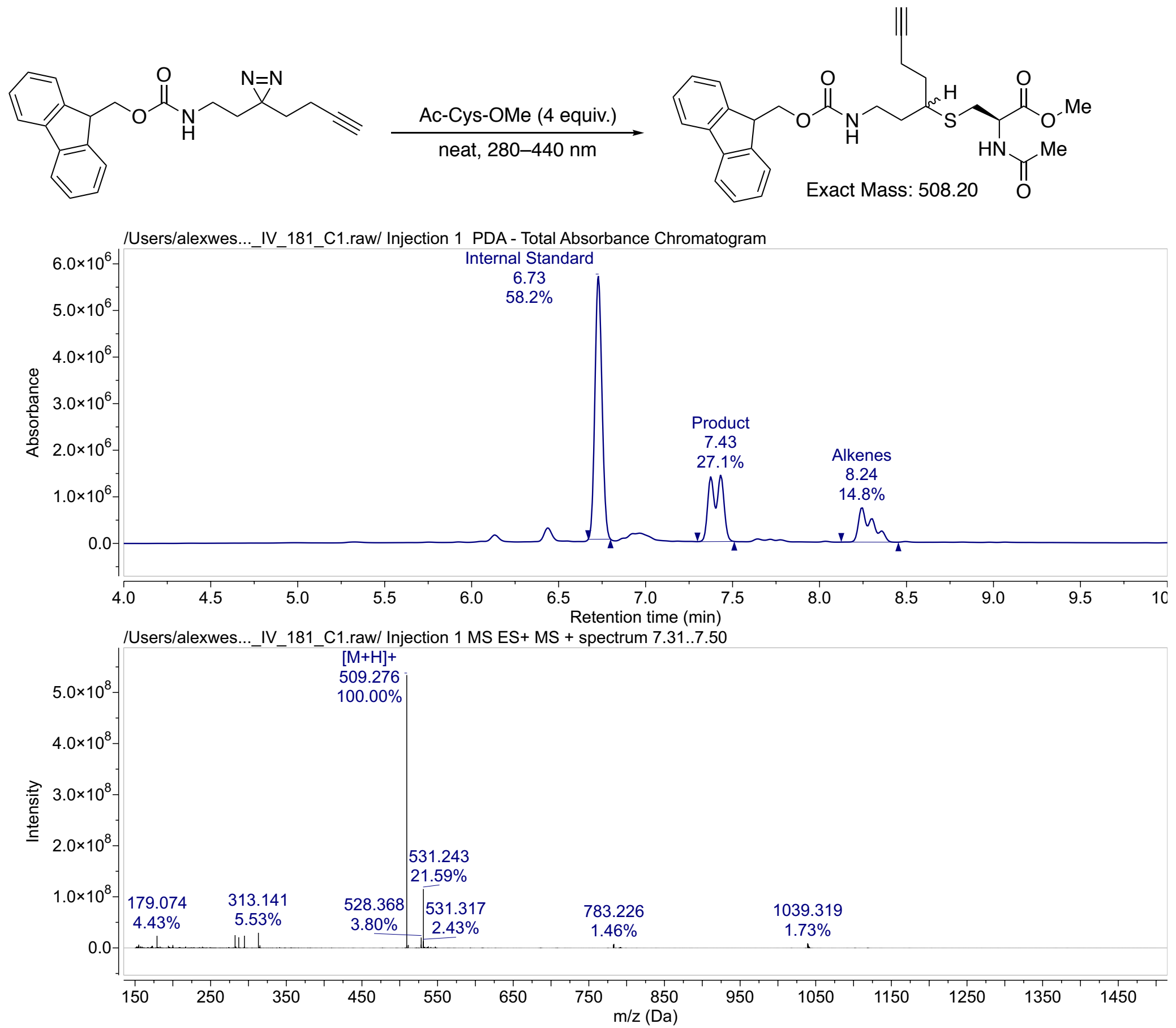

West, A. et al. Labeling preferences of diazirines with protein biomolecules, J. Am. Chem. Soc. 2021. 


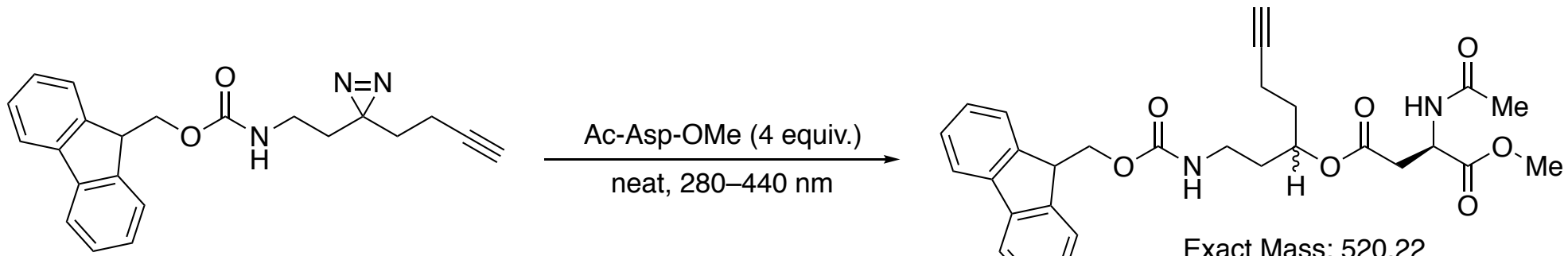

Exact Mass: 520.22
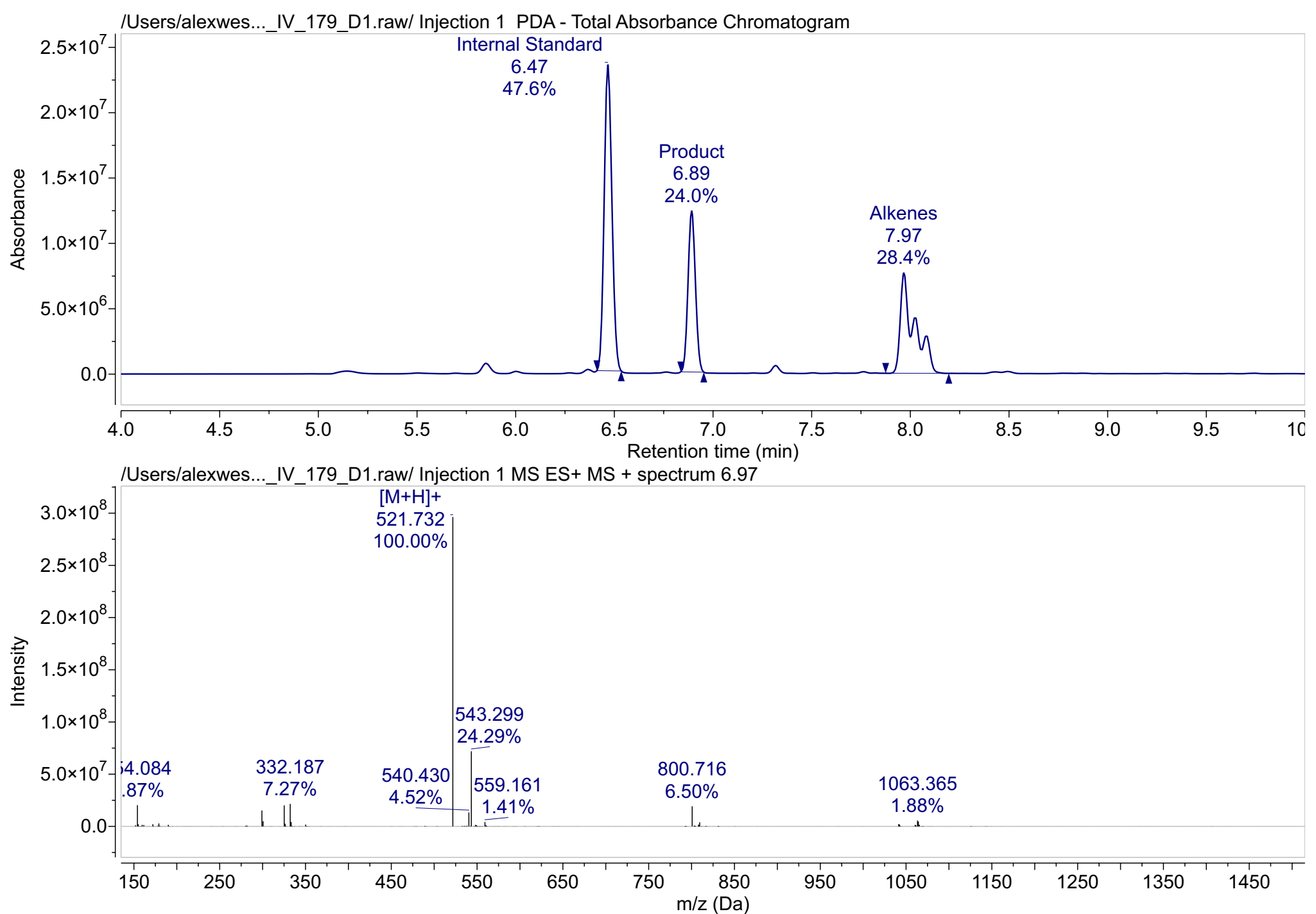

West, A. et al. Labeling preferences of diazirines with protein biomolecules, J. Am. Chem. Soc. 2021. 

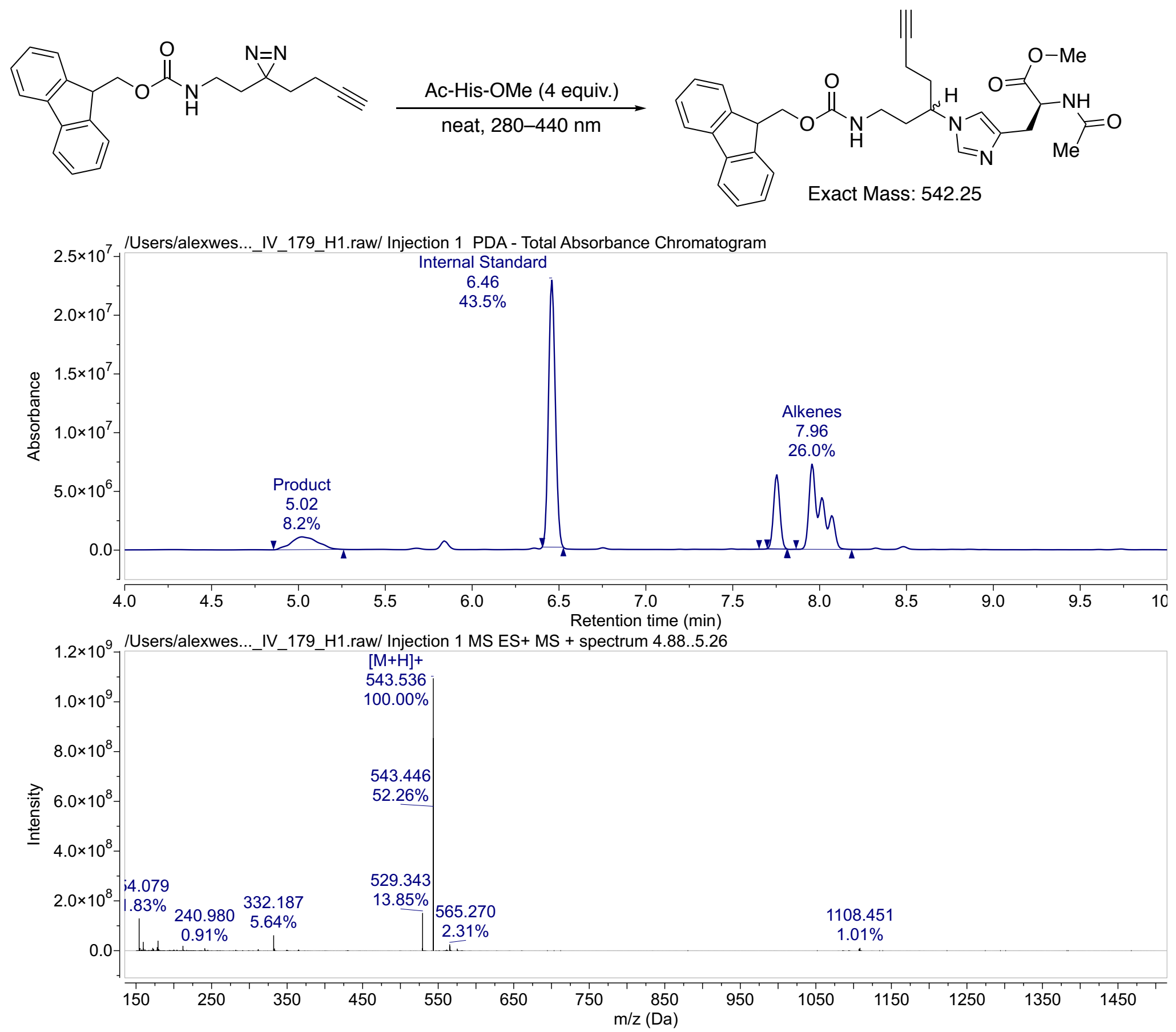

West, A. et al. Labeling preferences of diazirines with protein biomolecules, J. Am. Chem. Soc. 2021. 

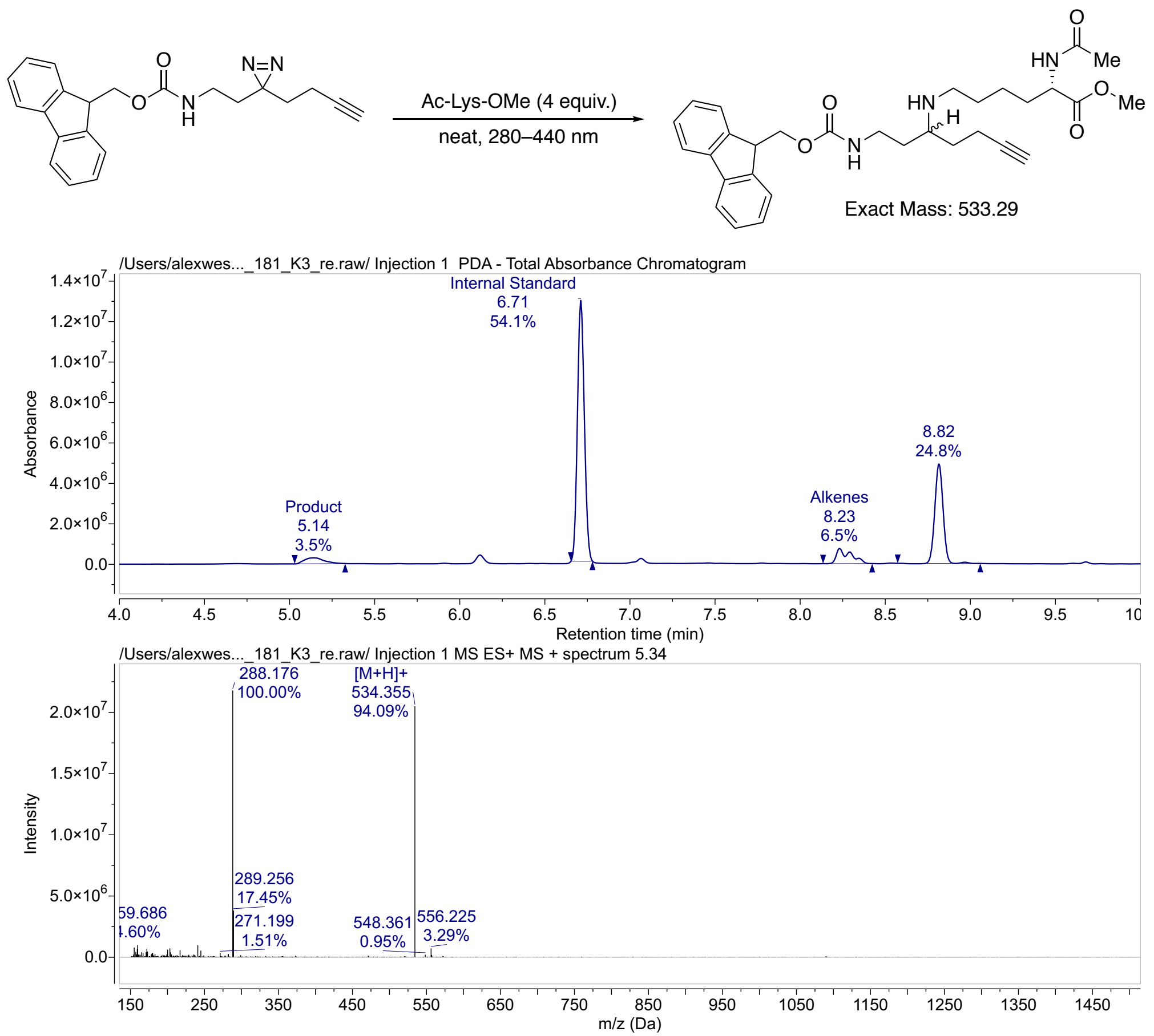

West, A. et al. Labeling preferences of diazirines with protein biomolecules, J. Am. Chem. Soc. 2021. 

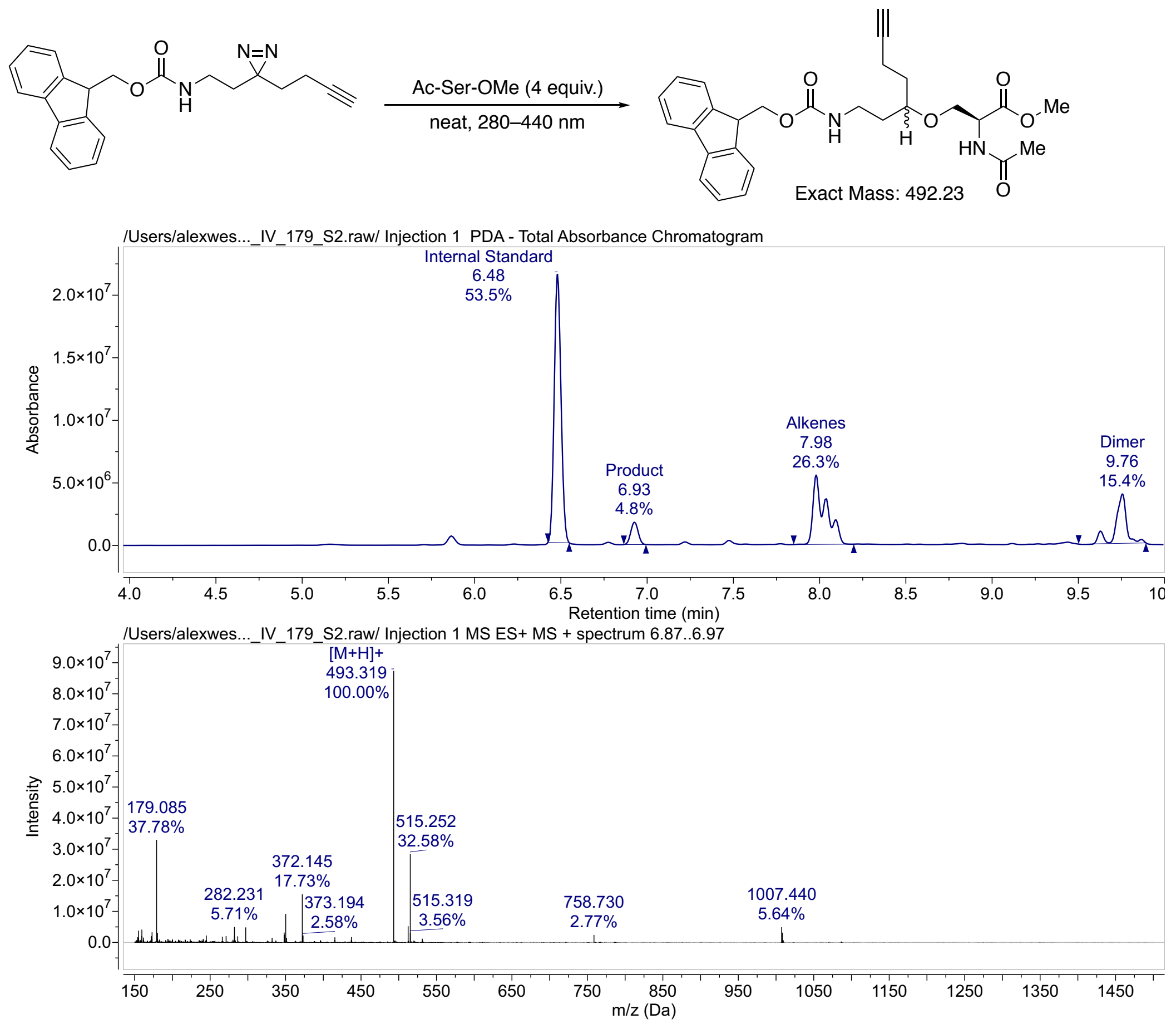

West, A. et al. Labeling preferences of diazirines with protein biomolecules, J. Am. Chem. Soc. 2021. 

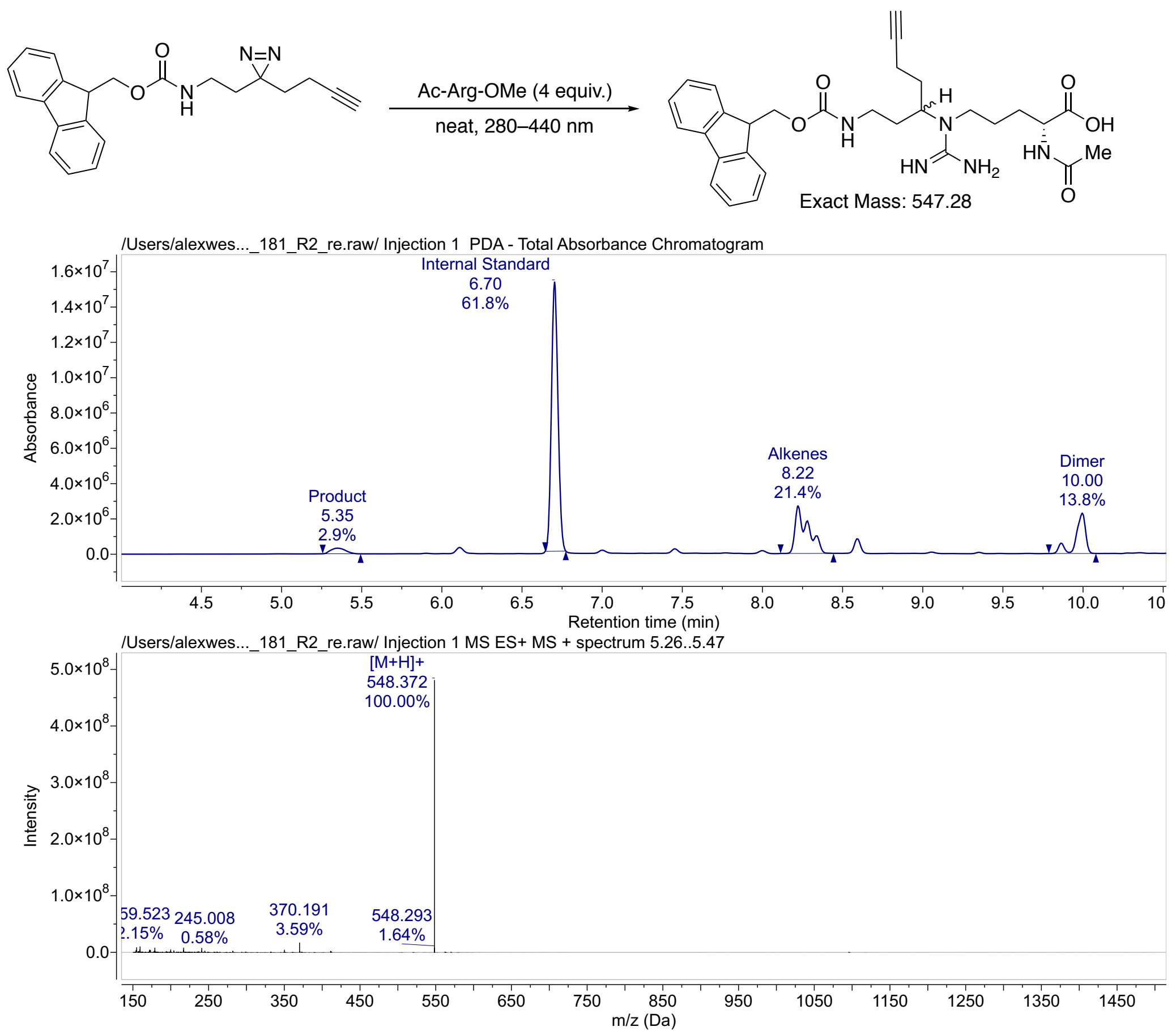

West, A. et al. Labeling preferences of diazirines with protein biomolecules, J. Am. Chem. Soc. 2021. 


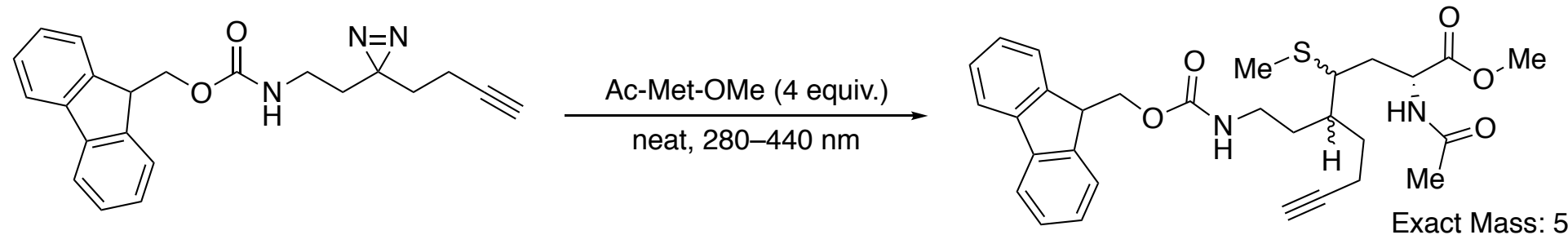

Exact Mass: 536.23
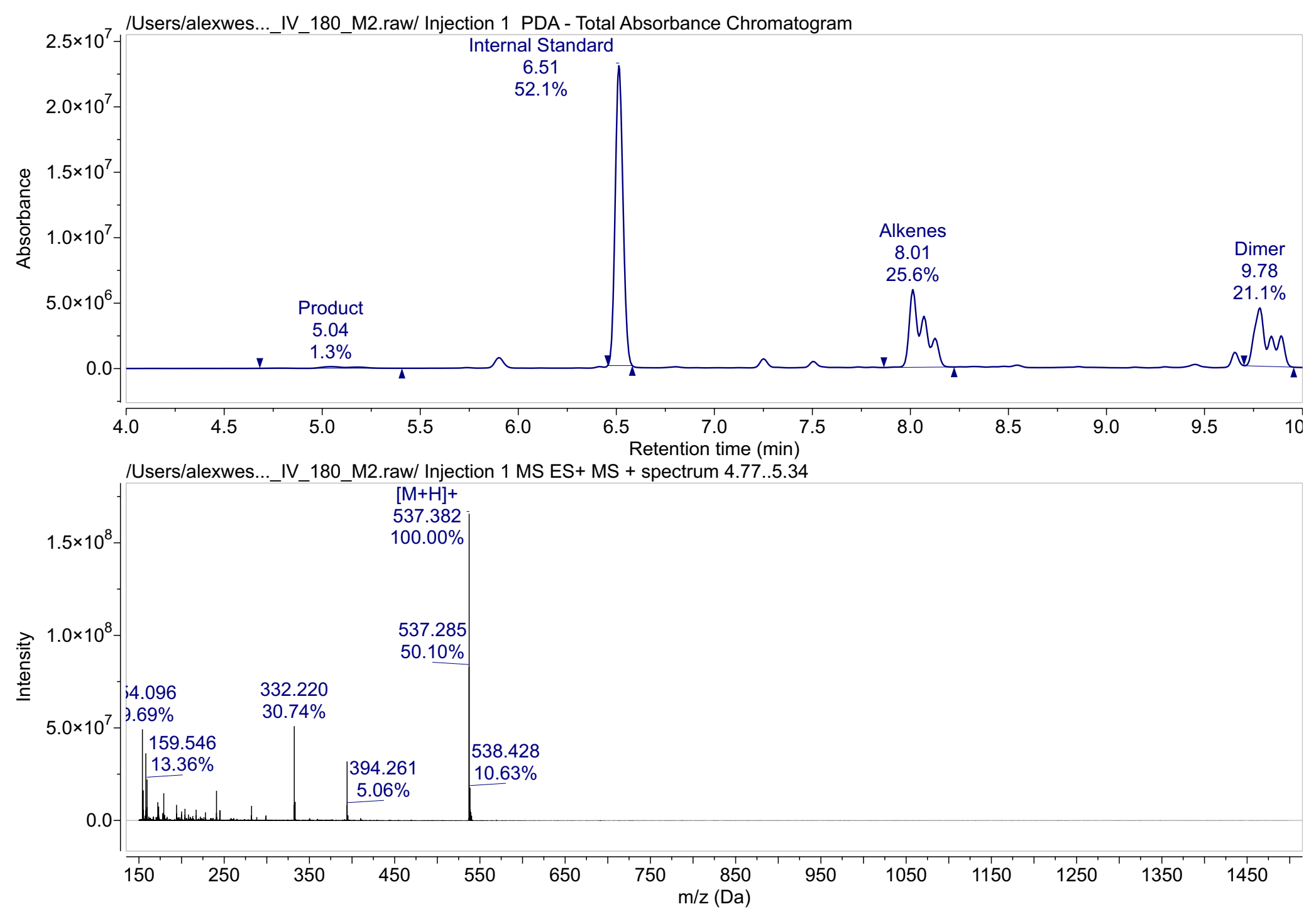

West, A. et al. Labeling preferences of diazirines with protein biomolecules, J. Am. Chem. Soc. 2021. 

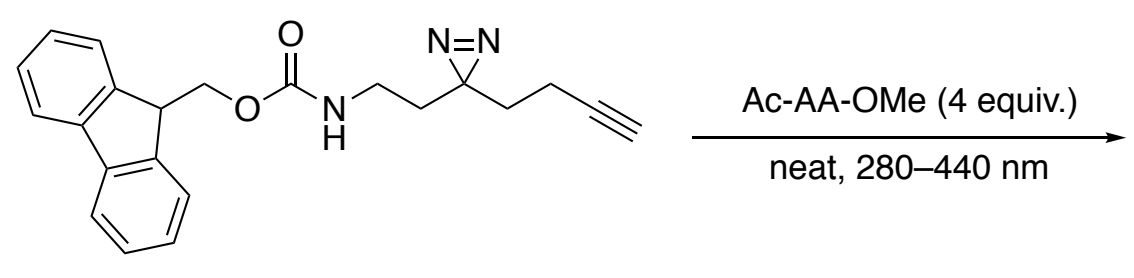

$A A=V, I, L, T, W, G, F, A, P, N, Q$
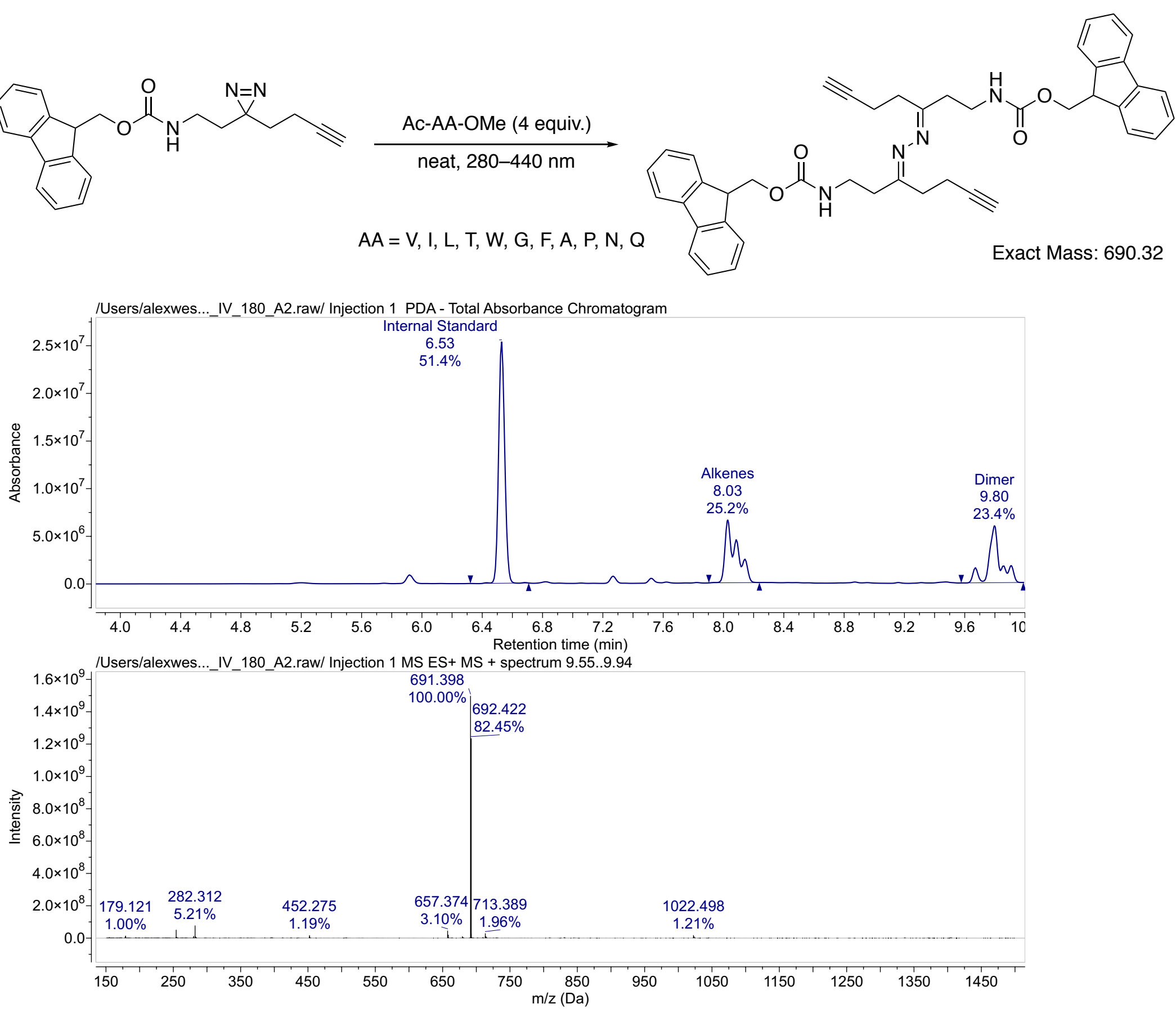

West, A. et al. Labeling preferences of diazirines with protein biomolecules, J. Am. Chem. Soc. 2021. 

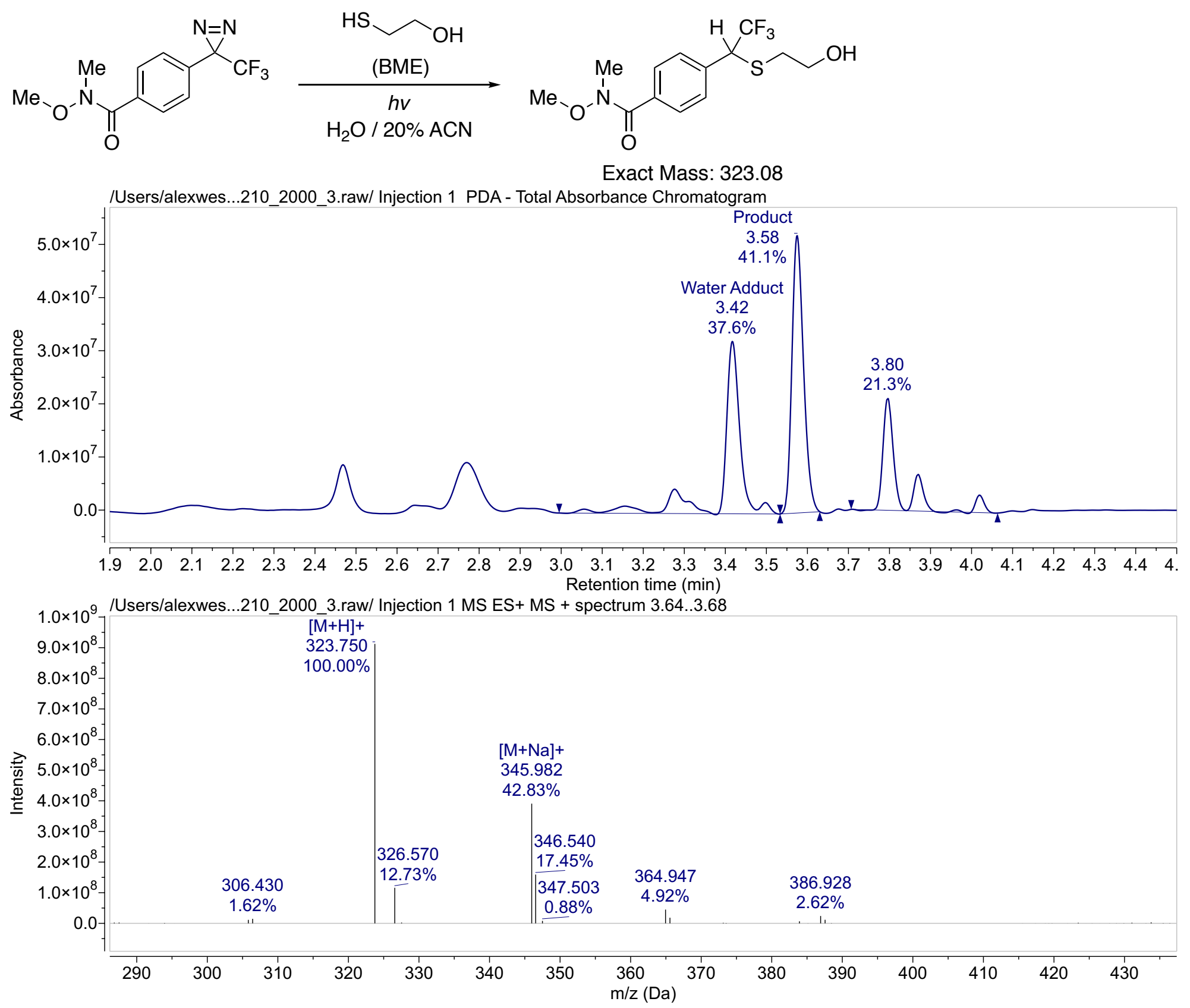
<smiles>C#CCCC1(CCC(=O)NC)N=N1</smiles>

JN Mod: JN-0001-NX-1

PSMs: 22

Unique Sites: 6

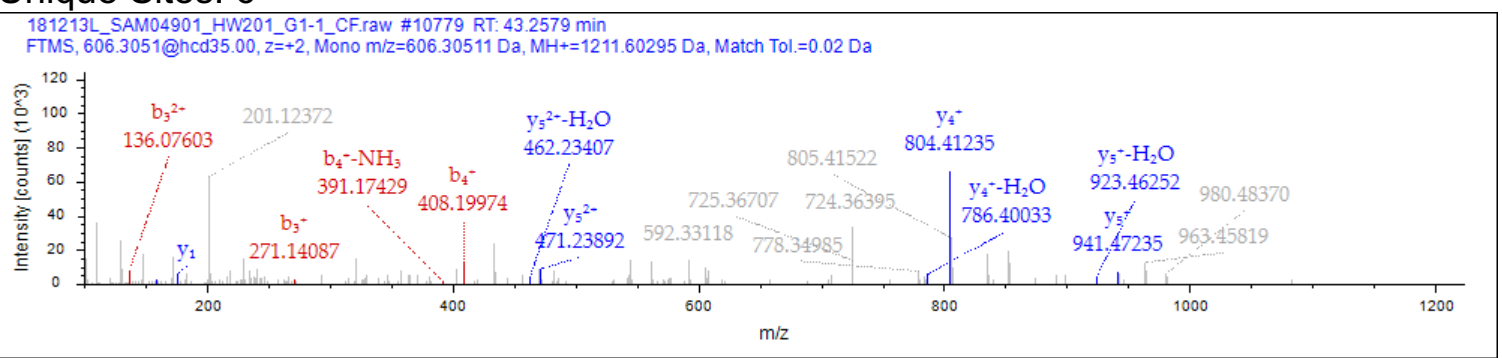

Sequence: AQAHAADR

Charge: +2 ,

Monoisotopic m/z: $606.30511 \mathrm{Da}(+0.06 \mathrm{mmu} /+0.09 \mathrm{ppm})$,

$\mathrm{MH}+:$ 1211.60295 Da,

RT: $43.2579 \mathrm{~min}$,

Identified with: Sequest HT (v1.17);

XCorr:1.21,

Fragment match tolerance used for search: $0.02 \mathrm{Da}$

Fragments used for search: - $\mathrm{H}_{2} \mathrm{O} ; \mathrm{y} ;-\mathrm{NH}_{3} ; \mathrm{y} ; \mathrm{b} ; \mathrm{b} ;-\mathrm{H}_{2} \mathrm{O} ; \mathrm{b} ;-\mathrm{NH}_{3} ; \mathrm{y}$

Proteins (1):

- Putative uncharacterized protein C15orf54 OS=Homo sapiens OX=9606 $\mathrm{GN}=\mathrm{C} 15$ orf54 $\mathrm{PE}=2 \mathrm{SV}=1$

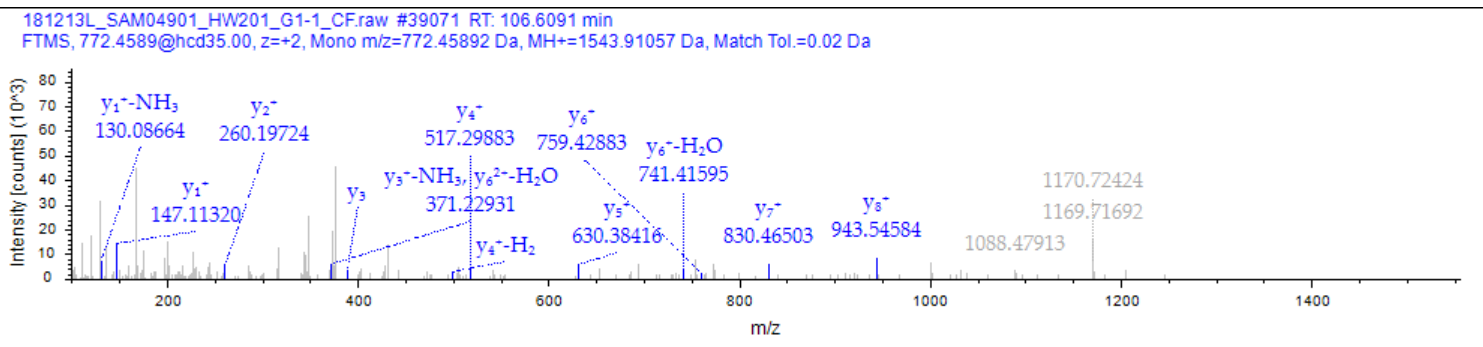

Sequence: ILLAELEQLK

Charge: +2 ,

Monoisotopic m/z: $772.45892 \mathrm{Da}(-0.57 \mathrm{mmu} /-0.73 \mathrm{ppm})$,

$\mathrm{MH}+:$ 1543.91057 Da,

RT: $106.6091 \mathrm{~min}$,

Identified with: Sequest HT (v1.17);

XCorr:1.57,

Fragment match tolerance used for search: $0.02 \mathrm{Da}$

Fragments used for search: - $\mathrm{H}_{2} \mathrm{O} ; \mathrm{y} ;-\mathrm{NH}_{3} ; \mathrm{y} ; \mathrm{b} ; \mathrm{b} ;-\mathrm{H}_{2} \mathrm{O} ; \mathrm{b} ;-\mathrm{NH}_{3} ; \mathrm{y}$

Proteins (1):

- Vimentin OS=Homo sapiens OX=9606 GN=VIM PE=1 SV=4

West, A. et al. Labeling preferences of diazirines with protein biomolecules, J. Am. Chem. S147 Soc. 2021. 


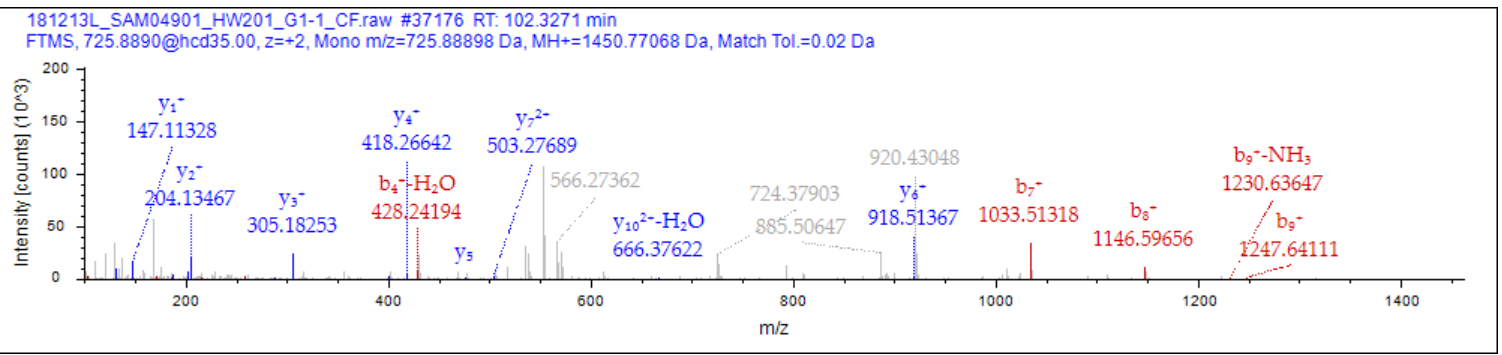

Sequence: TTRSSAGLTGK

Charge: +2 ,

Monoisotopic m/z: $725.88898 \mathrm{Da}(-2.72 \mathrm{mmu} /-3.75 \mathrm{ppm})$,

$\mathrm{MH}+:$ 1450.77068 Da,

RT: $102.3271 \mathrm{~min}$,

Identified with: Sequest HT (v1.17);

XCorr:1.98,

Fragment match tolerance used for search: $0.02 \mathrm{Da}$

Fragments used for search: - $\mathrm{H}_{2} \mathrm{O} ; \mathrm{y} ;-\mathrm{NH}_{3} ; \mathrm{y} ; \mathrm{b} ; \mathrm{b} ;-\mathrm{H}_{2} \mathrm{O} ; \mathrm{b} ;-\mathrm{NH}_{3} ; \mathrm{y}$

Proteins (1):

- Mucin-19 OS=Homo sapiens OX=9606 GN=MUC19 PE=1 SV=3

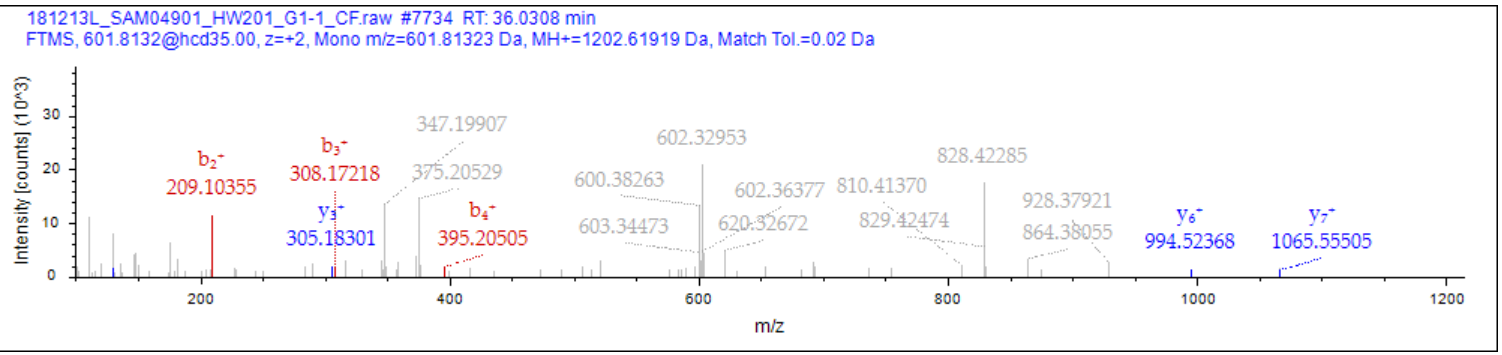

Sequence: HAVSEGTK

Charge: +2 ,

Monoisotopic m/z: $601.81323 \mathrm{Da}(+0.24 \mathrm{mmu} /+0.4 \mathrm{ppm})$,

$\mathrm{MH}+:$ 1202.61919 Da,

RT: 36.0308 min,

Identified with: Sequest HT (v1.17);

XCorr:1.55,

Fragment match tolerance used for search: $0.02 \mathrm{Da}$

Fragments used for search: $-\mathrm{H}_{2} \mathrm{O} ; \mathrm{y} ;-\mathrm{NH}_{3} ; \mathrm{y} ; \mathrm{b} ; \mathrm{b} ;-\mathrm{H}_{2} \mathrm{O} ; \mathrm{y}$

Proteins (15):

- Histone H2B type 2-E OS=Homo sapiens OX=9606 GN=HIST2H2BE PE=1 SV=3

- Histone H2B type 2-F OS=Homo sapiens OX=9606 GN=HIST2H2BF PE=1 SV=3

- Histone H2B type 1-M OS=Homo sapiens OX=9606 GN=HIST1H2BM PE=1 SV=3

- Histone H2B type 1-B OS=Homo sapiens OX=9606 GN=HIST1H2BB PE=1 SV=2

- Histone H2B type 1-D OS=Homo sapiens OX=9606 GN=HIST1H2BD PE=1 SV=2

- Histone H2B type 1-A OS=Homo sapiens OX=9606 GN=HIST1H2BA PE=1 SV=3

- Histone H2B type 1-H OS=Homo sapiens OX=9606 GN=HIST1H2BH PE=1 SV=3

- Histone H2B type 1-K OS=Homo sapiens OX=9606 GN=HIST1H2BK PE=1 SV=3

- Histone H2B type 1-L OS=Homo sapiens OX=9606 GN=HIST1H2BL PE=1 SV=3

West, A. et al. Labeling preferences of diazirines with protein biomolecules, J. Am. Chem. S148 Soc. 2021. 
<smiles>C#CCCC1(CCC(=O)N2CCC(c3ccccc3)CC2)N=N1</smiles>

JN Mod: JN-0003

PSMs: 267

Unique Sites: 46

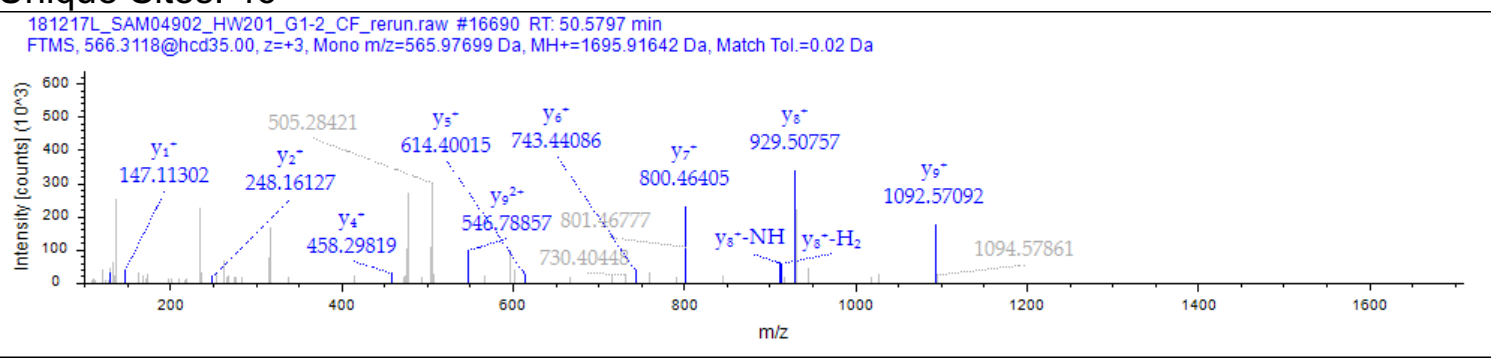

\section{Sequence: VYEGERPLTK}

Charge: +3, Monoisotopic m/z: $565.97699 \mathrm{Da}(+1.24 \mathrm{mmu} /+2.18 \mathrm{ppm})$,

$\mathrm{MH}+:$ 1695.91642 Da,

RT: $50.5797 \mathrm{~min}$,

Identified with: Sequest HT (v1.17);

XCorr:1.35,

Fragment match tolerance used for search: $0.02 \mathrm{Da}$

Fragments used for search: - $\mathrm{H}_{2} \mathrm{O} ; \mathrm{y} ;-\mathrm{NH}_{3} ; \mathrm{y} ; \mathrm{b} ; \mathrm{b} ;-\mathrm{H}_{2} \mathrm{O} ; \mathrm{b} ;-\mathrm{NH}_{3} ; \mathrm{y}$

Proteins (1):

- Endoplasmic reticulum chaperone BiP OS=Homo sapiens OX=9606 GN=HSPA5 $\mathrm{PE}=1 \mathrm{SV}=2$

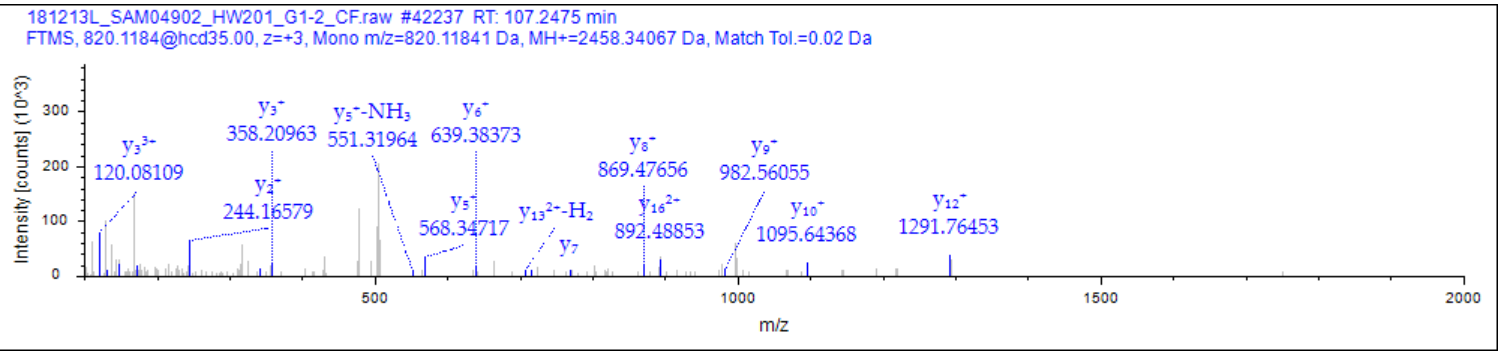

Sequence: VAPEEHPVLLTEAPLNPK

Charge: +3 ,

Monoisotopic m/z: 820.11841 Da (+0.12 mmu/+0.15 ppm),

$\mathrm{MH}+:$ 2458.34067 Da,

RT: $107.2475 \mathrm{~min}$,

Identified with: Sequest HT (v1.17);

XCorr:2.67,

Fragment match tolerance used for search: $0.02 \mathrm{Da}$

Fragments used for search: - $\mathrm{H}_{2} \mathrm{O} ; \mathrm{y} ;-\mathrm{NH}_{3} ; \mathrm{y} ; \mathrm{b} ; \mathrm{b} ;-\mathrm{H}_{2} \mathrm{O} ; \mathrm{b} ;-\mathrm{NH}_{3} ; \mathrm{y}$

Proteins (2):

- Actin, cytoplasmic 1 OS=Homo sapiens OX=9606 GN=ACTB PE=1 SV=1

- Actin, cytoplasmic 2 OS=Homo sapiens OX=9606 GN=ACTG1 PE=1 SV=1

West, A. et al. Labeling preferences of diazirines with protein biomolecules, J. Am. Chem. S149 Soc. 2021. 


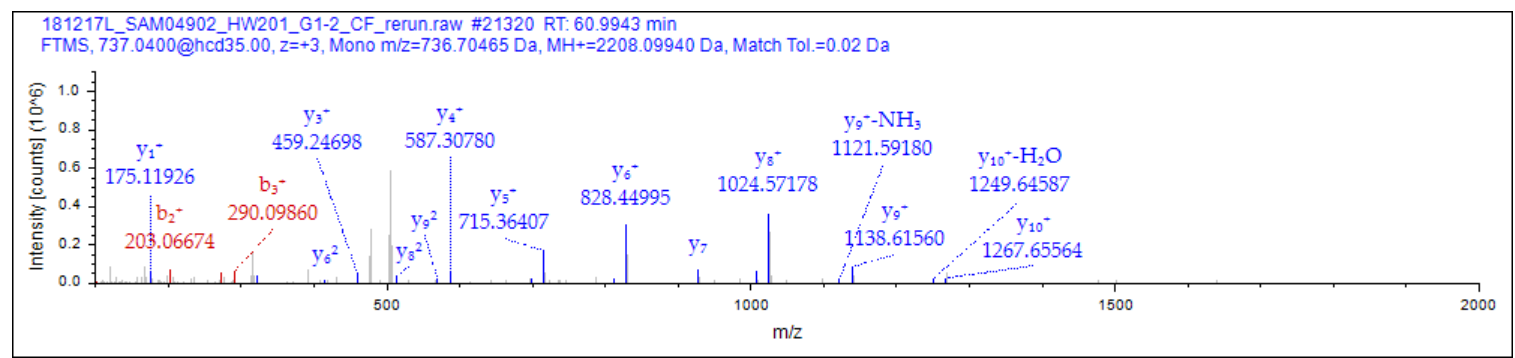

Sequence: SDSFENPVLQQHFR

Charge: +3, Monoisotopic m/z: 736.70465 Da (+3.3 mmu/+4.48 ppm), $\mathrm{MH+:} 2208.09940$

Da, RT: $60.9943 \mathrm{~min}$,

Identified with: Sequest HT (v1.17); XCorr:2.41,

Fragment match tolerance used for search: $0.02 \mathrm{Da}$

Fragments used for search: - $\mathrm{H}_{2} \mathrm{O} ; \mathrm{y} ;-\mathrm{NH}_{3} ; \mathrm{y} ; \mathrm{b} ; \mathrm{b} ;-\mathrm{H}_{2} \mathrm{O} ; \mathrm{b} ;-\mathrm{NH}_{3} ; \mathrm{y}$

Proteins (1):

- X-ray repair cross-complementing protein 6 OS=Homo sapiens OX=9606 $\mathrm{GN}=\mathrm{XRCC} 6 \mathrm{PE}=1 \mathrm{SV}=2$

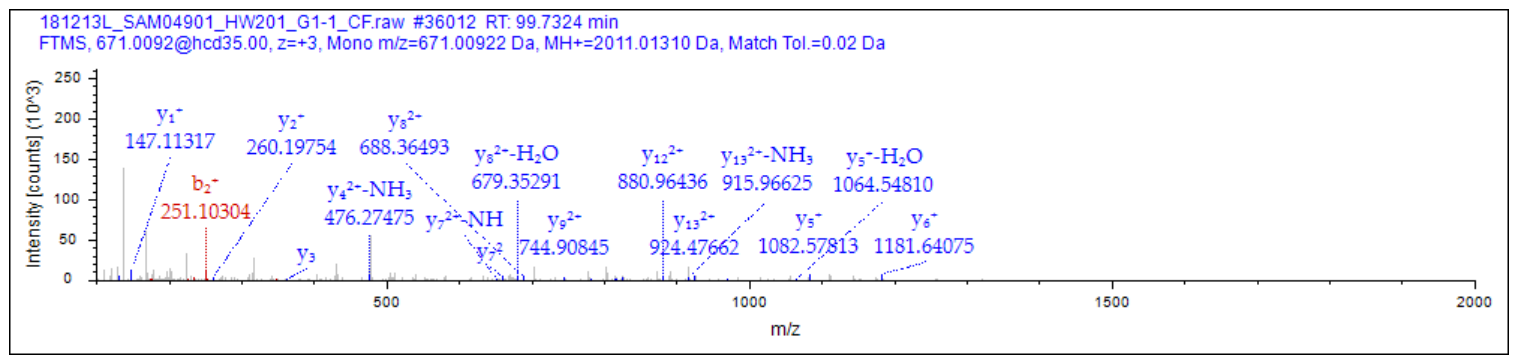

Sequence: YSNSALGHVNCTIK

Charge: +3 ,

Monoisotopic m/z: $671.00922 \mathrm{Da}(+0.09 \mathrm{mmu} /+0.13 \mathrm{ppm})$,

$\mathrm{MH}+:$ 2011.01310 Da,

RT: 99.7324 min,

Identified with: Sequest HT (v1.17);

XCorr:2.46,

Fragment match tolerance used for search: $0.02 \mathrm{Da}$

Fragments used for search: - $\mathrm{H}_{2} \mathrm{O} ; \mathrm{y} ;-\mathrm{NH}_{3} ; \mathrm{y} ; \mathrm{b} ; \mathrm{b} ;-\mathrm{H}_{2} \mathrm{O} ; \mathrm{b} ;-\mathrm{NH}_{3} ; \mathrm{y}$

Proteins (1):

- Reticulon-4 OS=Homo sapiens OX=9606 GN=RTN4 PE=1 SV=2

West, A. et al. Labeling preferences of diazirines with protein biomolecules, J. Am. Chem. S150 Soc. 2021. 
<smiles>C#CCCC1(CCC(=O)N2CCC(C(c3ccccc3)c3ccccc3)CC2)N=N1</smiles>

JN Mod: JN-0004

PSMs: 19

Unique Sites: 10

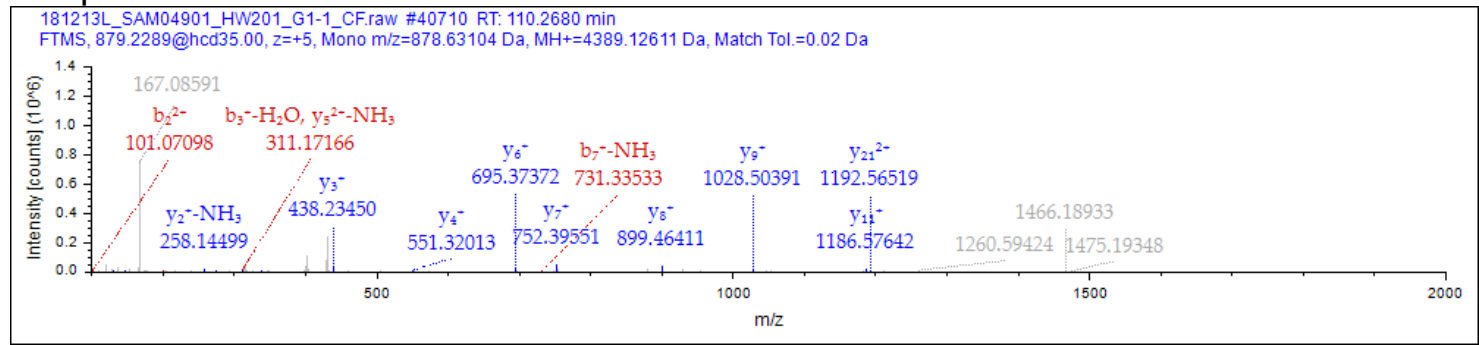

\section{Sequence: VTQSNFAVGYKTDEFQLHTNVNDGTEFGGSIYQK}

Charge: +5 ,

Monoisotopic m/z: 878.63104 Da (+1.99 mmu/+2.26 ppm),

$\mathrm{MH}+:$ 4389.12611 Da,

RT: 110.2680 min,

Identified with: Sequest HT (v1.17); XCorr:2.90,

Fragment match tolerance used for search: $0.02 \mathrm{Da}$

Fragments used for search: - $\mathrm{H}_{2} \mathrm{O} ; \mathrm{y} ;-\mathrm{NH}_{3} ; \mathrm{y} ; \mathrm{b} ; \mathrm{b} ;-\mathrm{H}_{2} \mathrm{O} ; \mathrm{b} ;-\mathrm{NH}_{3} ; \mathrm{y}$

Proteins (1):

- Voltage-dependent anion-selective channel protein 1 OS=Homo sapiens OX=9606 $\mathrm{GN}=\mathrm{VDAC} 1 \mathrm{PE}=1 \mathrm{SV}=2$

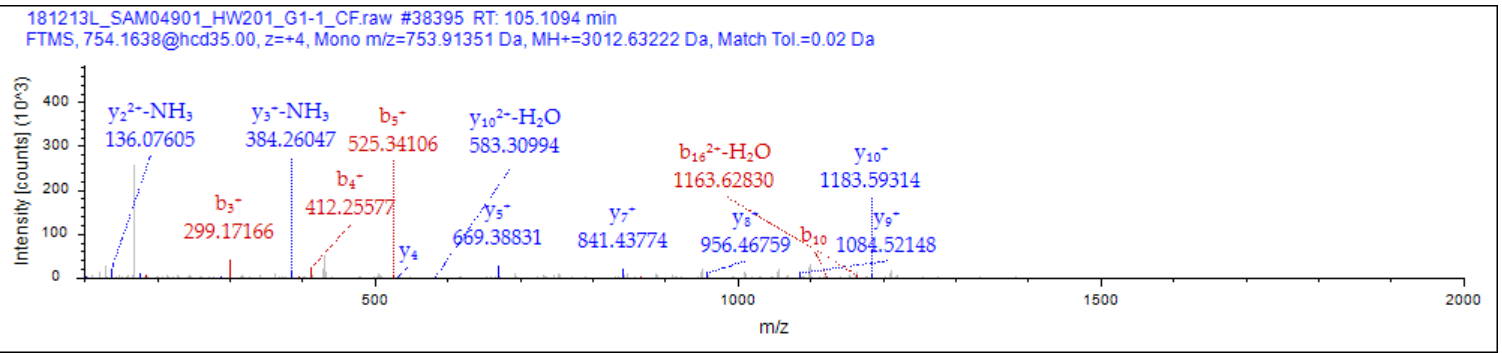

Sequence: IANLLKPDKEIVQDGDHMIIR

Charge: +4 ,

Monoisotopic m/z: 753.91351 Da (-2.01 mmu/-2.66 ppm),

$\mathrm{MH}+: 3012.63222 \mathrm{Da}$,

RT: $105.1094 \mathrm{~min}$,

Identified with: Sequest HT (v1.17);

XCorr:2.74,

Fragment match tolerance used for search: $0.02 \mathrm{Da}$

Fragments used for search: - $\mathrm{H}_{2} \mathrm{O} ; \mathrm{y} ;-\mathrm{NH}_{3} ; \mathrm{y} ; \mathrm{b} ; \mathrm{b} ;-\mathrm{H}_{2} \mathrm{O} ; \mathrm{b} ;-\mathrm{NH}_{3} ; \mathrm{y}$

Proteins (1):

- Retinol-binding protein 1 OS=Homo sapiens OX=9606 GN=RBP1 PE=1 SV=2

West, A. et al. Labeling preferences of diazirines with protein biomolecules, J. Am. Chem. S151 Soc. 2021. 


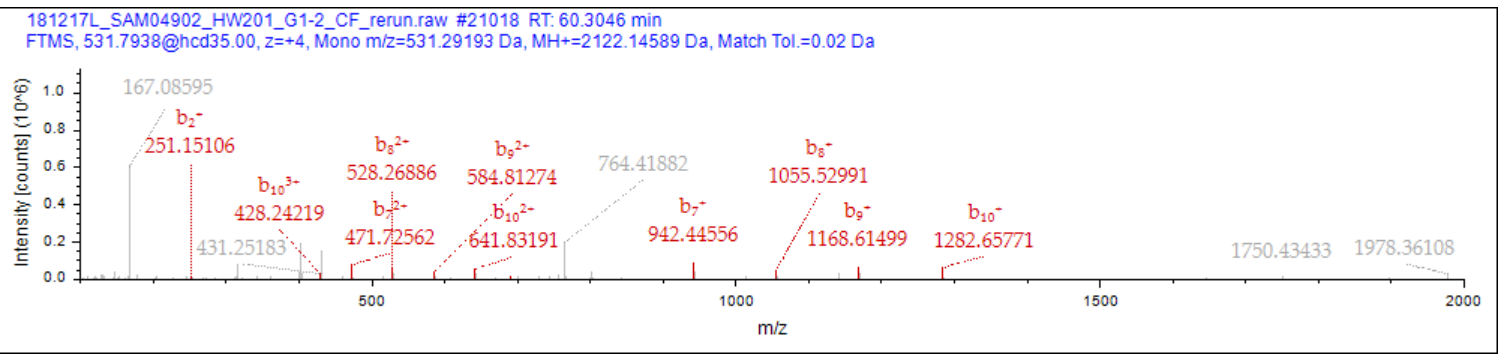

Sequence: HLREYQDLLNVK

Charge: +4 ,

Monoisotopic m/z: $531.29193 \mathrm{Da}(-1.2 \mathrm{mmu} /-2.27 \mathrm{ppm})$,

$\mathrm{MH}+:$ 2122.14589 Da,

RT: 60.3046 min,

Identified with: Sequest HT (v1.17);

XCorr:1.91,

Fragment match tolerance used for search: $0.02 \mathrm{Da}$

Fragments used for search: - $\mathrm{H}_{2} \mathrm{O} ; \mathrm{y} ;-\mathrm{NH}_{3} ; \mathrm{y} ; \mathrm{b} ; \mathrm{b} ;-\mathrm{H}_{2} \mathrm{O} ; \mathrm{b} ;-\mathrm{NH}_{3} ; \mathrm{y}$

Proteins (4):

- Desmin OS=Homo sapiens OX=9606 GN=DES PE=1 SV=3

- Vimentin OS=Homo sapiens OX=9606 GN=VIM PE=1 SV=4

- Neurofilament medium polypeptide OS=Homo sapiens OX=9606 GN=NEFM PE=1 $\mathrm{SV}=3$

- Alpha-internexin OS=Homo sapiens OX=9606 GN=INA PE=1 SV=2

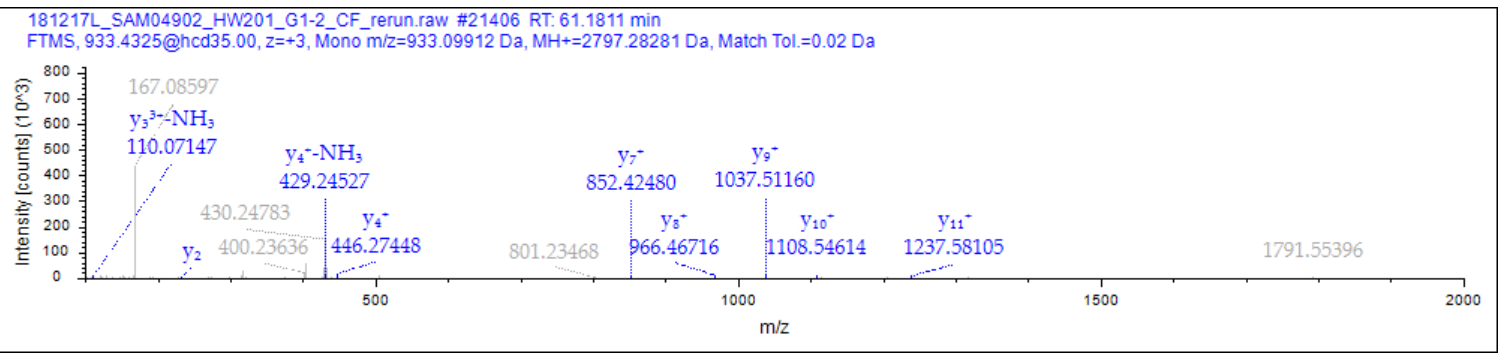

Sequence: EMEENFAVEAANYQDTIGR

Charge: +3 ,

Monoisotopic m/z: 933.09912 Da (-0.3 mmu/-0.32 ppm),

$\mathrm{MH}+:$ 2797.28281 Da,

RT: $61.1811 \mathrm{~min}$,

Identified with: Sequest HT (v1.17);

XCorr:1.27,

Fragment match tolerance used for search: $0.02 \mathrm{Da}$

Fragments used for search: $-\mathrm{H}_{2} \mathrm{O} ; \mathrm{y} ;-\mathrm{NH}_{3} ; \mathrm{y} ; \mathrm{b} ; \mathrm{b} ;-\mathrm{H}_{2} \mathrm{O} ; \mathrm{b} ;-\mathrm{NH}_{3} ; \mathrm{y}$

Proteins (1):

- Vimentin OS=Homo sapiens OX=9606 GN=VIM PE=1 SV=4

West, A. et al. Labeling preferences of diazirines with protein biomolecules, J. Am. Chem. S152 Soc. 2021. 
<smiles>C#CCCC1(CCn2cc3c(=O)n(C)c(=O)n(C)c3c2)N=N1</smiles>

JN Mod: JN-0005-NX-1

PSMs: 102

Unique Sites: 23

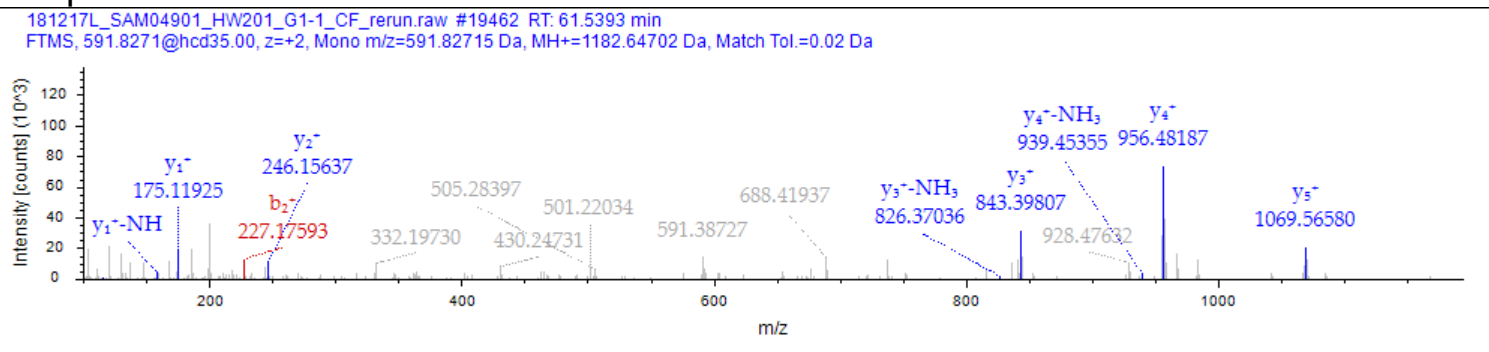

\section{Sequence: LLLCAR}

Charge: +2 ,

Monoisotopic m/z: 591.82715 Da (-0.26 mmu/-0.44 ppm),

$\mathrm{MH}+:$ 1182.64702 Da,

RT: 61.5393 min,

Identified with: Sequest HT (v1.17);

XCorr:1.82,

Fragment match tolerance used for search: $0.02 \mathrm{Da}$

Fragments used for search: $-\mathrm{NH}_{3} ; \mathrm{y} ; \mathrm{b} ; \mathrm{y}$

Proteins (1):

- Protein-glutamine gamma-glutamyltransferase 2 OS=Homo sapiens OX=9606 $\mathrm{GN}=\mathrm{TGM} 2 \mathrm{PE}=1 \mathrm{SV}=2$

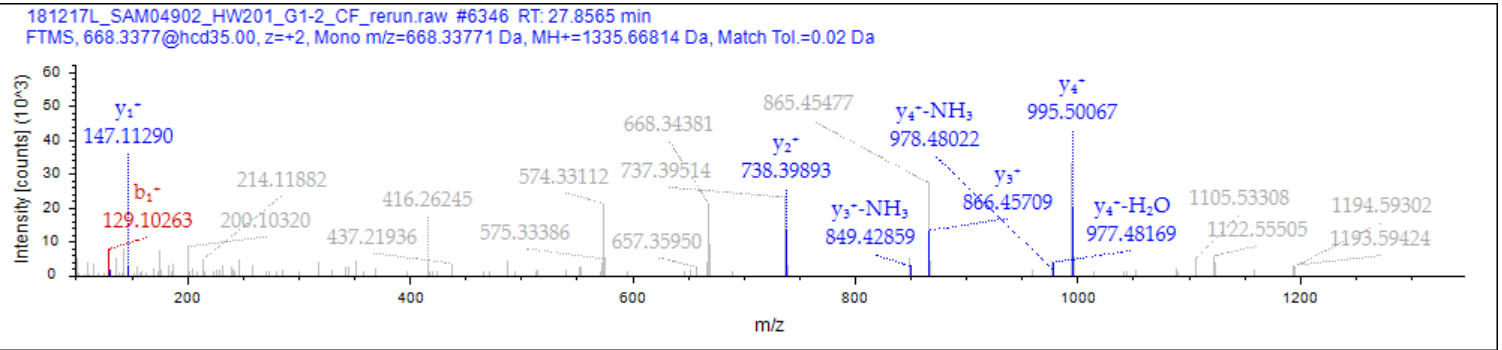

Sequence: KPDEQPK

Charge: +2 ,

Monoisotopic m/z: 668.33771 Da (-1.69 mmu/-2.52 ppm),

$\mathrm{MH}+: 1335.66814 \mathrm{Da}$,

RT: 27.8565 min,

Identified with: Sequest HT (v1.17);

XCorr:1.66,

Fragment match tolerance used for search: $0.02 \mathrm{Da}$

Fragments used for search: $-\mathrm{H}_{2} \mathrm{O} ; \mathrm{y} ;-\mathrm{NH}_{3} ; \mathrm{y} ; \mathrm{b} ; \mathrm{b} ;-\mathrm{H}_{2} \mathrm{O} ; \mathrm{b} ;-\mathrm{NH}_{3} ; \mathrm{y}$

Proteins (1):

- Sodium channel protein type 8 subunit alpha OS=Homo sapiens OX=9606 $\mathrm{GN}=\mathrm{SCN} 8 \mathrm{~A} \mathrm{PE}=1 \mathrm{SV}=1$

West, A. et al. Labeling preferences of diazirines with protein biomolecules, J. Am. Chem. S153 Soc. 2021. 


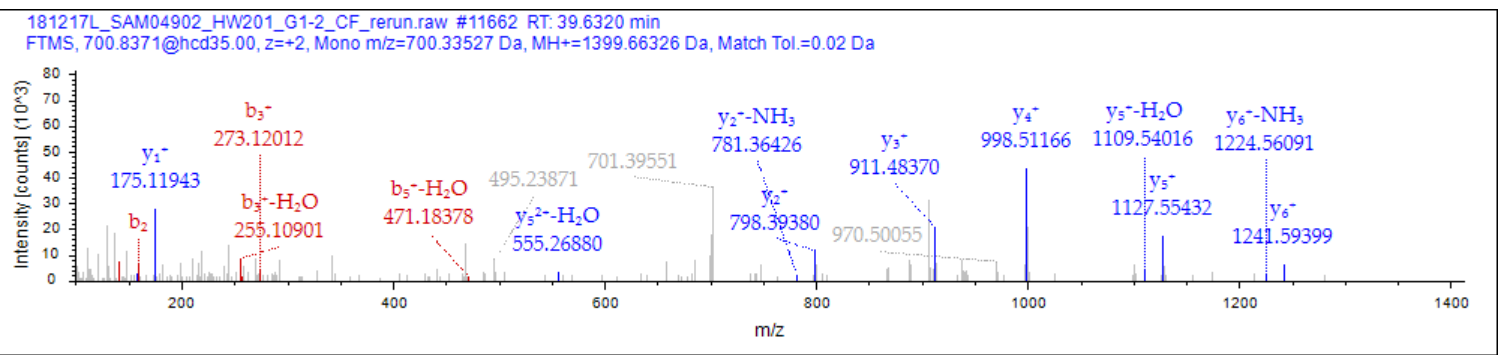

Sequence: GTNESLER

Charge: +2 ,

Monoisotopic m/z: 700.33527 Da (+0.43 mmu/+0.61 ppm),

$\mathrm{MH}+:$ 1399.66326 Da,

RT: 39.6320 min,

Identified with: Sequest HT (v1.17);

XCorr:2.06,

Fragment match tolerance used for search: $0.02 \mathrm{Da}$

Fragments used for search: - $\mathrm{H}_{2} \mathrm{O} ; \mathrm{y} ;-\mathrm{NH}_{3} ; \mathrm{y} ; \mathrm{b} ; \mathrm{b} ;-\mathrm{H}_{2} \mathrm{O} ; \mathrm{b} ;-\mathrm{NH}_{3} ; \mathrm{y}$

Proteins (2):

- Glial fibrillary acidic protein OS=Homo sapiens OX=9606 GN=GFAP PE=1 SV=1

- Vimentin OS=Homo sapiens OX=9606 GN=VIM PE=1 SV=4

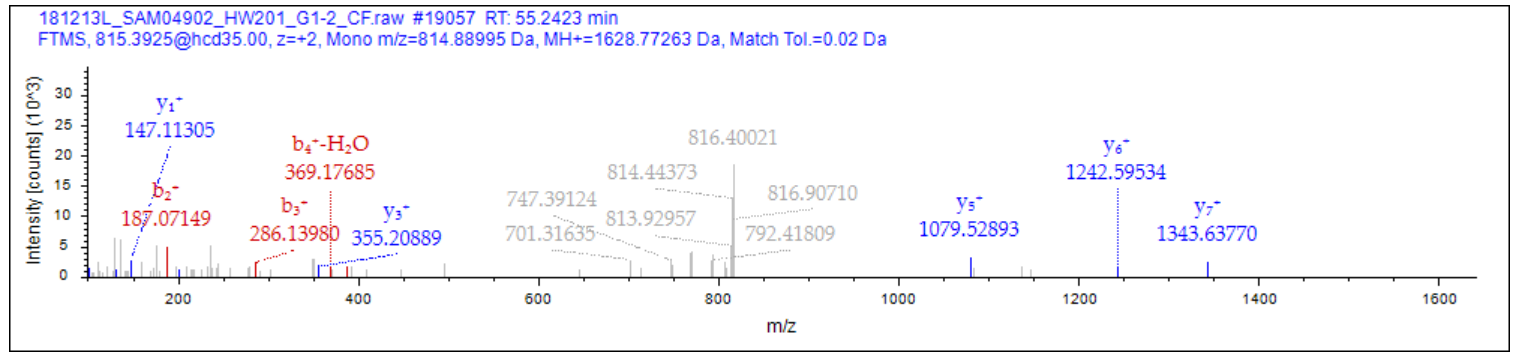

Sequence: DAVTYTEHAK

Charge: +2 ,

Monoisotopic m/z: 814.88995 Da (-0.02 mmu/-0.03 ppm),

$\mathrm{MH}+:$ 1628.77263 Da,

RT: 55.2423 min,

Identified with: Sequest HT (v1.17);

XCorr:1.61,

Fragment match tolerance used for search: $0.02 \mathrm{Da}$

Fragments used for search: $-\mathrm{H}_{2} \mathrm{O} ; \mathrm{y} ;-\mathrm{NH}_{3} ; \mathrm{y} ; \mathrm{b} ; \mathrm{b} ;-\mathrm{H}_{2} \mathrm{O}$; y

Proteins (1):

- Histone H4 OS=Homo sapiens OX=9606 GN=HIST1H4A PE=1 SV=2

West, A. et al. Labeling preferences of diazirines with protein biomolecules, J. Am. Chem. S154 Soc. 2021. 
<smiles>C#CCCC1(CCOc2ccc(C(=O)O)cc2)N=N1</smiles>

JN Mod: JN-0012-NX-1

PSMs: 39

Unique Sites: 12

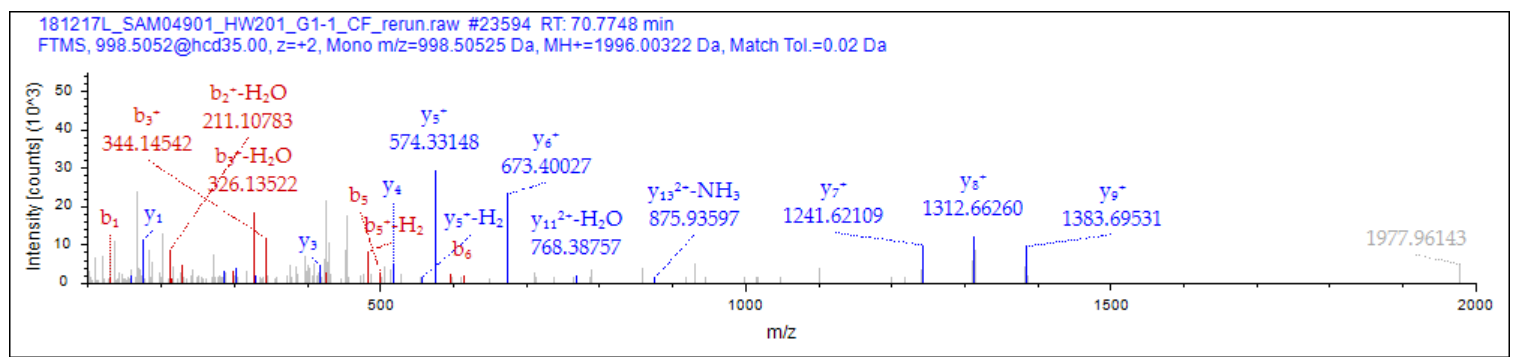

\section{Sequence: EVDVGLAADVGTLQR}

Charge: +2 ,

Monoisotopic m/z: 998.50525 Da (-0.65 mmu/-0.65 ppm),

$\mathrm{MH}+:$ 1996.00322 Da,

RT: 70.7748 min,

Identified with: Sequest HT (v1.17);

XCorr:2.95,

Fragment match tolerance used for search: $0.02 \mathrm{Da}$

Fragments used for search: - $\mathrm{H}_{2} \mathrm{O} ; \mathrm{y} ;-\mathrm{NH}_{3} ; \mathrm{y} ; \mathrm{b} ; \mathrm{b} ;-\mathrm{H}_{2} \mathrm{O} ; \mathrm{b} ;-\mathrm{NH}_{3} ; \mathrm{y}$

Proteins (1):

- Delta(3,5)-Delta(2,4)-dienoyl-CoA isomerase, mitochondrial OS=Homo sapiens $\mathrm{OX}=9606 \mathrm{GN}=\mathrm{ECH} 1 \mathrm{PE}=1 \mathrm{SV}=2$

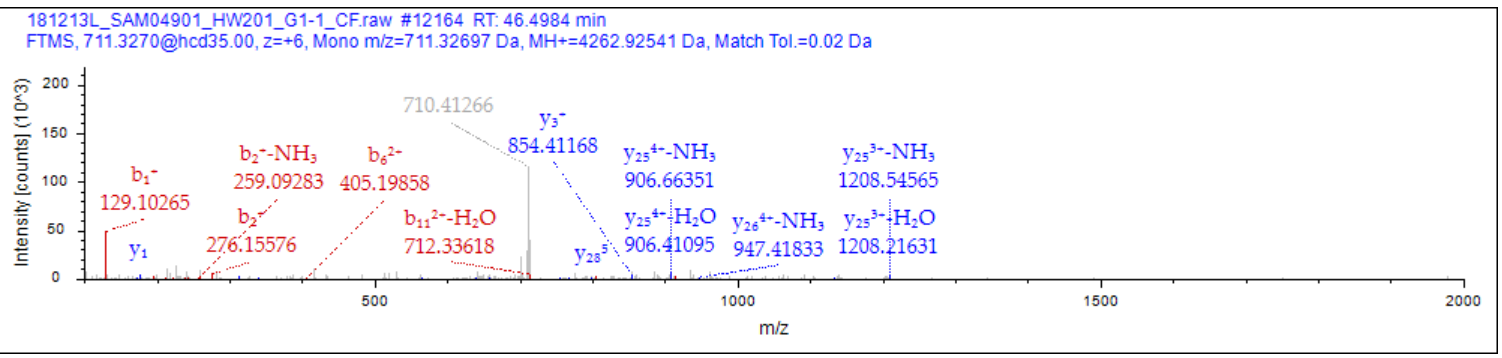

Sequence: KMLAYWMLEVCEEQRCEEEVFPLAMNYLDR

Charge: +6 ,

Monoisotopic m/z: 711.32697 Da (+1.69 mmu/+2.37 ppm),

$\mathrm{MH}+:$ 4262.92541 Da,

RT: 46.4984 min,

Identified with: Sequest HT (v1.17);

XCorr:2.02,

Fragment match tolerance used for search: $0.02 \mathrm{Da}$

Fragments used for search: - $\mathrm{H}_{2} \mathrm{O} ; \mathrm{y} ;-\mathrm{NH}_{3} ; \mathrm{y} ; \mathrm{b} ; \mathrm{b} ;-\mathrm{H}_{2} \mathrm{O} ; \mathrm{b} ;-\mathrm{NH}_{3} ; \mathrm{y}$

Proteins (1):

- G1/S-specific cyclin-D3 OS=Homo sapiens OX=9606 GN=CCND3 PE=1 SV=2

West, A. et al. Labeling preferences of diazirines with protein biomolecules, J. Am. Chem. S155 Soc. 2021. 


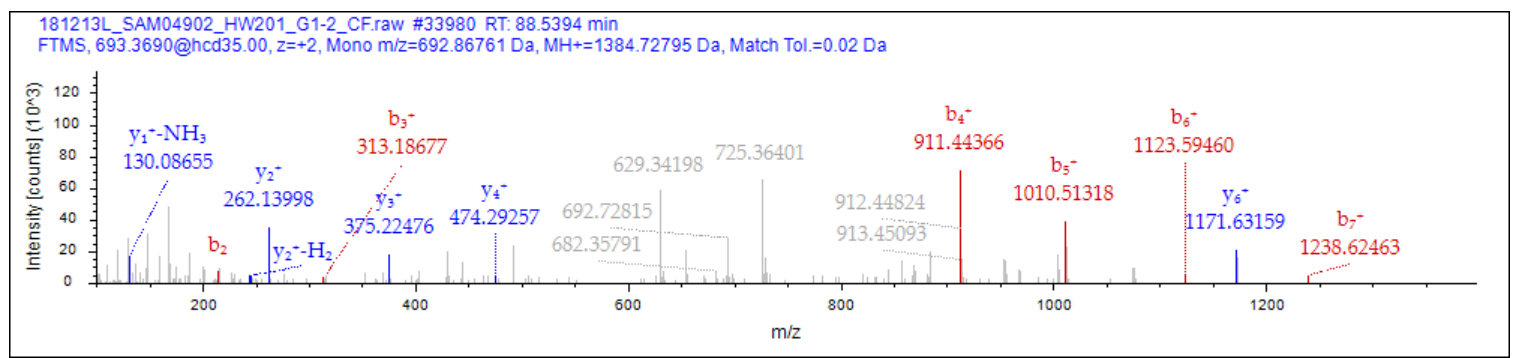

Sequence: NVVFVIDK

Charge: +2 ,

Monoisotopic m/z: $692.86761 \mathrm{Da}$ (+0.98 mmu/+1.41 ppm),

$\mathrm{MH}+:$ 1384.72795 Da,

RT: 88.5394 min,

Identified with: Sequest HT (v1.17);

XCorr:1.78,

Fragment match tolerance used for search: $0.02 \mathrm{Da}$

Fragments used for search: - $\mathrm{H}_{2} \mathrm{O} ; \mathrm{y} ;-\mathrm{NH}_{3} ; \mathrm{y} ; \mathrm{b} ; \mathrm{b} ;-\mathrm{H}_{2} \mathrm{O} ; \mathrm{b} ;-\mathrm{NH}_{3} ; \mathrm{y}$

Proteins (1):

- Inter-alpha-trypsin inhibitor heavy chain H4 OS=Homo sapiens OX=9606 $\mathrm{GN}=\mathrm{ITIH} 4 \mathrm{PE}=1 \mathrm{SV}=4$

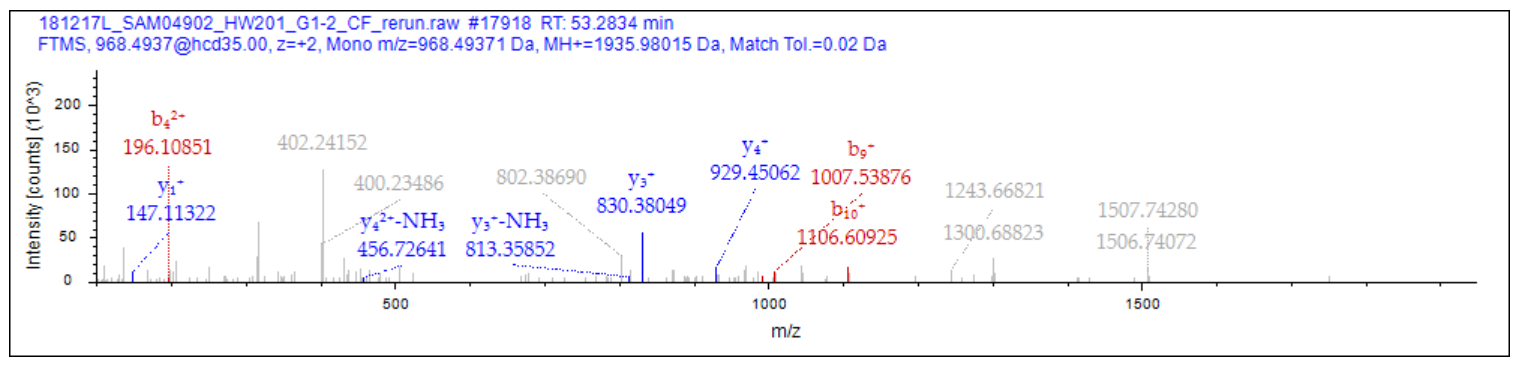

Sequence: IGGYTKKVCVMTK

Charge: +2 ,

Monoisotopic m/z: $968.49371 \mathrm{Da}(-0.91 \mathrm{mmu} /-0.94 \mathrm{ppm})$,

$\mathrm{MH}+:$ 1935.98015 Da,

RT: $53.2834 \mathrm{~min}$,

Identified with: Sequest HT (v1.17);

XCorr:1.41,

Fragment match tolerance used for search: $0.02 \mathrm{Da}$

Fragments used for search: - $\mathrm{H}_{2} \mathrm{O} ; \mathrm{y} ;-\mathrm{NH}_{3} ; \mathrm{y} ; \mathrm{b} ; \mathrm{b} ;-\mathrm{H}_{2} \mathrm{O} ; \mathrm{b} ;-\mathrm{NH}_{3} ; \mathrm{y}$

Proteins (2):

- Schlafen family member 12 OS=Homo sapiens OX=9606 GN=SLFN12 PE=2 SV=2

- Schlafen family member 12-like OS=Homo sapiens OX=9606 GN=SLFN12L PE=2 SV=4

Group 2:

West, A. et al. Labeling preferences of diazirines with protein biomolecules, J. Am. Chem. S156 Soc. 2021. 
<smiles>C#CCCC1(CCOc2cccc(C(=O)O)c2)N=N1</smiles>

JN Mod: JN-0020

PSMs: 9

Unique Sites: 4

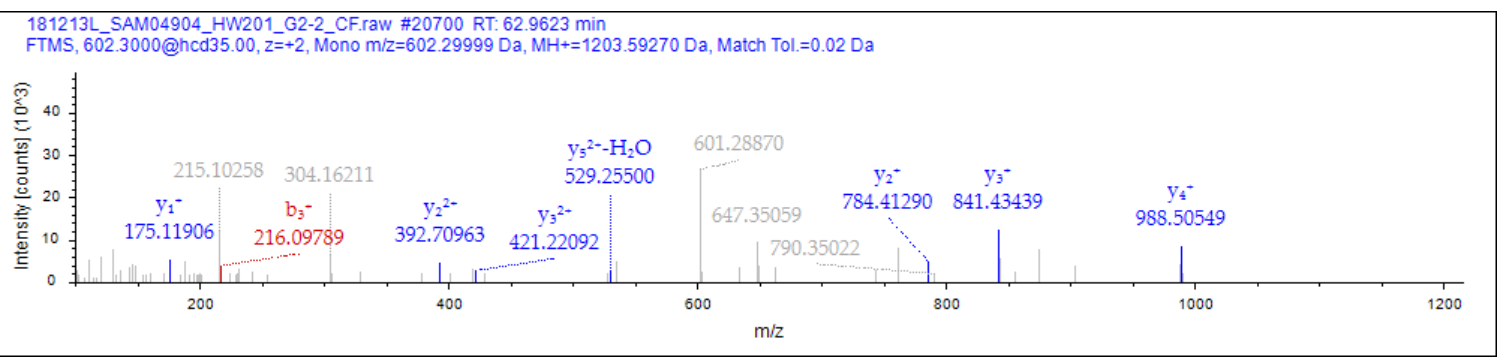

Sequence: AGSFGRR

Charge: +2 ,

Monoisotopic m/z: 602.29999 Da (-0.07 mmu/-0.12 ppm),

$\mathrm{MH}+:$ 1203.59270 Da,

RT: $62.9623 \mathrm{~min}$,

Identified with: Sequest HT (v1.17); XCorr:1.14,

Fragment match tolerance used for search: $0.02 \mathrm{Da}$

Fragments used for search: $-\mathrm{H}_{2} \mathrm{O} ; \mathrm{y} ;-\mathrm{NH}_{3} ; \mathrm{y} ; \mathrm{b} ; \mathrm{b} ;-\mathrm{H}_{2} \mathrm{O} ; \mathrm{y}$

Proteins (1):

- Dolichyl-diphosphooligosaccharide--protein glycosyltransferase 48 kDa subunit OS=Homo sapiens OX=9606 GN=DDOST PE=1 SV=4

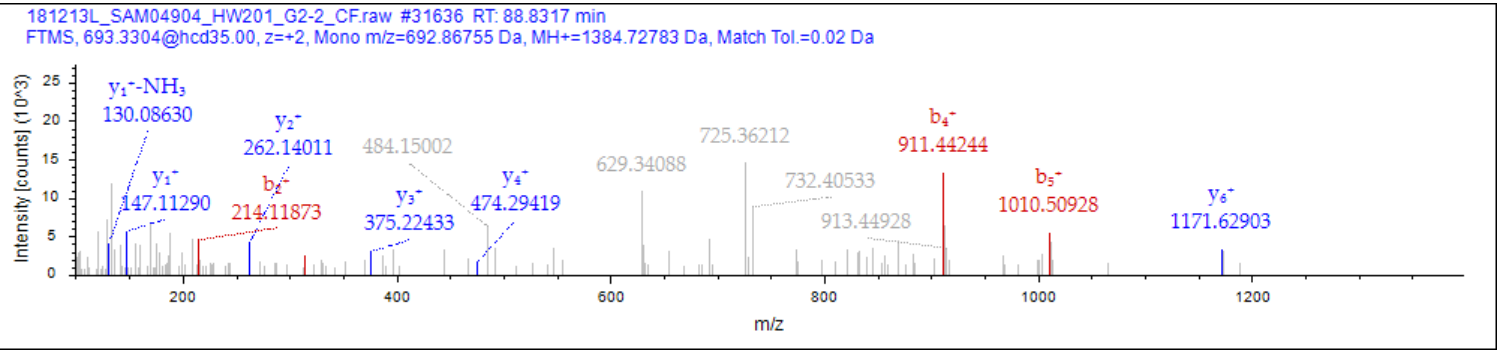

Sequence: NVVFVIDK

Charge: +2, Monoisotopic m/z: 692.86755 Da (+0.92 mmu/+1.33 ppm), $\mathrm{MH}+$ : 1384.72783 Da, RT: $88.8317 \mathrm{~min}$,

Identified with: Sequest HT (v1.17); XCorr:2.19,

Fragment match tolerance used for search: $0.02 \mathrm{Da}$

Fragments used for search: $-\mathrm{H}_{2} \mathrm{O} ; \mathrm{y} ;-\mathrm{NH}_{3} ; \mathrm{y} ; \mathrm{b} ; \mathrm{b} ;-\mathrm{H}_{2} \mathrm{O} ; \mathrm{b} ;-\mathrm{NH}_{3} ; \mathrm{y}$

Proteins (1):

- Inter-alpha-trypsin inhibitor heavy chain H4 OS=Homo sapiens OX=9606 GN=ITIH4 $P E=1 S V=4$

West, A. et al. Labeling preferences of diazirines with protein biomolecules, J. Am. Chem. S157 Soc. 2021. 


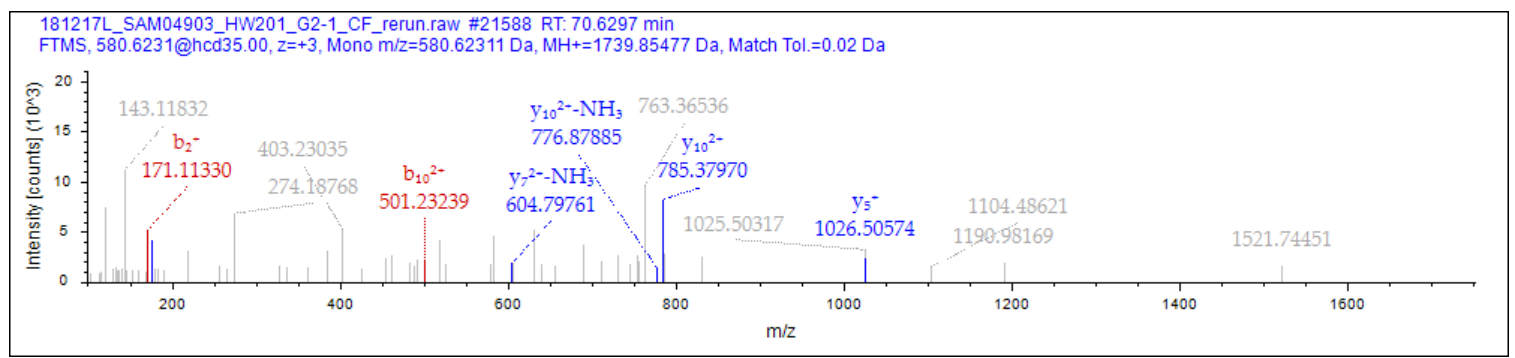

Sequence: GLCAIAQAESLR

Charge: +3 ,

Monoisotopic m/z: $580.62311 \mathrm{Da}$ (+0.46 mmu/+0.79 ppm),

$\mathrm{MH}+:$ 1739.85477 Da,

RT: $70.6297 \mathrm{~min}$,

Identified with: Sequest HT (v1.17); XCorr:1.18,

Fragment match tolerance used for search: $0.02 \mathrm{Da}$

Fragments used for search: $-\mathrm{H}_{2} \mathrm{O} ; \mathrm{y} ;-\mathrm{NH}_{3} ; \mathrm{y} ; \mathrm{b} ; \mathrm{b} ;-\mathrm{H}_{2} \mathrm{O} ; \mathrm{b} ;-\mathrm{NH}_{3} ; \mathrm{y}$

Proteins (1):

- 40S ribosomal protein S3 OS=Homo sapiens OX=9606 GN=RPS3 PE=1 SV=2<smiles>C#CCCC1(CCOc2cncc(C(=O)O)c2)N=N1</smiles>

JN Mod: JN-0021

PSMs: 0

Unique Sites: 0

West, A. et al. Labeling preferences of diazirines with protein biomolecules, J. Am. Chem. S158 Soc. 2021. 
<smiles>C#CCCC1(CCOc2ccc(C[C@H](N)C(=O)O)cc2)N=N1</smiles>

JN Mod: JN-0022

PSMs: 19

Unique Sites: 6

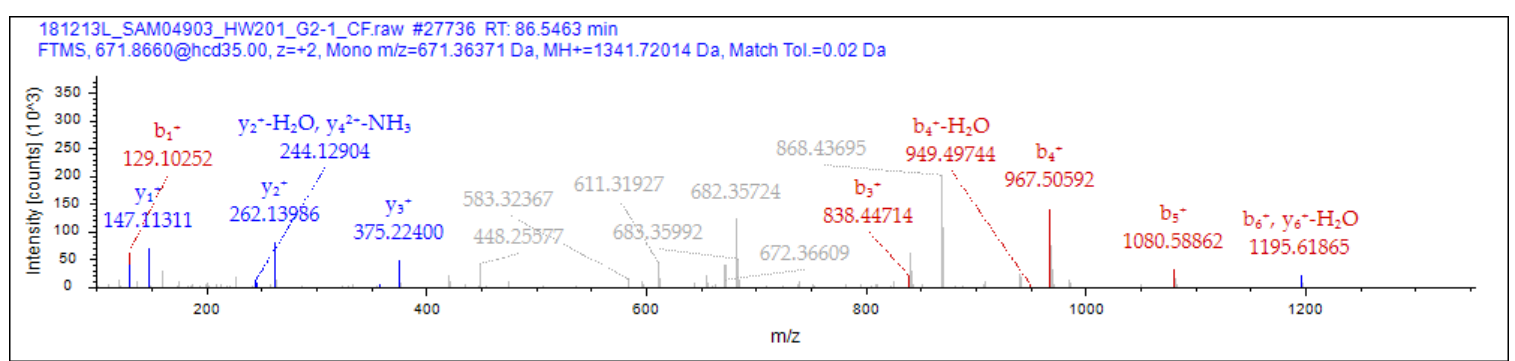

Sequence: KSKEIDK

Charge: +2 ,

Monoisotopic m/z: $671.36371 \mathrm{Da}(+1.99 \mathrm{mmu} /+2.97 \mathrm{ppm})$,

$\mathrm{MH}+:$ 1341.72014 Da,

RT: $86.5463 \mathrm{~min}$,

Identified with: Sequest HT (v1.17); XCorr:2.11,

Fragment match tolerance used for search: $0.02 \mathrm{Da}$

Fragments used for search: - $\mathrm{H}_{2} \mathrm{O} ; \mathrm{y} ;-\mathrm{NH}_{3} ; \mathrm{y} ; \mathrm{b} ; \mathrm{b} ;-\mathrm{H}_{2} \mathrm{O} ; \mathrm{b} ;-\mathrm{NH}_{3} ; \mathrm{y}$

Proteins (1):

- Guanine nucleotide-binding protein subunit alpha-13 OS=Homo sapiens OX=9606 $\mathrm{GN}=\mathrm{GNA} 13 \mathrm{PE}=1 \mathrm{SV}=2$

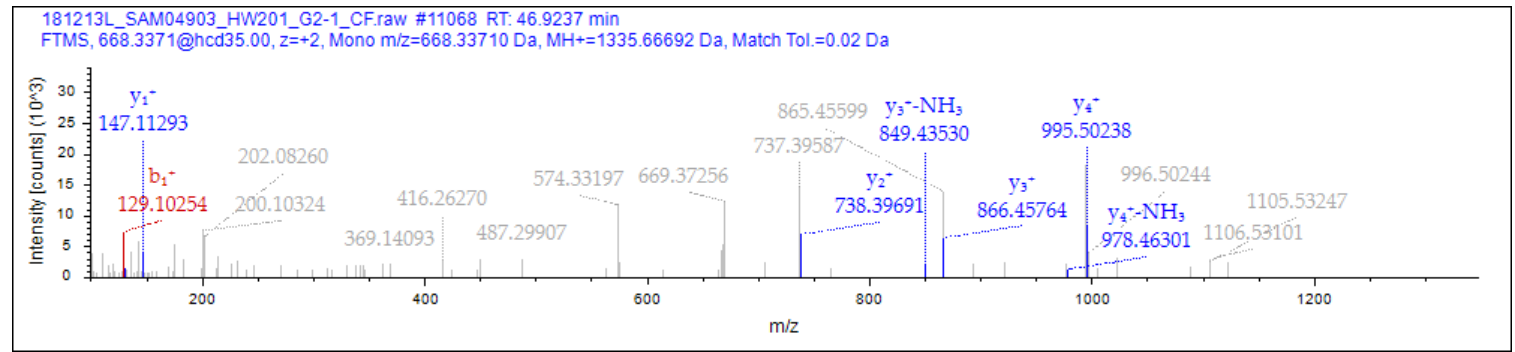

Sequence: KPDEQPK

Charge: +2 ,

Monoisotopic m/z: $668.33710 \mathrm{Da}(-1.15 \mathrm{mmu} /-1.71 \mathrm{ppm})$,

$\mathrm{MH}+:$ 1335.66692 Da,

RT: $46.9237 \mathrm{~min}$,

Identified with: Sequest HT (v1.17); XCorr:1.36,

Fragment match tolerance used for search: $0.02 \mathrm{Da}$

Fragments used for search: - $\mathrm{H}_{2} \mathrm{O} ; \mathrm{y} ;-\mathrm{NH}_{3} ; \mathrm{y} ; \mathrm{b} ; \mathrm{b} ;-\mathrm{H}_{2} \mathrm{O} ; \mathrm{b} ;-\mathrm{NH}_{3} ; \mathrm{y}$

Proteins (1):

- Sodium channel protein type 8 subunit alpha OS=Homo sapiens OX=9606 GN=SCN8A $\mathrm{PE}=1 \mathrm{SV}=1$

West, A. et al. Labeling preferences of diazirines with protein biomolecules, J. Am. Chem. S159 Soc. 2021. 


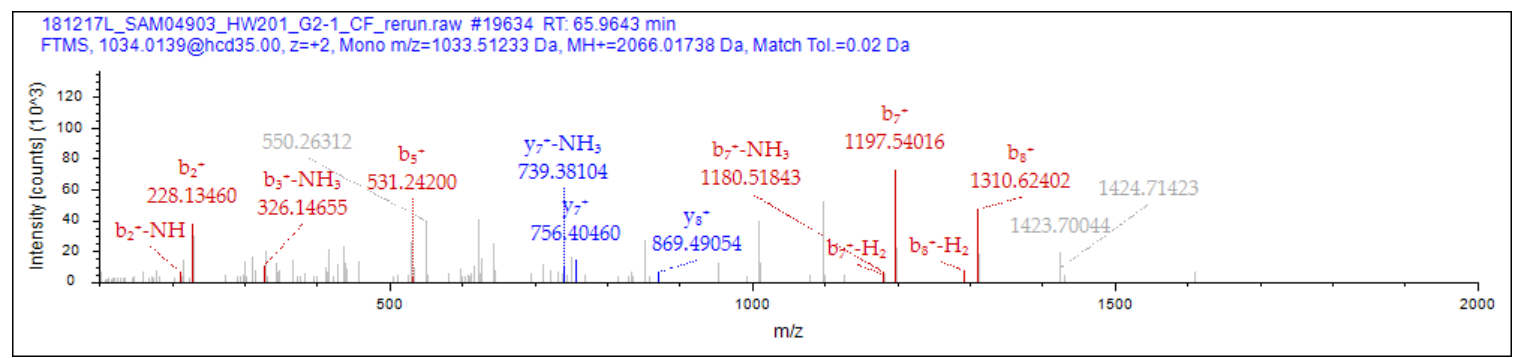

\section{Sequence: ARDGMTALHKAACAR}

Charge: +2 ,

Monoisotopic m/z: $1033.51233 \mathrm{Da}(+0.13 \mathrm{mmu} /+0.12 \mathrm{ppm})$,

$\mathrm{MH}+: 2066.01738 \mathrm{Da}$,

RT: $65.9643 \mathrm{~min}$,

Identified with: Sequest HT (v1.17); XCorr:1.13,

Fragment match tolerance used for search: $0.02 \mathrm{Da}$

Fragments used for search: - $\mathrm{H}_{2} \mathrm{O} ; \mathrm{y} ;-\mathrm{NH}_{3} ; \mathrm{y} ; \mathrm{b} ; \mathrm{b} ;-\mathrm{H}_{2} \mathrm{O} ; \mathrm{b} ;-\mathrm{NH}_{3} ; \mathrm{y}$

Proteins (1):

- $\quad$ SH3 and multiple ankyrin repeat domains protein 1 OS=Homo sapiens OX=9606 $\mathrm{GN}=\mathrm{SHANK} 1 \mathrm{PE}=1 \mathrm{SV}=2$

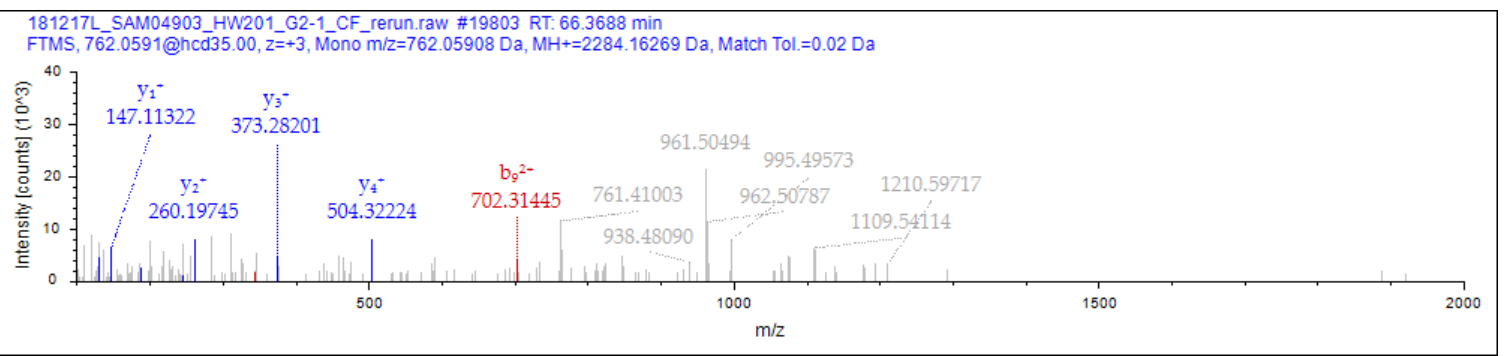

Sequence: AEAAGHRDTLYTMLIK

Charge: +3 ,

Monoisotopic m/z: $762.05908 \mathrm{Da}(+2.79 \mathrm{mmu} /+3.66 \mathrm{ppm})$,

$\mathrm{MH}+: 2284.16269 \mathrm{Da}$,

RT: $66.3688 \mathrm{~min}$,

Identified with: Sequest HT (v1.17); XCorr:1.42,

Fragment match tolerance used for search: $0.02 \mathrm{Da}$

Fragments used for search: - $\mathrm{H}_{2} \mathrm{O} ; \mathrm{y} ;-\mathrm{NH}_{3} ; \mathrm{y} ; \mathrm{b} ; \mathrm{b} ;-\mathrm{H}_{2} \mathrm{O} ; \mathrm{b} ;-\mathrm{NH}_{3} ; \mathrm{y}$

Proteins (1):

- Tumor necrosis factor receptor superfamily member 10B OS=Homo sapiens OX=9606 $\mathrm{GN}=\mathrm{TNFRSF} 10 \mathrm{~B} \mathrm{PE}=1 \mathrm{SV}=2$

West, A. et al. Labeling preferences of diazirines with protein biomolecules, J. Am. Chem. S160 Soc. 2021. 
<smiles>C#CCCC1(CCOc2ccc(CC(=O)O)cc2)N=N1</smiles>

JN Mod: JN-0013 and

PSMs: 26

Unique Sites: 7<smiles>C#CCCC1(CCOc2cccc(CC(=O)O)c2)N=N1</smiles>

$\mathrm{JN}-0017$

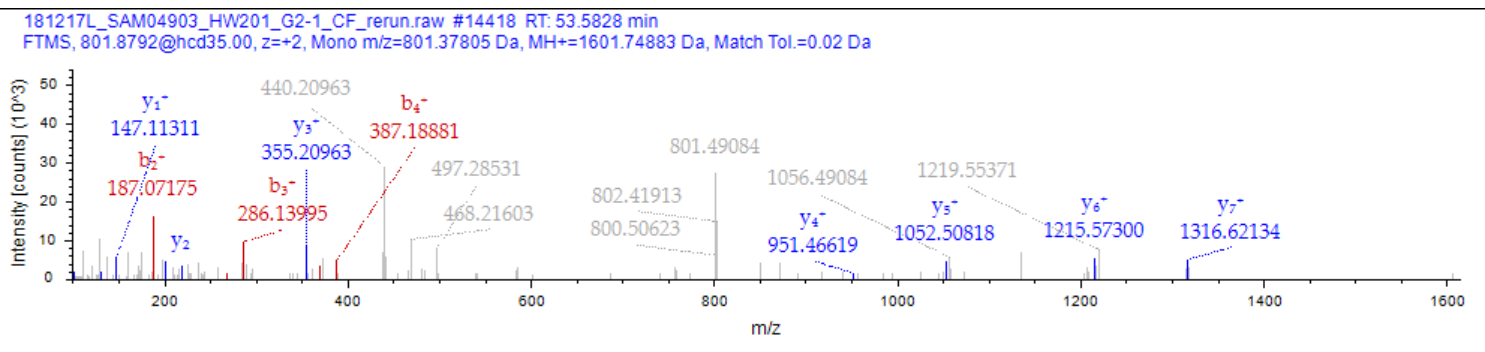

\section{Sequence: DAVTYTEHAK}

Charge: +2 ,

Monoisotopic m/z: 801.37805 Da (-0.83 mmu/-1.03 ppm),

$\mathrm{MH}+:$ 1601.74883 Da,

RT: $53.5828 \mathrm{~min}$,

Identified with: Sequest HT (v1.17); XCorr:2.10,

Fragment match tolerance used for search: $0.02 \mathrm{Da}$

Fragments used for search: $-\mathrm{H}_{2} \mathrm{O} ; \mathrm{y} ;-\mathrm{NH}_{3} ; \mathrm{y} ; \mathrm{b} ; \mathrm{b} ;-\mathrm{H}_{2} \mathrm{O} ; \mathrm{y}$

Proteins (1):

- Histone H4 OS=Homo sapiens OX=9606 GN=HIST1H4A PE=1 SV=2

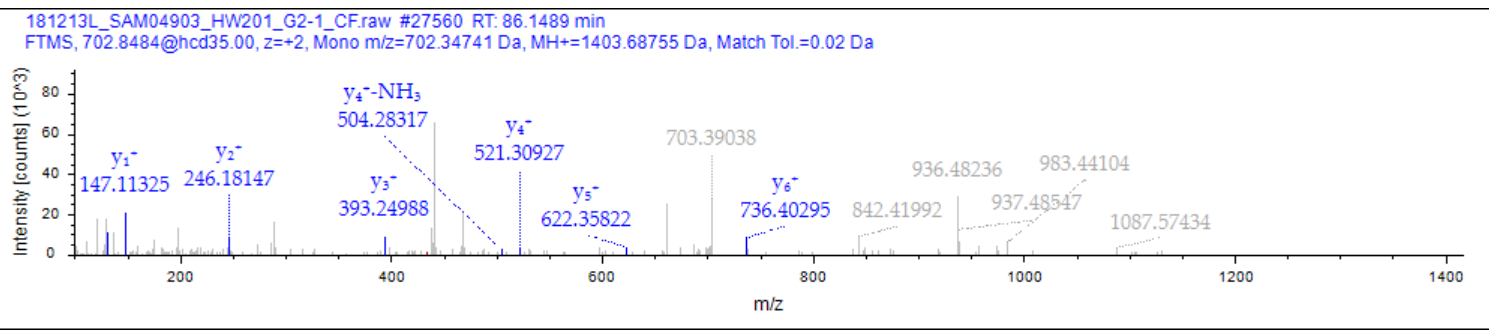

Sequence: AENTQFVK

Charge: +2, Monoisotopic m/z: $702.34741 \mathrm{Da}$ (+0.56 mm/+0.8 ppm),

$\mathrm{MH}+:$ 1403.68755 Da,

RT: 86.1489 min,

Identified with: Sequest HT (v1.17); XCorr:1.05,

Fragment match tolerance used for search: $0.02 \mathrm{Da}$

Fragments used for search: - $\mathrm{H}_{2} \mathrm{O} ; \mathrm{y} ;-\mathrm{NH}_{3} ; \mathrm{y} ; \mathrm{b} ; \mathrm{b} ;-\mathrm{H}_{2} \mathrm{O} ; \mathrm{b} ;-\mathrm{NH}_{3} ; \mathrm{y}$

Proteins (1):

- Heme oxygenase 2 OS=Homo sapiens OX=9606 GN=HMOX2 PE=1 SV=2

West, A. et al. Labeling preferences of diazirines with protein biomolecules, J. Am. Chem. S161 Soc. 2021. 


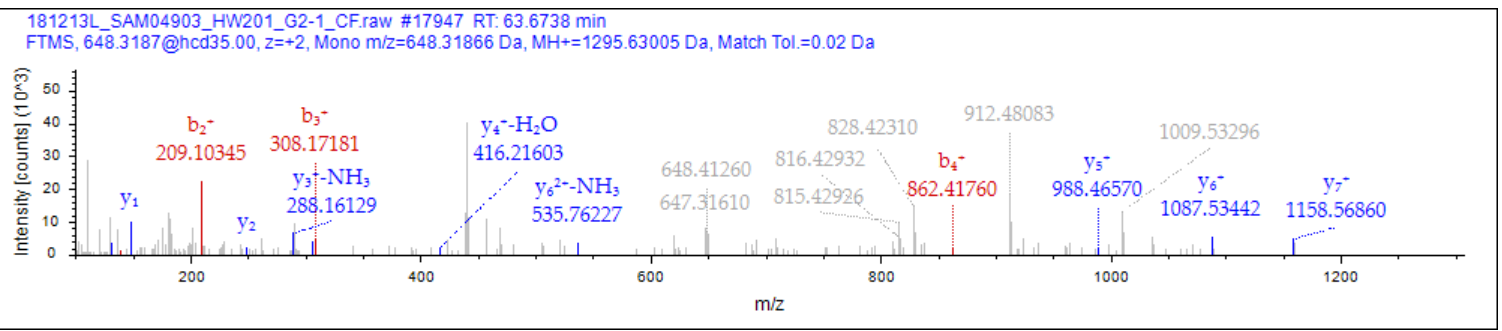

Sequence: HAVSEGTK

Charge: +2 ,

Monoisotopic m/z: $648.31866 \mathrm{Da}(+0.57 \mathrm{mmu} /+0.88 \mathrm{ppm})$,

$\mathrm{MH}+:$ 1295.63005 Da,

RT: $63.6738 \mathrm{~min}$,

Identified with: Sequest HT (v1.17); XCorr:1.95,

Fragment match tolerance used for search: $0.02 \mathrm{Da}$

Fragments used for search: $-\mathrm{H}_{2} \mathrm{O} ; \mathrm{y} ;-\mathrm{NH}_{3} ; \mathrm{y} ; \mathrm{b} ; \mathrm{b} ;-\mathrm{H}_{2} \mathrm{O} ; \mathrm{y}$

Proteins (15):

- Histone H2B type 2-E OS=Homo sapiens OX=9606 GN=HIST2H2BE PE=1 SV=3

- Histone H2B type 2-F OS=Homo sapiens $O X=9606 \mathrm{GN}=H I S T 2 H 2 B F P E=1 \mathrm{SV}=3$

- Histone H2B type 1-M OS=Homo sapiens OX=9606 GN=HIST1H2BM PE=1 SV=3

- Histone H2B type 1-B OS=Homo sapiens OX=9606 GN=HIST1H2BB PE=1 SV=2

- Histone H2B type 1-D OS=Homo sapiens $O X=9606 \mathrm{GN}=H I S T 1 H 2 B D P E=1 \mathrm{SV}=2$

- Histone H2B type 1-A OS=Homo sapiens OX=9606 GN=HIST1H2BA PE=1 SV=3

- Histone H2B type 1-H OS=Homo sapiens OX=9606 GN=HIST1H2BH PE=1 SV=3

- Histone H2B type 1-K OS=Homo sapiens OX=9606 GN=HIST1H2BK PE=1 SV=3

- Histone H2B type 1-L OS=Homo sapiens OX=9606 GN=HIST1H2BL PE=1 SV=3

- Histone H2B type 1-C/E/F/G/I OS=Homo sapiens OX=9606 GN=HIST1H2BC PE=1 $\mathrm{SV}=4$

- Histone H2B type F-S OS=Homo sapiens OX=9606 GN=H2BFS PE=1 SV=2

- Histone H2B type 3-B OS=Homo sapiens OX=9606 GN=HIST3H2BB PE=1 SV=3

- Histone H2B type 1-J OS=Homo sapiens OX=9606 GN=HIST1H2BJ PE=1 SV=3

- Histone H2B type 1-O OS=Homo sapiens OX=9606 GN=HIST1H2BO PE=1 SV=3

- Histone H2B type 1-N OS=Homo sapiens OX=9606 GN=HIST1H2BN PE=1 SV=3

West, A. et al. Labeling preferences of diazirines with protein biomolecules, J. Am. Chem. S162 Soc. 2021. 


\section{Group 3:}<smiles>C#CCCC(N)(CCN)CCCOc1ccc(CCN)cc1</smiles>

JN Mod: JN-00026

PSMs: 719

Unique Sites: 68

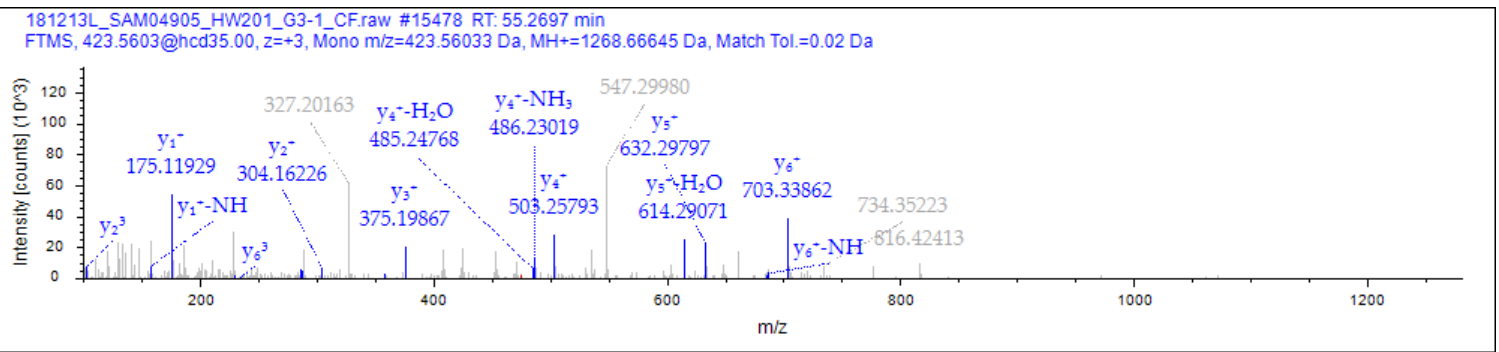

Sequence: LAEQAER

Charge: +3 ,

Monoisotopic m/z: $423.56152 \mathrm{Da}(+1.47 \mathrm{mmu} /+3.48 \mathrm{ppm})$,

$\mathrm{MH}+:$ 1268.67002 Da,

RT: $41.0711 \mathrm{~min}$,

Identified with: Sequest HT (v1.17);

XCorr:1.80,

Fragment match tolerance used for search: $0.02 \mathrm{Da}$

Fragments used for search: - $\mathrm{H}_{2} \mathrm{O} ; \mathrm{y} ;-\mathrm{NH}_{3} ; \mathrm{y} ; \mathrm{b} ; \mathrm{b} ;-\mathrm{H}_{2} \mathrm{O} ; \mathrm{b} ;-\mathrm{NH}_{3} ; \mathrm{y}$

Proteins (7):

- 14-3-3 protein zeta/delta OS=Homo sapiens OX=9606 GN=YWHAZ PE=1 SV=1

- 14-3-3 protein sigma OS=Homo sapiens $\mathrm{OX}=9606 \mathrm{GN}=\mathrm{SFN} P E=1 \mathrm{SV}=1$

- 14-3-3 protein beta/alpha OS=Homo sapiens OX=9606 GN=YWHAB PE=1 SV=3

- 14-3-3 protein eta OS=Homo sapiens OX=9606 GN=YWHAH PE=1 SV=4

- 14-3-3 protein gamma OS=Homo sapiens OX=9606 GN=YWHAG PE=1 SV=2

- 14-3-3 protein theta OS=Homo sapiens $\mathrm{OX}=9606 \mathrm{GN}=\mathrm{YWHAQ} P E=1 \mathrm{SV}=1$

- 14-3-3 protein epsilon OS=Homo sapiens OX=9606 GN=YWHAE PE=1 SV=1

West, A. et al. Labeling preferences of diazirines with protein biomolecules, J. Am. Chem. S163 Soc. 2021. 


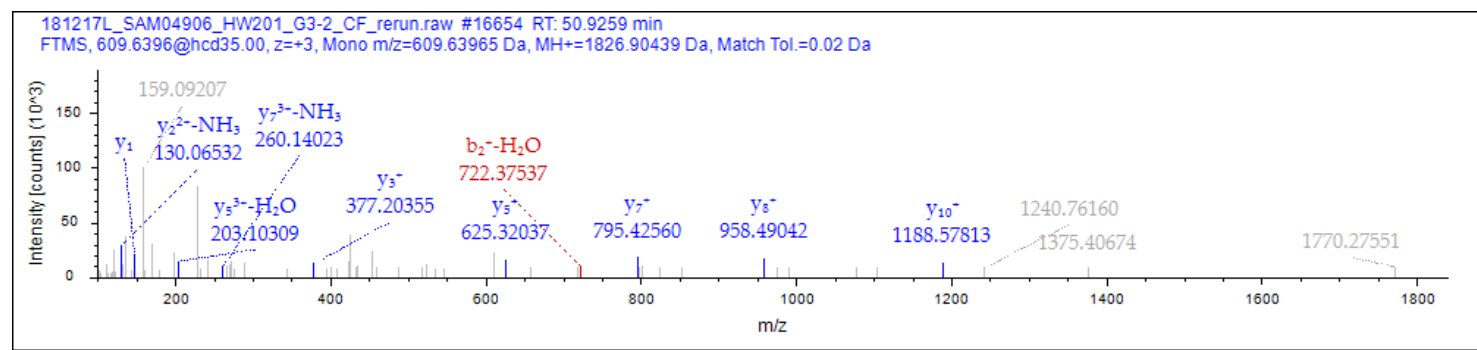

Sequence: WTEYGLTFTEK

Charge: +3 ,

Monoisotopic m/z: $609.63965 \mathrm{Da}(+0.73 \mathrm{mmu} /+1.2 \mathrm{ppm}), \mathrm{MH}+$ : $1826.90439 \mathrm{Da}$,

RT: 50.9259 min,

Identified with: Sequest HT (v1.17);

XCorr:1.05,

Fragment match tolerance used for search: $0.02 \mathrm{Da}$

Fragments used for search: - $\mathrm{H}_{2} \mathrm{O} ; \mathrm{y} ;-\mathrm{NH}_{3} ; \mathrm{y} ; \mathrm{b} ; \mathrm{b} ;-\mathrm{H}_{2} \mathrm{O} ; \mathrm{y}$

Proteins (1):

- Voltage-dependent anion-selective channel protein 1 OS=Homo sapiens $\mathrm{OX}=9606 \mathrm{GN}=\mathrm{VDAC} 1 \mathrm{PE}=1 \mathrm{SV}=2$

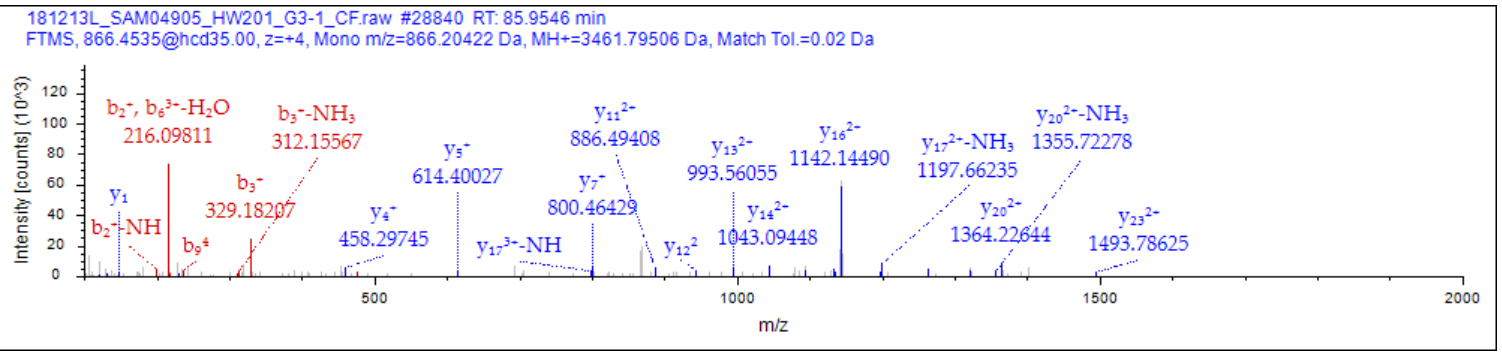

Sequence: SQIFSTASDNQPTVTIKVYEGERPLTK

Charge: +4 ,

Monoisotopic m/z: 866.20422 Da (-0.57 mmu/-0.66 ppm),

$\mathrm{MH}+: 3461.79506 \mathrm{Da}$,

RT: 85.9546 min,

Identified with: Sequest HT (v1.17);

XCorr:3.83,

Fragment match tolerance used for search: $0.02 \mathrm{Da}$

Fragments used for search: - $\mathrm{H}_{2} \mathrm{O} ; \mathrm{y} ;-\mathrm{NH}_{3} ; \mathrm{y} ; \mathrm{b} ; \mathrm{b} ;-\mathrm{H}_{2} \mathrm{O} ; \mathrm{b} ;-\mathrm{NH}_{3} ; \mathrm{y}$

Proteins (1):

- Endoplasmic reticulum chaperone BiP OS=Homo sapiens OX=9606 GN=HSPA5 PE=1 $\mathrm{SV}=2$

West, A. et al. Labeling preferences of diazirines with protein biomolecules, J. Am. Chem. S164 Soc. 2021. 


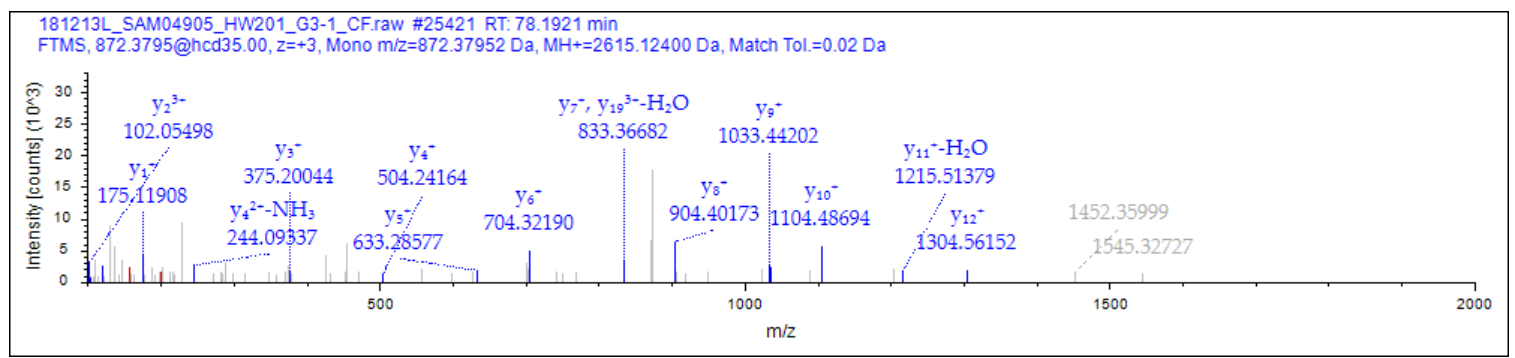

\section{Sequence: VGEEDEEAAEAEAEAEEAER}

Charge: +3 ,

Monoisotopic m/z: 872.37952 Da (-1.66 mmu/-1.91 ppm),

$\mathrm{MH}+:$ 2615.12400 Da,

RT: $78.1921 \mathrm{~min}$,

Identified with: Sequest HT (v1.17); XCorr:2.43,

Fragment match tolerance used for search: $0.02 \mathrm{Da}$

Fragments used for search: $-\mathrm{H}_{2} \mathrm{O} ; \mathrm{y} ;-\mathrm{NH}_{3} ; \mathrm{y} ; \mathrm{b} ; \mathrm{b} ;-\mathrm{H}_{2} \mathrm{O} ; \mathrm{y}$

Proteins (1):

- Neurosecretory protein VGF OS=Homo sapiens OX=9606 GN=VGF PE=1 SV=2

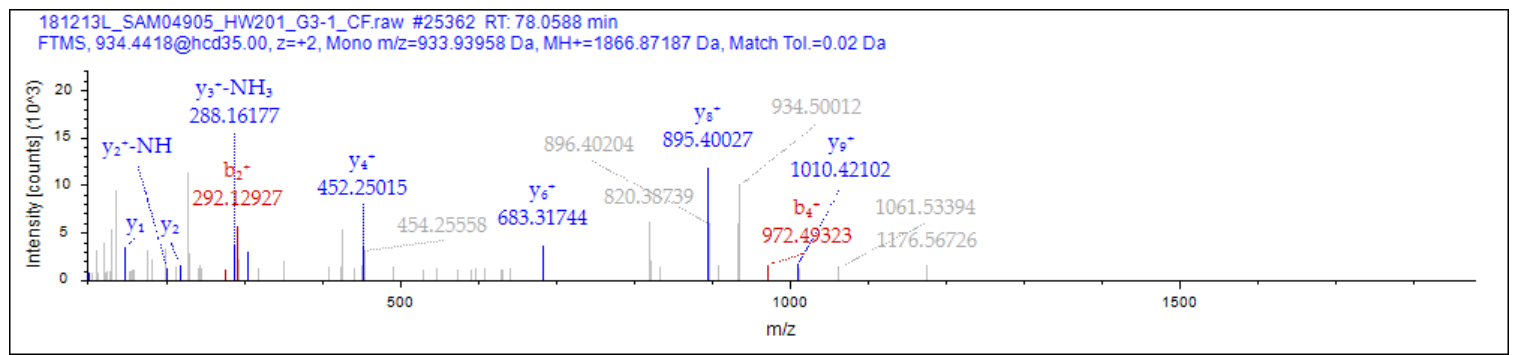

Sequence: YQIDPDACFSAK

Charge: +2 ,

Monoisotopic m/z: $933.93958 \mathrm{Da}(-1.74 \mathrm{mmu} /-1.86 \mathrm{ppm})$,

$\mathrm{MH}+:$ 1866.87187 Da,

RT: $78.0588 \mathrm{~min}$,

Identified with: Sequest HT (v1.17); XCorr:2.05,

Fragment match tolerance used for search: $0.02 \mathrm{Da}$

Fragments used for search: $-\mathrm{H}_{2} \mathrm{O} ; \mathrm{y} ;-\mathrm{NH}_{3} ; \mathrm{y} ; \mathrm{b} ; \mathrm{b} ;-\mathrm{H}_{2} \mathrm{O} ; \mathrm{b} ;-\mathrm{NH}_{3} ; \mathrm{y}$

Proteins (1):

- Voltage-dependent anion-selective channel protein 1 OS=Homo sapiens $\mathrm{OX}=9606 \mathrm{GN}=\mathrm{VDAC} 1 \mathrm{PE}=1 \mathrm{SV}=2$

West, A. et al. Labeling preferences of diazirines with protein biomolecules, J. Am. Chem. S165 Soc. 2021. 
<smiles>C#CCCC1(CCOc2ccc(CCC(=O)O)cc2)N=N1</smiles>

JN Mod: JN-00033

PSMs: 25

Unique Sites: 13

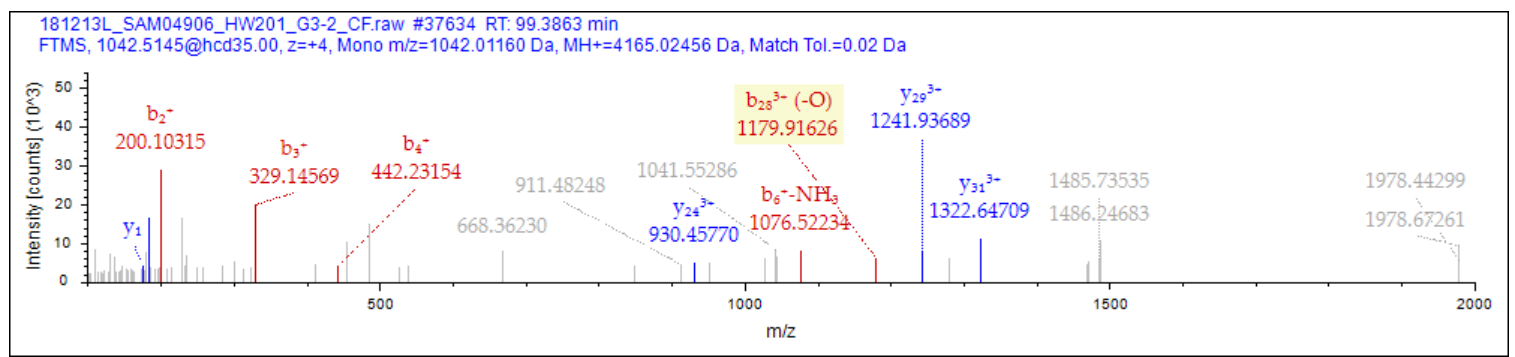

Sequence: AQELGLAPDMFFCLRLLEETGICVVPGSGFGQR

Charge: +4 ,

Monoisotopic m/z: $1042.01160 \mathrm{Da}(-0.23 \mathrm{mmu} /-0.22 \mathrm{ppm})$,

$\mathrm{MH}+:$ 4165.02456 Da,

RT: $99.3863 \mathrm{~min}$,

Identified with: Sequest HT (v1.17); XCorr:1.29,

Fragment match tolerance used for search: $0.02 \mathrm{Da}$

Fragments used for search: - $\mathrm{H}_{2} \mathrm{O} ; \mathrm{y} ;-\mathrm{NH}_{3} ; \mathrm{y} ; \mathrm{b} ; \mathrm{b} ;-\mathrm{H}_{2} \mathrm{O} ; \mathrm{b} ;-\mathrm{NH}_{3} ; \mathrm{y}$

Proteins (1):

- Alanine aminotransferase $1 \mathrm{OS}=$ Homo sapiens $\mathrm{OX}=9606 \mathrm{GN}=\mathrm{GPT} P E=1 \mathrm{SV}=3$

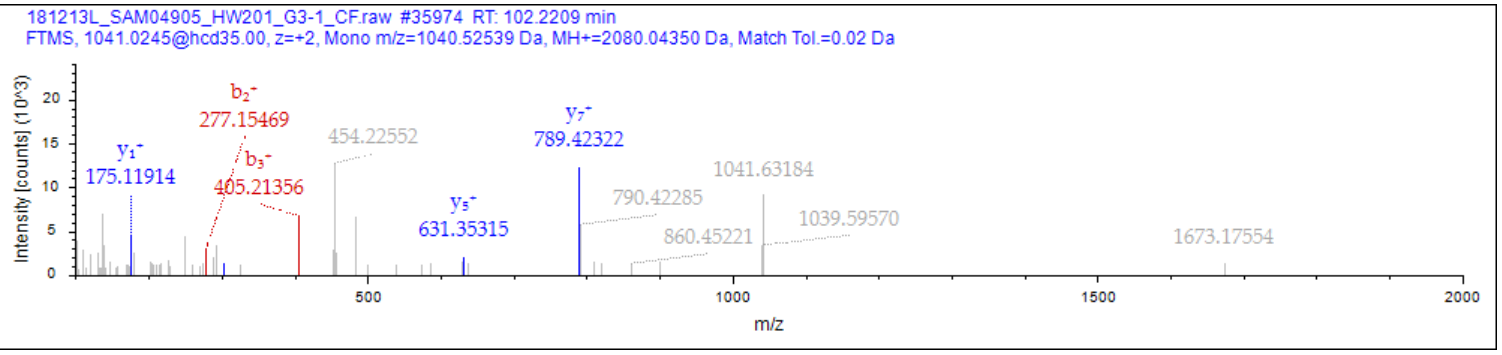

Sequence: IYQIYEGTSQIQR

Charge: +2 ,

Monoisotopic m/z: $1040.52539 \mathrm{Da}(+1.3 \mathrm{mmu} /+1.25 \mathrm{ppm})$,

$\mathrm{MH}+:$ 2080.04350 Da,

RT: $102.2209 \mathrm{~min}$,

Identified with: Sequest HT (v1.17); XCorr:1.45,

Fragment match tolerance used for search: $0.02 \mathrm{Da}$

Fragments used for search: - $\mathrm{H}_{2} \mathrm{O} ; \mathrm{y} ;-\mathrm{NH}_{3} ; \mathrm{y} ; \mathrm{b} ; \mathrm{b} ;-\mathrm{H}_{2} \mathrm{O} ; \mathrm{b} ;-\mathrm{NH}_{3} ; \mathrm{y}$

Proteins (1):

- Medium-chain specific acyl-CoA dehydrogenase, mitochondrial OS=Homo sapiens $\mathrm{OX}=9606 \mathrm{GN}=\mathrm{ACADM} \mathrm{PE}=1 \mathrm{SV}=1$

West, A. et al. Labeling preferences of diazirines with protein biomolecules, J. Am. Chem. S166 Soc. 2021. 


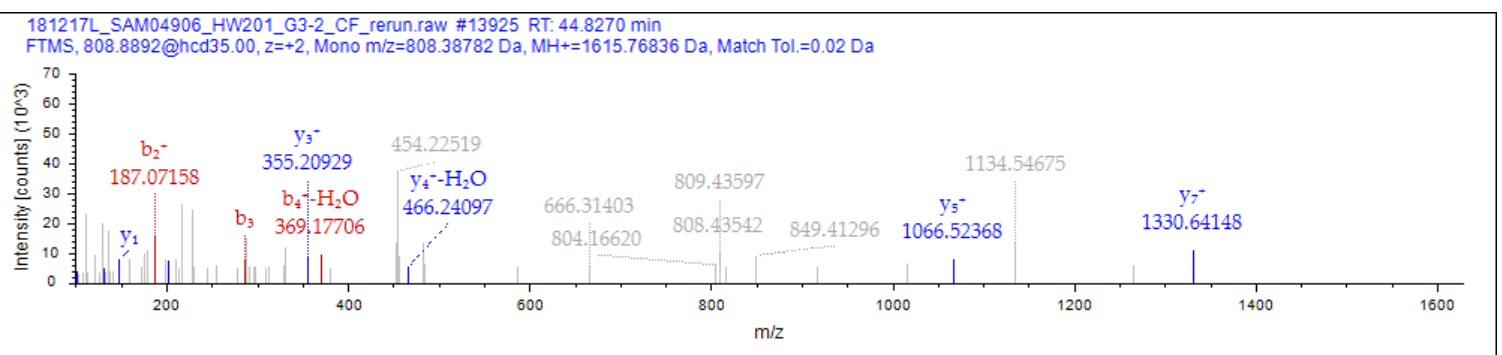

Sequence: DAVTYTEHAK

Charge: +2 ,

Monoisotopic m/z: 808.38782 Da (+1.09 mmu/+1.35 ppm),

$\mathrm{MH}+:$ 1615.76836 Da,

RT: $44.8270 \mathrm{~min}$,

Identified with: Sequest HT (v1.17); XCorr:1.33,

Fragment match tolerance used for search: $0.02 \mathrm{Da}$

Fragments used for search: $-\mathrm{H}_{2} \mathrm{O} ; \mathrm{y} ;-\mathrm{NH}_{3} ; \mathrm{y} ; \mathrm{b} ; \mathrm{b} ;-\mathrm{H}_{2} \mathrm{O} ; \mathrm{y}$

Proteins (1):

- Histone H4 OS=Homo sapiens OX=9606 GN=HIST1H4A PE=1 SV=2

West, A. et al. Labeling preferences of diazirines with protein biomolecules, J. Am. Chem. S167 Soc. 2021. 


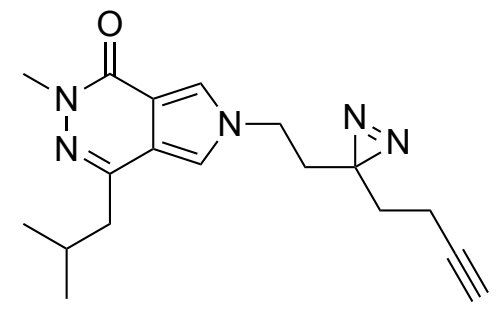

JN Mod: JN-0024

PSMs: 98

Unique Sites: 22

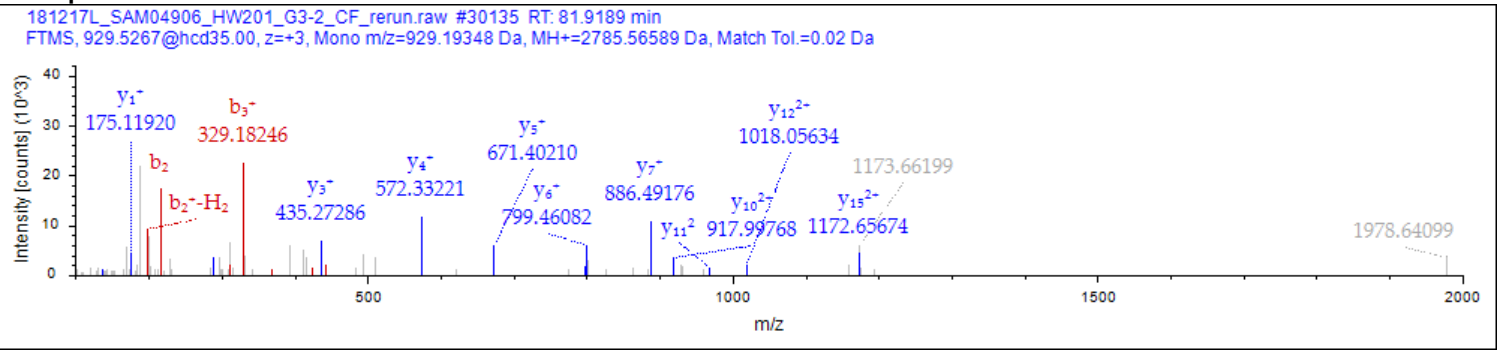

Sequence: TLNIPVLTVIEWSQVHFLR

Charge: +3 ,

Monoisotopic m/z: $929.19348 \mathrm{Da}(+2.8 \mathrm{mmu} /+3.01 \mathrm{ppm})$,

$\mathrm{MH}+:$ 2785.56589 Da,

RT: 81.9189 min,

Identified with: Sequest HT (v1.17); XCorr:3.04,

Fragment match tolerance used for search: $0.02 \mathrm{Da}$

Fragments used for search: $-\mathrm{H}_{2} \mathrm{O} ; \mathrm{y} ;-\mathrm{NH}_{3} ; \mathrm{y} ; \mathrm{b} ; \mathrm{b} ;-\mathrm{H}_{2} \mathrm{O} ; \mathrm{b} ;-\mathrm{NH}_{3} ; \mathrm{y}$

Proteins (1):

- Lysosome membrane protein 2 OS=Homo sapiens OX=9606 GN=SCARB2 PE=1 SV=2

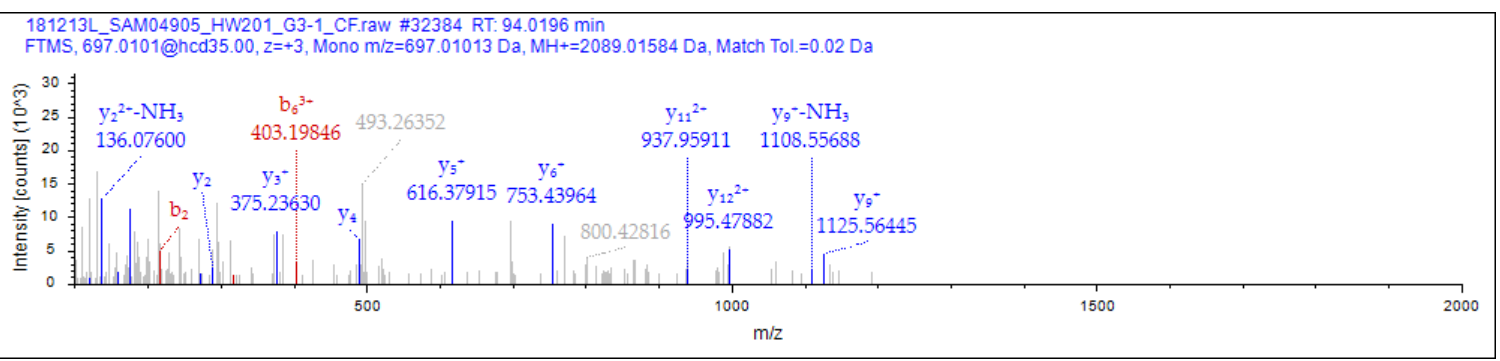

Sequence: VDNDENEHQLSLR

Charge: +3 ,

Monoisotopic m/z: 697.01013 Da (+1.27 mmu/+1.82 ppm),

$\mathrm{MH}+: 2089.01584 \mathrm{Da}$,

RT: $94.0196 \mathrm{~min}$,

Identified with: Sequest HT (v1.17); XCorr:2.80,

Fragment match tolerance used for search: $0.02 \mathrm{Da}$

Fragments used for search: - $\mathrm{H}_{2} \mathrm{O} ; \mathrm{y} ;-\mathrm{NH}_{3} ; \mathrm{y} ; \mathrm{b} ; \mathrm{b} ;-\mathrm{H}_{2} \mathrm{O} ; \mathrm{b} ;-\mathrm{NH}_{3} ; \mathrm{y}$

Proteins (1):

- Nucleophosmin OS=Homo sapiens OX=9606 GN=NPM1 PE=1 SV=2

West, A. et al. Labeling preferences of diazirines with protein biomolecules, J. Am. Chem. S168 Soc. 2021. 


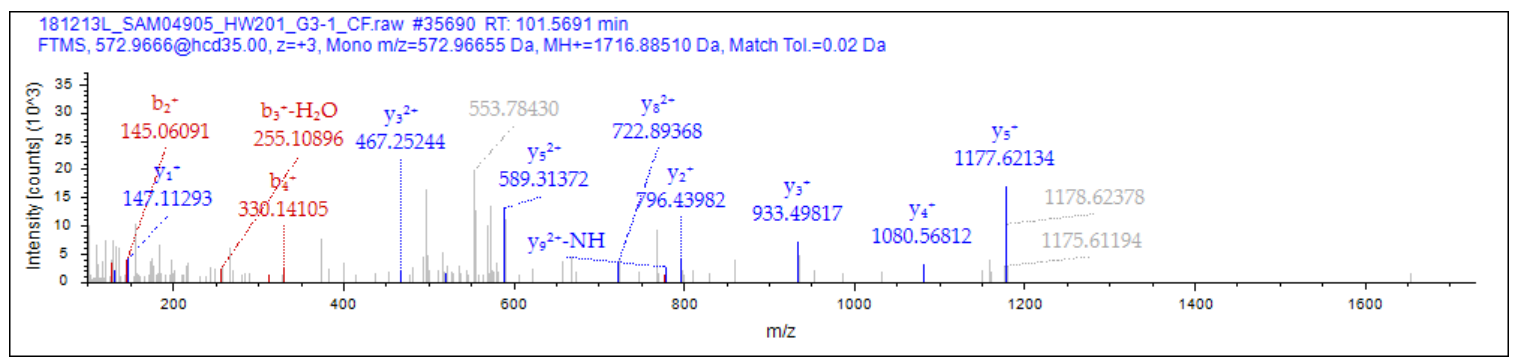

\section{Sequence: GSQGPLPFHEK}

Charge: +3 ,

Monoisotopic m/z: 572.96655 Da (-0.95 mmu/-1.66 ppm),

$\mathrm{MH}+:$ 1716.88510 Da,

RT: $101.5691 \mathrm{~min}$,

Identified with: Sequest HT (v1.17); XCorr:2.42,

Fragment match tolerance used for search: $0.02 \mathrm{Da}$

Fragments used for search: - $\mathrm{H}_{2} \mathrm{O} ; \mathrm{y} ;-\mathrm{NH}_{3} ; \mathrm{y} ; \mathrm{b} ; \mathrm{b} ;-\mathrm{H}_{2} \mathrm{O} ; \mathrm{b} ;-\mathrm{NH}_{3} ; \mathrm{y}$

Proteins (1):

- Mitochondrial dicarboxylate carrier OS=Homo sapiens OX=9606 GN=SLC25A10 PE=1 $\mathrm{SV}=2$

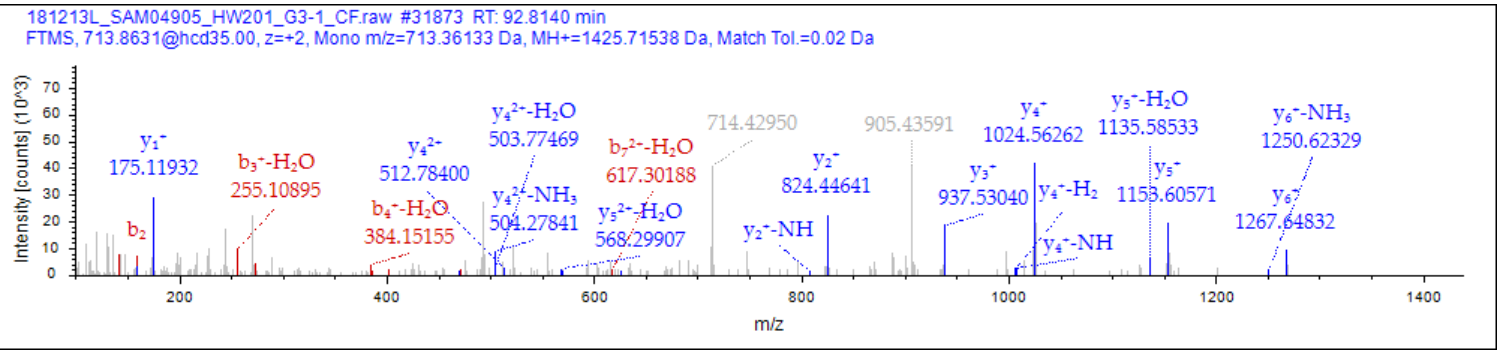

Sequence: GTNESLER

Charge: +2 ,

Monoisotopic m/z: 713.36133 Da (+0.49 mmu/+0.69 ppm),

$\mathrm{MH}+:$ 1425.71538 Da,

RT: $92.8140 \mathrm{~min}$,

Identified with: Sequest HT (v1.17); XCorr:2.12,

Fragment match tolerance used for search: $0.02 \mathrm{Da}$

Fragments used for search: - $\mathrm{H}_{2} \mathrm{O} ; \mathrm{y} ;-\mathrm{NH}_{3} ; \mathrm{y} ; \mathrm{b} ; \mathrm{b} ;-\mathrm{H}_{2} \mathrm{O} ; \mathrm{b} ;-\mathrm{NH}_{3} ; \mathrm{y}$

Proteins (2):

- Glial fibrillary acidic protein OS=Homo sapiens OX=9606 GN=GFAP PE=1 SV=1

- Vimentin OS=Homo sapiens OX=9606 GN=VIM PE=1 SV=4

West, A. et al. Labeling preferences of diazirines with protein biomolecules, J. Am. Chem. S169 Soc. 2021. 
<smiles>C#CCCC1(CCOc2ccc(C(=O)C(=O)O)cc2)N=N1</smiles>

JN Mod: JN-00032

PSMs: 2

Unique Sites: 1

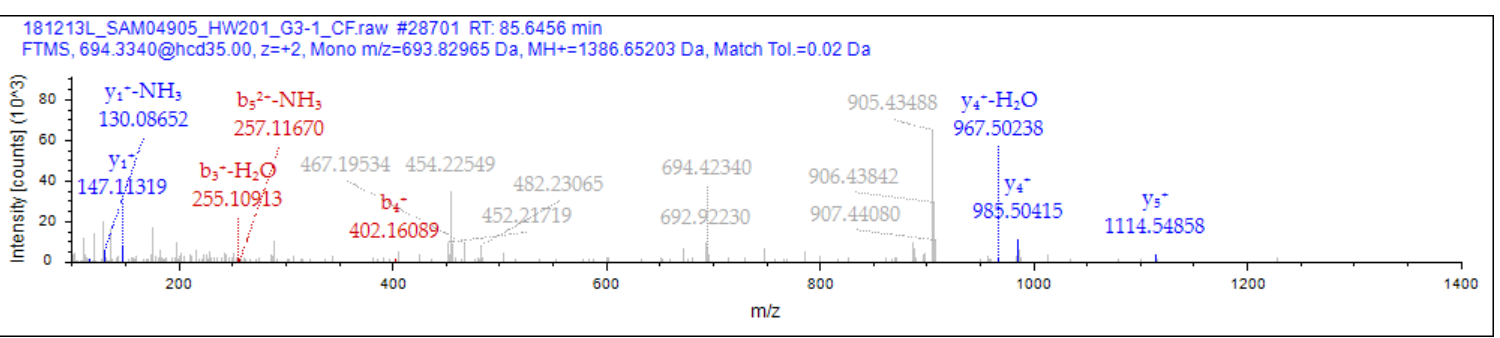

Sequence: GSQEKQTK

Charge: +2 ,

Monoisotopic m/z: $693.82965 \mathrm{Da}(-1.93 \mathrm{mmu} /-2.78 \mathrm{ppm})$,

$\mathrm{MH}+:$ 1386.65203 Da,

RT: 85.6456 min,

Identified with: Sequest HT (v1.17); XCorr:1.02,

Fragment match tolerance used for search: $0.02 \mathrm{Da}$

Fragments used for search: - $\mathrm{H}_{2} \mathrm{O} ; \mathrm{y} ;-\mathrm{NH}_{3} ; \mathrm{y} ; \mathrm{b} ; \mathrm{b} ;-\mathrm{H}_{2} \mathrm{O} ; \mathrm{b} ;-\mathrm{NH}_{3} ; \mathrm{y}$

Proteins (1):

- Bcl-2-associated transcription factor $1 \mathrm{OS}=$ Homo sapiens $\mathrm{OX}=9606 \mathrm{GN}=\mathrm{BCLAF} 1 \mathrm{PE}=1$ $\mathrm{SV}=2$ 
<smiles>C#CCCC1(CCOc2cccc(C[C@H](N)C(=O)O)c2)N=N1</smiles>

JN Mod: JN-00028

PSMs: 29

Unique Sites: 7

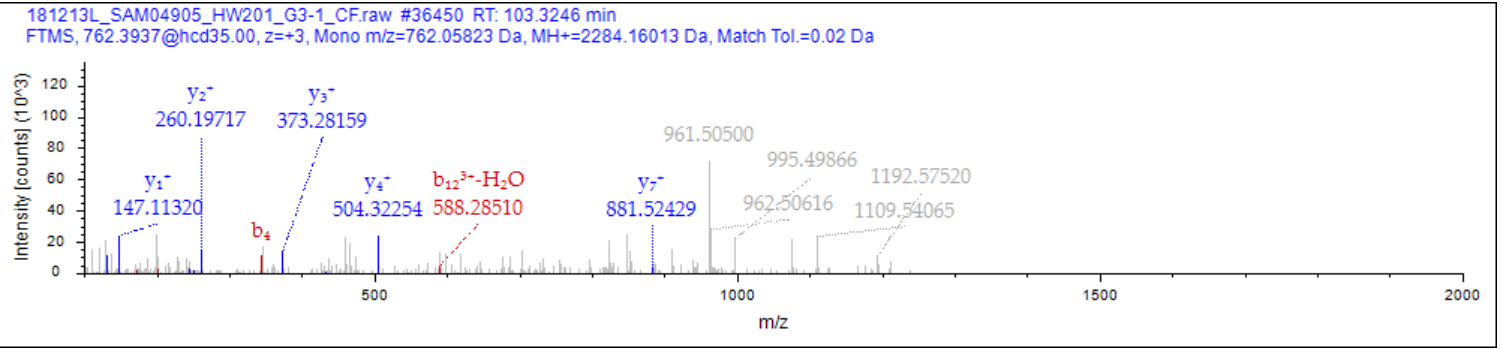

Sequence: AEAAGHRDTLYTMLIK

Charge: +3 ,

Monoisotopic m/z: $762.05823 \mathrm{Da}$ (+1.93 mmu/+2.54 ppm),

$\mathrm{MH}+:$ 2284.16013 Da,

RT: 103.3246 min,

Identified with: Sequest HT (v1.17); XCorr:2.13,

Fragment match tolerance used for search: $0.02 \mathrm{Da}$

Fragments used for search: - $\mathrm{H}_{2} \mathrm{O} ; \mathrm{y} ;-\mathrm{NH}_{3} ; \mathrm{y} ; \mathrm{b} ; \mathrm{b} ;-\mathrm{H}_{2} \mathrm{O} ; \mathrm{b} ;-\mathrm{NH}_{3} ; \mathrm{y}$

Proteins (1):

- Tumor necrosis factor receptor superfamily member 10B OS=Homo sapiens OX=9606 $\mathrm{GN}=\mathrm{TNFRSF} 10 \mathrm{~B} \mathrm{PE}=1 \mathrm{SV}=2$

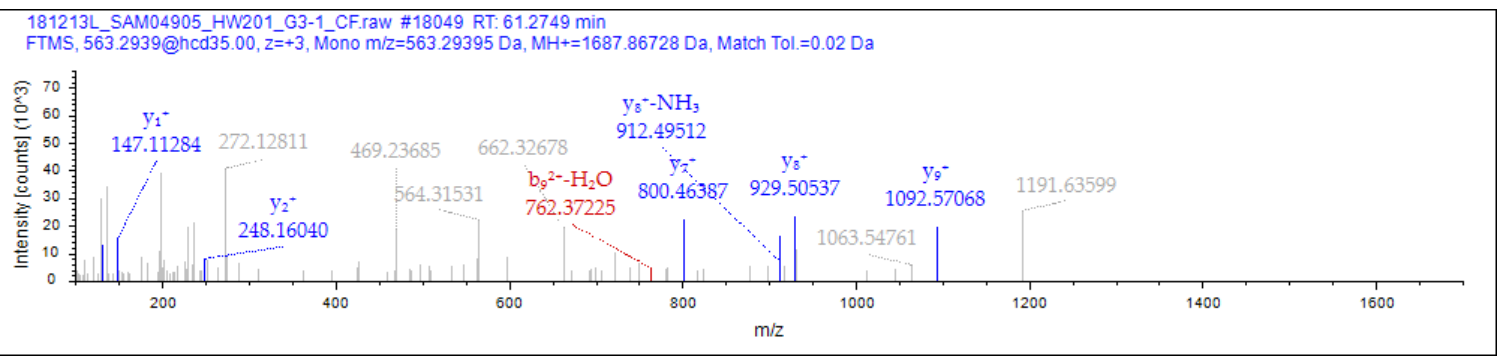

Sequence: VYEGERPLTK

Charge: +3, Monoisotopic m/z: 563.29395 Da (-1.35 mmu/-2.39 ppm),

$\mathrm{MH}+: 1687.86728 \mathrm{Da}$,

RT: 61.2749 min,

Identified with: Sequest HT (v1.17); XCorr:1.32,

Fragment match tolerance used for search: $0.02 \mathrm{Da}$

Fragments used for search: - $\mathrm{H}_{2} \mathrm{O} ; \mathrm{y} ;-\mathrm{NH}_{3} ; \mathrm{y} ; \mathrm{b} ; \mathrm{b} ;-\mathrm{H}_{2} \mathrm{O} ; \mathrm{b} ;-\mathrm{NH}_{3} ; \mathrm{y}$

Proteins (1):

- Endoplasmic reticulum chaperone BiP OS=Homo sapiens OX=9606 GN=HSPA5 PE=1 $\mathrm{SV}=2$ 


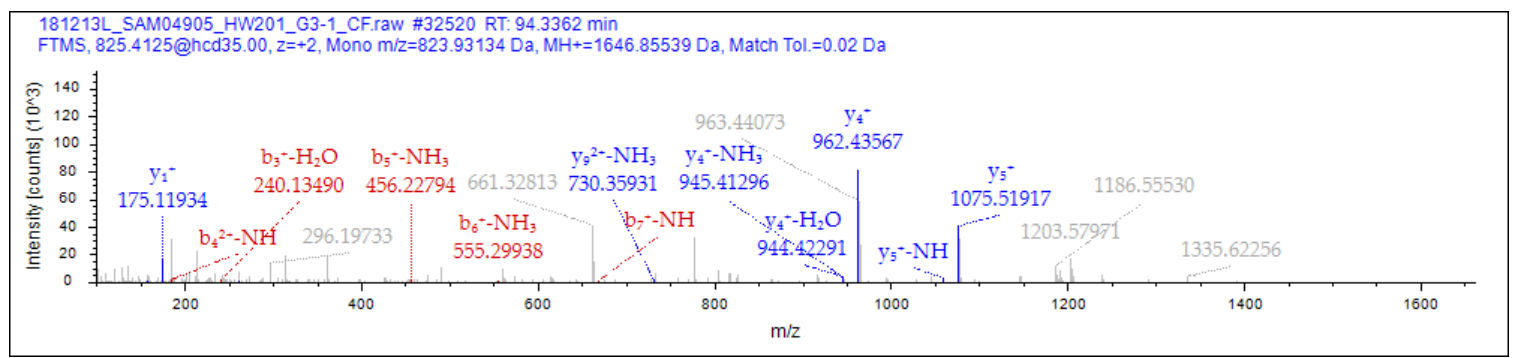

Sequence: LGSKSVLSTCR

Charge: +2 ,

Monoisotopic m/z: 823.93134 Da (-1.99 mmu/-2.41 ppm),

$\mathrm{MH}+:$ 1646.85539 Da,

RT: $94.3362 \mathrm{~min}$,

Identified with: Sequest HT (v1.17); XCorr:1.02,

Fragment match tolerance used for search: $0.02 \mathrm{Da}$

Fragments used for search: - $\mathrm{H}_{2} \mathrm{O} ; \mathrm{y} ;-\mathrm{NH}_{3} ; \mathrm{y} ; \mathrm{b} ; \mathrm{b} ;-\mathrm{H}_{2} \mathrm{O} ; \mathrm{b} ;-\mathrm{NH}_{3} ; \mathrm{y}$

Proteins (1):

- Reelin OS=Homo sapiens OX=9606 GN=RELN PE=1 SV=3

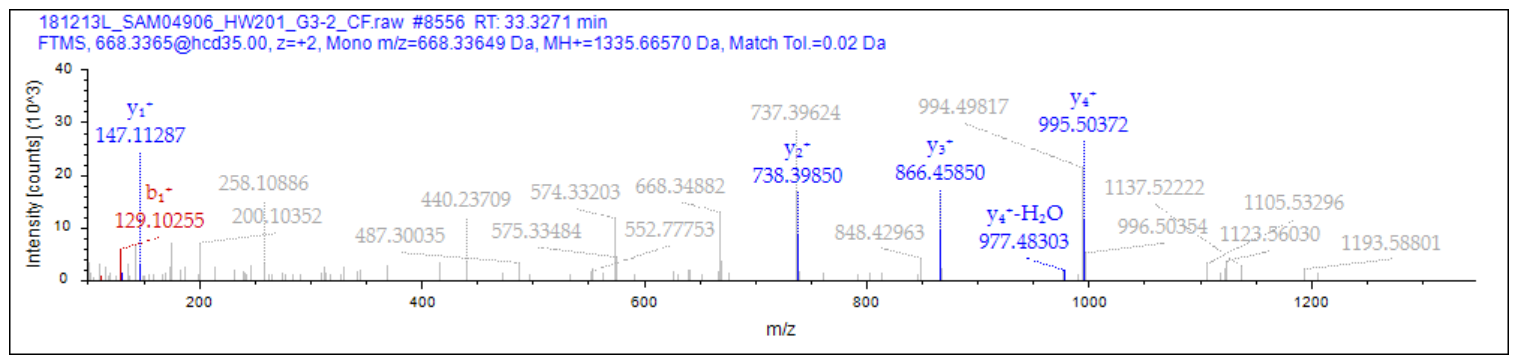

Sequence: KPDEQPK

Charge: +2 ,

Monoisotopic m/z: 668.33649 Da (-1.76 mmu/-2.63 ppm),

$\mathrm{MH}+:$ 1335.66570 Da,

RT: $33.3271 \mathrm{~min}$,

Identified with: Sequest HT (v1.17); XCorr:1.46,

Fragment match tolerance used for search: $0.02 \mathrm{Da}$

Fragments used for search: $-\mathrm{H}_{2} \mathrm{O} ; \mathrm{y} ;-\mathrm{NH}_{3} ; \mathrm{y} ; \mathrm{b} ; \mathrm{b} ;-\mathrm{H}_{2} \mathrm{O} ; \mathrm{b} ;-\mathrm{NH}_{3} ; \mathrm{y}$

Proteins (1):

- Sodium channel protein type 8 subunit alpha OS=Homo sapiens OX=9606 GN=SCN8A $\mathrm{PE}=1 \mathrm{SV}=1$

West, A. et al. Labeling preferences of diazirines with protein biomolecules, J. Am. Chem. S172 Soc. 2021. 


\section{Group 4:}

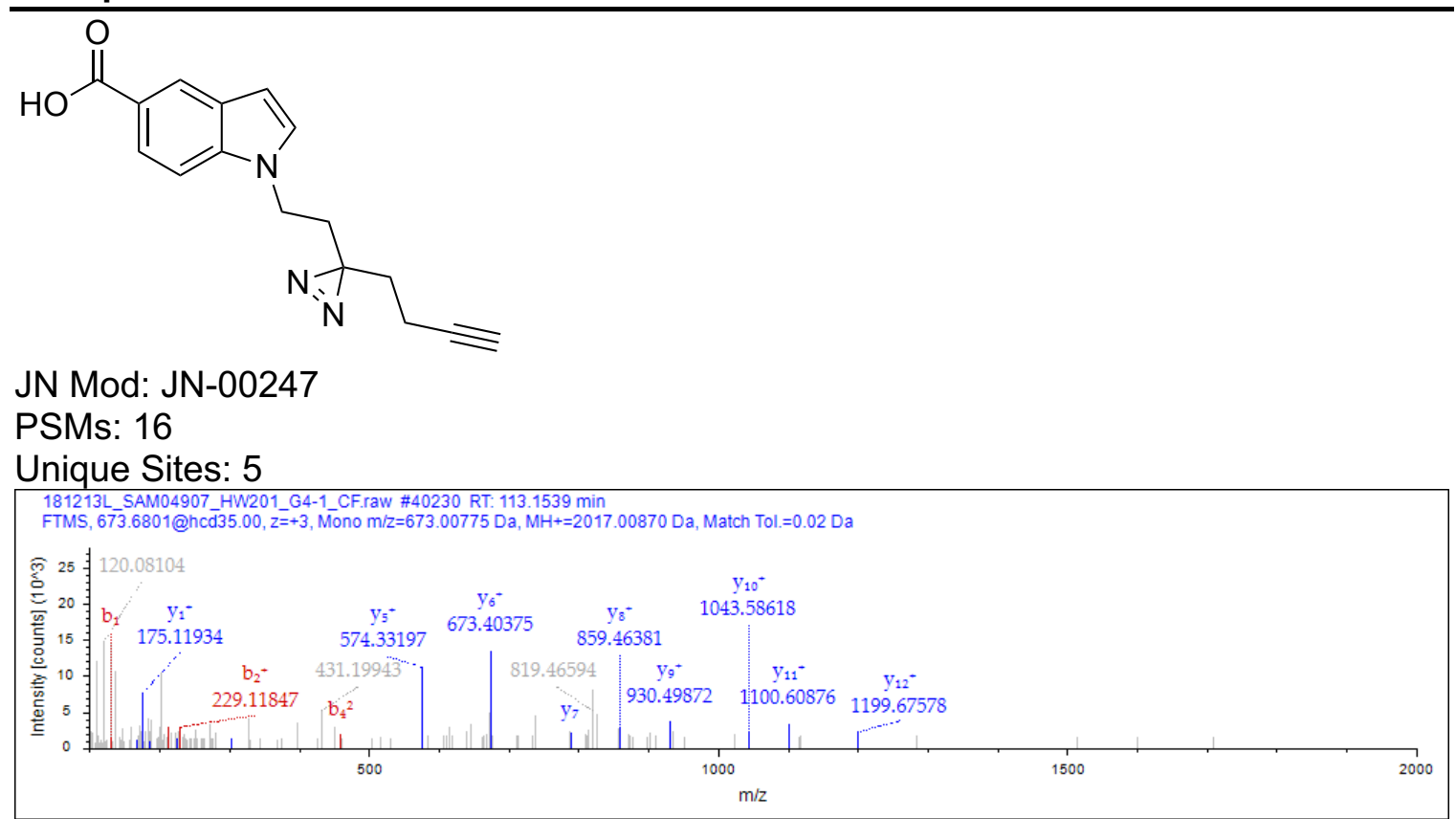

Sequence: EVDVGLAADVGTLQR

Charge: +3 ,

Monoisotopic m/z: $673.00775 \mathrm{Da}(-1.71 \mathrm{mmu} /-2.53 \mathrm{ppm})$,

$\mathrm{MH}+:$ 2017.00870 Da,

RT: 113.1539 min,

Identified with: Sequest HT (v1.17); XCorr:2.47,

Fragment match tolerance used for search: $0.02 \mathrm{Da}$

Fragments used for search: - $\mathrm{H}_{2} \mathrm{O} ; \mathrm{y} ;-\mathrm{NH}_{3} ; \mathrm{y} ; \mathrm{b} ; \mathrm{b} ;-\mathrm{H}_{2} \mathrm{O} ; \mathrm{b} ;-\mathrm{NH}_{3} ; \mathrm{y}$

Proteins (1):

- Delta(3,5)-Delta(2,4)-dienoyl-CoA isomerase, mitochondrial OS=Homo sapiens $\mathrm{OX}=9606 \mathrm{GN}=\mathrm{ECH} 1 \mathrm{PE}=1 \mathrm{SV}=2$

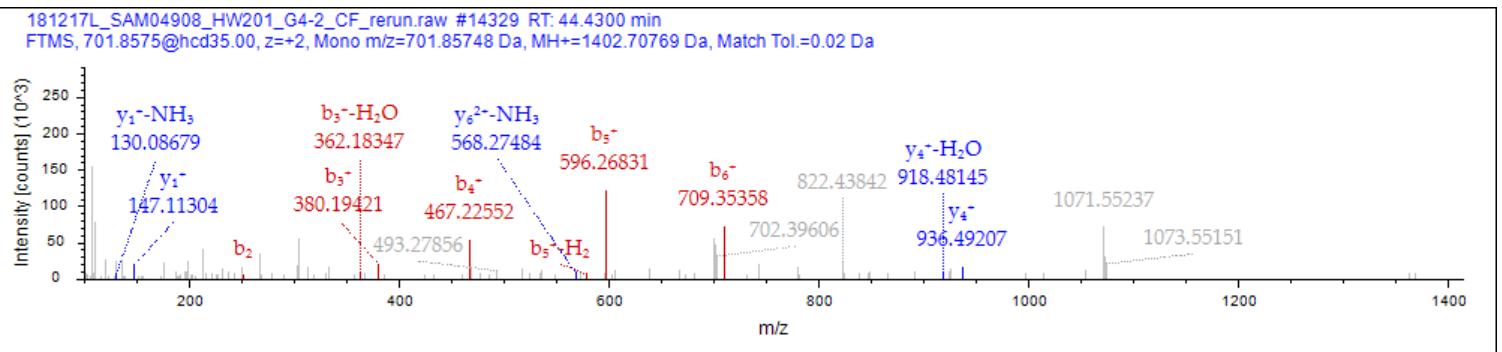

Sequence: HLESELAK

Charge: +2, Monoisotopic m/z: $701.85748 \mathrm{Da}(+2.61 \mathrm{mmu} /+3.72 \mathrm{ppm})$,

$\mathrm{MH}+:$ 1402.70769 Da,

RT: 44.4300 min,

Identified with: Sequest HT (v1.17); XCorr:1.51,

Fragment match tolerance used for search: $0.02 \mathrm{Da}$

Fragments used for search: $-\mathrm{H}_{2} \mathrm{O} ; \mathrm{y} ;-\mathrm{NH}_{3} ; \mathrm{y} ; \mathrm{b} ; \mathrm{b} ;-\mathrm{H}_{2} \mathrm{O} ; \mathrm{y}$

Proteins (1):

- Protein Aster-B OS=Homo sapiens OX=9606 GN=GRAMD1B PE=1 SV=1

West, A. et al. Labeling preferences of diazirines with protein biomolecules, J. Am. Chem. S173 Soc. 2021. 


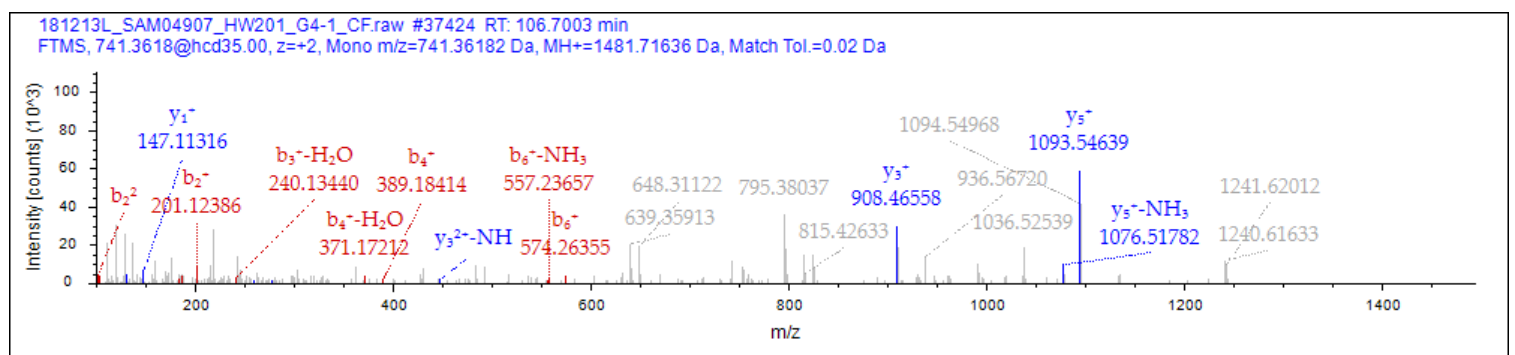

\section{Sequence: TVGMANREK}

Charge: +2 ,

Monoisotopic m/z: 741.36182 Da (-3.27 mmu/-4.41 ppm),

$\mathrm{MH}+:$ 1481.71636 Da,

RT: $106.7003 \mathrm{~min}$,

Identified with: Sequest HT (v1.17); XCorr:1.92,

Fragment match tolerance used for search: $0.02 \mathrm{Da}$

Fragments used for search: - $\mathrm{H}_{2} \mathrm{O} ; \mathrm{y} ;-\mathrm{NH}_{3} ; \mathrm{y} ; \mathrm{b} ; \mathrm{b} ;-\mathrm{H}_{2} \mathrm{O} ; \mathrm{b} ;-\mathrm{NH}_{3} ; \mathrm{y}$

Proteins (1):

- Vacuolar protein sorting-associated protein 13D OS=Homo sapiens OX=9606 $\mathrm{GN}=\mathrm{VPS} 13 \mathrm{D} \mathrm{PE}=1 \mathrm{SV}=2$

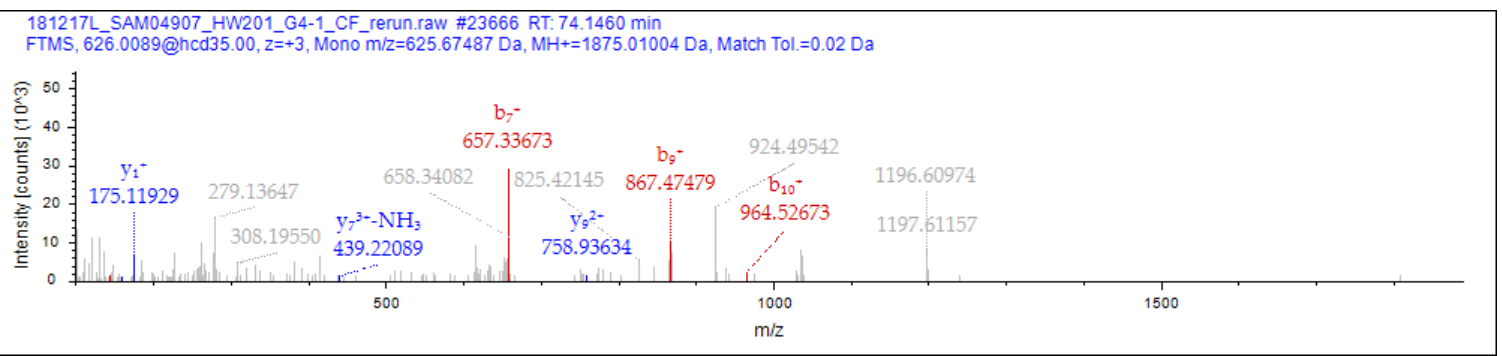

Sequence: AALCALNPLPFLR

Charge: +3 ,

Monoisotopic m/z: 625.67487 Da (+3.05 mmu/+4.88 ppm),

$\mathrm{MH}+:$ 1875.01004 Da,

RT: 74.1460 min,

Identified with: Sequest HT (v1.17); XCorr:1.07,

Fragment match tolerance used for search: $0.02 \mathrm{Da}$

Fragments used for search: $-\mathrm{NH}_{3} ; \mathrm{y} ; \mathrm{b} ; \mathrm{b} ;-\mathrm{NH}_{3} ; \mathrm{y}$

Proteins (1):

- Photoreceptor cilium actin regulator OS=Homo sapiens OX=9606 GN=PCARE PE=1 $\mathrm{SV}=1$

West, A. et al. Labeling preferences of diazirines with protein biomolecules, J. Am. Chem. S174 Soc. 2021. 
<smiles>C#CCCC1(CCC(=O)N/N=C(/C[C@H](N)C(=O)O)c2ccccc2N)N=N1</smiles>

JN Mod: JN-00248

PSMs: 1

Unique Sites: 1

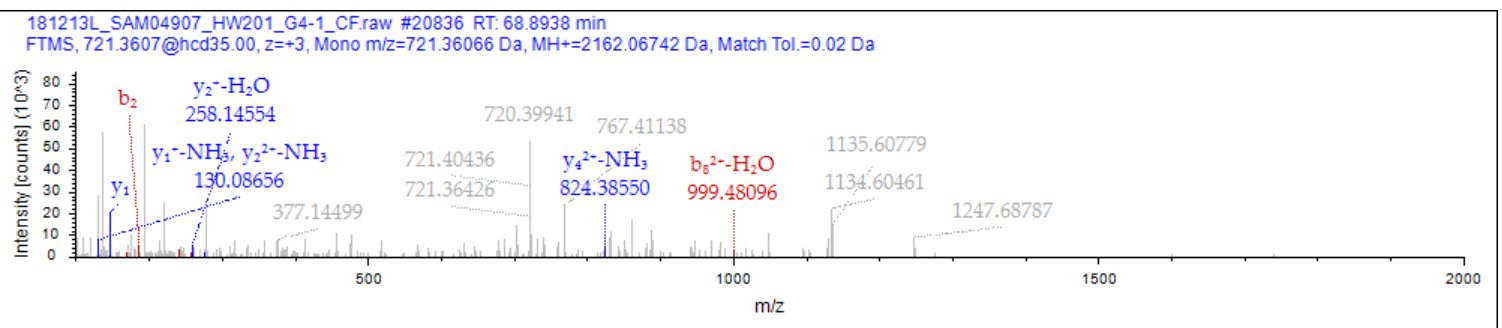

Sequence: QGAQLYVEK

Charge: +3 ,

Monoisotopic m/z: $721.36066 \mathrm{Da}(-0.17 \mathrm{mmu} /-0.24 \mathrm{ppm})$,

$\mathrm{MH}+:$ 2162.06742 Da,

RT: 68.8938 min,

Identified with: Sequest HT (v1.17); XCorr:1.34,

Fragment match tolerance used for search: $0.02 \mathrm{Da}$

Fragments used for search: $-\mathrm{H}_{2} \mathrm{O} ; \mathrm{y} ;-\mathrm{NH}_{3} ; \mathrm{y} ; \mathrm{b} ; \mathrm{b} ;-\mathrm{H}_{2} \mathrm{O} ; \mathrm{b} ;-\mathrm{NH}_{3} ; \mathrm{y}$

Proteins (1):

- Ubiquitin carboxyl-terminal hydrolase 24 OS=Homo sapiens OX=9606 GN=USP24 $\mathrm{PE}=1 \mathrm{SV}=3$

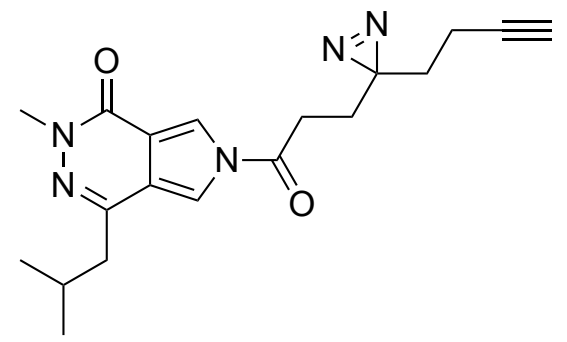

JN Mod: JN-835

PSMs: 0

Unique Sites: 0

West, A. et al. Labeling preferences of diazirines with protein biomolecules, J. Am. Chem. S175 Soc. 2021. 


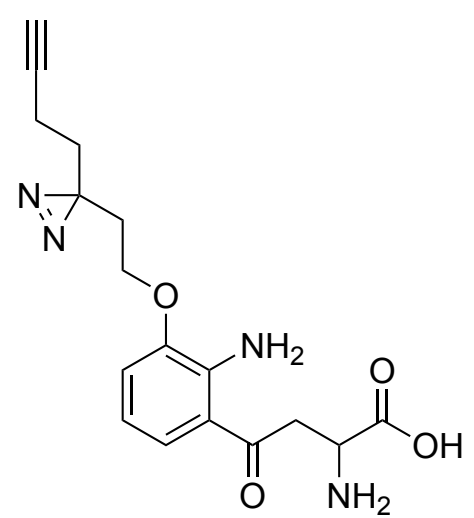

JN Mod: JN-00245

PSMs: 10

Unique Sites: 4

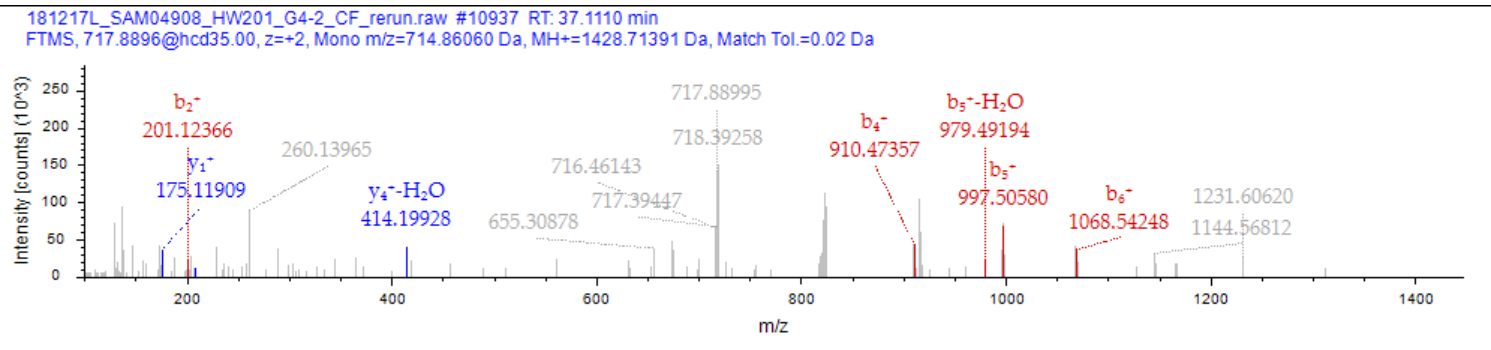

Sequence: LSLGSAGER

Charge: +2 ,

Monoisotopic m/z: 714.86060 Da (-0.08 mmu/-0.11 ppm),

$\mathrm{MH}+:$ 1428.71391 Da,

RT: $37.1110 \mathrm{~min}$,

Identified with: Sequest HT (v1.17); XCorr:1.29,

Fragment match tolerance used for search: $0.02 \mathrm{Da}$

Fragments used for search: $-\mathrm{H}_{2} \mathrm{O} ; \mathrm{y} ;-\mathrm{NH}_{3} ; \mathrm{y} ; \mathrm{b} ; \mathrm{b} ;-\mathrm{H}_{2} \mathrm{O}$; y

Proteins (1):

- Disrupted in schizophrenia 1 protein OS=Homo sapiens OX=9606 GN=DISC1 PE=1 $\mathrm{SV}=3$ 


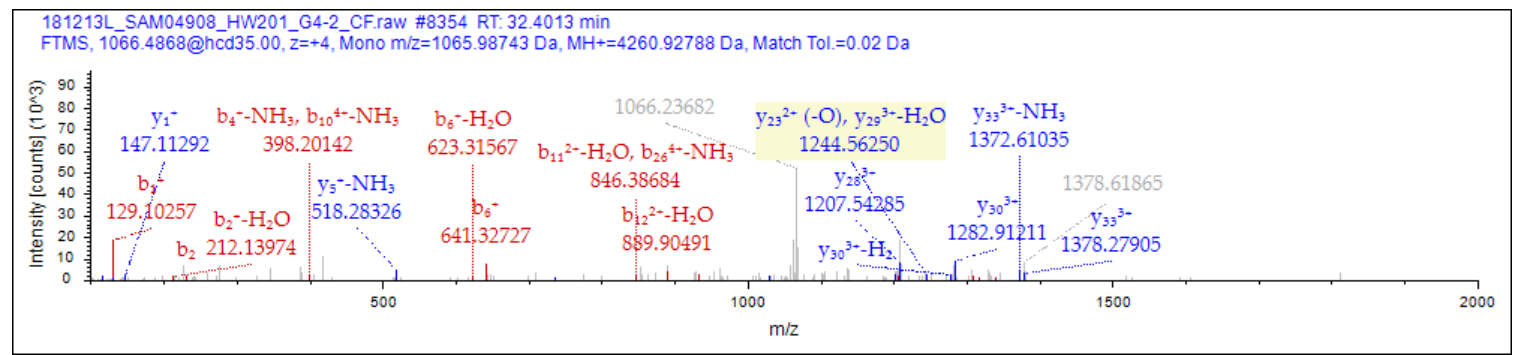

Sequence: KTGQPEELVSCSDCGRSGHPSCLQFTPVMMAAVK

Charge: +4 ,

Monoisotopic m/z: 1065.98743 Da (-2.83 mmu/-2.66 ppm),

$\mathrm{MH}+:$ 4260.92788 Da,

RT: 32.4013 min,

Identified with: Sequest HT (v1.17); XCorr:2.58,

Fragment match tolerance used for search: $0.02 \mathrm{Da}$

Fragments used for search: - $\mathrm{H}_{2} \mathrm{O} ; \mathrm{y} ;-\mathrm{NH}_{3} ; \mathrm{y} ; \mathrm{b} ; \mathrm{b} ;-\mathrm{H}_{2} \mathrm{O} ; \mathrm{b} ;-\mathrm{NH}_{3} ; \mathrm{y}$

Proteins (1):

- Zinc finger protein ubi-d4 OS=Homo sapiens OX=9606 GN=DPF2 PE=1 SV=2

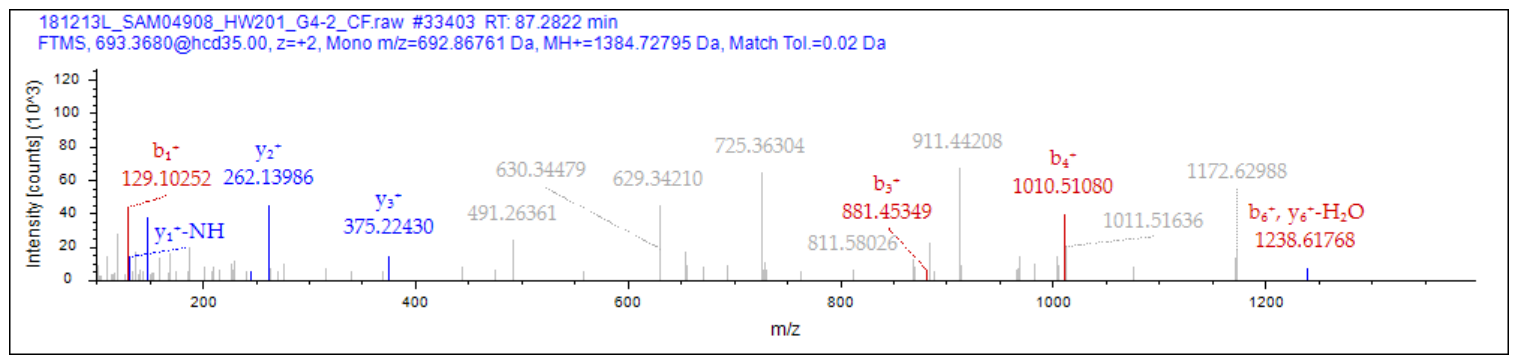

Sequence: KSKEIDK

Charge: +2 ,

Monoisotopic m/z: $692.86761 \mathrm{Da}(+3 \mathrm{mmu} /+4.33 \mathrm{ppm})$,

$\mathrm{MH}+:$ 1384.72795 Da,

RT: 87.2822 min,

Identified with: Sequest HT (v1.17); XCorr:1.17,

Fragment match tolerance used for search: $0.02 \mathrm{Da}$

Fragments used for search: - $\mathrm{H}_{2} \mathrm{O} ; \mathrm{y} ;-\mathrm{NH}_{3} ; \mathrm{y} ; \mathrm{b} ; \mathrm{b} ;-\mathrm{H}_{2} \mathrm{O} ; \mathrm{b} ;-\mathrm{NH}_{3} ; \mathrm{y}$

Proteins (1):

- Guanine nucleotide-binding protein subunit alpha-13 OS=Homo sapiens OX=9606 $\mathrm{GN}=\mathrm{GNA} 13 \mathrm{PE}=1 \mathrm{SV}=2$

West, A. et al. Labeling preferences of diazirines with protein biomolecules, J. Am. Chem. S177 Soc. 2021. 


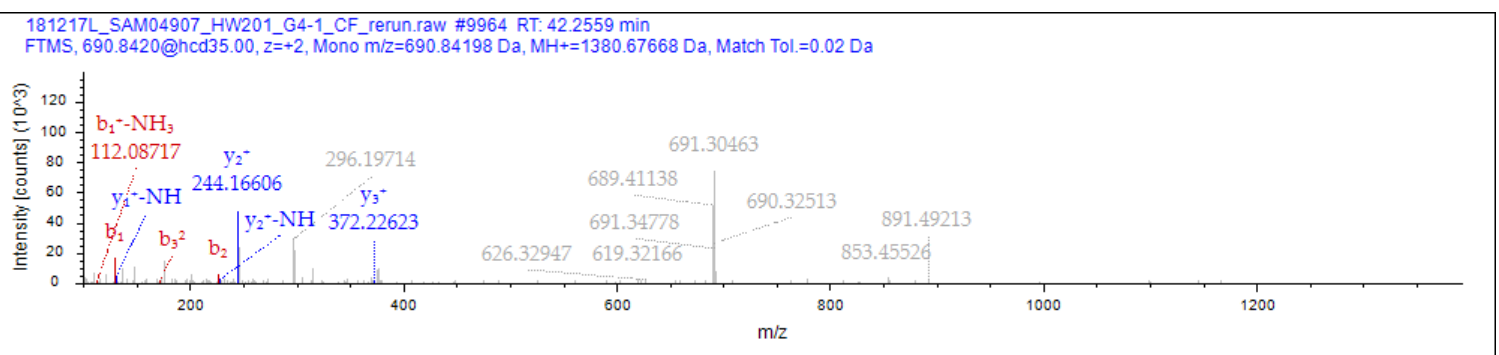

Sequence: KPDEQPK

Charge: +2 ,

Monoisotopic m/z: $690.84198 \mathrm{Da}(-2.51 \mathrm{mmu} /-3.64 \mathrm{ppm})$,

$\mathrm{MH}+:$ 1380.67668 Da,

RT: 42.2559 min,

Identified with: Sequest HT (v1.17); XCorr:1.18,

Fragment match tolerance used for search: $0.02 \mathrm{Da}$

Fragments used for search: - $\mathrm{H}_{2} \mathrm{O} ; \mathrm{y} ;-\mathrm{NH}_{3} ; \mathrm{y} ; \mathrm{b} ; \mathrm{b} ;-\mathrm{H}_{2} \mathrm{O} ; \mathrm{b} ;-\mathrm{NH}_{3} ; \mathrm{y}$

Proteins (1):

- Sodium channel protein type 8 subunit alpha OS=Homo sapiens OX=9606 GN=SCN8A $\mathrm{PE}=1 \mathrm{SV}=1$ 
<smiles>C#CCNC(=O)CC[C@H](C)[C@H]1CC[C@H]2[C@@H]3[C@H](C[C@H](O)[C@]21C)[C@@]1(C)CCC2(C[C@@H]31)N=N2</smiles>

JN Mod: JN-00038

PSMs: 223

Unique Sites: 36

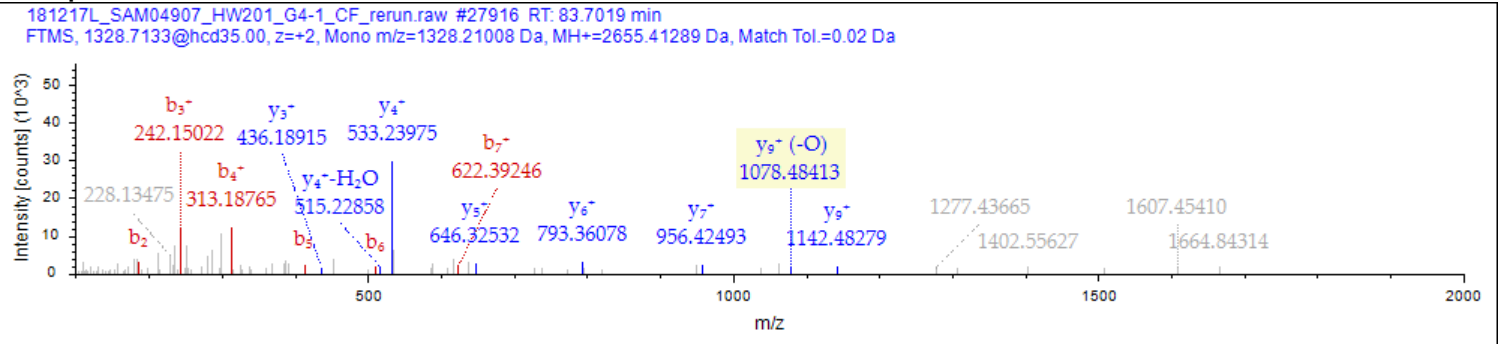

Sequence: AIGAVPLIQGEYMIPCEK

Charge: +2 ,

Monoisotopic m/z: 1328.21008 Da (-3.5 mmu/-2.64 ppm),

$\mathrm{MH}+:$ 2655.41289 Da,

RT: $83.7019 \mathrm{~min}$,

Identified with: Sequest HT (v1.17);

XCorr:2.18,

Fragment match tolerance used for search: $0.02 \mathrm{Da}$

Fragments used for search: - $\mathrm{H}_{2} \mathrm{O} ; \mathrm{y} ;-\mathrm{NH}_{3} ; \mathrm{y} ; \mathrm{b} ; \mathrm{b} ;-\mathrm{H}_{2} \mathrm{O} ; \mathrm{b} ;-\mathrm{NH}_{3} ; \mathrm{y}$

Proteins (1):

- Cathepsin D OS=Homo sapiens OX=9606 GN=CTSD PE=1 SV=1

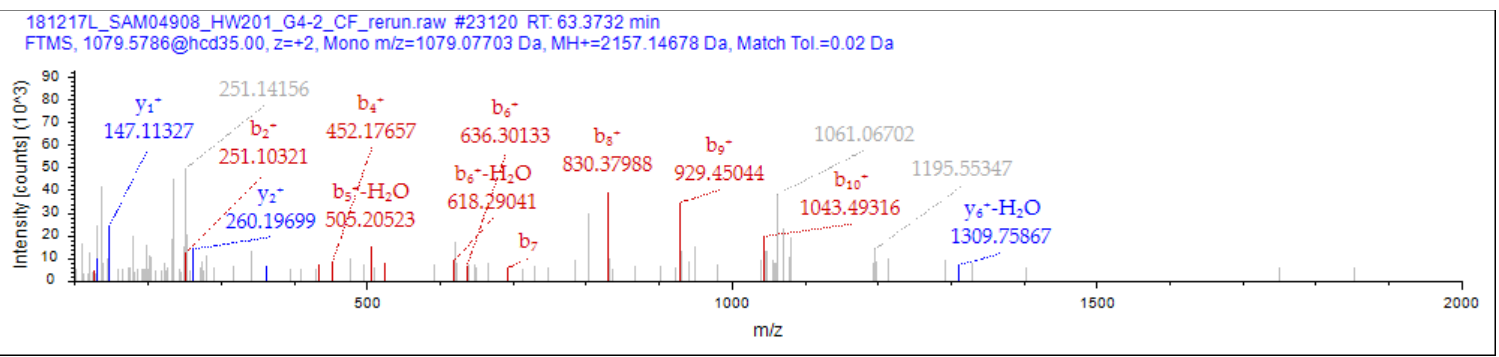

\section{Sequence: YSNSALGHVNCTIK}

Charge: +2 ,

Monoisotopic m/z: $1079.07703 \mathrm{Da}(+1.62 \mathrm{mmu} /+1.5 \mathrm{ppm})$,

$\mathrm{MH}+:$ 2157.14678 Da, RT: $63.3732 \mathrm{~min}$,

Identified with: Sequest HT (v1.17); XCorr:2.26,

Fragment match tolerance used for search: $0.02 \mathrm{Da}$

Fragments used for search: - $\mathrm{H}_{2} \mathrm{O} ; \mathrm{y} ;-\mathrm{NH}_{3} ; \mathrm{y} ; \mathrm{b} ; \mathrm{b} ;-\mathrm{H}_{2} \mathrm{O} ; \mathrm{b} ;-\mathrm{NH}_{3} ; \mathrm{y}$

Proteins (1):

- Reticulon-4 OS=Homo sapiens OX=9606 GN=RTN4 PE=1 SV=2

West, A. et al. Labeling preferences of diazirines with protein biomolecules, J. Am. Chem. S179 Soc. 2021. 


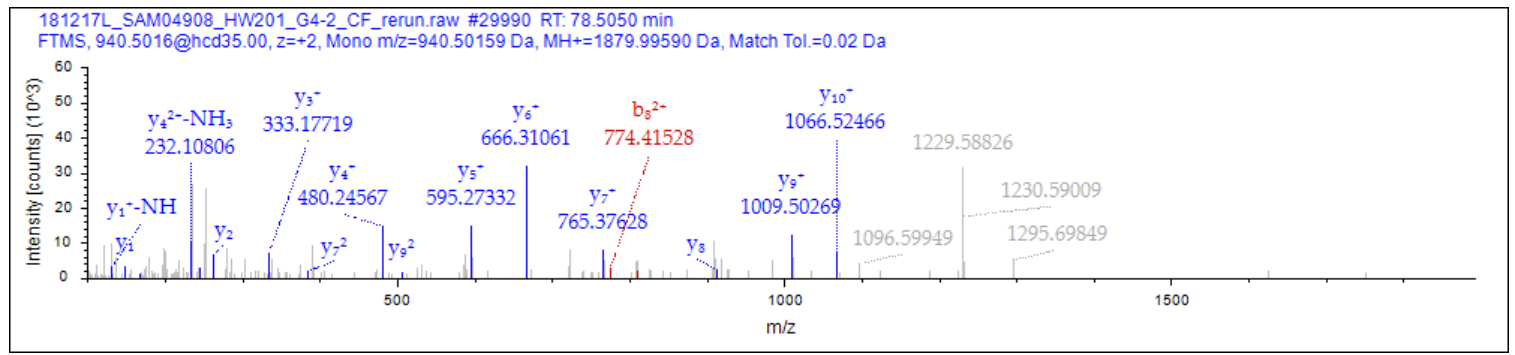

Sequence: YGPFVADFADK

Charge: +2 ,

Monoisotopic m/z: $940.50159 \mathrm{Da}(+2.79 \mathrm{mmu} /+2.97 \mathrm{ppm})$,

$\mathrm{MH}+:$ 1879.99590 Da,

RT: 78.5050 min,

Identified with: Sequest HT (v1.17); XCorr:2.07,

Fragment match tolerance used for search: $0.02 \mathrm{Da}$

Fragments used for search: $-\mathrm{H}_{2} \mathrm{O} ; \mathrm{y} ;-\mathrm{NH}_{3} ; \mathrm{y} ; \mathrm{b} ; \mathrm{b} ;-\mathrm{H}_{2} \mathrm{O} ; \mathrm{y}$

Proteins (1):

- ATP synthase $F(0)$ complex subunit B1, mitochondrial OS=Homo sapiens OX=9606 $\mathrm{GN}=\mathrm{ATP} 5 \mathrm{~PB} P \mathrm{PE}=1 \mathrm{SV}=2$

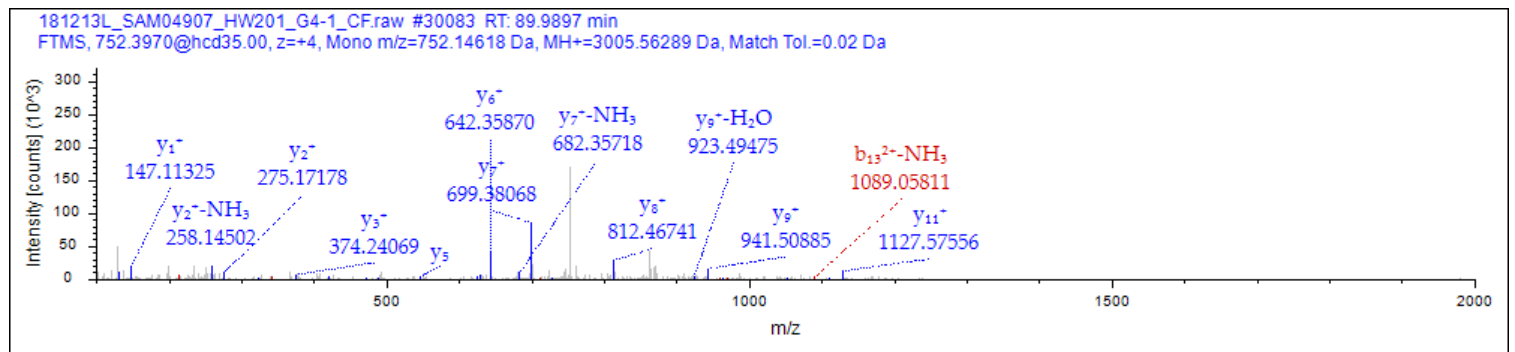

Sequence: VLQHYQESDKGEELGPGNVQK

Charge: +4 ,

Monoisotopic m/z: $752.14618 \mathrm{Da}(-0.31 \mathrm{mmu} /-0.41 \mathrm{ppm})$,

$\mathrm{MH}+: 3005.56289 \mathrm{Da}$,

RT: 89.9897 min,

Identified with: Sequest HT (v1.17); XCorr:3.16,

Fragment match tolerance used for search: $0.02 \mathrm{Da}$

Fragments used for search: - $\mathrm{H}_{2} \mathrm{O} ; \mathrm{y} ;-\mathrm{NH}_{3} ; \mathrm{y} ; \mathrm{b} ; \mathrm{b} ;-\mathrm{H}_{2} \mathrm{O} ; \mathrm{b} ;-\mathrm{NH}_{3} ; \mathrm{y}$

Proteins (1):

- Mitochondrial carrier homolog 2 OS=Homo sapiens OX=9606 GN=MTCH2 PE=1 SV=1

West, A. et al. Labeling preferences of diazirines with protein biomolecules, J. Am. Chem. S180 Soc. 2021. 


\section{Group 5:}<smiles>C#CCCC1(CCOc2ccc(C(=O)NC(=N)N)cc2)N=N1</smiles>

JN Mod: JN-935

PSMs: 589

Unique Sites: 67

181213L SAM04910. HW201 G5-2 CFraw \#33977 RT: 943440 min

FTMS, 759.8682@hcd35.00, z=+4, Mono m/z=759.86816 Da, MH+=3036.45083 Da, Match Tol. $=0.02 \mathrm{Da}$

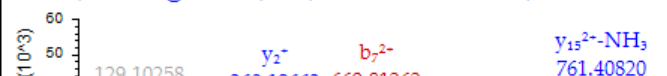

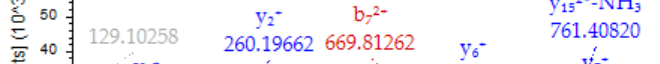

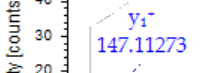

648.39294

$\mathrm{y}^{+}$

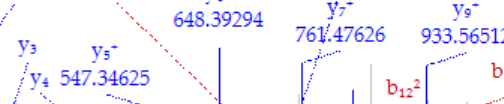

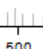

$\mathrm{b}_{13^{2+}-\mathrm{H}_{2}}$

$\mathrm{m} / 2$

Sequence: TFHETLDCCGSSTLTALTTSVLK

Charge: +4 ,

Monoisotopic m/z: 759.86816 Da (+1.09 mmu/+1.44 ppm),

$\mathrm{MH}+: 3036.45083 \mathrm{Da}$,

RT: $94.3440 \mathrm{~min}$,

Identified with: Sequest HT (v1.17);

XCorr:2.85,

Fragment match tolerance used for search: $0.02 \mathrm{Da}$

Fragments used for search: $-\mathrm{H}_{2} \mathrm{O} ; \mathrm{y} ;-\mathrm{NH}_{3} ; \mathrm{y} ; \mathrm{b} ; \mathrm{b} ;-\mathrm{H}_{2} \mathrm{O} ; \mathrm{y}$

Proteins (1):

- CD81 antigen OS=Homo sapiens OX=9606 GN=CD81 PE=1 SV=1

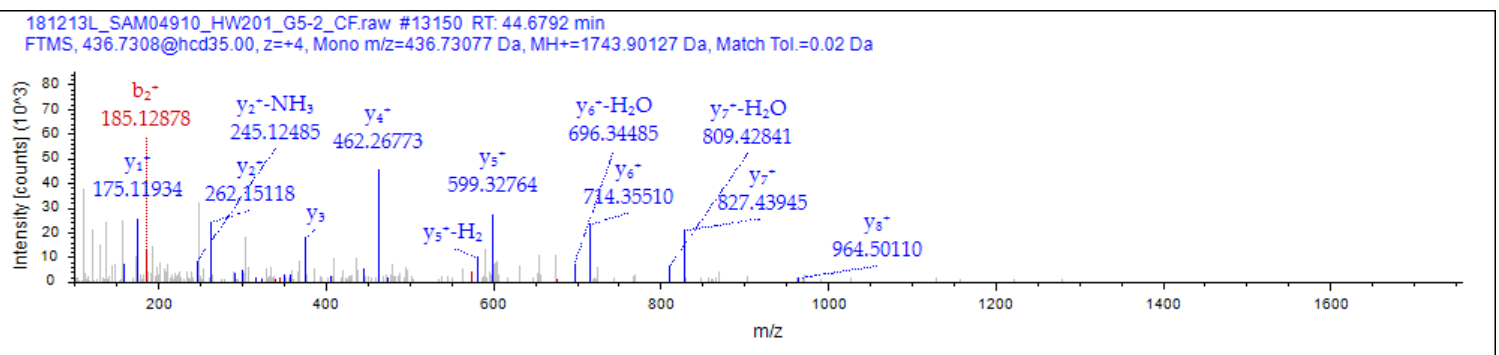

Sequence: ALTHIDHSLSR

Charge: +4 ,

Monoisotopic m/z: 436.73077 Da (+1.6 mmu/+3.67 ppm),

$\mathrm{MH}+:$ 1743.90127 Da,

RT: $44.6792 \mathrm{~min}$,

Identified with: Sequest HT (v1.17); XCorr:3.00,

Fragment match tolerance used for search: $0.02 \mathrm{Da}$

Fragments used for search: $-\mathrm{H}_{2} \mathrm{O} ; \mathrm{y} ;-\mathrm{NH}_{3} ; \mathrm{y} ; \mathrm{b} ; \mathrm{b} ;-\mathrm{H}_{2} \mathrm{O}$; y

Proteins (1):

- Methionine--tRNA ligase, cytoplasmic OS=Homo sapiens OX=9606 GN=MARS PE=1 $\mathrm{SV}=2$

West, A. et al. Labeling preferences of diazirines with protein biomolecules, J. Am. Chem. S181 Soc. 2021. 


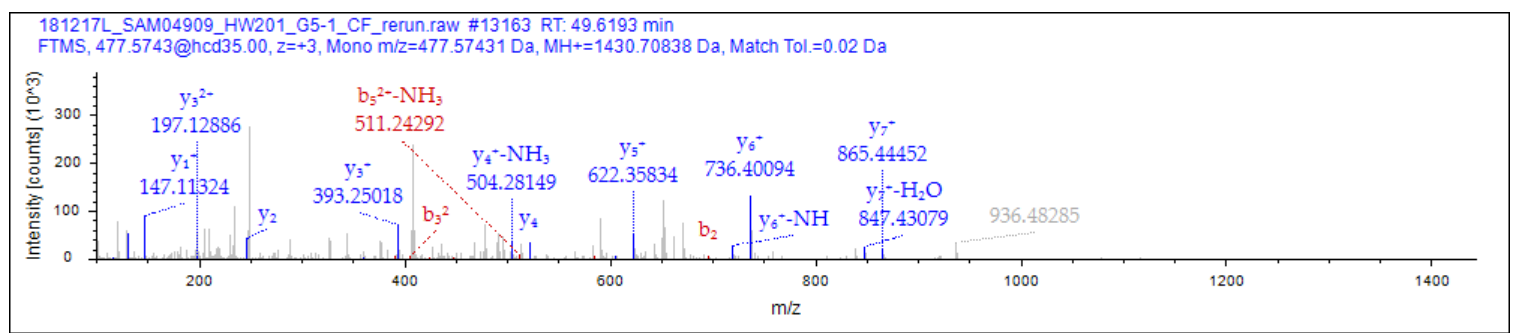

Sequence: AENTQFVK

Charge: +3 ,

Monoisotopic m/z: 477.57431 Da (-0.08 mmu/-0.17 ppm),

$\mathrm{MH}+:$ 1430.70838 Da,

RT: 49.6193 min,

Identified with: Sequest HT (v1.17); XCorr:1.98,

Fragment match tolerance used for search: $0.02 \mathrm{Da}$

Fragments used for search: $-\mathrm{H}_{2} \mathrm{O} ; \mathrm{y} ;-\mathrm{NH}_{3} ; \mathrm{y} ; \mathrm{b} ; \mathrm{b} ;-\mathrm{H}_{2} \mathrm{O} ; \mathrm{b} ;-\mathrm{NH}_{3} ; \mathrm{y}$

Proteins (1):

- Heme oxygenase 2 OS=Homo sapiens OX=9606 GN=HMOX2 PE=1 SV=2

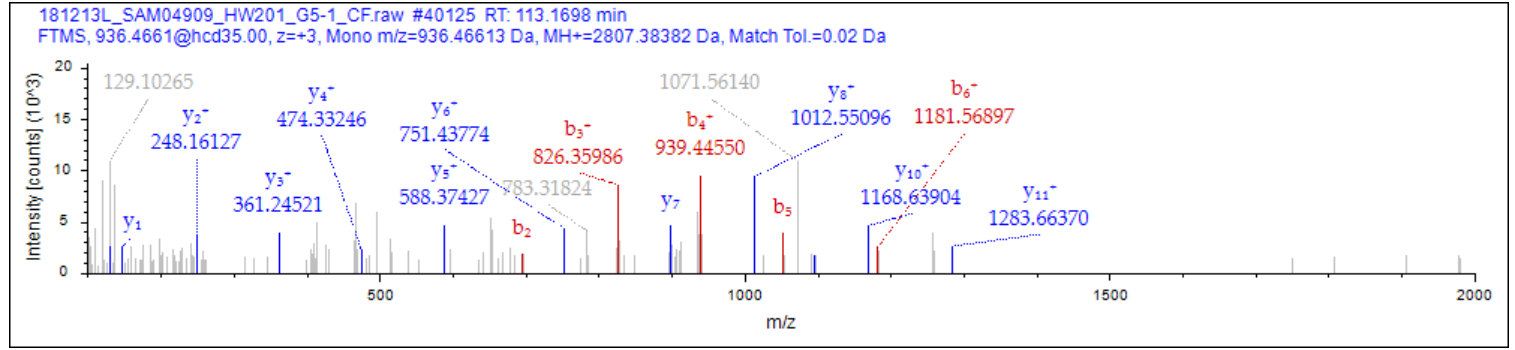

Sequence: AEMIIEQNTDGVNFYNILTK

Charge: +3 ,

Monoisotopic m/z: $936.46613 \mathrm{Da}(+3.54 \mathrm{mmu} /+3.78 \mathrm{ppm})$,

$\mathrm{MH}+:$ 2807.38382 Da,

RT: 113.1698 min,

Identified with: Sequest HT (v1.17); XCorr:3.42,

Fragment match tolerance used for search: $0.02 \mathrm{Da}$

Fragments used for search: - $\mathrm{H}_{2} \mathrm{O} ; \mathrm{y} ;-\mathrm{NH}_{3} ; \mathrm{y} ; \mathrm{b} ; \mathrm{b} ;-\mathrm{H}_{2} \mathrm{O} ; \mathrm{b} ;-\mathrm{NH}_{3} ; \mathrm{y}$

Proteins (1):

- Retinoid-inducible serine carboxypeptidase OS=Homo sapiens OX=9606 GN=SCPEP1 $\mathrm{PE}=1 \mathrm{SV}=1$

West, A. et al. Labeling preferences of diazirines with protein biomolecules, J. Am. Chem. S182 Soc. 2021. 
<smiles>C#CCCC1(CCn2cnc3c(=O)[nH]c(N)nc32)N=N1</smiles>

JN Mod: JN-846

PSMs: 55

Unique Sites: 10

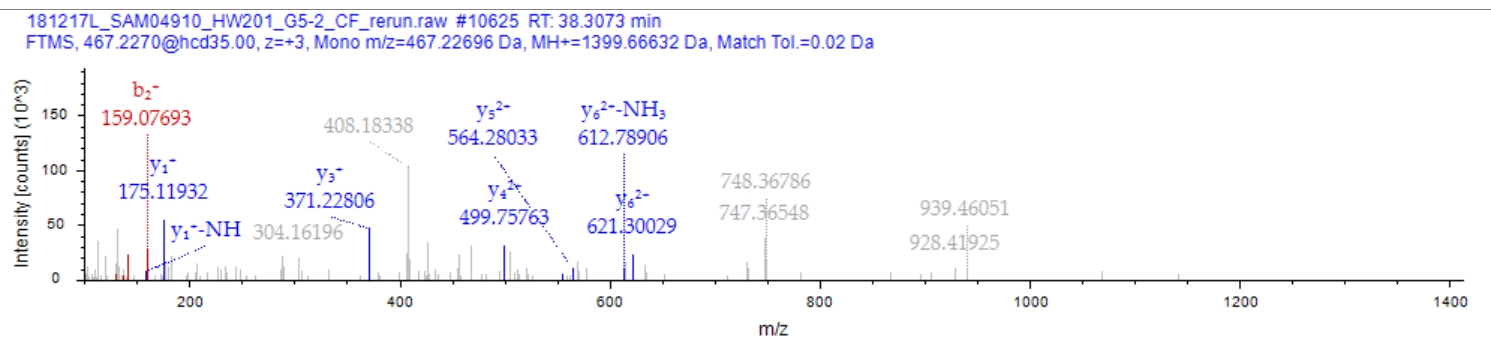

Sequence: GTNEYVPR

Charge: +3 ,

Monoisotopic m/z: 467.22696 Da (+1.64 mmu/+3.5 ppm),

$\mathrm{MH}+:$ 1399.66632 Da,

RT: 38.3073 min,

Identified with: Sequest HT (v1.17); XCorr:1.41,

Fragment match tolerance used for search: $0.02 \mathrm{Da}$

Fragments used for search: - $\mathrm{H}_{2} \mathrm{O} ; \mathrm{y} ;-\mathrm{NH}_{3} ; \mathrm{y} ; \mathrm{b} ; \mathrm{b} ;-\mathrm{H}_{2} \mathrm{O} ; \mathrm{b} ;-\mathrm{NH}_{3} ; \mathrm{y}$

Proteins (1):

- Protocadherin Fat 4 OS=Homo sapiens OX=9606 GN=FAT4 PE=1 SV=2

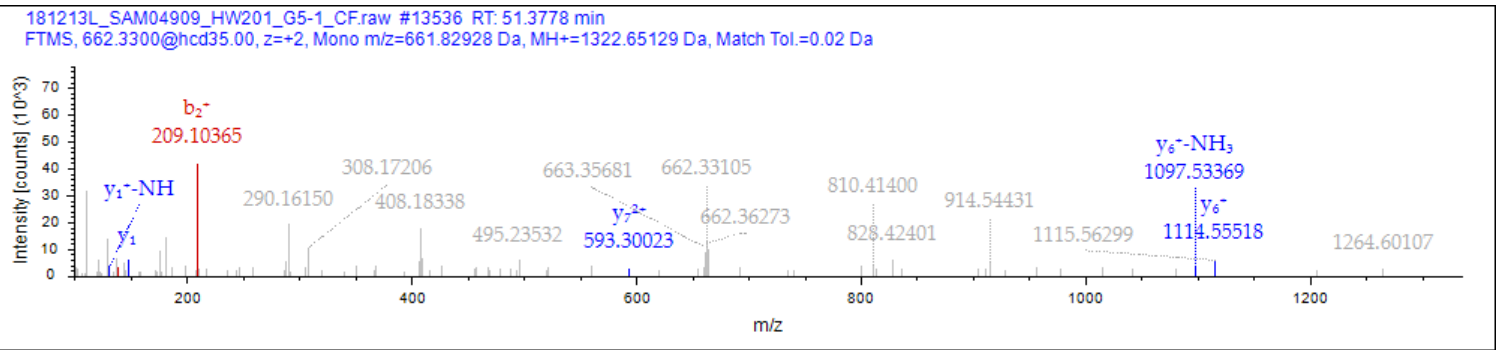

Sequence: HACSSIIK

Charge: +2 ,

Monoisotopic m/z: 661.82928 Da (-1.1 mmu/-1.67 ppm),

$\mathrm{MH}+: 1322.65129 \mathrm{Da}$,

RT: $51.3778 \mathrm{~min}$,

Identified with: Sequest HT (v1.17); XCorr:0.94,

Fragment match tolerance used for search: $0.02 \mathrm{Da}$

Fragments used for search: $-\mathrm{H}_{2} \mathrm{O} ; \mathrm{y} ;-\mathrm{NH}_{3} ; \mathrm{y} ; \mathrm{b} ; \mathrm{b} ;-\mathrm{H}_{2} \mathrm{O} ; \mathrm{y}$

Proteins (1):

- Exportin-5 OS=Homo sapiens OX=9606 GN=XPO5 PE=1 SV=1

West, A. et al. Labeling preferences of diazirines with protein biomolecules, J. Am. Chem. S183 Soc. 2021. 


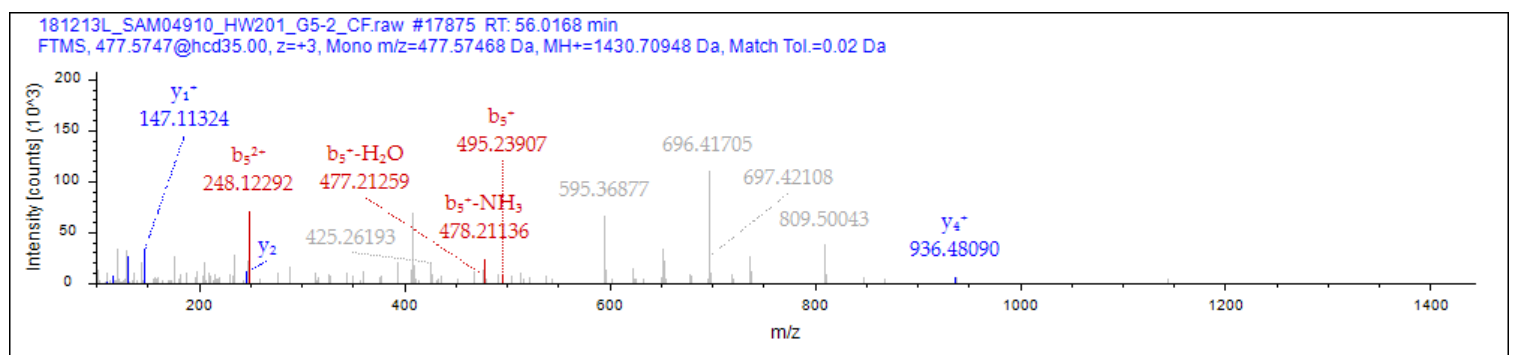

Sequence: GHDGKEPVK

Charge: +3 ,

Monoisotopic m/z: 477.57468 Da (+1.95 mmu/+4.09 ppm),

$\mathrm{MH}+:$ 1430.70948 Da,

RT: $56.0168 \mathrm{~min}$,

Identified with: Sequest HT (v1.17); XCorr:1.34,

Fragment match tolerance used for search: $0.02 \mathrm{Da}$

Fragments used for search: - $\mathrm{H}_{2} \mathrm{O} ; \mathrm{y} ;-\mathrm{NH}_{3} ; \mathrm{y} ; \mathrm{b} ; \mathrm{b} ;-\mathrm{H}_{2} \mathrm{O} ; \mathrm{b} ;-\mathrm{NH}_{3} ; \mathrm{y}$

Proteins (1):

- Fatty-acid amide hydrolase 2 OS=Homo sapiens OX=9606 GN=FAAH2 PE=2 SV=1

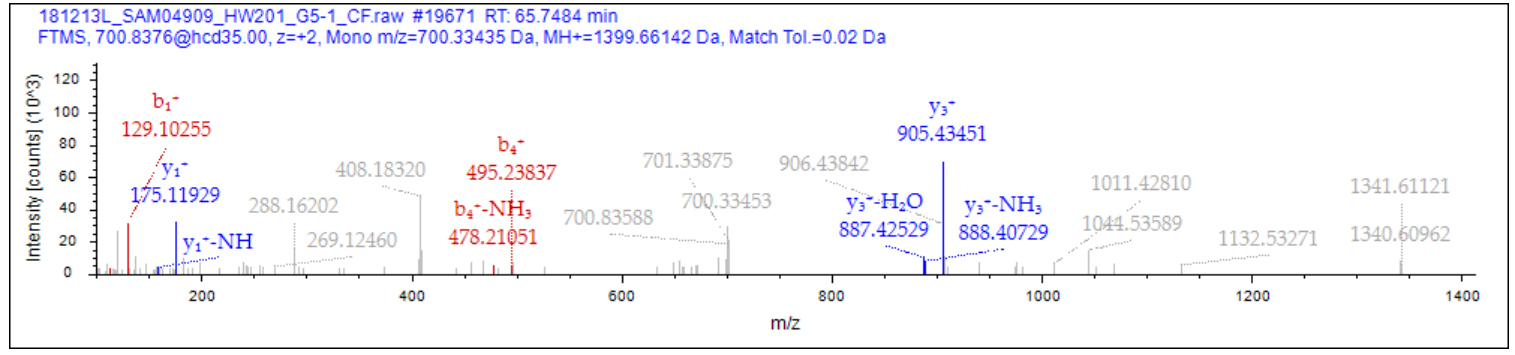

Sequence: KNDHTYR

Charge: +2 ,

Monoisotopic m/z: $700.33435 \mathrm{Da}(-1.14 \mathrm{mmu} /-1.63 \mathrm{ppm})$,

$\mathrm{MH}+:$ 1399.66142 Da,

RT: 65.7484 min,

Identified with: Sequest HT (v1.17); XCorr:1.00,

Fragment match tolerance used for search: $0.02 \mathrm{Da}$

Fragments used for search: - $\mathrm{H}_{2} \mathrm{O} ; \mathrm{y} ;-\mathrm{NH}_{3} ; \mathrm{y} ; \mathrm{b} ; \mathrm{b} ;-\mathrm{H}_{2} \mathrm{O} ; \mathrm{b} ;-\mathrm{NH}_{3} ; \mathrm{y}$

Proteins (1):

- 5-aminolevulinate synthase, nonspecific, mitochondrial OS=Homo sapiens OX=9606 $\mathrm{GN}=\mathrm{ALAS} 1 \mathrm{PE}=1 \mathrm{SV}=2$

West, A. et al. Labeling preferences of diazirines with protein biomolecules, J. Am. Chem. S184 Soc. 2021. 
<smiles>C#CCCC1(CCn2cnc3c(N)ncnc32)N=N1</smiles>

JN Mod: JN-847

PSMs: 43

Unique Sites: 14

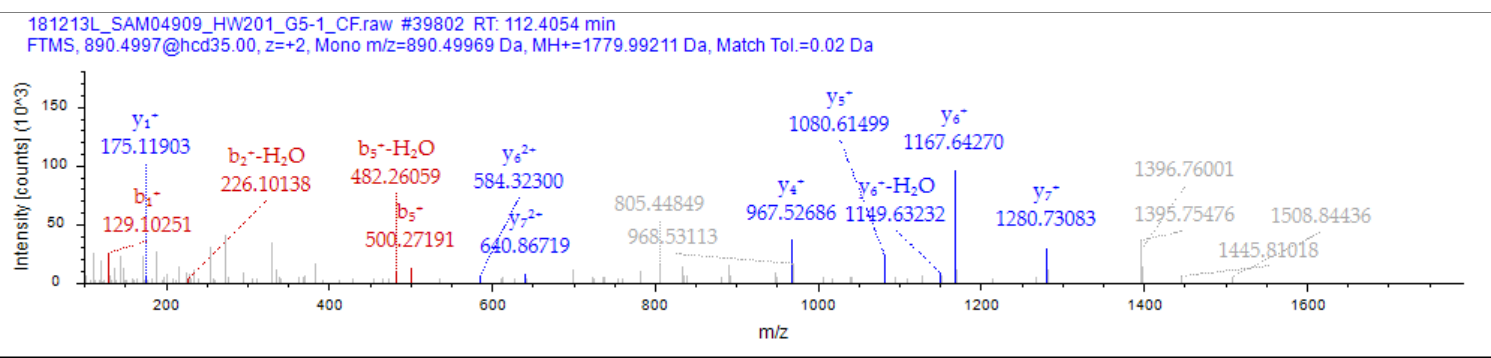

Sequence: KDAKGISIQTLR

Charge: +2 ,

Monoisotopic m/z: 890.49969 Da (-3.93 mmu/-4.42 ppm),

$\mathrm{MH}+:$ 1779.99211 Da,

RT: 112.4054 min,

Identified with: Sequest HT (v1.17); XCorr:1.30,

Fragment match tolerance used for search: $0.02 \mathrm{Da}$

Fragments used for search: - $\mathrm{H}_{2} \mathrm{O} ; \mathrm{y} ;-\mathrm{NH}_{3} ; \mathrm{y} ; \mathrm{b} ; \mathrm{b} ;-\mathrm{H}_{2} \mathrm{O} ; \mathrm{b} ;-\mathrm{NH}_{3} ; \mathrm{y}$

Proteins (1):

- Zinc finger protein DZIP1L OS=Homo sapiens OX=9606 GN=DZIP1L PE=1 SV=2

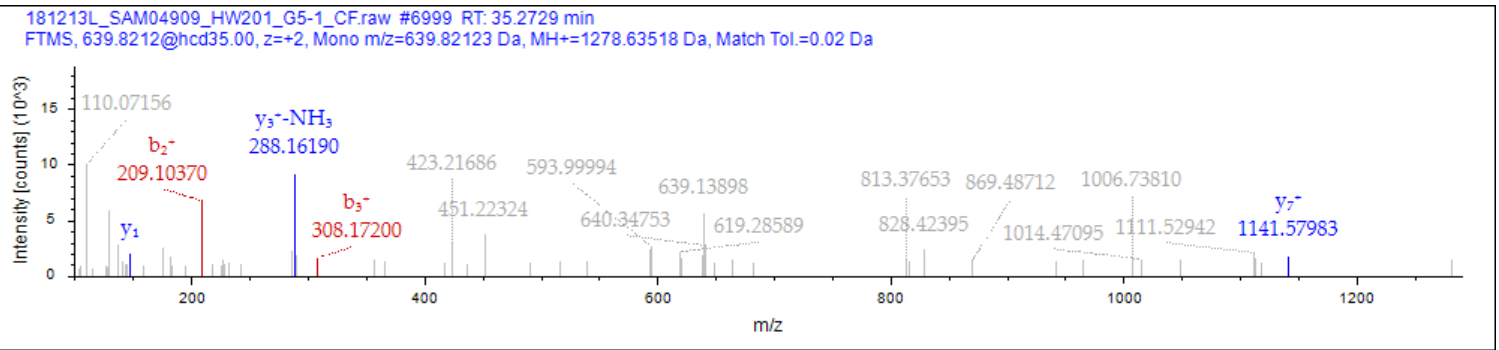

Sequence: HAVSEGTK

Charge: +2 ,

Monoisotopic m/z: 639.82123 Da (-0.46 mmu/-0.73 ppm),

$\mathrm{MH}+:$ 1278.63518 Da,

RT: 35.2729 min,

Identified with: Sequest HT (v1.17); XCorr:0.87,

Fragment match tolerance used for search: $0.02 \mathrm{Da}$

Fragments used for search: $-\mathrm{H}_{2} \mathrm{O} ; \mathrm{y} ;-\mathrm{NH}_{3} ; \mathrm{y} ; \mathrm{b} ; \mathrm{b} ;-\mathrm{H}_{2} \mathrm{O} ; \mathrm{y}$

Proteins (15):

- Histone H2B type 2-E OS=Homo sapiens OX=9606 GN=HIST2H2BE PE=1 SV=3

- Histone H2B type 2-F OS=Homo sapiens $O X=9606 \mathrm{GN}=H I S T 2 H 2 B F$ PE=1 SV=3

- Histone H2B type 1-M OS=Homo sapiens OX=9606 GN=HIST1H2BM PE=1 SV=3

- Histone H2B type 1-B OS=Homo sapiens OX=9606 GN=HIST1H2BB PE=1 SV=2

- Histone H2B type 1-D OS=Homo sapiens OX=9606 GN=HIST1H2BD PE=1 SV=2

West, A. et al. Labeling preferences of diazirines with protein biomolecules, J. Am. Chem. S185 Soc. 2021. 
- Histone H2B type 1-A OS=Homo sapiens OX=9606 GN=HIST1H2BA PE=1 SV=3

- Histone H2B type 1-H OS=Homo sapiens OX=9606 GN=HIST1H2BH PE=1 SV=3

- Histone H2B type 1-K OS=Homo sapiens OX=9606 GN=HIST1H2BK PE=1 SV=3

- Histone H2B type 1-L OS=Homo sapiens OX=9606 GN=HIST1H2BL PE=1 SV=3

- Histone H2B type 1-C/E/F/G/I OS=Homo sapiens OX=9606 GN=HIST1H2BC PE=1 $\mathrm{SV}=4$

- Histone H2B type F-S OS=Homo sapiens OX=9606 GN=H2BFS PE=1 SV=2

- Histone H2B type 3-B OS=Homo sapiens OX=9606 GN=HIST3H2BB PE=1 SV=3

- Histone H2B type 1-J OS=Homo sapiens OX=9606 GN=HIST1H2BJ PE=1 SV=3

- Histone H2B type 1-O OS=Homo sapiens OX=9606 GN=HIST1H2BO PE=1 SV=3

- Histone H2B type 1-N OS=Homo sapiens OX=9606 GN=HIST1H2BN PE=1 SV=3

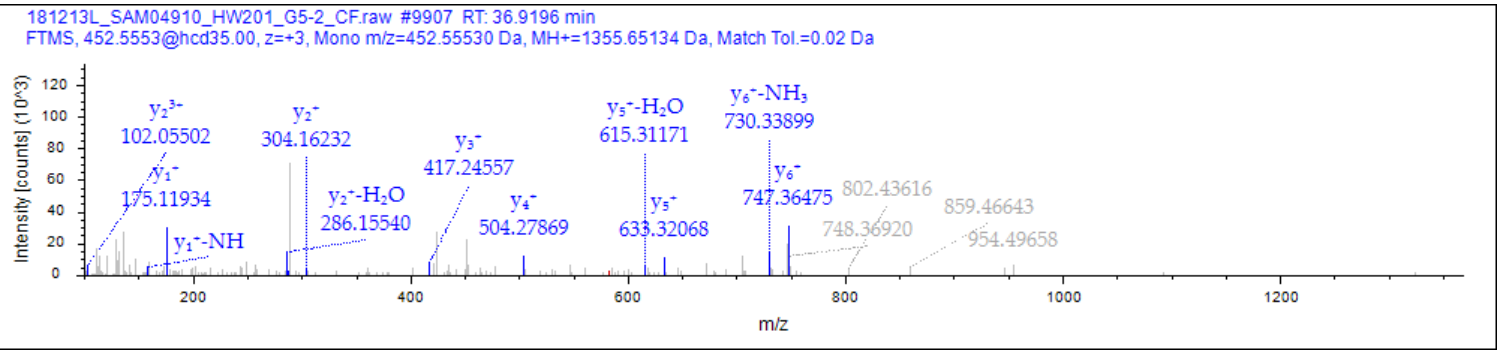

Sequence: GTNESLER

Charge: +3 ,

Monoisotopic m/z: $452.55530 \mathrm{Da}(+1.32 \mathrm{mmu} /+2.91 \mathrm{ppm})$,

$\mathrm{MH}+:$ 1355.65134 Da,

RT: 36.9196 min,

Identified with: Sequest HT (v1.17); XCorr:1.30,

Fragment match tolerance used for search: $0.02 \mathrm{Da}$

Fragments used for search: - $\mathrm{H}_{2} \mathrm{O} ; \mathrm{y} ;-\mathrm{NH}_{3} ; \mathrm{y} ; \mathrm{b} ; \mathrm{b} ;-\mathrm{H}_{2} \mathrm{O} ; \mathrm{b} ;-\mathrm{NH}_{3} ; \mathrm{y}$

Proteins (2):

- Glial fibrillary acidic protein OS=Homo sapiens OX=9606 GN=GFAP PE=1 SV=1

- Vimentin OS=Homo sapiens OX=9606 GN=VIM PE=1 SV=4

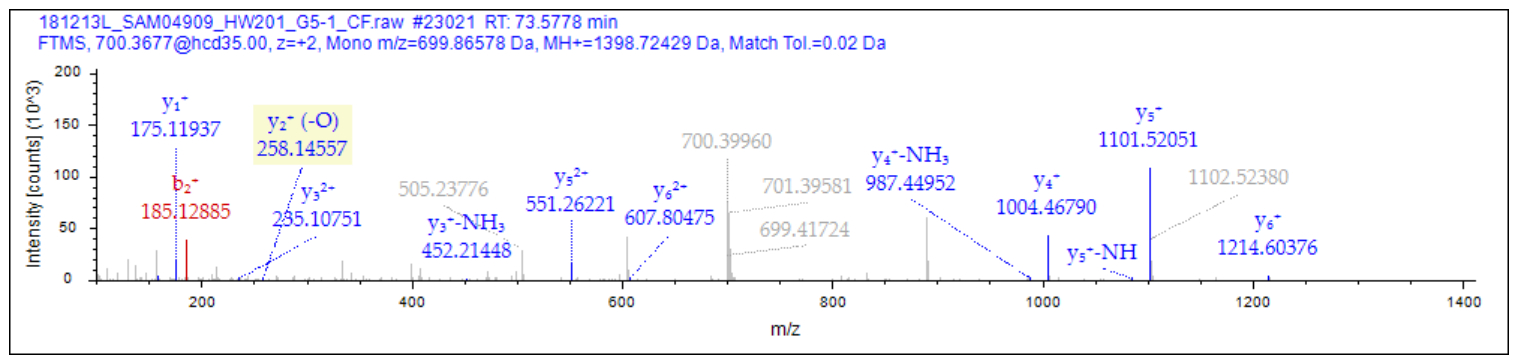

Sequence: AILPSFMR

Charge: +2 ,

Monoisotopic m/z: 699.86578 Da (+1.55 mmu/+2.21 ppm),

$\mathrm{MH+:} 1398.72429 \mathrm{Da}, \mathrm{RT}: 73.5778 \mathrm{~min}$,

Identified with: Sequest HT (v1.17); XCorr:1.19,

Fragment match tolerance used for search: $0.02 \mathrm{Da}$

Fragments used for search: $-\mathrm{NH}_{3} ; \mathrm{y} ; \mathrm{b} ; \mathrm{y}$

Proteins (1):

- Protein SSX3 OS=Homo sapiens OX=9606 GN=SSX3 PE=1 SV=2

West, A. et al. Labeling preferences of diazirines with protein biomolecules, J. Am. Chem. S186 Soc. 2021. 
<smiles>C#CCCC1(CCn2cnc3c(=O)[nH]cnc32)N=N1</smiles>

JN Mod: JN-845

PSMs: 6

Unique Sites: 4

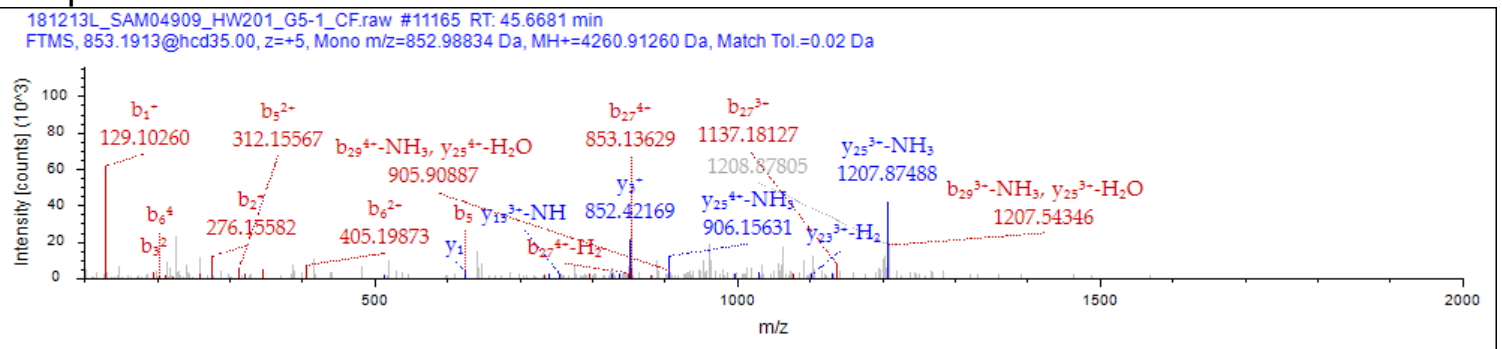

Sequence: KMLAYWMLEVCEEQRCEEEVFPLAMNYLDR

Charge: +5 ,

Monoisotopic m/z: 852.98834 Da (-1.9 mmu/-2.23 ppm),

$\mathrm{MH}+:$ 4260.91260 Da,

RT: $45.6681 \mathrm{~min}$,

Identified with: Sequest HT (v1.17); XCorr:2.64,

Fragment match tolerance used for search: $0.02 \mathrm{Da}$

Fragments used for search: - $\mathrm{H}_{2} \mathrm{O} ; \mathrm{y} ;-\mathrm{NH}_{3} ; \mathrm{y} ; \mathrm{b} ; \mathrm{b} ;-\mathrm{H}_{2} \mathrm{O} ; \mathrm{b} ;-\mathrm{NH}_{3} ; \mathrm{y}$

Proteins (1):

- G1/S-specific cyclin-D3 OS=Homo sapiens OX=9606 GN=CCND3 PE=1 SV=2

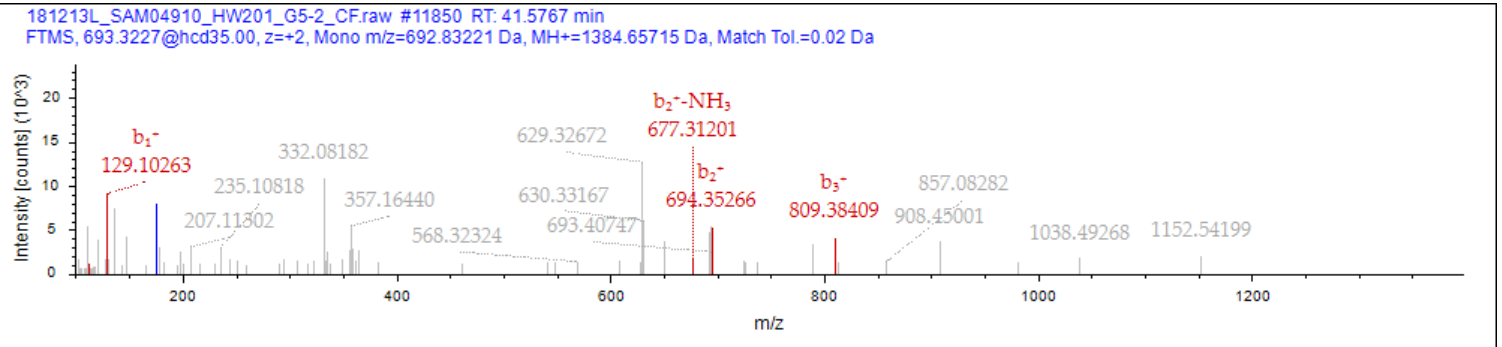

Sequence: KNDHTYR

Charge: +2 ,

Monoisotopic m/z: $692.83221 \mathrm{Da}(+2.18 \mathrm{mmu} /+3.14 \mathrm{ppm})$,

$\mathrm{MH}+:$ 1384.65715 Da,

RT: 41.5767 min,

Identified with: Sequest HT (v1.17); XCorr:1.07,

Fragment match tolerance used for search: $0.02 \mathrm{Da}$

Fragments used for search: - $\mathrm{H}_{2} \mathrm{O} ; \mathrm{y} ;-\mathrm{NH}_{3} ; \mathrm{y} ; \mathrm{b} ; \mathrm{b} ;-\mathrm{H}_{2} \mathrm{O} ; \mathrm{b} ;-\mathrm{NH}_{3} ; \mathrm{y}$

Proteins (1):

- 5-aminolevulinate synthase, nonspecific, mitochondrial OS=Homo sapiens OX=9606 $\mathrm{GN}=\mathrm{ALAS} 1 \mathrm{PE}=1 \mathrm{SV}=2$

West, A. et al. Labeling preferences of diazirines with protein biomolecules, J. Am. Chem. S187 Soc. 2021. 


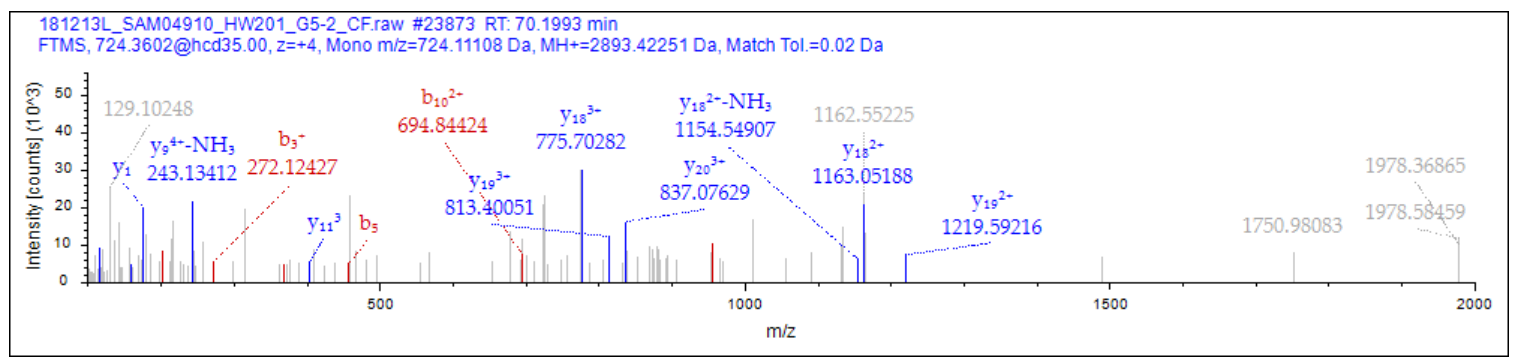

\section{Sequence: AEALALLPCGLGMAFSQSHVMAAR}

Charge: +4 ,

Monoisotopic m/z: $724.11108 \mathrm{Da}(+1.35 \mathrm{mmu} /+1.87 \mathrm{ppm})$,

$\mathrm{MH}+:$ 2893.42251 Da,

RT: $70.1993 \mathrm{~min}$,

Identified with: Sequest HT (v1.17); XCorr:2.92,

Fragment match tolerance used for search: $0.02 \mathrm{Da}$

Fragments used for search: - $\mathrm{H}_{2} \mathrm{O} ; \mathrm{y} ;-\mathrm{NH}_{3} ; \mathrm{y} ; \mathrm{b} ; \mathrm{b} ;-\mathrm{H}_{2} \mathrm{O} ; \mathrm{b} ;-\mathrm{NH}_{3} ; \mathrm{y}$

Proteins (1):

- Metallophosphoesterase domain-containing protein 1 OS=Homo sapiens OX=9606 $\mathrm{GN}=\mathrm{MPPED} 1 \mathrm{PE}=2 \mathrm{SV}=3$

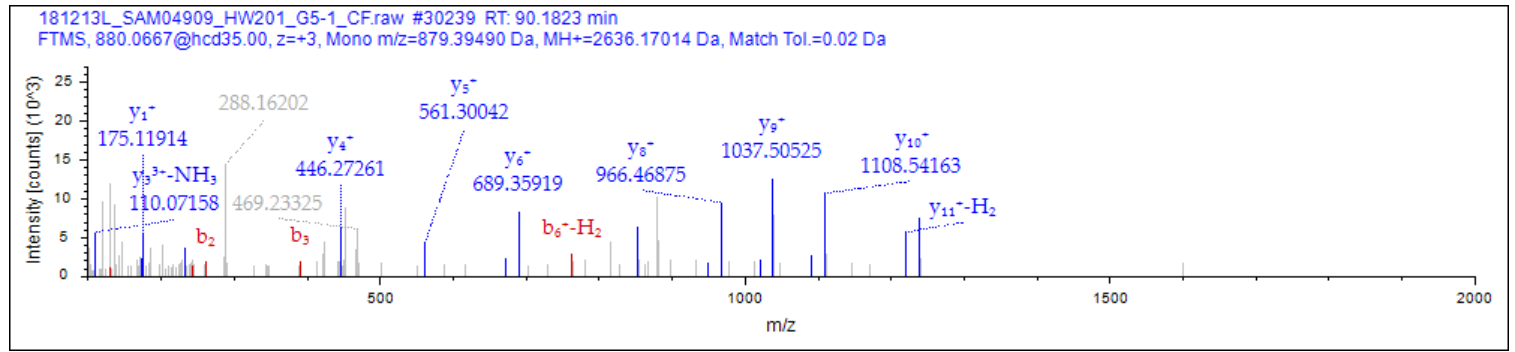

Sequence: EMEENFAVEAANYQDTIGR

Charge: +3 ,

Monoisotopic m/z: $879.39490 \mathrm{Da}(+3.95 \mathrm{mmu} /+4.5 \mathrm{ppm})$,

$\mathrm{MH}+:$ 2636.17014 Da,

RT: 90.1823 min,

Identified with: Sequest HT (v1.17); XCorr:2.95,

Fragment match tolerance used for search: $0.02 \mathrm{Da}$

Fragments used for search: - $\mathrm{H}_{2} \mathrm{O} ; \mathrm{y} ;-\mathrm{NH}_{3} ; \mathrm{y} ; \mathrm{b} ; \mathrm{b} ;-\mathrm{H}_{2} \mathrm{O} ; \mathrm{b} ;-\mathrm{NH}_{3} ; \mathrm{y}$

Proteins (1):

- Vimentin OS=Homo sapiens OX=9606 GN=VIM PE=1 SV=4

West, A. et al. Labeling preferences of diazirines with protein biomolecules, J. Am. Chem. S188 Soc. 2021. 
<smiles>C#CCCC1(CCn2cnc3c(=O)[nH]c(=O)[nH]c32)N=N1</smiles>

JN Mod: JN-936

PSMs: 32

Unique Sites: 6

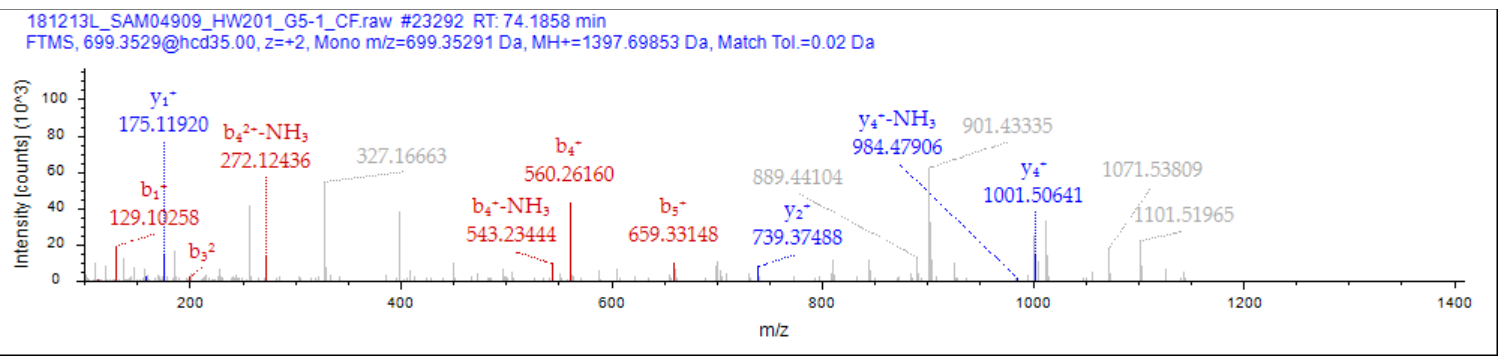

Sequence: KHMYVVR

Charge: +2 ,

Monoisotopic m/z: 699.35291 Da (-1.12 mmu/-1.6 ppm),

$\mathrm{MH}+:$ 1397.69853 Da,

RT: 74.1858 min,

Identified with: Sequest HT (v1.17); XCorr:1.93,

Fragment match tolerance used for search: $0.02 \mathrm{Da}$

Fragments used for search: $-\mathrm{NH}_{3} ; \mathrm{y} ; \mathrm{b} ; \mathrm{b} ;-\mathrm{NH}_{3} ; \mathrm{y}$

Proteins (1):

- Protein mono-ADP-ribosyltransferase PARP15 OS=Homo sapiens OX=9606 $\mathrm{GN}=\mathrm{PARP} 15 \mathrm{PE}=1 \mathrm{SV}=2$

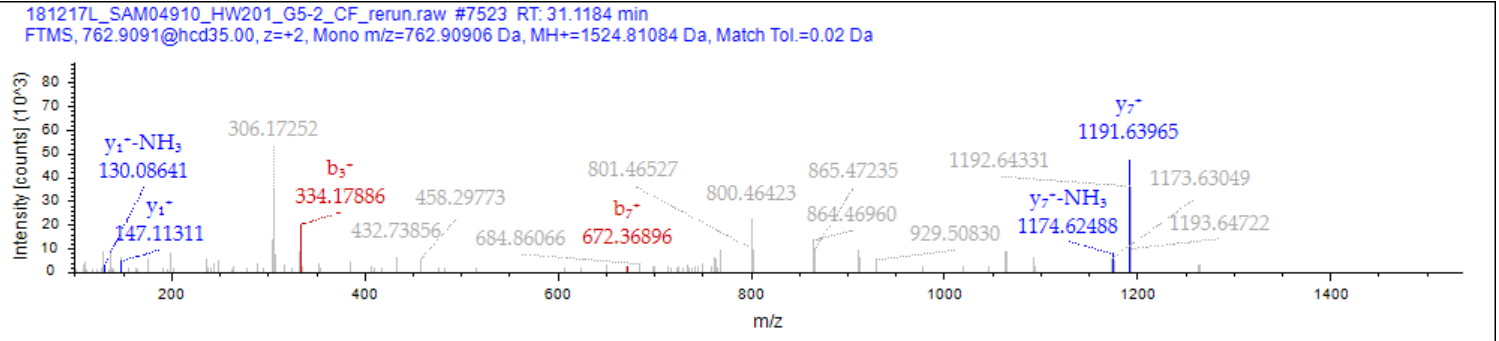

Sequence: YGLPGLAQLK

Charge: +2 ,

Monoisotopic m/z: $762.90906 \mathrm{Da}(+1.92 \mathrm{mmu} /+2.51 \mathrm{ppm})$,

$\mathrm{MH}+:$ 1524.81084 Da,

RT: $31.1184 \mathrm{~min}$,

Identified with: Sequest HT (v1.17); XCorr:1.06,

Fragment match tolerance used for search: $0.02 \mathrm{Da}$

Fragments used for search: $-\mathrm{NH}_{3} ; \mathrm{y} ; \mathrm{b} ; \mathrm{y}$

Proteins (1):

- Endonuclease G, mitochondrial OS=Homo sapiens OX=9606 GN=ENDOG PE=1 SV=4

West, A. et al. Labeling preferences of diazirines with protein biomolecules, J. Am. Chem. S189 Soc. 2021. 


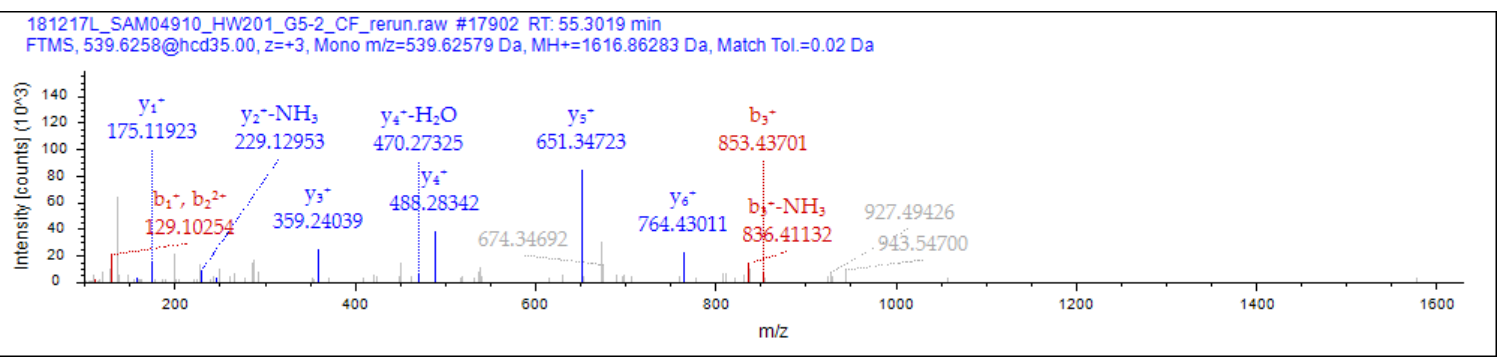

Sequence: KKEIYELAR

Charge: +3 ,

Monoisotopic m/z: $539.62579 \mathrm{Da}$ (+2.07 mmu/+3.83 ppm),

$\mathrm{MH}+:$ 1616.86283 Da,

RT: 55.3019 min,

Identified with: Sequest HT (v1.17); XCorr:1.74,

Fragment match tolerance used for search: $0.02 \mathrm{Da}$

Fragments used for search: - $\mathrm{H}_{2} \mathrm{O} ; \mathrm{y} ;-\mathrm{NH}_{3} ; \mathrm{y} ; \mathrm{b} ; \mathrm{b} ;-\mathrm{H}_{2} \mathrm{O} ; \mathrm{b} ;-\mathrm{NH}_{3} ; \mathrm{y}$

Proteins (1):

- Kynurenine/alpha-aminoadipate aminotransferase, mitochondrial OS=Homo sapiens $O X=9606 \mathrm{GN}=\mathrm{AADAT} \mathrm{PE}=1 \mathrm{SV}=2$

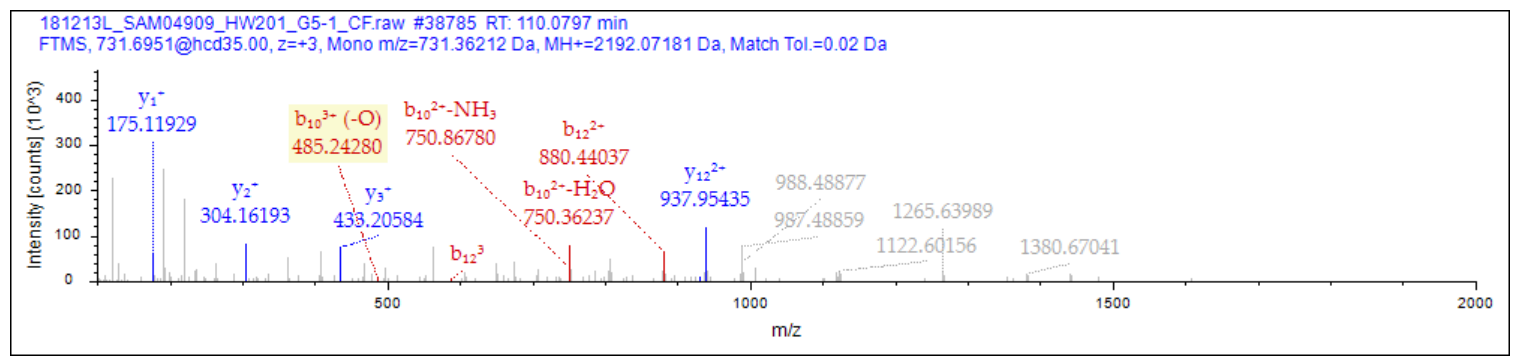

Sequence: VMARSLSPPPELEER

Charge: +3 ,

Monoisotopic m/z: $731.36212 \mathrm{Da}(+1.76 \mathrm{mmu} /+2.4 \mathrm{ppm})$,

$\mathrm{MH}+:$ 2192.07181 Da,

RT: 110.0797 min,

Identified with: Sequest HT (v1.17); XCorr:1.40,

Fragment match tolerance used for search: $0.02 \mathrm{Da}$

Fragments used for search: - $\mathrm{H}_{2} \mathrm{O} ; \mathrm{y} ;-\mathrm{NH}_{3} ; \mathrm{y} ; \mathrm{b} ; \mathrm{b} ;-\mathrm{H}_{2} \mathrm{O} ; \mathrm{b} ;-\mathrm{NH}_{3} ; \mathrm{y}$

Proteins (1):

- Tuberin OS=Homo sapiens OX=9606 GN=TSC2 PE=1 SV=2

West, A. et al. Labeling preferences of diazirines with protein biomolecules, J. Am. Chem. S190 Soc. 2021. 
<smiles>C#CCCC1(CCC(=O)Nc2ccc(OCCN(C)CC(=O)O)cc2)N=N1</smiles>

JN Mod: JN-836

PSMs: 16

Unique Sites: 4

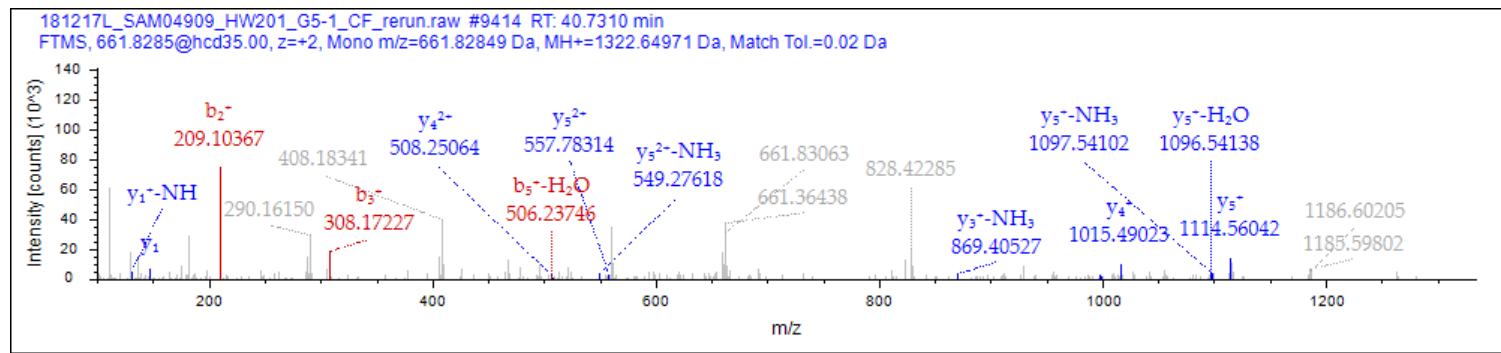

Sequence: AHVESSK

Charge: +2 ,

Monoisotopic m/z: $661.82849 \mathrm{Da}(+0.46 \mathrm{mmu} /+0.69 \mathrm{ppm})$,

$\mathrm{MH}+:$ 1322.64971 Da,

RT: $40.7310 \mathrm{~min}$,

Identified with: Sequest HT (v1.17); XCorr:1.22,

Fragment match tolerance used for search: $0.02 \mathrm{Da}$

Fragments used for search: $-\mathrm{H}_{2} \mathrm{O} ; \mathrm{y} ;-\mathrm{NH}_{3} ; \mathrm{y} ; \mathrm{b} ; \mathrm{b} ;-\mathrm{H}_{2} \mathrm{O} ; \mathrm{y}$

Proteins (1):

- Melanoma-associated antigen H1 OS=Homo sapiens OX=9606 GN=MAGEH1 PE=1 $\mathrm{SV}=1$

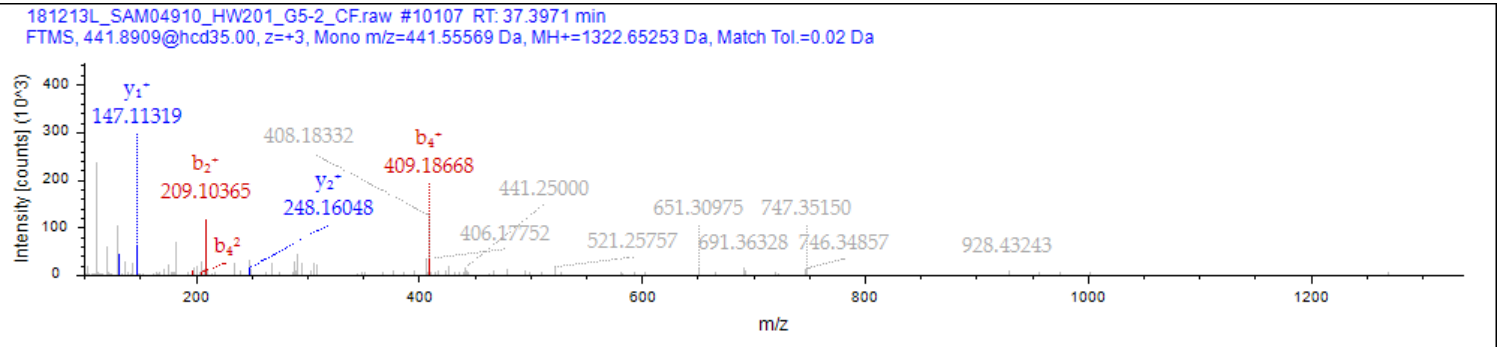

Sequence: AHAETTK

Charge: +3 ,

Monoisotopic m/z: $441.55569 \mathrm{Da}(+1.25 \mathrm{mmu} /+2.83 \mathrm{ppm})$,

$\mathrm{MH}+:$ 1322.65253 Da,

RT: $37.3971 \mathrm{~min}$,

Identified with: Sequest HT (v1.17); XCorr:1.22,

Fragment match tolerance used for search: $0.02 \mathrm{Da}$

Fragments used for search: $-\mathrm{H}_{2} \mathrm{O} ; \mathrm{y} ;-\mathrm{NH}_{3} ; \mathrm{y} ; \mathrm{b} ; \mathrm{b} ;-\mathrm{H}_{2} \mathrm{O}$; y

Proteins (1):

- Putative MAGE domain-containing protein MAGEA13P OS=Homo sapiens OX=9606 $\mathrm{GN}=\mathrm{MAGEA} 13 \mathrm{P}$ PE=5 SV=1

West, A. et al. Labeling preferences of diazirines with protein biomolecules, J. Am. Chem. S191 Soc. 2021. 


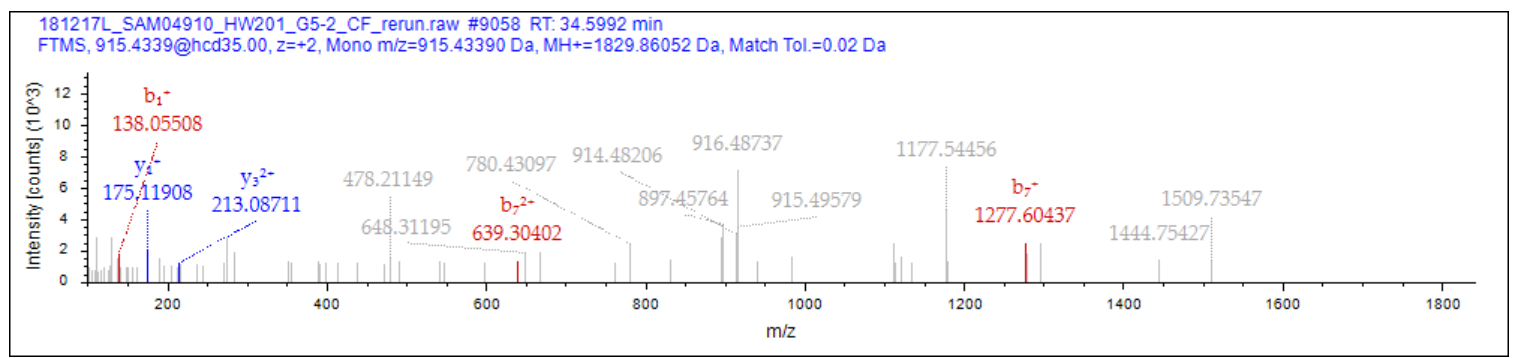

Sequence: HACSSIIKMCR

Charge: +2 ,

Monoisotopic m/z: $915.43390 \mathrm{Da}(-0.14 \mathrm{mmu} /-0.15 \mathrm{ppm})$,

$\mathrm{MH}+:$ 1829.86052 Da,

RT: 34.5992 min,

Identified with: Sequest HT (v1.17); XCorr:0.93,

Fragment match tolerance used for search: $0.02 \mathrm{Da}$

Fragments used for search: - $\mathrm{H}_{2} \mathrm{O} ; \mathrm{y} ;-\mathrm{NH}_{3} ; \mathrm{y} ; \mathrm{b} ; \mathrm{b} ;-\mathrm{H}_{2} \mathrm{O} ; \mathrm{b} ;-\mathrm{NH}_{3} ; \mathrm{y}$

Proteins (1):

- Exportin-5 OS=Homo sapiens OX=9606 GN=XPO5 PE=1 SV=1

West, A. et al. Labeling preferences of diazirines with protein biomolecules, J. Am. Chem. S192 Soc. 2021. 


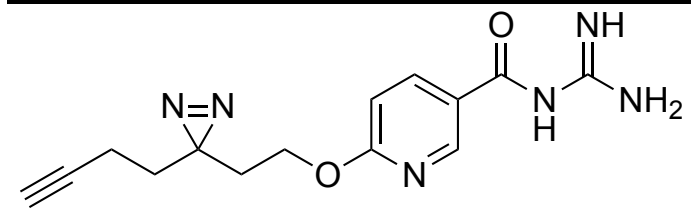

JN Mod: JN-942

PSMs: 21

Unique Sites: 11

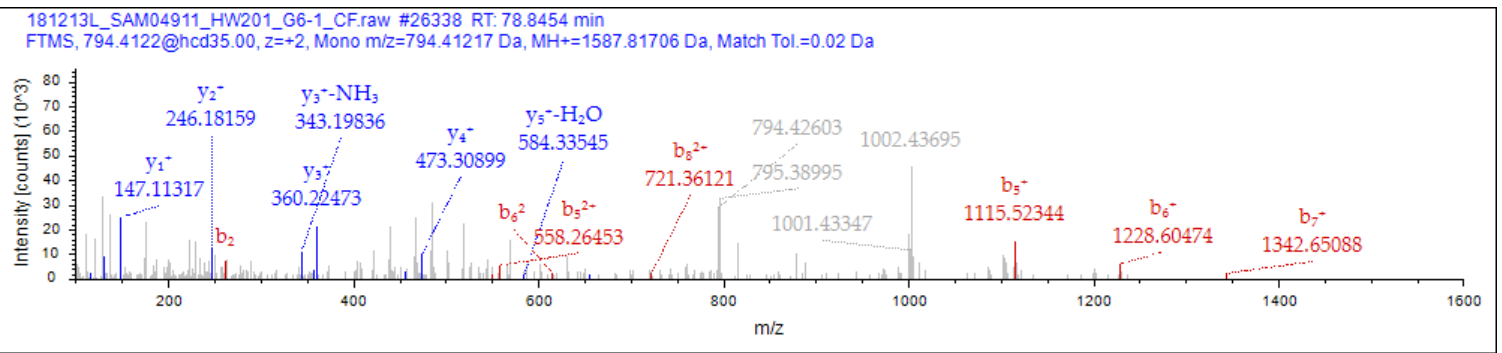

Sequence: FLTEEINVK

Charge: +2 ,

Monoisotopic m/z: 794.41217 Da (-0.89 mmu/-1.13 ppm),

$\mathrm{MH}+:$ 1587.81706 Da,

RT: 78.8454 min,

Identified with: Sequest HT (v1.17); XCorr:1.96,

Fragment match tolerance used for search: $0.02 \mathrm{Da}$

Fragments used for search: - $\mathrm{H}_{2} \mathrm{O} ; \mathrm{y} ;-\mathrm{NH}_{3} ; \mathrm{y} ; \mathrm{b} ; \mathrm{b} ;-\mathrm{H}_{2} \mathrm{O} ; \mathrm{b} ;-\mathrm{NH}_{3} ; \mathrm{y}$

Proteins (1):

- Protocadherin gamma-A7 OS=Homo sapiens OX=9606 GN=PCDHGA7 PE=2 SV=1

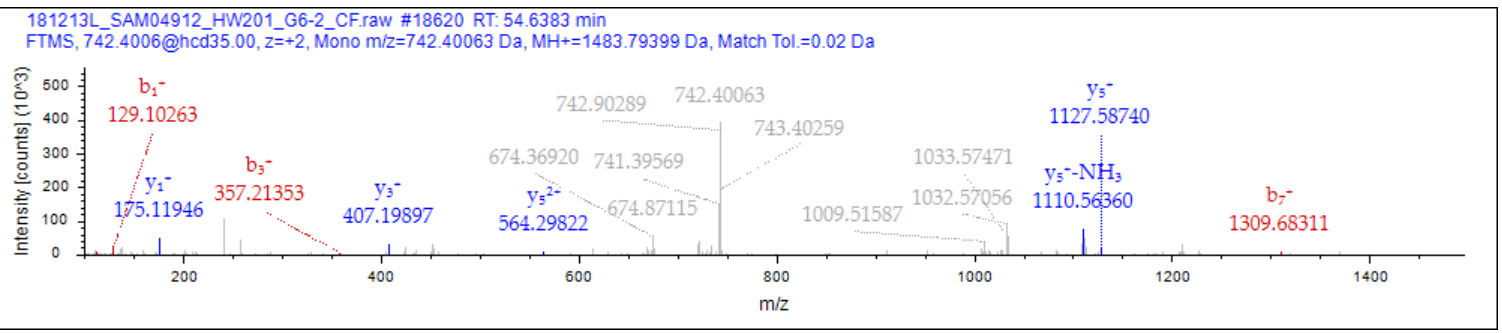

Sequence: KDLVKTMR

Charge: +2 ,

Monoisotopic m/z: $742.40063 \mathrm{Da}(-0.53 \mathrm{mmu} /-0.72 \mathrm{ppm})$,

$\mathrm{MH}+:$ 1483.79399 Da,

RT: $54.6383 \mathrm{~min}$,

Identified with: Sequest HT (v1.17); XCorr:1.34,

Fragment match tolerance used for search: $0.02 \mathrm{Da}$

Fragments used for search: - $\mathrm{H}_{2} \mathrm{O} ; \mathrm{y} ;-\mathrm{NH}_{3} ; \mathrm{y} ; \mathrm{b} ; \mathrm{b} ;-\mathrm{H}_{2} \mathrm{O} ; \mathrm{b} ;-\mathrm{NH}_{3} ; \mathrm{y}$

Proteins (1):

- Acetyl-CoA carboxylase 1 OS=Homo sapiens OX=9606 GN=ACACA PE=1 SV=2

West, A. et al. Labeling preferences of diazirines with protein biomolecules, J. Am. Chem. S193 Soc. 2021. 


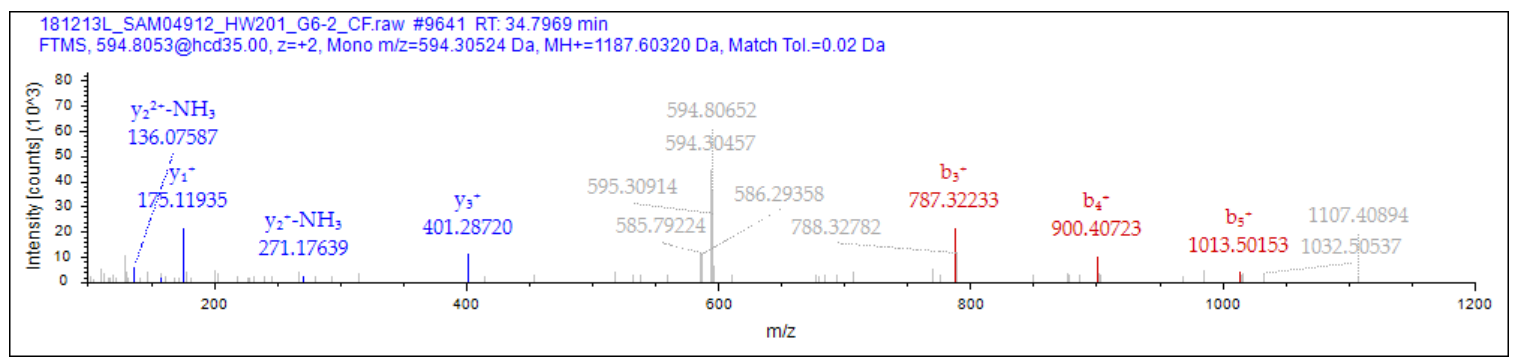

Sequence: GMSILR

Charge: +2 ,

Monoisotopic m/z: 594.30524 Da (+0.96 mmu/+1.62 ppm),

$\mathrm{MH}+:$ 1187.60320 Da,

RT: 34.7969 min,

Identified with: Sequest HT (v1.17); XCorr:1.40,

Fragment match tolerance used for search: $0.02 \mathrm{Da}$

Fragments used for search: $-\mathrm{H}_{2} \mathrm{O} ; \mathrm{y} ;-\mathrm{NH}_{3} ; \mathrm{y} ; \mathrm{b} ; \mathrm{b} ;-\mathrm{H}_{2} \mathrm{O}$; y

Proteins (1):

- Cerebellar degeneration-related protein 2-like OS=Homo sapiens OX=9606 GN=CDR2L $\mathrm{PE}=1 \mathrm{SV}=2$

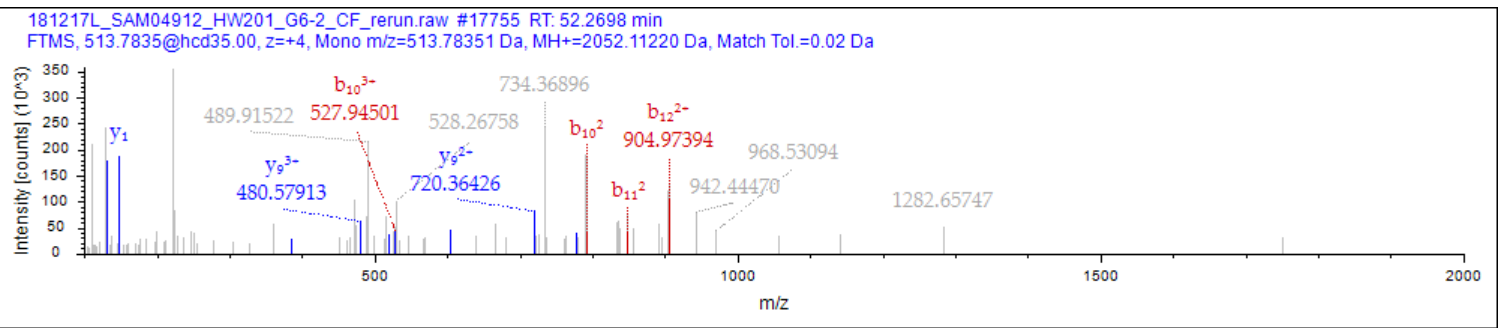

Sequence: MLRVIVESASNIPK

Charge: +4 ,

Monoisotopic m/z: $513.78351 \mathrm{Da}(+1.01 \mathrm{mmu} /+1.96 \mathrm{ppm})$,

$\mathrm{MH}+:$ 2052.11220 Da,

RT: $52.2698 \mathrm{~min}$,

Identified with: Sequest HT (v1.17); XCorr:1.60,

Fragment match tolerance used for search: $0.02 \mathrm{Da}$

Fragments used for search: - $\mathrm{H}_{2} \mathrm{O} ; \mathrm{y} ;-\mathrm{NH}_{3} ; \mathrm{y} ; \mathrm{b} ; \mathrm{b} ;-\mathrm{H}_{2} \mathrm{O} ; \mathrm{b} ;-\mathrm{NH}_{3} ; \mathrm{y}$

Proteins (1):

- Myoferlin OS=Homo sapiens OX=9606 GN=MYOF PE=1 SV=1

West, A. et al. Labeling preferences of diazirines with protein biomolecules, J. Am. Chem. S194 Soc. 2021. 
<smiles>C#CCCC1(CCOc2ccc(C(=N)N)cc2)N=N1</smiles>

JN Mod: JN-945

PSMs: 441

Unique Sites: 48

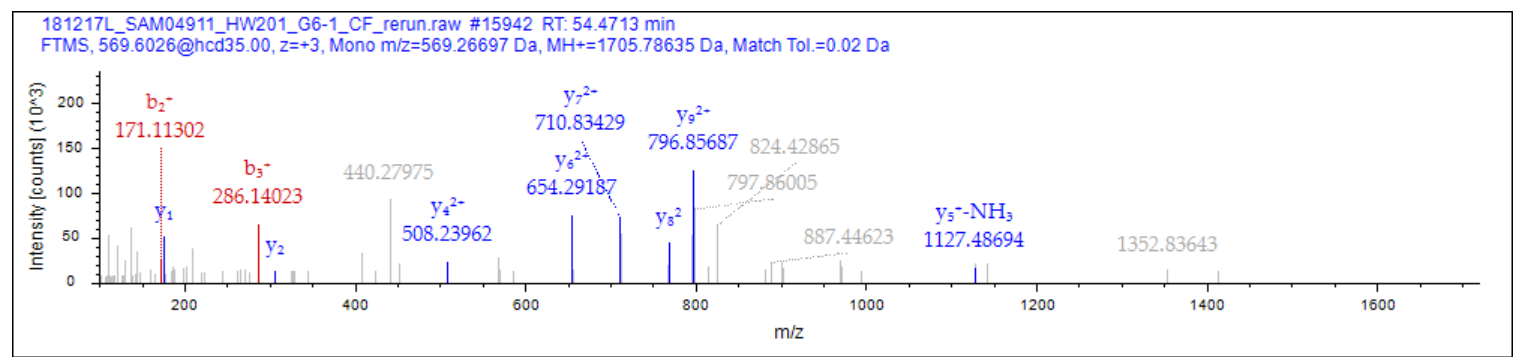

Sequence: LGDLYEEEMR

Charge: +3 ,

Monoisotopic m/z: $569.26697 \mathrm{Da}(-1.64 \mathrm{mmu} /-2.89 \mathrm{ppm})$,

$\mathrm{MH}+:$ 1705.78635 Da,

RT: $54.4713 \mathrm{~min}$,

Identified with: Sequest HT (v1.17); XCorr:2.31,

Fragment match tolerance used for search: $0.02 \mathrm{Da}$

Fragments used for search: $-\mathrm{H}_{2} \mathrm{O} ; \mathrm{y} ;-\mathrm{NH}_{3} ; \mathrm{y} ; \mathrm{b} ; \mathrm{b} ;-\mathrm{H}_{2} \mathrm{O} ; \mathrm{y}$

Proteins (1):

- Vimentin OS=Homo sapiens OX=9606 GN=VIM PE=1 SV=4

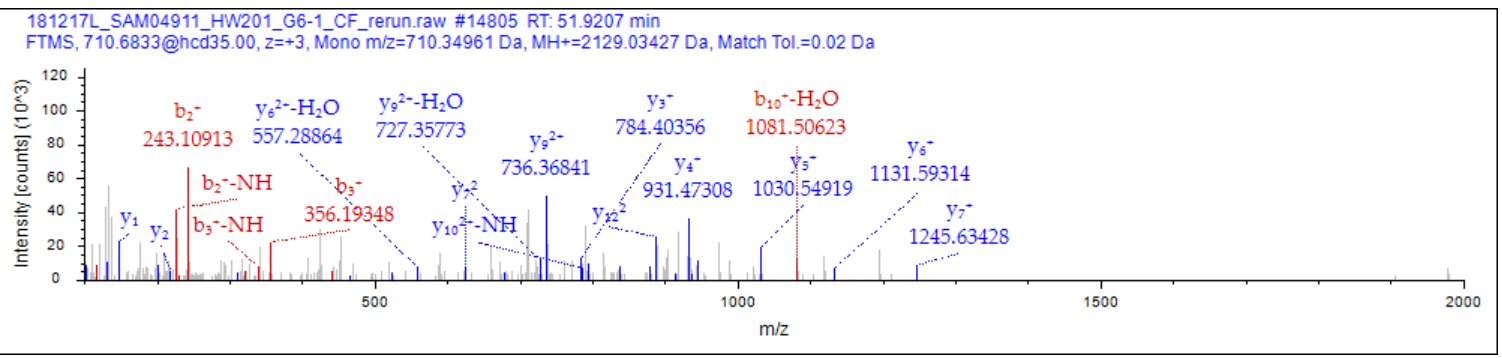

Sequence: NQLTSNPENTVFDAK

Charge: +3 ,

Monoisotopic m/z: $710.34943 \mathrm{Da}$ (+0.56 mm/+0.79 ppm),

$\mathrm{MH}+:$ 2129.03373 Da,

RT: 41.4659 min,

Identified with: Sequest HT (v1.17); XCorr:3.08,

Fragment match tolerance used for search: $0.02 \mathrm{Da}$

Fragments used for search: - $\mathrm{H}_{2} \mathrm{O} ; \mathrm{y} ;-\mathrm{NH}_{3} ; \mathrm{y} ; \mathrm{b} ; \mathrm{b} ;-\mathrm{H}_{2} \mathrm{O} ; \mathrm{b} ;-\mathrm{NH}_{3} ; \mathrm{y}$

Proteins (1):

- Endoplasmic reticulum chaperone BiP OS=Homo sapiens OX=9606 GN=HSPA5 PE=1 $\mathrm{SV}=2$

West, A. et al. Labeling preferences of diazirines with protein biomolecules, J. Am. Chem. S195 Soc. 2021. 


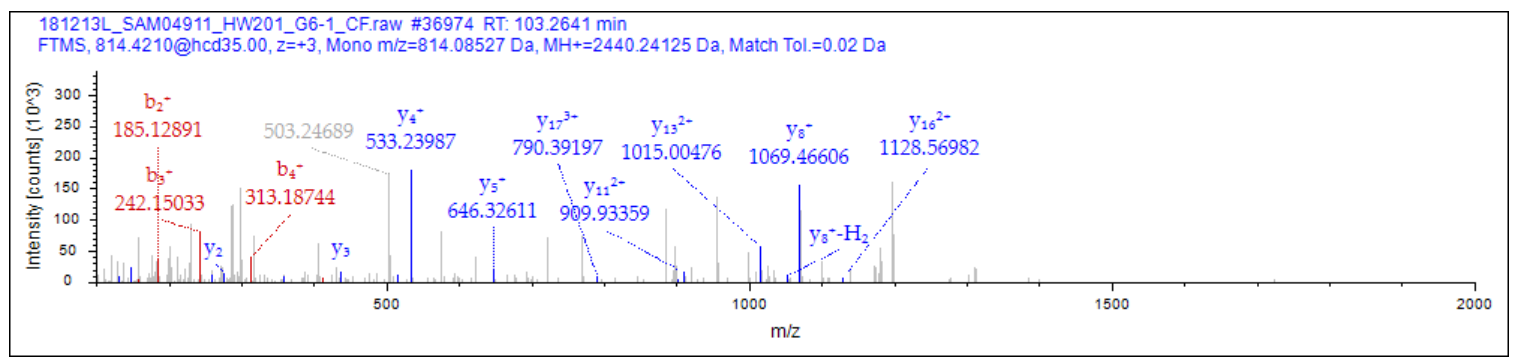

\section{Sequence: AIGAVPLIQGEYMIPCEK}

Charge: +3 ,

Monoisotopic m/z: $814.08527 \mathrm{Da}(-0.45 \mathrm{mmu} /-0.55 \mathrm{ppm})$,

$\mathrm{MH}+:$ 2440.24125 Da,

RT: $103.2641 \mathrm{~min}$,

Identified with: Sequest HT (v1.17); XCorr:1.87,

Fragment match tolerance used for search: $0.02 \mathrm{Da}$

Fragments used for search: $-\mathrm{H}_{2} \mathrm{O} ; \mathrm{y} ;-\mathrm{NH}_{3} ; \mathrm{y} ; \mathrm{b} ; \mathrm{b} ;-\mathrm{H}_{2} \mathrm{O} ; \mathrm{y}$

Proteins (1):

- Cathepsin D OS=Homo sapiens OX=9606 GN=CTSD PE=1 SV=1

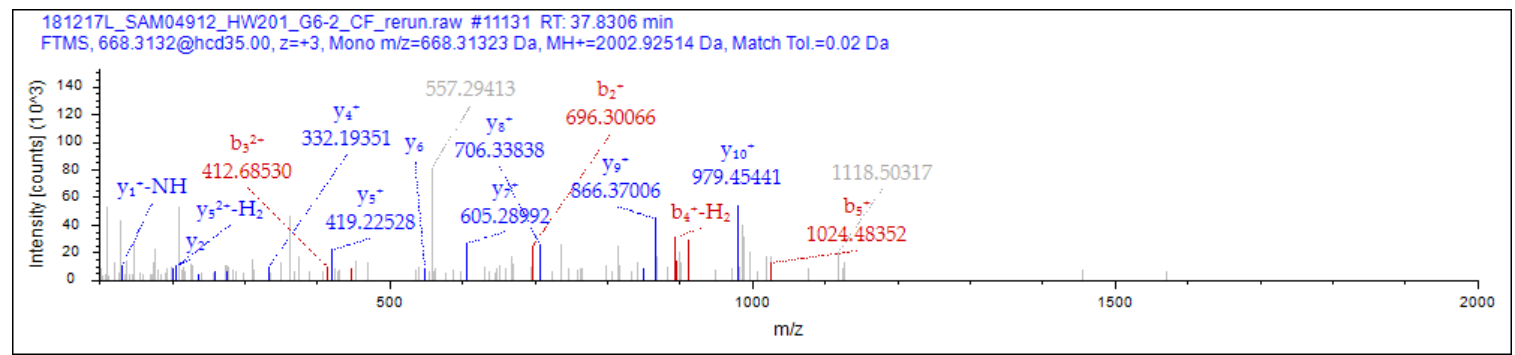

Sequence: EDQSILCTGESGAGK

Charge: +3 ,

Monoisotopic m/z: $668.31323 \mathrm{Da}(+1.81 \mathrm{mmu} /+2.7 \mathrm{ppm})$,

$\mathrm{MH}+:$ 2002.92514 Da,

$\mathrm{RT}: 37.8306 \mathrm{~min}$,

Identified with: Sequest HT (v1.17); XCorr:2.80,

Fragment match tolerance used for search: $0.02 \mathrm{Da}$

Fragments used for search: - $\mathrm{H}_{2} \mathrm{O} ; \mathrm{y} ;-\mathrm{NH}_{3} ; \mathrm{y} ; \mathrm{b} ; \mathrm{b} ;-\mathrm{H}_{2} \mathrm{O} ; \mathrm{b} ;-\mathrm{NH}_{3} ; \mathrm{y}$

Proteins (4):

- Myosin-10 OS=Homo sapiens OX=9606 GN=MYH10 PE=1 SV=3

- Myosin-11 OS=Homo sapiens OX=9606 GN=MYH11 PE=1 SV=3

- Myosin-9 OS=Homo sapiens OX=9606 GN=MYH9 PE=1 SV=4

- Myosin-14 OS=Homo sapiens OX=9606 GN=MYH14 PE=1 SV=2

West, A. et al. Labeling preferences of diazirines with protein biomolecules, J. Am. Chem. S196 Soc. 2021. 
<smiles>C#CCCC1(CCOc2ccc(NC(=N)N)cc2)N=N1</smiles>

JN Mod: JN-940

SI number: 37

PSMs: 333

Unique Sites: 45

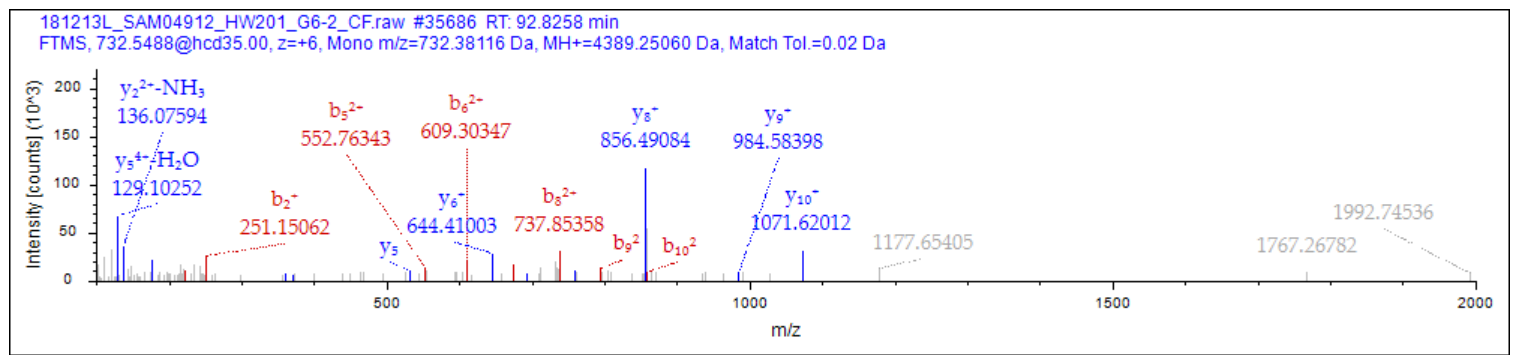

Sequence: LHEEEIQELQAQIQEQHVQIDVDVSKPDLTAALR

Charge: +6 ,

Monoisotopic m/z: $732.38116 \mathrm{Da}(+0.17 \mathrm{mmu} /+0.23 \mathrm{ppm})$,

$\mathrm{MH}+:$ 4389.25060 Da,

RT: $92.8258 \mathrm{~min}$,

Identified with: Sequest HT (v1.17); XCorr:2.91,

Fragment match tolerance used for search: $0.02 \mathrm{Da}$

Fragments used for search: - $\mathrm{H}_{2} \mathrm{O} ; \mathrm{y} ;-\mathrm{NH}_{3} ; \mathrm{y} ; \mathrm{b} ; \mathrm{b} ;-\mathrm{H}_{2} \mathrm{O} ; \mathrm{b} ;-\mathrm{NH}_{3} ; \mathrm{y}$

Proteins (1):

- Vimentin OS=Homo sapiens OX=9606 GN=VIM PE=1 SV=4

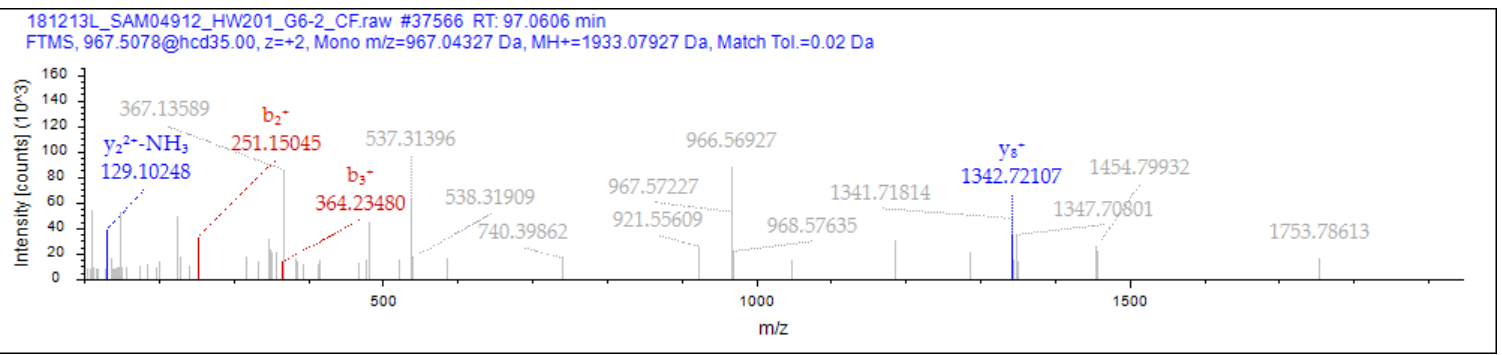

Sequence: HLIQVDFGVLAVR

Charge: +2 ,

Monoisotopic m/z: $967.04327 \mathrm{Da}(-1.82 \mathrm{mmu} /-1.88 \mathrm{ppm})$,

$\mathrm{MH}+:$ 1933.07927 Da,

RT: 97.0606 min,

Identified with: Sequest HT (v1.17); XCorr:0.83,

Fragment match tolerance used for search: $0.02 \mathrm{Da}$

Fragments used for search: - $\mathrm{H}_{2} \mathrm{O} ; \mathrm{y} ;-\mathrm{NH}_{3} ; \mathrm{y} ; \mathrm{b} ; \mathrm{b} ;-\mathrm{H}_{2} \mathrm{O} ; \mathrm{b} ;-\mathrm{NH}_{3} ; \mathrm{y}$

Proteins (1):

- E3 ubiquitin-protein ligase RNF213 OS=Homo sapiens OX=9606 GN=RNF213 PE=1 $\mathrm{SV}=3$

West, A. et al. Labeling preferences of diazirines with protein biomolecules, J. Am. Chem. S197 Soc. 2021. 


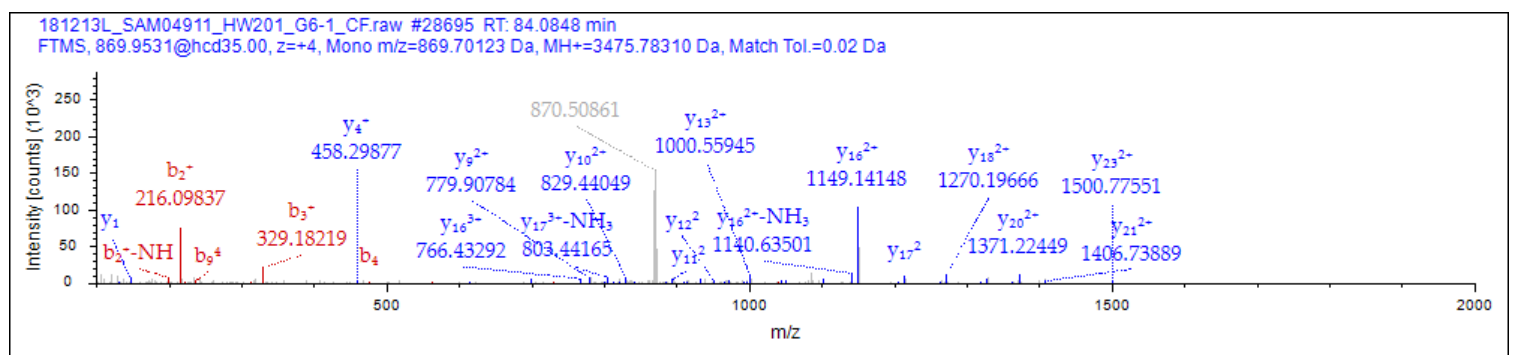

Sequence: SQIFSTASDNQPTVTIKVYEGERPLTK

Charge: +4 ,

Monoisotopic m/z: 869.70123 Da (-1.19 mmu/-1.37 ppm),

$\mathrm{MH}+: 3475.78310 \mathrm{Da}$,

RT: $84.0848 \mathrm{~min}$,

Identified with: Sequest HT (v1.17); XCorr:4.87,

Fragment match tolerance used for search: $0.02 \mathrm{Da}$

Fragments used for search: - $\mathrm{H}_{2} \mathrm{O} ; \mathrm{y} ;-\mathrm{NH}_{3} ; \mathrm{y} ; \mathrm{b} ; \mathrm{b} ;-\mathrm{H}_{2} \mathrm{O} ; \mathrm{b} ;-\mathrm{NH}_{3} ; \mathrm{y}$

Proteins (1):

- Endoplasmic reticulum chaperone BiP OS=Homo sapiens OX=9606 GN=HSPA5 PE=1 $\mathrm{SV}=2$

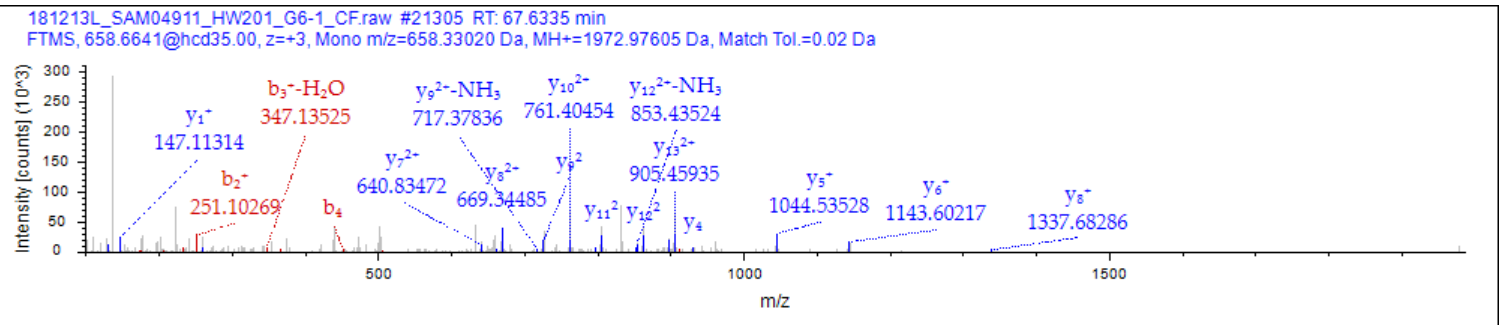

Sequence: YSNSALGHVNCTIK

Charge: +3 ,

Monoisotopic m/z: $658.33020 \mathrm{Da}(+1.34 \mathrm{mmu} /+2.03 \mathrm{ppm})$,

$\mathrm{MH}+:$ 1972.97605 Da,

RT: $67.6335 \mathrm{~min}$,

Identified with: Sequest HT (v1.17); XCorr:3.43,

Fragment match tolerance used for search: $0.02 \mathrm{Da}$

Fragments used for search: - $\mathrm{H}_{2} \mathrm{O} ; \mathrm{y} ;-\mathrm{NH}_{3} ; \mathrm{y} ; \mathrm{b} ; \mathrm{b} ;-\mathrm{H}_{2} \mathrm{O} ; \mathrm{b} ;-\mathrm{NH}_{3} ; \mathrm{y}$

Proteins (1):

- Reticulon-4 OS=Homo sapiens OX=9606 GN=RTN4 PE=1 SV=2

West, A. et al. Labeling preferences of diazirines with protein biomolecules, J. Am. Chem. S198 Soc. 2021. 
<smiles>C#CCCC1(CCN2Cc3ccccc3C(c3ccccc3)C2)N=N1</smiles>

JN Mod: JN-849

PSMs: 570

Unique Sites: 63

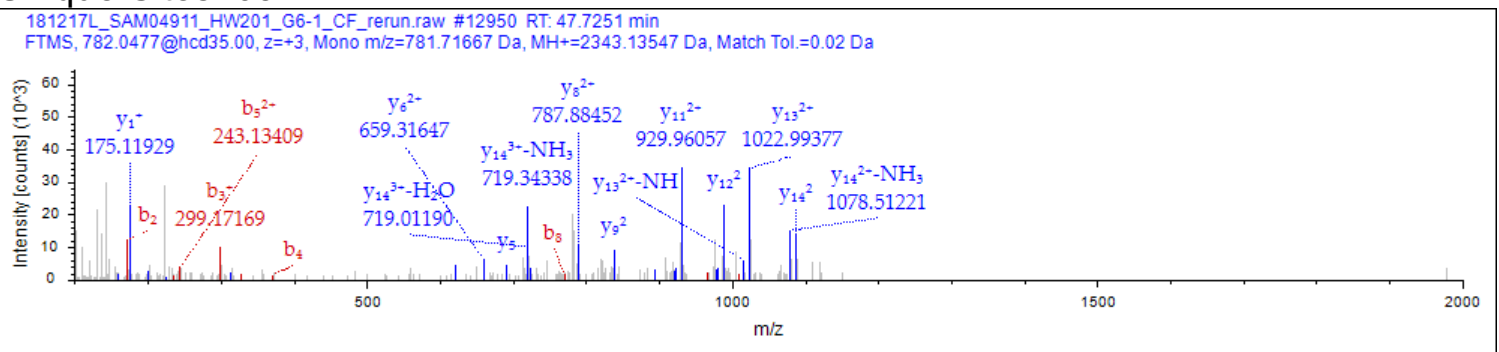

\section{Sequence: AVQADGQVKECYQSHR}

Charge: +3 ,

Monoisotopic m/z: 781.71667 Da (-0.23 mmu/-0.29 ppm),

$\mathrm{MH}+:$ 2343.13547 Da,

RT: $47.7251 \mathrm{~min}$,

Identified with: Sequest HT (v1.17); XCorr:3.07,

Fragment match tolerance used for search: $0.02 \mathrm{Da}$

Fragments used for search: - $\mathrm{H}_{2} \mathrm{O} ; \mathrm{y} ;-\mathrm{NH}_{3} ; \mathrm{y} ; \mathrm{b} ; \mathrm{b} ;-\mathrm{H}_{2} \mathrm{O} ; \mathrm{b} ;-\mathrm{NH}_{3} ; \mathrm{y}$

Proteins (1):

- Programmed cell death 6-interacting protein OS=Homo sapiens OX=9606 $\mathrm{GN}=\mathrm{PDCD} 6 \mathrm{IP} P E=1 \mathrm{SV}=1$

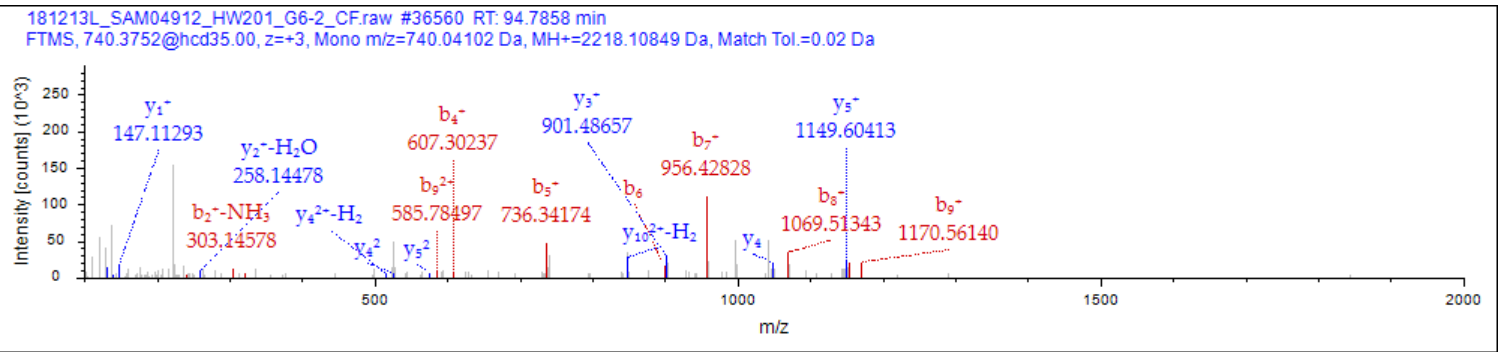

Sequence: YRWTEYGLTFTEK

Charge: +3 ,

Monoisotopic m/z: 740.04102 Da (+1.82 mm/+2.46 ppm),

$\mathrm{MH}+:$ 2218.10849 Da,

RT: $94.7858 \mathrm{~min}$,

Identified with: Sequest HT (v1.17); XCorr:2.93,

Fragment match tolerance used for search: $0.02 \mathrm{Da}$

Fragments used for search: - $\mathrm{H}_{2} \mathrm{O} ; \mathrm{y} ;-\mathrm{NH}_{3} ; \mathrm{y} ; \mathrm{b} ; \mathrm{b} ;-\mathrm{H}_{2} \mathrm{O} ; \mathrm{b} ;-\mathrm{NH}_{3} ; \mathrm{y}$

Proteins (1):

- Voltage-dependent anion-selective channel protein 1 OS=Homo sapiens $\mathrm{OX}=9606 \mathrm{GN}=\mathrm{VDAC} 1 \mathrm{PE}=1 \mathrm{SV}=2$

West, A. et al. Labeling preferences of diazirines with protein biomolecules, J. Am. Chem. S199 Soc. 2021. 


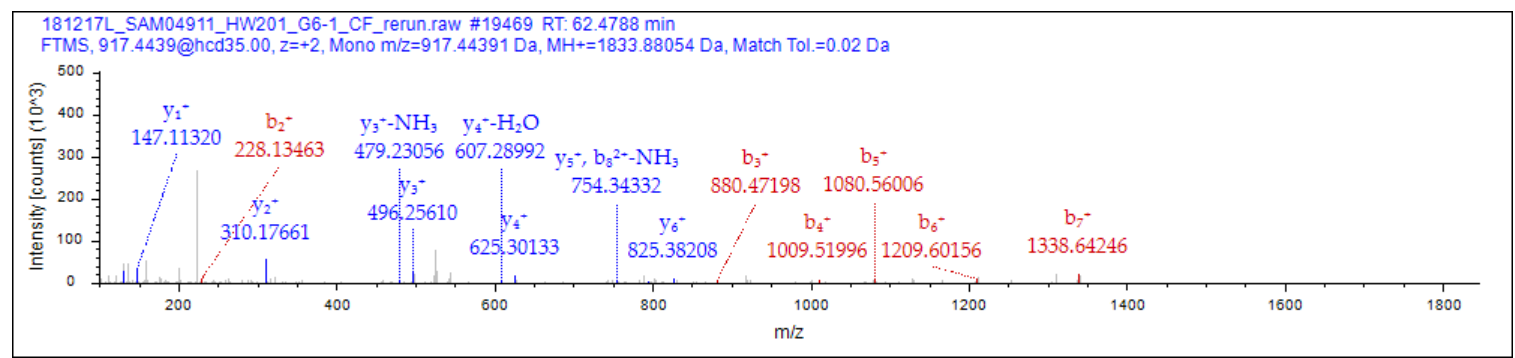

Sequence: NLQEAEEWYK

Charge: +2 ,

Monoisotopic m/z: $917.44391 \mathrm{Da}(-3.18 \mathrm{mmu} /-3.47 \mathrm{ppm})$,

$\mathrm{MH}+:$ 1833.88054 Da,

RT: $62.4788 \mathrm{~min}$,

Identified with: Sequest HT (v1.17); XCorr:2.77,

Fragment match tolerance used for search: $0.02 \mathrm{Da}$

Fragments used for search: - $\mathrm{H}_{2} \mathrm{O} ; \mathrm{y} ;-\mathrm{NH}_{3} ; \mathrm{y} ; \mathrm{b} ; \mathrm{b} ;-\mathrm{H}_{2} \mathrm{O} ; \mathrm{b} ;-\mathrm{NH}_{3} ; \mathrm{y}$

Proteins (2):

- Vimentin OS=Homo sapiens OX=9606 GN=VIM PE=1 SV=4

- Peripherin OS=Homo sapiens OX=9606 GN=PRPH PE=1 SV=2

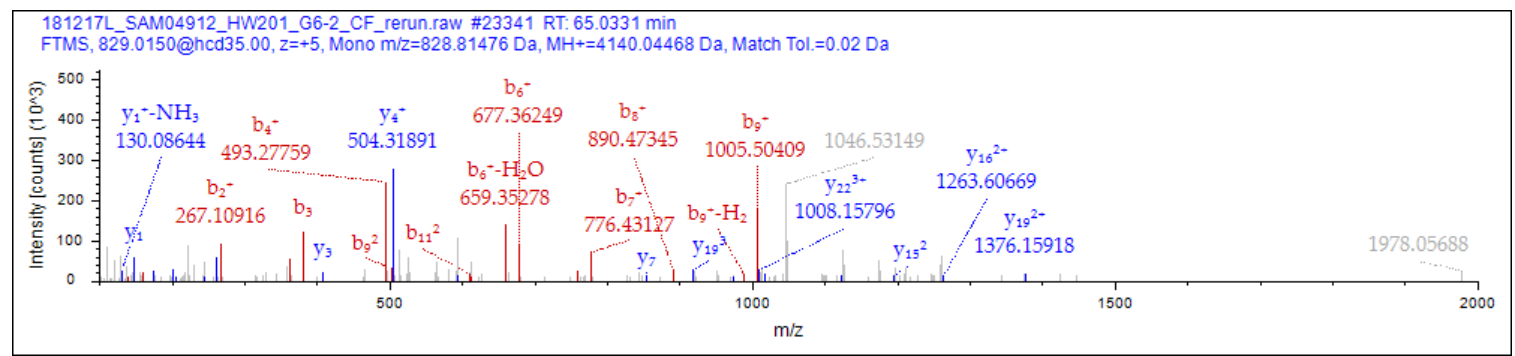

Sequence: HELLPSVNDITAVGPAHFYATNDHYFSDPFLK

Charge: +5 ,

Monoisotopic m/z: 828.81476 Da (+1.88 mmu/+2.26 ppm),

$\mathrm{MH}+: 4140.04468 \mathrm{Da}$,

RT: $65.0331 \mathrm{~min}$,

Identified with: Sequest HT (v1.17); XCorr:4.86,

Fragment match tolerance used for search: $0.02 \mathrm{Da}$

Fragments used for search: - $\mathrm{H}_{2} \mathrm{O} ; \mathrm{y} ;-\mathrm{NH}_{3} ; \mathrm{y} ; \mathrm{b} ; \mathrm{b} ;-\mathrm{H}_{2} \mathrm{O} ; \mathrm{b} ;-\mathrm{NH}_{3} ; \mathrm{y}$

Proteins (1):

- Serum paraoxonase/arylesterase 2 OS=Homo sapiens OX=9606 GN=PON2 PE=1 $\mathrm{SV}=4$

West, A. et al. Labeling preferences of diazirines with protein biomolecules, J. Am. Chem. S200 Soc. 2021. 
<smiles>C#CCCC1(CCN2CCC(C(O)(c3ccccc3)c3ccccc3)CC2)N=N1</smiles>

JN Mod: JN-938

PSMs: 244

Unique Sites: 45

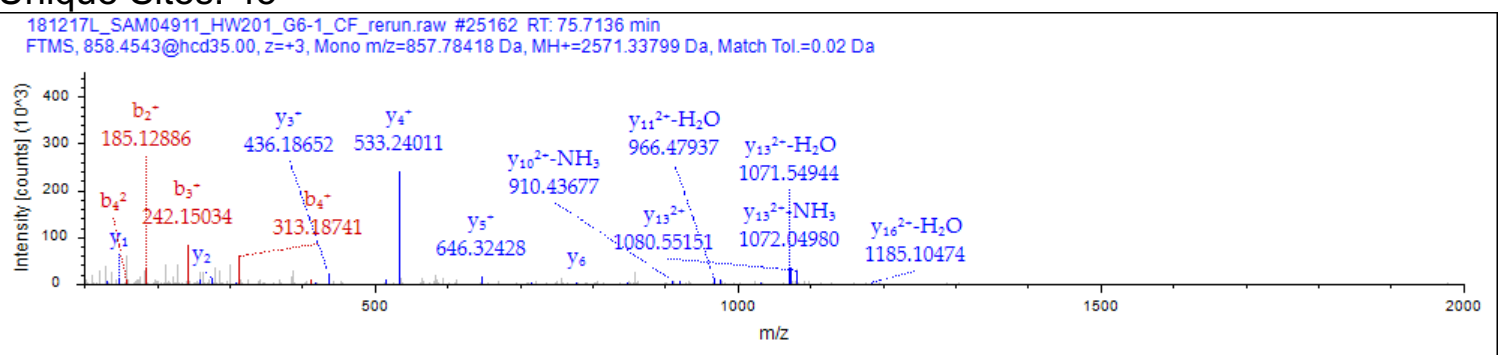

\section{Sequence: AIGAVPLIQGEYMIPCEK}

Charge: +3 ,

Monoisotopic m/z: 857.78418 Da (-1.1 mmu/-1.28 ppm),

$\mathrm{MH}+:$ 2571.33799 Da,

RT: 75.7136 min,

Identified with: Sequest HT (v1.17); XCorr:2.91,

Fragment match tolerance used for search: $0.02 \mathrm{Da}$

Fragments used for search: - $\mathrm{H}_{2} \mathrm{O} ; \mathrm{y} ;-\mathrm{NH}_{3} ; \mathrm{y} ; \mathrm{b} ; \mathrm{b} ;-\mathrm{H}_{2} \mathrm{O} ; \mathrm{b} ;-\mathrm{NH}_{3} ; \mathrm{y}$

Proteins (1):

- Cathepsin D OS=Homo sapiens OX=9606 GN=CTSD PE=1 SV=1

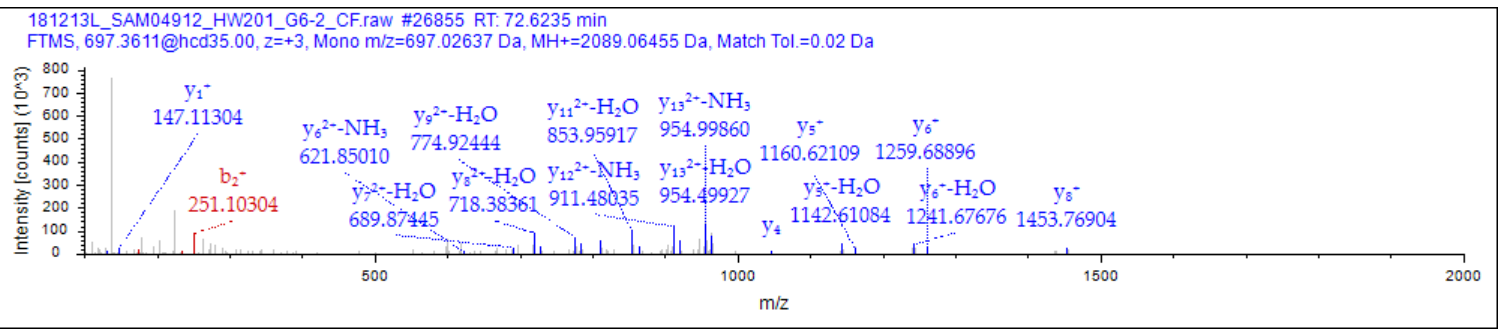

\section{Sequence: YSNSALGHVNCTIK}

Charge: +3 ,

Monoisotopic m/z: 697.02637 Da (+1.57 mmu/+2.25 ppm),

$\mathrm{MH}+: 2089.06455 \mathrm{Da}$,

RT: 72.6235 min,

Identified with: Sequest HT (v1.17); XCorr:2.60,

Fragment match tolerance used for search: $0.02 \mathrm{Da}$

Fragments used for search: $-\mathrm{H}_{2} \mathrm{O} ; \mathrm{y} ;-\mathrm{NH}_{3} ; \mathrm{y} ; \mathrm{b} ; \mathrm{b} ;-\mathrm{H}_{2} \mathrm{O} ; \mathrm{b} ;-\mathrm{NH}_{3} ; \mathrm{y}$

Proteins (1):

- Reticulon-4 OS=Homo sapiens OX=9606 GN=RTN4 PE=1 SV=2

West, A. et al. Labeling preferences of diazirines with protein biomolecules, J. Am. Chem. S201 Soc. 2021. 


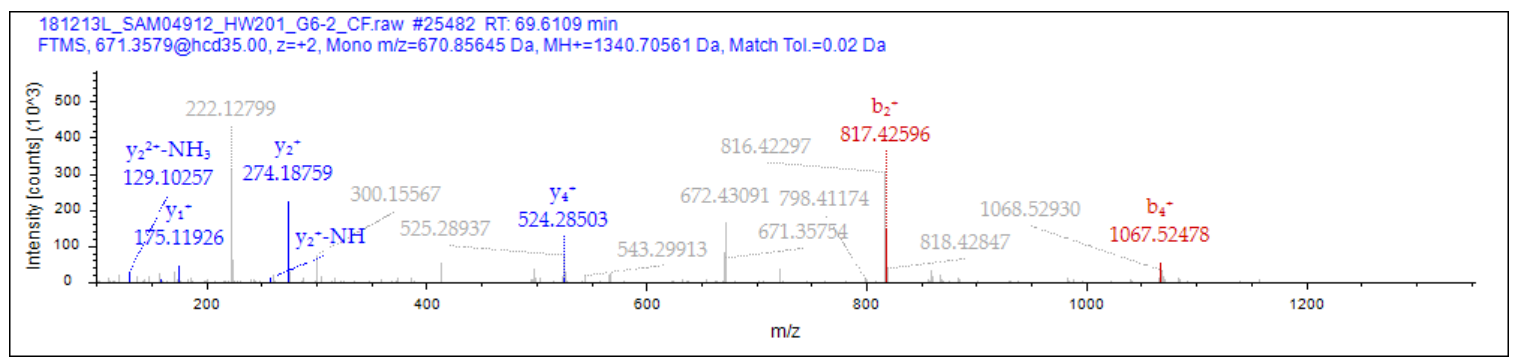

Sequence: YAYSVR

Charge: +2 ,

Monoisotopic m/z: $670.85645 \mathrm{Da}(-0.23 \mathrm{mmu} /-0.34 \mathrm{ppm})$,

$\mathrm{MH}+: 1340.70561 \mathrm{Da}$,

RT: 69.6109 min,

Identified with: Sequest HT (v1.17); XCorr:1.23,

Fragment match tolerance used for search: $0.02 \mathrm{Da}$

Fragments used for search: $-\mathrm{H}_{2} \mathrm{O} ; \mathrm{y} ;-\mathrm{NH}_{3} ; \mathrm{y} ; \mathrm{b} ; \mathrm{b} ;-\mathrm{H}_{2} \mathrm{O} ; \mathrm{y}$

Proteins (1):

- Probable ATP-dependent DNA helicase HFM1 OS=Homo sapiens OX=9606 $\mathrm{GN}=\mathrm{HFM} 1 \mathrm{PE}=1 \mathrm{SV}=2$

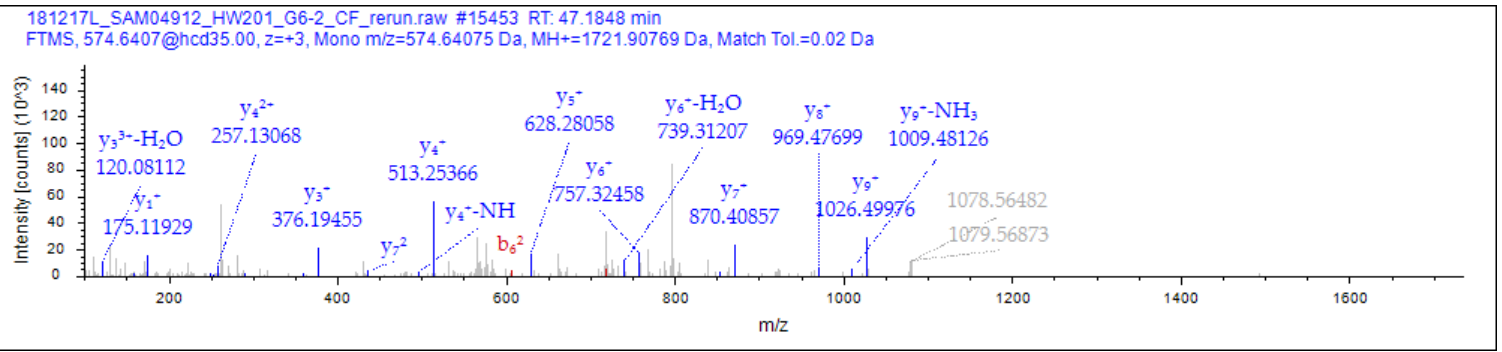

Sequence: LGVIEDHSNR

Charge: +3 ,

Monoisotopic m/z: 574.64075 Da (+1.48 mmu/+2.57 ppm),

$\mathrm{MH}+:$ 1721.90769 Da,

RT: 47.1848 min,

Identified with: Sequest HT (v1.17); XCorr:2.05,

Fragment match tolerance used for search: $0.02 \mathrm{Da}$

Fragments used for search: - $\mathrm{H}_{2} \mathrm{O} ; \mathrm{y} ;-\mathrm{NH}_{3} ; \mathrm{y} ; \mathrm{b} ; \mathrm{b} ;-\mathrm{H}_{2} \mathrm{O} ; \mathrm{b} ;-\mathrm{NH}_{3} ; \mathrm{y}$

Proteins (2):

- Endoplasmin OS=Homo sapiens OX=9606 GN=HSP90B1 PE=1 SV=1

- Putative endoplasmin-like protein OS=Homo sapiens OX=9606 GN=HSP90B2P PE=5 $\mathrm{SV}=1$

West, A. et al. Labeling preferences of diazirines with protein biomolecules, J. Am. Chem. S202 Soc. 2021. 
<smiles>C#CCCC1(CCC(=O)N2CCC(C(O)(c3ccccc3)c3ccccc3)CC2)N=N1</smiles>

JN Mod: JN-939

PSMs: 208

Unique Sites: 43

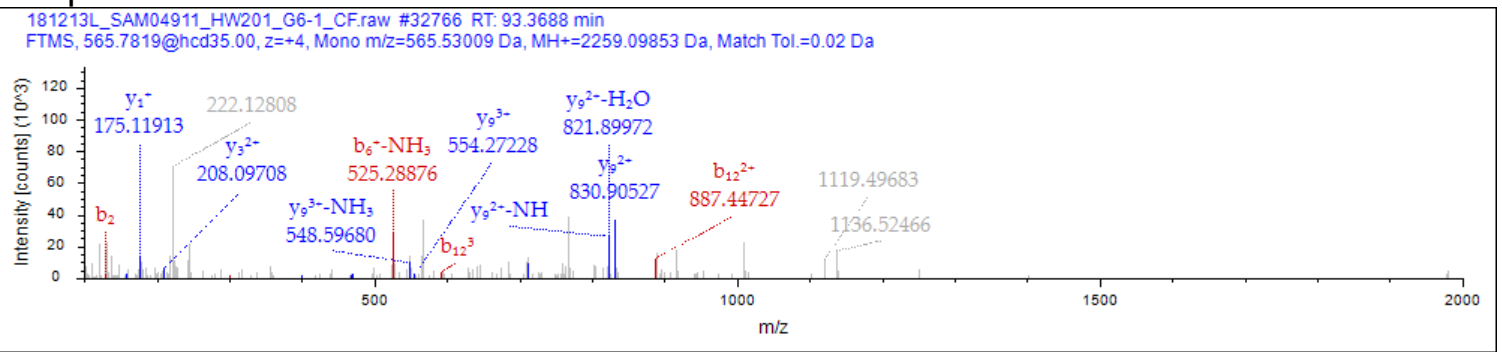

Sequence: AGRSAVGTKMTCAHCR

Charge: +4 ,

Monoisotopic m/z: $565.53009 \mathrm{Da}(-0.53 \mathrm{mmu} /-0.94 \mathrm{ppm})$,

$\mathrm{MH}+: 2259.09853 \mathrm{Da}, \mathrm{RT}: 93.3688 \mathrm{~min}$,

Identified with: Sequest HT (v1.17); XCorr:1.50,

Fragment match tolerance used for search: $0.02 \mathrm{Da}$

Fragments used for search: - $\mathrm{H}_{2} \mathrm{O} ; \mathrm{y} ;-\mathrm{NH}_{3} ; \mathrm{y} ; \mathrm{b} ; \mathrm{b} ;-\mathrm{H}_{2} \mathrm{O} ; \mathrm{b} ;-\mathrm{NH}_{3} ; \mathrm{y}$

Proteins (1):

- Zinc finger MYM-type protein 3 OS=Homo sapiens OX=9606 GN=ZMYM3 PE=1 SV=2

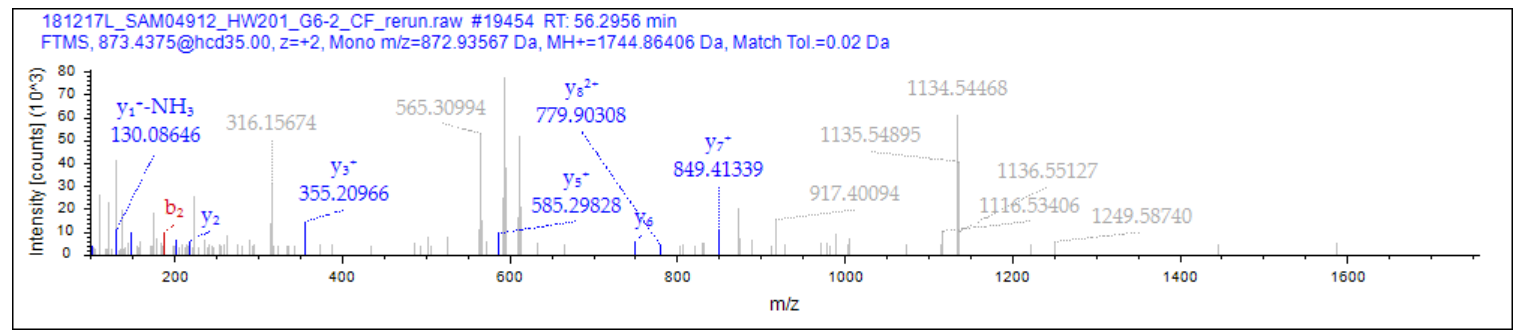

\section{Sequence: DAVTYTEHAK}

Charge: +2 ,

Monoisotopic m/z: 872.93567 Da (+1.84 mmu/+2.11 ppm),

$\mathrm{MH}+:$ 1744.86406 Da,

RT: 56.2956 min,

Identified with: Sequest HT (v1.17); XCorr:1.24,

Fragment match tolerance used for search: $0.02 \mathrm{Da}$

Fragments used for search: $-\mathrm{H}_{2} \mathrm{O} ; \mathrm{y} ;-\mathrm{NH}_{3} ; \mathrm{y} ; \mathrm{b} ; \mathrm{b} ;-\mathrm{H}_{2} \mathrm{O} ; \mathrm{y}$

Proteins (1):

- Histone H4 OS=Homo sapiens OX=9606 GN=HIST1H4A PE=1 SV=2

West, A. et al. Labeling preferences of diazirines with protein biomolecules, J. Am. Chem. S203 Soc. 2021. 


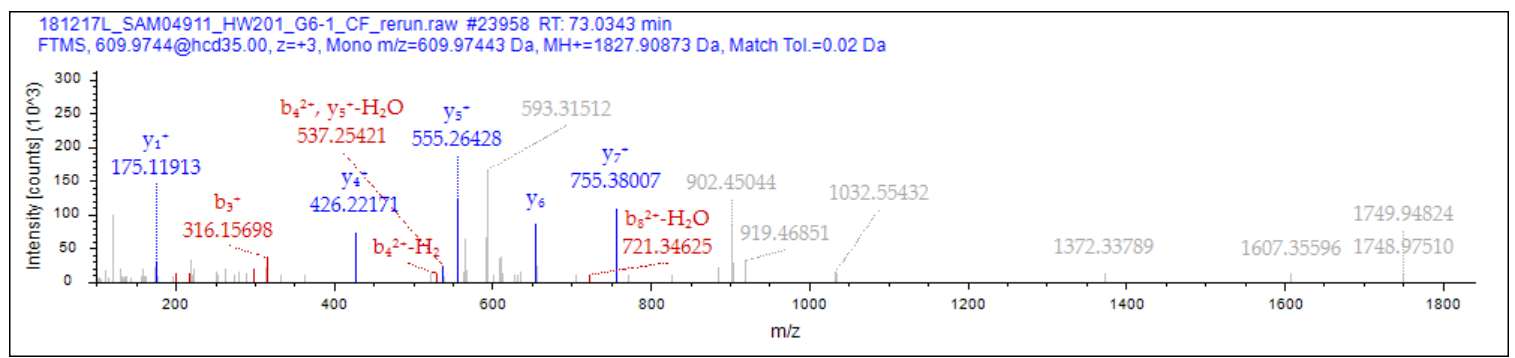

Sequence: ESVFTVEGGHR

Charge: +3 ,

Monoisotopic m/z: $609.97443 \mathrm{Da}$ (0 mmu/0 ppm),

$\mathrm{MH}+:$ 1827.90873 Da,

RT: $73.0343 \mathrm{~min}$,

Identified with: Sequest HT (v1.17); XCorr:1.84,

Fragment match tolerance used for search: $0.02 \mathrm{Da}$

Fragments used for search: $-\mathrm{H}_{2} \mathrm{O} ; \mathrm{y} ;-\mathrm{NH}_{3} ; \mathrm{y} ; \mathrm{b} ; \mathrm{b} ;-\mathrm{H}_{2} \mathrm{O} ; \mathrm{y}$

Proteins (1):

- Prohibitin-2 OS=Homo sapiens OX=9606 GN=PHB2 PE=1 SV=2

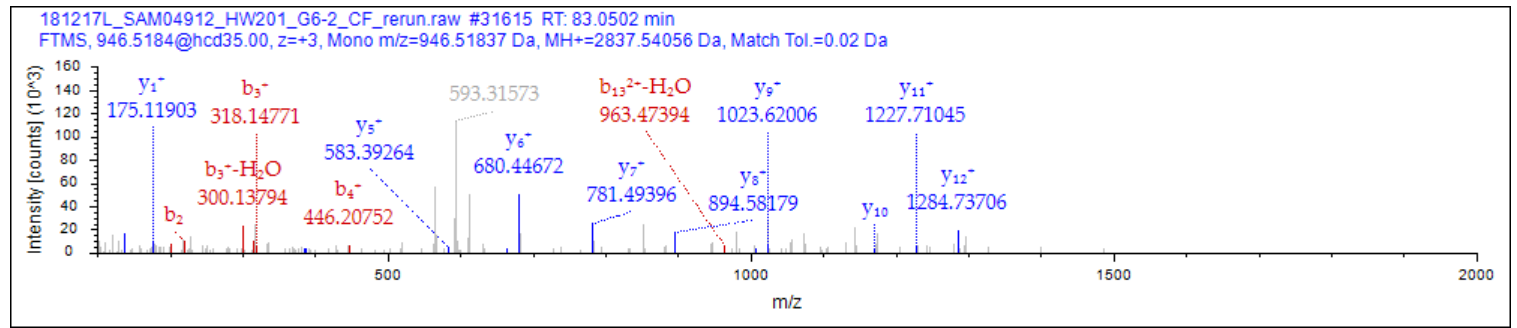

Sequence: MSVQPTVSLGGFEITPPVVLR

Charge: +3 ,

Monoisotopic m/z: $946.51837 \mathrm{Da}(+2.42 \mathrm{mmu} /+2.56 \mathrm{ppm})$,

$\mathrm{MH}+: 2837.54056 \mathrm{Da}$,

RT: $83.0502 \mathrm{~min}$,

Identified with: Sequest HT (v1.17); XCorr:2.53,

Fragment match tolerance used for search: $0.02 \mathrm{Da}$

Fragments used for search: - $\mathrm{H}_{2} \mathrm{O} ; \mathrm{y} ;-\mathrm{NH}_{3} ; \mathrm{y} ; \mathrm{b} ; \mathrm{b} ;-\mathrm{H}_{2} \mathrm{O} ; \mathrm{b} ;-\mathrm{NH}_{3} ; \mathrm{y}$

Proteins (1):

- Nucleophosmin OS=Homo sapiens OX=9606 GN=NPM1 PE=1 SV=2

West, A. et al. Labeling preferences of diazirines with protein biomolecules, J. Am. Chem. S204 Soc. 2021. 


\section{References:}

1. Miyamoto, D. K.; Flaxman, H. A.; Wu, H.-Y.; Gao, J.; Woo, C. M., Discovery of a Celecoxib Binding Site on Prostaglandin E Synthase (PTGES) with a Cleavable ChelationAssisted Biotin Probe. ACS Chem. Biol. 2019, 14 (12), 2527-2532.

2. Parker, C. G.; Galmozzi, A.; Wang, Y.; Correia, B. E.; Sasaki, K.; Joslyn, C. M.; Kim, A. S.; Cavallaro, C. L.; Lawrence, R. M.; Johnson, S. R., et al., Ligand and Target Discovery by Fragment-Based Screening in Human Cells. Cell 2017, 168 (3), 527541.e29.

3. Mitra, W. C. S. M. K. A., Rapid Chromatographic Technique for Preparative Separations with Moderate Resolution. J. Org. Chem 1978, 43 (14).

4. $\quad$ Pangborn, A. B. G., M. A.; Grubbs, R. H.; Rosen, R. K.; Timmers, F. J., Safe and Convenient Procedure for Solvent Purification. Organometallics 1996, 15 (5), 1518-1520. 5. Li, Z.; Hao, P.; Li, L.; Tan, C. Y.; Cheng, X.; Chen, G. Y.; Sze, S. K.; Shen, H. M.; Yao, S. Q., Design and synthesis of minimalist terminal alkyne-containing diazirine photo-crosslinkers and their incorporation into kinase inhibitors for cell- and tissue-based proteome profiling. Angew. Chem. Int. Ed. Engl. 2013, 52 (33), 8551-6.

6. Woo, C. M.; Bertozzi, C. R., Isotope Targeted Glycoproteomics (IsoTaG) to Characterize Intact, Metabolically Labeled Glycopeptides from Complex Proteomes. Curr. Protoc. Chem. Biol. 2016, 8 (1), 59-82.

7. Nassal, M., 4-(1-Azi-2,2,2-trifluoroethyl)benzoic Acid, a Highly Photolabile Carbene Generating Label Readily Fixable to Biochemical Agents. Liebigs Ann. Chem. 1983, 1510-1523.

8. Fustero, S.; Fernández, B.; Bello, P.; del Pozo, C.; Arimitsu, S.; Hammond, G. B., Intramolecular Hydroamination of Difluoropropargyl Amides: Regioselective Synthesis of Fluorinated $\beta$ - and $\gamma$-Lactams. Org. Lett. 2007, 9 (21), 4251-4253. 